University of Louisville

ThinkIR: The University of Louisville's Institutional Repository

Electronic Theses and Dissertations

$5-2020$

\title{
Functional nanomaterials by combining aminooxy chemistry and iodine activation at momolayer protected clusters.
}

Tirtha Raj Sibakoti

University of Louisville

Follow this and additional works at: https://ir.library.louisville.edu/etd

Part of the Analytical Chemistry Commons, and the Organic Chemistry Commons

\section{Recommended Citation}

Sibakoti, Tirtha Raj, "Functional nanomaterials by combining aminooxy chemistry and iodine activation at momolayer protected clusters." (2020). Electronic Theses and Dissertations. Paper 3366.

https://doi.org/10.18297/etd/3366

This Doctoral Dissertation is brought to you for free and open access by ThinkIR: The University of Louisville's Institutional Repository. It has been accepted for inclusion in Electronic Theses and Dissertations by an authorized administrator of ThinkIR: The University of Louisville's Institutional Repository. This title appears here courtesy of the author, who has retained all other copyrights. For more information, please contact thinkir@louisville.edu. 


\title{
FUNCTIONAL NANOMATERIALS BY COMBINING AMINOOXY CHEMISTRY AND IODINE ACTIVATION AT MONOLAYER PROTECTED CLUSTERS
}

\author{
By \\ Tirtha Raj Sibakoti \\ B.S. (Chemistry, Biology): Truman State University, 2012 \\ M.S (Chemistry): Northern Illinois University, 2015
}

\author{
A Dissertation \\ Submitted to the Faculty of the \\ College of Arts and Sciences of the University of Louisville \\ in Partial Fulfilment of the Requirements \\ for the Degree of \\ Doctor of Philosophy \\ in Chemistry \\ Department of Chemistry \\ University of Louisville \\ Louisville, KY
}

May 2020 



\title{
FUNCTIONAL NANOMATERIALS BY COMBINING AMINOOXY CHEMISTRY AND IODINE ACTIVATION AT MONOLAYER PROTECTED CLUSTERS
}

\author{
By \\ Tirtha Raj Sibakoti \\ B.S. (Chemistry, Biology): Truman State University, 2012 \\ M.S (Chemistry): Northern Illinois University, 2015
}

A Dissertation Approved on

May 08, 2020

by the following Dissertation Committee:

Dissertation Director: Dr. Michael H. Nantz

Dissertation Director: Dr. Francis P. Zamborini

Dr. Xiang Zhang

Dr. Farshid Ramezanipour

Dr. Gamini Sumanasekera 


\section{DEDICATION}

This dissertation is dedicated to my lovely and wonderful parents:

my father Krishna Prasad Sibakoti, my mother Rama Kumari Sibakoti,

my beautiful wife and cherubic son:

my wife Urusha Kumari Lamsal

my son Tejash Sibakoti

my amazing elder brother:

Mr. Chandra Prakash (CP) Sibakoti

and all my supportive family members 


\section{ACKNOWLEDGEMENTS}

I would like to dedicate this thesis to my parents (Krishna Prasad Sibakoti and Rama Kumari Sibakoti) for their treasurable love and overwhelming support. I am very thankful to my research advisors, Dr. Michael Nantz and Dr. Francis Zamborini, for accepting me as their combined student and providing me an opportunity to be involved in numerous interesting research projects in the past 4 years. I am always thankful to both of my advisors for encouraging and inspiring me throughout my time here. Dr. Nantz and Dr. Zamborini are the best examples of research mentors because of their excellent mentoring qualities and abilities to lead their students in the right paths. I have been extremely fortunate to have had chance to work with them, which is interdisciplinary field and an excellent collaborative works. My initial projects would not have been possible without the help of Dr. Raju Mandapati and Dr. Sadakatali Gori. I am forever indebted to their help and for teaching me different techniques for my project. I would like to thank my committee members Dr. Xiang Zhang, Dr. Farshid Ramezanipour and Dr. Gamini Sumanasekera for their precious time on being in my committee and providing me valuable suggestions. I would like to thank Dr. Zamborini, Director of Graduate Studies, for always being there when I needed help. I would also like to thank all my lab members from both Dr. Zamborini's and Dr. Nantz's research group. They all have been supportive and fun to work in a team. I am also very thankful to Dr. Neal Stolowich for helping me with the NMR operation, Mr. Ben Hutcherson for helping with 
the analytical instruments, Mrs. Renu Kakkar for helping with the chemicals ordering and Mr. Steve Riley for always being available to help when something comes up. I would also like to thank Dr. Marta Cecilia Yappert for being in my committee and providing me valuable suggestions with my literature seminar and original research proposal defense. I am also very thankful to my friend Anthony Ewurum for always being available to help me operate the MALDI-TOF MS instrument. I am very thankful to all the professors (Dr. Zamborini, Dr. Liu, Dr. Baldwin, Dr. Zhang, Dr. Luzzio), with whom I had an opportunity to take a course work with. I am also thankful to all the professor with whom I had pleasure to work as a teaching assistant, Dr. Baldwin, Dr. Zhang, Dr. Zamborini, Dr. Powe, Dr. Richter, Dr. Rich, and Dr. Wittebort. I learnt a lot of teaching skills from them. I would also like to thank Dr. Xiao-An Fu for allowing me to collaborate with his group in sensing project and for providing me feedbacks.

I would like to thank my teachers from higher secondary and secondary schools, Dr. Swagat Shrestha, Dr. Birendra Babu Adhikari, Gokul Silwal, R. K. Panta, Rajendra Sedhain, Biswo Dallakoti, Dipak Dhakal, Sabitri Neupane, etc. for always loving and inspiring me. I would like to thank my undergraduate research mentor Dr. David McCurdy and my graduate mentor Dr. Amartya Chakrabarti for their enormous support and faith in me. I would like to specially thank my grandparents Devi Prasad Sibakoti (late), and Januka Sibakoti for caring me. I would also like to thank my uncle Ganesh Sibakoti, Udhab Sibakoti, Kedar Sibakoti, Chet Raj (Dipak) Regmi, and Dipak Raj Oli for their enormous support. My deepest gratitude goes to my elder brother CP (Chandra Prakash) who has always supported me in every step of my life not only as a brother but also as a best friend. I am forever thankful to my mother's elder sister (Devi Sibakoti) 
who is also my mother figure as she was integral to raise me up and have always supported me. I would also like to thank my younger brothers (Dolraj, Ramit), sisters (Ambika, Sabitri, Astha and Asmi), my brother in laws (Udhab Prasad Upreti, Yadav Raj Pathak), my niece (Ankita), my nephews (Ankit and Asnav), my sister in laws (Sabina and Ayushma) and all friends for always supporting me when needed. Most importantly, I would like to thank my extremely wonderful wife Urusha Lamsal for her enormous support and love towards me. I would not be here without her unconditional love and patience towards me. The most beautiful thing that have happened throughout my $\mathrm{PhD}$ career was a birth of my son (Tejash Sibakoti) and I am incredibly lucky to have him. He has been a cheerful presence and fun bud to be around with his cutest smile and adorable activities. Tejash's support and love towards me is undefinable and unmeasurable. All these wonderful people have made this journey worthwhile and cheerful for me. 


\title{
ABSTRACT \\ FUNCTIONAL NANOMATERIALS BY COMBINING AMINOOXY CHEMISTRY AND IODINE ACTIVATION AT MONOLAYER PROTECTED CLUSTERS
}

\author{
Tirtha Raj Sibakoti
}

May 08, 2020

Aminooxy $\left(-\mathrm{ONH}_{2}\right)$ groups are most commonly known for their chemoselective reaction with carbonyl compounds (aldehydes/ketones) under mild reaction conditions. Aminooxy-based click chemistry is a versatile means of ligation as evidenced by broad application in material science, biology, biochemistry, analytical chemistry, and nanoscience. Our work exploits the facile reaction of aminooxy groups presented on the surface of gold $(\mathrm{Au})$ or palladium $(\mathrm{Pd})$ monolayer protected clusters (MPCs) with various aldehydes via oximation reactions, which form the robust oxime ether adducts. The functionalization of hexanethiolate-stabilized $\mathrm{Au}$ MPCs with a newly developed trifunctional amine-containing aminooxy alkanethiol ligand by thiol place-exchange affords aminooxylated mixed monolayer protected clusters (MMPCs). The Au MMPCsbound aminooxy groups react readily with various aldehydes in aqueous or non-polar organic solvents through oximation chemistry to form diversely functionalized $\mathrm{Au}$ 
MMPCs containing redox, aromatic, and fluorescent groups as well as chemical receptors that bind acetone. In addition, ligand and $\mathrm{pH}$ modification allows tuning of the solubility properties of the aminooxy functionalized Au MMPCs. Moreover, aminooxyfunctionalized $\mathrm{Au}$ and Pd MMPCs readily bind to commercially available aldehydefunctionalized polystyrene and agarose gel spherical microbeads, which serves as a unique method for the preparation of solid-supported metal clusters. The goal is to use these materials as catalysts with both high turnover frequency (TOF) and long-term recyclability compared to unsupported homogeneous catalysts of the same clusters. Studies of Au and Pd MPCs as homogeneous catalysts reveal that although the MPCs are quite stable, the thiolate capping agent strongly inhibits the catalyst activity. Partial thiolate ligand removal by straightforward in-situ addition of iodine significantly enhances the catalytic activity while maintaining good stability under optimized conditions. An increased rate constant for $\mathrm{NaBH}_{4}$ reduction of 4-nitrophenol to 4aminophenol demonstrates the effect of partial ligand removal on the catalytic activity of glutathione (SG)-capped Au MPCs serving as the catalyst. Similarly, an increased TOF for the hydrogenation/isomerization of allyl alcohol shows the benefit of iodine-activated partial ligand removal on the activity of SG-capped Pd MPCs. In both reactions, as the amount of $I_{2}$ increases, the catalytic activity increases (due to partial ligand dissociation) while the catalyst recyclability decreases (due to MPC aggregation). Low equivalents of $\mathrm{I}_{2}$ relative to SG ligands on the MPCs are optimal when considering both reaction rate and catalyst recyclability. The results on SG Au and SG Pd MPCs as homogeneous catalysts combined with successful loading of aldehyde-functionalized microbeads with aminooxy-functionalized Au and Pd MPCs demonstrates the feasibility of preparing 
unique MPC-loaded porous microbeads activated with iodine for use as stable, efficient heterogeneous catalysts. The combination of aminooxylation chemistry on metal MPCs and iodine activation provides a general approach to new catalyst design. The strategy also shows promise for creating nanomaterials useful for surface-enhanced Raman and chemiresistive selective sensing of carbonyl-containing volatile organic compounds (VOCs) of medical and environmental importance. 
TABLE OF CONTENTS

PAGE

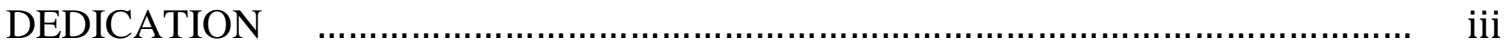

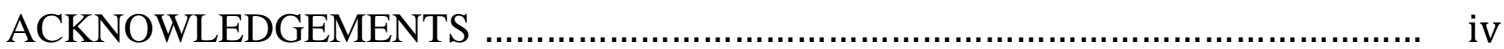

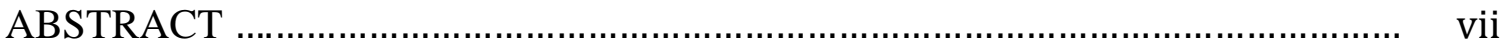

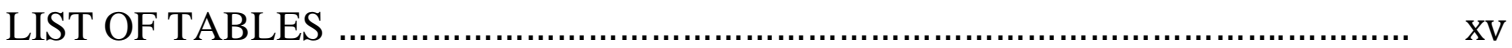

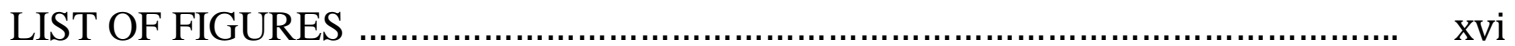

LIST OF SCHEMES …........................................................................

\section{CHAPTER 1 INTRODUCTION}

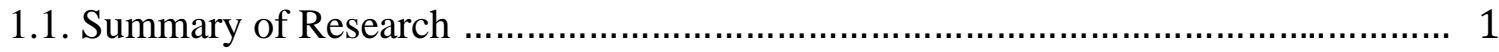

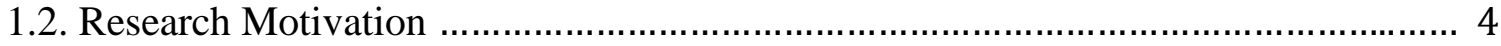

1.3. Introduction to Aminooxy Chemistry ................................................................ 5

1.4. Importance of Monolayer-Protected Clusters (MPCs) ...................................... 10

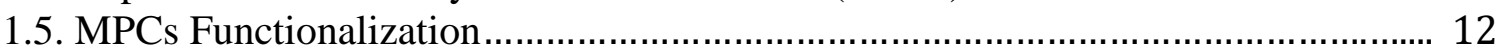

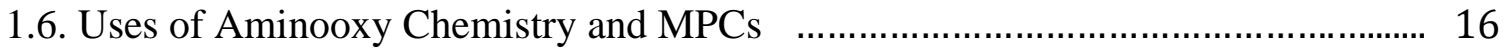

1.7. Catalytic Applications of MPCs ....................................................................... 21

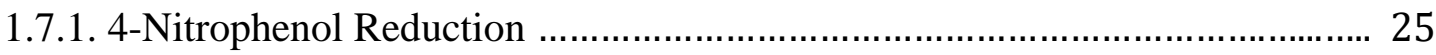

1.7.2. Hydrogenation/Isomerization of Allyl Alcohols ...................................... 29

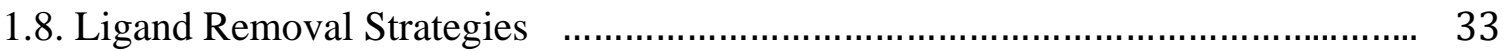

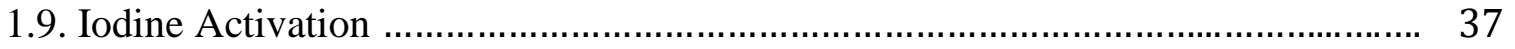

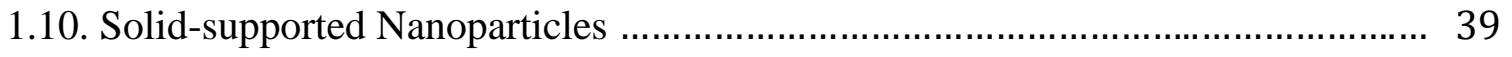

CHAPTER 2 SYNTHESIS OF AMINOOXY-THIOL LIGANDS AND MPCs

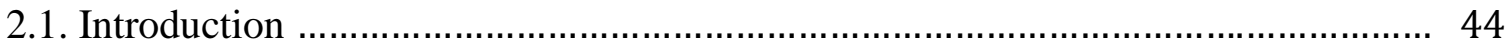

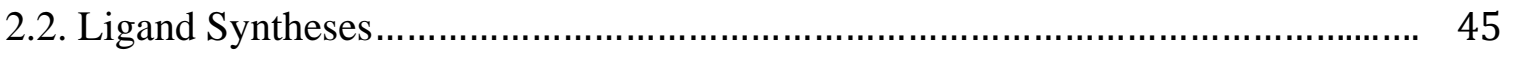

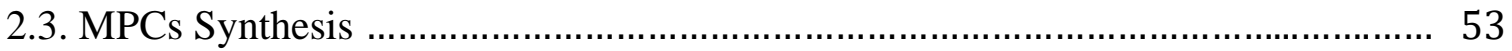




\section{CHAPTER 3 FUCNTIONALIZATION OF MPCs WITH AMINOOXY-}

\section{THIOL LIGANDS \& OXIMATION REACTIONS}

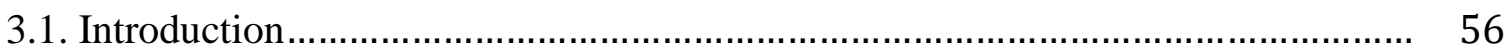

3.2. Ligand Place Exchange Reactions ……………...................................... 57

3.3. Oximation Reactions on $\mathrm{Au}_{144}\left(\mathrm{C}_{6} \mathrm{~S}\right)_{30}\left(\mathrm{AOT}_{\text {bis }}\right)_{30}$ MMPCs ................................. 64

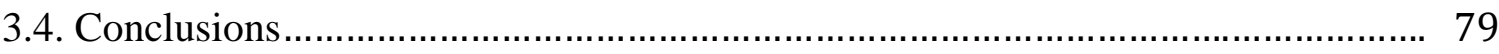

CHAPTER 4 ANCHORING MPCS ON A SOLID SUPPORT TO IMPROVE CATALYST SELECTIVITY AND RECYCLABILITY

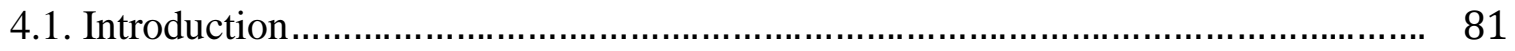

4.2. Attachment Strategy ............................................................................ 82

4.2.1. Au Cluster- Functionalized Polystyrene (PS) Beads ............................. 84

4.2.2. Pd Cluster- Functionalized Agarose Beads ........................................... 88

\section{CHAPTER 5 IODINE ACTIVATION OF METAL MPCs FOR CATALYTIC ENHANCEMENT}

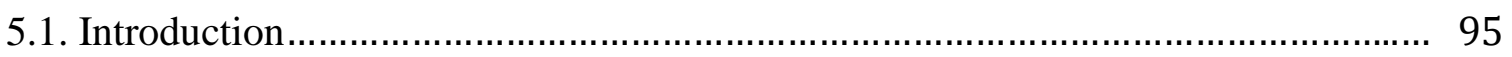

5.2. Results and Discussion ................................................................................. 99

5.2.1. Characterization of AuSG MPCs and PdSG MPCs ............................... 99

5.2.2. Catalytic Reduction of 4-Nitrophenol …………………………….... 105

5.2.3. Iodine Activation of AuSG MPCs .................................................... 106

5.2.4. Recyclability of AuSG MPCs ........................................................ 109

5.2.5. NMR Studies of $I_{2}$ Addition to AuSG MPCs...................................... 111

5.2.6. Study of AuSG MPC Stability by UV-Vis, SEM and ASV .................. 114

5.2.7. Catalytic Hydrogenation/Isomerization of Allyl Alcohol ...................... 118

5.2.8. Iodine Activation of PdSG MPCs ...................................................... 123

5.2.9. Example Calculation of TOF Values ................................................ 125

5.2.10 Recyclability of PdSG MPCs ..................................................... 127

5.2.11. NMR Studies of $\mathrm{I}_{2}$ Addition to PdSG MPCs .................................... 133

5.2.12. Activation of AuSG and PdSG MPCs.............................................. 137 
5.2.13. Heterogeneous Catalytic Reactions.....

CHAPTER 6

EXPERIMENTAL PROCEDURES

6.1. Experimental Procedures for Chapter 2 2........................................................ 145

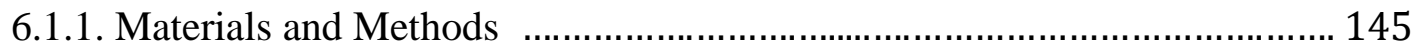

6.1.2. Spectroscopic Measurements ........................................................... 145

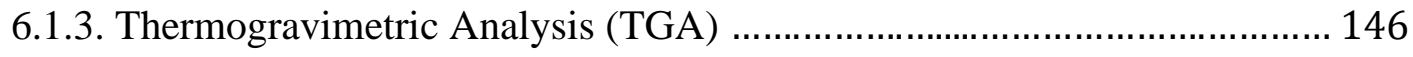

6.1.4. Synthesis Procedures for Aminooxy-

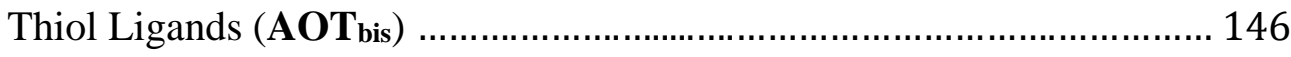

6.1.5. Synthesis Procedures for Water-Soluble Bis-Aminooxy-

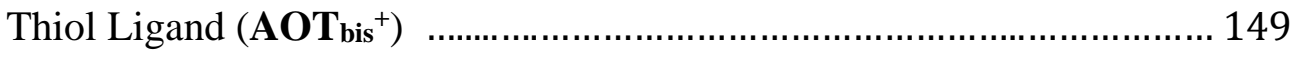

6.1.6. Synthesis Procedures for Aminooxy-Hexane

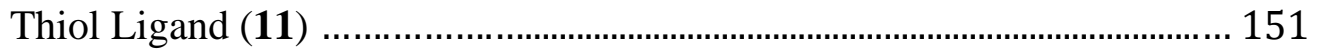

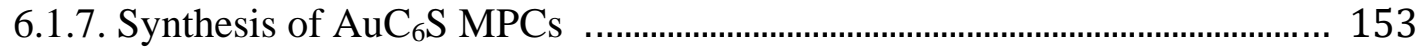

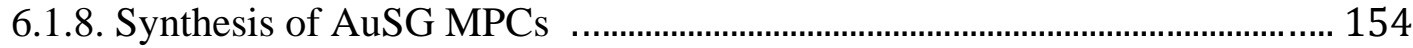

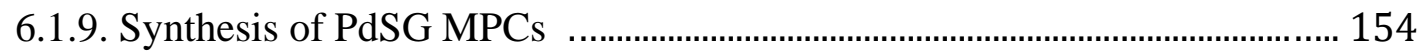

6.2. Experimental Procedures for Chapter 3........................................................ 155

6.2.1. Spectroscopic Measurements .......................................................................... 155

6.2.2. Electrochemical Measurements ................................................................... 156

6.2.3. Fluorescence Measurements ........................................................................... 156

6.2.4. MALDI-TOF MS Measurements ................................................................ 157

6.2.5. Place Exchange Reaction to Prepare

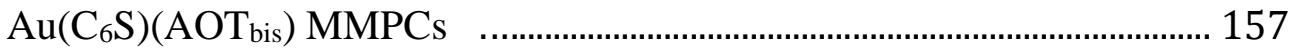

6.2.6. Synthesis Procedures for Urea-Aldehyde 15 .................................................. 158

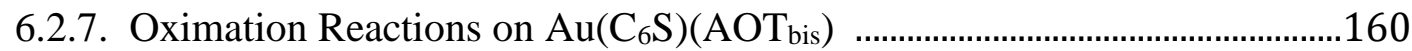

6.2.8. Place Exchange Reactions to Prepare

$\mathrm{Au}(\mathrm{SG})\left(\mathrm{AOT}_{\mathrm{bis}}{ }^{+}\right) \mathrm{MMPCs}$...........................................................................162

6.2.9. Oximation Reactions on $\mathrm{Au}(\mathrm{SG})\left(\mathrm{AOT}_{\text {bis }}{ }^{+}\right) \mathrm{MMPCs}$.........................................163 


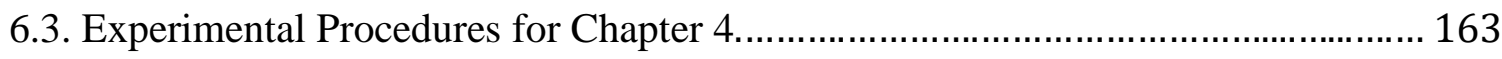

6.3.1. Materials and Methods ……………………………………………………......163

6.3.2. Optical Microscopic Image …………………………………………………...163

6.3.3. FTIR Measurements .........................................................................................164

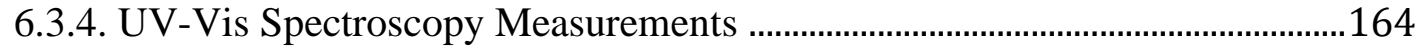

6.3.5. Thermogravimetric Analysis ……………………………………………....164

6.3.6. Place Exchange Reactions to Prepare

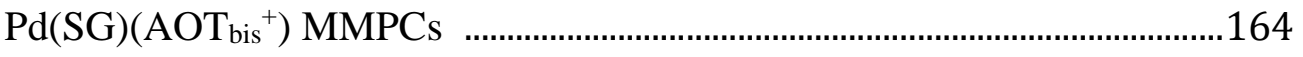

6.3.7. Synthesis of Au Cluster Functionalized Polystyrene Beads ........................165

6.3.8. Synthesis of Pd Cluster Functionalized Agarose Beads ...................................166

6.4. Experimental Procedures for Chapter 5 ……………………………………... 167

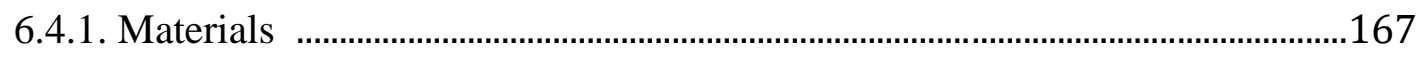

6.4.2. Electron Microscopy Measurements ..................................................................167

6.4.3. Thermogravimetric Analysis ...........................................................................168

6.4.4. X-ray Photoelectron Spectroscopy (XPS) Measurements .............................. 168

6.4.5. FTIR Measurements ..................................................................................... 169

6.4.6. UV-Vis Spectroscopy Measurements .............................................................169

6.4.7. ${ }^{1} \mathrm{H}$ NMR Measurements .............................................................................. 170

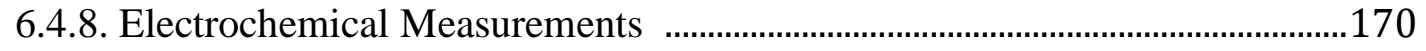

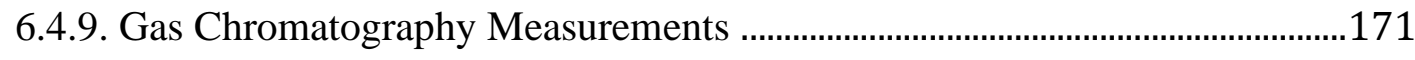

6.4.10. 4-Nitrophenol Reduction ............................................................................. 171

6.4.11. Allyl Alcohol Hydrogenation/Isomerization (Homogenous System)

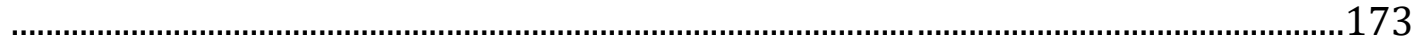

6.4.12. Allyl Alcohol Hydrogenation/Isomerization (Heterogenous System) 174

CHAPTER 7 SUMMARY AND FUTURE DIRECTIONS

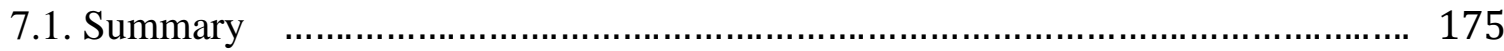

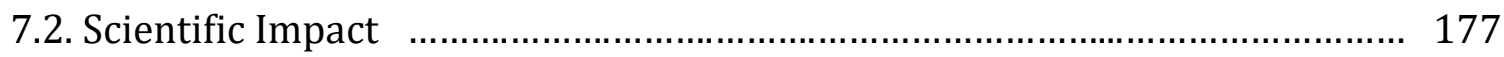

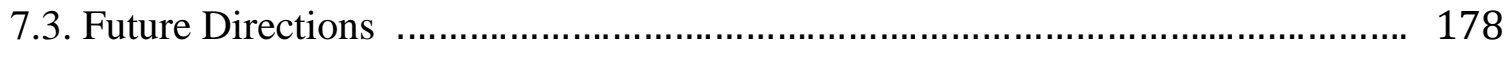




\section{APPENDIX-A}

Lists of Acronym and Abbreviations ...................................................................... 195

APPENDIX- B Spectra $\left({ }^{1} \mathrm{H}\right.$ NMR, ${ }^{13} \mathrm{C}$ NMR, FTIR, and HRMS $)$

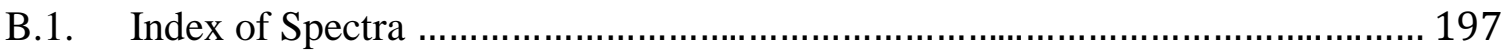

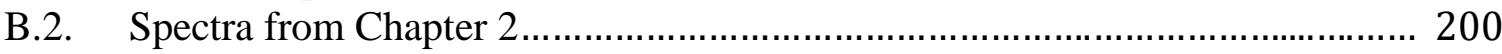

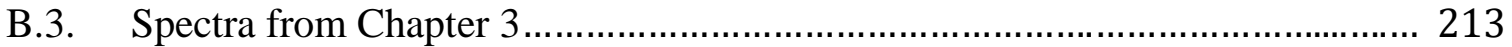

\section{APPENDIX-C}

C.1. List of Publications ................................................................................. 224

C.2. Published Papers and Previous Researches Title Pages .......................................... 225

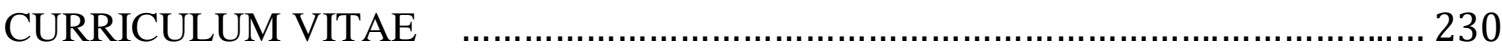




\section{LIST OF TABLES}

TABLE

PAGE

3.1. Aldehydes examined in oximation reactions with

$\mathrm{Au}\left(\mathrm{C}_{6} \mathrm{~S}\right)\left(\mathrm{AOT}_{\mathrm{bis}}\right) \mathrm{MMPCs}$.

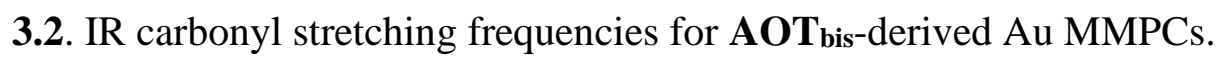

3.3. Proposed chemical structure of fragments generated from

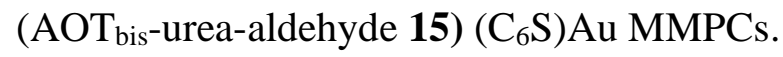

78

5.1. Quantitative results from the NMR data (Figure 5.10) showing the percent of ligand removal from AuSG MPCs as a function of added $\mathrm{I}_{2}$ equivalents after $40 \mathrm{~min}$. reaction.

5.2. Turnover frequency and catalyst recyclability for the PdSG MPCs-catalyzed hydrogenation/isomerization of allyl alcohol with and without added I2. TOFs are determined from the slopes of the plots at $<60 \%$ allyl alcohol conversion to products. 


\section{LIST OF FIGURES}

FIGURE

PAGE

1.1. A typical oximation reaction scheme showing the formation of oxime ethers.

1.2. Resonance structure of an oxime ether shows that hydrolysis is deterred relative to an imine due to greater electron density at the carbonyl center.

1.3. $\mathrm{pK}_{\mathrm{a}}$ of protonated primary amine and aminooxy compounds.

1.4. Schematic of generation of diverse oxime-based libraries based on aminooxy chemistry.

1.5. Oximation reaction of ATM coated on micropreconcentrator micropillars with aldehyde- and ketone-VOCs yield oxime ether adducts for analysis by MS after elution from the device.

1.6. A typical metal MPCs with alkane-thiolate (S-R) as a stabilizer.

1.7. A typical ligand-place exchange reaction to from metal MMPCs. -SR: parent thiolate, and - $\mathrm{R}$ 'SH: a new ligand.

1.8. Schematic diagram of strategy deployed by Thygesen et al, as a model for the detection of glycoproteins. $\mathrm{Pg}=$ Trimellitoyl

1.9. A schematic of "glycoblotting" concept introduced by Nagahori et al.

1.10. A scheme of photodeprotection and bioconjugation strategy studied by Maynard and co-workers. 
1.11. A schematic of nanoparticles self-assembly via a stable oxime-linkage formation at the interface.

1.12. A general schematic of solid-supported NPs.

2.1. A general representation of aminooxy-functionalized $\mathrm{Au}\left(\mathrm{C}_{6} \mathrm{~S}\right)\left(\mathrm{AOT}_{\mathrm{bis}}\right)$ MMPCs.

2.2. ${ }^{1} \mathrm{H}$ NMR spectrum $\left(400 \mathrm{MHz}, \mathrm{CDCl}_{3}\right)$ of compound $\mathbf{A O T}_{\text {bis. }}$

2.3. $\quad{ }^{13} \mathrm{C}$ NMR spectrum $\left(100 \mathrm{MHz}, \mathrm{CDCl}_{3}\right)$ of compound $\mathbf{A O T}$ bis.

2.4. ${ }^{1} \mathrm{H}$ NMR spectrum $\left(400 \mathrm{MHz}, \mathrm{CDCl}_{3}\right)$ of compound $\mathbf{A O T}_{\text {bis }}{ }^{+}$.

2.5. ${ }^{1} \mathrm{H}$ NMR spectrum $\left(400 \mathrm{MHz}, \mathrm{CDCl}_{3}\right)$ of aminooxy-hexanethiol (11).

2.6. ${ }^{13} \mathrm{C}$ NMR spectrum $\left(400 \mathrm{MHz}, \mathrm{CDCl}_{3}\right)$ of aminooxy-hexanethiol (11).

2.7. General structure of $\mathrm{Au}_{144}\left(\mathrm{C}_{6} \mathrm{~S}\right)_{60}, \mathrm{Au}_{\mathrm{x}}(\mathrm{SG})_{\mathrm{y}}$, and $\mathrm{Pd}_{\mathrm{x}}(\mathrm{SG})_{\mathrm{y}} \mathrm{MPCs}$.

3.1. Ligand place-exchange reactions for Au MPCs to afford corresponding MMPCs. $\mathrm{C}_{6} \mathrm{~S}=$ Hexane-thiolate, $\mathrm{SG}=$ Glutathiolate

3.2. A schematic of the synthesis of $\mathrm{AuC}_{6} \mathrm{~S}$ MPC following the Brust method.

3.3. TGA of $\mathrm{Au}_{144}\left(\mathrm{C}_{6} \mathrm{~S}\right)_{60} \mathrm{MPCs}$.

3.4. UV-Vis of $\mathrm{Au}_{144}\left(\mathrm{C}_{6} \mathrm{~S}\right)_{60} \mathrm{MPCs}$ (sample was prepared in $\mathrm{THF}$, concentration of MMPCs was $0.0188 \mathrm{mM})$.

3.5. $\quad{ }^{1} \mathrm{H}$ NMR spectrum of $\mathrm{Au}_{144}\left(\mathrm{C}_{6} \mathrm{~S}\right)_{60} \mathrm{MPCs}$.

3.6. FTIR spectrum of $\mathrm{Au}_{144}\left(\mathrm{C}_{6} \mathrm{~S}\right)_{60} \mathrm{MPCs}$.

3.7. A schematic of ligand place exchange reaction between $\mathrm{Au}_{144}\left(\mathrm{C}_{6} \mathrm{~S}\right)_{60} \mathrm{MPCs}$ and aminooxy ligand (AOT bis) to form $\mathrm{Au}_{144}\left(\mathrm{C}_{6} \mathrm{~S}\right)_{30}\left(\mathrm{AOT}_{\text {bis }}\right)_{30}$ MMPCs.

3.8. UV-VIS spectrum of $\mathrm{Au}_{144}\left(\mathrm{C}_{6} \mathrm{~S}\right)_{30}\left(\mathrm{AOT}_{\text {bis }}\right)_{30}$ MMPCs. (sample was prepared in THF, concentration of MMPCs was $0.0161 \mathrm{mM}$ ). 
3.9. FTIR spectrum of $\mathrm{Au}_{144}\left(\mathrm{C}_{6} \mathrm{~S}\right)_{30}\left(\mathrm{AOT}_{\text {bis }}\right)_{30} \mathrm{MMPCs}$.

3.10. ${ }^{1} \mathrm{H}$ NMR spectra $\left(400 \mathrm{MHz}, \mathrm{CDCl}_{3}\right.$ ) of (A) $\mathrm{Au} \mathrm{C}_{6} \mathrm{~S} \mathrm{MPCs}$, (B) thiol ligand $\mathrm{AOT}_{\text {bis, }}(\mathrm{C}) \mathrm{Au}\left(\mathrm{C}_{6} \mathrm{~S}\right)\left(\mathrm{AOT}_{\text {bis }}\right)$ MMPCs. Peaks at $\delta 7.24 \mathrm{ppm}$ are residual chloroform (solvent) peaks.

3.11. ${ }^{1} \mathrm{H}$ NMR spectrum $\left(500 \mathrm{MHz}, \mathrm{CDCl}_{3}\right)$ of urea-aldehyde 15.

3.12. ${ }^{13} \mathrm{C}$ NMR spectrum $\left(500 \mathrm{MHz}, \mathrm{CDCl}_{3}\right)$ of urea-aldehyde 15.

3.13. ${ }^{1} \mathrm{H}$ NMR spectra $\left(400 \mathrm{MHz}, \mathrm{CDCl}_{3}\right)$ of $(\mathrm{A}) \mathrm{Au}\left(\mathrm{C}_{6} \mathrm{~S}\right)\left(\mathrm{AOT}_{\text {bis }}\right) \mathrm{MMPCs}$, and (B) benzaldehyde- and (C) propanal-adducts obtained on reaction with $\mathrm{Au}\left(\mathrm{C}_{6} \mathrm{~S}\right)\left(\mathrm{AOT}_{\text {bis }}\right) \mathrm{MMPCs}$. Peaks at $\delta 7.24 \mathrm{ppm}$ are residual chloroform (solvent) peaks.

3.14. ${ }^{1} \mathrm{H}$ NMR spectra (400 MHz, $\left.\mathrm{CD}_{3} \mathrm{OD}\right)$ of (A) glyceraldehyde- and (B) urea-aldehyde 15-adducts obtained on reaction with $\mathrm{Au}\left(\mathrm{C}_{6} \mathrm{~S}\right)\left(\mathrm{AOT}_{\text {bis }}\right)$ MMPCs. Peaks at $\delta 4.87$ and $3.34 \mathrm{ppm}$ are residual methanol (solvent) peaks.

3.15. UV-VIS spectrum of $\mathrm{Au}\left(\mathrm{C}_{6} \mathrm{~S}\right)\left(\mathrm{AOT}_{\text {bis-benzaldehyde) MMPCs (sample was }}\right.$ prepared in THF, concentration of MMPCs was $2.82 \mathrm{mM})$.

3.16. Concept for tuning solubility properties of $\mathrm{Au}(\mathrm{C} 6 \mathrm{~S})\left(\mathrm{AOT}_{\mathrm{bis}}\right) \mathrm{MMPCs}$.

3.17. (A) Structure of ferrocenecarboxaldehyde adduct obtained on reaction with $\mathrm{Au}\left(\mathrm{C}_{6} \mathrm{~S}\right)\left(\mathrm{AOT}_{\text {bis }}\right)$ MMPCs and (B) cyclic voltammograms of $\mathrm{Au}\left(\mathrm{C}_{6} \mathrm{~S}\right)\left(\mathrm{AOT}_{\text {bis }^{-}}\right.$ ferrocene) MMPCs in a $0.10 \mathrm{M}$ TBAHFP/ $\mathrm{CH}_{2} \mathrm{Cl}_{2}$ solution with an overall ferrocene concentration of $1.7 \mathrm{mM}$ (red) and of the electrode after having been removed from the Au MMPCs solution and then placed in a $0.10 \mathrm{M}$ TBAHFP/ $\mathrm{CH}_{2} \mathrm{Cl}_{2}$ electrolyte solution (blue). Scan rate $=100 \mathrm{mV} / \mathrm{s}$, working 
electrode $=\mathrm{Pt}$ disk $(2 \mathrm{~mm}$ diameter $)$, counter electrode $=\mathrm{Pt}$ wire, and reference electrode $=\mathrm{Ag}$ wire quasi-reference electrode.

3.18. Fluorescence emission spectra of 1-pyrenecarboxaldehyde (1.0 E-07 M in

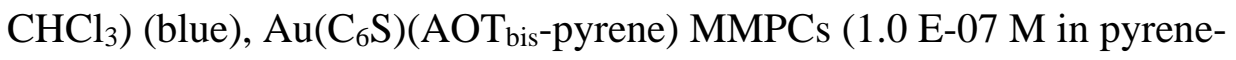
CHO) (pink), and $\mathrm{CHCl}_{3}$ (red). Excitation $\lambda=375 \mathrm{~nm}$.

3.19. Schematic illustration of imino ions formation via oxime bond breakdown upon laser irradiation during MALDI process. (PNA= p-nitroaniline)

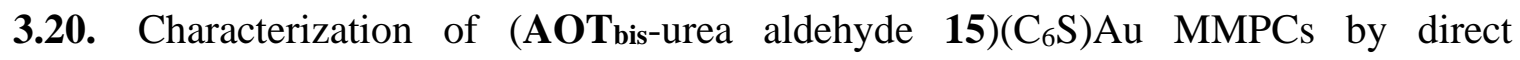
MALDI-TOFMS. Chemical formulas of major signals are listed in Table 3.3. 77

4.1. A general strategy of anchoring MPCs on a solid support via oximation reaction

4.2. A general representation of oximation reaction between $A u\left(\mathrm{C}_{6} \mathrm{~S}\right)\left(\mathrm{AOT}_{\text {bis }}\right)$ MMPCs and PS-CHO beads.

4.3. Optical image of (A) PS-aldehyde beads and (B) agglomerated Au cluster functionalized-PS beads.

4.4. FTIR spectra of $\mathrm{AuC}_{6} \mathrm{~S}$ MPCs (black plot) and $\mathrm{Au}\left(\mathrm{C}_{6} \mathrm{~S}\right)\left(\mathrm{AOT}_{\text {bis }}\right)$ MMPCs (red plot) reacted with PS-CHO beads.

4.5. Place exchange reaction between $\mathrm{Pd}(\mathrm{SG})$ MPCs and $\mathrm{AOT}_{\text {bis }}{ }^{+}$to afford $\mathrm{Pd}(\mathrm{SG})\left(\mathrm{AOT}_{\mathrm{bis}}{ }^{+}\right)$MMPCs.

4.6. ${ }^{1} \mathrm{H}$ NMR spectra $\left(\mathrm{D}_{2} \mathrm{O}, 500 \mathrm{MHz}\right)$ of PdSG MPCs and Pd(SG)(AOT $\left.{ }_{\text {bis }}{ }^{+}\right)$MMPCs. 1:1 exchange ratio (estimated ratio) was based on the ratio of integration values of $\mathrm{CH}_{2}$ peak of $\mathrm{SG}$ to the $\mathrm{CH}_{2}$ peak of $\mathrm{AOT}_{\text {bis }}{ }^{+}$. 
4.7. Schematic of oximation reaction between $\mathrm{Pd}(\mathrm{SG})\left(\mathrm{AOT}_{\text {bis }}{ }^{+}\right)$MMPCs and agarose$\mathrm{CHO}$ beads to afford Pd-cluster functionalized agarose bead.

4.8 Optical image of agarose-aldehyde beads (A) and Pd cluster- functionalized agarose beads $(\mathbf{B})$.

4.9. UV-Vis absorbance spectra of $\mathrm{Pd}(\mathrm{SG})($ AOTbis+) MMPCs, Pd clusterfunctionalized beads and supernatant of Pd cluster-functionalized beads (nanopure water was used as a solvent).

4.10. Digital images of $\operatorname{Pd}(\mathrm{SG})\left(\mathrm{AOT}_{\mathrm{bis}^{+}}{ }^{+}\right)$MMPCs solution(A), $\mathrm{Pd}$ clusterfunctionalized agarose beads (black precipitate) with supernatant (clear) on top (B), and PdSG MPC only reacted with agarose aldehyde beads (C)

4.11. FTIR spectra of as-purchased agarose-CHO beads and Pd-cluster functionalized agarose beads (AOT: $\mathrm{CHO}=1: 10)$.

4.12. Thermogravimetric analysis (TGA) of PdSG MPCs, $\mathrm{Pd}(\mathrm{SG})\left(\mathrm{AOT}_{\mathrm{bis}}{ }^{+}\right) \mathrm{MMPCs}$, and Pd cluster- functionalized agarose- beads.

5.1. TGA of synthesized AuSG MPCs (A) and PdSG MPCs (B).

101

5.2. UV-Vis of synthesized AuSG MPCs (A) and PdSG MPCs (B) (nanopure water was used as a blank).

5.3. Characterization of synthesized MPCs. A: Brightfield TEM image of AuSG MPCs, and B: Darkfield TEM image of PdSG MPCs (red, circled aggregated sphere was not counted in the size analysis). Corresponding histogram plot of the particle size analyses are below the image.

103

5.4. FTIR spectrum of glutathione (A), AuSG MPCs (B) and PdSG MPCs (C). 104 
5.5. UV-Vis absorption spectra of 4-nitrophenol, 4-nitrophenolate (4-NP) and 4-

aminophenol (4-AP), each at a concentration of $3 \times 10^{-5} \mathrm{M}$.

5.6. A:Changes in absorbance during $\mathrm{NaBH}_{4}$ reduction of $4-\mathrm{NP}$ in the presence of AuSG MPCs as a catalyst over 5-minute intervals (no $\mathrm{I}_{2}$ added). B:Changes in absorbance during the AuSG MPC-catalyzed $\mathrm{NaBH}_{4}$ reduction of 4-NP with 0.25 equivalents of $\mathrm{I}_{2}$ addition.

5.7. Schematic of 4-NP reduction on AuSG MPC surface upon $I_{2}$ activation.

5.8. A: Plots of $\ln (\mathrm{Ao} / \mathrm{At})$ vs. time for the AuSG MPC-catalyzed reduction of 4-NP with different amounts of added $\mathrm{I}_{2} ; \mathbf{B}$ : plot of reaction rate constants (left y-axis, red) calculated from Fig. 5.8 A and correlation with recyclability (right y-axis, blue).

108

5.9. A:Plot of rate constant values vs. recyclability for 4-NP reduction with varying equivalents of added $\mathrm{I}_{2} ; \mathbf{B}$ :plot of rate constant values vs. number of cycles for the reduction with 0.5 equivalents of added $I_{2}$, with and without products removed in subsequent cycles.

5.10. ${ }^{1} \mathrm{H}$ NMR spectra of AuSG MPCs in $\mathrm{D}_{2} \mathrm{O}$ without added $\mathrm{I}_{2}$ and 40 min after the addition of various equivalents of $\mathrm{I}_{2}$. The signals at $\delta 2.93-2.99$ and 3.27-3.32 ppm (marked by blue arrows) correspond to the methylene protons of the cysteine residues of glutathione disulfide (GS-SG), and the signal at $84.72-4.76$ ppm corresponds to the adjacent methine proton (far left blue arrow). The increase in these signals on addition of $\mathrm{I}_{2}$, relative to the internal standard $t$ butanol singlet at $\delta 1.19 \mathrm{ppm}$, indicates that the disulfide forms and dissociates 
from the AuSG MPCs. With no $I_{2}$, the methylene protons next to $S$ of bound glutathione are not visible in the NMR spectrum and the signal for the immediately adjacent methine proton appears at $\delta 4.44 \mathrm{ppm}$ (red arrow) as a broad peak, indicating that the SG is bound to the Au cluster. The peak height decreases with added $I_{2}$ in agreement with ligand removal upon disulfide formation. The NMR spectra of free glutathione (GSH) and GSH oxidized to disulfide by $\mathrm{I}_{2}$ are shown for comparison. Some differences in chemical shifts for the signals of GS-SG formed on reaction of AuSG MPCs vs. $\mathrm{I}_{2}$-oxidation of free GSH can be expected, particularly for protons adjacent to $\mathrm{N}$ groups, due to differences in $\mathrm{pH}$ and the possible presence of metal ions.

5.11. (Top) Digital image of aqueous solutions of AuSG MPCs after reaction with $I_{2}$ for 30 seconds and 3 hours. The change to red color and then disappearance of the color is consistent with aggregation or increased size by ripening followed by dissolution at higher equivalents of $\mathrm{I}_{2}$. (Bottom) UV-Vis absorption spectra of aqueous solutions of AuSG MPCs treated with A: $0.25 \mathrm{eq}, \mathbf{B}: 0.5 \mathrm{eq}, \mathbf{C}: 1.0$ eq, and $\mathbf{D}: 2.0$ eq of $\mathrm{I}_{2}$ for the times indicated.

5.12. SEM images of iodine-activated AuSG MPCs; A. 0.25 eq $I_{2}$ (cannot be observed in SEM), B. 0.5 eq $\mathrm{I}_{2}(68 \pm 7 \mathrm{~nm})$, C. 1.0 eq $\mathrm{I}_{2}(497 \pm 33 \mathrm{~nm})$, D. 2.0 eq $\mathrm{I}_{2}$ (Au dissolved, only ITO observed, E. Histogram of AuSG MPCs with 0.5 eq $\mathrm{I}_{2}$, and $\mathbf{F}$. Histogram of AuSG MPCs with 1.0 eq $\mathrm{I}_{2}$.

5.13. Influence of added $I_{2}$ ( 0.25 to 2.0 eq) on the anodic stripping voltammetry (ASV) of AuSG MPCs attached to glass/ITO electrodes. Measurements were taken after 3 hours reaction time. 
5.14. Digital images of PdSG MPCs in water (A), after adding chlorobenzene (B), and the reaction set-up for isomerization/hydrogenation of allyl alcohol (C).

5.15. UV-Vis spectra of $0.123 \mathrm{M}$ aqueous solutions of reactant (allyl alcohol) and products (1-propanol and propanal).

5.16. Gas chromatogram of a standard solution containing mixture of $10 \mathrm{mM}$ each of reactant (allyl alcohol), and products (Propanal, 1-Propanol) in organic phase (chlorobenzene), and aqueous phase.

5.17. GC-FID chromatograms of allyl alcohol hydrogenation/ isomerization using PdSG MPCs in chlorobenzene: water (1:1) with a $\mathrm{H}_{2}$ flow rate of $20.0 \pm 0.5 \mathrm{~mL} / \mathrm{min}$. A:organic phase, in absence of $\mathrm{I}_{2} ; \mathbf{B}$ :organic phase, with 0.05 eq $\mathrm{I}_{2}$ addition. 121

5.18. GC-FID chromatograms of allyl alcohol hydrogenation/ isomerization using PdSG MPCs in chlorobenzene: water (1:1) with a $\mathrm{H}_{2}$ flow rate of $20.0 \pm 0.5$ $\mathrm{mL} / \mathrm{min}$. A:aqueous phase, in absence of $\mathrm{I}_{2}$, and $\mathbf{B}$ :aqueous phase, with 0.05 eq $\mathrm{I}_{2}$ addition.

5.19. Reaction completion percentage (hydrogenated plus isomer) for the PdSG MPCcatalyzed hydrogenation/isomerization of allyl alcohol for different amounts of added $\mathrm{I}_{2} . \mathrm{H}_{2}$ flow rate $=20.0 \pm 0.5 \mathrm{~mL} / \mathrm{min}$.

5.20. Plot of the $\%$ hydrogenation and $\%$ isomerization of allyl alcohol versus reaction time with the intercept of the line forced through zero, using PdSG MPC as a catalyst (no $\mathrm{I}_{2}$ added). The slope of the plots ( $<60 \%$ corresponding conversions) were used to determine the TOF values.

5.21. UV- UV-Vis spectra of the aqueous phase separated from the biphasic reaction mixture before and after extraction of the products (propanal and 1-propanol) with 
$\mathrm{CH}_{2} \mathrm{Cl}_{2}$. $4.0 \mathrm{~mL}$ of $\mathrm{CH}_{2} \mathrm{Cl}_{2}$ was used to extract the products, and the process was repeated 2 more times.

5.22. GC plots of the aqueous phase separated from the biphasic reaction mixture before and after extraction of the products (propanal and 1-propanol) with $\mathrm{CH}_{2} \mathrm{Cl}_{2} .4 .0$ $\mathrm{mL}$ of $\mathrm{CH}_{2} \mathrm{Cl}_{2}$ was used to extract the products, and the process was repeated 2 more times.

5.23. Plot of total TOF values vs. number of reaction cycles for hydrogenation/isomerization of allyl alcohol using catalytic PdSG MPCs with and without added $\mathrm{I}_{2}$. Successful cycle completion required that $100 \%$ of allyl alcohol be transformed into products within 60 minutes.

5.24. Gas chromatograms of cycle 1 to cycle 5 using PdSG NPs as a catalyst for allyl alcohol hydrogenation/isomerization (no I2 added); A]. organic layer, and B]. aqueous layer.

5.25. UV-Vis spectra showing the stability of PdSG MPCs after (A) completion of the first cycle and (B) completion of the last cycle (with no $\mathrm{I}_{2}$ and different equivalents of $I_{2}$ addition) in the hydrogenation/isomerization of allyl alcohol (\# cycles shown in $\mathbf{B})$.

5.26. UV-Vis spectra of PdSG MPCs treated with 0.3 eq $\mathrm{I}_{2}$ and 0.6 eq $\mathrm{I}_{2}$. (compared with that of no $\mathrm{I}_{2}$ added). The high absorbance at higher wavelengths is a clear indication of aggregation.

5.27. ${ }^{1} \mathrm{H}$ NMR (400 MHz, $\left.\mathrm{D}_{2} \mathrm{O}\right)$ spectra of particle stability studies of PdSG MPCs upon $I_{2}$ activation. Each spectrum was obtained on a separate sample after 40 
minutes of reaction with $I_{2}$ except for 0.1 equivalents $I_{2}$, which was obtained on the sample with 0.05 equivalents $\mathrm{I}_{2}$ after adding a second 0.05 equivalents $(0.1$ total) and obtaining the spectrum after another 40 minutes.

5.28. ${ }^{1} \mathrm{H}$ NMR spectra of GSH only and GSH treated with 2.0 equivalents $\mathrm{I}_{2}, 1$ equivalent of $\mathrm{K}_{2} \mathrm{PdCl}_{4}$, and both $\mathrm{I}_{2}$ and $\mathrm{K}_{2} \mathrm{PdCl}_{4}$ after $40 \mathrm{~min}$.

5.29. TGA of PdSG MPCs obtained after reaction with varying equivalents of $I_{2}$ addition for $60 \mathrm{~min}$. There is less organic weight percent as the amount of $I_{2}$ increased. The estimated ligand loss is 5, 17, 31, and $38 \%$ for the 0.025 eq, 0.05 eq, $0.1 \mathrm{eq}$, and $0.2 \mathrm{eq}$, respectively.

5.30. XPS survey spectra of the iodide region (BE 610-640 eV) of a sample of (A) AuSG MPCs and (B) PdSG MPCs after one reaction cycle with no $I_{2}$ and various $\mathrm{I}_{2}$ additions as indicated.

5.31. XPS survey spectra from $50-450 \mathrm{eV}$ BE of a sample of (A) AuSG MPCs and (B) PdSG MPCs after one reaction cycle with no $I_{2}$ and various $I_{2}$ additions as indicated.

5.32. Pd-cluster functionalized agarose bead as a heterogenous catalyst for allyl alcohol hydrogenation/isomerization reaction (biphasic condition: $\mathrm{H}_{2} \mathrm{O} / \mathrm{EtOAc}$ ).

5.33. GC-FID chromatogram of allyl alcohol hydrogenation/isomerization reaction (Pd functionalized agarose bead catalyst) under monophasic condition $\left(\mathrm{H}_{2} \mathrm{O}\right) . \quad 142$

5.34. GC-FID chromatogram of allyl alcohol hydrogenation/isomerization reaction (Pdcluster functionalized agarose bead catalyst) under biphasic condition (EtOAc and $\mathrm{H}_{2} \mathrm{O}$ ). 


\section{LIST OF SCHEMES}

SCHEMES

PAGE

1.1. A schematic model of processes that takes place during ligands-place exchange reaction on thiolated-Au MPCs.

1.2. A diagram representing three different types of catalytic system.

1.3. General schematic of catalytic cycle in a biphasic system $(R=$ Reactant, $P=$ Product).

1.4. General schematic of catalytic activity and stability of nanoparticles.

1.5. AuSG catalyzed- model reaction of $4-\mathrm{NP}$ reduction to $4-\mathrm{AP}$ by $\mathrm{NaBH}_{4}$. $(\mathrm{SG}=$ glutathione)

1.6. A mechanistic route for the Au MPCs catalyzed 4-NP reduction in presence of excessive $\mathrm{NaBH}_{4}$.

1.7. A general schematic of allyl alcohol hydrogenation/isomerization reaction. 30

1.8. Proposed mechanism showing the two routes for the hydrogenation/isomerization of an allyl alcohol.

1.9. A schematic of biphasic system, for an allyl alcohol hydrogenation/isomerization reaction of allyl alcohol

1.10. Iodine activation concept for the partial removal of thiolated ligands from the MPCs surface to promote catalytic enhancement of 4-NP reduction reaction. 39 


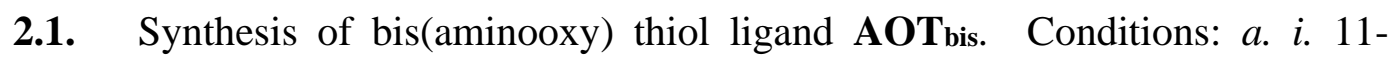
bromo-1-undecene, $\mathrm{CH}_{3} \mathrm{CN}$ : $\mathrm{MeOH}$ (7:3), reflux, 48 h; ii. aq. $\mathrm{NH}_{4} \mathrm{OH}$, $88 \% ; b . \mathrm{N}$-hydroxyphthalimide (NHP), $\mathrm{PPh}_{3}$, diisopropyl azodicarboxylate (DIAD), toluene, $0{ }^{\circ} \mathrm{C}$ to $\mathrm{rt}, 16 \mathrm{~h}, 95 \%$; c. i. camphorsulfonic acid (CSA), THF, $0{ }^{\circ} \mathrm{C}, 2 \mathrm{~h}, i i$. azobisisobutyronitrile (AIBN), thioacetic acid, reflux, 20 h, iii. aq. $\mathrm{NaHCO}_{3}, 78 \%$; . hydrazine monohydrate, $\mathrm{CH}_{2} \mathrm{Cl}_{2}, 0{ }^{\circ} \mathrm{C}$ to rt, $12 \mathrm{~h}, 82 \%$.

2.2. Synthesis of water-soluble bis(aminooxy) thiol AOT bis $^{+}$. Conditions: $a$. $\mathrm{CH}_{3} \mathrm{I}, \mathrm{CH}_{2} \mathrm{Cl}_{2}$, sealed tube, $50{ }^{\circ} \mathrm{C}, 24 \mathrm{~h}, 88 \%$ b. hydrazine monohydrate, $\mathrm{CH}_{2} \mathrm{Cl}_{2}, 0{ }^{\circ} \mathrm{C}$ to rt, $12 \mathrm{~h}, 84 \%$.

2.3. Synthesis of aminooxy hexane-thiol ligand (11). Conditions: $a$. Nhydroxyphthalimide (NHP), $\mathrm{PPh}_{3}$, diisopropyl azodicarboxylate (DIAD), THF, $0{ }^{\circ} \mathrm{C}$ to $\mathrm{rt}, 12 \mathrm{~h}, 82 \%$; $b$. Anhydrous THF, Azobisisobutyronitrile $(\mathrm{AIBN})$, thioacetic acid, reflux, $16 \mathrm{~h}, 88 \%$; $c$. hydrazine monohydrate, $\mathrm{CH}_{2} \mathrm{Cl}_{2}, 0{ }^{\circ} \mathrm{C}$ to $\mathrm{rt}, 12 \mathrm{~h}, 91 \%$.

3.1. Synthesis of urea-aldehyde 15; $\mathrm{TMSCl}=$ trimethylsilyl chloride, $\mathrm{t}-$ $\mathrm{BuNCO}=$ tert-Butyl isocyanate, $\mathrm{TBAF}=$ tetra- $n$-butylammonium fluoride, PCC $=$ pyridinium chlorochromate. Overall yield $(3$ steps $)=28 \% . \quad 66$

3.2. Schematic representation of place exchange and oximation reactions using the

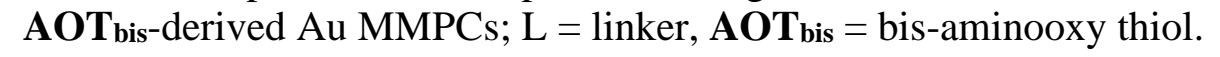
68

3.2. Graphical representation of aminooxy-functionalized Au MMPCs tunability to generate corresponding oxime- ether clusters. 80

5.1. Schematic of Au MPCs synthesis using Bunte salts as a ligand precursor. 97

5.2. Schematic of branched- thiol ligand (glutathione) capped- Pd MPCs. 97

5.3. Iodine activation at the surface of a thiolate-coated metal cluster. We postulate neighboring thiolate displacement of iodide from a sulfonium ion intermediate results in disulfide formation and ligand dissociation, making the metal surface 
accessible for substrate binding (shaded area). Iodide can presumably adsorb to the metal surface or dissolve into solution.

5.4. Model reduction and hydrogenation/isomerization reactions subjected to catalyst activation using $\mathrm{I}_{2}$. 


\section{CHAPTER 1}

\section{INTRODUCTION}

\subsection{Summary of Research}

The main goal of this research was to synthesize aminooxy compounds and use them to prepare novel, stable metal monolayer protected clusters (MPCs) for applications in catalysis and sensing. Place-exchange reactions were exploited for the incorporation of the new aminooxy-containing compounds onto the surface of MPCs to afford aminooxylated metal mixed-monolayer protected clusters (MMPCs). These metal MMPCs were then subjected to click-type reactions, 'oximation' reactions between aminooxy functionality and different aldehyde/ketone carbonyls, to generate libraries of oxime ether adducts. The obtained adducts were examined for their potential catalytic and sensing applications. This dissertation is divided in to seven chapters. Chapter 1 describes detailed background information about all the related topics of this research, such as the significance of aminooxy chemistry, importance of smaller sized and highly stable metal MPCs in catalytic applications, ligand removal strategies, and advantages of MPC anchored solid supported catalyst.

Chapter 2 includes the synthetic pathways to various aminooxy target compounds, referred to as 'ligands' in this dissertation. Synthesis strategies for making several classes of aminooxy ligands, such as trifunctional bis-aminooxy amino thiol, mono-aminooxy amino thiol, straight chain aminooxy thiol, and aromatic aminooxy thiols, as well as synthesis routes to metal MPCs containing different stabilizers are presented in this chapter. A synthetic pathway to a novel urea motif-containing aldehyde, which is used in 
an oximation reaction to form a urea-adduct for use as a sensor, is also included in this chapter. The methods and techniques implemented for the characterization of the synthesized compounds and MPCs are discussed as well. In chapter 3, the functionalization of gold $(\mathrm{Au}) \mathrm{MPCs}$ with aminooxy ligands to afford Au MMPCs is reported along with the details of characterization techniques. The subsequent oximation reactions of aminooxy-functionalized MPCs with different aldehydes/ketones is demonstrated and, importantly, the tunable properties of the MPCs is investigated.

In chapter 4, the method of preparing a solid-supported Au or Pd MPC catalyst is discussed. Aldehyde-activated polystyrene (PS) and agarose beads were used as a solidsupport material. Click-reaction of aminooxylated MPCs and aldehyde-activated solid material afforded a heterogenous-type material which may have potential use in various organic reactions, such as 4-NP reduction, alkyne hydration, allyl alcohol hydrogenation/isomerization reaction, and Suzuki-coupling reaction. Initial studies have suggested that click chemistry is indeed a powerful technique to prepare solid-supported $\mathrm{Au}$ or Pd MPCs. Iodine activation, a general method for ligand removal from MPCs, is introduced in chapter 5. The results of $I_{2}$-promoted catalytic enhancement of glutathione (GSH) stabilized Au and palladium (Pd) MPCs are presented. These MPCs were used as catalysts for the reduction of 4-nitrophenol (4-NP) and allyl alcohol hydrogenation/isomerization reactions, respectively. The catalytic activities of such reactions were then evaluated by monitoring the reaction progress using ultra-violet visible (UV-Vis) spectroscopy and gas chromatography (GC) measurements. The results were reported in terms of rate constant $(\mathrm{k})$ values for 4-NP reduction while allyl alcohol hydrogenation/isomerization reaction results were reported in terms of turnover 
frequency (TOF) values. Improvement of the catalytic performance through ligand dissociation promoted via disulfide formation as well as the MPC stability studies upon $\mathrm{I}_{2}$ activation is described in this chapter.

Chapter 6 summarizes the experimental procedures used in chapters $2-5$. Materials and methods are discussed in general and detailed experimental protocols for preparation of aminooxy ligands, urea-aldehyde substrates, derived MPCs, place exchange reactions, and oximation reactions are presented along with characterization data (where applicable), such as melting point (m.p), proton $\left({ }^{1} \mathrm{H}\right) \mathrm{NMR}$, carbon $\left({ }^{13} \mathrm{C}\right) \mathrm{NMR}$, FourierTransform Infrared Spectroscopy (FTIR), high resolution mass spectrometry (HRMS) data. UV-Vis measurements, GC measurements, other ${ }^{1} \mathrm{H}$ NMR studies, anodic stripping voltammetry (ASV), and X-ray photoelectron spectroscopy (XPS) of iodine-activated AuSG and PdSG MPCs are also included. Likewise, the experimental details of preparation of two types of solid-supported MPCs are presented in this chapter. Finally, chapter 7 concludes this dissertation with a summary of the research and future perspectives. The potential applications of this research have not been fully studied yet, but much promise lies in the development of aminooxylated metal MPCs based sensors. The present work details some useful means of designing MPCs-based sensors using the key results obtained from this research. Primarily, such type of MPCs have a high promise in their use as a chemiresistor based sensor as well as a surface enhanced Raman spectroscopy (SERS) based sensor. 


\subsection{Research Motivation}

Aminooxy $\left(-\mathrm{ONH}_{2}\right)$ groups are most commonly known for their chemoselective reactions with carbonyl compounds (aldehydes/ketones). The well-known "clickoximation" reaction ${ }^{1}$ already has been shown to be a powerful method for the attachment of smaller molecules onto nanoparticles assemblies. ${ }^{2}, 3$ Oximation facilitates a straightforward way of attaching carbonyl substrates, such as aldehydes and ketones, onto materials by forming a stable and robust oxime ether adduct (Figure 1.1). Our research work was motivated from an idea of exploiting the surface chemistry of nanoparticles by functionalizing them with compounds containing the aminooxy functionality. The feasible synthesis of smaller sized nanoparticles, termed as MPCs in this dissertation, has turned our attention to exploit the surface properties of these particles. We are enthused by the idea of using place-exchange reactions to afford aminooxy-functionalized MPCs for applications in nanocatalysis and sensing. An initial idea of synthesizing MPCs fully decorated with aminooxy compounds did not proceed as planned due to issues such as MPC aggregation caused via the intermolecular forces between the aminooxy functionalities on the metal surface. The key issue to synthesize stable aminooxy MPCs was solved by controlling the amount of incoming and outgoing ligand via the placeexchange reaction, resulting in stable MMPCs. This allowed a new synthetic route for preparing metal MPCs with many useful functionalities (redox groups, chromophores, fluorophores, chemical receptors, etc). Then we believed we could apply these findings $n$ catalysis. Consequently, we began the preparation of solid-supported catalysts prepared by reacting aldehyde-functionalized beads with aminooxy MPCs. 
To enhance the catalytic activity, we later developed an interest in using $I_{2}$ as a means to affect ligand surface density. This led to a method for improving the catalytic activity of MPCs by $\mathrm{I}_{2}$-induced ligand dissociation. Ultimately, this concept directed us to carry out fundamental studies on $\mathrm{I}_{2}$ controlled ligand removal and catalysis using glutathione-stabilized MPCs. Finally, we were motivated to utilize the $\mathrm{I}_{2}$ activation approach on heterogenous systems to improve catalyst recyclability. Moreover, we are also interested in exploring the sensing applications of such functional nanomaterials.

\subsection{Introduction to Aminooxy Chemistry}

The aminooxy-carbonyl oximation reaction is one of the most popular types of “click chemistry" conceptualized by Nobel laureate K. Barry Sharpless. ${ }^{1}$ Click chemistry is promoted as a method to form stable carbon-heteroatom bonds. ${ }^{4}$ In general, click reactions liberate products in high yields, do not generate biproducts, and are popular in the pharmaceutical industry. There are several classes of reactions that fit into the category of click reactions, such as azide-alkyne cycloaddition, ${ }^{5,} 6 \mathrm{~S}_{\mathrm{N}} 2$-type reactions, ${ }^{7}$ epoxidation, ${ }^{8}$ etc. Among various conjugation strategies, oxime formation is widely employed in the scientific field because of its feasibility and versatility. Aminooxy groups $\left(\mathrm{R}-\mathrm{ONH}_{2}\right)$ react chemoselectively with the carbonyl groups of aldehydes and ketones under mild conditions to form stable oxime ether (OE) adducts (Figure 1.1). Oxime ether linkages also possess greater intrinsic hydrolytic stability as compared to imine or enamine linkages. The resonance form in oxime ethers shows the reduced electrophilicity and greater hydrolytic stability as shown in Figure 1.2. Despite the chemoselectivity of aminooxy group towards aldehyde/ketones, there are many examples 
of other forms of reactivity for this functionality. ${ }^{9}$ It is indeed because of the $\alpha$-effect, ${ }^{10}$ the aminooxy group is a good nucleophile that reacts with different electrophiles, such as acyl chlorides, carboxylic acids and esters (yields hydroxamates), anhydrides, imides, etc.
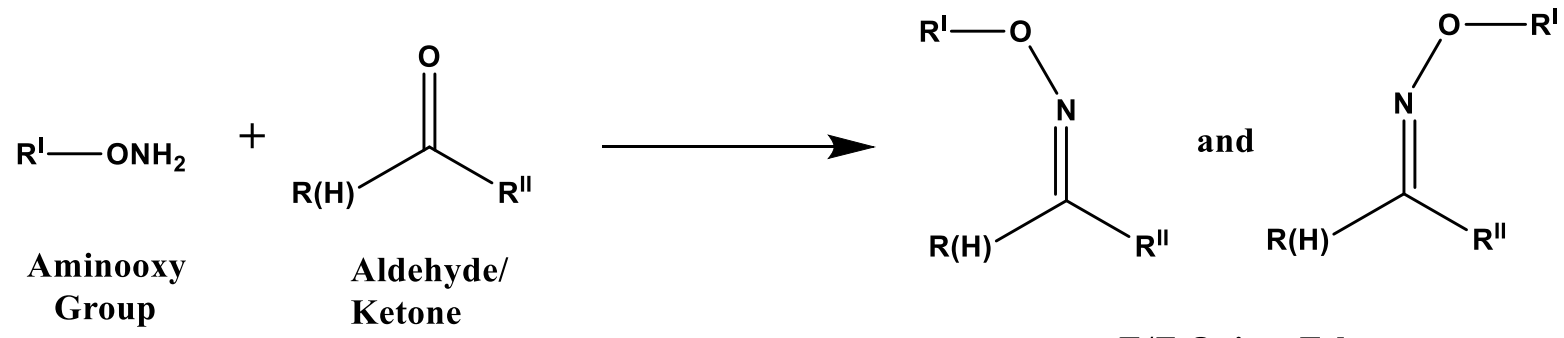

E/Z Oxime Ethers

Figure 1.1. A typical oximation reaction scheme showing the formation of oxime ethers.

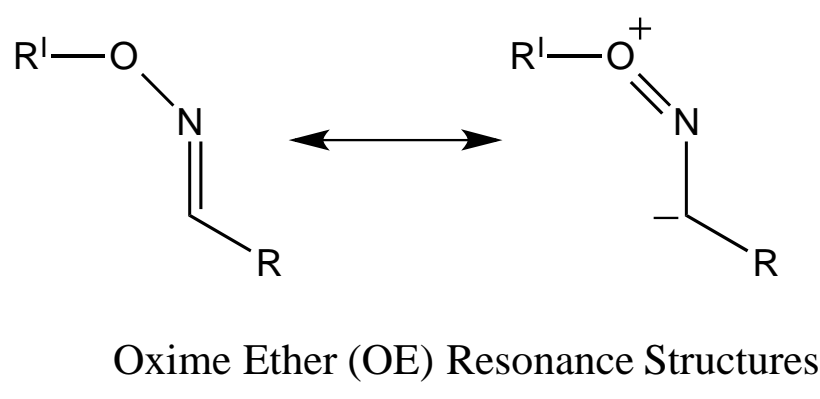

Figure 1.2. Resonance structure of an oxime ether shows that hydrolysis is deterred relative to an imine due to greater electron density at the carbonyl center.

There are indeed some chemical differences between the structurally similar primary amine and aminooxy group. Primary amines react with aldehydes/ketones at room temperature under mild condition to yield imines and react with esters to form secondary imides. The imine linkage exhibits weak hydrolytic stability and is readily hydrolyzed compared to the hydrolytically stable oxime ether linkage. Due to the $\alpha$ effect, the protonated form of aminooxy compounds have a lower $\mathrm{pK}_{\mathrm{a}}(5-6)$ compared to 
the $\mathrm{pK}_{\mathrm{a}}$ of the primary aminium ion (9-10) as shown in Figure 1.3. Aminooxy compounds having more than one lone pair of electrons ( $\mathrm{N}$ and $\mathrm{O}$ ), are known to be highly nucleophilic due to the presence of the electronegative oxygen atom adjacent to nitrogen, the so called $\alpha$-effect in aminooxy compounds. ${ }^{11}$

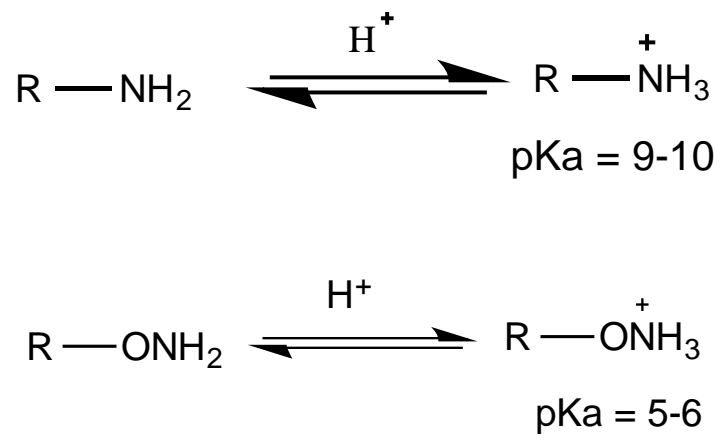

Figure 1.3. $\mathrm{pK}_{\mathrm{a}}$ of protonated primary amine and aminooxy compounds.

This selectivity has increased attention toward exploiting the formation of oxime ethers as a ligation approach. As reported by Rose in 1994, oximation chemistry was used for the conjugation of aminooxy peptides onto a linear polypeptide possessing aldehyde groups. ${ }^{12}$ Such reaction usually proceeds at $\mathrm{pH}$ of $3-4$, where the intermediate formed is "carbinolamine" that subsequently undergoes dehydration to form the oxime bond. This reaction is known for being fast and quantitative, favoring the formation of the Zstereoisomers. ${ }^{13}$ Aminooxy chemistry is utilized in various applications, including pharmaceutical, nanoscience, sensing, biomolecular labeling, ${ }^{14}$ bioconjugation, and drug discovery and development. For example, Bahta and coworkers ${ }^{15}$ described an oximebased library protocol where they synthesized 12 different peptides containing an aminooxy group (P1-P12). They then reacted P1-P12 with 12 different commercially 
available aldehydes (A1-A12). Their methodology generated a diverse library of 144 different peptide-oxime ethers as shown in Figure 1.4. Their studies suggested that the peptide-aldehyde oxime ether adducts show 15 to 20-fold binding enhancements compared to wild-type parent peptide based on their biological assay and structural activity relationship (SAR) results. ${ }^{16}$

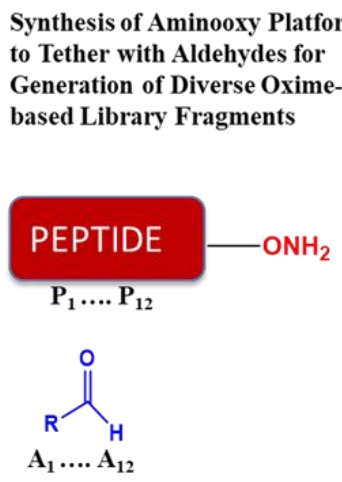

Oximation Reaction in Microplates
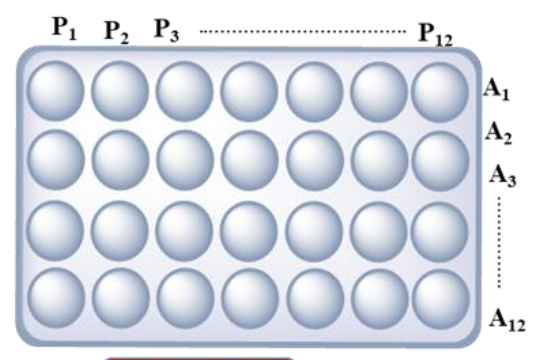

PEPTIDE
Analysis and High-

Throughput Screening

GC/LC-MS

Structural Activity Relationship (SAR)

Data Analysis

Identification

Figure 1.4. Schematic of generation of diverse oxime ether-based libraries based on aminooxy chemistry.

Li et al. demonstrated that a preconcentration device based on silicon microreactor chips functionalized with the aminooxy compound [2-(aminooxy)ethyl]$\mathrm{N}, \mathrm{N}, \mathrm{N}$-trimethylammonium iodide (ATM) is suitable for quantitative analysis of trace volatile aldehydes and ketones in ambient air as well as in human breath. ${ }^{17}$ The trapping of VOCs via the oximation reaction and elution of the formed oxime ether adducts was followed by analysis using Fourier transform ion cyclotron resonance (FTICR) mass spectrometry with the detection levels of $1 \mathrm{ppbv}$ (Figure 1.5). 


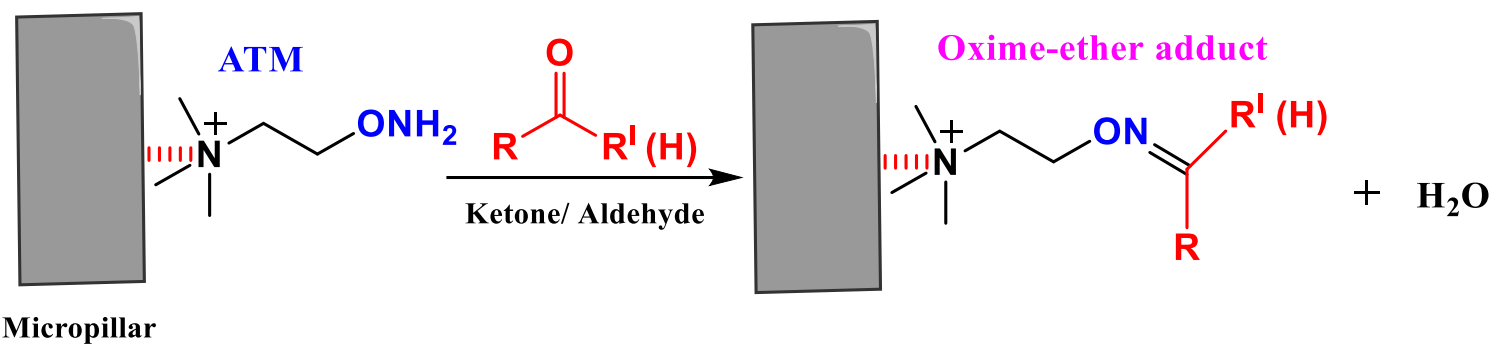

Figure 1.5. Oximation reaction of ATM coated on micropreconcentrator micropillars with aldehyde- and ketone-VOCs yield oxime ether adducts for analysis by MS after elution from the device.

Aminooxy chemistry has recently been widely used with other biomolecules, including carbohydrates and glycoconjugates, because of their several biological functions. A review article published by Pifferi et al. in 2016 details the in-depth overview related to the synthesis and application of so-called aminooxylated carbohydrates. ${ }^{13}$ There are variety of methodologies known for the introduction of the aminooxy functionality at different positions of the sugar rings. For example, Mitsunobu reaction first introduced by Gorochowski and Jurczak ${ }^{18}$ in 1976, was used to synthesize aminooxylated furanoid derivative compounds. Whereas direct oxidation of sugar amines using dimethyldioxirane (DMDO), deprotections of oxyamines protected with protecting groups such as phthalimide (Phth), carboxybenzyl (Cbz), Boc, Fmoc, etc., and addition of hydroxylamine across the double bond of glycals in a stereochemical fashion are commonly employed in the synthesis of aminooxylated carbohydrates. Oxime ligation has truly been a significant development in the synthesis of modified oligosaccharides, glycoproteins, antibiotics, polymers, cyclopeptides, and oligonucleotides, as such 
reactions exhibit good stability, high yield, excellent purity, high reproducibility, short times, and mild reaction conditions.

\subsection{Importance of Monolayer-Protected Clusters (MPCs)}

In general, metal nanoparticles (MNPs) can be defined as any metal materials with at least one dimension in the $1-100 \mathrm{~nm}$ range. Because of their unique electronic structure, optical properties ${ }^{19}$ and size dependent stability, nanoparticles have found broad applications in optical devices, ${ }^{20}$ electrochemical systems, ${ }^{21}, 22$ magnetic devices, ${ }^{23}$ fuel/solar cells, ${ }^{24}$ biomedical technology, ${ }^{25}$ sensors ${ }^{26,} 27$ and catalysis. ${ }^{28}$ Not only can they display unique size-tunable properties, their small size also provides a high surfaceto-volume ratio, which can be favorably exploited for various applications. ${ }^{29}$ Our research focused on the synthesis of smaller sized NPs. NPs synthesized and used in different projects mentioned in this dissertation are of $1.5-2.5 \mathrm{~nm}$ and termed monolayer protected clusters (MPCs). The term "cluster" indicates their small size $(<3.0 \mathrm{~nm}$ diameter), while the term "monolayer-protected" indicates that they are stabilized with a single monolayer of some type of ligand (usually a thiol), which typically has a strong binding interaction with the metal cluster surface. The control of nucleation and growth of MPCs to afford uniform-sized particles is challenging. Although many techniques are known to synthesize metal MPCs, the $\mathrm{NaBH}_{4}$ reduction of metal ions in the presence of organomercaptan stabilizers is the most common and widely used method. Other stabilizers used in MPC synthesis include various heteroatom-based ligands, surfactants, polymers, ions and dendrimers. In addition to thiols, amines, phosphines, and carboxylic acid functional groups are common. Brust and co-workers ${ }^{30}$ developed a straightforward 
method to prepare organic soluble Au NPs stabilized with thiolate ligands $\left(\mathrm{RS}^{-}\right)$that involves the use of a phase-transfer catalyst, such as tetraoctyl ammonium bromide (TOAB), for the transfer of tetrachloroaurate complex anions from an aqueous phase to organic phase. The two-step process delivers Au MPCs coated with a monolayer of alkane-thiolate as a stabilizer (Figure 1.6). The relevant reactions are shown in equations 1.1 and 1.2 below.

$$
\begin{aligned}
& \mathrm{AuCl}_{4^{-}} \text {(solvent) }+\mathrm{RSH} \longrightarrow\left(-\mathrm{Au}^{\mathrm{I}} \mathrm{SR}-\right)_{\mathrm{n}} \text { (polymer) } \\
& \left(-\mathrm{Au}^{\mathrm{I}} \mathrm{SR}-\right)_{\mathrm{n}}+\mathrm{BH}_{4}^{-} \longrightarrow(\mathrm{Au})_{\mathrm{x}}(\mathrm{SR})_{\mathrm{y}} \mathrm{MPCs}
\end{aligned}
$$

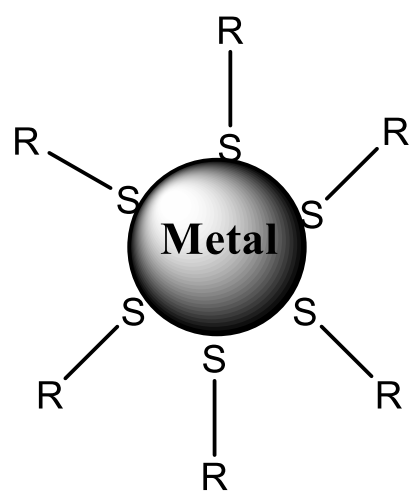

Thiolated-Metal MPCs

Figure 1.6. A typical metal MPCs with alkane-thiolate (S-R) as a stabilizer.

MPCs are formed by the nucleation and growth of metallic $\mathrm{Au}$ upon $\mathrm{BH}_{4}{ }^{-}$reduction, while the thiolate ligands are adsorbed onto the surface of the NP to form a single selfassembled monolayer (SAM) coating. Murray and co-workers ${ }^{31}$ studied the synthesis, reactivity and electrochemistry of Au MPCs, where they demonstrated the importance of 
the thiol/Au mole ratios, rate of $\mathrm{NaBH}_{4}$ addition, and reaction temperature as critical factors to determine the size and size dispersity of the formed particles. All the work presented in this dissertation is based on the use of Au and Pd metal core MPCs. Alkanethiolate-protected Pd MPCs are also synthesized using the Brust method, where the thiol/Pd ratio was normally kept at $0.35: 1.32$ The stabilizer used for both metals is either non-polar hexanethiol $\left(\mathrm{C}_{6} \mathrm{~S}\right)$ or water-soluble glutathione $(\mathrm{GSH})$, a tripeptide molecule. Both organic soluble and water soluble MPCs synthesized for this research work are highly stable, <3.0 nm in diameter, and can be stored for many weeks at room temperature before their use in catalysis or sensing studies. These MPCs are usually first characterized by UV-Vis spectroscopy to determine the approximate size based on the appearance of the localized surface plasmon resonance peaks. ${ }^{33}$ TEM images of clusters synthesized by the Brust method generally consist of spherical metal cores surrounded by the organic monolayer. Synthesized MPCs were further characterized by proton $\left({ }^{1} \mathrm{H}\right)$ NMR and FTIR spectroscopy to understand the fate of thiolates on the metal surface by looking at the broadening of select peaks and/or disappearance of the methylene $\left(\mathrm{CH}_{2}\right)$ protons of the cysteine residues in NMR spectrum and the disappearance of the - $\mathrm{SH}$ stretching in FTIR spectrum.

\subsection{MPCs Functionalization}

Metals such as Au and Pd are known to bind strongly with thiol. ${ }^{34,35}$ A key step in MPCs functionalization is the ligand place exchange reaction. A new functional group could be feasibly introduced onto the surface of metal MPCs via a simple ligand place-exchange reaction. ${ }^{36}$ In a typical ligand place-exchange reaction, a solution of parent thiolate-(SR) 
protected Au MPC is mixed with a solution of a different thiol (-R'-SH) ligand as show by the equation 1.3 below (Figure 1.7).

$(\mathrm{RS})_{\mathrm{m}} \mathrm{Au} \mathrm{MPCs}+\mathrm{x}\left(\mathrm{R}^{\prime}-\mathrm{SH}\right) \longrightarrow\left(\mathrm{R}^{\prime} \mathrm{S}\right)_{\mathrm{y}}(\mathrm{RS})_{\mathrm{m}-\mathrm{y}} \mathrm{Au} \mathrm{MPCs}+\mathrm{y}(\mathrm{R}-\mathrm{SH})+(\mathrm{x}-\mathrm{y}) \mathrm{R}^{\prime}-\mathrm{SH}$

During the reaction, some of the incoming ligands $\left(\mathrm{R}^{\prime}-\mathrm{SH}\right)$ ligands replace the parent $\mathrm{RS}$ ligands on the MPC surface, via the transfer of the $\mathrm{H}$ from the incoming thiol to the replaced thiolate. This will lead to the formation of the free thiols and possibly some disulfide species during the reaction, and these can be removed upon work-up following reaction completion. The resultant MPCs are generally referred to as mixed-MPCs (MMPCs). As Murray and co-workers studied the rate and equilibrium stoichiometry, $\mathrm{y}:(\mathrm{m}-\mathrm{y})$ ratio on the MPC product, following the place exchange reaction, they understood that indeed these are controlled by the R'-SH:SR mole ratio , their relative steric bulk, and $\mathrm{R}$ versus $\mathrm{R}^{\prime}$ chain lengths. These factors are critical and are totally responsible to determine the fate of the final ratio between parent and new ligand, y:(my). In our studies, we first optimized the mole ratio between original ligands (determined by calculating the organic fraction by thermogravimetric analysis) and new thiol ligands to determine the optimal condition to afford the desired final ratio of $1: 1$ on the final MPC product. 


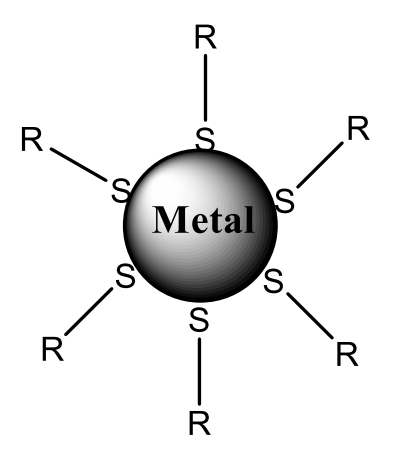

Thiolated-Metal MPCs
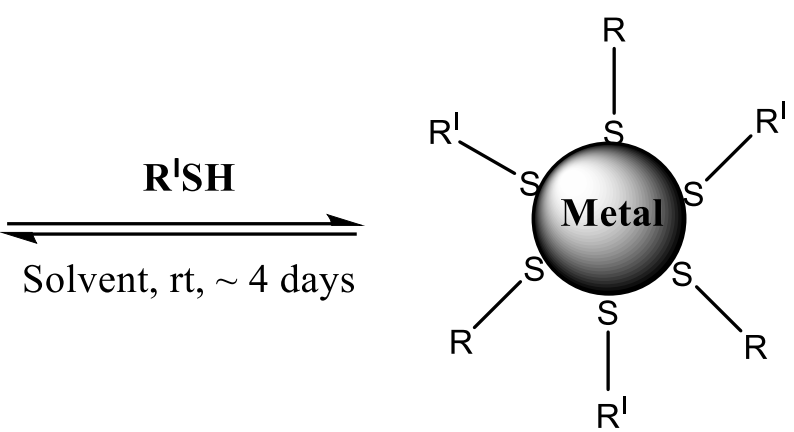

Thiolated-Metal MMPCs

Figure 1.7. A typical ligand-place exchange reaction to from metal MMPCs. -SR: parent thiolate, and -R'SH: a new ligand.

Several studies aimed at understanding the mechanism of the place-exchange reaction have suggested that the mechanism is somewhat comparable to ligand substitution reactions in metal complexes, ${ }^{37}$ but it is still not conclusive as to the exact pathways involved. The possible mechanisms are associative, dissociative, and intermediate mechanisms. An associative mechanism is like the $\mathrm{S}_{\mathrm{N}} 2$ type reaction in organic chemistry, where one incoming ligand first interacts and adsorbs onto the $\mathrm{Au}$ MPC with simultaneous desorption of one of the parent ligands from the Au MPC surface. In the associative rate-determining step, an incoming ligand enters the monolayer and protonates a bound thiolate ligand. A dissociative mechanism resembles the $\mathrm{S}_{\mathrm{N}} 1$-type reaction mechanism, where one of the parent ligands first dissociates from the Au MPC surface and leaves a vacant site for the incoming ligand to bind with the $\mathrm{Au}$ MPC. Moreover, it is also possible that both the incoming and outgoing ligands are incorporated into the Au MPCs, but they are not fully desorbed or adsorbed yet. Such weak interaction between metal core and ligands could potentially lead to the easy removal of ligands during their use in various applications and that could be either advantageous or could 
cause aggregation of particles. Hostetler and coworkers found that the presence of defect sites on the surface of MPCs causes inhomogeneous coverage of the ligands. ${ }^{38}$ These sites include vertices, edges, and grain boundaries on the MPC surface. Their studies revealed that the ligands are less crowded and less organized in these areas, giving the incoming ligands more opportunity to interact with the MPCs surface. The exchange is very fast at the beginning, presumably when the reaction occurs at the defect-rich sites, like the corners and edges of the MPCs. However, the reaction slows down dramatically later, possibly due to steric hindrance along the terrace and smooth regions of the $\mathrm{Au}$ MPC, where the ligands are well packed, organized, and crowded (Scheme 1.1). The kinetic studies of such reactions also revealed that rates of reaction also indeed depend on the concentration of parent and new ligands in the solution.

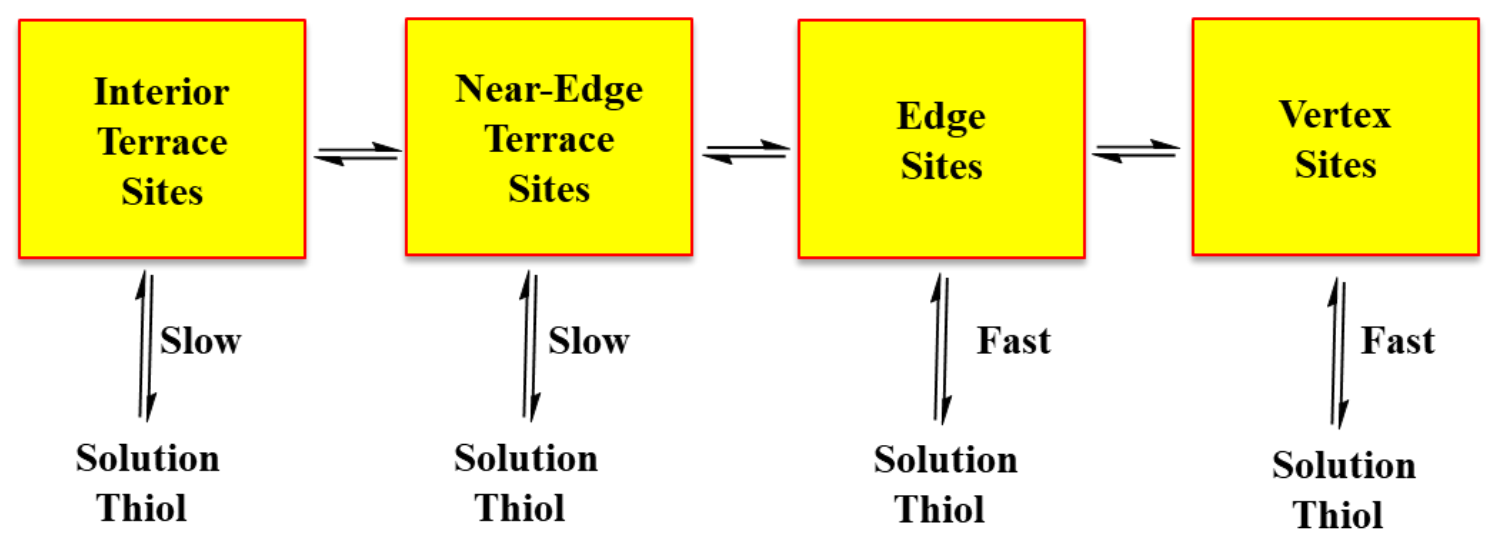

Scheme 1.1. A schematic model of processes that takes place during ligands-place exchange reaction on thiolated-Au MPCs.

We are aware that the development of aminooxy-functionalized thiol ligands to improve ease of carbonyl ligation as well as to provide flexibility in addressing solubility and adduct loading considerations could potentially enable new applications in this field. 
Such potential motivated us to design a synthesis pathway of aminooxy alkane thiol ligand for use in the functionalization of the metal MPCs.

\subsection{Uses of Aminooxy Chemistry and Nanoparticles}

The chemoselectivity of aminooxy reactions has attracted the attention of researchers to explore its application in the rapidly emerging field of MPCs with respect to monolayer functionalization. The features of metal MPCs, such as particle core and surface monolayer structure, organic vs. inorganic composition, particle size, and optical and electronic properties, often determine the application. Much research has been done involving MPCs surface containing aminooxy functionality. Indeed, due to the ease of oxime ether formation, aminooxy compounds have been used for surface decoration of nanoparticles via chemoselective conjugation with carbonyl substrates. A study by Zhang et al, ${ }^{39}$ established a colorimetric assay for $\alpha$-glucosidase activity and screening of its inhibitor. They used 4-aminophenyl- $\alpha$-D-glucopyranoside (pAPG) - functionalized Au NPs to obtain a color change of a test solution based upon aggregation of NPs induced by the interaction between 1,4-phenylenediboronic acid (PDBA) and pAPG. Such type of studies suggested that aminooxy functionality could be introduced onto the MPCs surface for understanding the specific binding activity with polypeptide aldehyde.

Thygesen et al. used the combination of material science and biological applications to prepare glycan-functionalized Au NPs in order to study the proteincarbohydrate interactions. ${ }^{40}$ Their study also was focused on the synthesis of complex Au-glyconanoparticles via click-chemistry. They reacted the aminooxy moiety of the 
linker with a carbohydrate, such as glucose, maltose and maltotriose. Then later, Thygesen and co-workers developed a bifunctional thiol-aminooxy oligo(ethylene glycol) ligand for preparation of aminooxy-conjugated gold glycanoparticles (Figure 1.8). ${ }^{2} \mathrm{~A}$ citrate Au NPs (diameter $~ 12 \mathrm{~nm})$ with a metal core $(\mathrm{Au})$ and a reactive shell $\left(-\mathrm{ONH}_{2}\right.$ functionality) was prepared to decorate the surface of NPs with the unmodified glycans via means of oximation reaction. Protecting groups $(\mathrm{Pg})$ were deprotected using hydrazine monohydrate to reveal the aminooxy group without compromising the NPs properties. An aldehyde-containing biomolecule (polypeptide aldehyde) was anchored onto the metal surface under mild aqueous conditions. A colorimetric assay consisting of a binding protein was used for the colorimetric detection of lectin interactions with high specificity. This strategy allows the direct quantification of glycan-protein interactions with high specificity. Such studies could facilitate the rapid screening of biomolecular interactions that could be potentially useful in drug development and discovery.

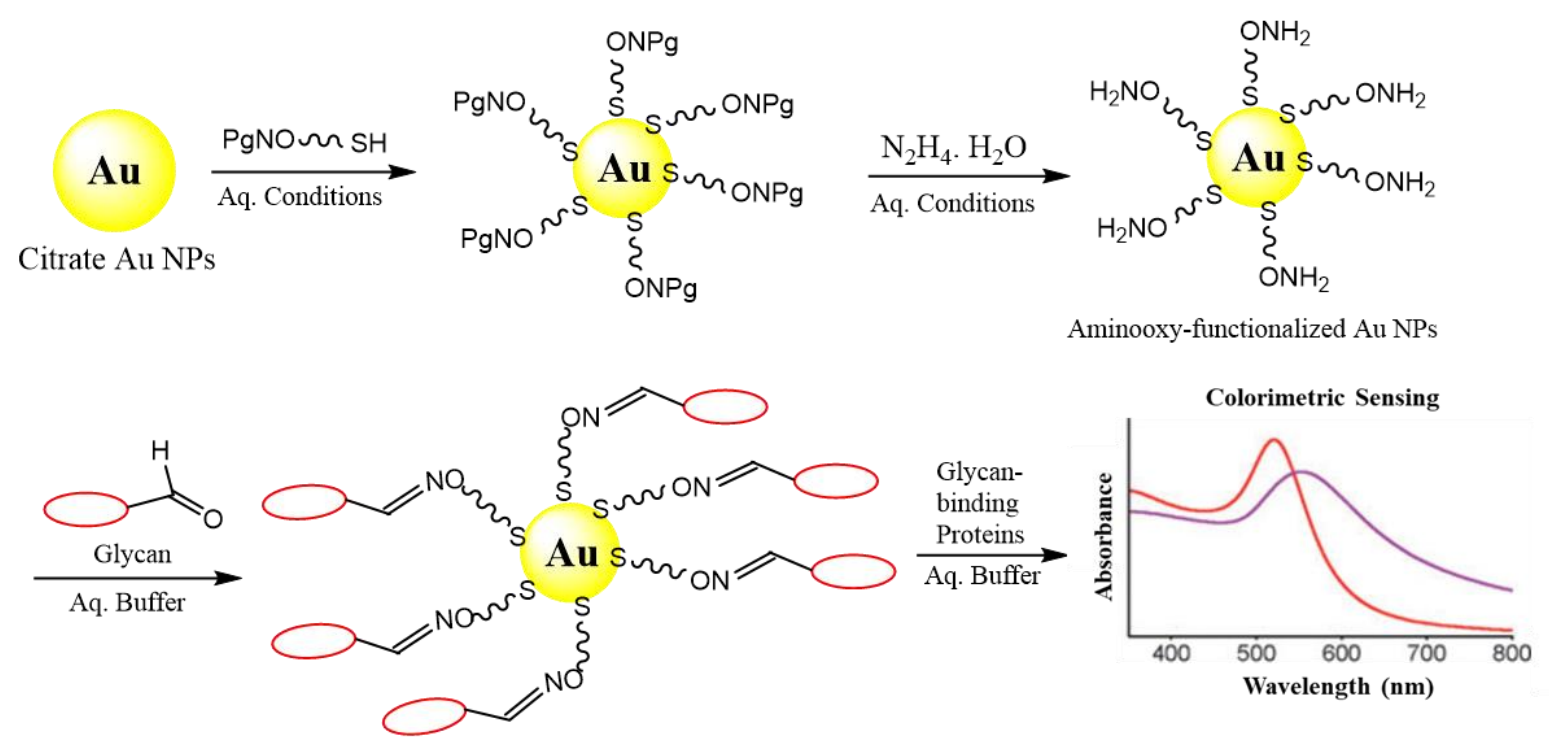

Figure 1.8. Schematic diagram of strategy deployed by Thygesen et al, as a model for the detection of glycoproteins. $\mathrm{Pg}=$ Trimellitoyl 
Nagahori et al. prepared aminooxy-functionalized Au MPCs for capture and enrichment of glycosphingolipid (GSL)-generated aldehydes as a means to characterize whole GSLs in living cells. ${ }^{3}$ A high-throughput structural profiling method, which they called "glycoblotting" was developed for a functional characterization of living cell surfaces for GSLs. Their method involves the extraction of GSLs from the cell, followed by selective ozonolysis of the $\mathrm{C}=\mathrm{C}$ bond to generate an aldehyde moiety. Subsequent enrichment on aminooxy-functionalized NPs then occurs via chemical ligation (i.e., oximation) (Figure 1.9). Surface plasmon resonance (SPR) based functional analysis and MALDI-TOFMS based structural analysis on mouse brain gangliosides suggested that this method of selective interaction of GSL directly from the living cells constitutes a novel and standardized method that enables simultaneous analysis of structural and functional GSLs.

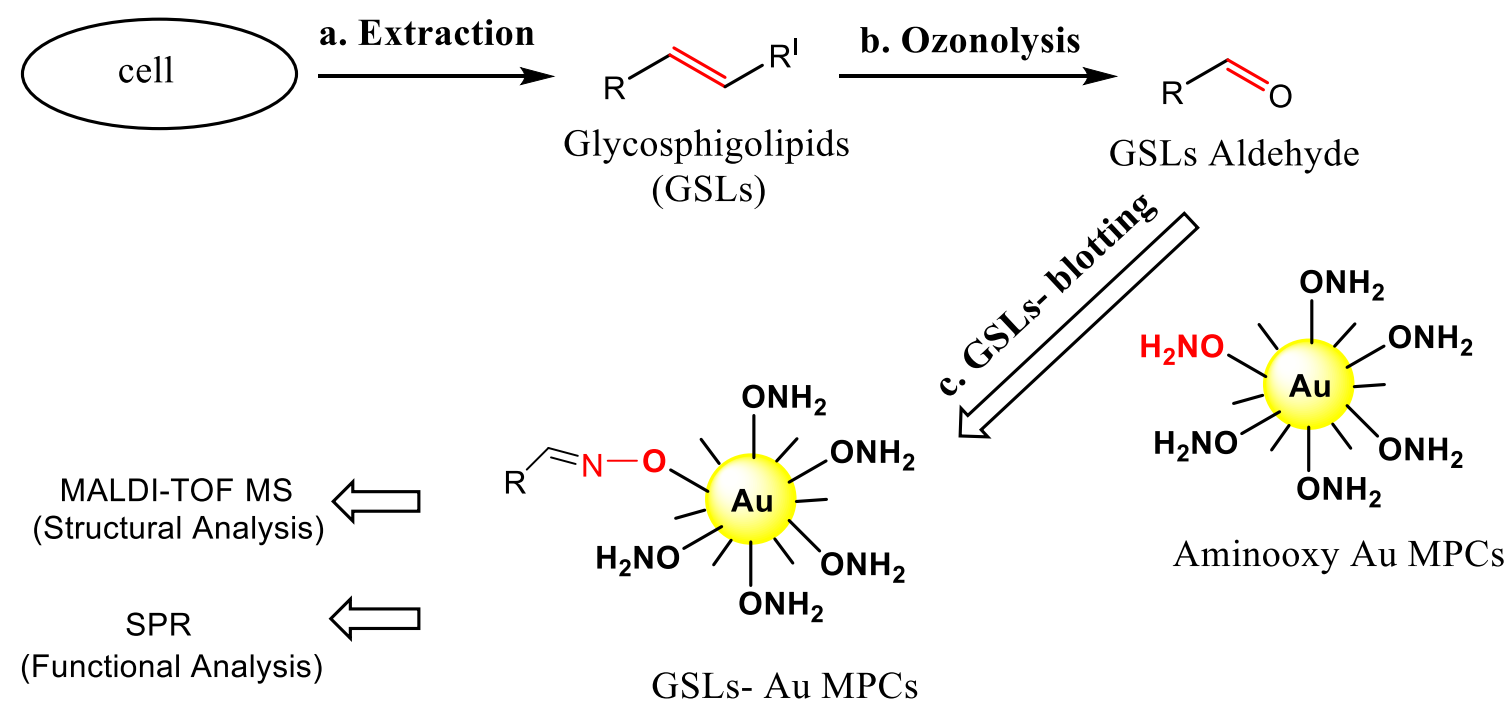

Figure 1.9. A schematic of "glycoblotting" concept introduced by Nagahori et al.

Maynard and coworkers synthesized a photo-caged aminooxy alkane thiol for conjugating carbonyl substrates to Au surfaces following photolysis. ${ }^{41}$ In their studies, an 
in-house synthesized 2-(2-nitrophenyl)propyloxycarbonyl (NPPOC) protected alkane aminooxy thiol ligand was used to form a SAM, followed by a deprotection via exposure to a hand held UV light $(365 \mathrm{~nm})$, to reveal the aminooxy groups on the SAM surface, and subsequent surface conjugation with ethyl levulinate (a small molecule with a ketone moiety-used to modify proteins) via oxime bond formation as shown in Figure 1.10. Evidence of successful deprotection and conjugation was monitored by FTIR spectroscopy and contact-angle measurements. This study provides a foundation for eventual studies focused on the immobilization of the biomolecule on the metal surfaces for a variety of applications.

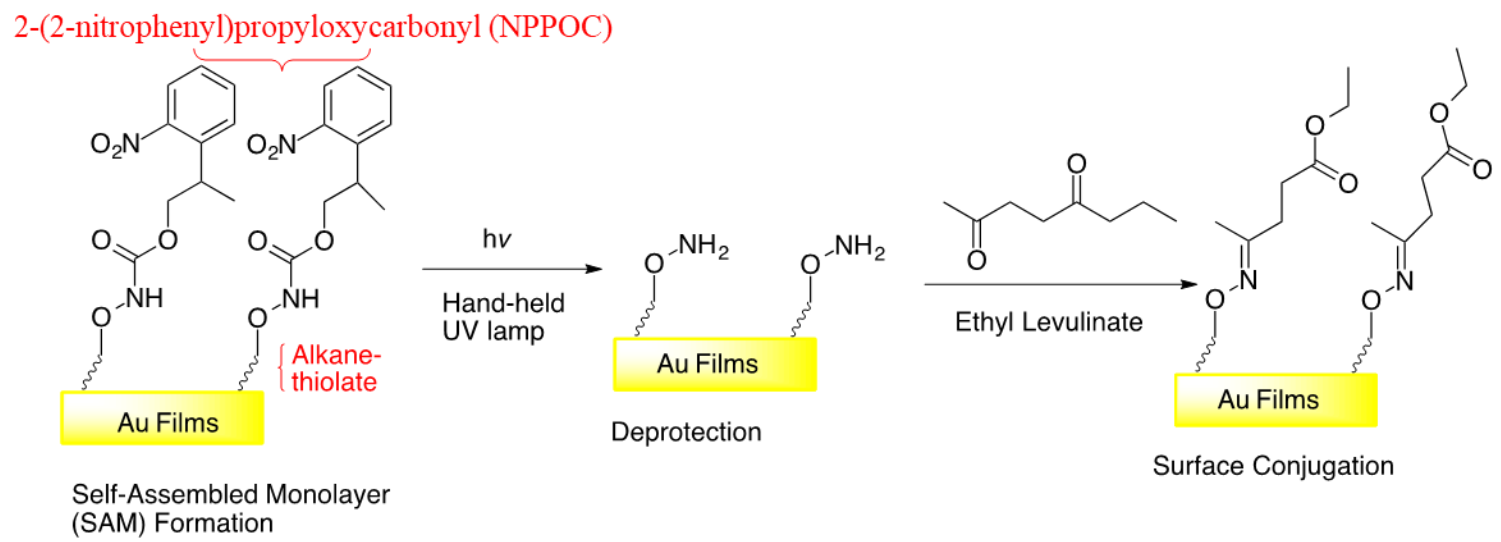

Figure 1.10. A scheme of photodeprotection and bioconjugation strategy studied by Maynard and co-workers.

Another interesting example relating to the ligation properties of the aminooxy functionality involves immobilization of Au NPs to form self-assembled NP monolayers, as demonstrated by Chan and $\mathrm{Yu}$ in $2002 .{ }^{42}$ In their studies, they coated Au NPs using a mixture of dodecanethiol and 11-mercapto-2-undecanone, hence forming a mixed monolayer on the surface consisting of alkane and ketone functionality. It is imminent 
that only the ketone functionality would react with the surface-confined aminooxy group (Figure 1.11). The Au MMPCs were added to the aminooxy-containing Au surface with stirring at room temperature for $\sim 12$ hours followed by rinsing the surface with dichloromethane and ethanol to remove any unreacted Au NPs. In this way, selfassembled NP monolayers were obtained. Cyclic voltammetry, FTIR, TEM and proton NMR measurements suggested that the chemoselective ligation strategy was successful as the Au MMPCs remained stable and uniformly distributed on the surface after immobilization. This is a simple and flexible method for the immobilization of Au NPs onto a solid support, which will be discussed in a later section.

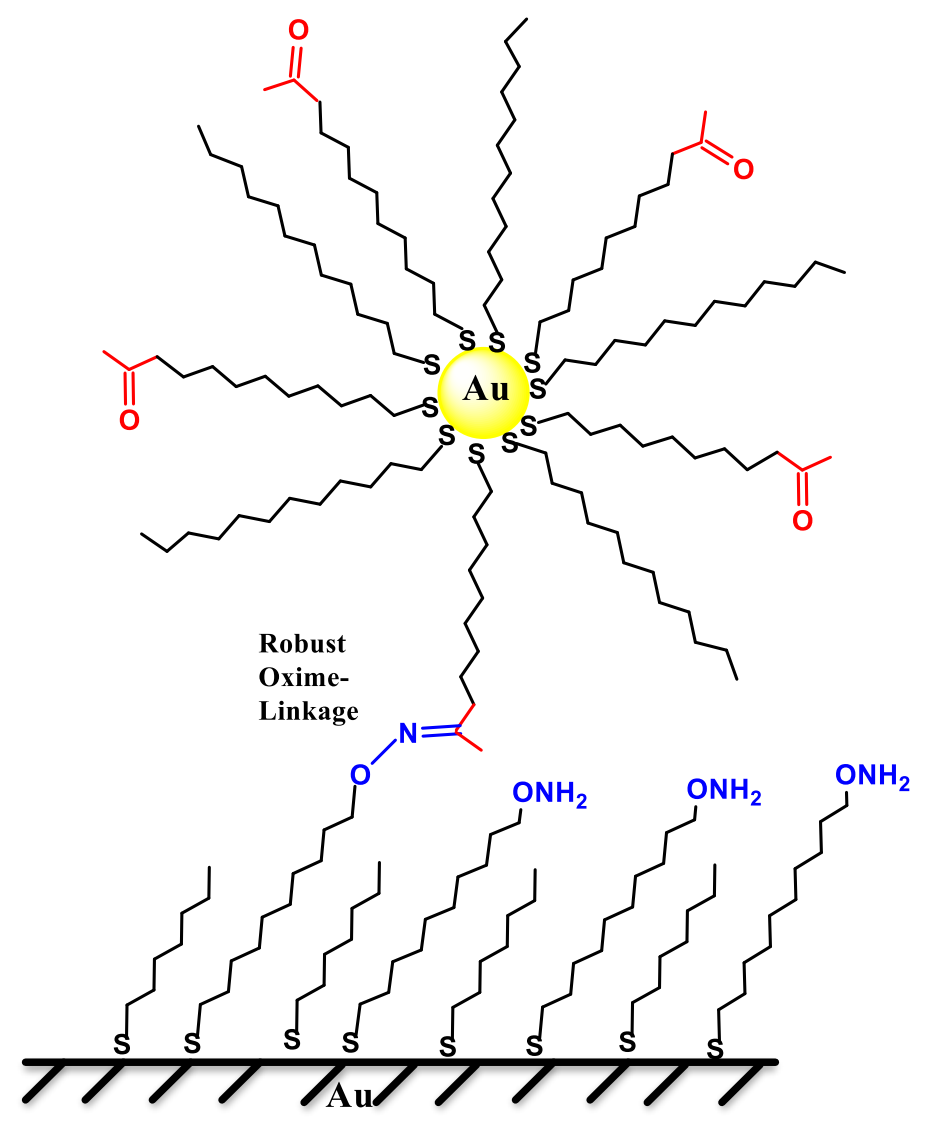

Figure 1.11. A schematic of NPs self-assembled onto an Au surface via stable oximelinkage formation at the interface. 


\subsection{Catalytic Applications of MPCs}

MPCs possess unique physical and chemical properties due to their nanoscale size and high surface area. ${ }^{43}$ Their size, shape and structure determine their reactivity, optical and electronic properties, and other properties, which in turn determines their applications. NPs are the leading edge of the rapidly developing field of nanoscience because of their applications in the field of catalysis, ${ }^{44}$ sensing, ${ }^{45}$ drug delivery, ${ }^{46}$ biology and medicine, ${ }^{47}$ electronics, ${ }^{48}$ optics. ${ }^{49}$ NPs can be formed from diverse chemical constituents such as metals, metal oxides, carbon, polymers, and semiconductors. ${ }^{50}$ NPs are also used to improve the photovoltaic performance of solar cells. ${ }^{51,52}$ This dissertation is mostly focused on the utilization of metal MPCs as catalysts and potentially as sensors. As described below, Au- and Pd-based glutathione-stabilized MPCs are used as homogenous catalysts ${ }^{53}$ and aminooxy chemistry is also examined to prepare a solid-supported Au MPCs catalyst. The potential of our work in the development of a sensor is described in Chapter 7 (Future Directions).

In a simple definition, a catalyst is a substance used in a reaction, often in substoichiometric amounts, to accelerate the rate of the reaction. The main advantage of using a catalyst is the reduction of time needed for completion of the reaction. To be cost effective, the catalyst ideally should be reusable. Generally, catalysts can be classified as homogenous or heterogenous; however, a pseudo-homogenous catalyst is also common nowadays. ${ }^{54,55}$ A homogenous catalyst is in the same phase (e.g., liquid) as the substrate, while a heterogenous catalyst is in a different phase to that of the reacting substrate (Scheme 1.2). In a typical pseudo-homogenous reaction, a biphasic solvent system is used with organic and aqueous phases, where the catalysis most likely occurs at the 
interface of the solvents. This type of catalyst allows the easy isolation of product and separation of the catalyst for use in multiple reaction cycles.
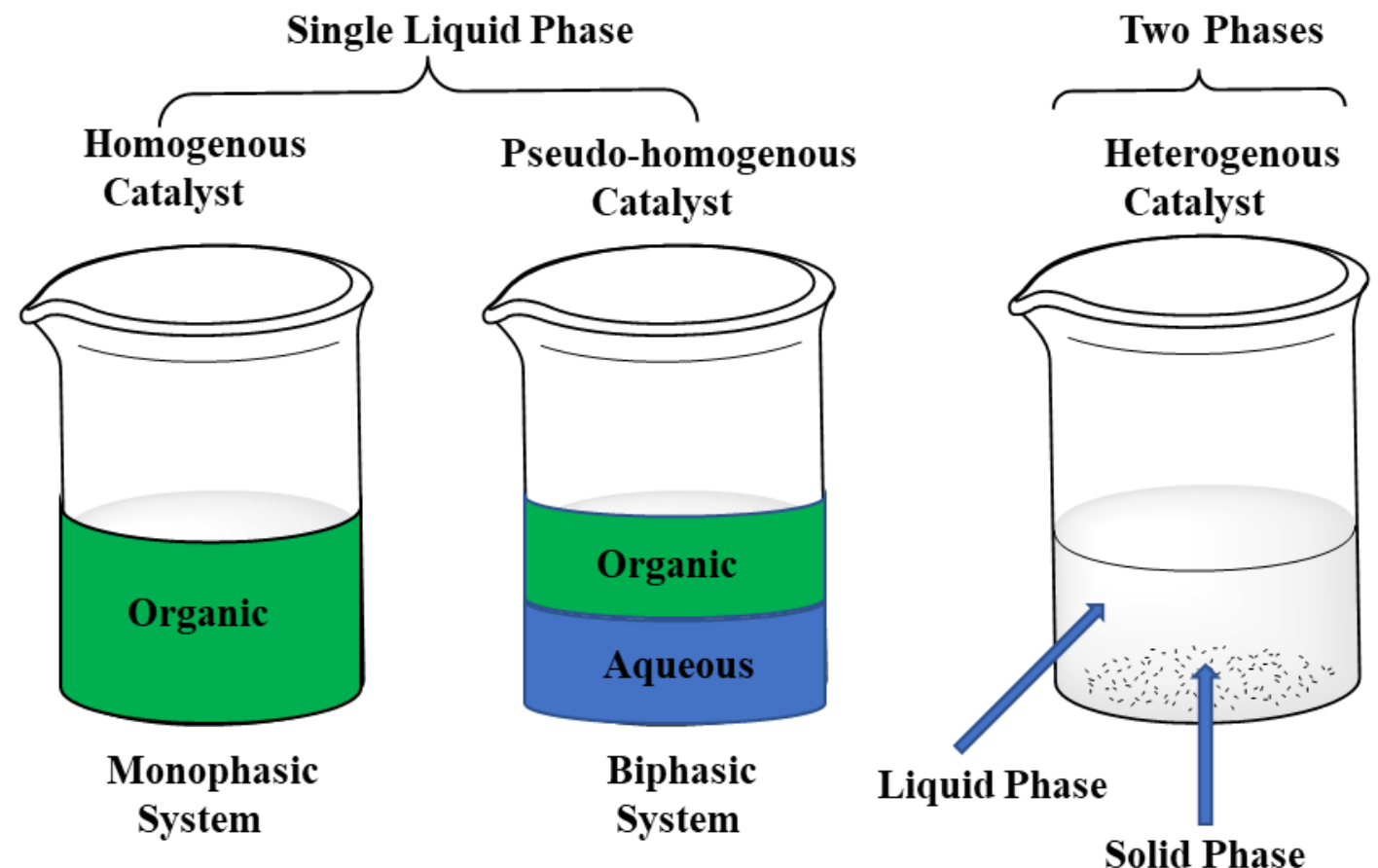

Scheme 1.2. A diagram representing three different types of catalytic system.

Catalysts are extremely useful on industrial scales as they can significantly decrease the overall production cost of manufactured chemicals. Improving the recyclability of catalysts is always a priority of researchers. Various groups tend to use heterogenous catalysts to obtain better recyclability, however heterogenous catalysts usually suffer from slower reaction rates compared to homogenous catalysts. ${ }^{56}, 57$ Usually, the phase containing most of the formed products are separated from the other phase using a general extraction procedure. For example, if the catalyst is in the aqueous phase and the reaction products favor the organic phase, the organic layer will be separated and additional organic solvent added in a second extraction to remove the 
remaining product from the aqueous layer as shown in Scheme 1.3. This is a useful strategy as one can simply separate the catalyst and reuse it many times by following the same extraction steps in consecutive reaction cycles.

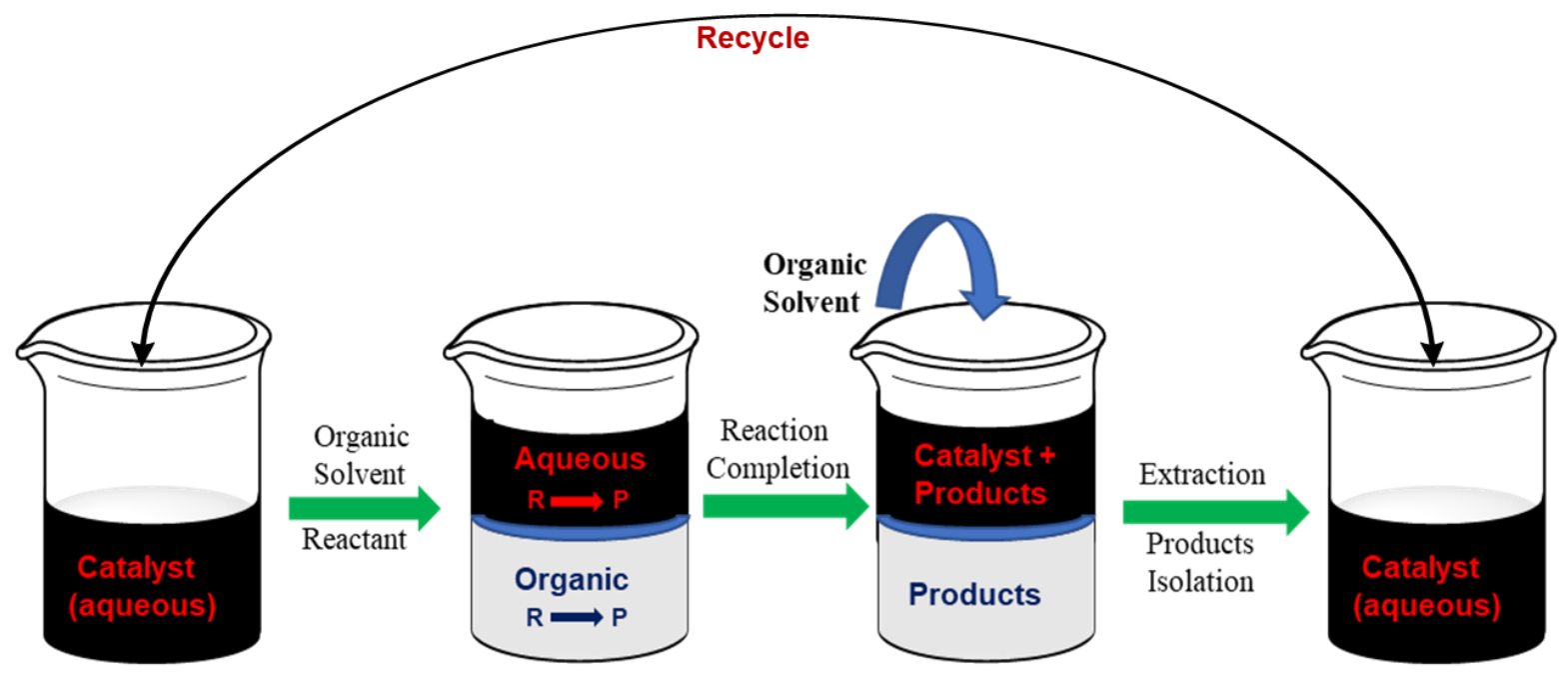

Scheme 1.3. General schematic of catalytic cycle in a biphasic system $(\mathrm{R}=$ Reactant, $\mathrm{P}$ $=$ Product $)$.

NPs are widely used as a catalyst because of their high surface area to volume ratio. Usually, NPs are synthesized in the presence of suitable stabilizers (ligands) to avoid aggregation of the particles, which is one of the key requirements for NP stability and ensuring it acts as a homogeneous catalyst. The ligands are known to exhibit less steric crowding on regions of the NPs with vertices, edges and grain boundaries and thus have higher surface energy to make those areas more reactive for catalysis to occur. When using metal NPs without stabilizers as a catalyst, a faster reaction rate would be achieved due to a more accessible surface; however, NP aggregation will occur, which lowers the accessible surface and makes the NPs heterogeneous (Scheme 1.4). While, in cases of the NPs with stabilizers, despite the slower reaction rate, the stability of the 
particles is mostly unchanged after completion of the catalytic cycle. Synthesizing metal NPs or MPCs that provide both a faster reaction rate as well as maintaining high stability is a considerable challenge. One of the methodologies introduced to do so is via ligand removal (discussed later). A capping agent (i.e., stabilizer) will cover the surface of the metal core and thus not provide much of an opportunity for an incoming substrate to interact with the NPs, resulting in little product/s formation but the reaction are usually slower. Therefore, a partial ligand removal strategy is found to be useful to enhance the catalytic activity without compromising the stability of the particles. An example is the partial removal of thiolate ligand stabilizers with iodine as illustrated in Scheme 1.4 and described in Chapter 5. 


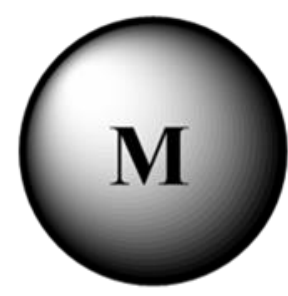

Metal Nanoparticles without stabilizers

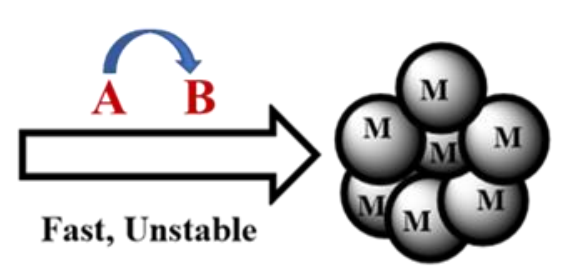

Aggregated

Nanoparticles

Heterogeneous

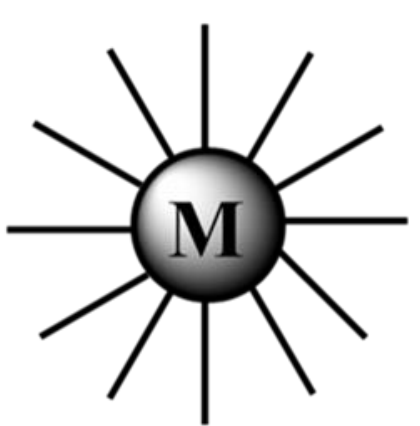

Metal Nanoparticles with stabilizers

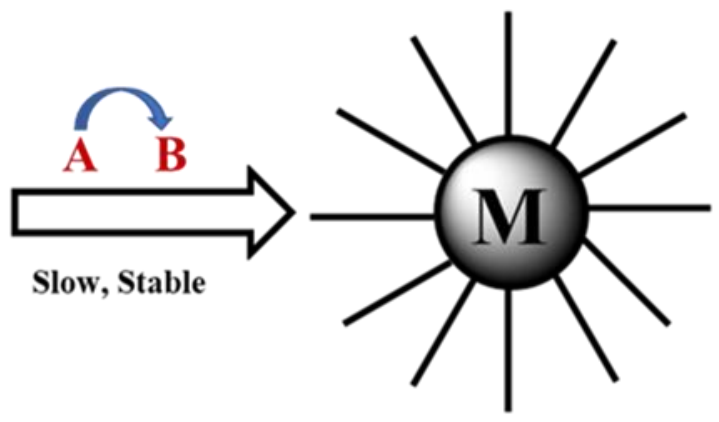

Stable Nanoparticles

Homogeneous

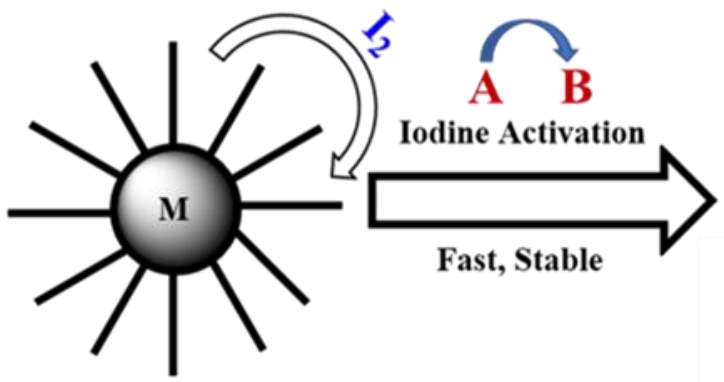

Metal Nanoparticles with stabilizers

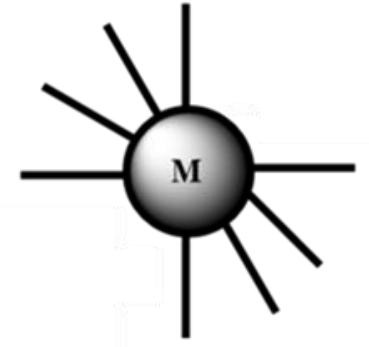

Stable

Nanoparticles

Scheme 1.4. General schematic of catalytic activity and stability of nanoparticles.

\subsubsection{4-Nitrophenol Reduction}

Reduction of nitro compounds to amino compounds is industrially important as the amino product that is formed is often a precursor material for the synthesis of drugs. ${ }^{58}$ For example, 4- aminophenol (reduced form of 4-nitrophenol) is reacted with acetic 
anhydride to form paracetamol. ${ }^{59}$ Although, $\mathrm{NaBH}_{4}$ itself can reduce a nitro compound, it is not found to be effective. Therefore, the presence of a catalyst is required for an efficient conversion. Au-based NPs are found to be a more effective catalyst for this reduction despite the usefulness of other catalysts such as $\mathrm{NiCl}_{2}, \mathrm{TiCl}_{4}, \mathrm{Cu}(\mathrm{OH})_{2}, \mathrm{Cu}^{\mathrm{II}}$, Pd-C, Ag NPs, etc. for nitro reduction. ${ }^{60}$ The reduction of 4-nitrophenol (4-NP) to 4aminophenol (4-AP) by sodium borohydride ${ }^{61}$ is a well-studied reaction using a variety of free or immobilized NPs/clusters as catalysts (Scheme 1.5).

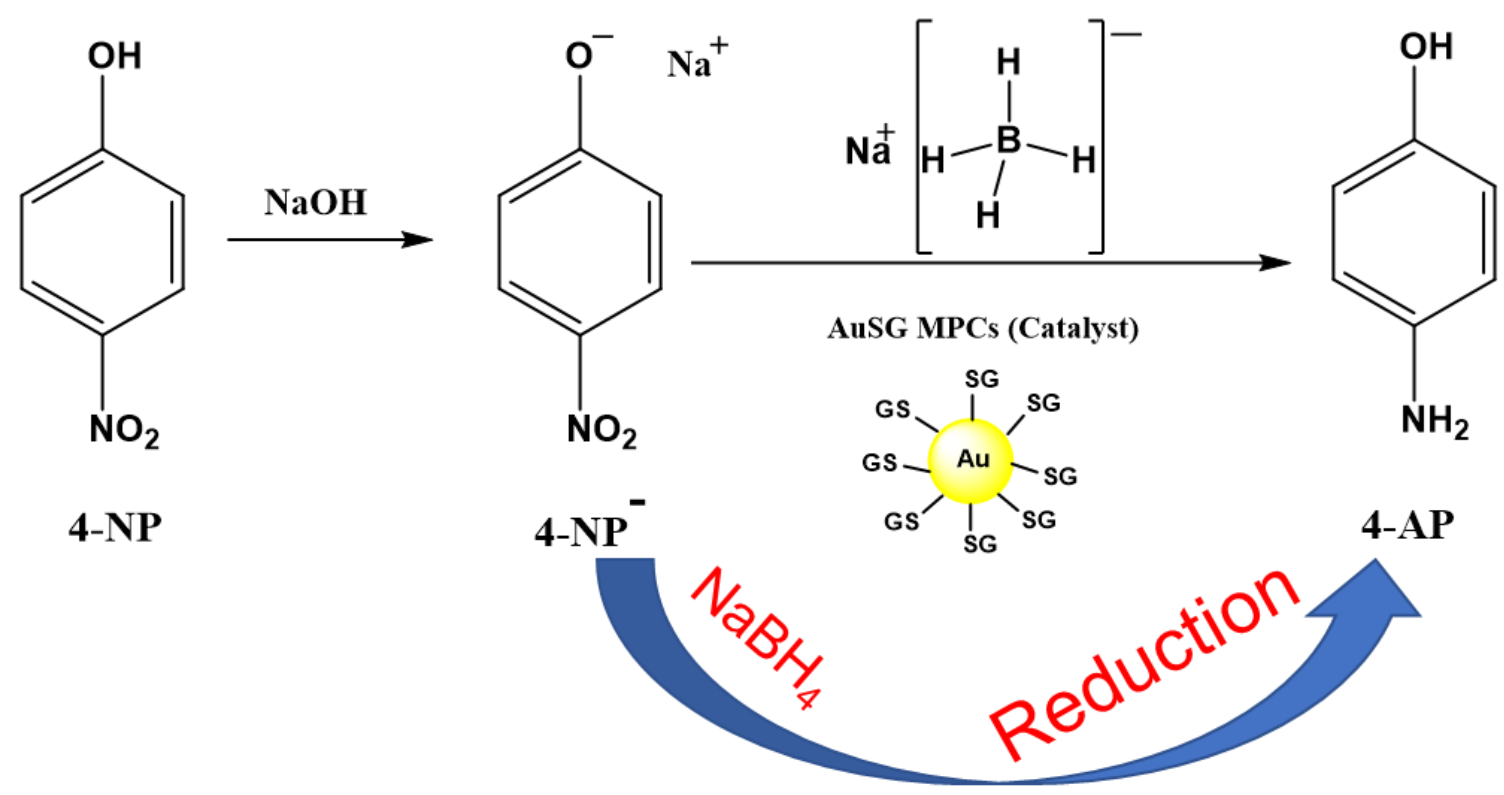

Scheme 1.5. AuSG-catalyzed model reaction of 4-NP reduction to 4-AP by $\mathrm{NaBH}_{4}$. (SG = glutathione)

Wunder and co-workers ${ }^{62}$ proposed a Langmuir-Hinshelwood mechanism, ${ }^{63}$ which suggests that catalytic reduction occurs at the surface of the metallic NPs. Firstly, borohydride $\left(\mathrm{BH}_{4}^{-}\right)$adsorbs and transfers a hydride to the surface of the MPCs, resulting 
in the development of Au-hydride bonds. Next, the 4-nitrophenolate ion also adsorbs onto the surface of the MPCs. The nitro group of the adsorbed 4-NP then accepts the hydride and electrons from the Au-hydride complex. Finally, after a series of hydrodeoxygenation reactions, 4-AP is formed as the product (Scheme 1.6). Ciganda and coworkers reported that ligand is displaced by the substrate during the catalytic process, eventually leading to longer induction times for MPCs with strongly bound ligands on surface. ${ }^{64}$ This led us to explore a general and effective ligand removal strategy that could potentially facilitate a ligand displacement process from the Au surface (Section 1.9).
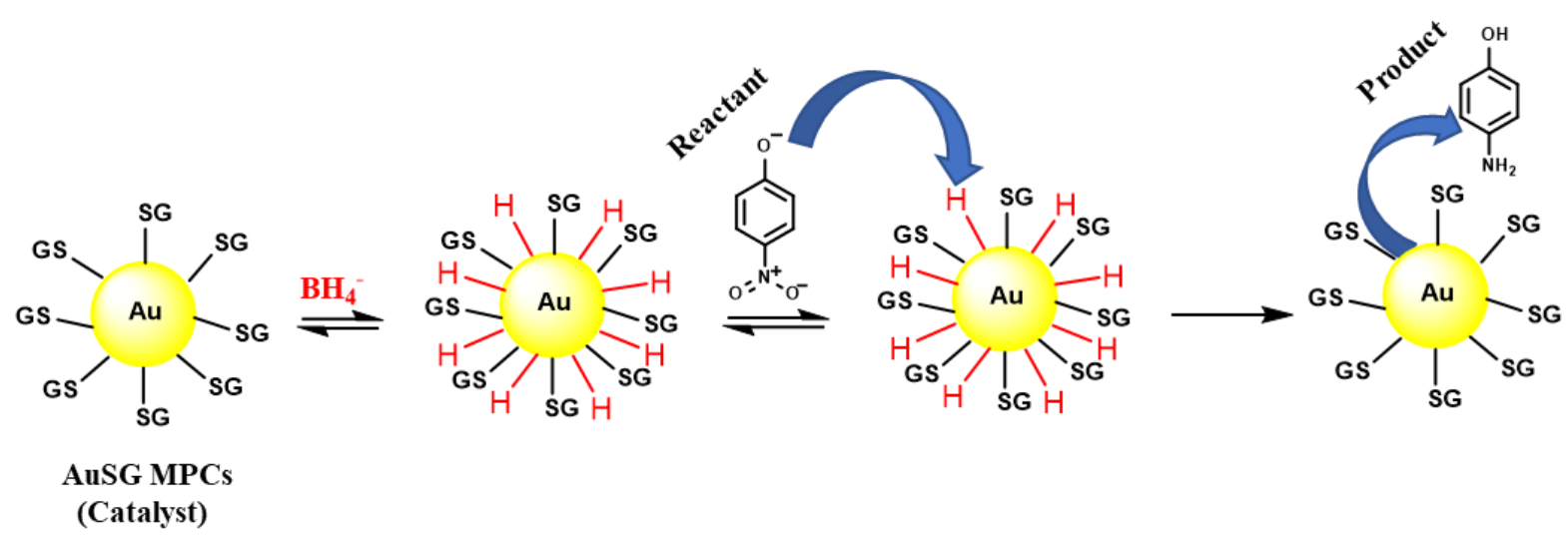

(Catalyst)

Scheme 1.6. A mechanistic route for the Au MPC-catalyzed 4-NP reduction in the presence of excess $\mathrm{NaBH}_{4}$.

4-NP reduction is a model reaction commonly used to understand the catalytic properties of Au-based NPs/MPCs. However, different types of nanostructures have been used to study this reaction. Among several previous studies, Haldar et al. synthesized 
bimetallic $\mathrm{Au} / \mathrm{Ag}$ core-shell NPs for use in the reduction of 4-NP to form 4-AP.65 Stabilizers such as PVP, cysteine, and cyclodextrin were used to synthesize core-shell structure with sizes ranging from 10-100 $\mathrm{nm}$ core Au NPs. In their studies, they found that the catalytic activity of the reaction increased with increasing core-size of the $\mathrm{Au} / \mathrm{Ag}$ core-shell NPs. A 12-fold enhancement was afforded on using $\mathrm{Au}_{100} / \mathrm{Ag}$ bimetallic NPs as compared to pure $\mathrm{Au}_{100} \mathrm{NPs}$. Dasog et al. showed that sterically-hindered active sites and rapid growth of MPC size resulted in different catalytic activity towards 4-NP reduction between Au MPCs and poly(vinylpyrrolidone)(PVP)-stabilized Au NPs. ${ }^{66}$ They noted that the thiolated MPCs they studied showed lower activities than PVPstabilized Au NPs, mostly due to the blocking of surface area by passivated MPCs, despite their small sizes. Yamamoto et al. examined the catalytic properties for the reduction of 4-NP to 4-AP of surfactant free Au nanoclusters (NCs) synthesized in N,Ndimethylformamide (DMF). DMF stabilized Au NCs were synthesized according to the DMF reduction method. ${ }^{67}$ They reported a rate constant of $0.18 \mathrm{~min}^{-1}$ observed for the 4NP reduction catalyzed by DMF-stabilized Au NCs. ${ }^{68}$ The coordinating effect of DMF in this case ${ }^{69}$ may be responsible for the slower reaction rate. However, $\mathrm{Au}_{25}(\mathrm{SG})_{18}$ clusters showed higher catalytic activity, suggesting that the active sites of such clusters were not sterically hindered, possibly due to the unique core-shell like structure. Recently, Tan and coworkers ${ }^{70}$ developed a highly efficient heterogenous catalyst using $\mathrm{Au}$ NPs incorporated into hyper-cross-linked polymers of triphenylmethanethiol (HCPTPMT) that have a tunable surface area and narrow pore size. By varying the ratio of $\mathrm{HAuCl}_{4} \cdot 4 \mathrm{H}_{2} \mathrm{O} / \mathrm{HCP}-\mathrm{TPMT}$, they controlled the size range and mol $\%$ of Au. Their work showed that the use of polymers as a catalyst support is an effective strategy to achieve 
high catalytic activity as well as recyclability toward 4-NP reduction (using Au NPs sized between 1.7-5.1 nm).

\subsubsection{Hydrogenation/Isomerization of Allyl Alcohols}

The hydrogenation/isomerization of allylic alcohols is one of the common and widely used model reactions to test the catalytic activity of various organic-stabilized $\mathrm{Pd}$ MPCs (Scheme 1.7). Various groups have studied the activity and selectivity for hydrogenation and isomerization of allyl alcohols either using a homogenous/pseudohomogenous system (monophasic or biphasic) or using a heterogenous system. Pd catalysts such as $\mathrm{Pd} / \mathrm{TiO}_{2}{ }^{71}$ or $\mathrm{Pd}$ NPs immobilized on composites or embedded in polymers or electrolyte films ${ }^{72}$ have been used as heterogenous catalysts for this reaction. Our work focused on both a homogenous and heterogenous system with the goal to improve the turnover frequency (TOF) and recyclability of the catalyst.

Very recently, Shon and co-workers synthesized water-soluble 6-mercaptohexanoic acid and 8-mercaptooctanoic acid-capped Pd NPs as water-soluble catalysts for use in a biphasic organic system. ${ }^{73}$ Their studies revealed that the catalytic activity and selectivity of allylic alcohols are dependent on the $\mathrm{pH}$ of the NPs solution and size of the substrates. Previously, Shon and co-workers reported the selectivity for the isomerization of allyl alcohol using dodecanethiolate-capped Pd NPs generated from thiosulfate, where

an $80 \%$ reaction completion was obtained after one hour. ${ }^{74,} 75$ Cliffel and co-workers described the synthesis of organomercaptan ligand-protected Pt NPs to study their role as a catalyst for hydrogenation of allyl alcohol to propanol and maleic acid to succinic acid. ${ }^{76}$ They found differences in activity due to variations in ligand chain length, branching, charge, packing density and core size. Similarly, Crooks and co-workers 
synthesized various Pd nanoparticles encapsulated within poly(amidoamine) dendrimers of different generations and functionalized them with different end groups and then showed their substrate size-selective catalytic activity. ${ }^{77}$ Their studies suggested that steric crowding on the dendrimer surface led to lower TOF values for larger substrates. Moreover, Pd/Pt dendrimer-encapsulated alloy NPs have higher catalytic activity $(\mathrm{TOF}=193)$ than dendrimer-encapsulated Pd or Pt NPs $(\mathrm{TOF}=50)$ for the hydrogenation of allyl alcohol..$^{78}$ The work of Shon and coworkers represents an example of thiol ligand-stabilized Pd NPs for catalysis, where they focused on understanding the mechanism and regioselectivity of hexanethiolate- and dodecanethiolate-capped Pd NPs in different environments. ${ }^{79}$ They concluded that alongside hydrogen, the choice of solvent is also an essential factor for the catalytic isomerization reaction.

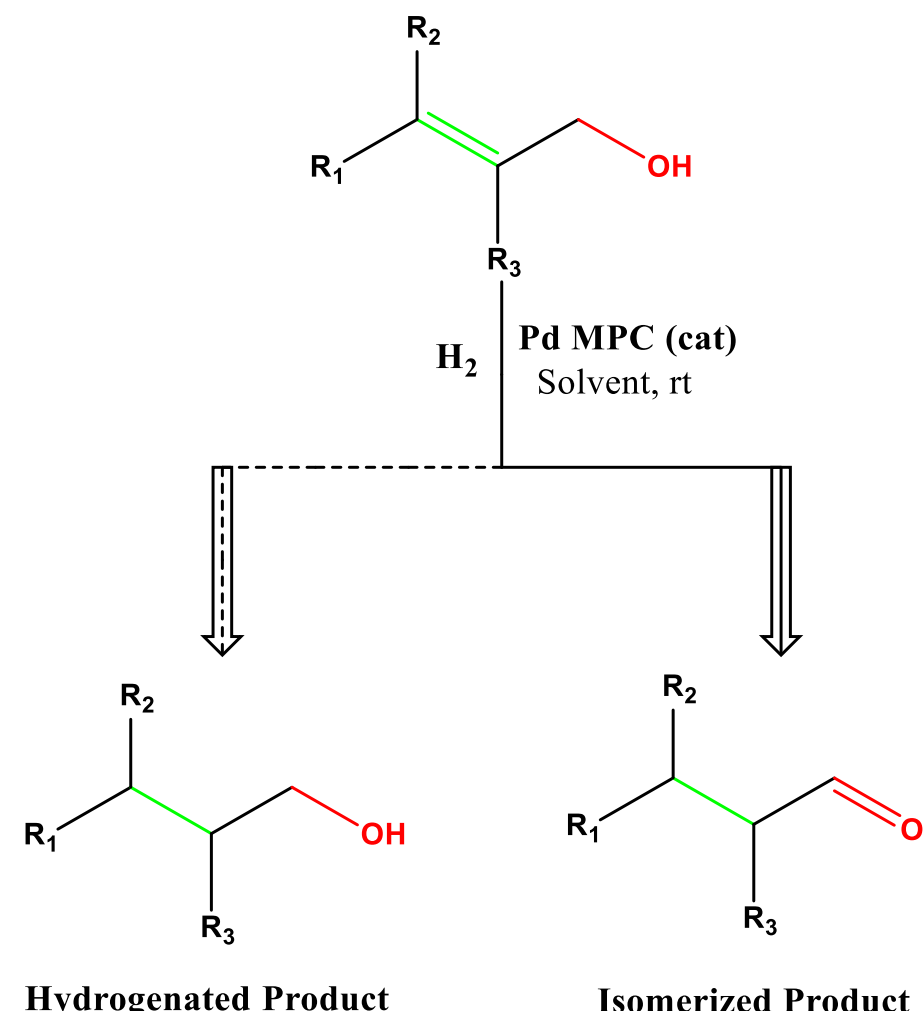

Scheme 1.7. A general schematic of the hydrogenation/isomerization of allyl alcohol. 
In order to explain the Pd-based catalytic isomerization and hydrogenation of allylic alcohol, two different mechanism routes —Pd-alkyl mechanism and $\pi$-allyl Pd hydride mechanism—-have been proposed. ${ }^{80} \mathrm{In}$ a Pd-alkyl mechanism, the presence of $\mathrm{H}_{2}$ gas is necessary to form the $\mathrm{Pd}-\mathrm{H}$ species that proceeds with an adsorption of substrate and the initiation of the catalytic reaction. The selectivity (hydrogenation vs isomerization) of the reaction depends on the insertion mode of Pd-H species to the $\pi$ bond of the allylic group. When the insertion mode follows a Markovnikov-type of addition, it will result in the formation of a branched Pd-alkyl intermediate and lead to an isomerized product (Propanal), whereas the anti-Markovnikov addition will yield a linear Pd-alkyl intermediate and lead to the hydrogenated product (1-Propanol), as shown in Scheme 1.8. In comparison, the $\pi$-allyl Pd hydride mechanism route (not shown) does not require the presence of $\mathrm{H}_{2}$ gas, as it follows oxidative addition of $\mathrm{Pd}$ to the $\mathrm{C}-\mathrm{H}$ bond adjacent to the OH group for generating the Pd-H species. Same products are expected to be formed through this route as well. In our study, presence of $\mathrm{H}_{2}$ gas was a requirement for reaction to occur, which suggests that the catalytic reaction of allyl alcohol followed Pd-alkyl mechanism. 


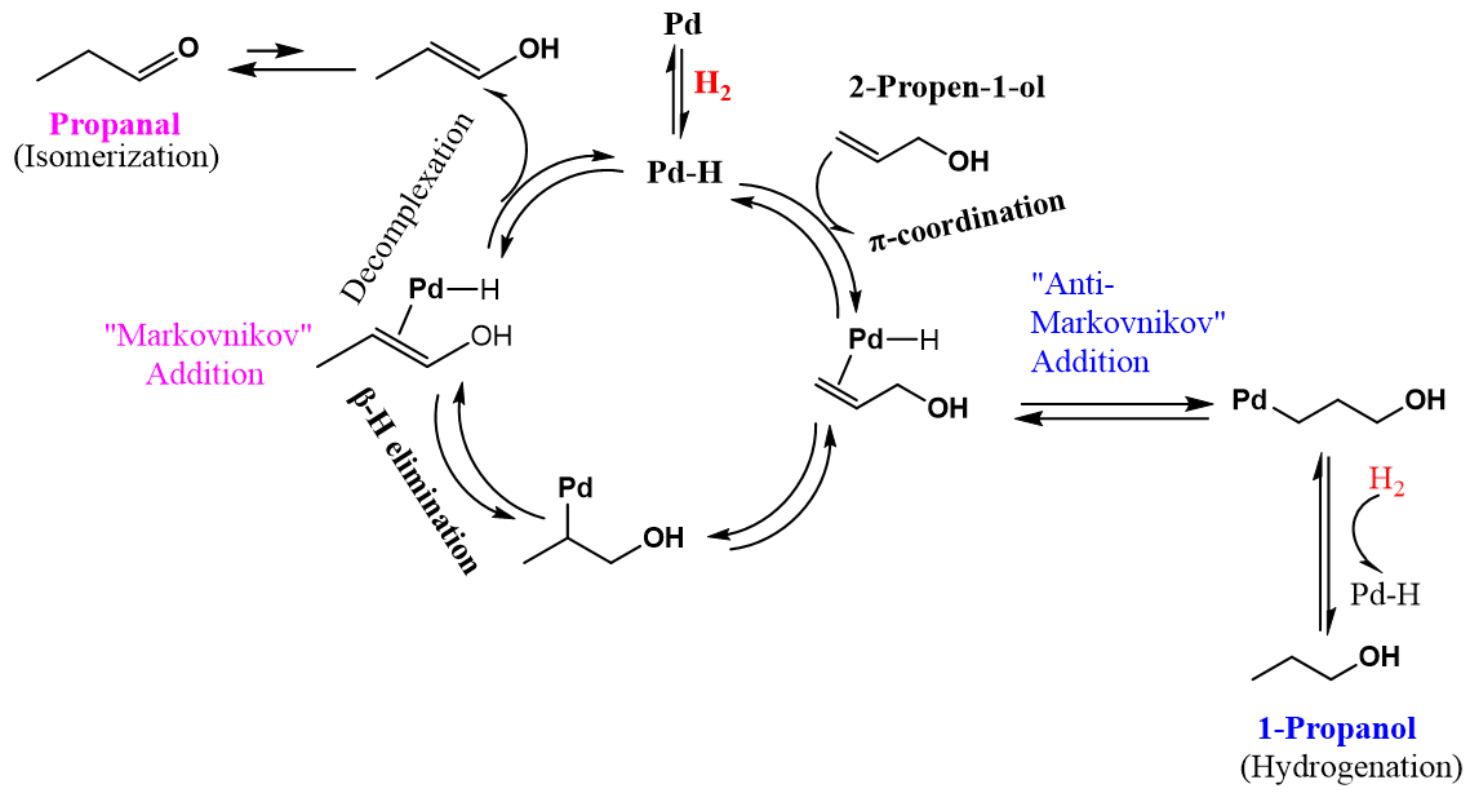

Scheme 1.8. Proposed mechanism (Pd-alkyl mechanism) showing the two routes for the hydrogenation/isomerization of an allyl alcohol.

Previously in our group, Moreno and coworkers synthesized Pd MPCs coated with amine, thiol, and mixed monolayers to understand the effect of mixed monolayers against $\mathrm{H}_{2}$ induced aggregation of MPCs. They found that MMPCs have the advantage of high $\mathrm{H}_{2}$ reactivity as suggested by minimal changes in the optical properties, minimal morphological changes, and conductivity measurements. ${ }^{81}$ Such stable MPCs are potentially useful in catalysis and sensing applications. Later, Moreno et al. studied the selectivity and reactivity of amine- and thiol-capped Pd and PdAg MPCs for hydrogenation and isomerization of allyl alcohol. Their study shows that by simply controlling the ligand and metal composition of the MPCs, one can optimize the selectivity and activity of the catalyst. ${ }^{82}$ Likewise, Bhama et al. recently demonstrated the use of branched, water-soluble glutathione (SG)-capped Pd and Au/Pd alloy MPCs as a 
catalyst for the hydrogenation/isomerization of allyl alcohol in a biphasic system (Scheme 1.9). This study demonstrated a turnover frequency (TOF) roughly 5 times greater than when using a linear chain hexanethiol-stabilized cluster. ${ }^{53}$ Following these achievements, our group became motivated to improve the catalytic activity further by utilizing a ligand-removal approach in a homogenous system as well as by combining solid-supported NPs and the ligand removal technique in a heterogenous system.

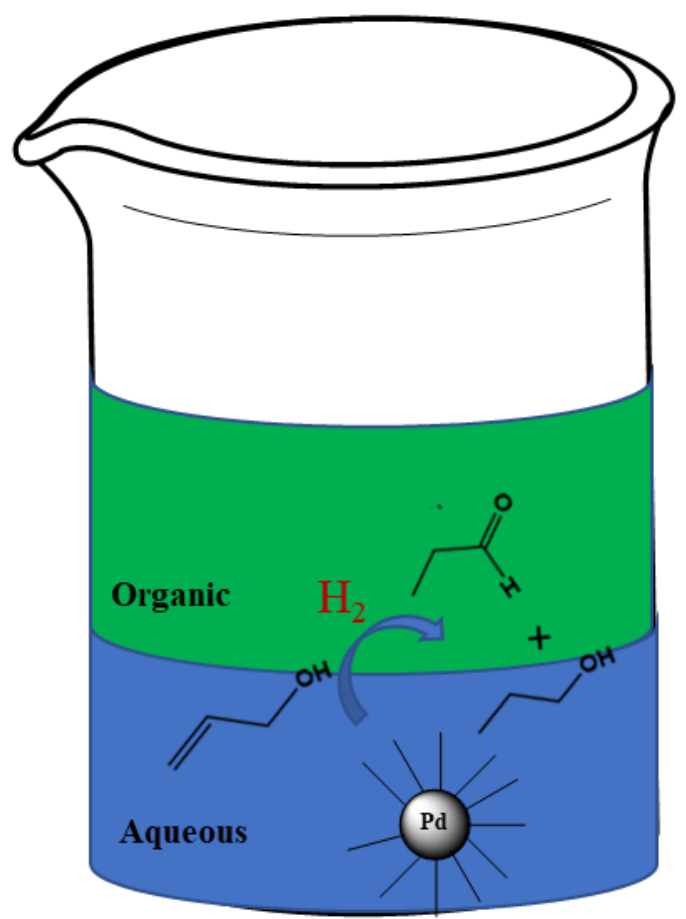

Scheme 1.9. A schematic of a biphasic system for hydrogenation/isomerization reaction of allyl alcohol.

\subsection{Ligand Removal Strategies}

Researchers desire to make small, stable NPs that are active homogeneous catalysts in various applications. This is very challenging since the stabilizing ligands (organic 
ligands or polymers) necessary for stability also inhibit the catalytic activity. Among other applications of MPCs, our goal is to exploit the MPC surface chemistry for catalyzing organic reactions. The strength of binding, unpredictable surface coverage of bound ligands, noncovalent interactions between ligands and the incoming reactant, and charge transfer at the ligand-metal surface interface could significantly affect the properties and catalytic activity of the metal NPs. ${ }^{83}$ Removing the ligand shell without destabilizing or changing the properties of the NPs is undoubtedly a very challenging task. The growth of the metal NP core is usually the outcome of ligand removal along with loss of the ligand density from the surface. In many cases, the size of the metal core are found to be very useful, especially in sensing and catalysis applications. Weaklybound stabilizers (citrate) can be partially removed simply by refluxing, while strongly bound ligands such as thiols require alternative methods such as oxidation or ligandexchange reactions that could also result in morphological changes as well as aggregation. ${ }^{84}$ Effective strategies of ligand removal to enhance the catalytic performance of metal NPs or MPCs are crucially important for improving applications in the field of catalysis. The understanding of the pros and cons of using capping agents in the NP synthesis and their effects on catalysis is significant. Many methods of ligand removal have been developed and include thermal and chemical treatments, ozone treatment, and electrochemical approaches. The influence of the ligand removal method on the surface chemistry and catalytic activity is particularly important to understand in order to assess the advantages of using such methods. Ligand removal strategies are an emerging field in nanoscience; past and current works in this field and future perspectives 
have suggested that the ligand removal concept is useful in catalytic applications of nanomaterials.

Examples of ligand removal strategies include electrochemically-induced ligand desorption, ${ }^{85}$ UV-ozone ligand decomposition, ${ }^{86}$ solvent extraction, ${ }^{87}$ plasma cleaning, ${ }^{88}$ thermal annealing, 89,90 and various chemical treatments. ${ }^{91,92}$ The presence of organic ligands adsorbed on the MPC surface has a big influence during catalytic reactions. Such ligands certainly impact the interactions between substrate and metal surface including chemoselectivity and stereoselectivity. Post-synthesis ligand addition techniques such as the ligand-place-exchange reaction, functionalization, and stripping techniques are commonly applied to understand their consequences on the catalytic activity. The electronic effect of functional groups, steric hindrance, and chain length effects decide the fate of ligand and substrate adsorption on facets, corner, or edges. Therefore, it is a challenging task to remove the organic ligands without changing the properties of the NPs or MPCs. Problems such as undesired particle size growth, metal leaching, catalyst poisoning, and excess loss of NPs or ligands can result from such processes.

Somorjai et al.93 examined the differences in catalytic activity using PVP-stabilized Rh NPs both calcined and uncalcined and found that the uncalcined catalysts exhibit a higher activity for $\mathrm{CO}$ oxidation than the calcined catalysts. They also reported that the catalytic activity of uncalcined Pt NPs capped with either PVP or oleic acid (OA) for ethylene hydrogenation increased when the organic ligand was removed. They speculated that upon removal of PVP, carbonaceous fragments are formed on the NP surface that reversibly restructures in $\mathrm{H}_{2}$ and $\mathrm{O}_{2}$. In the case of $\mathrm{O}_{2}$, these carbonaceous fragments form a tightly closed shell around the NPs and block Pt sites, leading to no catalytic 
activity. In $\mathrm{H}_{2}$, the shell opens, and the NPs are highly catalytic for ethylene hydrogenation. ${ }^{94}$ Moreover, this group also concluded that the improvement in catalytic activity is not related to the particle size growth resulting from calcination. Murray and coworkers $^{95}$ proposed that organic ligands from alumina supported Pd NPs could be removed via a rapid thermal treatment process. The heating mechanism in air involved very fast heating and cooling ramps, successfully activating the catalyst without changing the size, size distribution, or morphology of the supported particles. Likewise, LopezSanchez et $a l .{ }^{87}$ showed an efficient strategy to remove PVA ligands from Au NPs supported on $\mathrm{TiO}_{2}$ by simply refluxing the catalyst in water at $90{ }^{\circ} \mathrm{C}$, followed by calcination treatment at $300{ }^{\circ} \mathrm{C}$. Their water extraction procedure resulted in only minimal particle size growth $(3.0$ to $6.1 \mathrm{~nm})$ and found that washing at low temperature is a better way to enhance the catalytic activity in a $\mathrm{CO}$ oxidation reaction.

Despite the benefits of applying different ligand removal techniques, it is possible to suffer from low catalytic activity when there is unwanted or complete removal of ligands on applying such techniques. For example, Prati et al. ${ }^{96}$ obtained an unexpected lower activity for Au NPs when the organic ligands were completely removed by washing at 60 ${ }^{\circ} \mathrm{C}$, which suggested that the washing temperature is important when using it for ligand removal. Xu et al. ${ }^{97}$ applied UV irradiation and ozone treatment to control the removal and degradation of PVP from Au NP surface to better understand their effect on the catalytic activity for p-chloronitrobenzene and cinnamaldehyde hydrogenation. There was no change in the size and morphology of the NPs following the treatment. They restored the catalytic behavior of the NPs upon adding PVP and different capping ligands 
to the cleaned surface. Similarly, oxidation of thiolated ligands using $50 \mathrm{ppm}$ of ozone in nitrogen allowed the removal of ligands at room temperature after washing with water. ${ }^{98}$ The ozone treatment has been found to be more effective than thermal treatments. Many studies have been going on in this field to further understand the unexplored changes in the properties and catalytic activity of MPCs upon ligand removal.

\subsection{Iodine Activation}

To avoid the disadvantages suffered from aggressive ligand removal strategies, such as thermal pyrolysis, plasma etching, heating, etc., our group is motivated to develop a milder ligand removal approach. The ability to control the stoichiometry of ligand displacement from the MPC surface is important for achieving catalytic enhancement without altering cluster stability or other properties. One of the ideas we considered was to remove thiolate ligands via oxidation to disulfide using an oxidizing agent such as iodine $\left(\mathrm{I}_{2}\right)$. The first use of $\mathrm{I}_{2}$ in nanomaterials was first investigated by Templeton et al., ${ }^{99}$ who showed that the reaction of organothiolate Au MPCs with iodine results in decomposition of the MPCs with concomitant desorbed of the stabilizing ligands as disulfide species. The use of $I_{2}$ in this manner is often used as a means for confirming the identity of the ligated thiols on MPCs by NMR. Generally, a few crystals of $\mathrm{I}_{2}$ are added to an NMR tube containing thiolate-protected MPCs to 'knock off' the thiol ligands in order to obtain a ${ }^{1} \mathrm{H}$ NMR spectrum of the resultant, corresponding disulfide, which displays much sharper and well-resolved signals for all the protons as opposed to broadened, low intensity signals when on the Au clusters. Iodine-induced disulfide formation has been cleverly harnessed by Sun et al. in their report on the synthesis of 
hollow spheres comprised of polycyclodextrin. ${ }^{100}$ After preparing Au MPCs coated with a monolayer of thiolated $\beta$-cyclodextrin molecules, the ligated thiols were cross-linked by addition of $\mathrm{I}_{2}$ in aqueous $\mathrm{KI}$ to afford a polycyclodextrin structure comprising a hollow sphere, formed on oxidation and dissolution of the Au core by the excess iodide. In another example, Kim et al. cleaved thiol polymers ligated to Au nanoparticles (NPs) by addition of $\mathrm{I}_{2}$ to determine the molecular weight and polydispersity of the released polymers after disulfide reduction using $\mathrm{NaBH}_{4}{ }^{101}$ These examples of thiol ligand removal from the surface of $\mathrm{Au}$ nanostructures have motivated us to examine the reaction of $\mathrm{Au}$ and Pd MPCs with $\mathrm{I}_{2}$ as a means to enhance the their catalytic activity.

It could be hypothesized that treatment of a thiol-capped MPC catalyst with a small quantity of $\mathrm{I}_{2}$ would lead to well-controlled partial ligand removal by disulfide formation in-situ (Scheme 1.10), resulting in partial exposure of the metal surface to substrates for enhanced binding and reactivity. This $\mathrm{I}_{2}$-activation concept may have potential application in both homogenous and heterogenous catalysis. Many organic reactions such as 4-NP reduction, ${ }^{102}$ allyl alcohol hydrogenation/isomerization, alkyne hydration, ${ }^{103}$ and Suzuki-coupling reaction amongst others could be tested with this general method of ligand removal. This method is expected to work well for ligand removal from solidsupported NPs, where the solid-support assists to maintain NP stability for improvement in recyclability. 


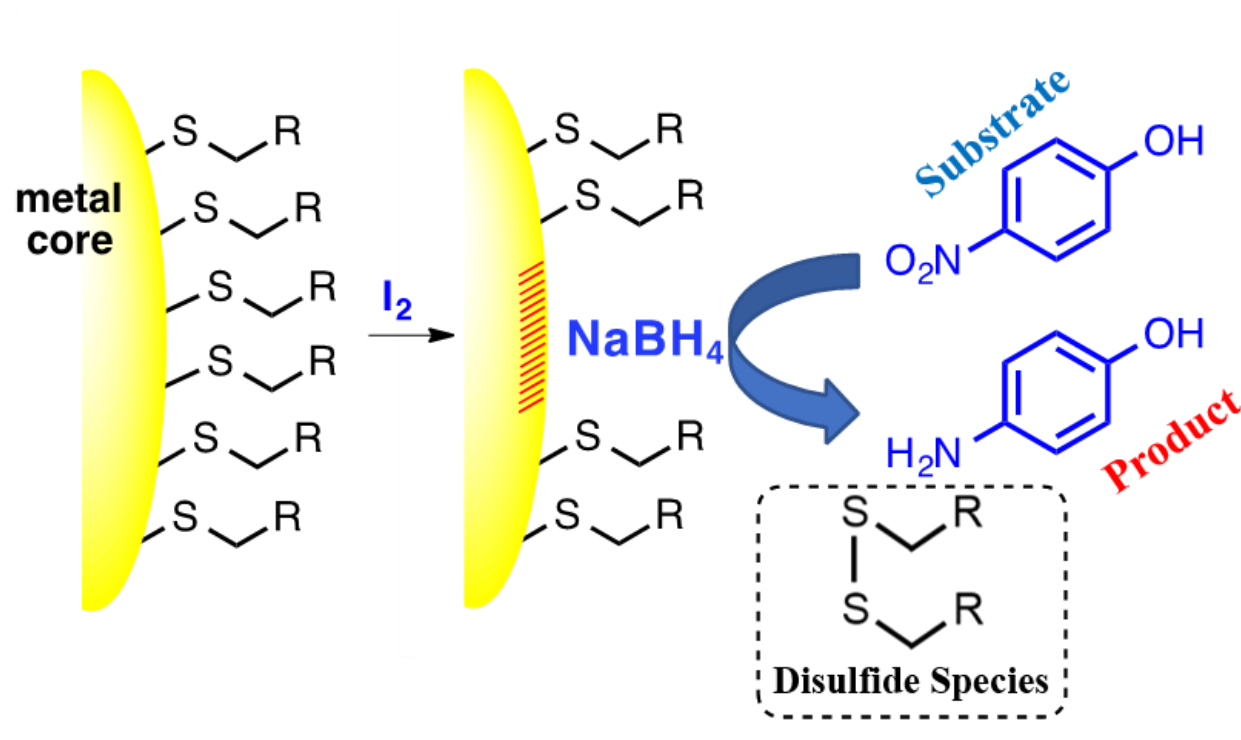

Scheme 1.10. Iodine activation concept for the partial removal of thiolated ligands from the MPCs surface to promote catalytic enhancement of 4-NP reduction reaction.

\subsection{Solid-Supported Nanoparticles}

Immobilization of NPs on a solid-support is a well-known practice to develop a heterogenous catalyst that can potentially overcome drawbacks associated with homogenous catalysts. Despite the advantages of using homogeneous catalysts, such as higher activity and selectivity, there still remains a challenge to separate the homogenous catalyst from products without compromising the mass loss of catalyst. Small-sized NPs possess a higher surface area to volume ratio $(\mathrm{SA} / \mathrm{V})$ and this unique property makes them a good candidate for active catalyst development. Problems in using NPs as catalysts, such as poisoning of the active sites or uncontrolled aggregation of particles, must be addressed. Other issues such as leaching of delicate core metals and inefficient recycling and recovery has raised the attention of researchers in search of alternatives. 
Therefore, development of heterogenous NP-based catalysts is rapidly emerging in the field of catalysis science and technology. The nature of the support materials and choice of metal/s type are important in preparation of heterogenous catalysts, which are decided on based on the intended application needs. For example, Au and Pd metals are suitable candidates for 4-NP reduction and allyl alcohol hydrogenation/isomerization, respectively (discussed in section 1.7). The role of solid-supported NPs as a catalyst is useful in industry, as it could potentially save time and money. A general schematic diagram of solid-supported NPs is shown in the Figure 1.12.

Encapsulation of the metal NPs in porous materials is a facile strategy used to prepare solid-supported NP-based catalysts. Support materials with different porosity include polymers, ${ }^{104}$ beads, ${ }^{105}$ zeolites, ${ }^{106}$ metal organic frameworks (MOFs), ${ }^{107}$ and zeolitic imidazolate frameworks (ZIFs). ${ }^{108}$ For example, Lee et al. ${ }^{106}$ Reported the preparation of size- and shape-controlled MNPs coated on unfunctionalized polystyrene (PS) beads. Their results suggested that prepared composite beads were stable due to a strong incorporation of metal NPs on the bead surface, and their properties could be controlled by simply adjusting the solvent and NP concentrations. Indeed, the solvent (THF) composition influenced the overall NP stability, surface coverage and coating morphology. Yu et al. ${ }^{106}$ investigated in situ encapsulation of Pd MPCs within the intersectional channels of nanosized zeolite and studied their catalytic activity. This catalyst showed excellent catalytic activity (TOF: $856 \mathrm{~h}^{-1}$ ) and recyclability for hydrogen generation from formic acid under mild conditions. Jia and coworkers found that porous polyacrylonitrile (PAN) bead-supported Pd NPs are an excellent candidate for the catalysis of formic acid dehydrogenation. ${ }^{109}$ They proposed that the $\mathrm{CN}$ functional group, 
which is present abundantly on the surface of the PAN beads, assisted in the immobilization of Pd NPs. Moreover, porous glass beads are also used as a support for the enhancement in catalytic performance. In the seminal work presented by Shen et al., ${ }^{110}$ glass beads were used as a support to load Pd NPs for hydrogenation reactions. They prepared their catalyst using the combination of a water treatment process and ionexchange method to form core-shell like structures, finding that the prepared catalyst showed superior activity in the hydrogenation of 2-ethylanthraquinone. Recently, the development of recoverable magnetic nanoparticle catalyst is highly studied to discover a strategy to attach non-magnetic catalytic active components. The progress on the preparation of chemically-modified (activated) magnetic NPs have suggested there is ultimate benefits of using such catalysts. ${ }^{111}$ It is obvious that magnetic separation is the best choice because it offers high efficiency and specificity when compared with traditional methods, such as filtration or centrifugation. The choice of magnetic materials or support used in the preparation of solid-supported MNPs can influence the efficiency of the separation process and eventually the catalytic performance as well. Activated magnetic beads are designed via surface-engineering to affix a catalytic material to a suitable magnetic catalyst. The recovery of catalyst facilitated by the magnetic separation would dramatically increase the efficiency. 


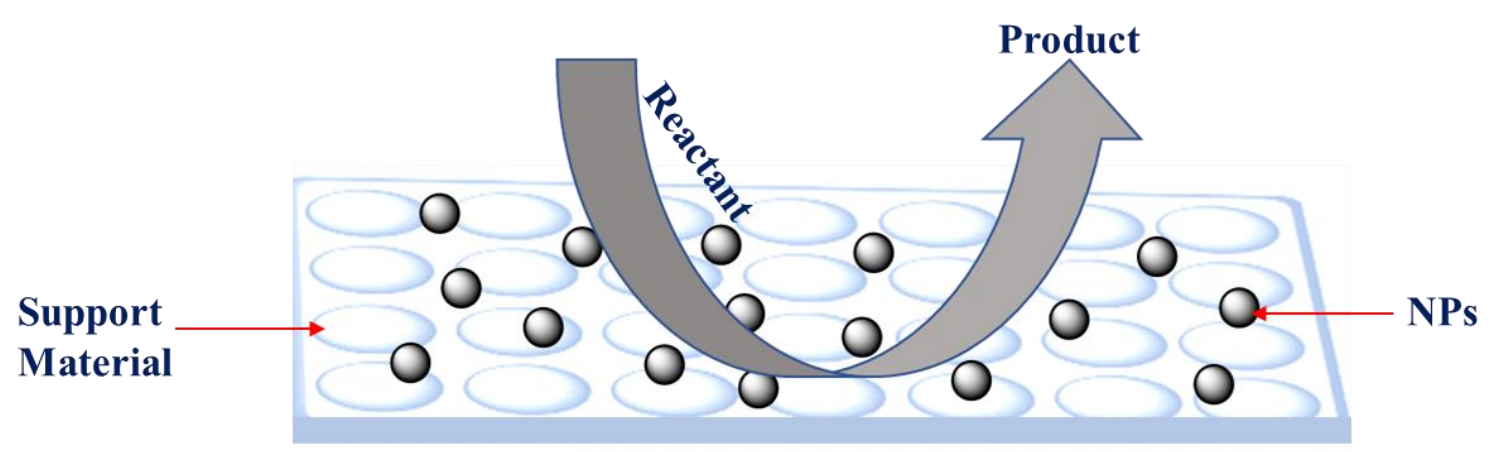

Solid- Supported NPs

Figure 1.12. A general schematic of solid-supported NPs.

Polymer-supported metal NPs are mainly synthesized via ex-situ and in-situ routes. In an ex-situ method, the synthesized NPs are dispersed in a polymer solution, and in an in-situ method a series of reactions is performed to generate the MNPs inside the polymer phase. The flexibility with which the particle size and morphology can be controlled gives the in-situ method an edge in popularity, however; ex-situ methodology is also commonly used to prepared polymer supported MNPs. For ex-situ synthesis, techniques such as solvent evaporation, co-precipitation, drop-casting and copolymerization are used to entrap the MNPs in the polymer network. The work presented in this dissertation utilized the ex-situ synthesis procedure for preparation of a heterogenous catalyst.

In our study, we are interested in the preparation of polymer-supported catalyst. After exploring different support materials, we found that the use of micrometer sized porous beads containing aldehyde functionalities on the surface could be potentially useful. Aldehyde- activated polystyrene (PS) beads (330-1225 $\mu \mathrm{m})$ and agarose beads 
(average of $145 \mu \mathrm{m}$ ) were purchased from commercial sources. Au or Pd MPCs were synthesized and functionalized with aminooxy groups by the place-exchange reactions discussed previously to afford the corresponding MMPCs. Click reactions between aminooxy-functionalized MMPCs and aldehyde-activated solid-support (beads) were performed to afford the solid-supported MPCs as a heterogenous catalyst. Therefore, utilizing the click chemistry we could attach MPCs onto the solid-support surface ex-situ. It is expected that the catalyst prepared in this way would provide higher recyclability and potentially lower TOF values compared to the homogenous counterparts. Different organic reactions could be tested using the heterogenous catalyst prepared by this methodology. Further details into the synthesis and catalytic activity of the heterogenous catalysts are discussed in Chapter 4 and 5 of this dissertation. 


\section{CHAPTER 2}

\section{SYNTHESIS OF AMINOOXY-THIOL LIGANDS AND MPCs}

\subsection{Introduction}

Aminooxy-based click $^{1}$ chemistry has proven to be a versatile means of ligation, as evidenced by broad application in the fields of material science, biology, biochemistry, analytical chemistry and nanoscience. $112,113,114$ Aminooxy groups $\left(\mathrm{RONH}_{2}\right)$ react chemoselectively with aldehydes and ketones under mild conditions to form highly stable oxime ether adducts. The chemoselectivity of the oximation reaction has stimulated efforts to exploit oxime ether formation not only as a straightforward, efficient coupling approach, but also as a means for identification and quantification of carbonyls in complex mixtures. For example, aminooxy derivatization reagents have been used to selectively tag and/or sequester carbonyl substrates in biological extracts, ${ }^{115,} 116$ environmental air and water, ${ }^{117}, 118$ exhaled breath, ${ }^{119,}{ }^{17}$ and even living organisms. ${ }^{120}$

As the usefulness of oximation chemistry has become apparent, several approaches exploiting nanoparticles fitted with aminooxy surfaces have been reported. ${ }^{121}$, 122, 123 The ease of functionalization of gold surfaces, clusters, and nanoparticles with a monolayer of thiol- or dithiol-containing molecules ${ }^{124,}{ }^{25}$ has guided some efforts toward developing and applying thiol ligands containing the aminooxy functionality. ${ }^{126}$ Given the rapidly growing interests in using gold monolayer-protected clusters (Au MPCs) and $\mathrm{Au}$ nanoparticles (NPs) for applications in catalysis, 127,128 sensing volatile organic compounds, ${ }^{129}$ analyzing small molecule mixtures using NP-mediated Raman and laser desorption/ionization spectroscopy, ${ }^{130}$ and drug delivery, ${ }^{131}, 132$ the development of aminooxy-functionalized thiol ligands to improve ease of carbonyl ligation as well as to 
provide flexibility in addressing solubility and adduct loading considerations could potentially enable new Au MPC and NP applications. With this in mind, we designed synthesis routes for the preparation of different types of ligands that contain the following functionalities: an aminooxy moiety, a thiol (RSH) group, and in some cases an amino group, depending upon the specific application. In our studies, aminooxy ligands with different hydrocarbon chain lengths, aminooxy density were synthesized as discussed below.

\subsection{Ligand Syntheses}

At the onset of this graduate research, we designed a synthetic pathway to a trifunctional amine-containing aminooxy thiol $\left(\mathrm{AOT}_{\text {bis, }}\right.$ Figure 2.1) for use as a ligand in monolayer functionalization of hexanethiolate $\left(\mathrm{C}_{6} \mathrm{~S}\right)$-protected $\mathrm{Au}$ clusters to produce $\mathrm{Au}\left(\mathrm{C}_{6} \mathrm{~S}\right)\left(\mathrm{AOT}_{\text {bis }}\right)$ mixed monolayer protected clusters (MMPCs) (Scheme 2.1). Incorporation of the amine group was motivated by several considerations: a) the solubility properties of the cluster may be adjusted by control over the amine protonation or alkylation state; b) the amine moiety enables convenient structural bifurcation to increase pendant aminooxy density; and c) an ammonium $\mathrm{NH}$ could potentially accelerate oximation reactions of the cluster. ${ }^{133}$ Herein we describe the synthesis of such a trifunctional thiol ligand and its use in formation of Au MMPCs. We also present our findings on representative oximation reactions of $\mathrm{Au}\left(\mathrm{C}_{6} \mathrm{~S}\right)\left(\mathrm{AOT}_{\text {bis }}\right)$ MMPCs under both non-polar and aqueous conditions. 


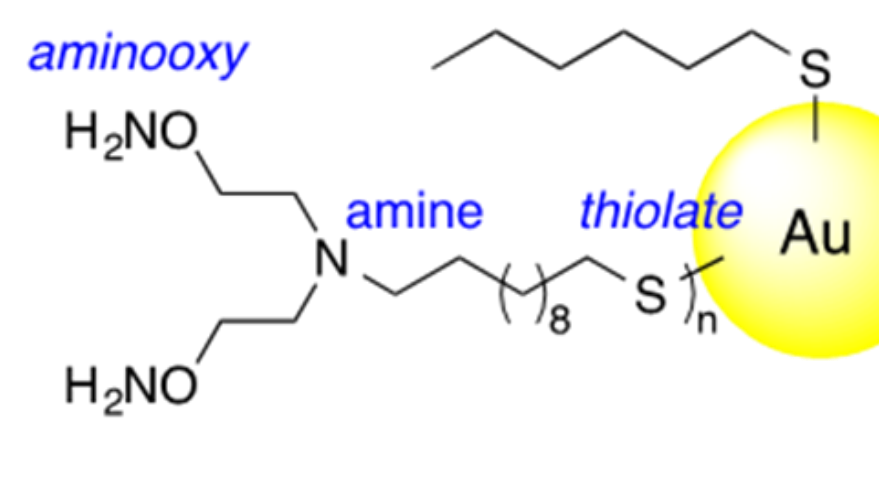

Figure 2.1. A general representation of aminooxy-functionalized $\mathrm{Au}\left(\mathrm{C}_{6} \mathrm{~S}\right)\left(\mathrm{AOT}_{\text {bis }}\right)$ MMPCs.

Synthesis of the bis(aminooxy) thiol ligand AOT bis was accomplished by first $N$ alkylating diethanolamine (1, Scheme 2.1) by heating with 11-bromo-1-undecene. Subsequent transformation of the primary alcohol moieties to phthalimido groups was performed using standard Mitsunobu conditions ${ }^{134}$ according to the method reported by Grochowski and Jurczak. ${ }^{18}$ Installation of the terminal thiol moiety to form $\mathbf{4}$ was next attempted, also using established conditions. ${ }^{135}$ AIBN-initiated radical addition of thioacetic acid to the terminal alkene of $\mathbf{3}$ proved troublesome; however, on protonation of the amine group using CSA prior to addition of AIBN, the reaction produced thioacetate $\mathbf{4}$ in good yield. Treatment of $\mathbf{4}$ with excess hydrazine hydrate cleaved both phthalimide groups to reveal the aminooxy moieties as well as cleaved the thioester group to afford $\mathbf{A O T}_{\text {bis. }}$ 


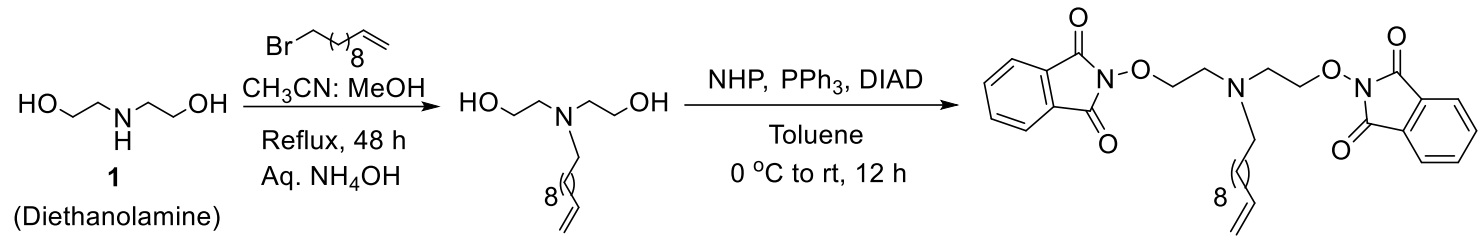

23

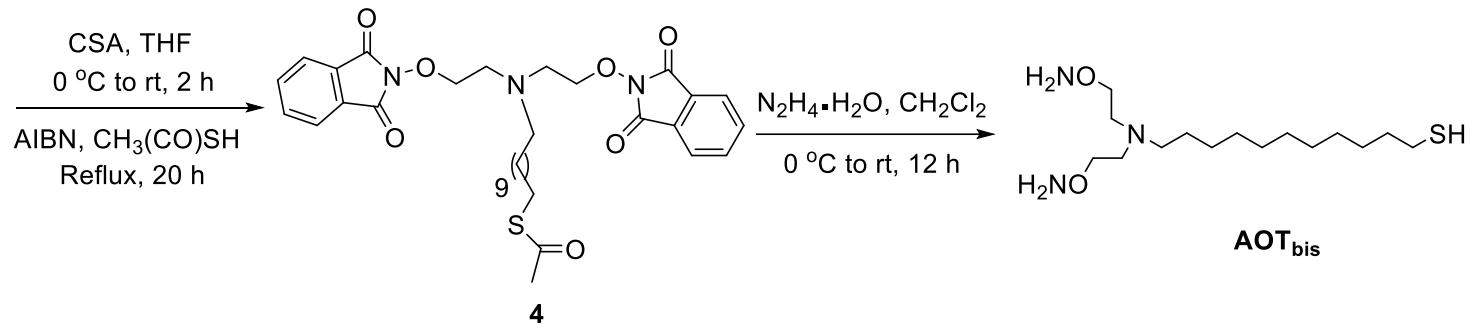

Scheme 2.1. Synthesis of bis(aminooxy) thiol ligand AOT bis. Conditions: a. i. 11-bromo-1-undecene, $\mathrm{CH}_{3} \mathrm{CN}$ : $\mathrm{MeOH}$ (7:3), reflux, $48 \mathrm{~h}$; ii. aq. $\mathrm{NH}_{4} \mathrm{OH}, \quad 88 \% ; \quad b . \quad N$-hydroxyphthalimide (NHP), $\mathrm{PPh}_{3}$, diisopropyl azodicarboxylate (DIAD), toluene, $0{ }^{\circ} \mathrm{C}$ to $\mathrm{rt}, 16 \mathrm{~h}, 95 \%$; c. $i$. camphorsulfonic acid (CSA), THF, $0{ }^{\circ} \mathrm{C}, 2 \mathrm{~h}$, ii. azobisisobutyronitrile (AIBN), thioacetic acid, reflux, $20 \mathrm{~h}$, iii. aq. $\mathrm{NaHCO}_{3}, 78 \%$; $d$. hydrazine monohydrate, $\mathrm{CH}_{2} \mathrm{Cl}_{2}, 0^{\circ} \mathrm{C}$ to rt, $12 \mathrm{~h}, 82 \%$.

The downfield signal of the methylene protons adjacent to the aminooxy moiety at $\delta$ $3.74 \mathrm{ppm}$ and the methylene protons adjacent to the thiol group at $\delta 2.48 \mathrm{ppm}$ in the ${ }^{1} \mathrm{H}$ NMR spectrum as well as the ${ }^{13} \mathrm{C}$ NMR signals from 15 carbon atoms (with 2 signals chemically equivalent) confirm the $\mathrm{AOT}_{\text {bis }}$ structure (Figures 2.2 and 2.3). On purification, $\mathrm{AuC}_{6} \mathrm{~S}$ MPCs were immediately subjected to a place exchange reaction with $\mathbf{A O T}_{\text {bis }}$ to form the corresponding $\mathrm{Au}\left(\mathrm{C}_{6} \mathrm{~S}\right)\left(\mathrm{AOT}_{\text {bis }}\right)$ MMPCs (Chapter 3). 


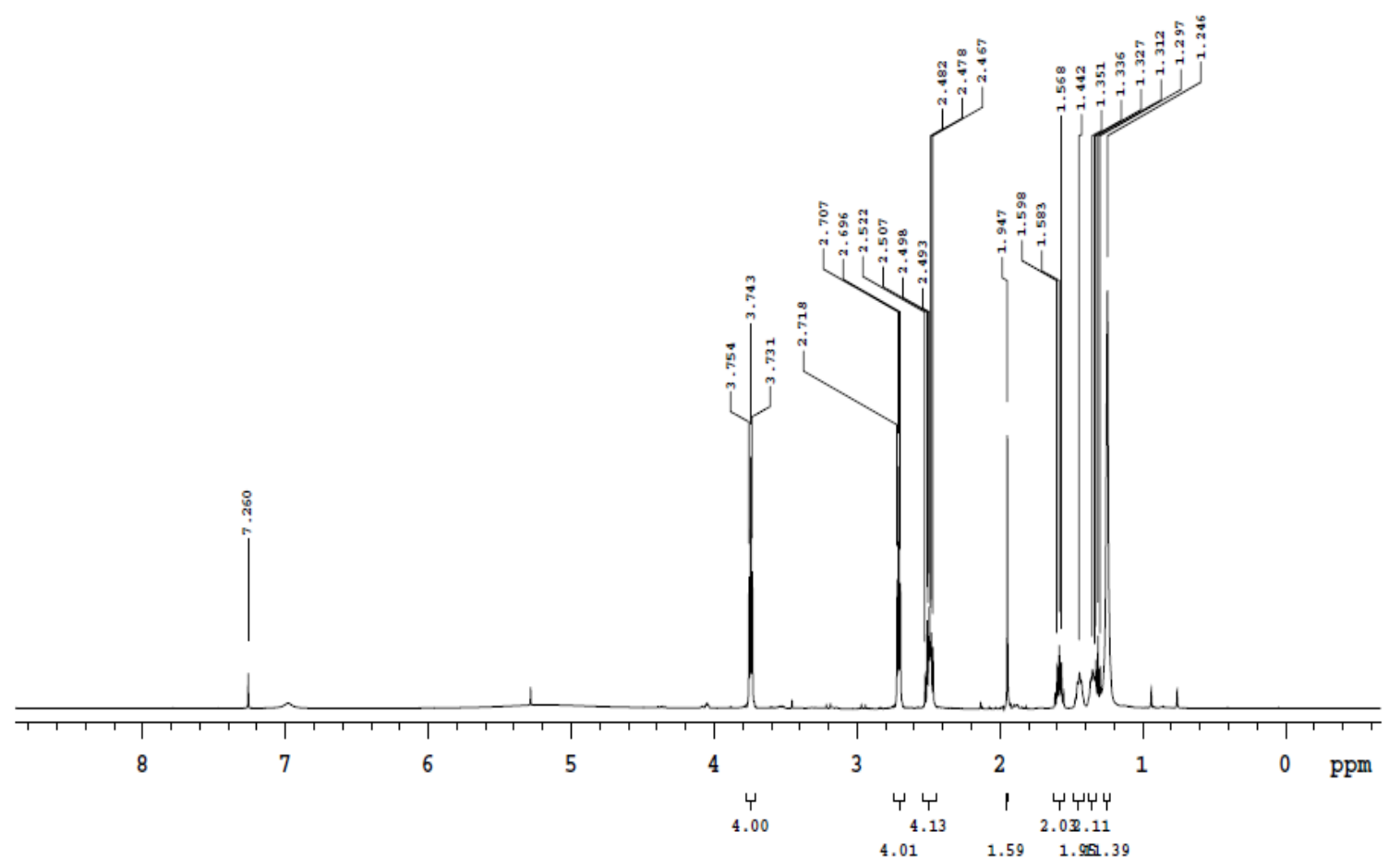

Figure 2.2. ${ }^{1} \mathrm{H}$ NMR spectrum $\left(400 \mathrm{MHz}, \mathrm{CDCl}_{3}\right)$ of compound $\mathrm{AOT}_{\text {bis. }}$

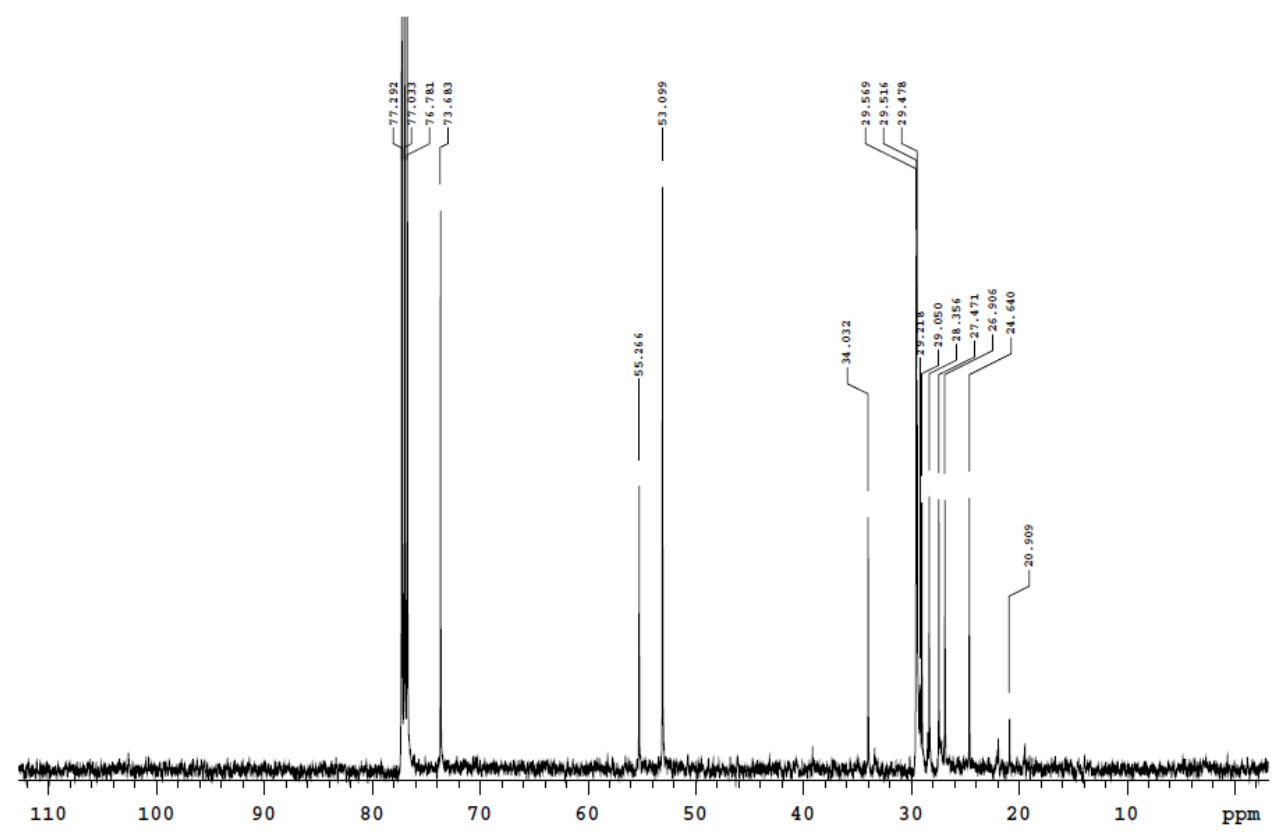

Figure 2.3. ${ }^{13} \mathrm{C}$ NMR spectrum $\left(100 \mathrm{MHz}, \mathrm{CDCl}_{3}\right)$ of compound $\mathrm{AOT}_{\text {bis. }}$ 
We found that reaction of thioacetate $\mathbf{4}$ with methyl iodide smoothly produced the corresponding quaternary ammonium iodide 6 (Scheme 2.2), which, on hydrazinolysis, gave cationic bis(aminooxy) thiol ligand $\mathrm{AOT}_{\text {bis }}{ }^{+}$. The ease of this synthesis allowed us to prepare a water-soluble aminooxy functionalized ligand that could be used for the functionalization of water soluble MPCs.
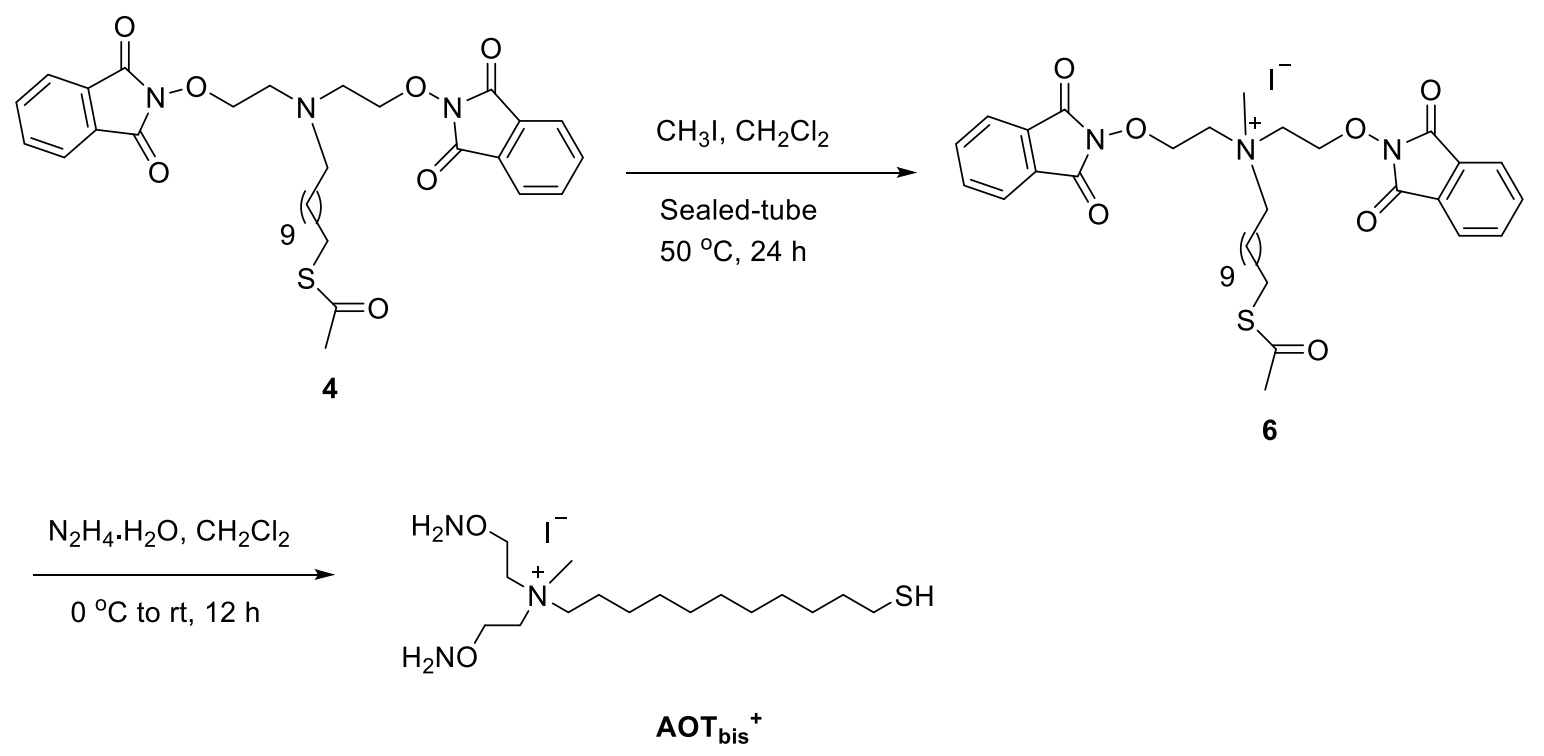

Scheme 2.2. Synthesis of water-soluble bis(aminooxy) thiol AOT $_{\text {bis }}{ }^{+}$. Conditions: $a . \mathrm{CH}_{3} \mathrm{I}, \mathrm{CH}_{2} \mathrm{Cl}_{2}$, sealed tube, $50{ }^{\circ} \mathrm{C}, 24 \mathrm{~h}, 88 \%$ b. hydrazine monohydrate, $\mathrm{CH}_{2} \mathrm{Cl}_{2}, 0{ }^{\circ} \mathrm{C}$ to $\mathrm{rt}, 12 \mathrm{~h}, 84 \%$.

The principal differences in the ${ }^{1} \mathrm{H}$ NMR signals of $\mathrm{AOT}_{\text {bis }}{ }^{+}$compared to $\mathrm{AOT}_{\text {bis }}$ include a downfield shift in the methylene protons adjacent to the aminooxy group from $\delta 3.66 \mathrm{ppm}$ to $\delta 4.12 \mathrm{ppm}$ and the methylene protons adjacent to the thiol group from $\delta$ $2.48 \mathrm{ppm}$ to $\delta 2.56 \mathrm{ppm}$. The singlet at $\delta 3.14 \mathrm{ppm}$ is due to the methyl group resonance of the ammonium ion, indicating successful alkylation (Figure 2.4). On purification 
AuSG MPCs were immediately subjected to a place exchange reaction with $\mathbf{A O T}_{\text {bis }}{ }^{+}$to form the corresponding $\mathrm{Au}(\mathrm{SG})\left(\mathbf{A O T}_{\mathbf{b i s}}{ }^{+}\right)$MMPCs (Chapter 3).

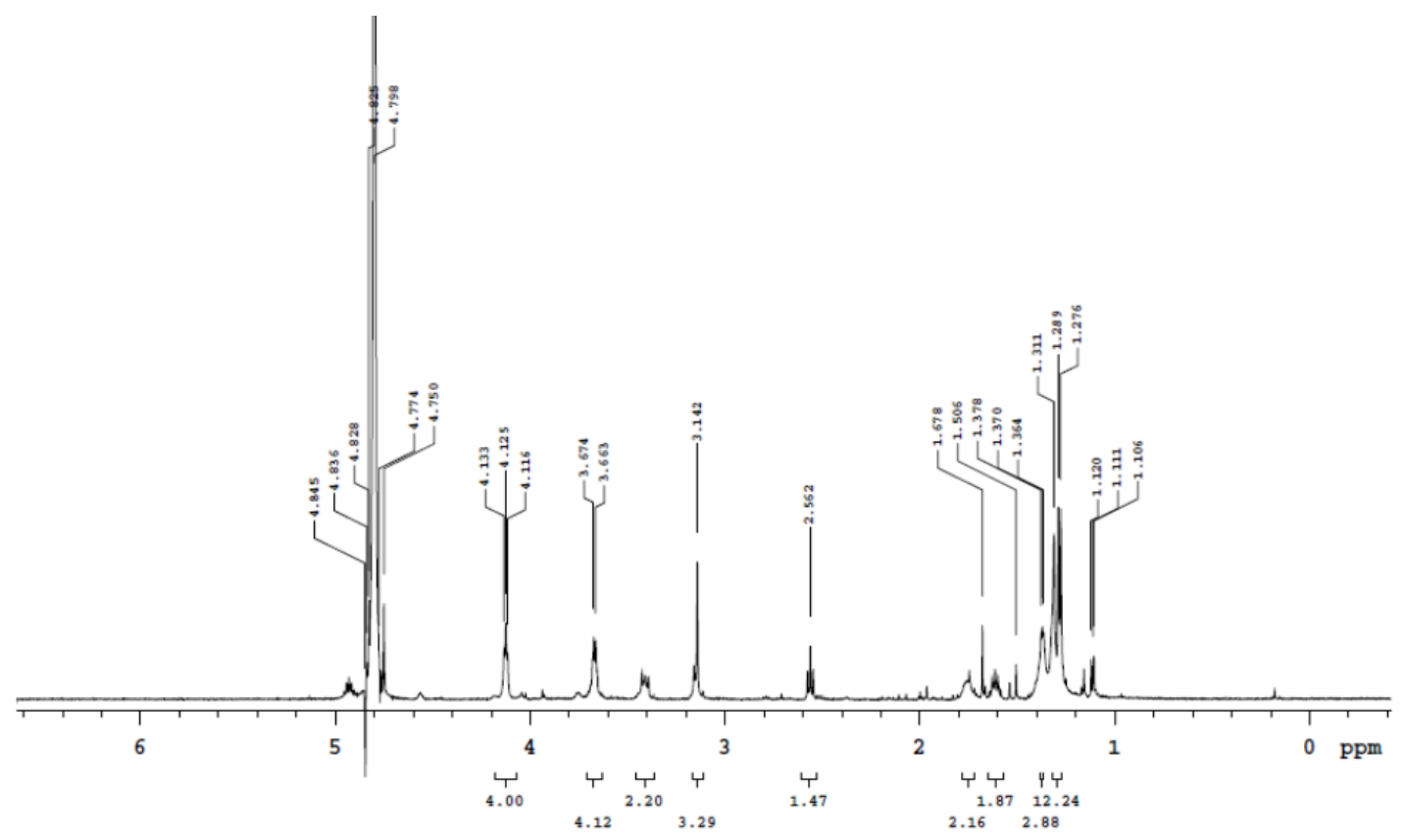

Figure 2.4. ${ }^{1} \mathrm{H}$ NMR spectrum $\left(400 \mathrm{MHz}, \mathrm{CDCl}_{3}\right.$ ) of compound $\mathrm{AOT}_{\text {bis }}{ }^{+}$.

Likewise, the synthesis of aminooxy-hexane thiol 11 was accomplished by first transforming the primary alcohol moieties of 5-hexen-1-ol (8) to phthalimido groups under standard Mitsunobu conditions. Installation of the terminal thioacetate moiety was performed via AIBN-initiated radical addition of thioacetic acid to the terminal alkene to form $\mathbf{1 0}$ using the established conditions. Compound $\mathbf{1 0}$ was afforded in pure form, after the purification of the crude product by silica gel chromatography (eluent: hexane/EtOAc, 8.5/1.5). Deprotection of both the thioacetate and phthalimido groups was achieved by treating compound $\mathbf{1 0}$ with excess hydrazine hydrate to reveal the thiol 
group and aminooxy group (Scheme 2.3). This type of ligand is shorter in carbon chain length $(n=6)$, does not contain an amine group, and only contains one aminooxy moiety.
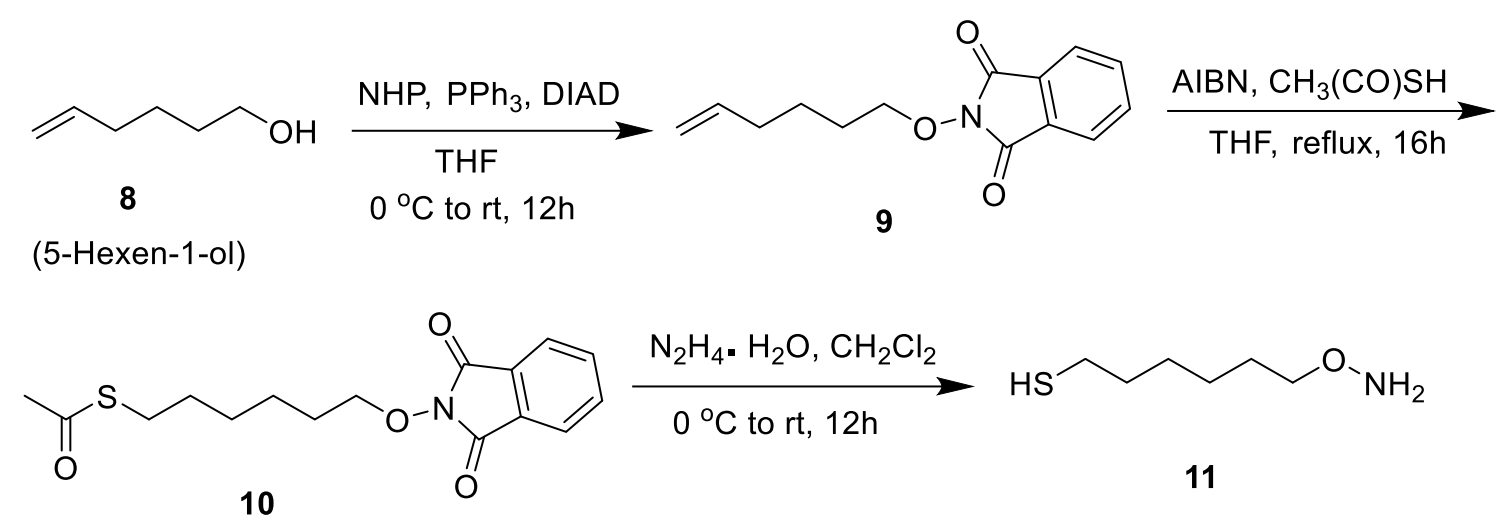

Scheme 2.3. Synthesis of aminooxy hexane-thiol ligand (11). Conditions: $a$. $\mathrm{N}$-hydroxyphthalimide (NHP), $\mathrm{PPh}_{3}$, diisopropyl azodicarboxylate (DIAD), THF, $0{ }^{\circ} \mathrm{C}$ to rt, $12 \mathrm{~h}, 82 \%$; $b$. Anhydrous THF, Azobisisobutyronitrile (AIBN), thioacetic acid, reflux, $16 \mathrm{~h}, 88 \%$; $c$. hydrazine monohydrate, $\mathrm{CH}_{2} \mathrm{Cl}_{2}, 0{ }^{\circ} \mathrm{C}$ to rt, $12 \mathrm{~h}, 91 \%$.

The upfield signals of the methylene protons towards the aminooxy end at $\delta 3.61$ ppm and adjacent to thiol group at $\delta 2.48 \mathrm{ppm}$ in ${ }^{1} \mathrm{H} \mathrm{NMR}$, and 6 carbon signals from ${ }^{13} \mathrm{C}$ NMR confirms the structure of compound 11 (Figures 2.5 and 2.6). Compound 11 could be immediately functionalized on the surface of Au MPCs and has potential application in their use as Au MPC-based chemiresistive sensors. 


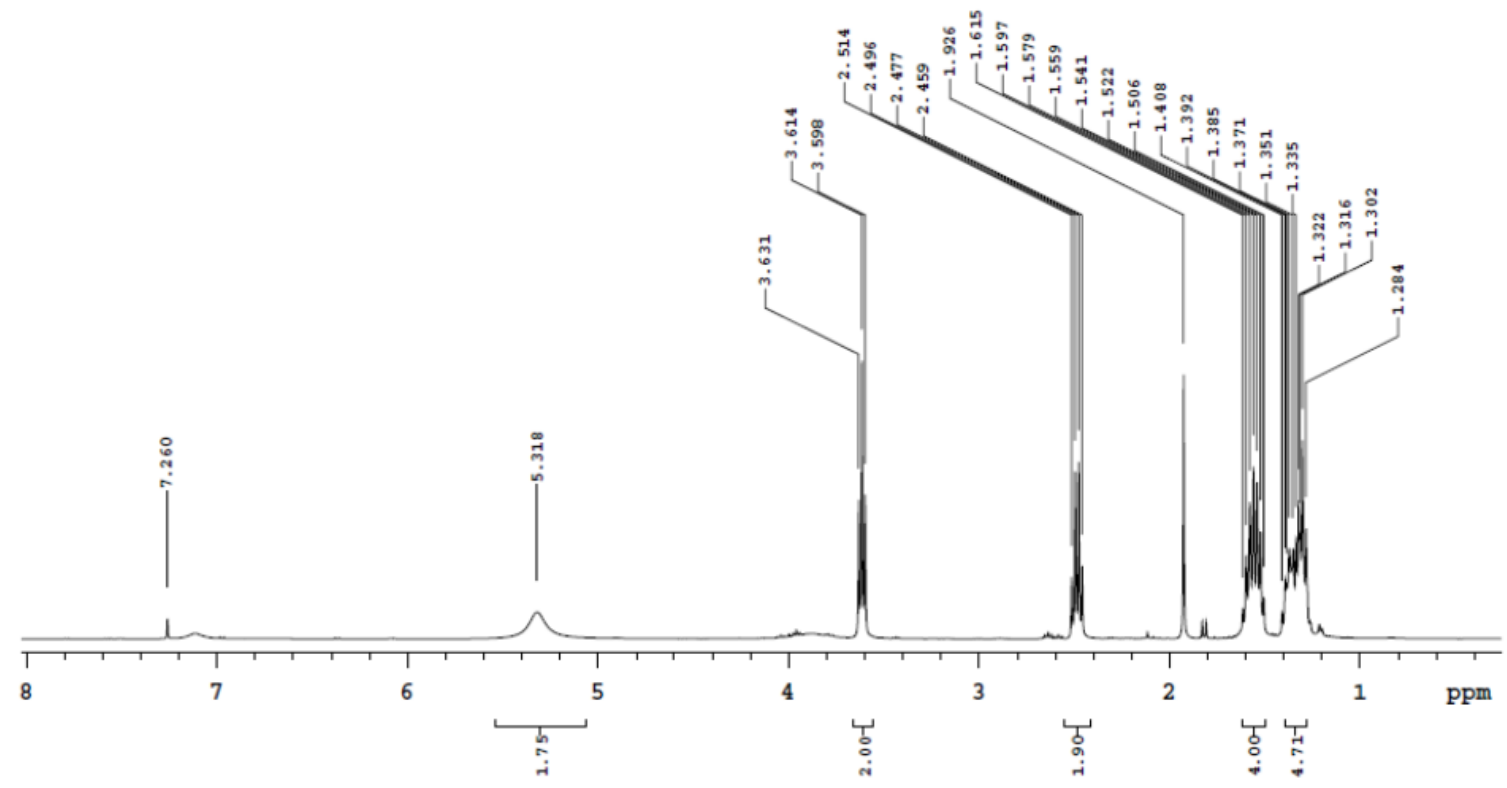

Figure 2.5. ${ }^{1} \mathrm{H}$ NMR spectrum $\left(400 \mathrm{MHz}, \mathrm{CDCl}_{3}\right)$ of aminooxy-hexanethiol (11).

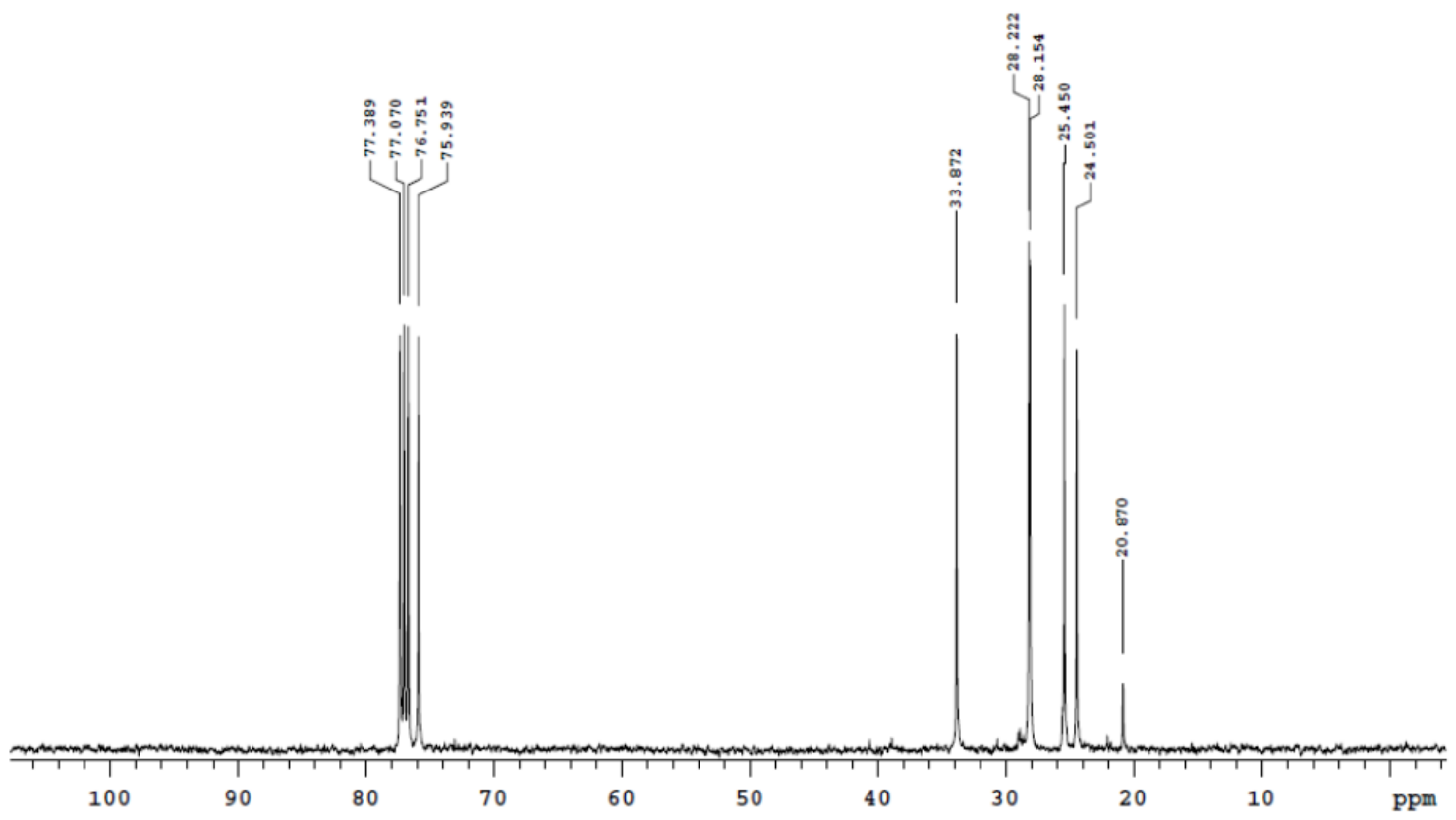

Figure 2.6. ${ }^{13} \mathrm{CNMR}$ spectrum $\left(400 \mathrm{MHz}, \mathrm{CDCl}_{3}\right)$ of aminooxy-hexanethiol (11). 
Overall, we synthesized aminooxy thiol ligands of two types for different intended applications. Initially, a trifunctional aminooxy ligand was synthesized in order to attach it to Au MPCs and demonstrate oximation reactions successfully on the Au MPC surface. This motivated us to further explore the synthesis of another thiol-containing ligand. With such ligands in hand, we were interested in understanding their use in catalysis and sensing applications, among others.

\subsection{MPCs Synthesis}

Monolayer protected clusters were synthesized using the reported protocols with a slight modification, which can be found in Chapter 6. Hexanethiolate-capped Au MPCs were synthesized following the well-known Brust method, ${ }^{30}$ where $t$-octylammonium bromide was used as a phase transfer catalyst to transfer $\mathrm{HAuCl}_{4} \bullet 3 \mathrm{H}_{2} \mathrm{O}$, the $\mathrm{Au}$ precursor, into an organic solvent, typically toluene or dichloromethane. Glutathionecapped water-soluble Au MPCs and Pd MPCs were also synthesized following the reported procedure under inert conditions. ${ }^{136,137}$ A general structure of synthesized MPCs along with the structure of the stabilizers used are shown in Figure 2.7.

$\mathrm{AuC}_{6} \mathrm{~S}$ MPCs were mostly used for their functionalization with $\mathrm{AOT}_{\text {bis }}$ ligand and subsequent oximation reactions. Hexane-thiolate Pd MPCs ( $\left.\mathrm{Pd} \mathrm{C}_{6} \mathrm{~S}\right)$ were also synthesized to prepare solid-supported MPCs, however; its utilization is not discussed in this dissertation. AuSG MPCs were used as a catalyst for the 4-NP reduction reaction to form 4-AP. The use of glutathione-capped water-soluble Pd MPCs as a catalyst in the allyl alcohol hydrogenation/isomerization reaction is exploited as well. 


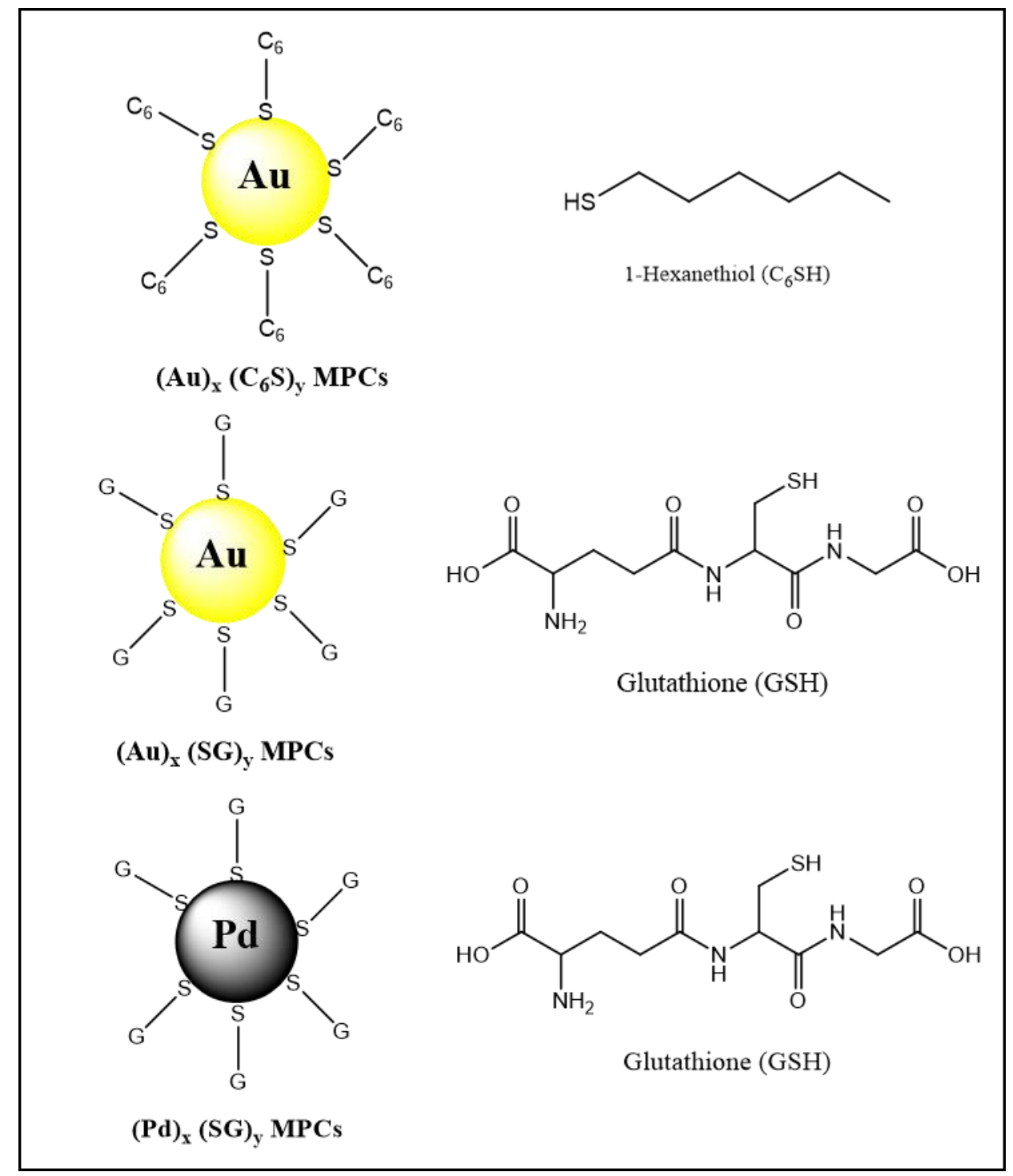

Figure 2.7. General structure of $\mathrm{Au}_{\mathrm{x}}\left(\mathrm{C}_{6} \mathrm{~S}\right)_{\mathrm{y}}, \mathrm{Au}_{\mathrm{x}}(\mathrm{SG})_{\mathrm{y}}$, and $\mathrm{Pd}_{\mathrm{x}}(\mathrm{SG})_{\mathrm{y}} \mathrm{MPCs}$.

All synthesized MPCs were fully characterized using the commonly used techniques: thermo-gravimetric analysis (TGA), nuclear magnetic resonance spectroscopy $\left({ }^{1} \mathrm{H}\right.$ NMR), UV-visible spectroscopy (UV-Vis), Fourier transform infrared spectroscopy (FTIR), and transmission electron microscopy (TEM). TGA analysis is generally performed in materials characterization because it can provide the organic percentage 
present on the surface of MPCs, which helps to determine the composition of the MPCs. In TGA, the weight of the MPCs is measured as a function of temperature, and at certain temperature all of the organic species are thermally decomposed, thereby the percentage of organic component can be determined simply by calculating the mass loss. ${ }^{1} \mathrm{H}$ NMR measurements provided the information about the successful attachments of the ligands or stabilizers on to the surface of metal core and elucidated the structure purity by confirming the removal of the non-bound ligands and disulfides. The signals for the organic ligands are usually observed as broad peaks in ${ }^{1} \mathrm{H}$ NMR due to the close association with the metal core, therefore the absence of sharp peaks in the spectrum indicates the successful ligand attachment on the metal surface. To further confirm, FTIR recordings were taken, where a disappearance of $-\mathrm{SH}$ stretching band $\left(\sim 2500 \mathrm{~cm}^{-1}\right)$ indicates that ligands are indeed attached. UV-Vis spectroscopy measurements were performed to approximate the metallic composition and stability of all synthesized MPCs. UV-Vis absorbance spectra measurements generally provide the estimated size of the $\mathrm{Au}$ MPCs which is correlated to the location of where the surface plasmon band is observed and the intensity. TEM was used to determine the size and morphology of the synthesized MPCs. X-ray photoelectron spectroscopy (XPS) was used to observe the elemental analysis of modified MPCs. The combination of these techniques can provide accurate information on the size and composition of synthesized MPCs. MMPCs were prepared by functionalizing the parent MPCs with a synthesized aminooxy thiol ligand. A detailed discussion of MMPCs synthesis and characterization are presented in Chapter 3. 


\section{CHAPTER 3}

FUCNTIONALIZATION OF MPCs WITH AMINOOXY-THIOL LIGANDS AND OXIMATION REACTIONS

\subsection{Introduction}

A key step in functionalization of Au or Pd MPCs with aminooxy groups is the ligand place-exchange reaction. All efforts to directly incorporate AOT $\mathbf{b i s}$ or $\mathbf{A O T}_{\text {bis }}{ }^{+}$ligands during Au or Pd MPCs formation uniformly failed due to problems with aggregation and cluster precipitation, likely due to the strong interparticle hydrogen bonding that increases as loading increases. Indeed, this challenge is evident from examination of the literature, which indicates that aminooxy-containing ligands are widely incorporated as aminooxyprotected ligands followed by deprotection of the resultant functionalized surfaces to reveal the aminooxy groups. ${ }^{3}$ Consequently this Chapter is devoted to the functionalization of $\mathrm{AuC}_{6} \mathrm{~S}$ MPCs with aminooxy-thiol ligands using the place-exchange reaction to form $\mathrm{AuC}_{6} \mathrm{~S} / \mathrm{AOT}_{\mathrm{bis}}$ mixed-monolayer protected clusters (MMPCs). Subsequent oximation reactions of the MMPCs with various aldehydes are also presented. More specifically, $\mathrm{AuC}_{6} \mathrm{~S}$ MPCs were functionalized with the bis-aminooxy thiol ligand to afford $\mathrm{AuC}_{6} \mathrm{~S} / \mathrm{AOT}_{\text {bis }} \mathrm{MMPCs}$ and these were subsequently used in oximation reactions with a panel of organic-soluble aldehydes. $\mathrm{AuC}_{6} \mathrm{~S} / \mathrm{AOT}_{\text {bis }} \mathrm{MMPCs}$ also were converted to a cationic form $\left(\mathrm{AuC}_{6} \mathrm{~S} / \mathrm{AOT}_{\mathrm{bis}}-\mathrm{H}^{+} \mathrm{MMPCs}\right)$ by protonating the amine functionality present in $\mathrm{AOT}_{\text {bis }}$ backbone using an acid as a way to demonstrate the tunability of the MMPCs. Also described is how water-soluble AuSG MPCs were functionalized with the aqueous-soluble bis-aminooxy thiol ligand $\mathrm{AOT}_{\text {bis }}{ }^{+}$to obtain AuSG/AOT ${ }_{\text {bis }}{ }^{+}$MMPCs for oximation reactions with water-soluble aldehydes. The 
syntheses and characterization of the $\mathrm{AuC}_{6} \mathrm{~S} / \mathrm{AOT}_{\text {bis }}$ and $\mathrm{AuSG} / \mathrm{AOT}_{\text {bis }}{ }^{+}$MMPCs (Figure 3.1) are discussed below.

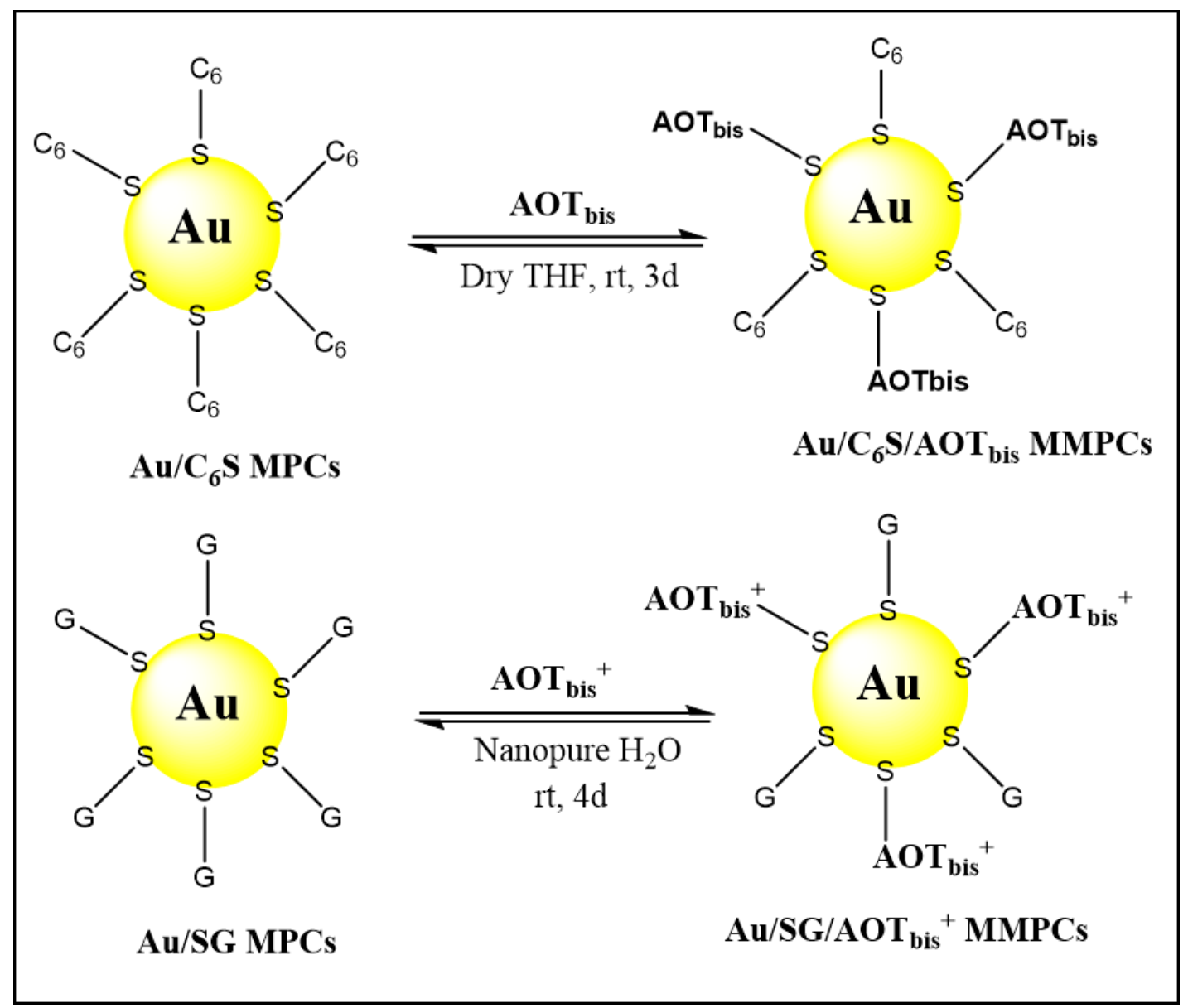

Figure 3.1. Ligand place-exchange reactions for Au MPCs to afford corresponding MMPCs. $\mathrm{C}_{6} \mathrm{~S}=$ Hexane-thiolate, $\mathrm{SG}=$ Glutathiolate.

\subsection{Ligand Place Exchange Reactions}

To avoid possible solubility issues associated with the synthesis of Au MPCs directly with AOT bis, we successfully used a ligand place exchange reaction to smoothly

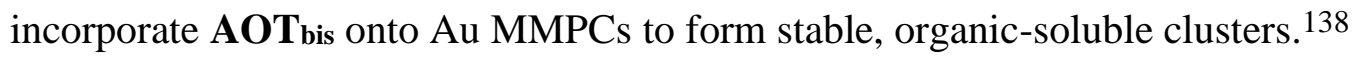


First, we used a literature procedure to synthesize hexanethiolate $\left(\mathrm{C}_{6} \mathrm{~S}\right)$-capped $\mathrm{Au}$ MPCs. ${ }^{30}$ Briefly, tetra-octyl ammonium bromide (TOABr) was used as a phase-transfer catalyst to transfer the aqueous $\mathrm{HAuCl}_{4} 3 \mathrm{H}_{2} \mathrm{O}$ into a non-polar organic solvent, such as toluene. Subsequent addition of hexane-thiol reduces the $\mathrm{Au}^{\mathrm{III}}$ ionic complex to a $-\left(\mathrm{Au}^{\mathrm{I}}-\right.$ $\left.\mathrm{SC}_{6}\right)_{\mathrm{n}^{-}}$- polymer, that can be further reduced to $\mathrm{Au}^{0}$ by using excess ice-cold $\mathrm{NaBH}_{4}$ to afford $\mathrm{AuC}_{6} \mathrm{~S}$ MPCs (Figure 3.2). Thermogravimetric analysis (TGA) on the resultant $\mathrm{AuC}_{6} \mathrm{~S}$ MPCs showed an organic composition of $20.25 \%$ (Figure 3.3), which is close to the expected $19.84 \%$ weight change for an $\mathrm{Au}_{144}\left(\mathrm{C}_{6} \mathrm{~S}\right)_{60}$ cluster. ${ }^{139}$ The size of this type of MPCs is $1.6 \mathrm{~nm}$ (representing $\mathrm{Au}_{144}$ atoms) based on TEM reported in literature. ${ }^{139}$ The UV-Vis spectra of $\mathrm{Au}_{6} \mathrm{~S}$ MPCs showed that the cluster is small $(<2 \mathrm{~nm})$ and stable (Figure 3.4). Indeed, characterization by TGA and UV-Vis revealed that the composition of the $\mathrm{AuC}_{6} \mathrm{~S}$ MPCs was approximately $\mathrm{Au}_{144}\left(\mathrm{C}_{6} \mathrm{~S}\right)_{60}$. Characterization by ${ }^{1} \mathrm{H}$ NMR spectroscopy gives information about the successful attachment of ligands onto the metal core of the synthesized MPCs. A residual solvent peak at $\delta 7.26 \mathrm{ppm}$ is of $\mathrm{CDCl}_{3} .{ }^{1} \mathrm{H}$ NMR of $\mathrm{Au}_{144}\left(\mathrm{C}_{6} \mathrm{~S}\right)_{60}$ cluster differed from that of pure $\mathrm{C}_{6} \mathrm{SH}$ in that the peaks for the thiol-coated Au MPCs were all broadened relative to those of pure $\mathrm{C}_{6} \mathrm{SH}$. The peak for the methylene protons $\alpha$ to the thiol group at $2.51 \mathrm{ppm}$ wasn't observed (peak a, Figure 3.5) in the hexanethiol-coated Au MPCs, due to the presence of Au core that creates large inhomogeneity in the magnetic field around the local chemical environment. Also, a lack of any sharp peaks in the acquired spectrum confirmed the absence of any other impurities or free (non-bound) ligand. Moreover, the disappearance of the $-\mathrm{SH}$ stretching band at $\sim 2500 \mathrm{~cm}^{-1}$ in the FTIR spectrum provides evidence that the synthesized $\mathrm{Au}$ MPCs is hexanethiolate-protected (Figure 3.6). 

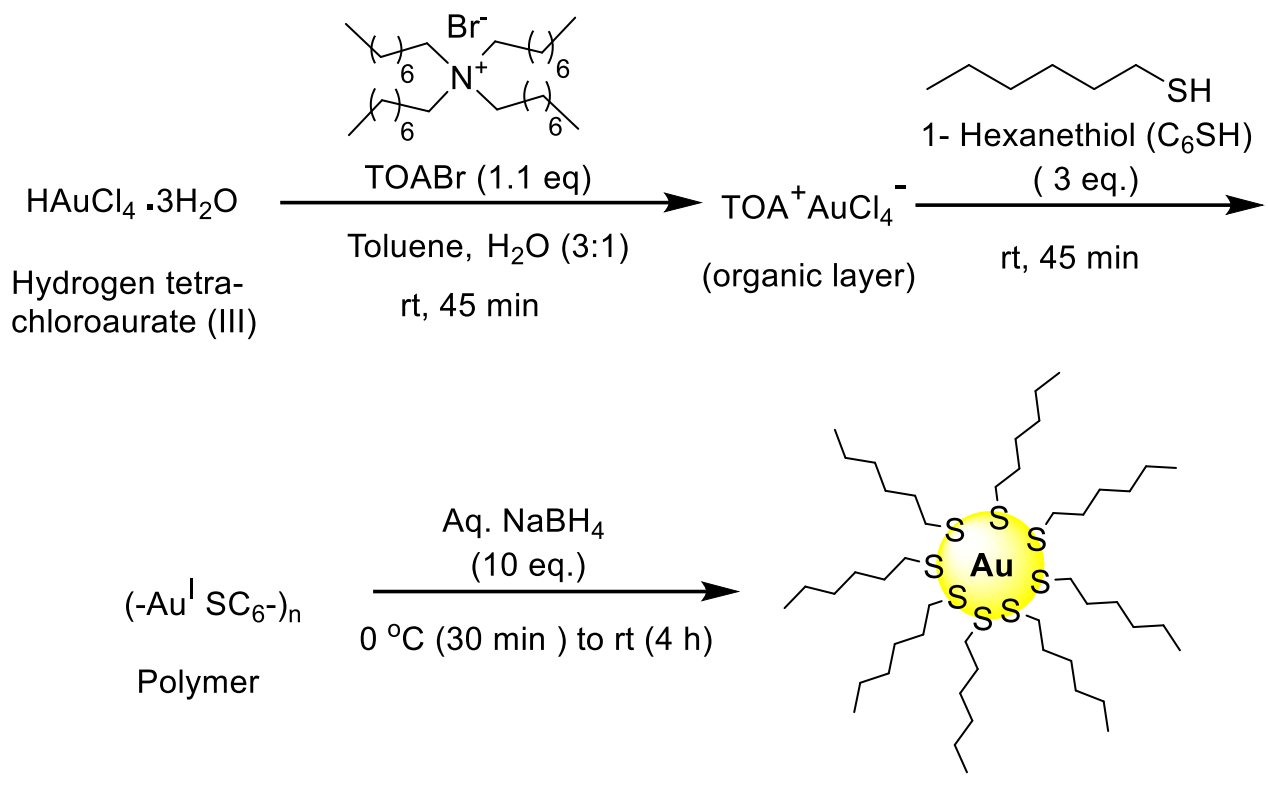

Au $\mathrm{C}_{6} \mathrm{~S}$ MPCs

Figure 3.2. A schematic of the synthesis of $\mathrm{AuC}_{6} \mathrm{~S}$ MPCs following the Brust method. ${ }^{30}$

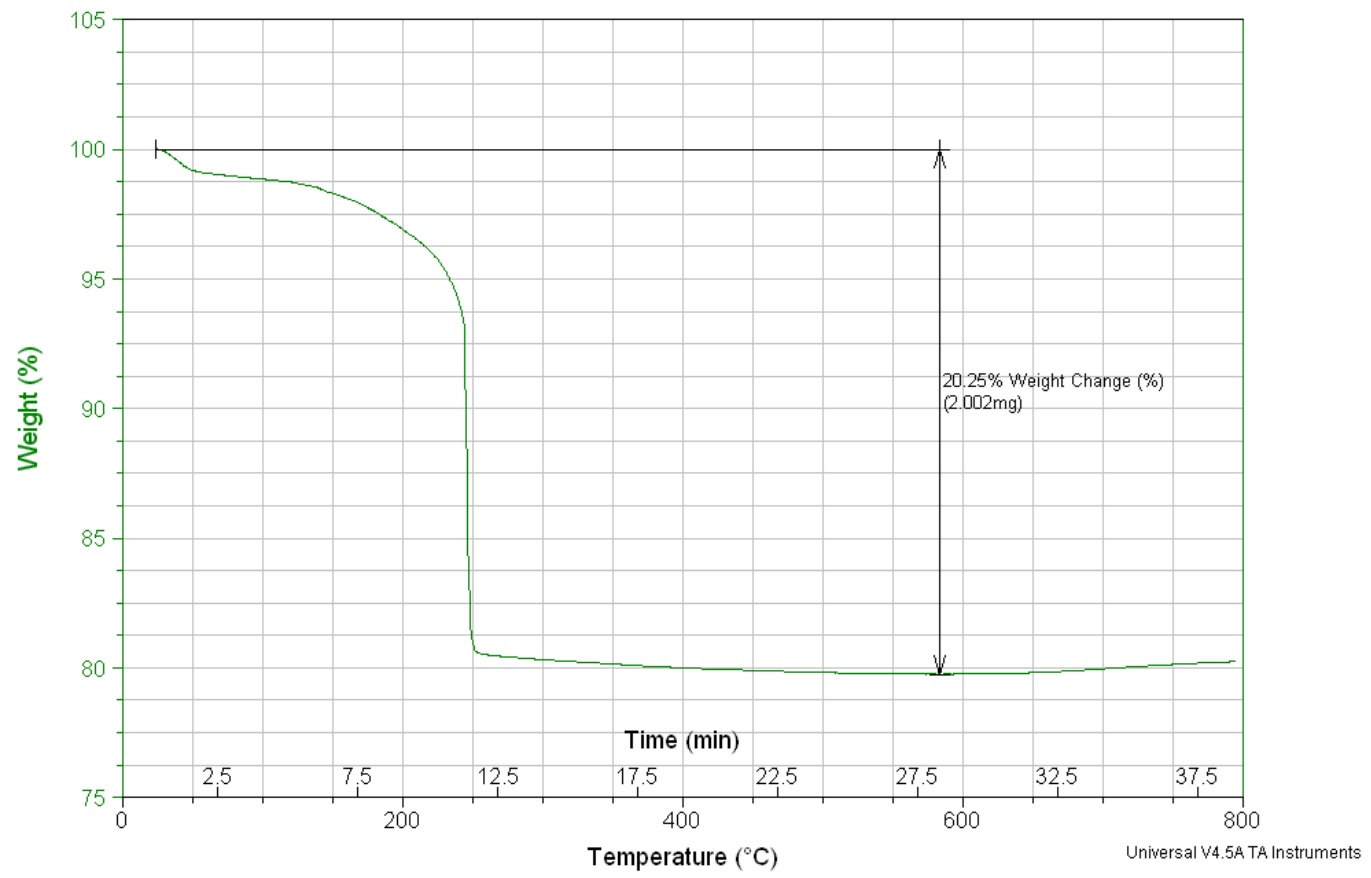

Figure 3.3. TGA of $\mathrm{Au}_{144}\left(\mathrm{C}_{6} \mathrm{~S}\right)_{60} \mathrm{MPCs}$. 


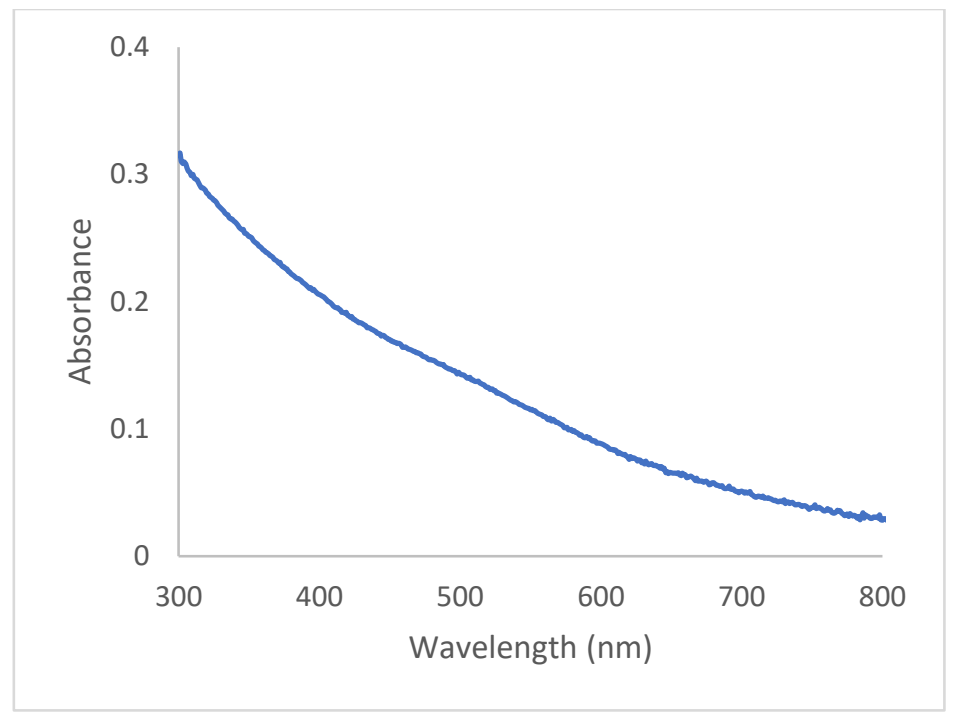

Figure 3.4. UV-Vis of $\mathrm{Au}_{144}\left(\mathrm{C}_{6} \mathrm{~S}\right)_{60}$ MPCs (sample was prepared in THF, concentration of MMPCs was $0.0188 \mathrm{mM}$ ).
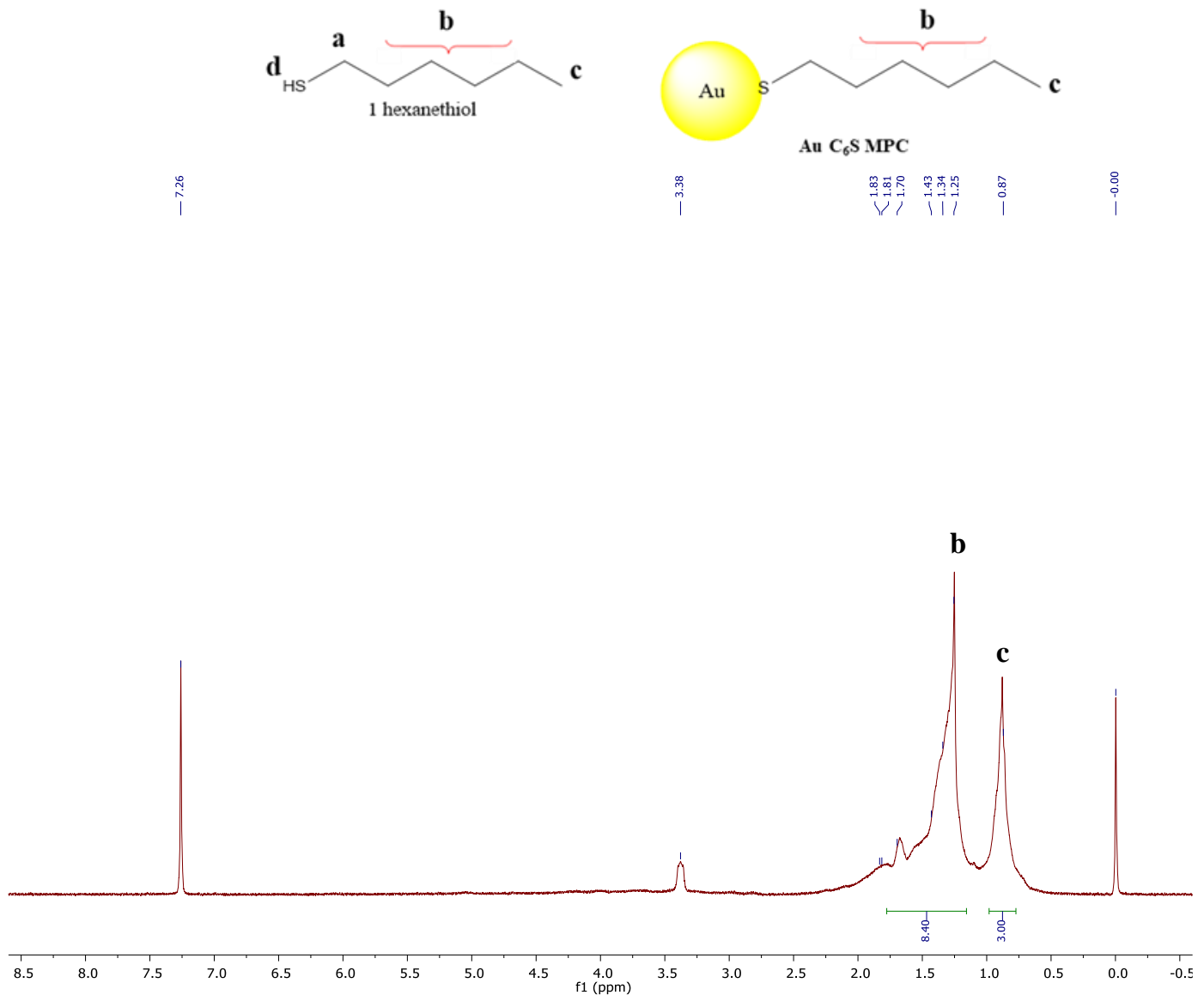

Figure 3.5. ${ }^{1} \mathrm{H}$ NMR spectrum of $\mathrm{Au}_{144}\left(\mathrm{C}_{6} \mathrm{~S}\right)_{60} \mathrm{MPCs}$ 


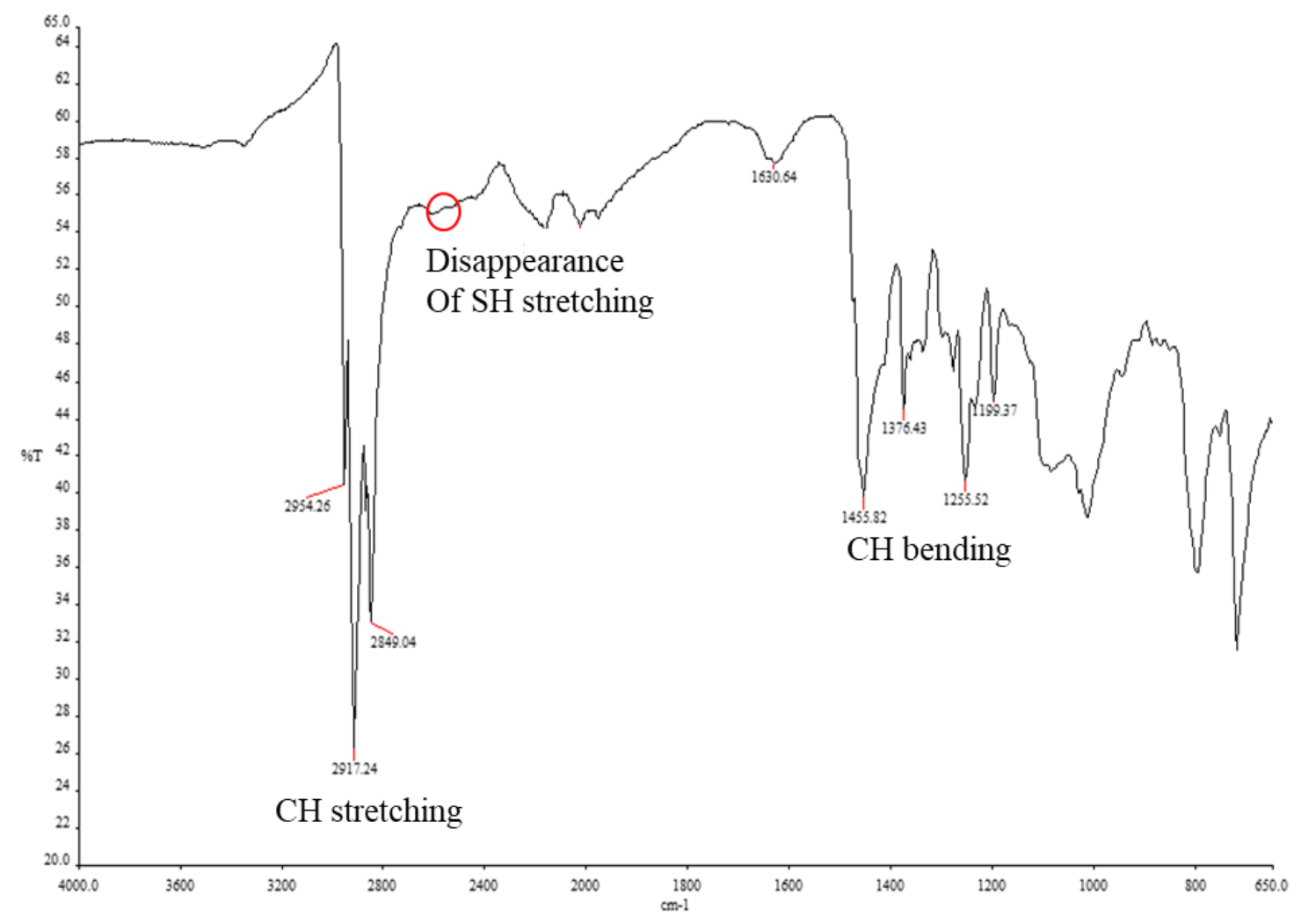

Figure 3.6. FTIR spectrum of $\mathrm{Au}_{144}\left(\mathrm{C}_{6} \mathrm{~S}\right)_{60} \mathrm{MPCs}$

With $\mathrm{AuC}_{6} \mathrm{~S}$ MPCs in hand, a ligand place exchange reaction was performed to prepare $\mathrm{Au}\left(\mathrm{C}_{6} \mathrm{~S}\right)\left(\mathrm{AOT}_{\text {bis }}\right)$ MMPCs with a $\sim 1: 1$ ratio of the reactant thiol ( $\left.\mathrm{AOT}_{\text {bis }}\right)$ : parent thiol $\left(\mathrm{C}_{6} \mathrm{~S}\right)$ by simple mixing with AOT $_{\text {bis. }}$ In this reaction, 4 equivalents of $\mathrm{AOT}_{\text {bis }}$ ligand relative to $\mathrm{SC}_{6}$ ligand, based on TGA data, was added to $\mathrm{AuC}_{6} \mathrm{~S}$ MPC solution to get 1 equivalent added to final cluster (Figure 3.7). UV-vis spectroscopy on the MMPCs showed that the size of the clusters remained less than $2 \mathrm{~nm}$ in diameter after the exchange reaction, as no plasmon band was observed (Figure 3.8). FTIR spectrum of aminooxy MMPCs, shows the broad band representing - $\mathrm{NH}$ stretching of $-\mathrm{ONH}_{2}$ functionality at $3300 \mathrm{~cm}^{-1}$. Absence of the $-\mathrm{SH}$ stretching band at $\sim 2500 \mathrm{~cm}^{-1}$ confirms that there are not any displaced parent ligands or unreacted new ligands remaining in the sample (Figure 3.9). 


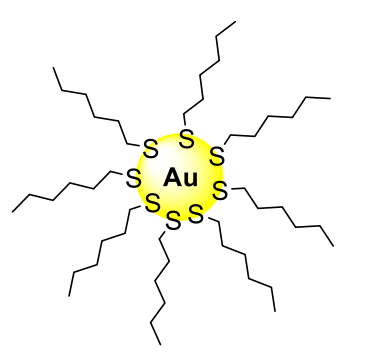

Au $\mathrm{C}_{6} \mathrm{~S}$ MPCs

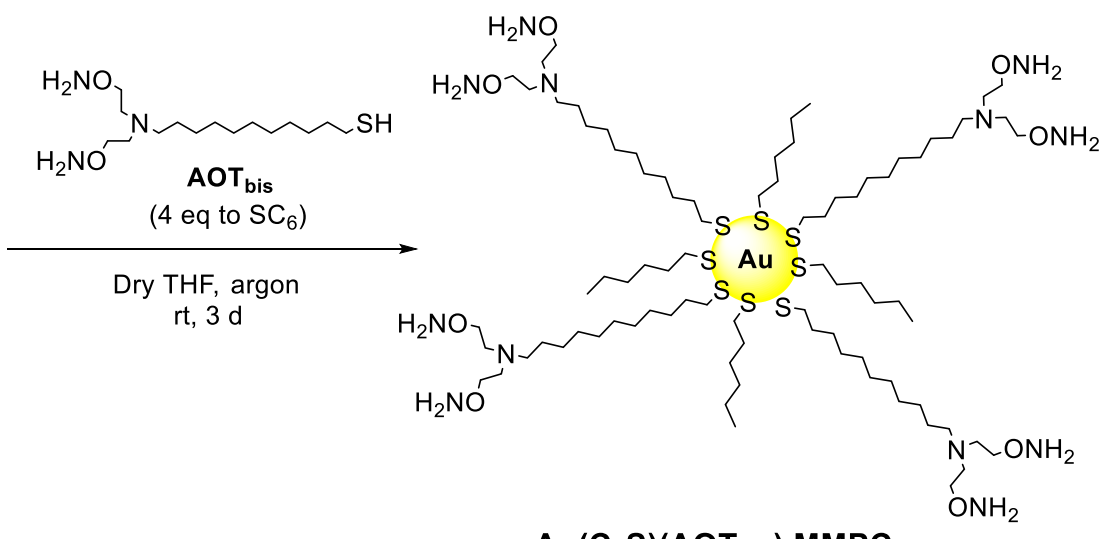

$\mathrm{Au}\left(\mathrm{C}_{6} \mathrm{~S}\right)\left(\mathrm{AOT}_{\mathrm{bis}}\right) \mathrm{MMPCs}$

Figure 3.7. A schematic of ligand place exchange reaction between $\mathrm{Au}_{144}\left(\mathrm{C}_{6} \mathrm{~S}\right)_{60} \mathrm{MPCs}$ and aminooxy ligand $\left(\mathrm{AOT}_{\text {bis }}\right)$ to form $\mathrm{Au}_{144}\left(\mathrm{C}_{6} \mathrm{~S}\right)_{30}\left(\mathrm{AOT}_{\text {bis }}\right)_{30} \mathrm{MMPCs}$.

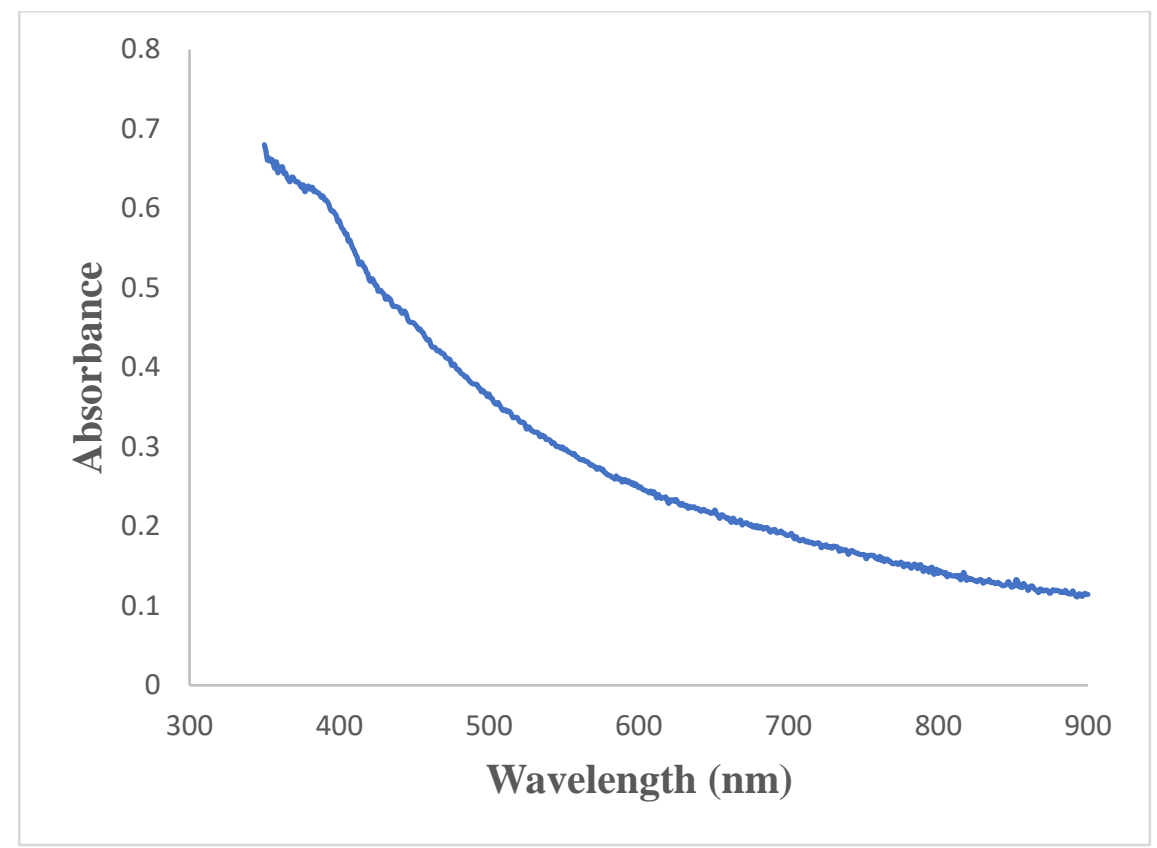

Figure 3.8. UV-VIS spectrum of $\mathrm{Au}_{144}\left(\mathrm{C}_{6} \mathrm{~S}\right)_{30}\left(\mathrm{AOT}_{\text {bis }}\right)_{30} \mathrm{MMPCs}$ (sample was prepared in THF, concentration of MMPCs was $0.0161 \mathrm{mM}$ ). 


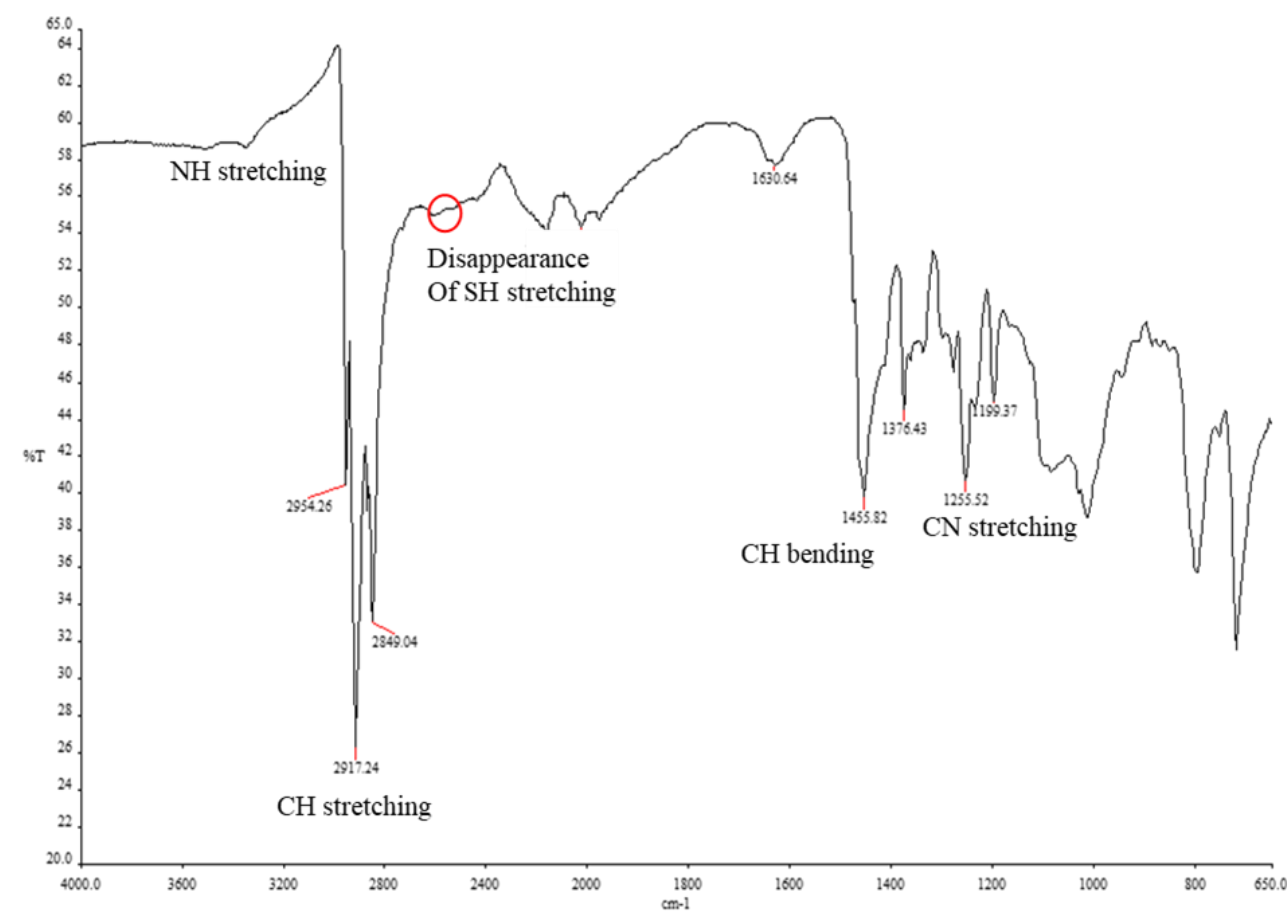

Figure 3.9. FTIR spectrum of $\mathrm{Au}_{144}\left(\mathrm{C}_{6} \mathrm{~S}\right)_{30}\left(\mathrm{AOT}_{\mathrm{bis}}\right)_{30} \mathrm{MMPCs}$.

Based on ${ }^{1} \mathrm{H}$ NMR analysis (Figure 3.10), there is clear evidence of ligand

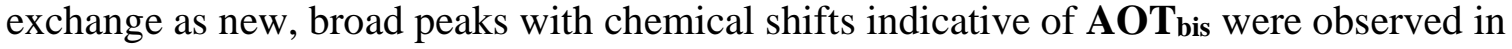
the spectrum of the isolated $\mathrm{Au}\left(\mathrm{C}_{6} \mathrm{~S}\right)\left(\mathbf{A O T}_{\text {bis }}\right)$ MMPCs. Specifically, the distinctive ${ }^{1} \mathrm{H}$ NMR signals at $\delta 3.76\left(-\mathrm{CH}_{2} \mathrm{~N}\left(\mathrm{CH}_{2} \mathrm{CH}_{2} \mathrm{ONH}_{2}\right)_{2}, 2.68\left(-\mathrm{CH}_{2} \mathrm{~N}\left(\mathrm{CH}_{2} \mathrm{CH}_{2} \mathrm{ONH}_{2}\right)_{2}\right)\right.$ and 2.53 $-\mathrm{CH}_{2} \mathrm{~N}\left(\mathrm{CH}_{2} \mathrm{CH}_{2} \mathrm{ONH}_{2}\right)_{2} \mathrm{ppm}$ in the spectrum of the organic soluble MMPCs confirm the replacement of $\mathrm{C}_{6} \mathrm{~S}$ with $\mathbf{A O T}_{\text {bis. }}$ Integration of the $\mathrm{C}_{6} \mathrm{~S}$ methyl resonance at $\delta$ 0.86-0.89 ppm with the unique downfield AOT $\mathbf{A r s}_{\text {bis }}$ signal at $\delta 3.76 \mathrm{ppm}$ (Figure $3.10 \mathrm{C}$, blue arrow) indicates incorporation of $\mathbf{A O T}_{\text {bis }}$ at a $\sim 1: 1$ ratio of $\mathbf{A O T}_{\text {bis: }}: \mathrm{C}_{6} \mathrm{~S}$. Therefore, the results from ${ }^{1} \mathrm{H}$ NMR, suggests the composition of newly formed Au MMPCs as $\mathrm{Au}_{144}\left(\mathrm{C}_{6} \mathrm{~S}\right)_{30}(\mathbf{A O T} \text { bis })_{30}$. To demonstrate the availability of the aminooxy groups in these MMPCs for oximation chemistry, we next examined the conjugation of various aldehydes. 


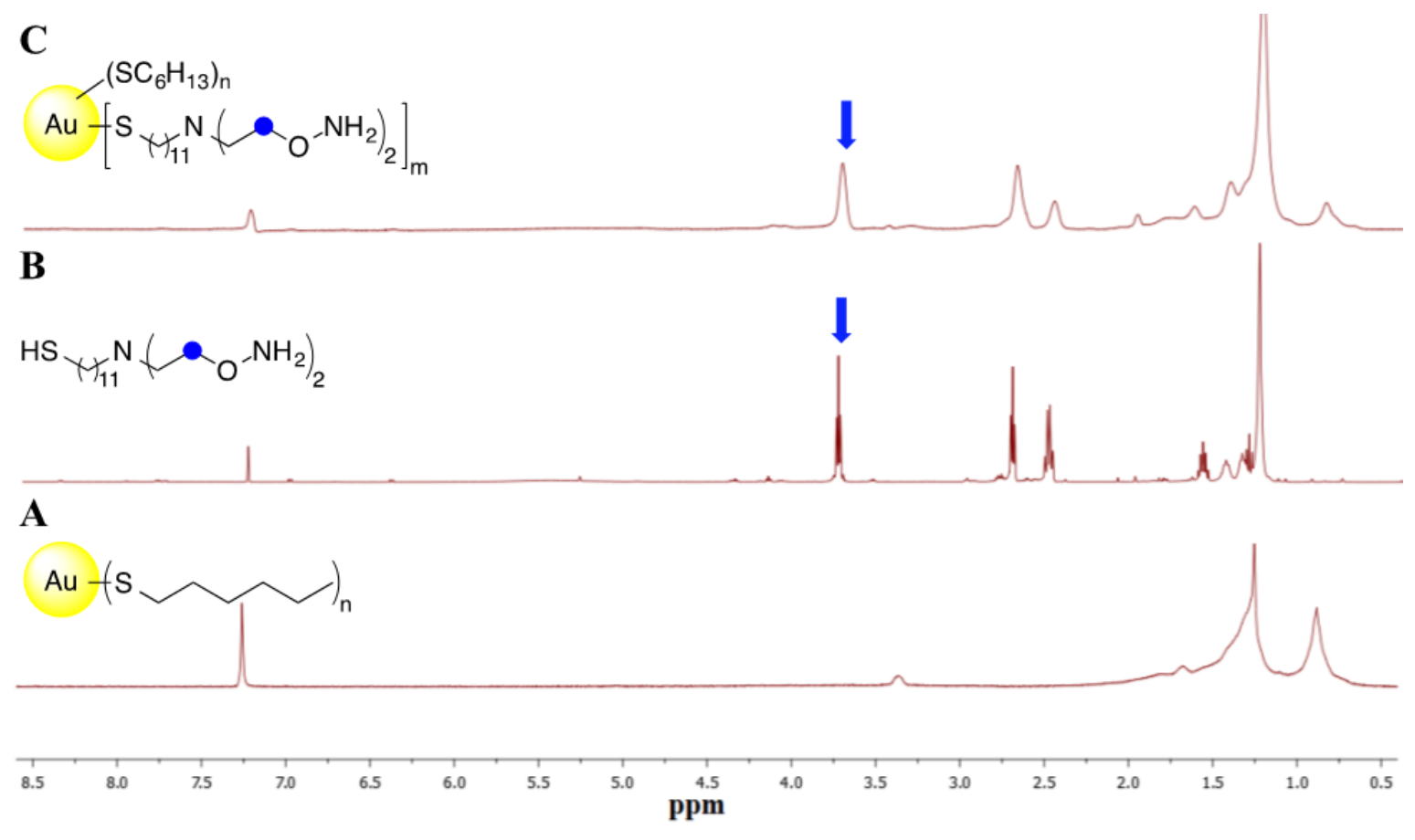

Figure 3.10. ${ }^{1} \mathrm{H}$ NMR spectra (400 $\mathrm{MHz}, \mathrm{CDCl}_{3}$ ) of (A) $\mathrm{Au} \mathrm{C}_{6} \mathrm{~S} \mathrm{MPCs}$, (B) aminooxythiol ligand $\mathrm{AOT}_{\text {bis }},(\mathrm{C}) \mathrm{Au}\left(\mathrm{C}_{6} \mathrm{~S}\right)\left(\mathrm{AOT}_{\text {bis }}\right)$ MMPCs. Peaks at $\delta 7.24 \mathrm{ppm}$ are residual chloroform (solvent) peaks.

\subsection{Oximation Reactions on $\operatorname{Au}_{144}\left(\mathrm{C}_{6} \mathrm{~S}_{30}\left(\mathrm{AOT}_{\text {bis }}\right) 30 \mathrm{MMPCs}\right.$}

We examined the conjugation of a representative panel of aldehydes that included benzaldehyde (organic-soluble aldehyde), ferrocene carboxaldehyde (redox-active aldehyde), pyrene carboxaldehyde (fluorophore), propanal and glyceraldehyde (watersoluble aldehydes), and urea-aldehyde 15 (a bifunctional aldehyde) as listed in Table 3.1.

As part of our program on Au MMPC-based chemiresistors for carbonyl detection, we sought to develop a convenient route for the assembly of urea-functionalized $\mathrm{Au}$ clusters. We have previously reported on the effectiveness of terminal urea functionality 
in sensing acetone based on its ability to form a H-bonded network. ${ }^{140}$ Thus, to investigate a new synthetic approach using aminooxy chemistry, we prepared a model urea-aldehyde for coupling with the $\mathrm{Au}\left(\mathrm{C}_{6} \mathrm{~S}\right)\left(\mathbf{A O T} \mathbf{b i s}_{\text {bs }}\right.$ MMPCs. Synthesis of ureaaldehyde 15 (Scheme 3.1) was performed by first masking the alcohol group of 8-amino1-octanol (12) by reaction with TMSCl to afford silyl ether 13, which was then treated with tert-butyl isocyanate to connect the urea moiety. Silyl ether deprotection using TBAF afforded urea alcohol $\mathbf{3 0}$ in good yield. Subsequent oxidation using PCC gave urea-aldehyde 15.

${ }^{1} \mathrm{H}$ NMR of urea-aldehyde 15 shows the distinct aldehyde proton at $\delta 9.73 \mathrm{ppm}$ confirming the conversion of alcohol group to aldehyde moiety. Likewise, the upfield signals of the methylene protons adjacent to the $-\mathrm{OH}$ group and $-\mathrm{NH}$ group towards $-\mathrm{OH}$ end further confirm product 15 formation (Figure 3.11). Whereas 11 carbon peaks (representing 13 carbon atoms), and especially two distinct carbonyl peaks at $\delta 203$ and $\delta$ $158 \mathrm{ppm}$ from ${ }^{13} \mathrm{C}$ NMR confirms the structure of urea-aldehyde 15 (Figure 3.12). Ureaaldehyde 15 was immediately subjected to an oximation reaction with $\mathrm{Au}\left(\mathrm{C}_{6} \mathrm{~S}\right)\left(\mathrm{AOT}_{\text {bis }}\right)$ MMPCs to form the corresponding urea-aldehyde adduct. 


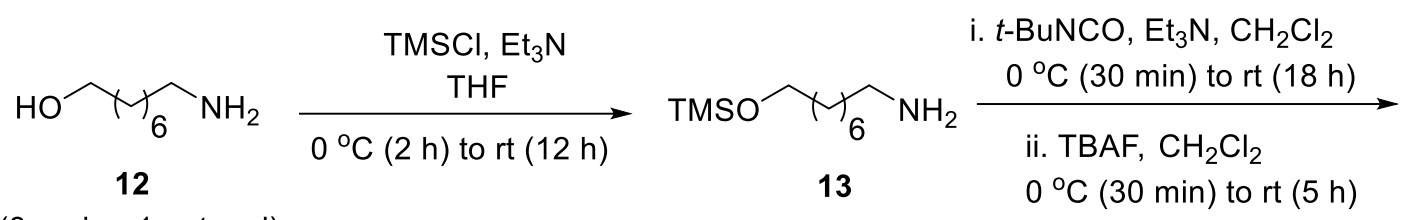

(8-amino-1-octanol)

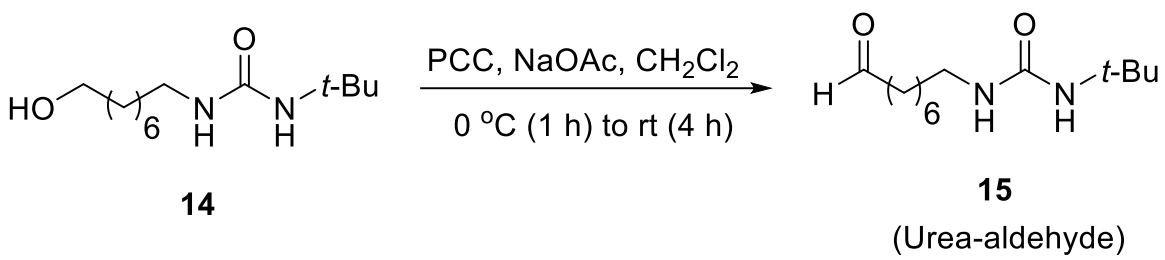

Scheme 3.1. Synthesis of urea-aldehyde 15; $\mathrm{TMSCl}=$ trimethylsilyl chloride, $\mathrm{t}$ $\mathrm{BuNCO}=$ tert $-\mathrm{Butyl}$ isocyanate, $\mathrm{TBAF}=$ tetra $-n$-butylammonium fluoride, $\mathrm{PCC}=$ pyridinium chlorochromate. Overall yield ( 3 steps $)=28 \%$.

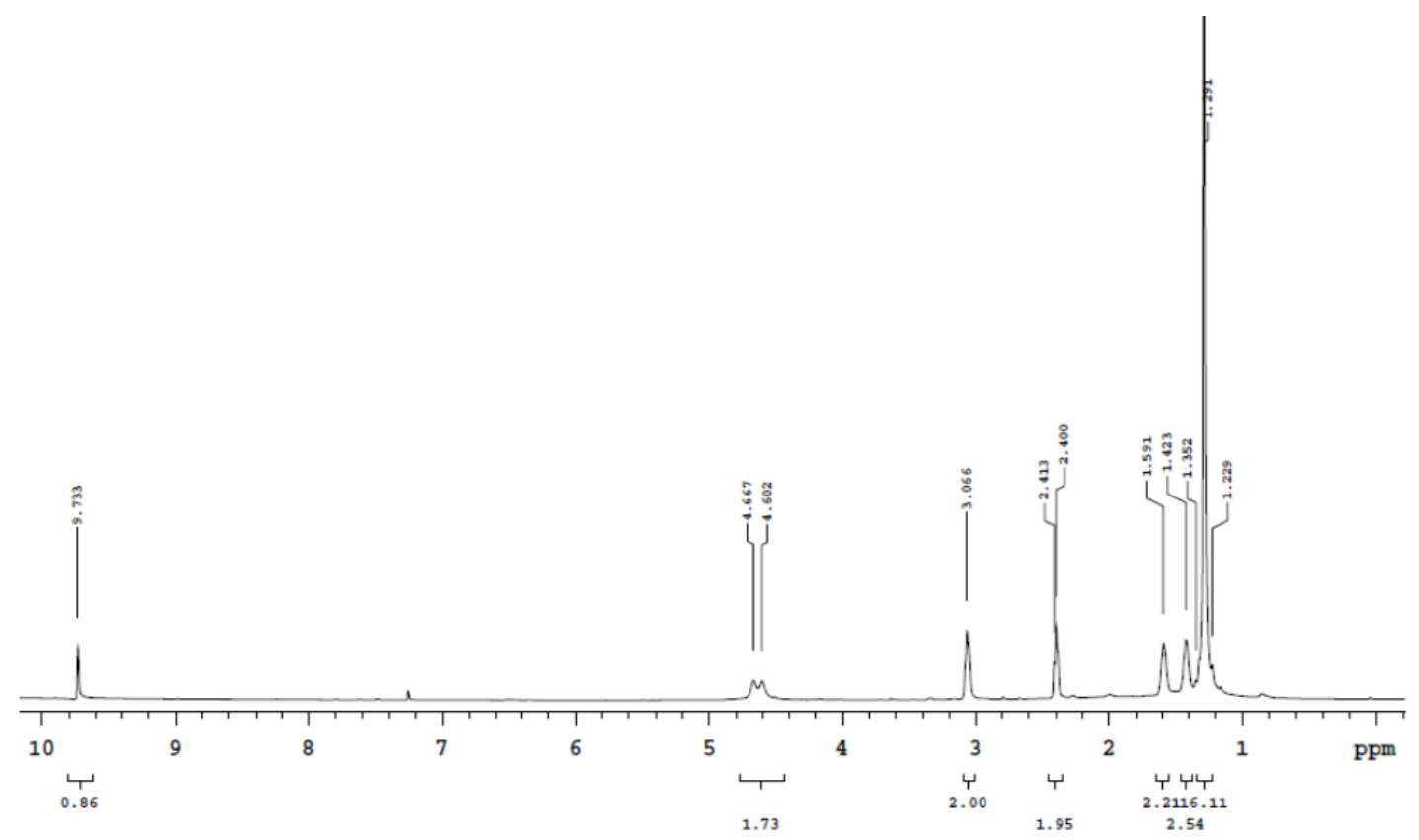

Figure 3.11. ${ }^{1} \mathrm{H}$ NMR spectrum $\left(500 \mathrm{MHz}, \mathrm{CDCl}_{3}\right.$ ) of urea-aldehyde 15. 


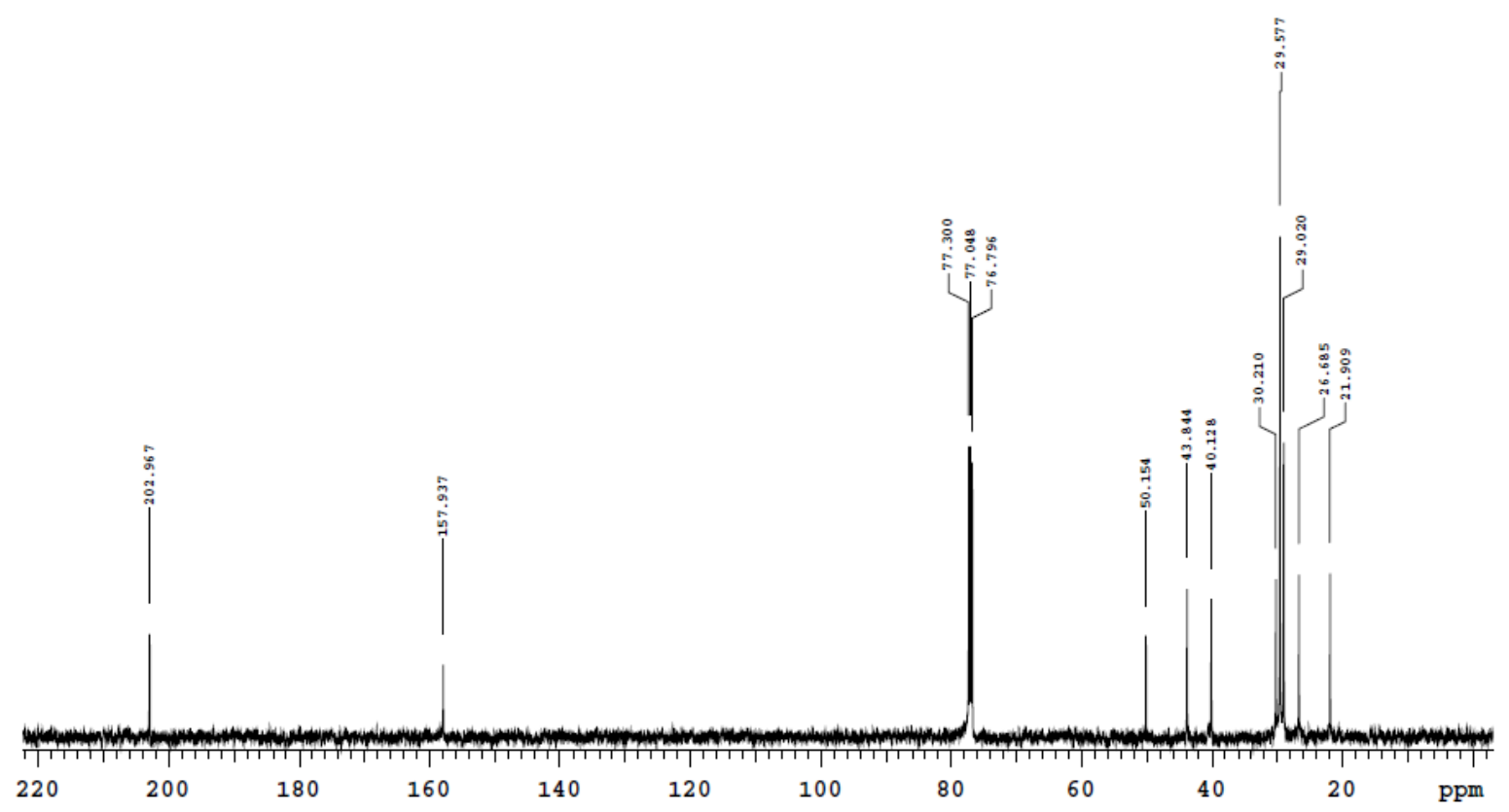

Figure 3.12. ${ }^{13} \mathrm{C}$ NMR spectrum $\left(500 \mathrm{MHz}, \mathrm{CDCl}_{3}\right)$ of urea-aldehyde 15.

The oximation reactions were performed under mild conditions in either THF or water as solvent to accommodate the specific carbonyl compounds (Scheme 3.2). The progress of the couplings was readily monitored by ${ }^{1} \mathrm{H}$ NMR analysis. Specifically, the distinctive downfield shifts of the methylene group adjacent to the aminooxy moiety on oxime ether formation (blue arrows, Figures 3.13 and 3.14) as well as appearances of characteristic oximyl protons (-CH=NOR, red arrows, Figures 3.13 and 3.14) for the $E$ and Z-oxime ether products show that the $\mathrm{Au}\left(\mathrm{C}_{6} \mathrm{~S}\right)\left(\mathbf{A O T}_{\text {bis }}\right)$ MMPCs reacted efficiently with the panel of aldehydes to afford the corresponding oxime ether adducts. Oxime ether formation was also confirmed by FTIR spectroscopy, which showed a consistent shift of carbonyl stretching frequency from $\geq 1700 \mathrm{~cm}^{-1}$ for the starting aldehyde substrate to near $1600 \mathrm{~cm}^{-1}$ for the oxime ether-functionalized MMPCs (Table 3.2). The sizes of 
the reacted $\mathrm{Au}\left(\mathrm{C}_{6} \mathrm{~S}\right)\left(\mathbf{A O T}_{\text {bis }}\right)$ MMPCs did not change as a result of the oximation as determined by UV-vis spectroscopy (Figure 3.15, $\mathrm{Au}\left(\mathrm{C}_{6} \mathrm{~S}\right)\left(\mathbf{A O T}_{\text {bis-benzaldehyde) }}\right.$ MMPCs).

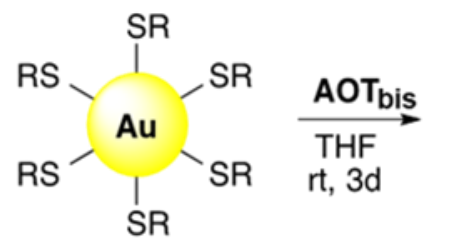

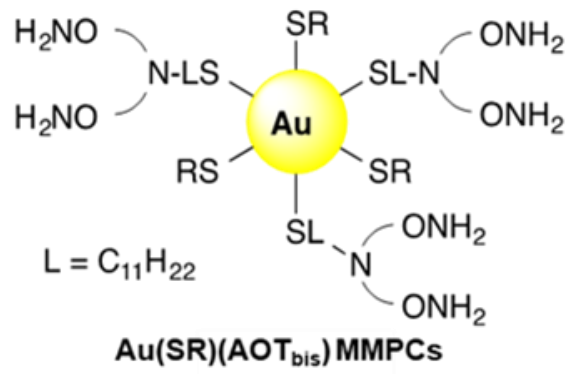

Au MPCs

$\mathrm{R}=\mathrm{C}_{6} \mathrm{H}_{13}$

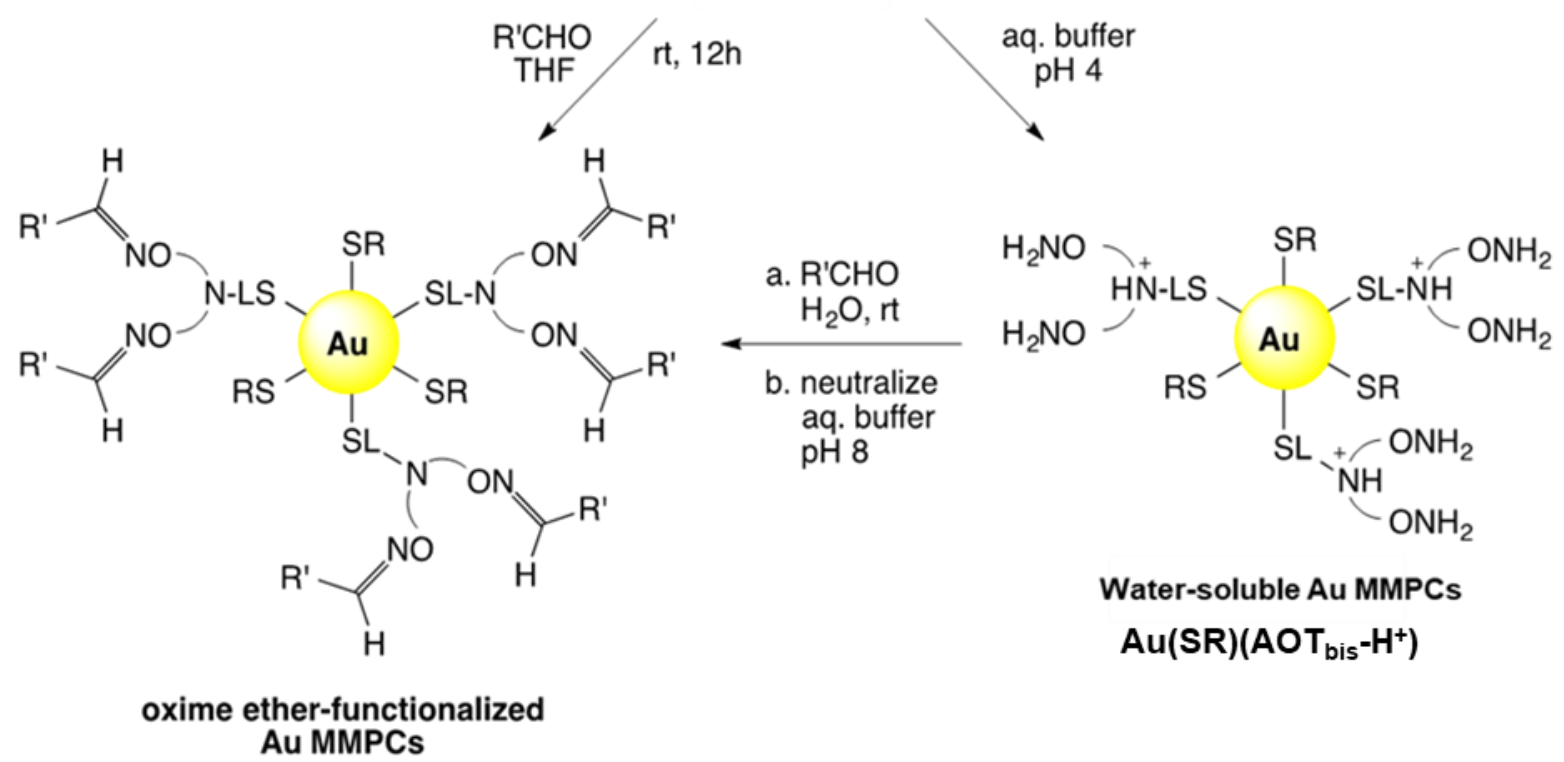

Scheme 3.2. Schematic representation of place exchange and oximation

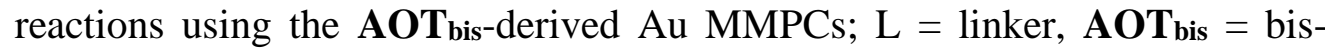
aminooxy thiol. 


\begin{tabular}{|c|c|c|c|}
\hline Aldehydes & Chemical Structure & $\begin{array}{c}\text { Nonpolar/ } \\
\text { Polar/ } \\
\text { Aqueous }\end{array}$ & Applications \\
\hline Benzaldehyde & & Non-polar & $\begin{array}{l}\text { Sensor (e.g. } \\
\text { Benzene) }\end{array}$ \\
\hline Ferrocenecarboxaldehyde & & Non-polar & $\begin{array}{l}\text { Redox-active } \\
\text { molecule } \\
\text { (electrochemical- } \\
\text { study) }\end{array}$ \\
\hline 1-Pyrenecarboxaldehyde & & Non-polar & $\begin{array}{c}\text { Fluorophore } \\
\text { (probe in biological } \\
\text { applications) }\end{array}$ \\
\hline $\begin{array}{l}\text { Urea-Aldehyde (15) } \\
\text { (Synthesized in lab) }\end{array}$ & & Polar & $\begin{array}{c}\text { Sensor } \\
\text { (Detection of VOCs) }\end{array}$ \\
\hline Propanal & & Aqueous & $\begin{array}{l}\text { Industrial } \\
\text { applications }\end{array}$ \\
\hline Glyceraldehyde & & Aqueous & $\begin{array}{c}\text { Biological } \\
\text { applications }\end{array}$ \\
\hline
\end{tabular}

Table 3.1. Aldehydes examined in oximation reactions with $\mathrm{Au}\left(\mathrm{C}_{6} \mathrm{~S}\right)\left(\mathrm{AOT}_{\mathrm{bis}}\right) \mathrm{MMPCs}_{\text {. }}$ 


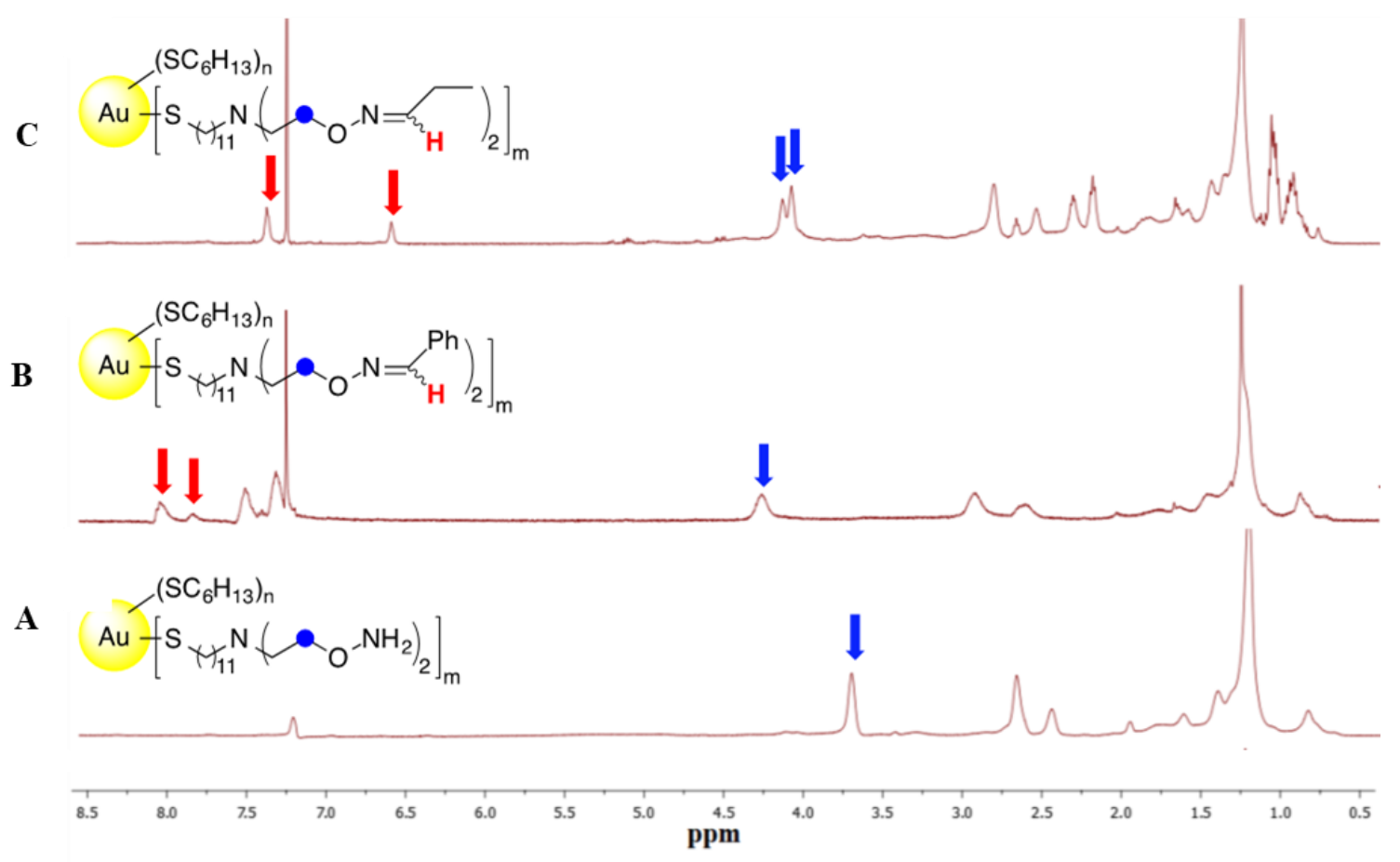

Figure 3.13. ${ }^{1} \mathrm{H}$ NMR spectra $\left(400 \mathrm{MHz}, \mathrm{CDCl}_{3}\right)$ of $(\mathrm{A}) \mathrm{Au}\left(\mathrm{C}_{6} \mathrm{~S}\right)\left(\mathrm{AOT}_{\mathrm{bis}}\right)$ MMPCs, and (B) benzaldehyde- and (C) propanal-adducts obtained on reaction with $\mathrm{Au}\left(\mathrm{C}_{6} \mathrm{~S}\right)\left(\mathrm{AOT}_{\text {bis }}\right) \mathrm{MMPCs}$. Peaks at $\delta 7.24 \mathrm{ppm}$ are residual chloroform (solvent) peaks. 


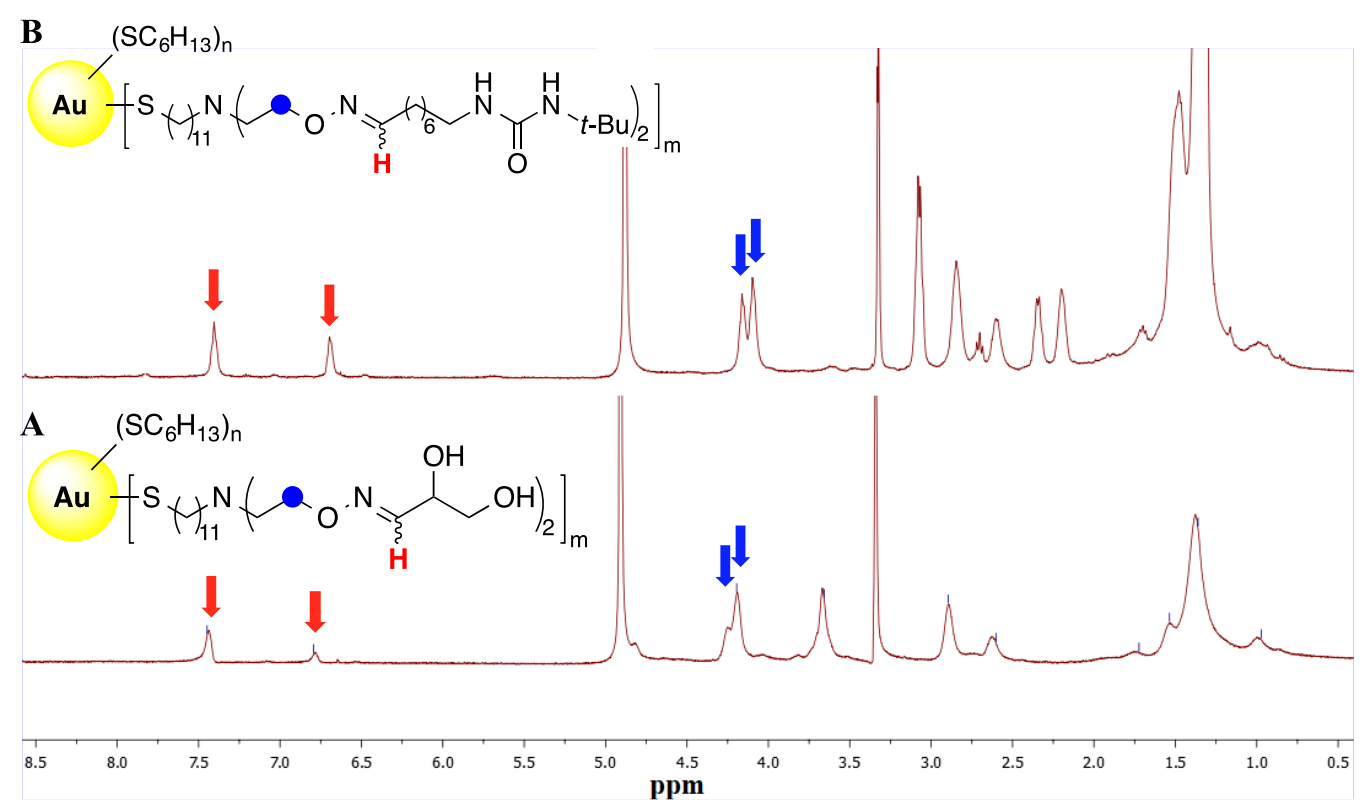

Figure 3.14. ${ }^{1} \mathrm{H}$ NMR spectra $\left(400 \mathrm{MHz}, \mathrm{CD}_{3} \mathrm{OD}\right)$ of (A) glyceraldehydeand (B) urea-aldehyde 15-adducts obtained on reaction with $\mathrm{Au}\left(\mathrm{C}_{6} \mathrm{~S}\right)\left(\mathrm{AOT}_{\text {bis }}\right)$ MMPCs. Peaks at $\delta 4.87$ and $3.34 \mathrm{ppm}$ are residual methanol (solvent) peaks.

\begin{tabular}{|l|c|c|}
\hline \multicolumn{1}{|c|}{$\mathrm{R}-\mathrm{CHO}$} & $\begin{array}{c}\text { starting aldehyde } \\
\mathrm{C}=\mathrm{O}\left(\mathrm{cm}^{-1}\right)\end{array}$ & $\begin{array}{c}\text { oxime ether MMPCs } \\
\mathrm{C}=\mathrm{N}\left(\mathrm{cm}^{-1}\right)\end{array}$ \\
\hline $\mathrm{Ph}-\mathrm{CHO}$ & 1696 & 1603 \\
\hline $\mathrm{CH}_{3} \mathrm{CH}_{2}-\mathrm{CHO}$ & 1734 & 1599 \\
\hline $\mathrm{CH}_{2}(\mathrm{OH}) \mathrm{CH}(\mathrm{OH})-\mathrm{CHO}$ & 1731 & 1599 \\
\hline $\mathrm{CpFe}\left(\mathrm{C}_{5} \mathrm{H}_{4}\right)-\mathrm{CHO}$ & 1658 & 1607 \\
\hline pyrene-CHO & 1678 & 1602 \\
\hline urea-aldehyde 31 & 1723 & 1634 \\
\hline
\end{tabular}

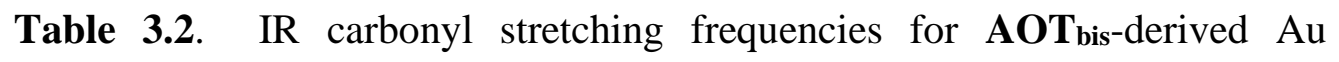
MMPCs. 


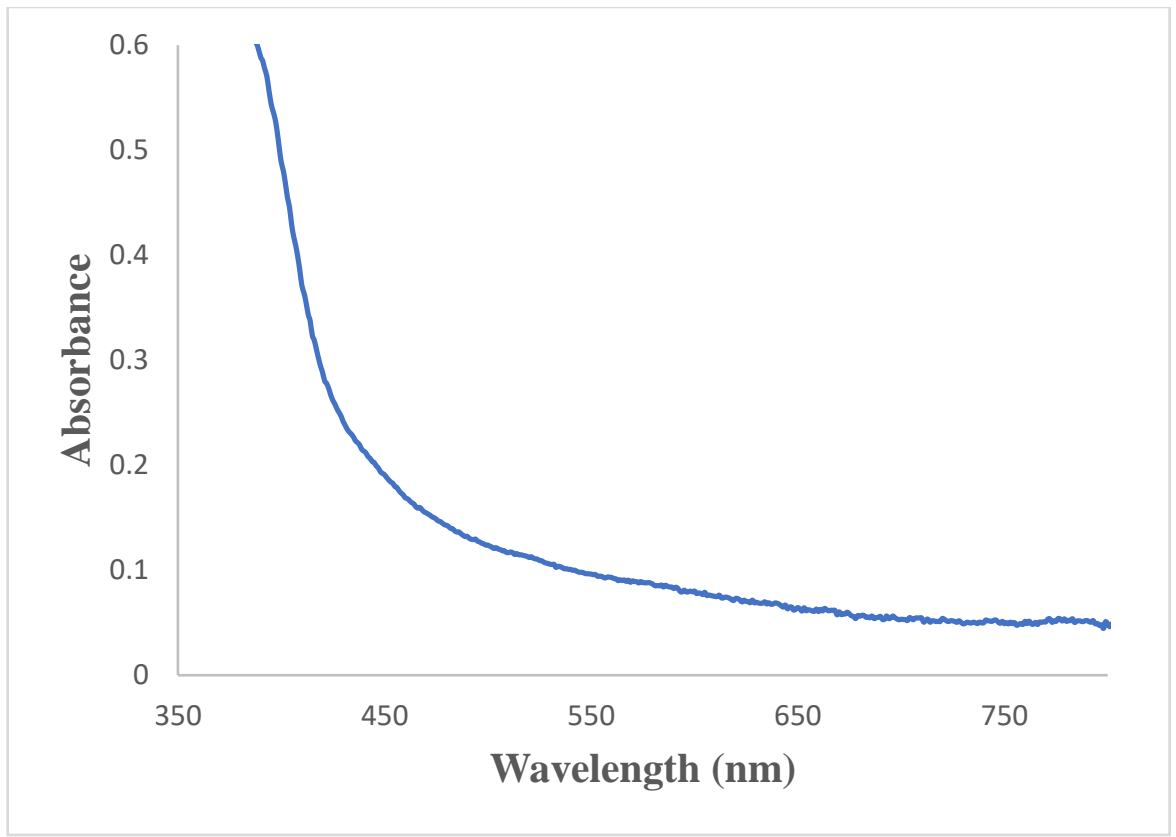

Figure 3.15. UV-VIS spectrum of $\mathrm{Au}\left(\mathrm{C}_{6} \mathrm{~S}\right)\left(\mathrm{AOT}_{\text {bis }}-\right.$ benzaldehyde) MMPCs (sample was prepared in THF, concentration of MMPCs was $2.82 \mathrm{mM}$ ).

To demonstrate oximation under aqueous conditions, $\mathrm{Au}_{144}\left(\mathrm{C}_{6} \mathrm{~S}\right)_{30}\left(\mathbf{A O T} \mathbf{T}_{\mathbf{b i s}}\right)_{30}$ MMPCs were reacted with propanal and glyceraldehyde in $\mathrm{pH} 4.0$ acetate buffer solutions. The clusters readily dissolved, likely due to protonation of the amino moiety rendering the $\mathrm{Au}$ MMPCs water-soluble $\left(\mathrm{Au}\left(\mathrm{C}_{6} \mathrm{~S}\right)\left(\mathbf{A O T}_{\text {bis }}-\mathbf{H}^{+}\right)\right.$, Scheme 3.2). Reaction with the water-soluble aldehydes yielded the corresponding oxime ether adducts as mixtures of $E / Z$ isomers (Figures $3.13 \mathrm{C}$ and $3.14 \mathrm{~A}$ ). In the case of propanal, neutralization of the adduct solution by washing with basic buffer restored the organic solubility and enabled extraction of the derivatized MMPCs into organic solvent for ease of isolation. The formation of the propanal and glyceraldehyde adducts on the MPC surface demonstrates the tunable properties of the $\mathrm{Au}_{144}\left(\mathrm{C}_{6} \mathrm{~S}\right)_{30}\left(\mathbf{A O T}_{\text {bis }}\right)_{30}$ MMPCs (Figure 3.16). This easy switchability of the MMPCs solublity suggested that 
functionalization of water soluble MPCs may not be essential depending on the intended application. For example, AuSG MPCs functionalization with $\mathbf{A O T}_{\text {bis }^{+}}$may not be required for performing aqueous oximation reactions with water-soluble aldehydes.

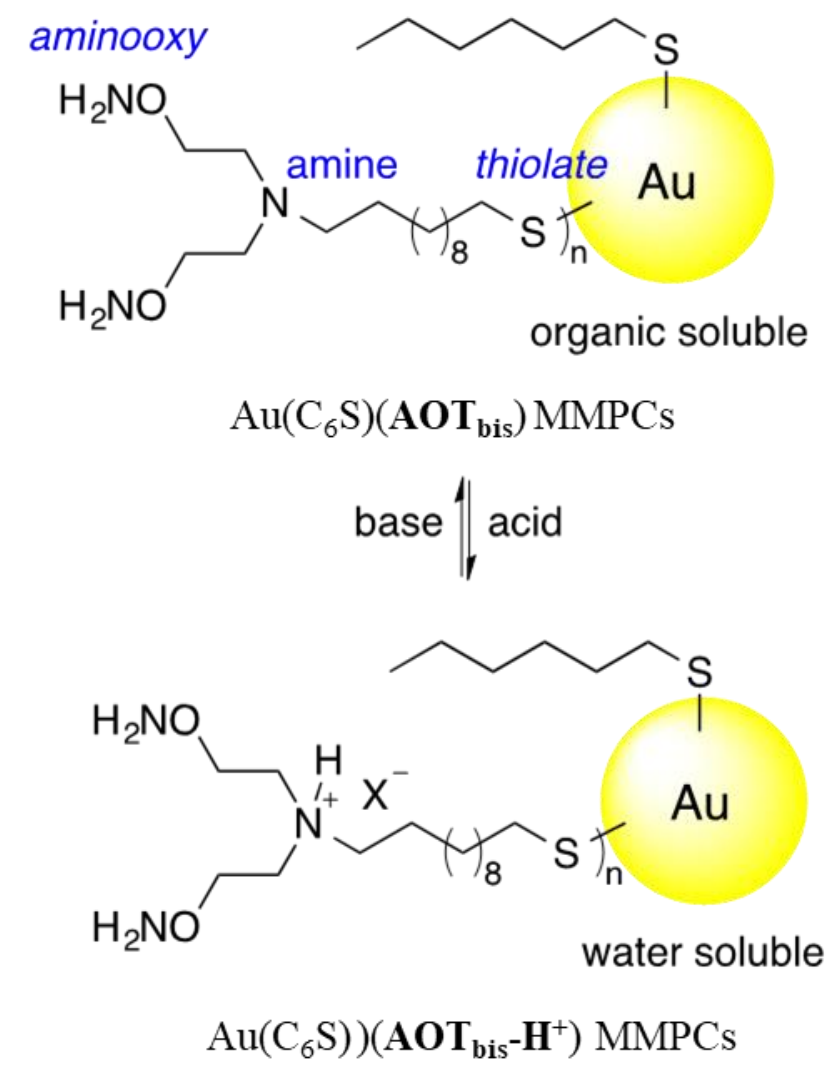

Figure 3.16. Concept for tuning the solubility of $\mathrm{Au}\left(\mathrm{C}_{6} \mathrm{~S}\right)\left(\mathrm{AOT}_{\mathrm{bis}}\right)$ MMPCs.

$\mathrm{Au}_{144}\left(\mathrm{C}_{6} \mathrm{~S}\right)_{30}\left(\mathbf{A O T}_{\text {bis }}\right)_{30}$ MMPCs surface functionalization was also assessed by electrochemical and fluorescent measurements. For example, after reaction of $\mathrm{Au}_{144}\left(\mathrm{C}_{6} \mathrm{~S}\right)_{30}\left(\mathbf{A O T}_{\text {bis }}\right)_{30}$ MMPCs with ferrocene-carboxaldehyde, electrochemical measurements on the Fc-derivatized MMPCs in solution (Figure $3.17 \mathrm{~B}$, red CV) demonstrated oxidation/reduction behaviour characteristic of ferrocene. The small peak 
splitting $(45 \mathrm{mV})$ and symmetric waves indicate that the $\mathrm{Au}\left(\mathrm{C}_{6} \mathrm{~S}\right)\left(\right.$ AOT $_{\text {bis-ferrocene) }}$ MMPCs are strongly adsorbed to the Pt electrode surface, similar to previous results on other Fc-MPCs with high loading141, 142 A CV of the working electrode after removal from the solution of $\mathrm{Au}\left(\mathrm{C}_{6} \mathrm{~S}\right)(\mathbf{A O T}$ bis-ferrocene) MMPCs (Figure $3.17 \mathrm{~B}$, blue $\mathrm{CV}$ ) confirms strong adsorption to the electrode surface since the Fc peaks remain in the $\mathrm{CV}$ and they are symmetric with small peak splitting.
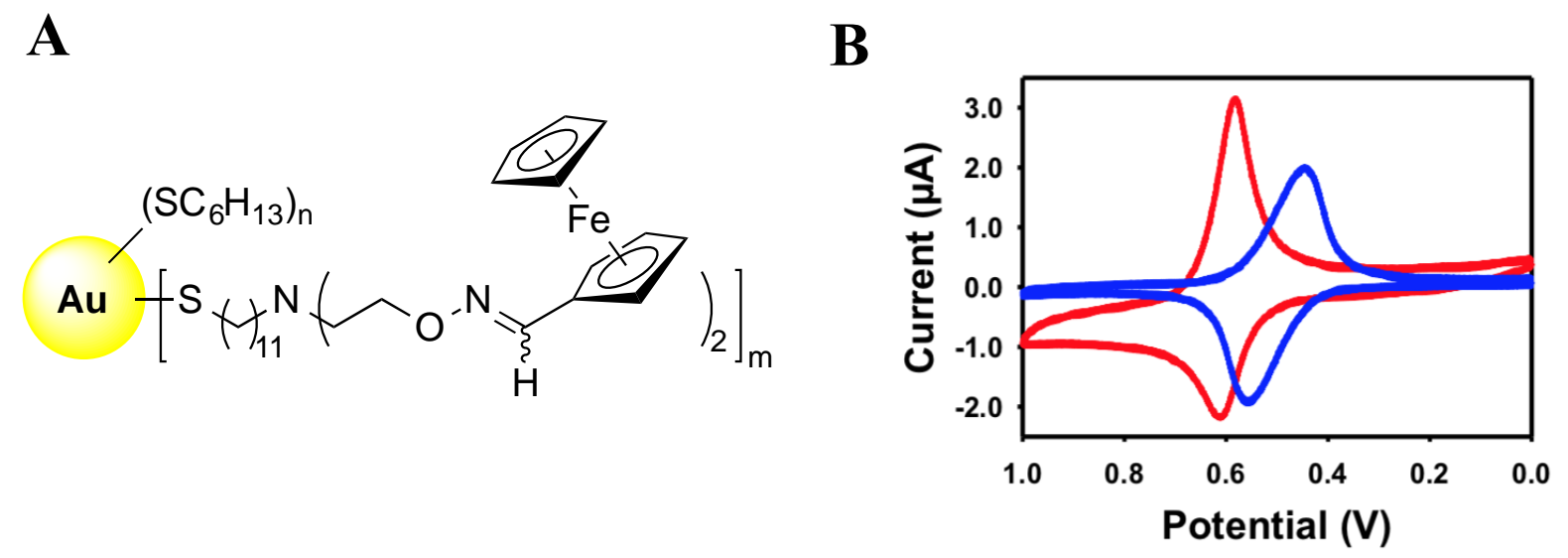

Figure 3.17. (A) Structure of ferrocenecarboxaldehyde adduct obtained on reaction with $\mathrm{Au}\left(\mathrm{C}_{6} \mathrm{~S}\right)\left(\mathrm{AOT}_{\text {bis }}\right) \mathrm{MMPCs}$ and (B) cyclic voltammograms of $\mathrm{Au}\left(\mathrm{C}_{6} \mathrm{~S}\right)\left(\mathrm{AOT}_{\text {bis-ferrocene }}\right) \mathrm{MMPCs}$ in a $0.10 \mathrm{M} \mathrm{TBAHFP} / \mathrm{CH}_{2} \mathrm{Cl}_{2}$ solution with an overall ferrocene concentration of $1.7 \mathrm{mM}$ (red) and of the electrode after having been removed from the Au MMPCs solution and then placed in a $0.10 \mathrm{M} \mathrm{TBAHFP} / \mathrm{CH}_{2} \mathrm{Cl}_{2}$ electrolyte solution (blue). Scan rate $=100 \mathrm{mV} / \mathrm{s}$, working electrode $=\mathrm{Pt}$ disk $(2 \mathrm{~mm}$ diameter $)$, counter electrode $=\mathrm{Pt}$ wire, and reference electrode $=\mathrm{Ag}$ wire quasi-reference electrode. 
The fluorophore 1-pyrenecarboxaldehyde was attached to the $\mathrm{Au}\left(\mathrm{C}_{6} \mathrm{~S}\right)\left(\mathrm{AOT}_{\text {bis }}\right)$ MMPCs by simple mixing and the fluoresence of the resulting MMPCs was measured. Comparison of the fluorescence emission spectra of 1-pyrenecarboxaldehyde with the

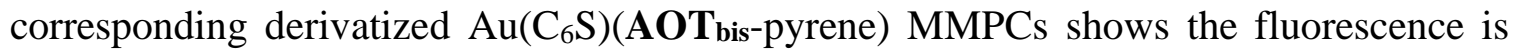
quenched by the binding to the Au cluster (Figure 3.18). The quenching of fluorophores by metal nanoparticles is well established, ${ }^{143}$ and serves in this case as an indication of successful coupling.

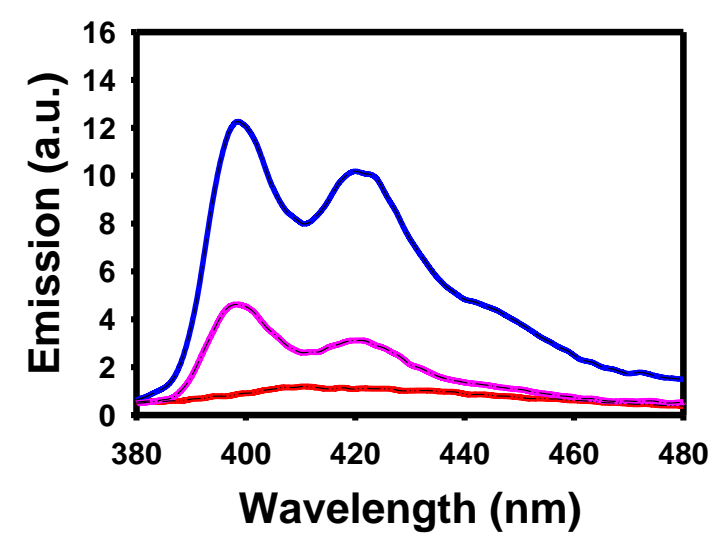

Figure 3.18. Fluorescence emission spectra of 1-pyrenecarboxaldehyde (1.0 x $10^{-7} \mathrm{M}$ in $\left.\mathrm{CHCl}_{3}\right)$ (blue), $\mathrm{Au}\left(\mathrm{C}_{6} \mathrm{~S}\right)\left(\mathrm{AOT}_{\text {bis }}\right.$-pyrene) MMPCs $\left(1.0 \times 10^{-7} \mathrm{M}\right.$ in pyrene-CHO) (pink), and $\mathrm{CHCl}_{3}$ (red). Excitation $\lambda=375 \mathrm{~nm}$.

Urea-aldehyde $\mathbf{1 5}$ was synthesized in a lab for their use in the development of $\mathrm{Au}$ MPC-urea motif-based gas sensor for the detection of VOCs. The appearances of characteristic oximyl protons (-CH=NOR, red arrows) for the E- and Z-oxime ether products indicated that the $\mathrm{Au}\left(\mathrm{C}_{6} \mathrm{~S}\right)(\mathrm{AOT})$ MMPCs reacted efficiently with the ureaaldehyde 15 to afford the corresponding urea-aldehyde adduct (Figure 3.13 B). Further 
characterization of this adduct using MALDI-TOF MS techniques demonstrated the successful coupling. Structure characterization of the obtained Au MPC-adduct using MALDI-TOF MS was aided by the fact that Au itself serves as a matrix, hence providing the higher ionization efficiency. Laser irradiation in the MALDI process induce the efficient cleavage of Au-S bond to release the ligands chemisorbed on the Au surface. Moreover, MPCs possessing higher surface area accelerates the ionization of chemisorbed molecules from the MPC surface.

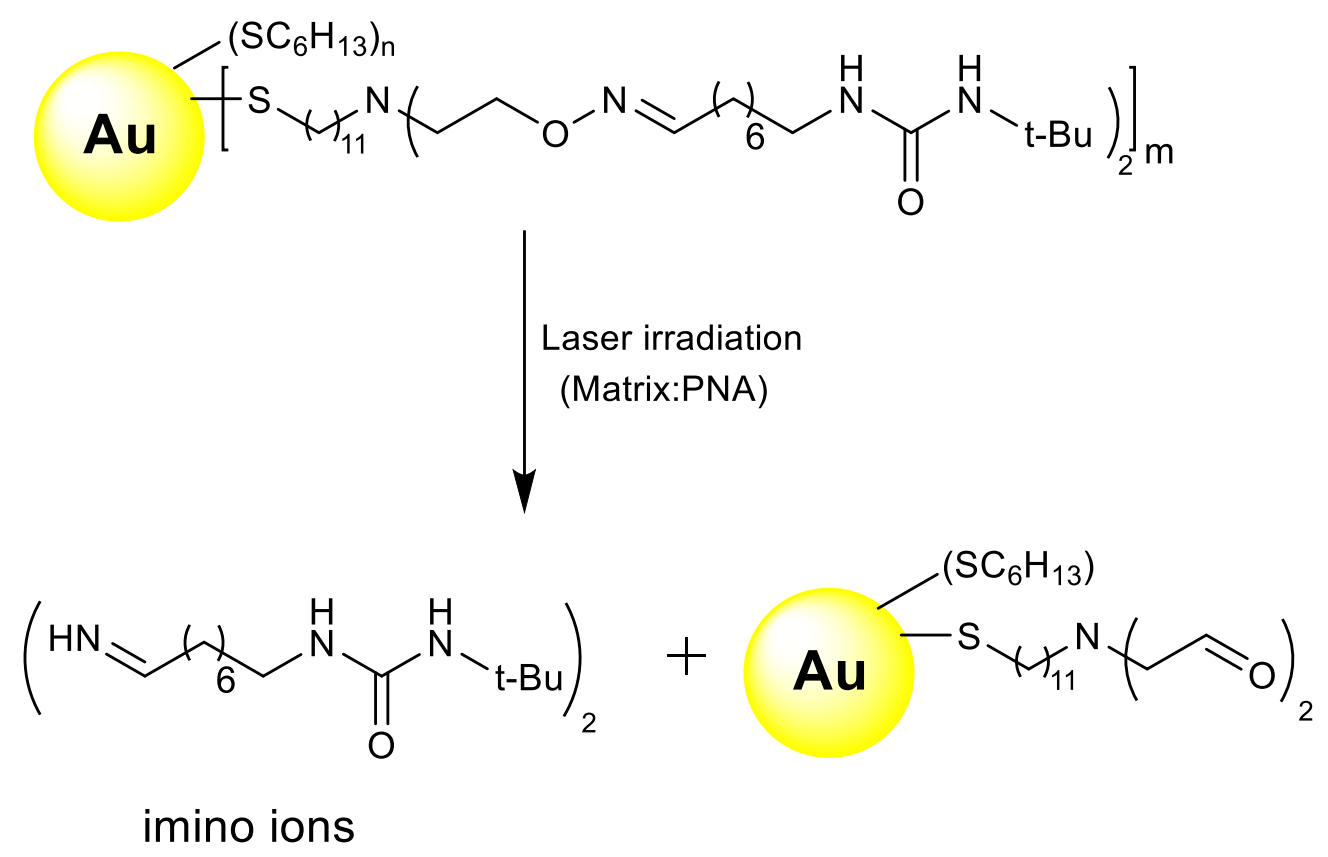

Figure 3.19. Schematic illustration of imino ions formation via oxime bond breakdown upon laser irradiation during MALDI process. (PNA $=$ pnitroaniline) 
As expected the laser irradiation caused the cleavage of the oxime bond formed between the aldehydes and aminooxy groups to liberate imino alcohol ions as shown in Figure 3.19. ${ }^{3}$ While the cleavage of Au-S bond is likely, other possible fragments formed upon laser irradiation are shown in the MALDI-TOF MS spectrum below (Figure 3.20). Summary of the proposed chemical structure of the fragment ions formed directly from the Au MMPC are listed in Table 3.3. Fragments corresponding to sodiatedhexanethiolate, aminooxy thiol ligand (AOT $\mathrm{Ais}_{\text {bis }}$, disulfides, imino ions are reported.

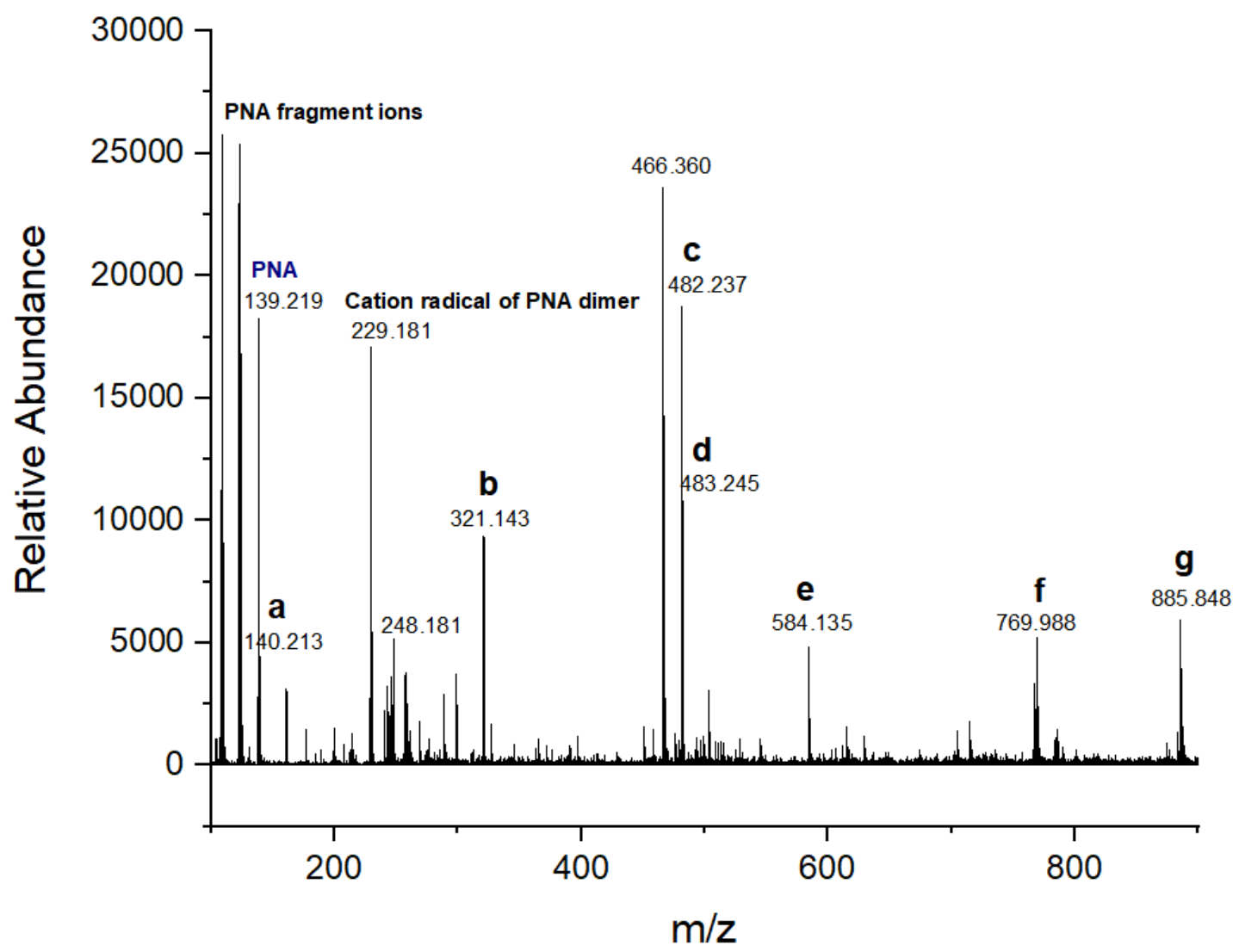

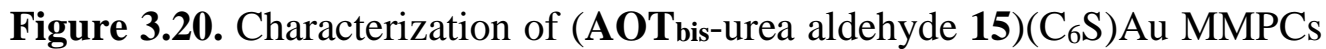
by direct MALDI-TOFMS. Chemical formulas of major signals are listed in Table 3.3. 


\begin{tabular}{|c|c|}
\hline Label & Proposed Chemical Structure (calculated $\mathbf{m} / \mathbf{z}$ ) \\
\hline$\stackrel{\mathbf{a}}{[\mathrm{M}+\mathrm{Na}]^{+}}$ & $\mathrm{C}_{6} \mathrm{H}_{13} \mathrm{NaS}$, calcd 140.2198 \\
\hline b & $\mathrm{C}_{15} \mathrm{H}_{35} \mathrm{~N}_{3} \mathrm{O}_{2} \mathrm{~S}$, calcd 321.2450 \\
\hline c & $\mathrm{C}_{26} \mathrm{H}_{54} \mathrm{~N}_{6} \mathrm{O}_{2}$, calcd 482.4308 \\
\hline d & $\mathrm{C}_{15} \mathrm{H}_{28} \mathrm{AuNO}_{2} \mathrm{~S}$, calcd 483.1506 \\
\hline e & $\mathrm{C}_{30} \mathrm{H}_{62} \mathrm{~N}_{7} \mathrm{O}_{4}{ }^{+}$, calcd 584.4859 \\
\hline f & 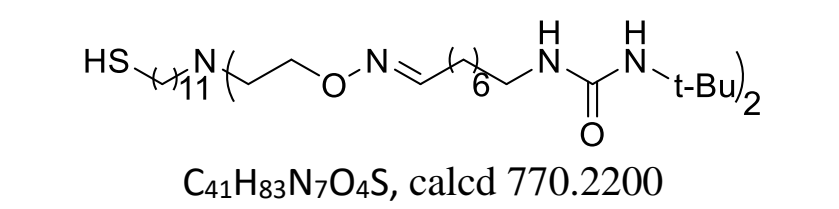 \\
\hline $\mathbf{g}$ & $\mathrm{C}_{47} \mathrm{H}_{95} \mathrm{~N}_{7} \mathrm{O}_{4} \mathrm{~S}_{2}$, calcd 885.6887 \\
\hline
\end{tabular}

Table 3.3. Proposed chemical structure of fragments generated from ( $\mathrm{AOT}_{\mathrm{bis}^{-}}$ urea-aldehyde 15) $\left(\mathrm{C}_{6} \mathrm{~S}\right) \mathrm{Au}$ MMPCs. 
Whereas bis(aminooxy) ligand AOT $\mathbf{A i s}_{\text {can }}$ be formulated to be positively charged using acid, ligand $\mathbf{A O T}$ bis $^{+}$has the advantage of a permanent positive charge in the form of a tetra-alkylammonium ion that is not susceptible to changes in $\mathrm{pH}$. $\mathbf{A O T}_{\text {bis }}{ }^{+}$was used for ligand place exchange reactions with AuSG MPCs to yield $\mathrm{Au}(\mathrm{SG})\left(\mathbf{A O T}_{\text {bis }}{ }^{+}\right)$ MMPCs in a similar way that was used to form $\mathrm{Au}\left(\mathrm{C}_{6} \mathrm{~S}\right)\left(\mathbf{A O T}_{\text {bis }}\right)$ MMPCs. To examine the oximation reactions on water-soluble aminooxy functionalized Au MMPCs, we first reacted $\mathrm{Au}(\mathrm{SG})\left(\mathbf{A O T}_{\text {bis }^{+}}{ }^{+}\right)$MMPCs with a water-soluble aldehyde, glucose under standard condition. However, this reaction failed due to the closed pyran ring structure of the glucose leading to difficulties in oxidation of the alcohol group to an aldehyde group. However, we studied the oximation reaction on $\mathrm{Au}(\mathrm{SG})\left(\mathbf{A O T}_{\text {bis }}{ }^{+}\right)$MMPCs with aldehydes such as Ferrocene carboxaldehyde, benzaldehyde, etc. under biphasic conditions. The characterization data of $\mathrm{Au}(\mathrm{SG}) \mathrm{MPCs}, \mathrm{Au}(\mathrm{SG})\left(\mathbf{A O T}_{\text {bis }}{ }^{+}\right)$MMPCs, and reacted aldehyde adducts are included in Appendix B.3. This study was not continued further due to the findings on the tunable properties on $\mathrm{Au}_{144}\left(\mathrm{C}_{6} \mathrm{~S}\right)_{30}\left(\mathrm{AOT}_{\mathrm{bis}}\right)_{30} \mathrm{MMPCs}$.

\subsection{Conclusion}

This work has presented a new aminooxy-thiol ligand for gold nanoparticle functionalization with significant loading (50\%) that features a linking tertiary amino group for solubility tuning. The aminooxy thiol ligand was directly loaded onto $\mathrm{Au}$ MPCs using a ligand place exchange protocol that obviates the need for aminooxy protection. Simple mixing of the derived aminooxy Au MMPCs in either organic or aqueous media with a panel of aldehydes demonstrated the ease of incorporation of a variety of functionalities onto the cluster surface. It is important to carry the reaction under inert conditions due to the chemoselectivity of aminooxy functionality to react with 
carbonyl substate present in atmosphere. Protonation of the amine group using a buffer solution showed that the synthesized Au MMPCs can be adjusted to be water soluble. A graphical representation demonstrating the tunable properties of this aminooxy Au cluster to generate oxime ether-derived Au clusters under both non-polar and aqueous conditions is depicted in Scheme 3.3 below. Multiple methods of characterization, including ${ }^{1} \mathrm{H}$ NMR, IR, CV, fluorescence, MS, and UV-vis spectroscopy, confirmed that oxime ether bond formation proceeded smoothly, and that particle size was not affected by the couplings. As functionalized $\mathrm{Au}$ nanostructures are important for a variety of applications. We intend to explore the present approach toward the development of $\mathrm{Au}$ MMPC-based sensors for the detection of volatile organic compounds and caged metal catalyst MMPCs, which is presented in later chapters.

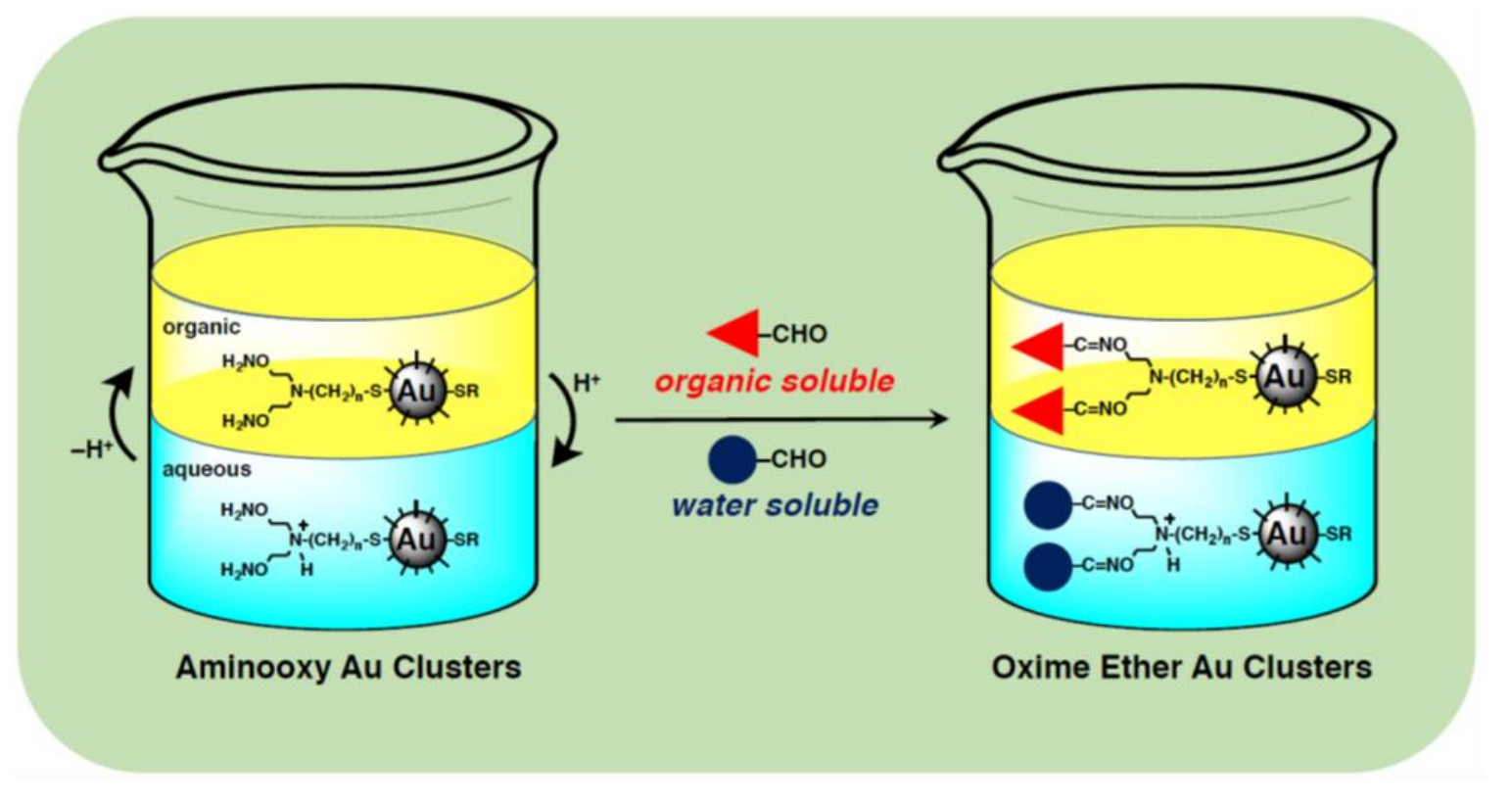

Scheme 3.3. Graphical representation of aminooxy-functionalized Au MMPCs tunability to generate the corresponding oxime-ether clusters. 


\section{CHAPTER 4}

\section{ANCHORING MPCs ON A SOLID SUPPORT TO IMPROVE CATALYST SELECTIVITY AND RECYCLABILITY}

\subsection{Introduction}

With aminooxy-functionalized Au MMPCs on hand, we are motivated to explore their use in catalysis applications. In a quest of achieving improvements in catalytic activity, such as greater reaction selectivity (i.e., hydrogenation vs. isomerization), turnover frequency, and recyclability of the catalyst for various organic transformations, we prepared solid-supported metal MPC-based catalyst (a heterogenous catalyst). To examine this possibility, we envisioned that aminooxy-functionalized metal MMPCs could be immobilized onto aldehyde-functionalized solid supports as shown in Figure 4.1. Therefore, utilizing the place exchange method, we first prepared the aminooxyfunctionalized Au MMPCs and Pd MMPCs as previously described (see Chapter 3).

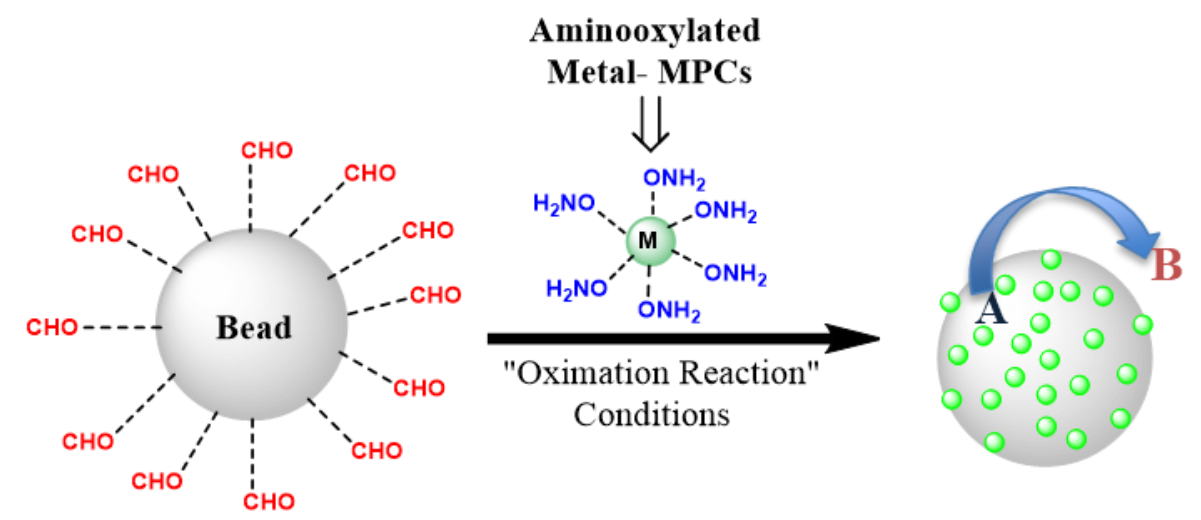

Aldehyde-activated Beads

Heterogenous Catalysis (Solid-support)

Figure 4.1. A general strategy of anchoring MPCs on a solid support via oximation reaction. 
The motivation behind attaching MMPCs to the aforementioned solid supports emanated from reports in the literature, $144,145,146,147,148,149$ showing that solid-supported catalysts have significantly different properties from their homogeneous counterparts. Heterogeneous catalysts can usually be easily recovered, which decreases the effort required for purification of the final product. They are often reusable, making them a more economically efficient choice than a homogeneous catalyst. 150 We also hypothesized that formation of a network of solid-support (e.g., polymeric chains) around the surface of MMPCs might serve to create a caged-like catalyst structure $151,152,153,154$ that would improve particle stability, and thus recyclability for use in multiple reactions. In an effort to develop a caged catalyst with improved features, we first decided to search for a suitable solid material containing aldehyde functionalities to use as a support. In our initial attempts, the aminooxy-functionalized Au and Pd MMPCs were subjected to oximation reactions to attach the MMPCs to either aldehyde-functionalized polystyrene (PS) beads or to aldehyde-containing agarose beads to prepare the targeted solidsupported catalysts. Figure 4.1 illustrates the attachment of aminooxy-functionalized metal MMPCs to aldehyde-functionalized beads through oximation chemistry.

\subsection{Attachment Strategy}

Some examples of aminooxy-functionalized nanoparticles include the work demonstrated by Mancini et al. and Chan et al., as discussed in Chapter 1 (Section 1.6). ${ }^{41}$, 42 Both groups showed that self-assembled monolayers (SAMs) on Au films (the support) are useful for surface conjugation, where the presented aminooxy groups and ketone groups undergo selective coupling to form a chemically stable oxime ether

linkage. 155 In our quest to prepare a solid-supported MPC-based catalyst, we attached 
aminooxy-functionalized MMPCs to aldehyde functionalized micrometer sized porous beads. . Commercially available aldehyde-activated polystyrene (PS) beads (SupraSciences, Catalog \# SPPS 28-25) and agarose beads (ThermoFisher scientific, Catalog \# 20381) were used in oximation reactions with the aminooxy-functionalized $\mathrm{Au}$ or Pd MMPCs. The PS beads are 330-1225 microns in average diameter with aldehyde loading of $1.0-1.2 \mathrm{mmol} / \mathrm{g}$, while the average size of the agarose beads is $125 \mu \mathrm{m}$ in diameter with an aldehyde loading of $40 \mu \mathrm{mol}$ per $\mathrm{mL}$.

Our attachment strategy relies on the straightforward addition of aminooxylated MMPCs to a suspension of aldehyde-activated beads, with a controlled mole ratio between the aminooxy and aldehyde functionality as previously described. ${ }^{184}$ This reaction occurred at room temperature and under mild conditions. Keeping the reaction under inert condition is important to prevent the reaction of aminooxy groups with carbonyls present in the atmosphere, which is undesirable because it would reduce the number of aminooxy groups available to react with the bead-confined aldehydes. After an overnight reaction, a simple work-up procedure was followed to remove the unreacted aminooxy MMPCs, where the MMPC-functionalized beads were isolated via centrifugation. A clear supernatant after centrifugation and color change of the beads (precipitate) from colorless to black (color of MMPCs) provide strong evidence of successful attachment. UV-Vis, FTIR, TGA, and optical microscopy provided evidence of success of the oximation reaction on the solid surface. UV-Vis studies of the isolated precipitate and supernatant provides information on the location of the MMPCs. TGA provides a change in the organic weight $\%$ of the sample, which would much higher after attachment of MMPCs to the entirely organic beads. Imaging techniques directly show 
the change in size and shape and other morphological changes. FTIR is a useful technique that shows a characteristic stretching frequency for oxime-ether bond formation. Also, unreacted functional groups $\left(-\mathrm{ONH}_{2}\right.$ or $\left.-\mathrm{CHO}\right)$ remained after the completion of the oximation reaction. Surface passivation techniques could be implemented to passivate further reaction of such groups. For example, hydroxylamine hydrochloride could be reacted with the aldehydes in beads to generate polyoxime, ${ }^{156}$ while acetone could react with aminooxy group on MMPCs to generate the oxime adduct, preventing oximation with aldehydes on beads.

\subsubsection{Au Cluster- Functionalized Polystyrene (PS) Beads}

Our first choice of solid-support, polystyrene aldehyde resin, possesses a hydrophobic surface which makes them a suitable candidate for protein adsorption. ${ }^{157}$ We employed them for MMPC attachment. In the first step, $\mathrm{Au}\left(\mathrm{C}_{6} \mathrm{~S}\right)\left(\mathrm{AOT}_{\text {bis }}\right)$ MMPCs were synthesized via $\mathrm{AuC}_{6} \mathrm{~S}$ MPC synthesis followed by place-exchange with $\mathrm{AOT}_{\text {bis }}$ as described in Chapter 3 (Figure 3.7). The estimated size of $\mathrm{Au}\left(\mathrm{C}_{6} \mathrm{~S}\right)\left(\mathrm{AOT}_{\mathrm{bis}}\right)$ is between 1$2 \mathrm{~nm}$ based on $\mathrm{UV}$-Vis and the $\mathrm{AOT}_{\text {bis }}$ loading is $1: 1$ relative to $\mathrm{C}_{6} \mathrm{~S}$ ligands based on proton NMR results. ${ }^{184}$ The resultant MMPCs were reacted with PS-CHO beads to afford Au MMPC- functionalized PS beads (Figure 4.2). In a typical reaction, we mixed $\mathrm{Au}_{144}\left(\mathrm{C}_{6} \mathrm{~S}\right)_{30}(\mathrm{AOT})_{30}$ and the PS-CHO beads in the stoichiometric ratio of 2:1 (based on $\mathrm{AOT}_{\text {bis }}$ loading on MMPCs obtained from NMR results and CHO loading on PS-bead provided by the supplier) under inert conditions, followed by a simple work-up (centrifugation) to isolate the bead/MMPC product. This showed good reactivity as evidenced by the beads changing into a black color. Reaction of non-aminooxy $\mathrm{AuC}_{6} \mathrm{~S}$ MPCs with the same beads in the same 2:1 ratio $\left(\mathrm{C}_{6} \mathrm{~S}: \mathrm{CHO}\right)$ resulted in little to no 
change in bead color, indicating little to no MMPC attachment without the aminooxy groups.
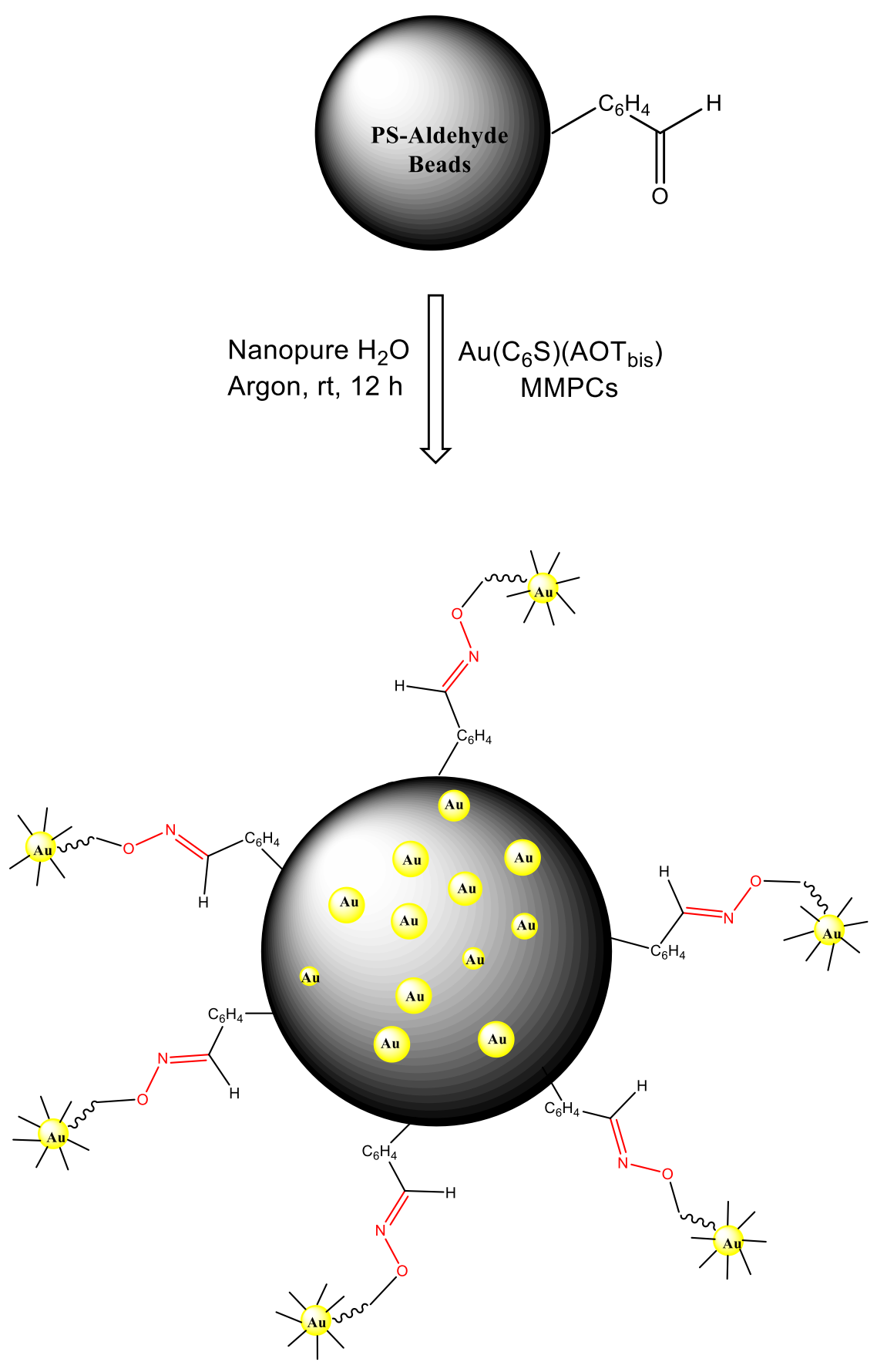

Au-cluster functionalized PS-beads

Figure 4.2. A general representation of oximation reaction between $\mathrm{Au}\left(\mathrm{C}_{6} \mathrm{~S}\right)\left(\mathrm{AOT}_{\mathrm{bis}}\right)$ MMPCs and PS-CHO beads. 
The obtained cluster was not suspendable in organic solvent, which we attributed to bead cross-linking by the Au MMPCs. The optical image of the PS-CHO bead is shown in Figure 4.3A which exhibits a spherical structure as expected. ${ }^{158}$ However, the reacted beads did not show the uniform spherical structure of the beads. Instead, the beads appear to be large aggregates, likely due to MMPC-initiated bead cross-linking (Figure 4.3 B).
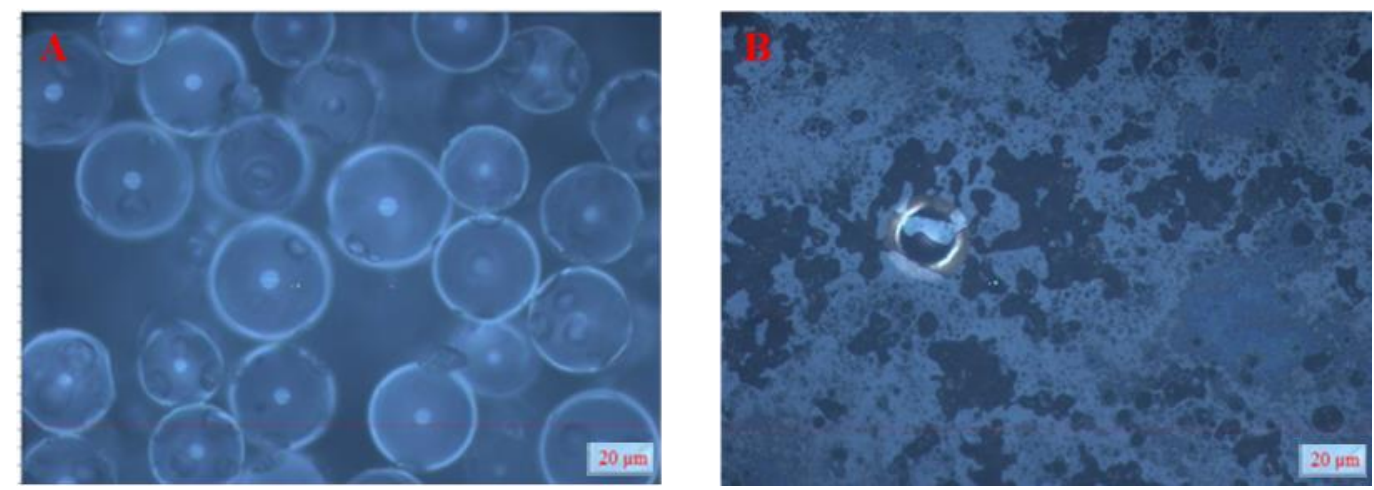

Figure 4.3. Optical image of (A) PS-aldehyde beads and (B) agglomerated Au cluster functionalized-PS beads.

In a control study, where we reacted $\mathrm{AuC}_{6} \mathrm{~S}$ MPCs (non-aminooxy) with PS-CHO beads, no reaction occurred as indicated by the dominant $-\mathrm{CHO}$ peak at $1701 \mathrm{~cm}^{-1}$ (blue curve, Figure 4.4). Upon reaction of the PS-SCHO bead with the $\mathrm{Au}\left(\mathrm{C}_{6} \mathrm{~S}\right)\left(\mathrm{AOT}_{\text {bis }}\right)$ MMPCs, the - $\mathrm{CHO}$ peak almost completely disappeared, and there was the appearance of an oxime ether stretching peak between $1580-1620 \mathrm{~cm}^{-1}$ and a broad peak at $3350 \mathrm{~cm}^{-1}$, representing the amine group of the AOT bis ligands (Figure 4.4). Moreover, the alkyl -CH stretching bands were more dominant in the case of $\mathrm{Au}\left(\mathrm{C}_{6} \mathrm{~S}\right)\left(\mathrm{AOT}_{\mathrm{bis}}\right)$ MMPCfunctionalized PS beads due to the fact that they contain several $\mathrm{CH}_{2}$ groups from the 
$\mathrm{AOT}_{\text {bis }}$ and $\mathrm{C}_{6} \mathrm{~S}$ ligands. With the FTIR results, we were convinced that anchoring of aminooxylated Au MMPCs on the surface of activated beads was successful. However, the stability of the beads was poor since they did not remain suspended in organic solvents. In order to afford a functioning catalyst using this bead, further optimization on the reaction condition is required, such as adjusting the $\mathrm{AOT}_{\text {bis: }} \mathrm{CHO}$ ratio to avoid bead cross-linking. . ${ }^{159}$ Concurrently, we were also interested in finding another commercially available bead (an aldehyde activated crosslinked agarose bead) for use in the immobilization of MPCs.

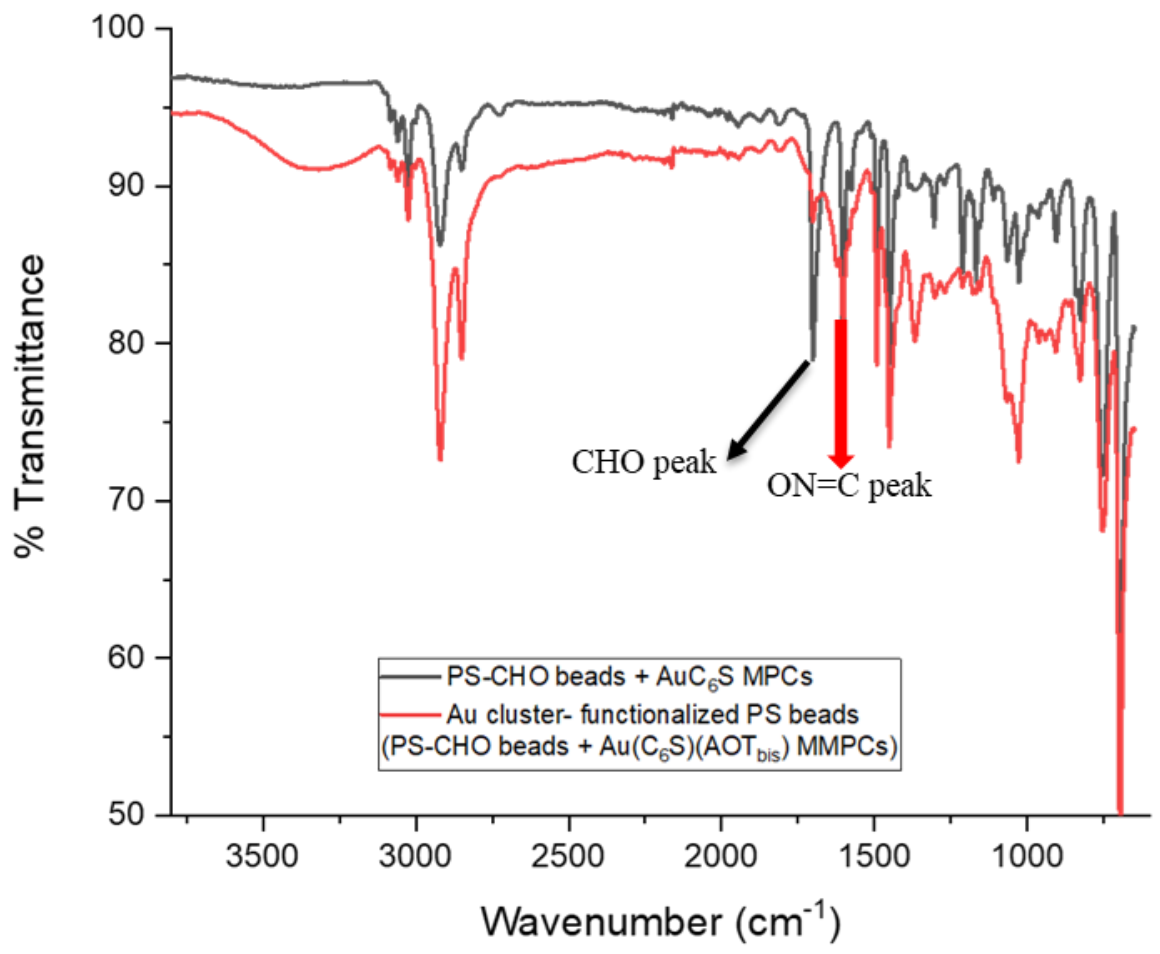

Figure 4.4. FTIR spectra of $\mathrm{AuC}_{6} \mathrm{~S}$ MPCs (black plot) and $\mathrm{Au}\left(\mathrm{C}_{6} \mathrm{~S}\right)\left(\mathrm{AOT}_{\text {bis }}\right) \mathrm{MMPCs}$ (red plot) reacted with PS-CHO beads. 


\subsubsection{Pd (SG)(AOT bis $\left.^{+}\right)$-Functionalized Agarose Beads}

Another type of solid material used was crosslinked $4 \%$ beaded agarose activated with aldehyde groups, which are generally used to immobilize proteins (antibodies) containing amine moieties. ${ }^{160}$ In our study, we are using this resin as a solid support to anchor aminooxylated Pd MMPCs. We first prepared $\mathrm{Pd}(\mathrm{SG})\left(\mathrm{AOT}_{\mathrm{bis}}{ }^{+}\right) \mathrm{MMPCs}$ via Pd SG MPC synthesis followed by place-exchange with $\mathrm{AOT}_{\text {bis }}{ }^{+}$(Figure 4.5). The estimated size of the MMPCs is between 2-3 nm based on TEM imaging, whereas the aminooxy loading is 1:1 relative to SG ligands based on proton NMR results (Figure 4.6). ${ }^{161}$ Again, the resultant MMPCs were reacted with agarose-CHO beads (1:10 AOT:CHO mole ratio based on TGA data of MMPCs and - $\mathrm{CHO}$ loading of bead) to afford Pd MMPCfunctionalized agarose beads (Figure 4.7). For a control study, PdSG MPC only was reacted with agarose aldehyde beads in the same 1:10 ratio (SG: $\mathrm{CHO}$ ).

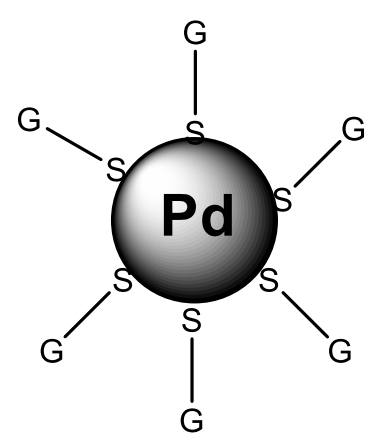

Pd(SG) MPCs

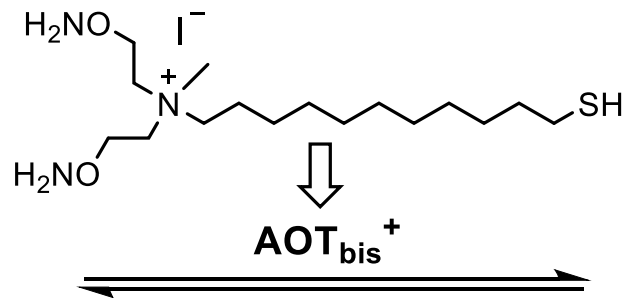

Nanopure $\mathrm{H}_{2} \mathrm{O}$

rt, $7 \mathrm{~d}$

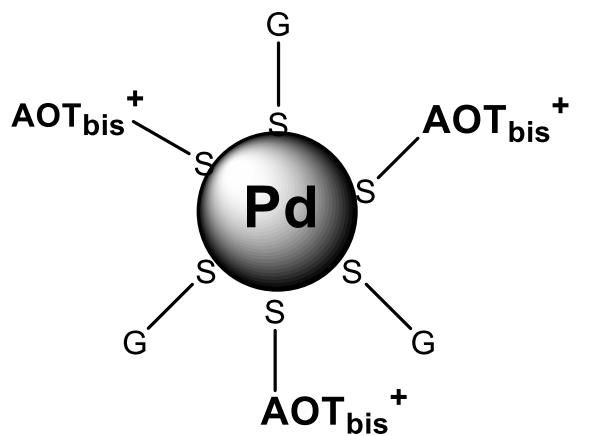

Pd(SG)(AOT $\left.{ }_{\text {bis }}^{+}\right)$MMPCs

Figure 4.5. Place exchange reaction between $\mathrm{Pd}(\mathrm{SG}) \mathrm{MPCs}$ and $\mathrm{AOT}_{\text {bis }}{ }^{+}$to afford $\mathrm{Pd}(\mathrm{SG})\left(\mathrm{AOT}_{\mathrm{bis}}{ }^{+}\right)$MMPCs. 

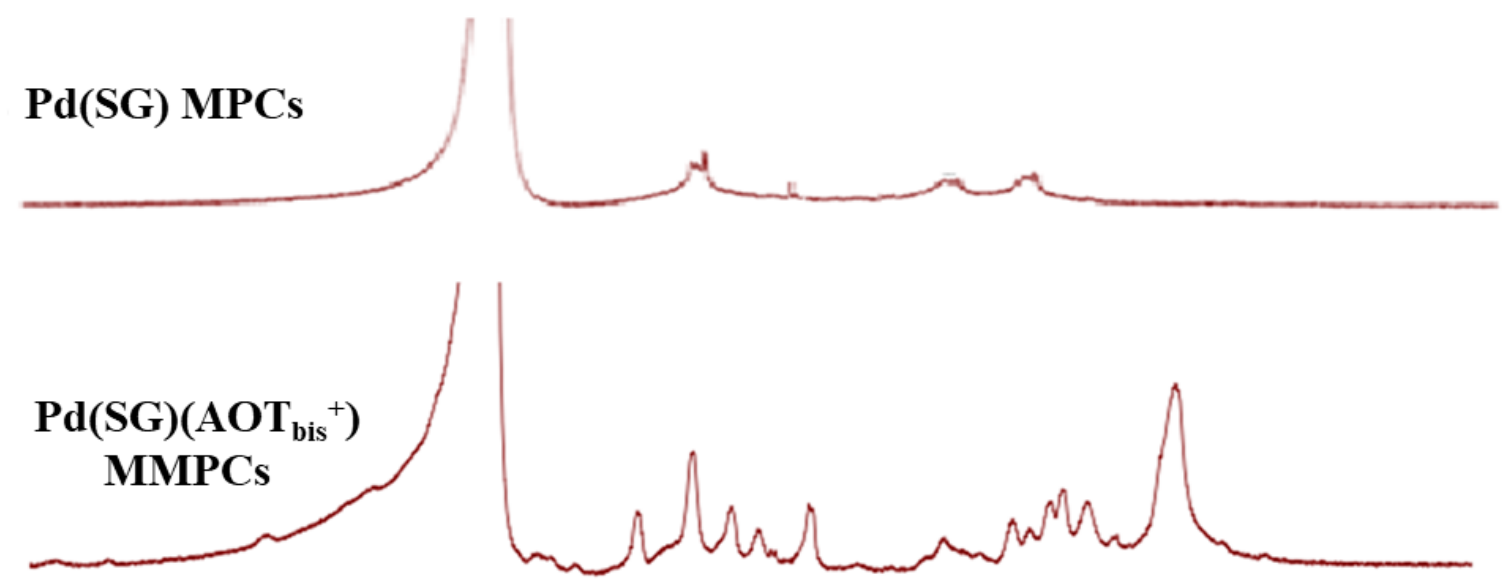

\begin{tabular}{|c|c|c|c|c|c|c|c|c|c|c|c|c|c|c|}
\hline 7 & 1 & 1 & 1 & 1 & 1 & 1 & 1 & 1 & $T$ & 1 & 1 & $T$ & 1 & 1 \\
\hline 7.0 & 6.5 & 6.0 & 5.5 & 5.0 & 4.5 & 4.0 & 3.5 & 3.0 & 2.5 & 2.0 & 1.5 & 1.0 & 0.5 & 0.0 \\
\hline
\end{tabular}

Figure 4.6. ${ }^{1} \mathrm{H}$ NMR spectra $\left(\mathrm{D}_{2} \mathrm{O}, 500 \mathrm{MHz}\right)$ of $\mathrm{PdSG} \mathrm{MPCs}$ and $\mathrm{Pd}(\mathrm{SG})\left(\mathrm{AOT}_{\mathrm{bis}}{ }^{+}\right)$ MMPCs. 1:1 exchange ratio (estimated ratio) was based on the ratio of integration values of $\mathrm{CH}_{2}$ peak of $\mathrm{SG}$ to the $\mathrm{CH}_{2}$ peak of $\mathrm{AOT}_{\text {bis }}{ }^{+}$.

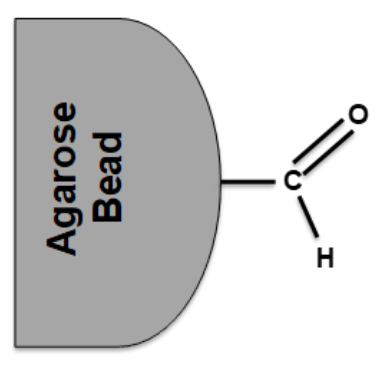

Agarose-CHO beads

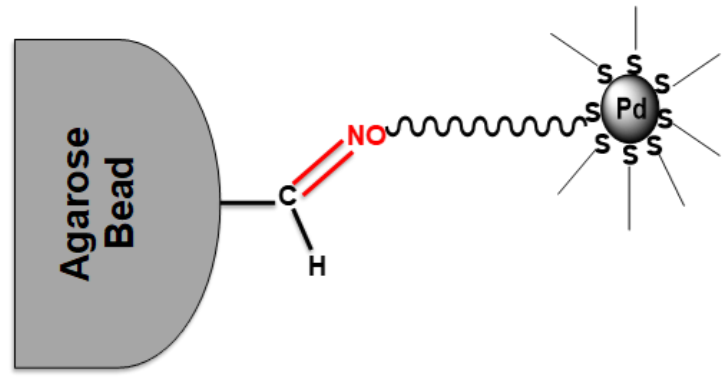

Pd-cluster functionalized agarose-beads

Figure 4.7. Schematic of oximation reaction between $\mathrm{Pd}(\mathrm{SG})\left(\mathrm{AOT}_{\mathrm{bis}}{ }^{+}\right) \mathrm{MMPCs}$ and agarose- $\mathrm{CHO}$ beads to afford $\mathrm{Pd}$-cluster functionalized agarose beads. 
First, the optical image of the agarose-CHO bead by itself was obtained, which exhibits a spherical structure as expected, while the optical image of the Pd-cluster functionalized agarose bead showed darker spheres after the reaction, which may have suggested successful coupling of the Pd MPC to the beads (Figure 4.8). Importantly, the agarose bead structure did not change like the PS beads did.
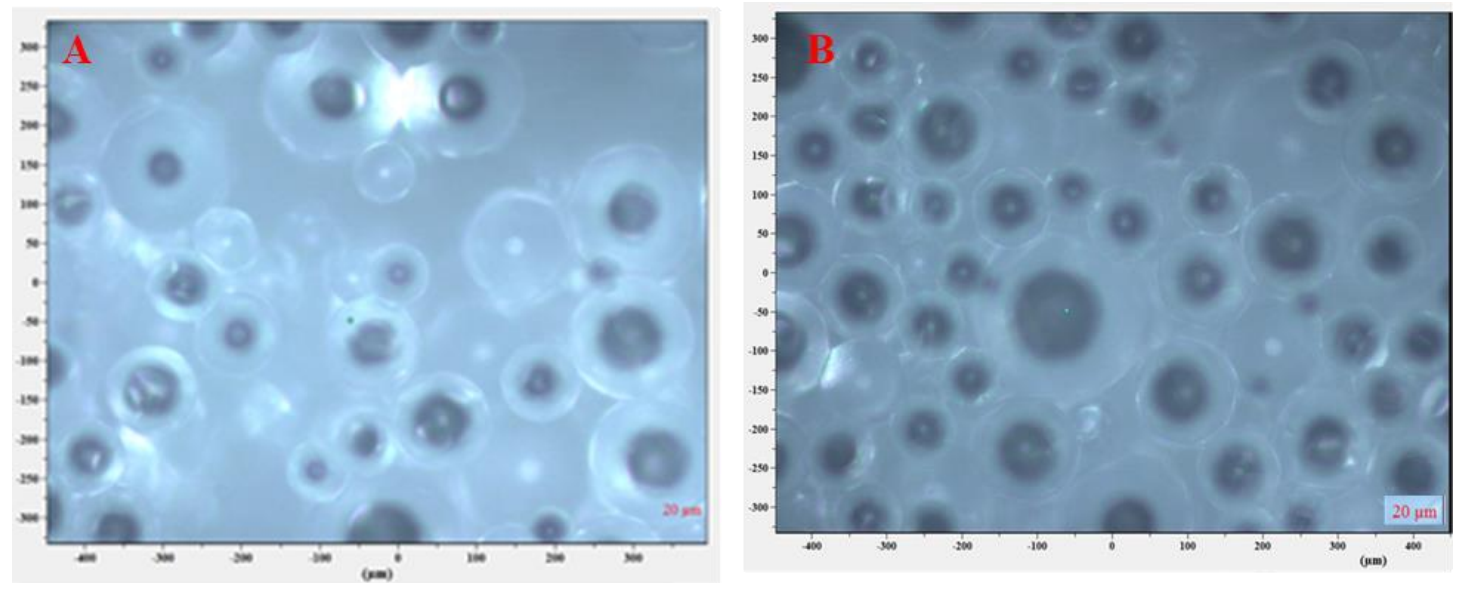

Figure 4.8. Optical image of agarose-aldehyde beads (A) and Pd clusterfunctionalized agarose beads (B).

To confirm, UV-Vis spectra of the aqueous supernatant after the reaction, centrifugation, and washing was recorded using nanopure $\mathrm{H}_{2} \mathrm{O}$ as the solvent. The UVVis spectrum of the clusters before reaction with beads shows the characteristic drop in absorbance with increasing wavelength (Figure 4.9, black plot). The same solution (or supernatant) after reacting with $\mathrm{CHO}$ agarose beads resembled the blank solution, signifying the absence of MPCs because they attached to the beads and went into the precipitate (Figure 4.9, blue plot). The spectrum of the bead precipitate shows a higher absorbance at high wavelength, indicative of aggregated MMPCs, as might be expected when anchored to the agarose beads. Pictures of the Pd MMPCs before reaction with the 
beads shows that the entire solution starts of brown/black, indicative of the Pd MMPCs in solution, but then shows the top supernatant layer is colorless after reaction with the beads while the bottom layer is brown/black (Figure $4.10 \mathrm{~A}$ and B). We also performed a control study with Pd MPCs (which do not consist $\mathrm{ONH}_{2}$ functionality on surface), the results show that without $\mathrm{ONH}_{2}$ functionality the reaction did not happen and hence we did not observe any precipitate after centrifugation (Figure $4.10 \mathrm{C}$ ). This indicates that the Pd MMPCs attached to the beads and completely settled down with the beads when there is presence of $\mathrm{ONH}_{2}$ functionality on the surface. The UV-Vis results and visual pictures clearly indicate that the PdSG MPC are attached on the beads. The optical microscope images show the beads remained intact, likely due to the low AOT:CHO ratio used (1:10).

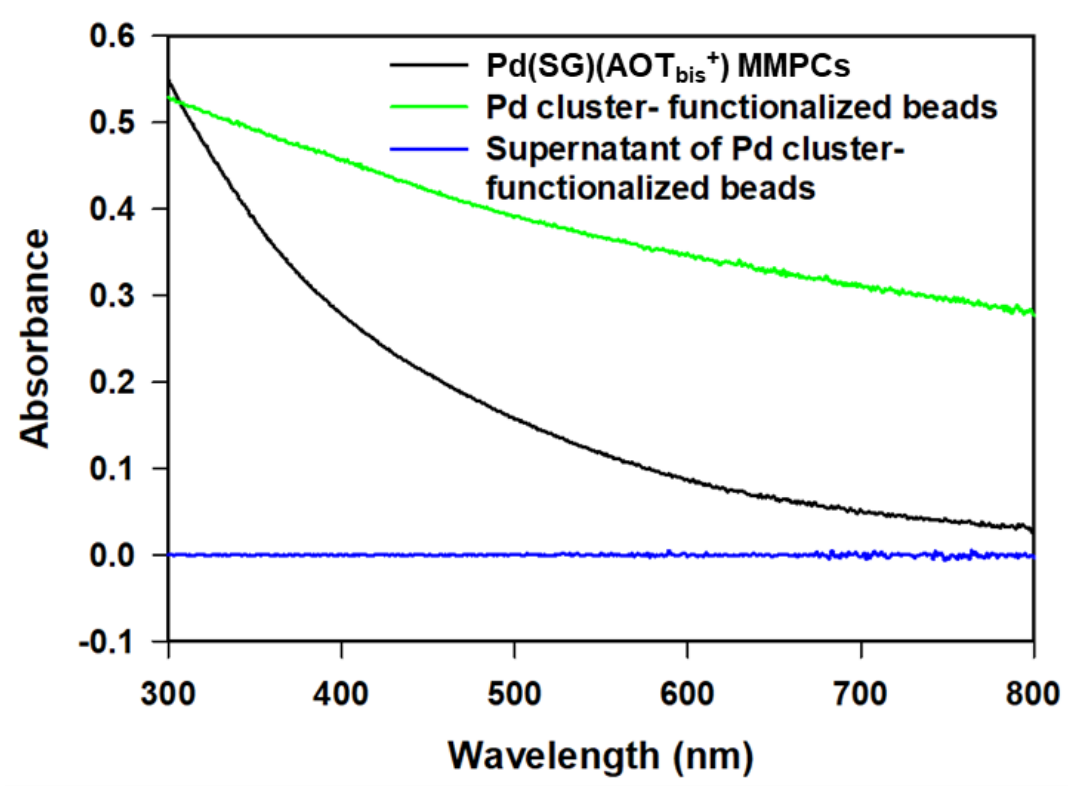

Figure 4.9. UV-Vis absorbance spectra of $\mathrm{Pd}(\mathrm{SG})\left(\mathrm{AOT}_{\text {bis }}{ }^{+}\right)$MMPCs, $\mathrm{Pd}$ cluster-functionalized beads and supernatant of Pd cluster-functionalized beads (nanopure water was used as a solvent). 


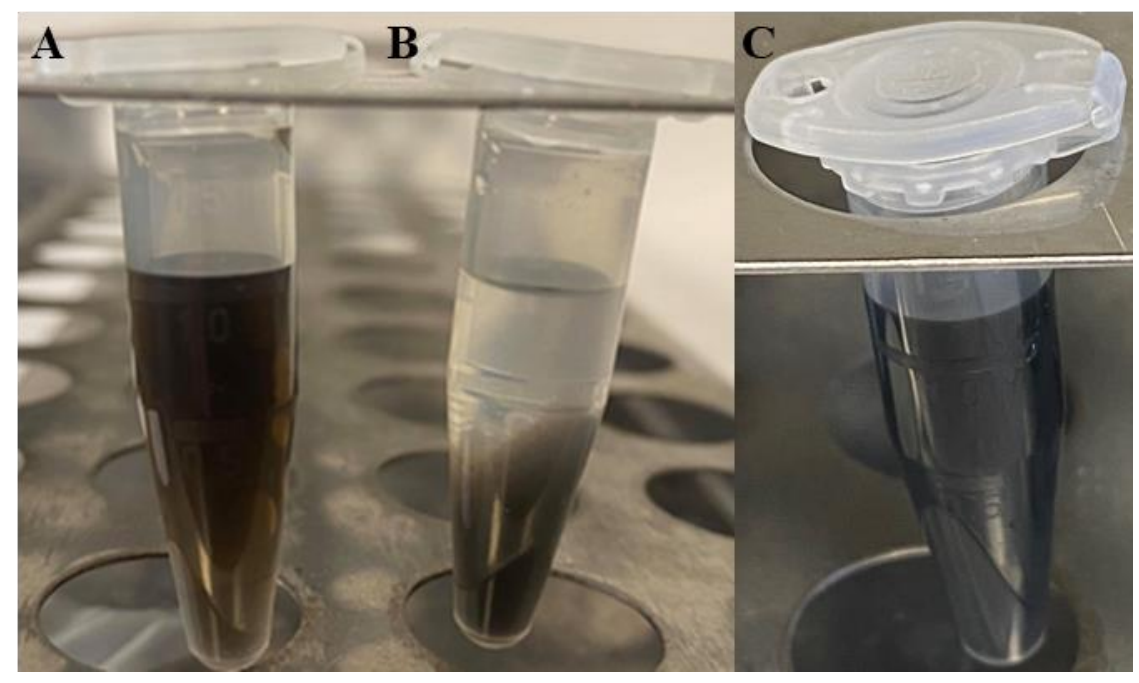

Figure 4.10. Digital images of $\mathrm{Pd}(\mathrm{SG})\left(\mathrm{AOT}_{\mathrm{bis}}{ }^{+}\right) \mathrm{MMPCs}$ solution (A), $\mathrm{Pd}$ cluster-functionalized agarose beads (black precipitate) with supernatant (clear) on top (B), and PdSG MPCs reacted with agarose aldehyde beads (C).

FTIR spectra show the formation of a new band between 1560-1610 $\mathrm{cm}^{-1}$ representing an oxime ether bond stretching (red plot) and the complete disappearance of - $\mathrm{CHO}$ peak at $1640 \mathrm{~cm}^{-1}$ (black plot) as shown in Figure 4.11. While $\mathrm{CH}$ stretching peaks at 2920 and $2848 \mathrm{~cm}-1$ are from $\mathrm{AOT}_{\text {bis }}{ }^{+}$and $\mathrm{SG}$ groups present on $\mathrm{Pd}$ surface. These evidences from the FTIR study provides an evidence of successful attachment of $\mathrm{Pd}(\mathrm{SG})\left(\mathrm{AOT}_{\mathrm{bis}}{ }^{+}\right) \mathrm{MMPCs}$ on to agarose-CHO bead surface resulting in the Pd-clusterfunctionalized bead. 


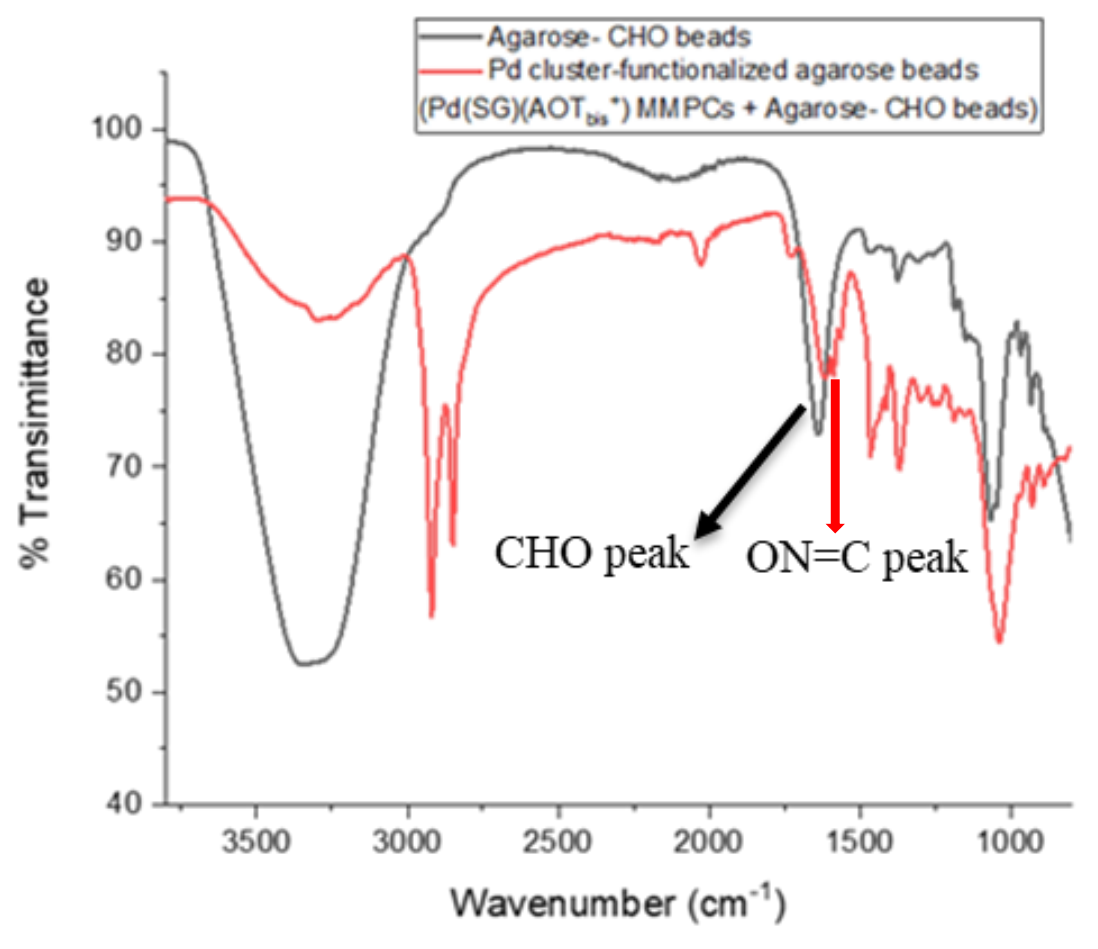

Figure 4.11. FTIR spectra of as-purchased agarose-CHO beads and Pdcluster functionalized agarose beads (AOT: $\mathrm{CHO}=1: 10)$.

TGA studies reveal that there is an increase in the organic weight percentage on the Pd-cluster functionalized beads. The Pd-cluster functionalized bead showed $65 \%$ organic component as compared to $50 \%$ for $\mathrm{Pd}(\mathrm{SG})\left(\mathrm{AOT}_{\text {bis }}{ }^{+}\right)$MMPCs (Figure 4.12). Overall, the combination of all these characterization techniques provided a solid evidence that the attachment of Pd MPCs on the bead surface was effective. 


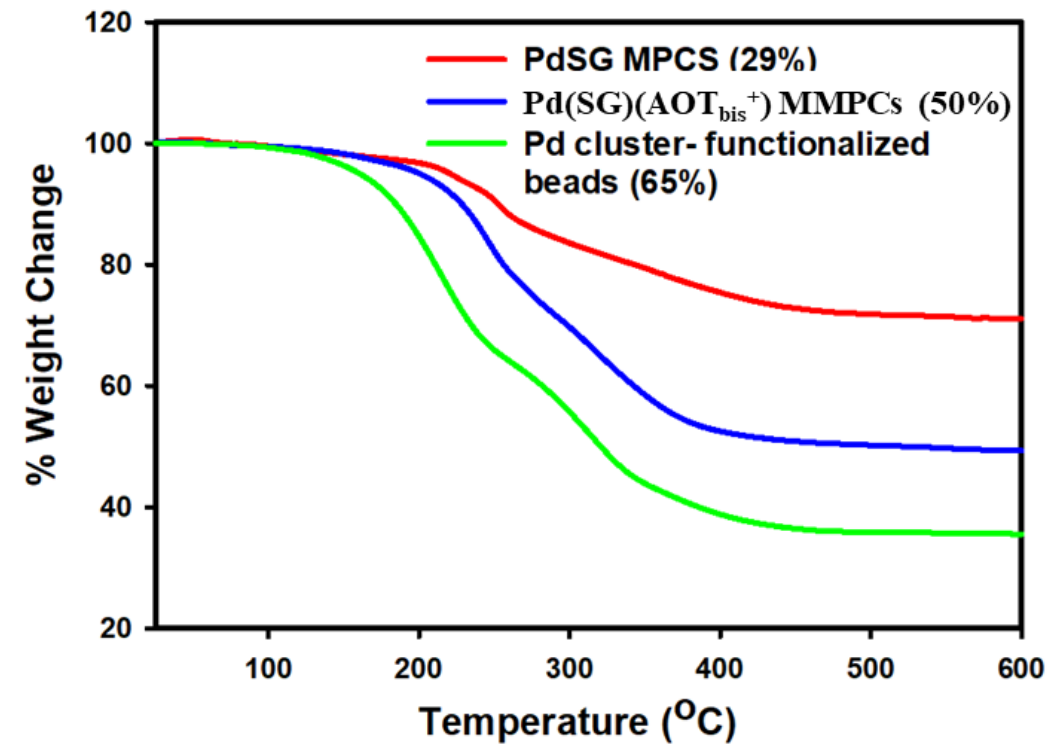

Figure 4.12. Thermogravimetric analysis (TGA) of PdSG MPCs, $\mathrm{Pd}(\mathrm{SG})\left(\mathrm{AOT}_{\mathrm{bis}}{ }^{+}\right)$MMPCs, and Pd cluster-functionalized agarose beads . 


\section{CHAPTER 5}

\section{IODINE ACTIVATION OF METAL MPCs FOR CATALYTIC ENHANCEMENT}

\subsection{Introduction}

Metal monolayer-protected clusters (MPCs) stabilized by organothiol monolayers have been used broadly as homogeneous and heterogeneous catalysts in numerous organic transformations, ${ }^{162}, 163$ including nitro group reduction, ${ }^{164}$ alkene isomerization, ${ }^{165}$ alkyne hydrogenation, 166,167 and Suzuki-Miyaura coupling reactions. ${ }^{168}$ The great advantage in using metal MPCs is the excellent synthetic control over the metal cluster size and composition, which allows strong control over the metal structural and electronic properties for tuning catalytic activity. The use of a wide variety of thiol-containing capping ligands enables high stability, tuning of the solubility properties, potential molecular gating properties, and the ability to treat these molecular metals as normal chemical reagents, thereby allowing characterization by standard analytical spectroscopic tools of the organic chemist (e.g., nuclear magnetic resonance spectroscopy (NMR), Fourier-transform infrared spectroscopy (FTIR), mass spectrometry (MS), and UV-vis). ${ }^{169}$ Additionally, improvements in separation methods have led to syntheses of highly uniform MPCs of one composition, also known as atomically-precise clusters, such as the widely studied $\mathrm{Au}_{25}(\mathrm{SR})_{18}$ cluster product. ${ }^{170,} 171$ While use of thiol ligands as capping agents affords tremendous synthetic control, they also strongly passivate the metal cluster surface and poison (or inhibit) the catalytic properties. As a result, there is much interest in developing new ligand systems or incorporating strategies to increase MPC catalytic activity without sacrificing stability. 
This is a tremendous challenge as the two - ligand-imparted stability vs. liganddiminished activity — work directly against each other.

One main strategy for balancing the stabilizing properties of a given ligand while increasing reactant access to the metal core, essential for catalytic activity to occur, ${ }^{172}$ is the use of lower coverage thiol ligands, such as those derived from Bunte salts (alkyl thiosulfates), ${ }^{173}, 174$ or branched, bulky thiol-containing ligands (glutathione-capped NPs), so as to decrease ligand surface density. ${ }^{53}$ In the synthesis of Au NPs using Bunte salts as ligand precursors, first Bunte salt physically adsorbs to the surface of the developing NPs, and then removes sulfite to form a thiolate monolayer on the surface of the Au core (Scheme 5.1). This synthesis takes advantage of the slow passivation kinetics of the Bunte salts during the reaction, resulting in large core NPs. Different types of ligands containing hydrophilic functional groups can be used in the synthesis of Bunte salt ligand precursors. Likewise, a branched thiol ligand such as glutathione possesses the advantage of a more open structure and hence reduces the surface passivation. Bhama et al. recently demonstrated the use of water-soluble glutathione (SG)-capped Pd MPCs (Scheme 5.2), which catalyzed the hydrogenation/isomerization of allyl alcohol with a turnover frequency (TOF) roughly 5 times greater than linear chain hexanethiolatestabilized Pd MPCs. ${ }^{53}$ In MPCs synthesized using Bunte salts ligand precursors and branched SG ligands, there is less surface passivation compared to MPCs synthesized using crowded alkanethiol ligands. Therefore, MPCs of these types possess catalytic space on the metal surface, which are useful for catalytic applications. Another common approach is to activate MPCs by post-synthesis ligand removal, such as electrochemically-induced ligand removal (stripping), ozone treatment, chemical 
treatments, and heating. These ligand removal strategies are more effective for heterogeneous catalysis applications with solid-supported metal MPCs because some of the ligand-removing approaches require either an electrode surface or gas-phase conditions. Otherwise, the approach is not controlled, and particle growth or other morphological changes quickly diminish the catalytic properties. Our interest in the synthesis of functionalized $\mathrm{Au}$ and $\mathrm{Pd}$ nanoclusters ${ }^{138}$ and their applications in sensing ${ }^{140}$ and catalysis ${ }^{53}$ have motivated us to consider an alternate method for catalytic activation.

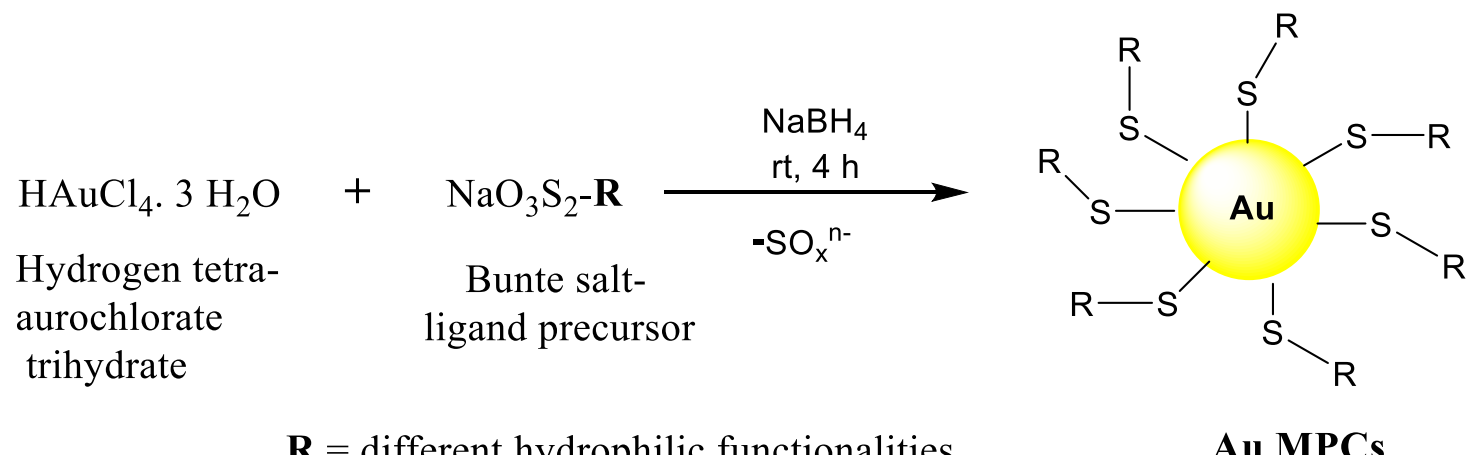

Scheme 5.1. Schematic of Au MPCs synthesis using Bunte salts as a ligand precursor.
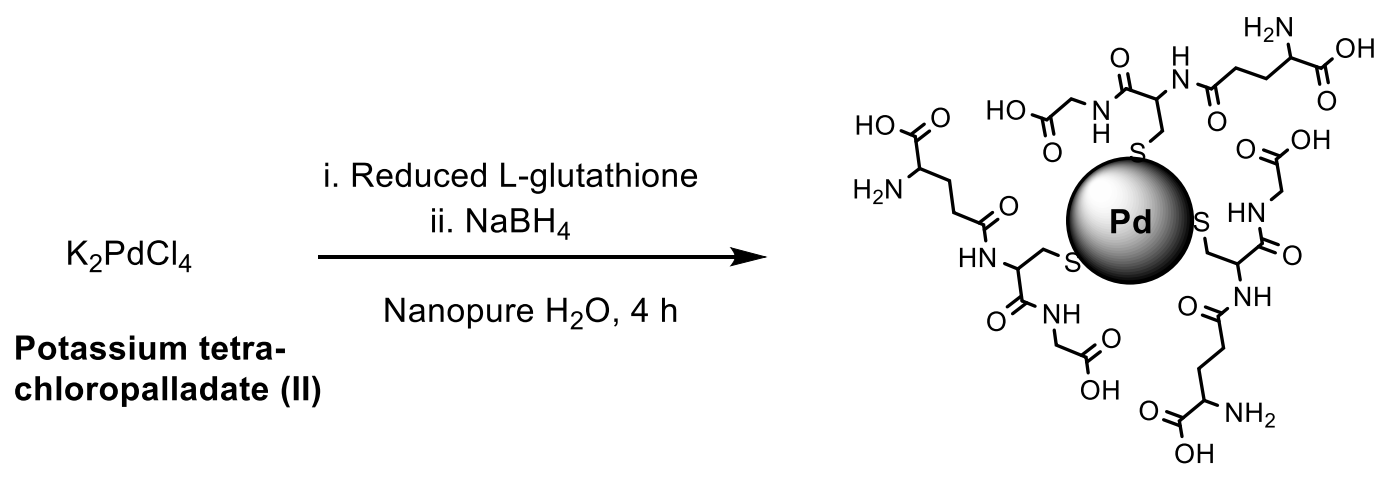

Glutathione-capped Pd MPCs

Scheme 5.2. Schematic of branched glutathione-capped Pd MPCs. 
We hypothesized that treatment of a thiol-capped MPC catalyst with a small quantity of $\mathrm{I}_{2}$ would lead to well-controlled partial ligand removal by disulfide formation in-situ (Scheme 5.3), resulting in partial exposure of the metal cluster surface to substrates for enhanced binding and reactivity. To test the $\mathrm{I}_{2}$-activation concept, we examined two model reactions (Scheme 5.4): Au MPC-catalyzed reduction of 4nitrophenol (4-NP) to 4-aminophenol (4-AP) by sodium borohydride, ${ }^{62}$ a reaction that has been well-studied using a variety of free or immobilized nanoparticles/clusters as catalysts, and the Pd MPC-catalyzed hydrogenation/isomerization of allyl alcohol in the presence of $\mathrm{H}_{2}$ to afford propanal and 1-propanol as the isomerized and hydrogenated products, respectively. ${ }^{53} \mathrm{We}$ report herein our findings on the influence of $I_{2}$ on both the rate of the reaction/turnover frequency (TOF) and recyclability using SG-stabilized Au and Pd MPCs as catalysts.

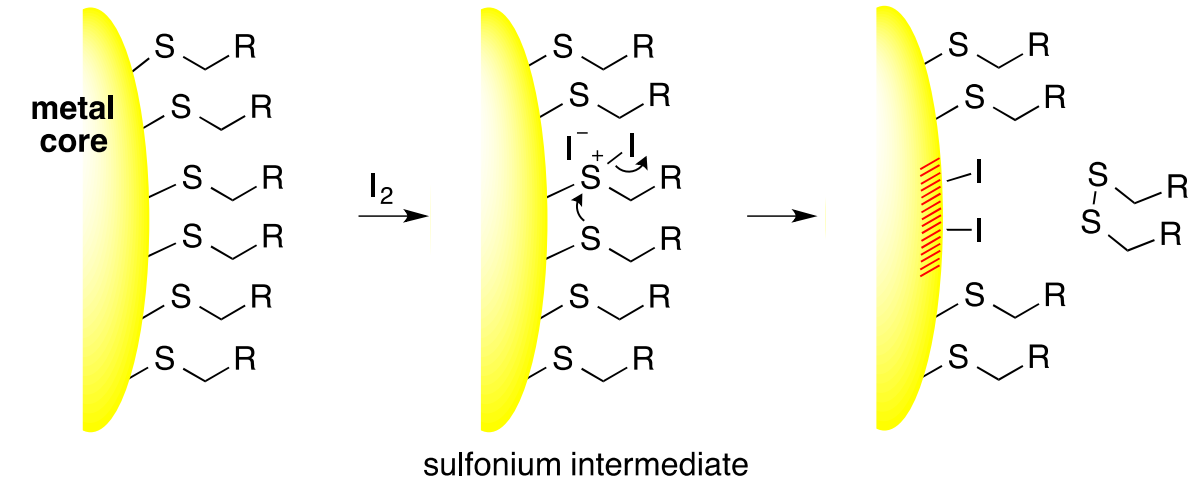

Scheme 5.3. Iodine activation at the surface of a thiolate-coated metal cluster. We postulate neighboring thiolate displacement of iodide from a sulfonium ion intermediate results in disulfide formation and ligand dissociation, making the metal surface accessible for substrate binding (shaded area). Iodide can presumably adsorb to the metal surface or dissolve into solution. 


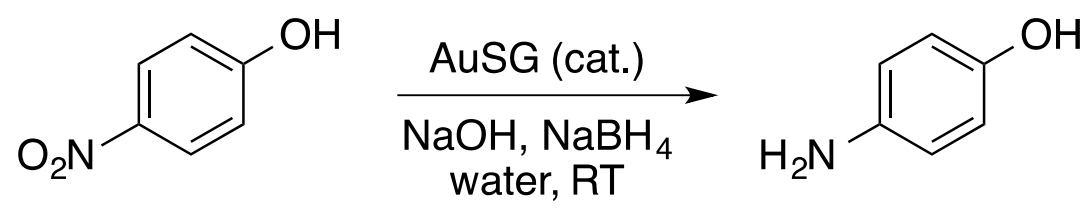

4-nitrophenol

4-aminophenol

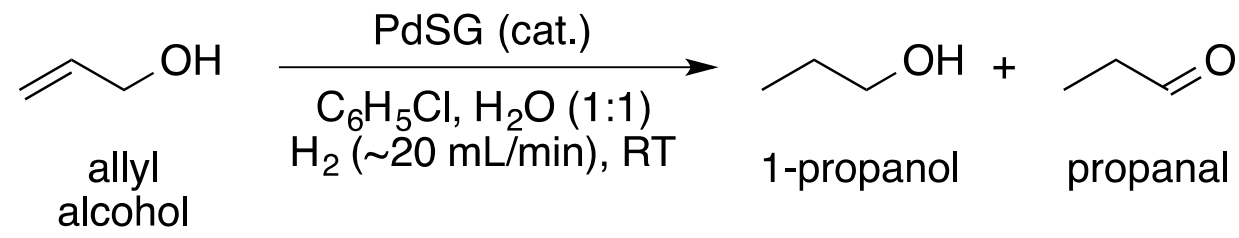

Scheme 5.4. Model reduction and hydrogenation/isomerization reactions subjected to catalyst activation using $\mathrm{I}_{2}$.

\subsection{Results and Discussion}

\subsubsection{Characterization of AuSG MPCs and PdSG MPCs}

AuSG and PdSG MPCs were synthesized following the previously reported procedure with slight modifications. ${ }^{175,}{ }^{137}$ The synthesis protocols are described in Chapter 6 (section 6.1.8 and 6.1.9 respectively for AuSG and PdSG MPCs). TGA analysis of AuSG MPCs indicated a composition of $\sim 33 \%$ organic and $67 \%$ Au metal (Figure $5.1 \mathrm{~A}$ ). The UV-Vis absorbance of the synthesized MPCs did not show the presence of a plasmon band (Figure 5.2 A), suggesting that the synthesized particles have diameters $<2$ nm. ${ }^{176}$ Indeed, TEM analysis of the AuSG MPCs show roughly spherical particles with an average diameter of $1.6 \pm 0.3 \mathrm{~nm}$ (Figure 5.3 A). TGA analysis of the PdSG MPCs indicated an organic composition of 29\% (Figure 5.1 B) and the TEM analysis showed an average particle diameter of $2.6 \pm 0.5 \mathrm{~nm}$ (Figure $5.3 \mathrm{~B}$ ), which is similar to the core diameter previously reported for glutathione-protected PdSG 
MPCs synthesized under these conditions. ${ }^{137}$ In agreement with prior observations, ${ }^{177}$ the UV-Vis absorbance of the PdSG MPCs did not show a plasmon band (Figure 5.2 B). FTIR spectra of AuSG and PdSG MPCs shows the disappearance of the thiol stretching band that would have appeared at $2500 \mathrm{~cm}^{-1}$ for free $\mathrm{GSH}$, hence confirming attachment to the cluster and removal of the non-bound ligands (Figure 5.4). Finally, the appearance of well-broadened glutathione peaks and specifically the disappearance of the methylene protons adjacent to the glutathione thiol group at $\delta \sim 2.95 \mathrm{ppm}$ from the ${ }^{1} \mathrm{H}$ NMR analyses on both the AuSG and PdSG clusters confirmed attachment of glutathione. 

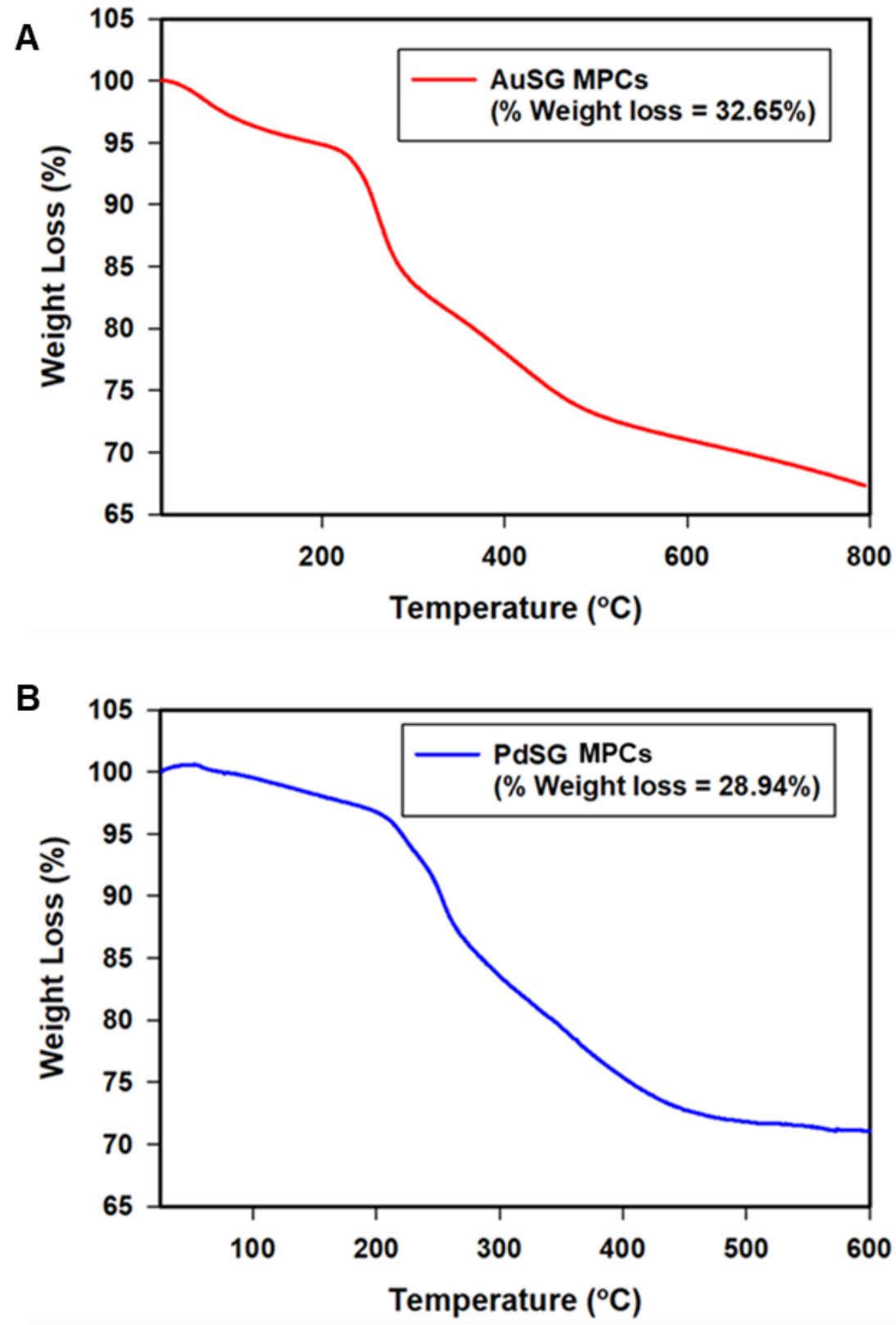

Figure 5.1. TGA of synthesized AuSG MPCs (A) and PdSG MPCs (B). 

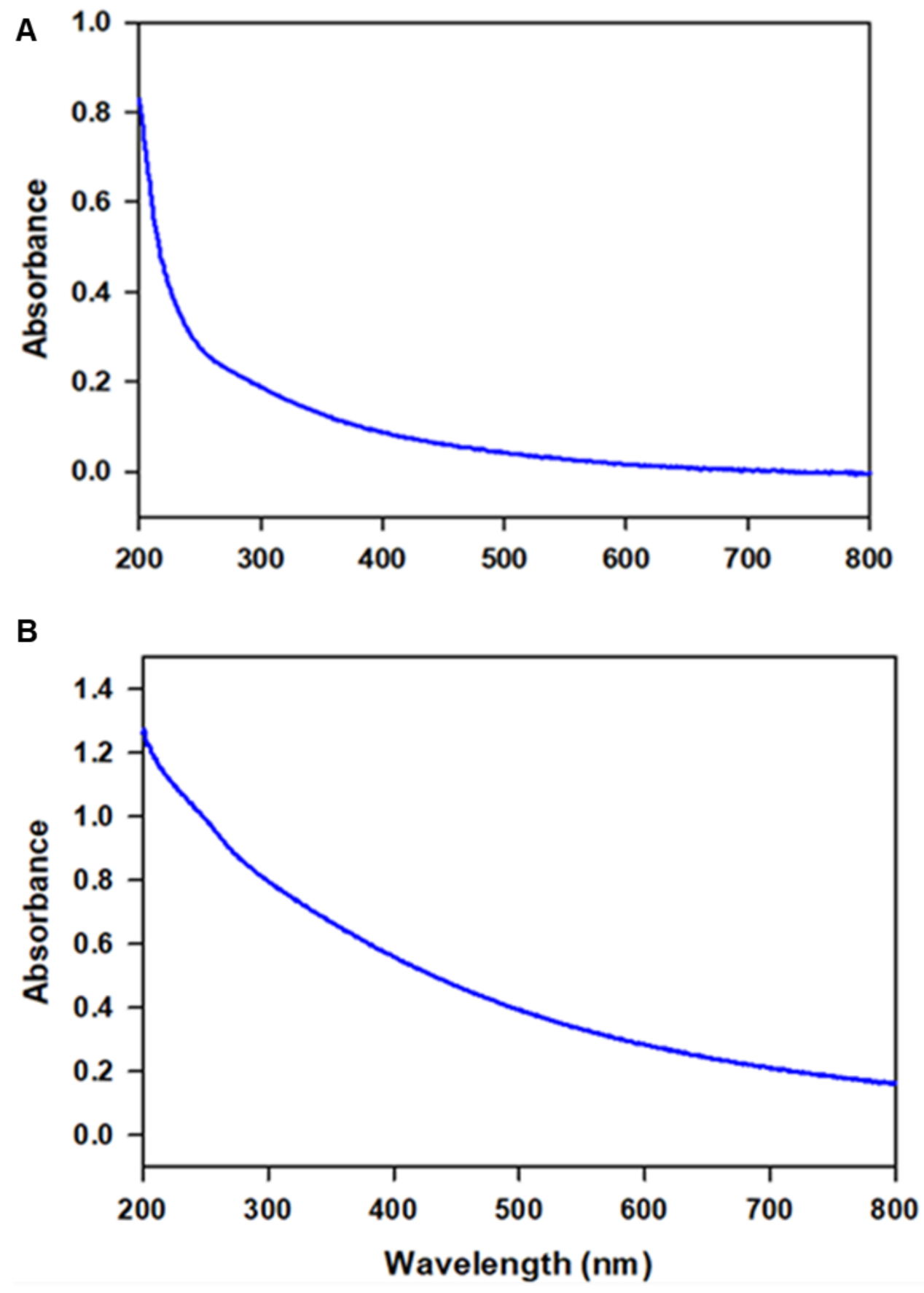

Figure 5.2. UV-Vis of synthesized AuSG MPCs (A) and PdSG MPCs (B) (nanopure water was used as a blank). 

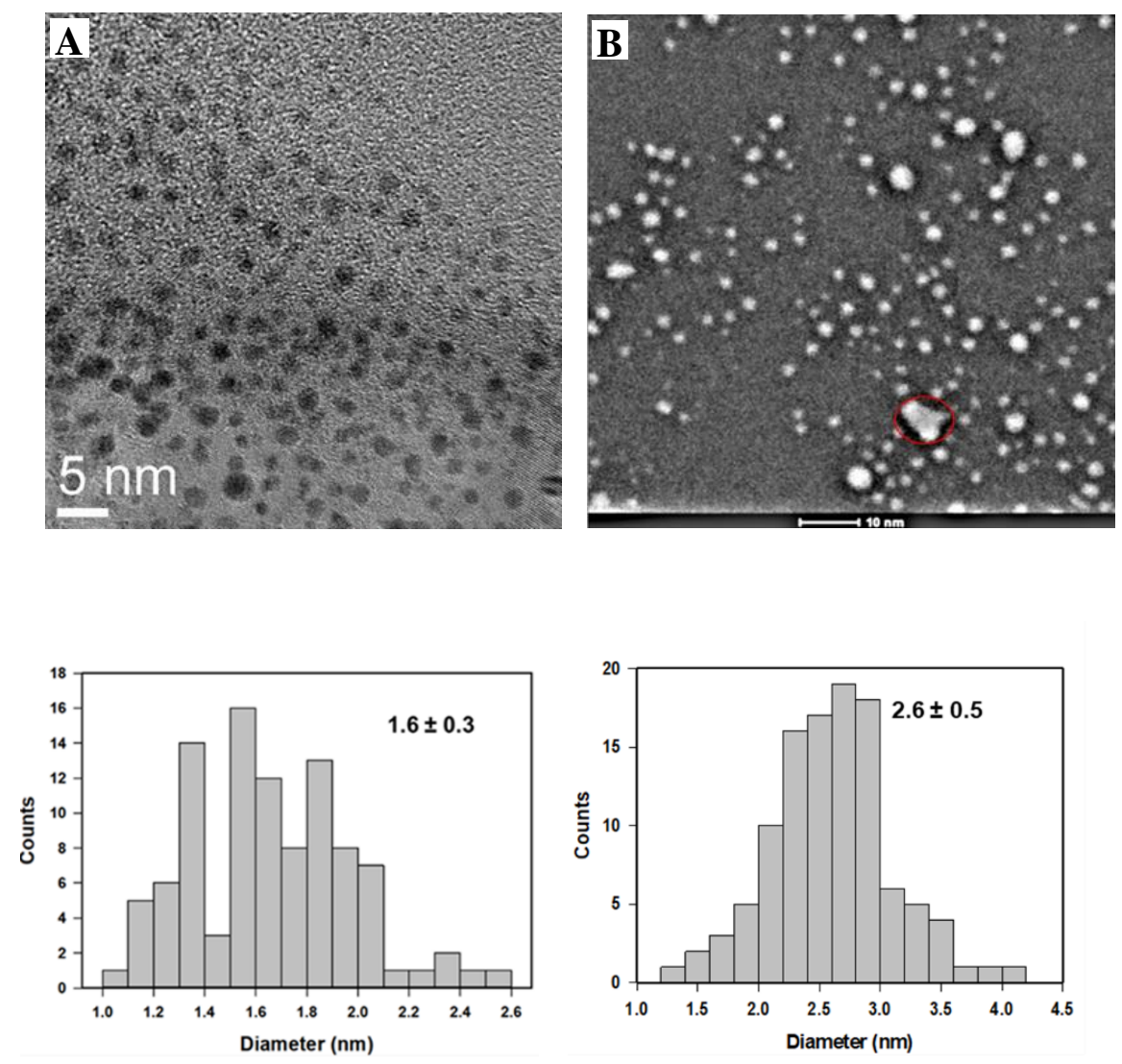

Figure 5.3. Characterization of synthesized MPCs. A: Brightfield TEM image of AuSG MPCs, and B: Darkfield TEM image of PdSG MPCs (red, circled aggregated sphere was not counted in the size analysis). Corresponding histogram plot of the particle size analyses are below the image. 

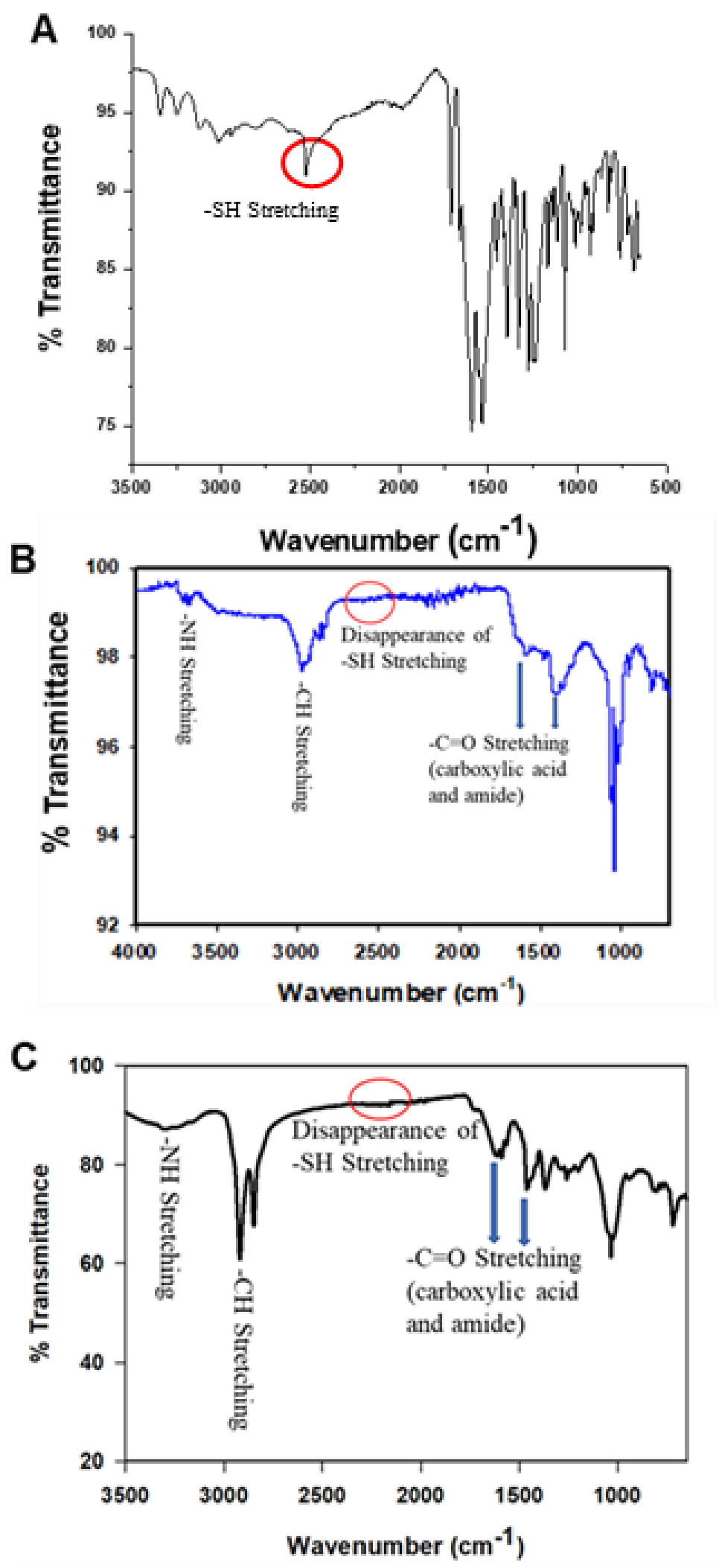

Figure 5.4. FTIR spectrum of glutathione (A), AuSG MPCs (B) and PdSG MPCs (C). 


\subsubsection{Catalytic reduction of 4-nitrophenol}

The reduction of 4-nitrophenol (4-NP) using $\mathrm{NaBH}_{4}$ (Scheme 5.4) proceeds slowly without addition of AuSG MPCs, requiring up to two days for completion. ${ }^{178}$ The reaction is conveniently followed using UV-Vis absorption spectroscopy by monitoring the disappearance of 4-nitrophenolate at $400 \mathrm{~nm}$ and the appearance of 4-aminophenolate (4-AP) at $300 \mathrm{~nm}$ (Figure 5.5). In the presence of a catalytic amount of an Au MPC catalyst, however, product formation ensues rapidly (Figure 5.6 A). The addition of catalytic AuSG MPCs resulted in complete reduction of 4-NP within 40 minutes (rate constant of $0.066 \mathrm{~min}^{-1}$ ). The catalytic activity has been attributed to a rate enhancement derived from association of both $\mathrm{NaBH}_{4}$ and 4-NP at the surface of the Au MPCs. ${ }^{62}$ Specifically, the reaction rate is related to the total metal surface atoms available for the reduction of adsorbed 4-NP by a surface-hydrogen species formed on reversible transfer of hydride by adsorbed $\mathrm{NaBH}_{4} .{ }^{179}$ With this in mind, we turned our attention to increasing the accessibility of the reactants to the metal surface.

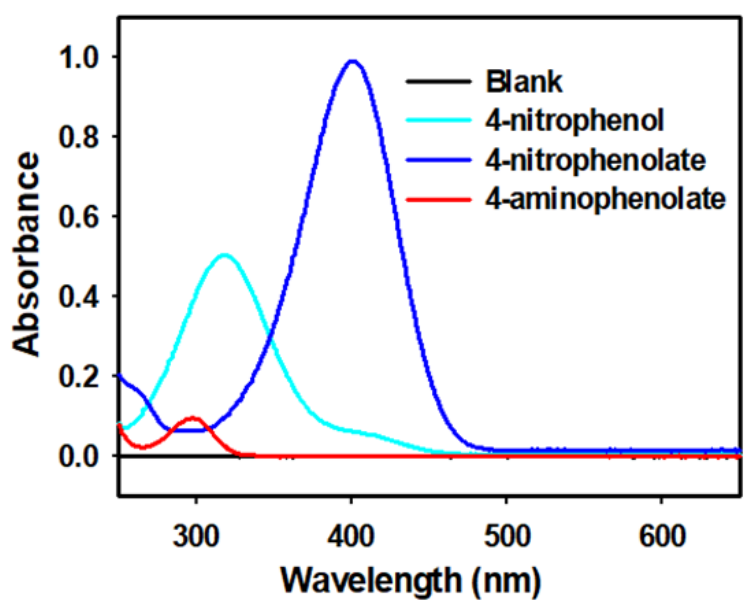

Figure. 5.5. UV-Vis absorption spectra of 4-nitrophenol, 4-nitrophenolate (4-NP) and 4aminophenolate (4-AP), each at a concentration of $3 \times 10^{-5} \mathrm{M}$. 

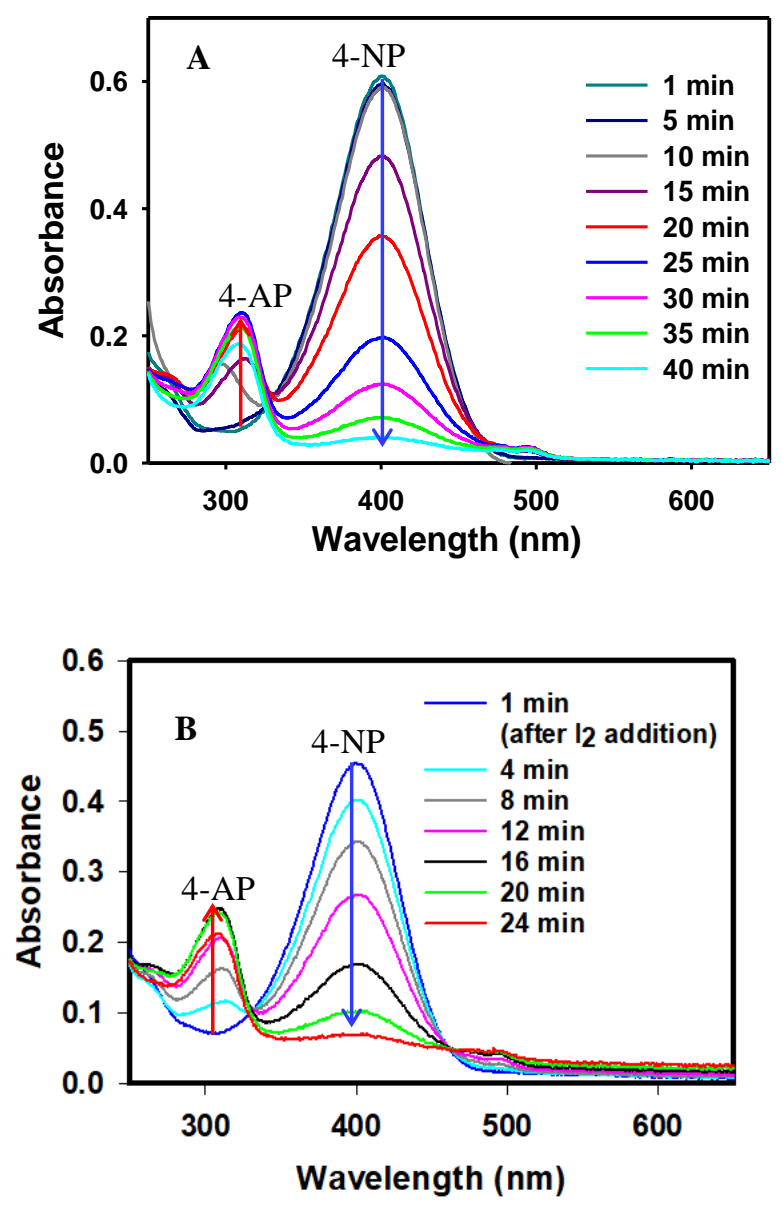

Figure 5.6. A:Changes in absorbance during $\mathrm{NaBH}_{4}$ reduction of 4-NP in the presence of AuSG MPCs as a catalyst over 5-minute intervals (no $\mathrm{I}_{2}$ added). B:Changes in absorbance during the AuSG MPC-catalyzed $\mathrm{NaBH}_{4}$ reduction of 4-NP with 0.25 equivalents of $\mathrm{I}_{2}$ addition.

\subsubsection{Iodine activation of AuSG MPCs}

The addition of $I_{2}$ to the reaction mixture containing AuSG MPCs dramatically increased the reaction rate. A schematic of in-situ 4-NP reduction on the Au surface upon $I_{2}$ treatment is shown in Figure 5.7. In comparison to the rate of reaction in the absence of added $\mathrm{I}_{2}$, addition of $\mathrm{I}_{2}$ at a $0.25 \mathrm{I}_{2}:$ SG ratio enhanced the catalytic activity of 
the AuSG MPCs to completely reduce the 4-NP within 24 minutes (Figure 5.6 B), as shown by the plot of $\ln \left(\right.$ absorbance $_{\text {initial }}\left(A_{o}\right) /$ absorbance $\left._{\text {time }}\left(A_{t}\right)\right)$ for the reaction (Figure $5.8 \mathrm{~A})$. Here, $\mathrm{A}_{\mathrm{o}}$ refers to the initial absorbance that was taken at the beginning of the reaction and $A_{t}$ refers to the absorbance at particular time. The reaction was modeled using pseudo first-order kinetics to determine the rate constant for the catalytic activity as reported previously. ${ }^{180}$ Addition of more $\mathrm{I}_{2}$ resulted in even faster reduction rates the addition of 2.0 equivalents of $\mathrm{I}_{2}$ resulted in complete reduction within 4 minutes.

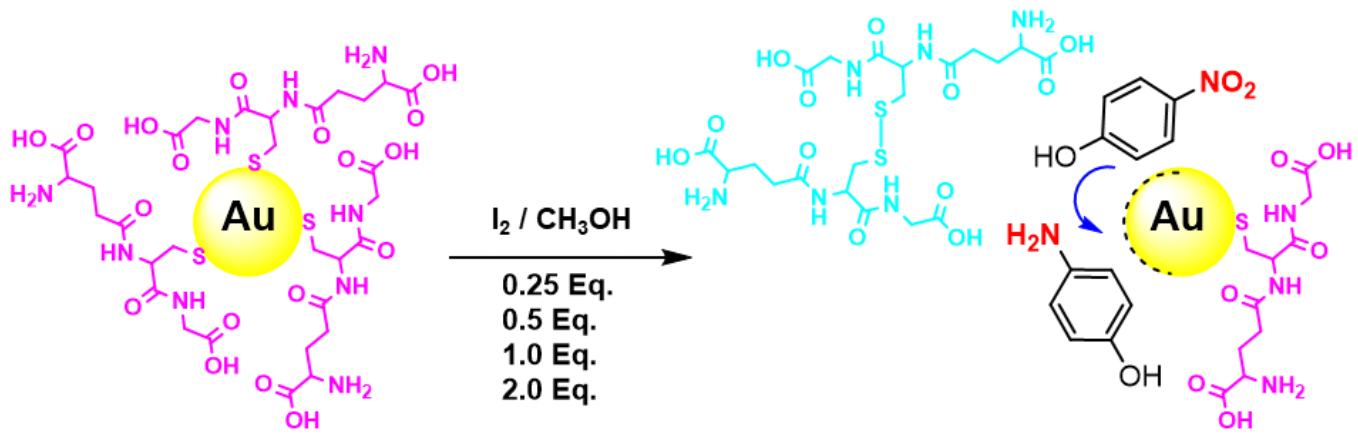

Au-SG MPCs

Reduction on the exposed Au Surface

Figure 5.7. Schematic of 4-NP reduction on AuSG MPC surface upon $I_{2}$ activation.

A control experiment in which $\mathrm{I}_{2}$ was added $\left(\mathrm{I}_{2}: \mathrm{SG}\right.$ ratio $\left.=2.0\right)$ to a mixture of 4 $\mathrm{NP}$ and $\mathrm{NaBH}_{4}$ in the absence of AuSG MPCs showed that $\mathrm{I}_{2}$ addition by itself did not affect the reaction rate. These data are consistent with the hypothesis that $I_{2}$ functions to remove glutathione ligands from the surface of the AuSG MPCs to provide better access of the reactants to the Au surface, which, in turn, enhances the catalytic activity. The rate constant for the AuSG MPC-catalyzed reaction in the absence of $\mathrm{I}_{2}$ was $0.066 \mathrm{~min}^{-1}$ (Figure 5.8 B), which is comparable to the previously reported rate constant observed for the 4-NP reduction catalyzed by DMF-stabilized Au MPCs. ${ }^{68}$ The coordinating effect of 
$\mathrm{DMF}^{69}$ was thought to be responsible for the relatively slow reaction rate. In our case, as more $\mathrm{I}_{2}$ was added, the reaction rate increased, reaching a nearly 8 -fold enhancement to $0.505 \mathrm{~min}^{-1}$ for a ratio of $2.0 \mathrm{I}_{2}: \mathrm{SG}$.
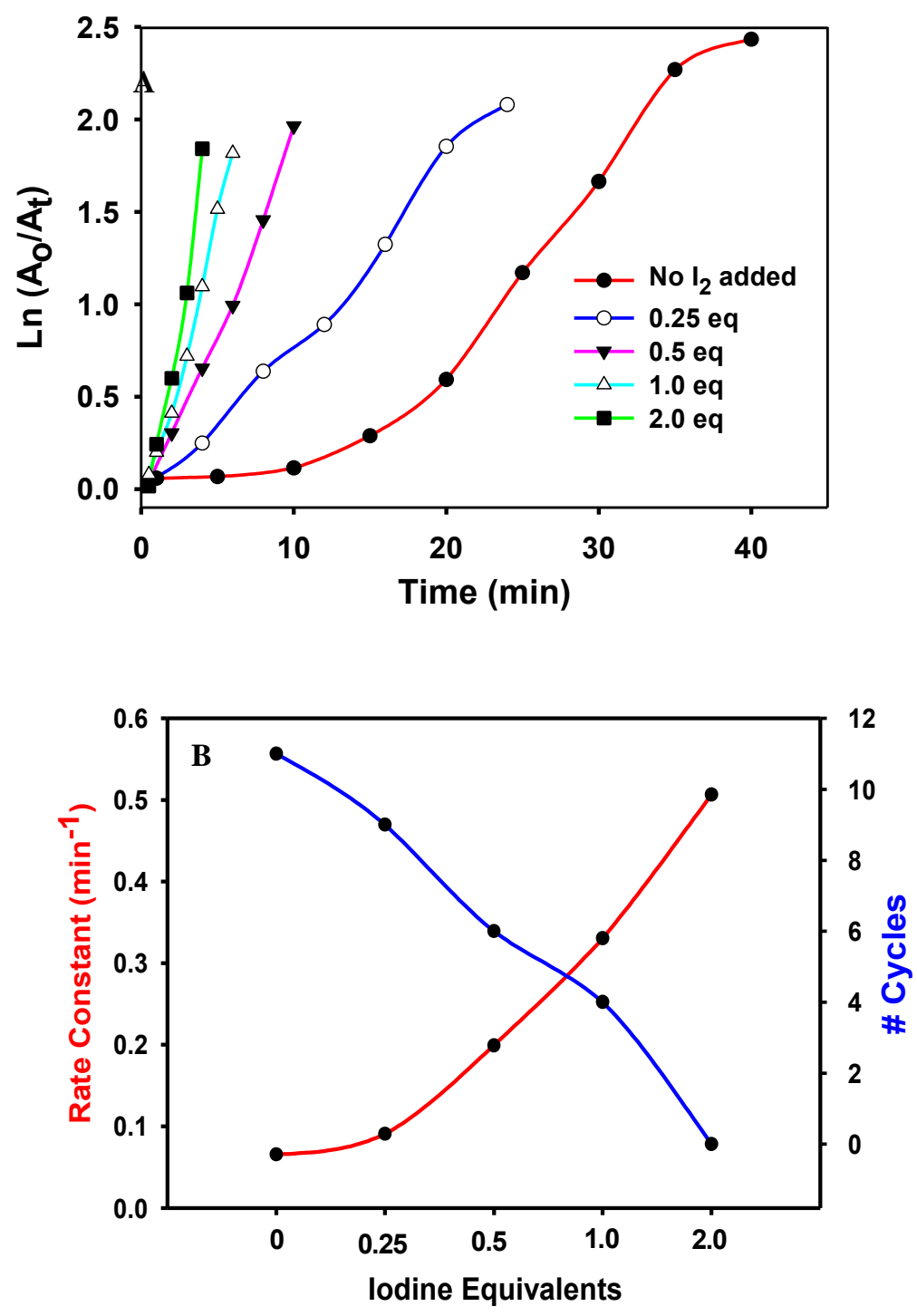

Figure 5.8. A: Plots of $\ln \left(A_{o} / A_{t}\right)$ vs. time for the AuSG MPC-catalyzed reduction of 4-NP with different amounts of added $\mathrm{I}_{2} ; \mathbf{B}$ : plot of reaction rate constants (left y-axis, red) calculated from Fig. 5.8 A and correlation with recyclability (right yaxis, blue). 


\subsubsection{Recyclability of AuSG MPCs}

While $I_{2}$ addition enhanced the catalytic activity, the $I_{2}$ negatively affected the AuSG MPCs as evidenced by the reduced recyclability of the $\mathrm{I}_{2}$-activated AuSG MPCs (Figure 5.8 B). The untreated AuSG MPCs showed the ability to reduce 4-NP to completion in eleven consecutive reduction cycles at a gradually decreasing reaction rate for each subsequent cycle (Figure 5.9 A). In contrast, when an $I_{2}$ ratio of 2.0 was added to the catalyst, no reactivity occurred on the second cycle, likely due to complete removal of SG ligands followed by Au core aggregation and dissolution. Addition of 0.25 equivalents, however showed comparable recyclability to the AuSG MPCs without $\mathrm{I}_{2}$ while significantly enhancing the catalytic activity.

To further explore the recyclability of $\mathrm{I}_{2}$-activated AuSG MPCs, a large-scale reaction was performed in which THF was added to the reaction mixture after each cycle to precipitate the AuSG MPCs for isolation via centrifugation and to remove the 4-AP product by separation of the THF supernatant. The collected MPCs then were used to catalyze the next reaction cycle. This experiment was designed to study the effect of 4AP on catalyst recyclability. We hypothesized that the build-up in concentration of 4AP, formed with each consecutive cycle, might hinder AuSG MPC activity. If this is true, then removing the product at the end of each cycle should increase the number of active catalytic cycles. Figure 5.9 B shows a comparison between the catalytic activity observed when 0.5 eq of $I_{2}$ are added and the product is allowed to amass continuously upon each reaction cycle vs. the experiment in which the AuSG MPCs are separated from the product by precipitation and centrifugation with each cycle before using again. The catalyst was active for 9 cycles when separating from the product after each cycle as 
compared to being active for 5 cycles when the product was allowed to accumulate in solution.
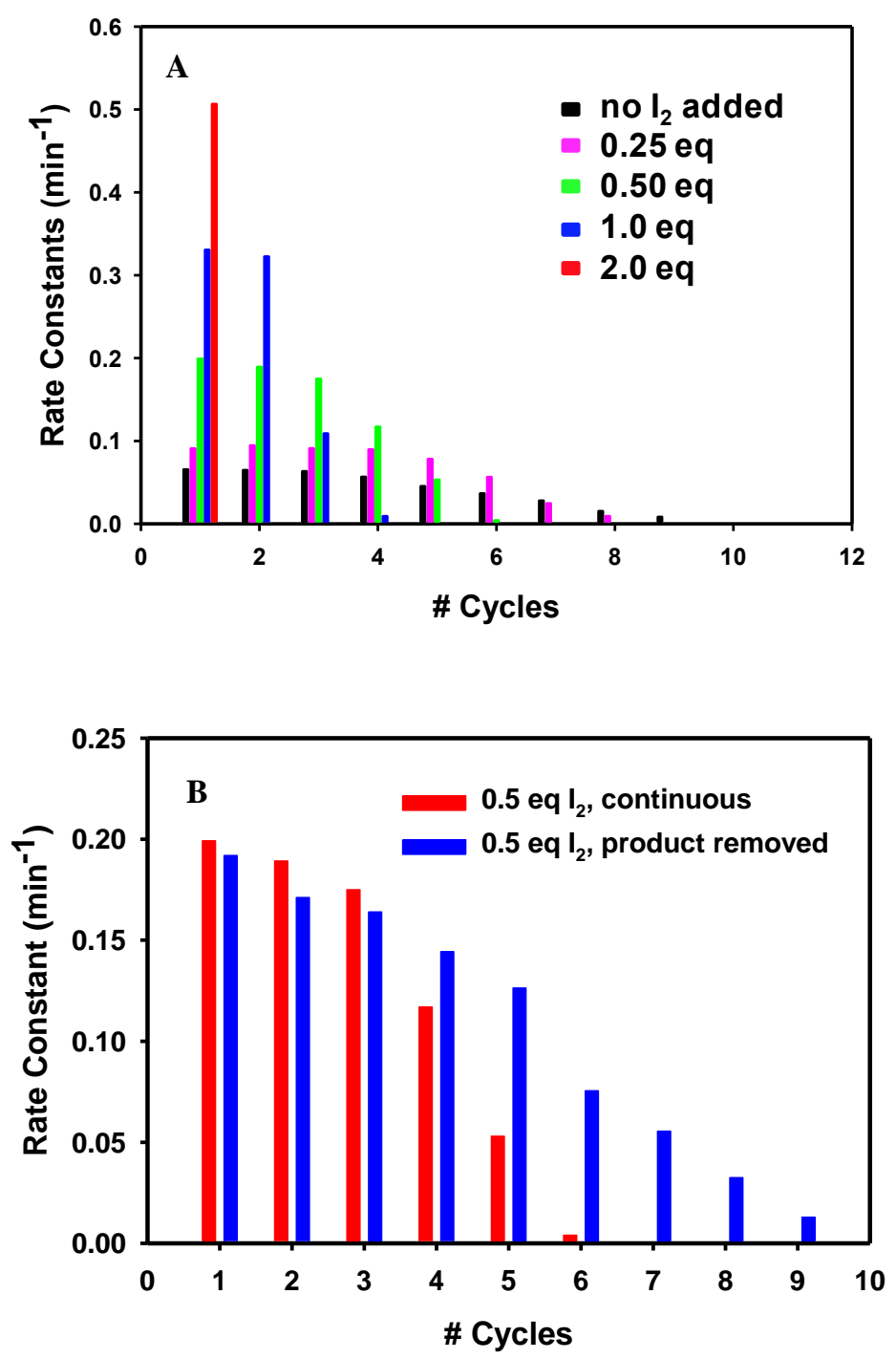

Figure 5.9. A: Plot of rate constant values vs. recyclability for 4-NP reduction with varying equivalents of added $\mathrm{I}_{2} ; \mathbf{B}$ : plot of rate constant values vs. number of cycles for the reduction with 0.5 equivalents of added $I_{2}$, with and without products removed in subsequent cycles. 


\subsubsection{NMR Studies of $I_{2}$ Addition to AuSG MPCs}

The rate enhancement data are consistent with the assertion that $I_{2}$ functions to remove glutathione ligands from the surface of the AuSG MPCs as disulfides (GS-SG) to provide better access of the reactants to the Au surface, which in turn enhances the catalytic activity. To confirm this and quantify the amount of SG ligand removal, we added varying equivalents of $\mathrm{I}_{2}$ to AuSG MPCs in $\mathrm{D}_{2} \mathrm{O}$ relative to the SG ligands and analyzed the amount of dissociated GS-SG by ${ }^{1} \mathrm{H}$ NMR spectroscopy after 40 min of reaction (Figure 5.10). The addition of $\mathrm{I}_{2}$ removed a proportionate amount of bound glutathione from the surface of the Au core, as evidenced by the appearance of signals corresponding to $\mathrm{CH}_{2}$ protons next to the disulfide group at $82.93-2.99$ and 3.27-3.32 ppm (two right blue arrows) in the ${ }^{1} \mathrm{H}$ NMR spectra. As the amount of $\mathrm{I}_{2}$ increased, the area of these signals increased. We also observed a decrease in the signal of the $\mathrm{CH}$ proton of the $-\mathrm{CHCH}_{2} \mathrm{~S}-\mathrm{Au}$ of bound glutathione at $\delta 4.44 \mathrm{ppm}$ (red arrow), which shifts to $84.72-4.76$ and sharpens for the unbound GS-SG disulfide (far left blue arrow), indicating ligand removal from the MPCs in correlation with added $\mathrm{I}_{2}$. We quantified the signal at $\delta 2.93-2.99 \mathrm{ppm}$ as a function of added $\mathrm{I}_{2}$ by comparing it to the intensity of the $t$-butanol internal standard signal at $\delta 1.19 \mathrm{ppm}$. Assuming $100 \%$ of the SG ligands were removed as GS-SG at 2.0 eq $\mathrm{I}_{2}$, we calculated that $37 \%$ of the SG ligands were removed from AuSG MPCs on addition of 0.125 eq $\mathrm{I}_{2}, 53 \%$ on addition of 0.25 eq, $83 \%$ on addition of $0.50 \mathrm{eq}$, and $97 \%$ on addition of $1.0 \mathrm{eq} \mathrm{I}_{2}$ (see Table 5.1). These calculations in combination with the rate data show that $37 \%$ ligand removal does not significantly enhance the catalytic activity, while 53\% ligand removal shows enhanced activity with high recyclability. $83 \%$ ligand removal is close to optimal in terms of high rate 
enhancement while maintaining recyclability. Beyond that, any benefit from rate enhancement is negated by very poor recyclability due to catalyst instability.

Table 5.1. Quantitative results from the NMR data (Figure 5.10) showing the percent of ligand removal from AuSG MPCs as a function of added $\mathrm{I}_{2}$ equivalents after $40 \mathrm{~min}$. reaction.

\begin{tabular}{|c|c|c|c|}
\hline I2 equivalents & $\begin{array}{c}\text { Ratio of Disulfide/t- } \\
\text { butanol Integration }\end{array}$ & $\begin{array}{c}\text { Ratio/Ratio at 2 } \\
\text { equiv. }\end{array}$ & $\begin{array}{c}\text { \% Ligand } \\
\text { Removal }\end{array}$ \\
\hline 0 & 0 & $0 / 0.57$ & 0 \\
\hline 0.125 & 0.21 & $0.21 / 0.57$ & 37 \\
\hline 0.25 & 0.3 & $0.30 / 0.57$ & 53 \\
\hline 0.5 & 0.47 & $0.47 / 0.57$ & 83 \\
\hline 1.0 & 0.55 & $0.55 / 0.57$ & 97 \\
\hline 2.0 & 0.57 & $0.57 / 0.57$ & 100 \\
\hline
\end{tabular}




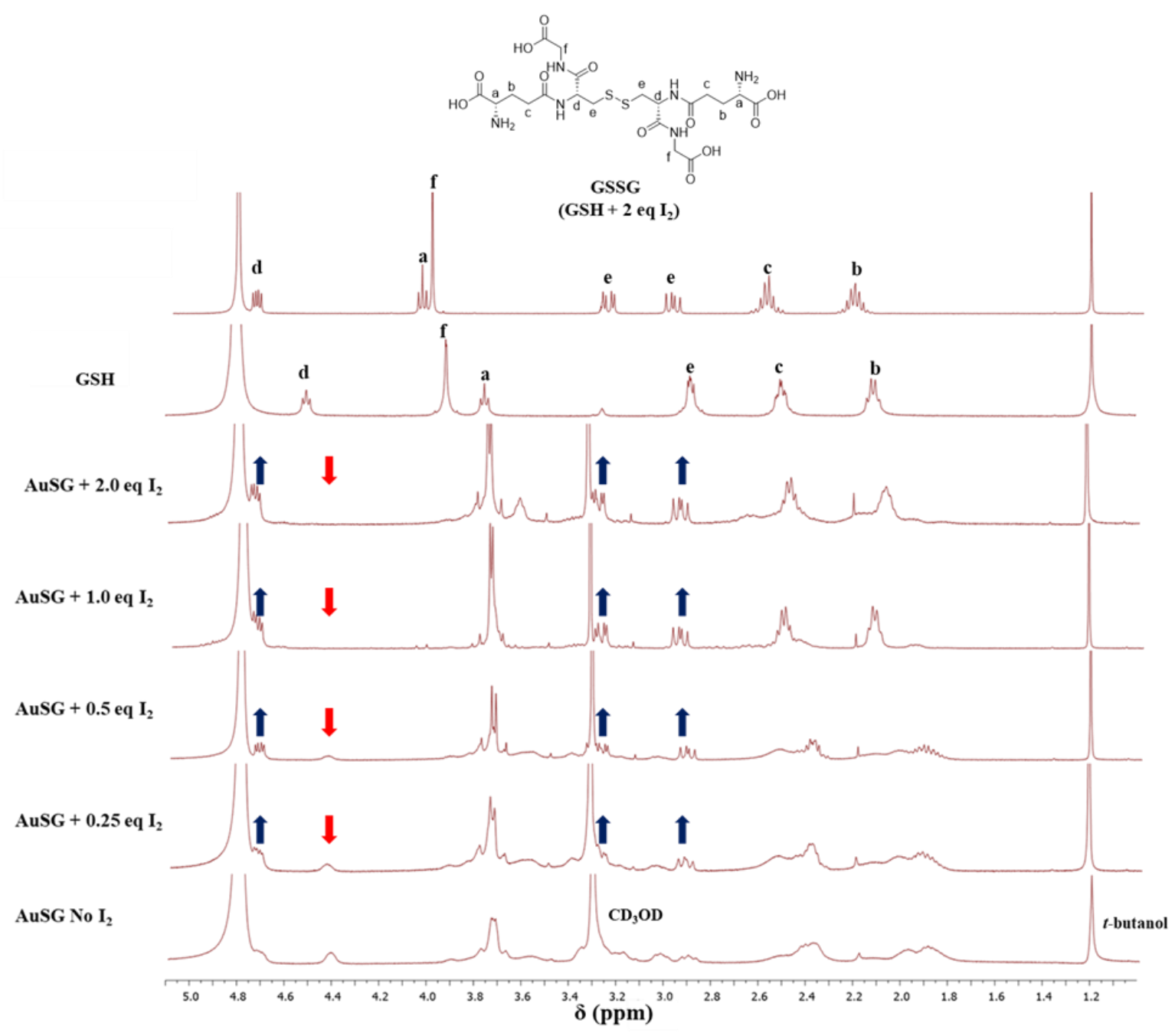

Figure 5.10. ${ }^{1} \mathrm{H}$ NMR spectra of AuSG MPCs in $\mathrm{D}_{2} \mathrm{O}$ without added $\mathrm{I}_{2}$ and 40 min after the addition of various equivalents of $\mathrm{I}_{2}$. The signals at $82.93-2.99$ and 3.27-3.32 ppm (marked by blue arrows) correspond to the methylene protons of the cysteine residues of glutathione disulfide (GS-SG), and the signal at $84.72-4.76 \mathrm{ppm}$ corresponds to the adjacent methine proton (far left blue arrow). The increase in these signals on addition of $\mathrm{I}_{2}$, relative to the internal standard $t$-butanol singlet at $\delta 1.19 \mathrm{ppm}$, indicates that the disulfide forms and dissociates from the AuSG MPCs. With no $\mathrm{I}_{2}$, the methylene protons next to $\mathrm{S}$ of bound glutathione are not visible in the NMR spectrum and the signal for the immediately adjacent methine proton appears at $\delta 4.44 \mathrm{ppm}$ (red arrow) as a broad peak, indicating that the SG is bound to the Au cluster. The peak height decreases with added $\mathrm{I}_{2}$ in agreement with ligand removal upon disulfide formation. The NMR spectra of free glutathione (GSH) and GSH oxidized to disulfide by $I_{2}$ are shown for comparison. Some differences in chemical shifts for the signals of GS-SG formed on reaction of AuSG MPCs vs. $\mathrm{I}_{2}$-oxidation of free GSH can be expected, particularly for protons adjacent to $\mathrm{N}$ groups, due to differences in $\mathrm{pH}$ and the possible presence of metal ions. 


\subsubsection{Study of AuSG MPC Stability by UV-Vis, SEM and ASV}

We measured the absorbance spectra of aqueous solutions of AuSG MPCs treated with different equivalents of $I_{2}$ and monitored their stability from $30 \mathrm{~s}$ to $3 \mathrm{~h}$ (Figure 5.11). In agreement with recyclability trends and noticeable changes in the MPCs with increasing $\mathrm{I}_{2}$ equivalents, the UV-vis spectra of the AuSG MPCs showed the development of a localized surface plasmon resonance (LSPR) peak for Au at $\sim 530 \mathrm{~nm}$ with 0.50 and 1.0 eq of $I_{2}$ added (Figure $5.11 \mathrm{~B}$ and C). This indicates enlargement of the Au MPCs by aggregation or ripening. The addition of 0.25 equivalents of $\mathrm{I}_{2}$ did not lead to any significant changes in the UV-vis spectra, indicating no growth or aggregation of the MPCs, consistent with their higher stability/recyclability (Figure 5.11 A). The addition of excess $I_{2}(2.0$ eq) resulted in a large decrease in absorbance and the appearance of two absorbance peaks at $352 \mathrm{~nm}$ and $454 \mathrm{~nm}$, indicative of dissolution of the $\mathrm{Au}$ to form soluble $\mathrm{Au}^{\mathrm{I}}$ or $\mathrm{Au}^{\mathrm{III}}$ iodide ionic complexes (Figure $5.11 \mathrm{D}$ ). The kinetics of Au dissolution in the presence of iodide has been previously reported. ${ }^{181}$ Furthermore, these observations are consistent with the changes we noted in the solution color of the AuSG MPCs (Figure 5.11, Top).

Iodine-activated AuSG MPCs characterized by scanning electron microscopy (SEM) agree with the UV-vis data. At an $\mathrm{I}_{2}: \mathrm{SG}$ ratio of 0.25 , no change in particle size was observed based on the MPCs being difficult to observe by the SEM since $1.6 \mathrm{~nm}$ is below our resolution (Figure 5.12 A). At an $\mathrm{I}_{2}: \mathrm{SG}$ ratio of 0.5, the SEM images showed nanoparticles with an average diameter of $68 \pm 7 \mathrm{~nm}$, indicative of MPCs growth into larger nanoparticles (Figure 5.12 B). At an $\mathrm{I}_{2}: \mathrm{SG}$ ratio of 1.0, the average diameter of the nanoparticles increased to $497 \pm 33 \mathrm{~nm}$ (Figure $5.12 \mathrm{C}$ ), consistent with the larger LSPR 
band in Figure 5.11 C. In agreement with the observed dissolution of $\mathrm{Au}$, no particles were observed at an $\mathrm{I}_{2}: \mathrm{SG}$ ratio of 2 .

Anodic stripping voltammetry is a useful technique to study size dependent properties and aggregation of nanoclusters or nanoparticles. In anodic stripping voltammetry (ASV) studies (Figure 5.13), we did not observe an oxidation peak for AuSG MPCs because the metal surface is monolayer-protected. Likewise, when 0.25 equivalents of $\mathrm{I}_{2}$ were added to the AuSG MPCs, no Au oxidation peak was observed, presumably because a significant amount of the SG ligands remained bound, passivating the Au. However, a peak for Au oxidation in KBr occurred for AuSG MPCs treated with 0.5 and 1.0 equivalents of $\mathrm{I}_{2}$ at $\sim 1.0 \mathrm{~V}$ with respective currents of $-2.8 \mu \mathrm{A}$ and $-4.4 \mu \mathrm{A}$. The appearance of this peak and the specific oxidation potential being at $1.0 \mathrm{Vis}$ consistent with the clusters losing the SG ligands and increasing in size well above $30 \mathrm{~nm}$ in diameter as a result of $I_{2}$ addition (Figure 5.13). Due to the complete dissolution of the AuSG MPCs when 2.0 equivalents of $I_{2}$ were added, there were no AuSG MPCs in solution to stick to the glass/ITO electrode. As a result, no oxidation peak was observed in the ASV at 2.0 equivalents $\mathrm{I}_{2}$. The ASV results are consistent with the UV-Vis measurements for particles treated with $\mathrm{I}_{2}$, which go through the steps of ligand removal, increase in size, and finally Au dissolution as the amount of $I_{2}$ increases. 

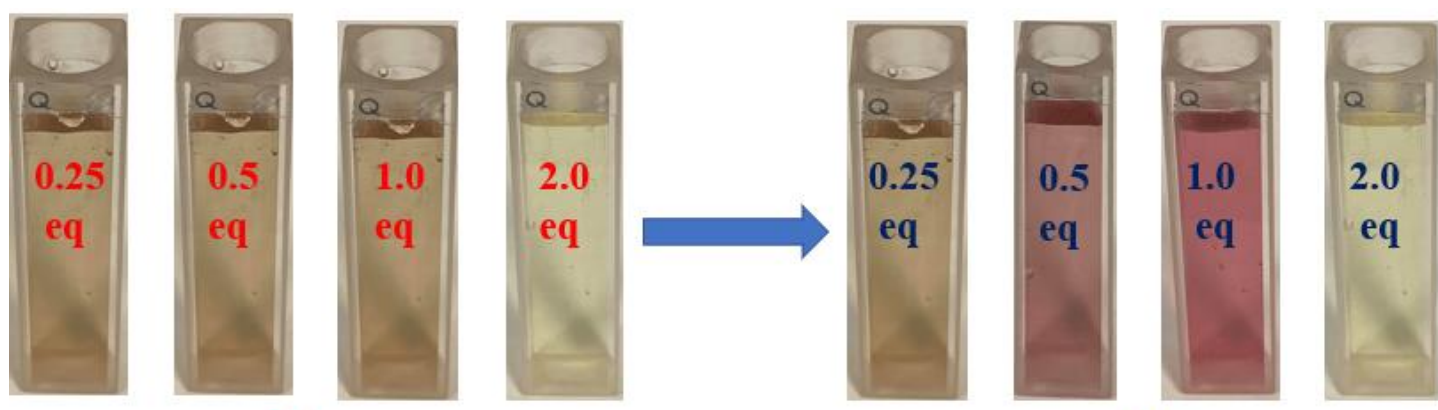

$30 \mathrm{~s}$

\section{$3 \mathbf{h r}$}
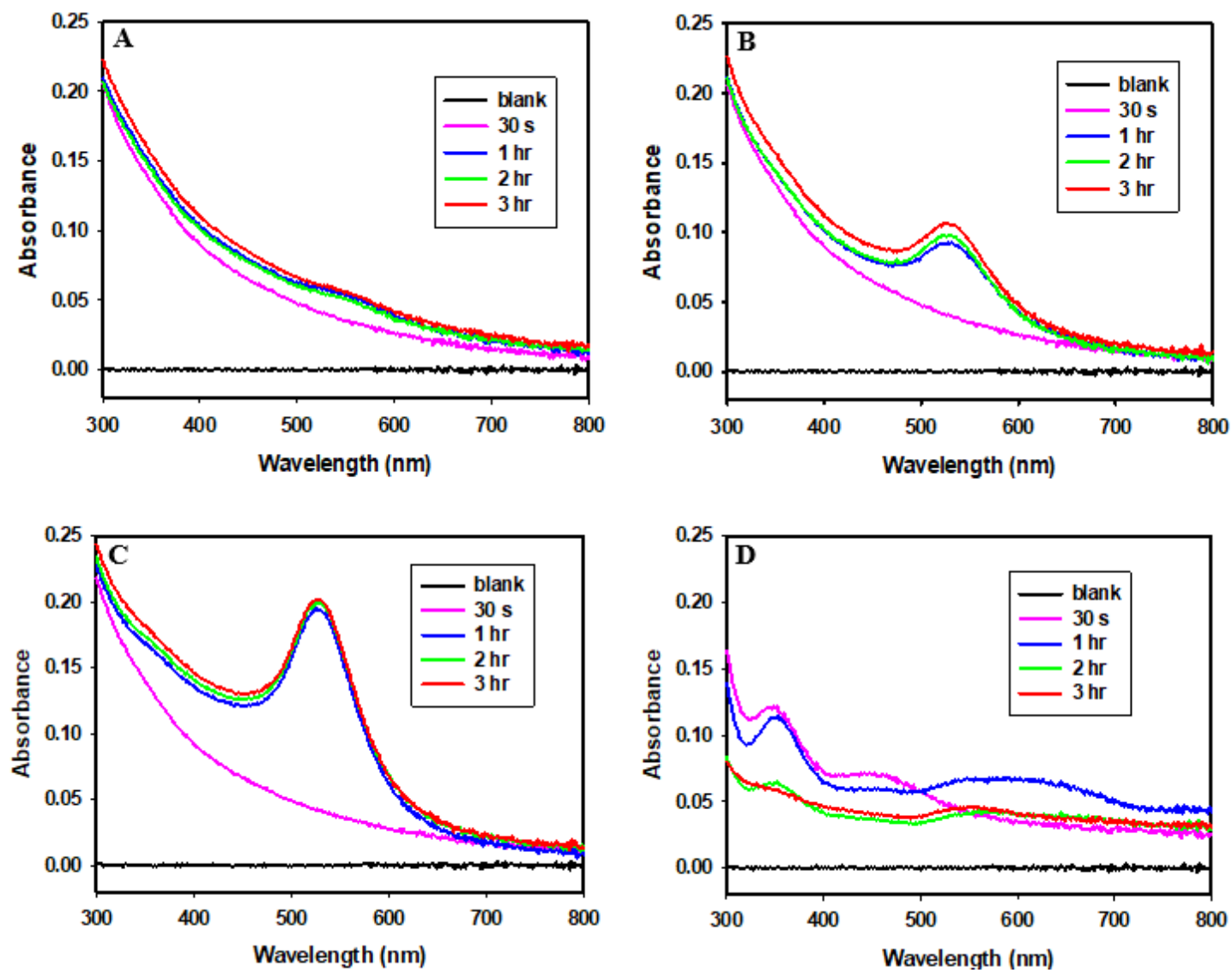

Figure 5.11. (Top) Digital image of aqueous solutions of AuSG MPCs after reaction with $\mathrm{I}_{2}$ for 30 seconds and 3 hours. The change to red color and then disappearance of the color is consistent with aggregation or increased size by ripening followed by dissolution at higher equivalents of $\mathrm{I}_{2}$. (Bottom) UV-Vis absorption spectra of aqueous solutions of AuSG MPCs treated with A: 0.25 eq, B: 0.5 eq, C: 1.0 eq, and D: 2.0 eq of $\mathrm{I}_{2}$ for the times indicated. 

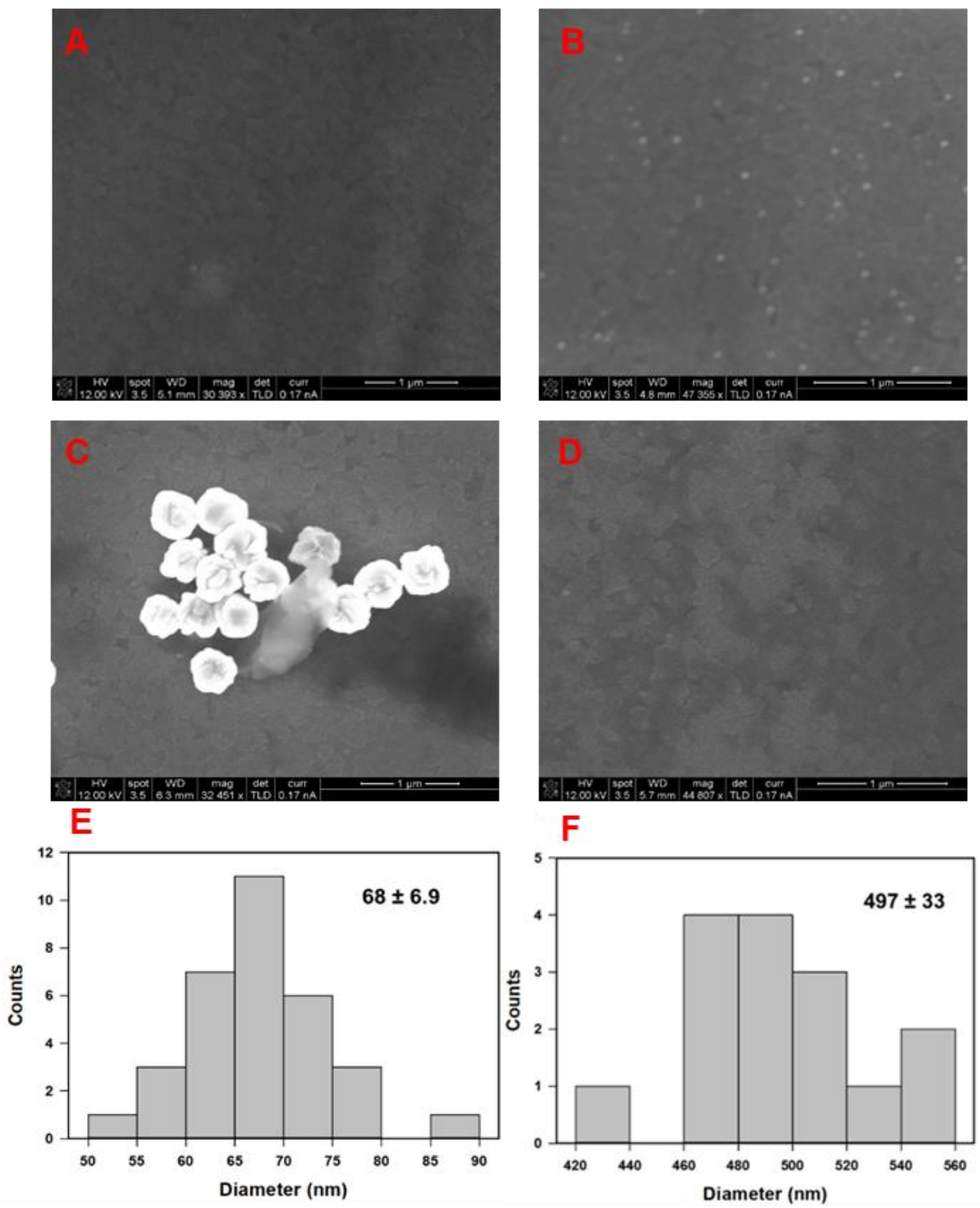

Figure 5.12. SEM images of iodine-activated AuSG MPCs; A. 0.25 eq $I_{2}$ (cannot be observed in SEM), B. 0.5 eq $\mathrm{I}_{2}(68 \pm 7 \mathrm{~nm})$, C. 1.0 eq $\mathrm{I}_{2}(497 \pm 33 \mathrm{~nm})$, D. 2.0 eq $\mathrm{I}_{2}$ (Au dissolved, only ITO observed), E. Histogram of AuSG MPCs with 0.5 eq $\mathrm{I}_{2}$, and $\mathbf{F}$. Histogram of AuSG MPCs with 1.0 eq $\mathrm{I}_{2}$. 


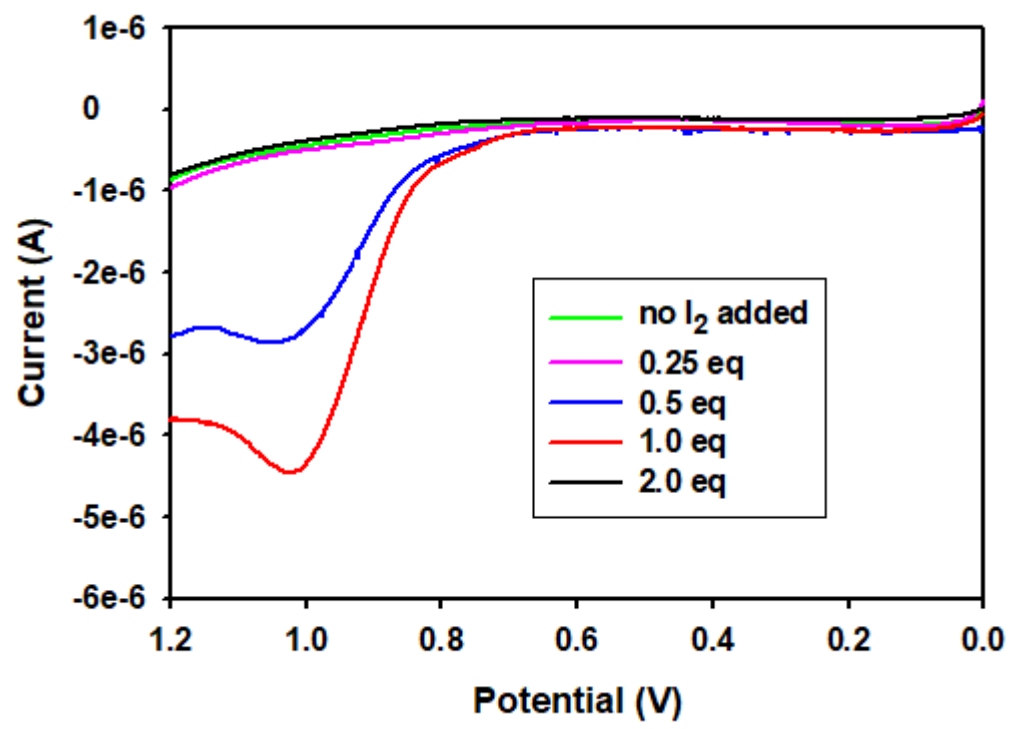

Figure 5.13. Influence of added $I_{2}(0.25$ to 2.0 eq) on the anodic stripping voltammetry (ASV) of AuSG MPCs attached to glass/ITO electrodes. Measurements were taken after 3 hours reaction time.

\subsubsection{Catalytic hydrogenation/isomerization of allyl alcohol}

The catalysis of allyl alcohol hydrogenation/isomerization (Scheme 5.4) to 1propanol (hydrogenation product) and propanal (isomerization product) using alkanethiolate-capped $\mathrm{Pd}$ nanoclusters is well understood. ${ }^{74,} 75$ We performed this reaction using PdSG MPCs in a chlorobenzene-water biphasic system while passing $\mathrm{H}_{2}$ through the mixture using a glass pipette (Figure 5.14). 

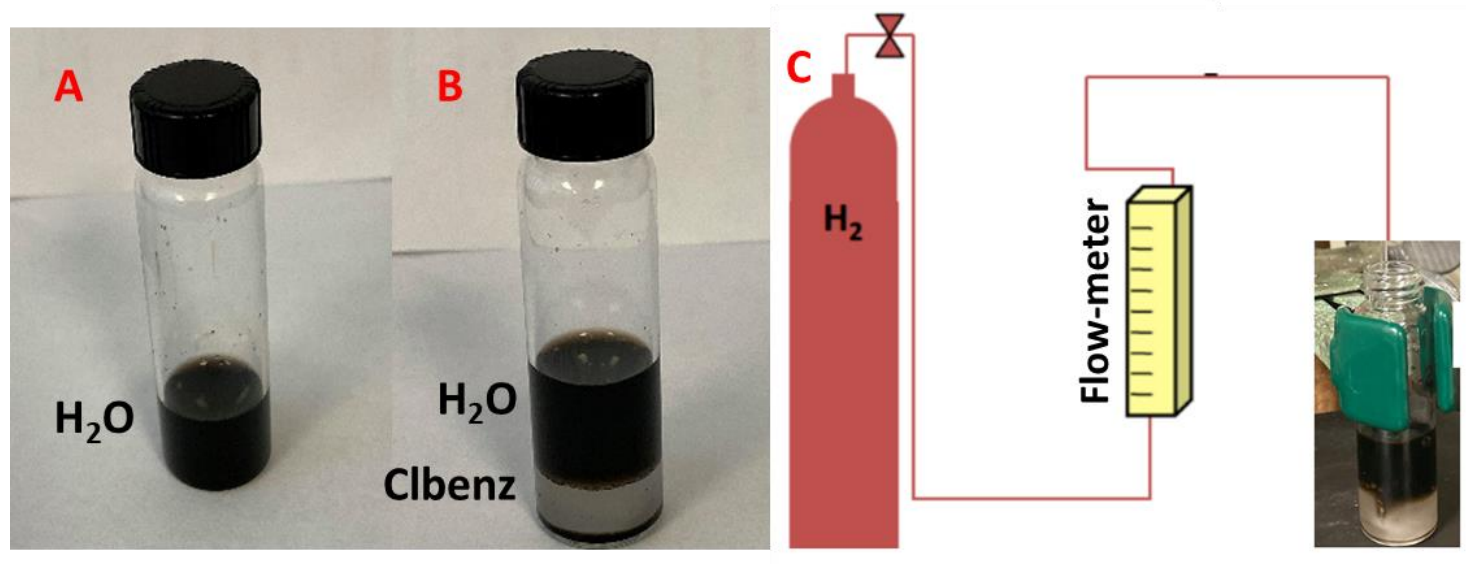

Figure 5.14. Digital images of PdSG MPCs in water (A), after adding chlorobenzene (B), and the reaction set-up for isomerization/hydrogenation of allyl alcohol (C).

Figure 5.15 shows the UV-Vis spectra of $0.123 \mathrm{M}$ aqueous solutions of substrate (allyl alcohol) and the products (propanal and 1-propanol) with a strong absorbance peak found for propanal at $280 \mathrm{~nm}$. Whereas, Figure 5.16 shows the GC-FID chromatograms of allyl alcohol, propanal and 1-propanol (all $10 \mathrm{mM}$ ), indicating the retention time of each component in both organic and aqueous phases. The progress of the PdSG MPCcatalyzed reaction in the absence of $I_{2}$ was monitored by examining both phases in 5minute intervals using GC (organic phase, Figure 5.17 A and aqueous phase, Figure 5.18 A). In both the organic and aqueous phases, the allyl alcohol $\left(t_{r}=3.6 \mathrm{~min}\right)$ disappeared within 35 minutes, while peaks corresponding to propanal $\left(t_{r}=1.1 \mathrm{~min}\right)$ and 1-propanol $\left(\mathrm{t}_{\mathrm{r}}\right.$ $=2.9 \mathrm{~min}$ ) increased over time. The distribution of the products formed depended on the phase, as the isomerized product was favored in the organic phase while a $\sim 1: 1$ mixture of isomerized:hydrogenated products was observed in the aqueous phase (overall selectivity was $\sim 90 \%$ isomerized product). We next turned our attention to examining the effects of adding varying amounts of $I_{2}$ on the reaction rate and catalyst recyclability. 


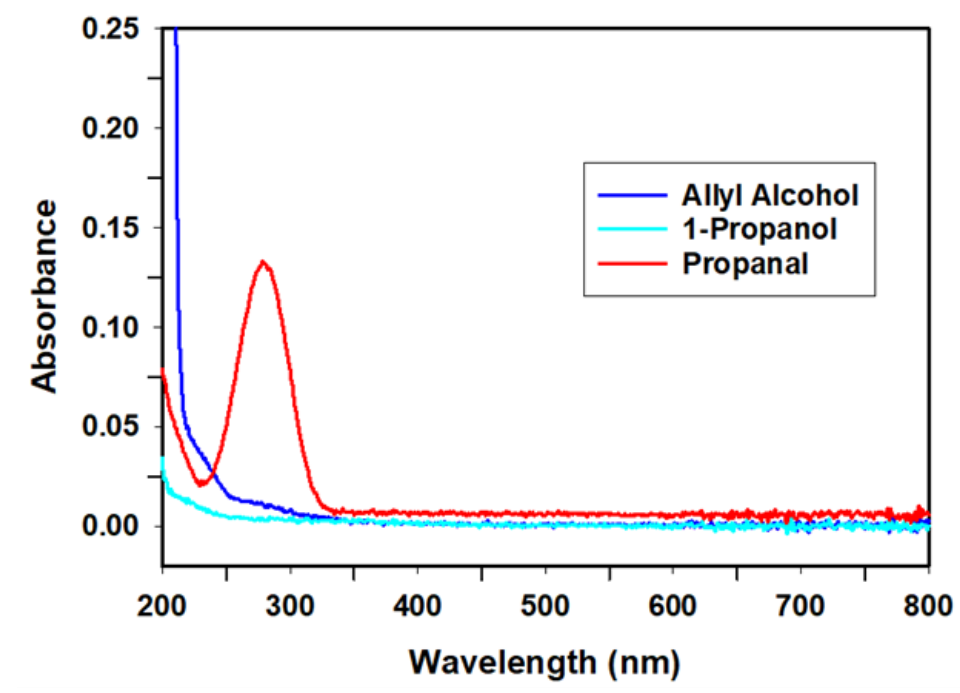

Figure 5.15. UV-Vis spectra of $0.123 \mathrm{M}$ aqueous solutions of reactant (allyl alcohol) and products (1-propanol and propanal).

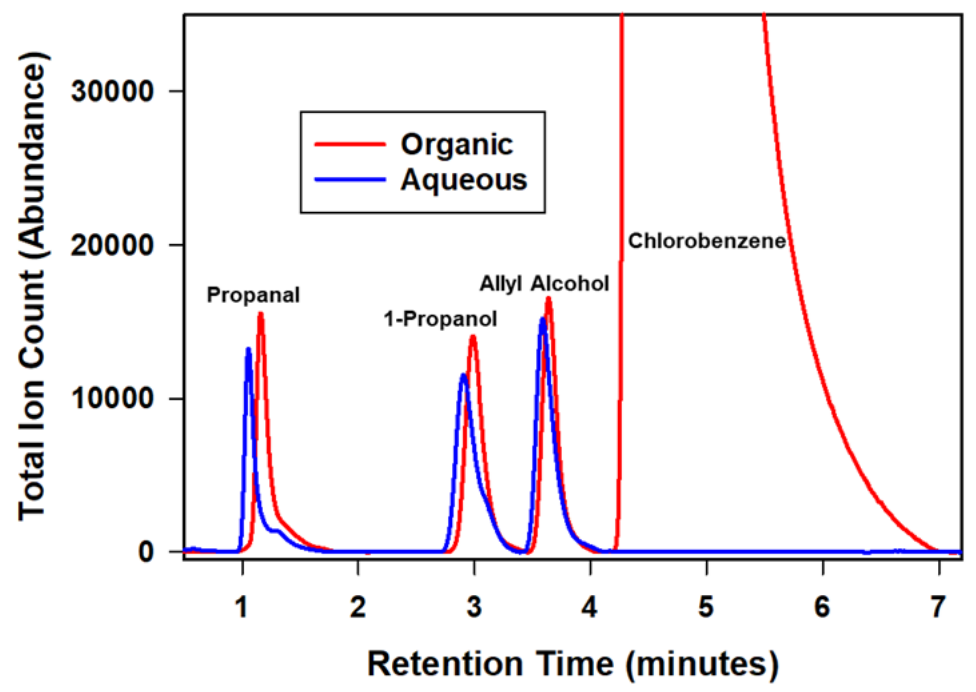

Figure 5.16. Gas chromatograms of standard solutions containing mixtures of $10 \mathrm{mM}$ each of reactant (allyl alcohol), and products (propanal, 1-propanol) in organic phase (chlorobenzene) and aqueous phase. Three chromatograms were averaged to determine the response factor $\left(\mathrm{R}_{\mathrm{f}}\right)$ in order to calculate final TOF values. 

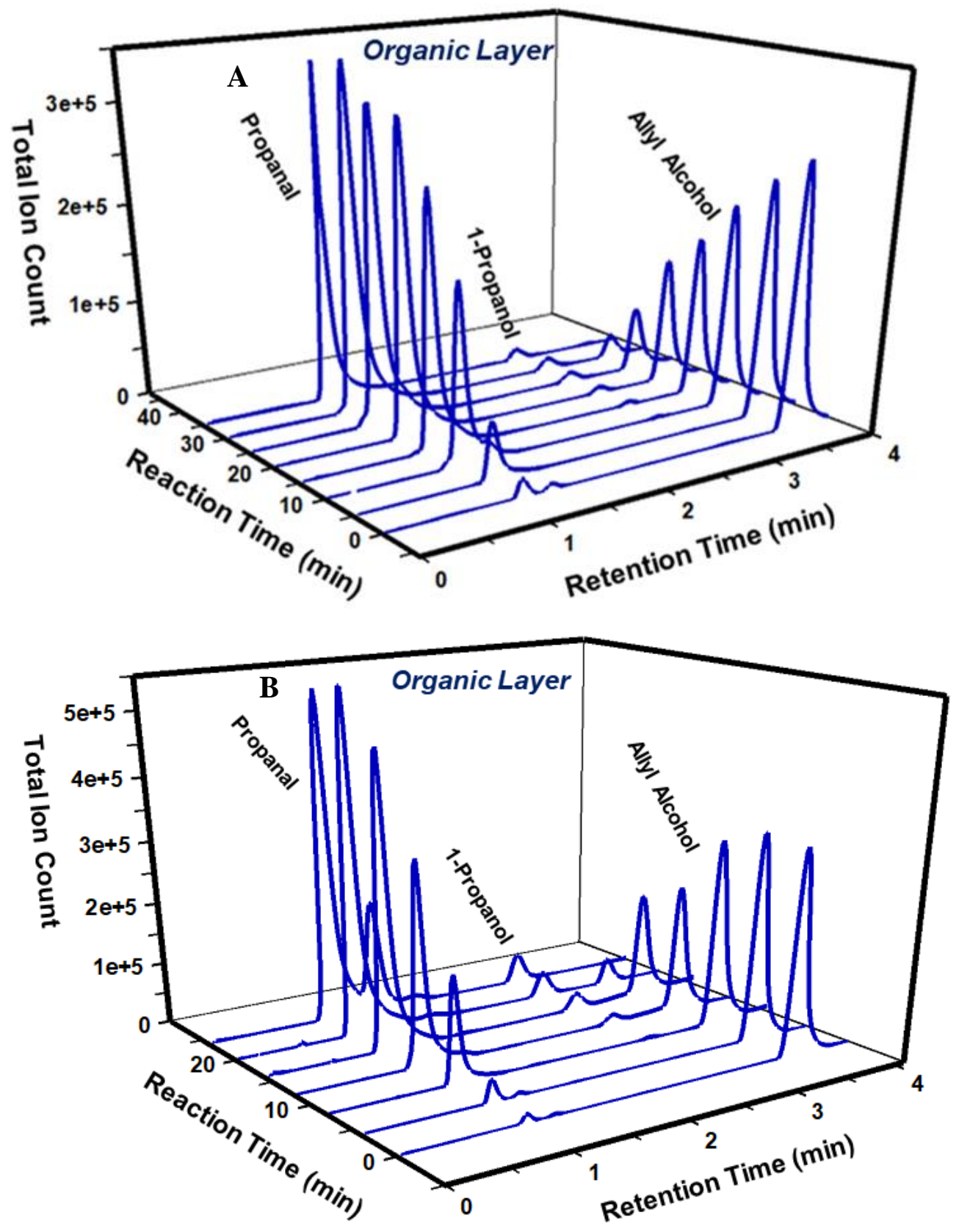

Figure 5.17. GC-FID chromatograms of allyl alcohol hydrogenation/isomerization using PdSG MPCs in chlorobenzene:water (1:1) with a $\mathrm{H}_{2}$ flow rate of $20.0 \pm 0.5$ $\mathrm{mL} / \mathrm{min}$. A:organic phase, in absence of $\mathrm{I}_{2} ; \mathbf{B}$ :organic phase, with 0.05 eq $\mathrm{I}_{2}$ addition. 

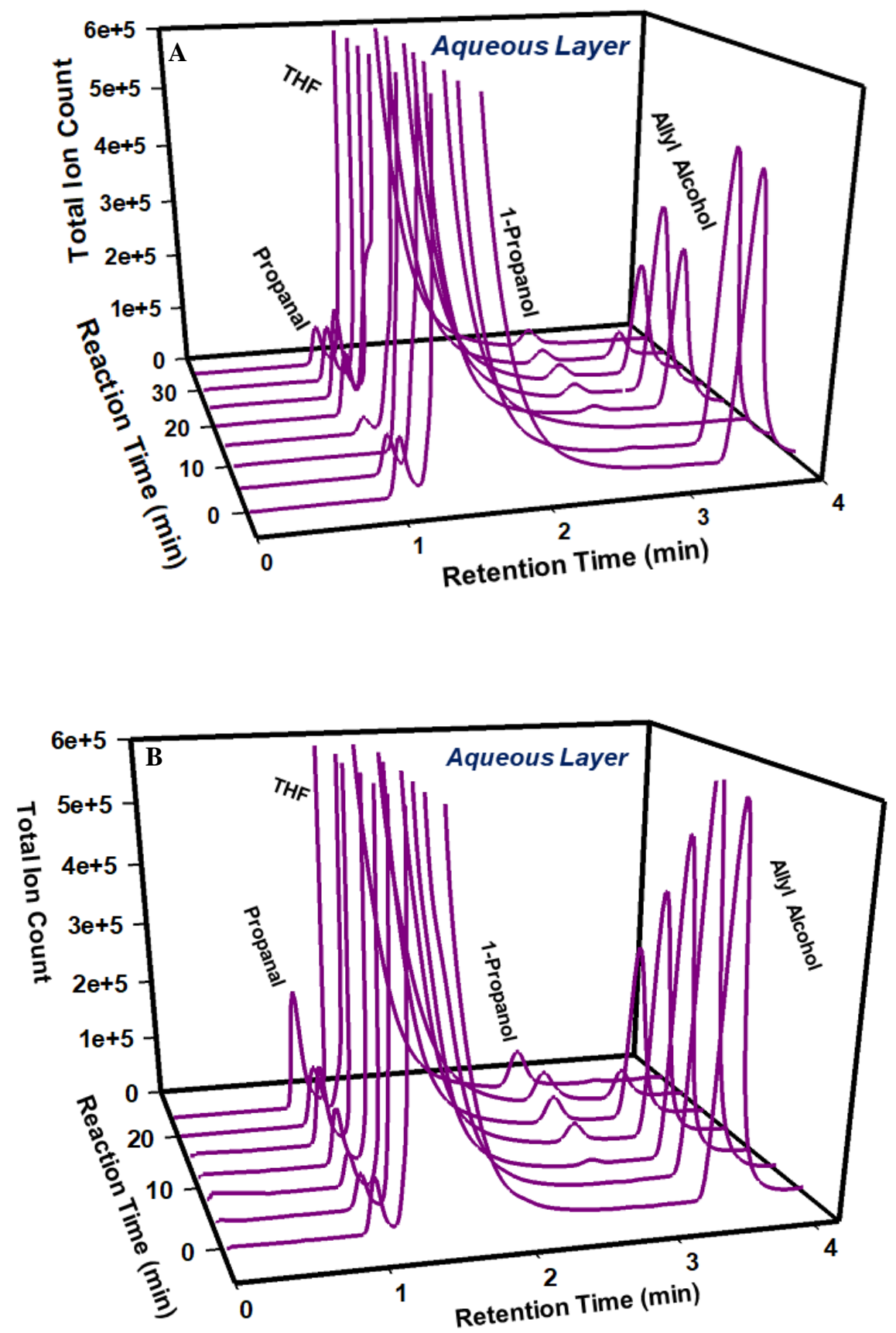

Figure 5.18. GC-FID chromatograms of allyl alcohol hydrogenation/isomerization using PdSG MPCs in chlorobenzene:water (1:1) with a $\mathrm{H}_{2}$ flow rate of $20.0 \pm 0.5 \mathrm{~mL} / \mathrm{min}$. A:aqueous phase, in absence of $\mathrm{I}_{2}$, and $\mathbf{B}$ :aqueous phase, with 0.05 eq $\mathrm{I}_{2}$ addition. 


\subsubsection{Iodine activation of PdSG MPCs}

The addition of $I_{2}$ to the reaction mixture containing PdSG MPCs and allyl alcohol significantly increased the reaction rate. Figure 5.17 B (organic phase) and 5.18 B (aqueous phase) shows that the addition of 0.05 equivalents of $I_{2}$ enhanced the catalytic activity as it only took 24 minutes in comparison to 35 minutes in the absence of $I_{2}$ for complete conversion. The total percentage completion plot shows that the reaction was completed nearly twice as fast when 0.2 equivalents of $I_{2}$ were added as compared to the reaction without added $\mathrm{I}_{2}$ (Figure 5.19). Catalytic enhancement is noted with addition of as little as 0.025 equivalents of $\mathrm{I}_{2}$. TOF values were determined from the plots of the \% hydrogenation and $\%$ isomerization vs reaction time and taking the slope of the plots with $<60 \%$ corresponding conversions (Figure 5.20). Table 5.2 summarizes the turnover frequencies (TOFs) for the reaction in the absence and presence of varying equivalents of added $\mathrm{I}_{2}$. The TOFs were calculated by measuring the response factor of 1-propanol and propanal relative to allyl alcohol from a GC of standard solutions of $10 \mathrm{mM}$ each (Figure 5.16), which allowed a measurement of the percent of allyl alcohol converted to 1propanol and propanal as a function of time according to a method reported in the literature. ${ }^{182}$ The total TOF (hydrogenation TOF plus isomerization TOF) significantly increased from 131 to 230 moles products/moles $\mathrm{Pd} / \mathrm{h}$ on increasing the $\mathrm{I}_{2}: \mathrm{SG}$ ratio from 0.025 to 0.2 . However, at a ratio higher than 0.3 , there was little conversion of allyl alcohol into products, even after 60 minutes of reaction time. 


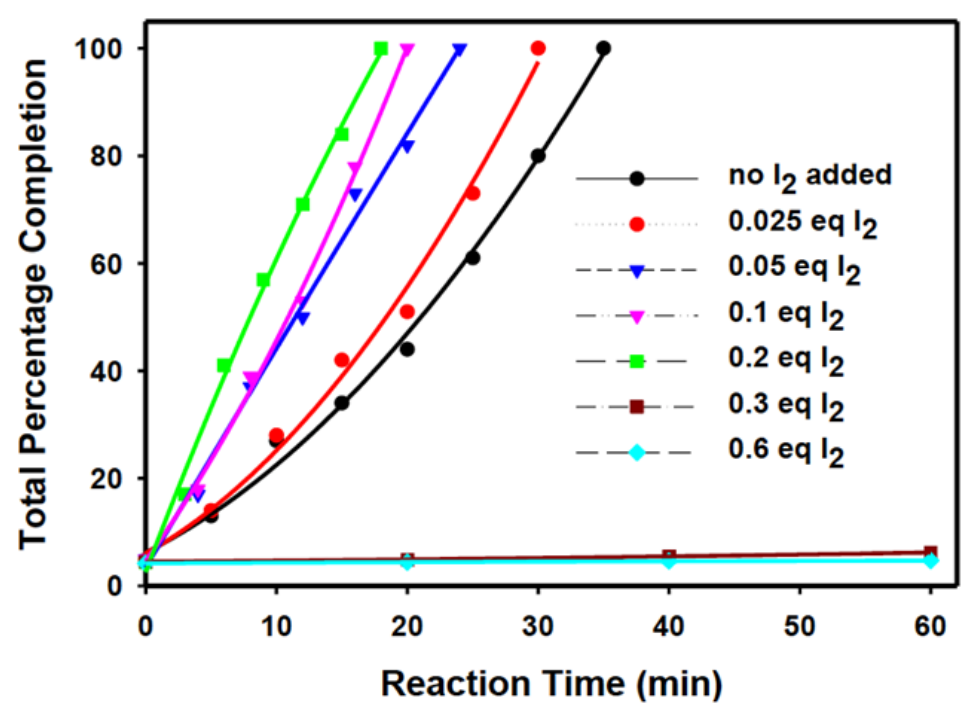

Figure 5.19. Reaction completion percentage (hydrogenated plus isomerized) for the PdSG MPC - catalyzed hydrogenation/isomerization of allyl alcohol for different amounts of added $\mathrm{I}_{2} . \mathrm{H}_{2}$ flow rate $=20.0 \pm 0.5 \mathrm{~mL} / \mathrm{min}$.

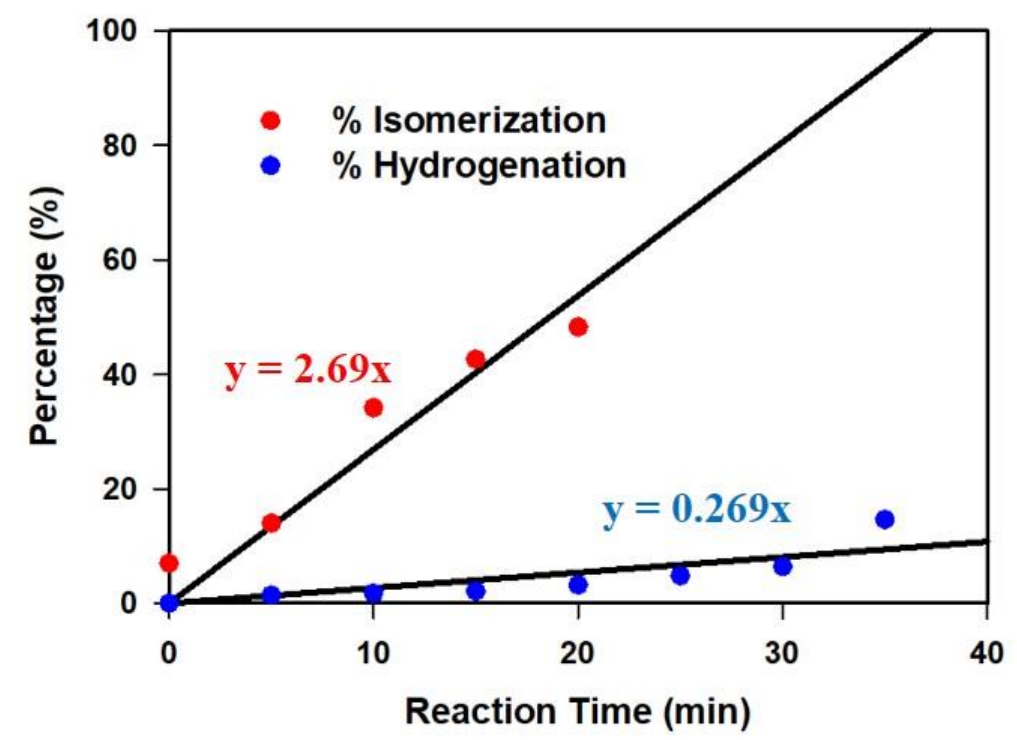

Figure 5.20. Plot of the $\%$ hydrogenation and $\%$ isomerization of allyl alcohol versus reaction time with the intercept of the line forced through zero, using PdSG MPC as a catalyst (no $\mathrm{I}_{2}$ added). The slopes for the points up to $60 \%$ isomerization and all of the points for hydrogenation were used to determine the TOF values. 


\subsubsection{Example calculation of TOF values}

The final TOF values were calculated based on the procedure reported in the literature. ${ }^{53,183}$ The following shows an example.

$100 \%$ hydrogenation/isomerization $=200 \mu \mathrm{L}$ allyl alcohol $=2.94 \times 10^{-3}$ moles

Mass of Pd in the $6.0 \mathrm{mg}$ of catalyst (PdSG MPCs) used for the reaction was calculated based on the organic composition (glutathiolate determined by TGA of $29 \%$ gives $71 \%$ of Pd metal)

Mass $\mathrm{Pd}=71 \%$ of $6.0 \mathrm{mg}=4.26 \mathrm{mg}$

moles Pd $=(4.26 \mathrm{mg}) /(106.42 \mathrm{mg} / \mathrm{mmol})=4.0 \times 10^{-5} \mathrm{~mol} \mathrm{Pd}$

$\%$ hydrogenation $=\left[\left(\mathrm{S}_{\mathrm{H}} * \mathrm{k}_{2}\right) /\left(\mathrm{S}_{\mathrm{H}} * \mathrm{k}_{2}+\mathrm{S}_{\mathrm{R}} * \mathrm{k}_{1} * \mathrm{k}_{2}+\mathrm{S}_{\mathrm{I}} * \mathrm{k}_{1}\right)\right] \mathrm{x} 100 \%$

$\%$ isomerization $=\left[\left(\mathrm{S}_{\mathrm{I}} * \mathrm{k}_{1}\right) /\left(\mathrm{S}_{\mathrm{H}} * \mathrm{k}_{2}+\mathrm{S}_{\mathrm{R}} * \mathrm{k}_{1} * \mathrm{k}_{2}+\mathrm{S}_{\mathrm{I}} * \mathrm{k}_{1}\right)\right] \times 100 \%$

Where, $S_{\mathrm{H}}=$ Peak area corresponding to hydrogenated product (1-propanol)

$\mathrm{S}_{\mathrm{I}}=$ Peak area corresponding to isomerized product (propanal)

$\mathrm{S}_{\mathrm{R}}=$ Peak area corresponding to reactant (allyl alcohol)

$\mathrm{k}_{1}=$ Response factor for hydrogenated product to reactant

$\mathrm{k}_{2}=$ Response factor for isomerized product to reactant

$\mathrm{k}_{1}=\left(\mathrm{S}_{\mathrm{H}} * \mathrm{C}_{\mathrm{R}}\right) /\left(\mathrm{S}_{\mathrm{R}} * \mathrm{C}_{\mathrm{H}}\right)$

$\mathrm{k}_{2}=\left(\mathrm{S}_{\mathrm{I}} * \mathrm{C}_{\mathrm{R}}\right) /\left(\mathrm{S}_{\mathrm{R}} * \mathrm{C}_{\mathrm{I}}\right)$ 
Where, $\mathrm{C}_{\mathrm{R}}=$ Concentration of reactant, $\mathrm{C}_{\mathrm{H}}=$ Concentration of hydrogenated product and $\mathrm{C}_{\mathrm{I}}=$ Concentration of isomerized product $(10 \mathrm{mM}$ each $)$

For example, PdSG MPCs (in absence of $\mathrm{I}_{2}$ ) as a catalyst (slope values from Figure 4.15):

$\mathrm{TOF}=\{$ slope $(\%$ hydrogenated or isomerized $/ \mathrm{min}) / 100\} *\left[\left\{2.94 \times 10^{-3}\right.\right.$ moles $* 60$ $(\mathrm{min} / \mathrm{h})\} / 4.0 \times 10^{-5}$ moles $\left.\mathrm{Pd}\right]$

TOF Hydrogenation $=0.002690(\%$ hydrogenated $/ \mathrm{min}) *\left[\left\{2.94 \times 10^{-3}\right.\right.$ moles $* 60$ $(\mathrm{min} / \mathrm{h})\} / 4.0 \times 10^{-5}$ moles $\left.\mathrm{Pd}\right]$

TOF Hydrogenation = 12 moles hydrogenated $/ \mathrm{moles} \mathrm{Pd} / \mathrm{h}$

TOF Isomerization $=0.026867(\%$ isomerized $/ \mathrm{min}) *\left[\left\{2.94 \times 10^{-3} \mathrm{moles} * 60(\mathrm{~min} / \mathrm{h})\right\} /\right.$ $4.0 \times 10^{-5}$ moles Pd]

TOF Isomerization $=119$ moles isomerized $/ \mathrm{moles} \mathrm{Pd} / \mathrm{h}$

Overall Conversion TOF $=$ TOF Hydrogenation + TOF Isomerization

Total TOF $=12+119$

Total TOF $=131$ moles products $/$ moles $\mathrm{Pd} / \mathrm{h}$

The TOFs were determined from the slopes of the plots when $<60 \%$ allyl alcohol was converted to the isomerized and hydrogenated products. 
Table 5.2. Turnover frequency and catalyst recyclability for the PdSG MPCscatalyzed hydrogenation/isomerization of allyl alcohol with and without added $\mathrm{I}_{2}$. TOFs were determined from the slope of the points obtained at $<60 \%$ isomerization and slope for all points obtained for hydrogenation.

\begin{tabular}{|c|c|c|c|c|c|c|}
\hline & \multirow{2}{*}{$\begin{array}{c}\mathrm{No}_{2} \\
\text { added }\end{array}$} & \multicolumn{5}{|c|}{ Equivalents of added $I_{2}$} \\
\hline & & 0.025 & 0.05 & 0.1 & 0.2 & 0.3 \\
\hline Hydrogenation $\mathrm{TOF}^{a}$ & 12 & 17 & 22 & 26 & 28 & $\begin{array}{c}<5 \% \\
\text { converted }\end{array}$ \\
\hline Isomerization $\mathrm{TOF}$ & 119 & 144 & 172 & 197 & 202 & $\begin{array}{c}<5 \% \\
\text { converted }\end{array}$ \\
\hline Total TOF & 131 & 161 & 194 & 223 & 230 & - \\
\hline Hydrogenation $\%^{b}$ & 9 & 11 & 11 & 12 & 12 & - \\
\hline Isomerization $\%^{c}$ & 91 & 89 & 89 & 88 & 88 & - \\
\hline Recyclability $^{d}$ & 4 & 4 & 3 & 2 & 1 & - \\
\hline
\end{tabular}

${ }^{a} \mathrm{TOF}$ given in moles product/moles $\mathrm{Pd} / \mathrm{h}$ (combined aqueous and organic phase); ${ }^{b}$ percentage of 1-propanol formed; ${ }^{c}$ percentage of propanal formed; ${ }^{d}$ number of cycles after initial reaction that the isolated catalyst completely transformed allyl alcohol into products within 60 minutes.

\subsubsection{Recyclability of PdSG MPCs}

In that we used a biphasic system for the reaction, the PdSG MPCs in the aqueous phase were readily separated from the products in the organic phase for re-use in subsequent cycles. For each subsequent reaction, complete extraction of products from the PdSG MPCs/aqueous phase occurred before fresh allyl alcohol was added for the next reaction to determine the recyclability of the catalyst. After each consecutive cycle, a 
simple work up was performed again for removal of products via extraction. The UV-Vis and GC-FID measurements taken after the work-up showed there is indeed a complete removal of products (Figures 5.21 and 5.22). We observed that the catalyst could be recycled up to 4 times in the absence of $\mathrm{I}_{2}$ (Table 5.2, Figure 5.23). Of particular note is that the addition of 0.025 eq $\mathrm{I}_{2}$ maintained higher TOFs in each subsequent cycle relative to the reaction without added $\mathrm{I}_{2}$ without affecting the recyclability of the catalyst. However, addition of greater amounts of $\mathrm{I}_{2}$ degraded the recyclability of the catalyst, presumably a consequence of particle aggregation, growth, and possibly even dissolution caused by the decrease in ligand surface density. GC-FID chromatograms of 5 different cycles (recyclability $=4$ ) of allyl alcohol hydrogenation/isomerization in the absence of $I_{2}$ are shown in Figure 5.24.

Although the particles were stable during their first cycle (Figure 5.25 A), the UV-Vis spectra of activated PdSG MPCs show aggregation induced by $I_{2}$ addition (Figure $5.25 \mathrm{~B}$ ) in higher amounts $(0.2 \mathrm{eq})$. Aggregation of the MPCs treated with 0.2 eq $\mathrm{I}_{2}$ limited their use to only one catalytic cycle (Table 5.2). Likewise, reactions attempted with even higher amounts of added $\mathrm{I}_{2}$, such as 0.3 eq and 0.6 eq, did not result in product formation even after 2 hours of $\mathrm{H}_{2}$ flow, suggesting severe instability due to aggregation or dissolution of the PdSG MPCs (Figure 5.26). 


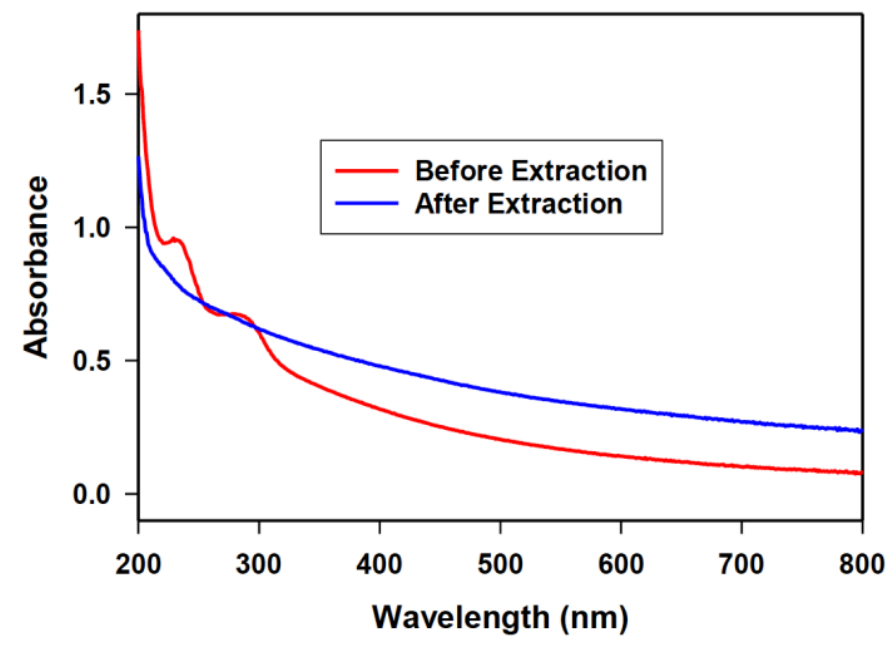

Figure 5.21. UV-Vis spectra of the aqueous phase separated from the biphasic reaction mixture before and after extraction of the products (propanal and 1-propanol) with $\mathrm{CH}_{2} \mathrm{Cl}_{2} .4 .0 \mathrm{~mL}$ of $\mathrm{CH}_{2} \mathrm{Cl}_{2}$ was used to extract the products, and the process was repeated 2 more times.

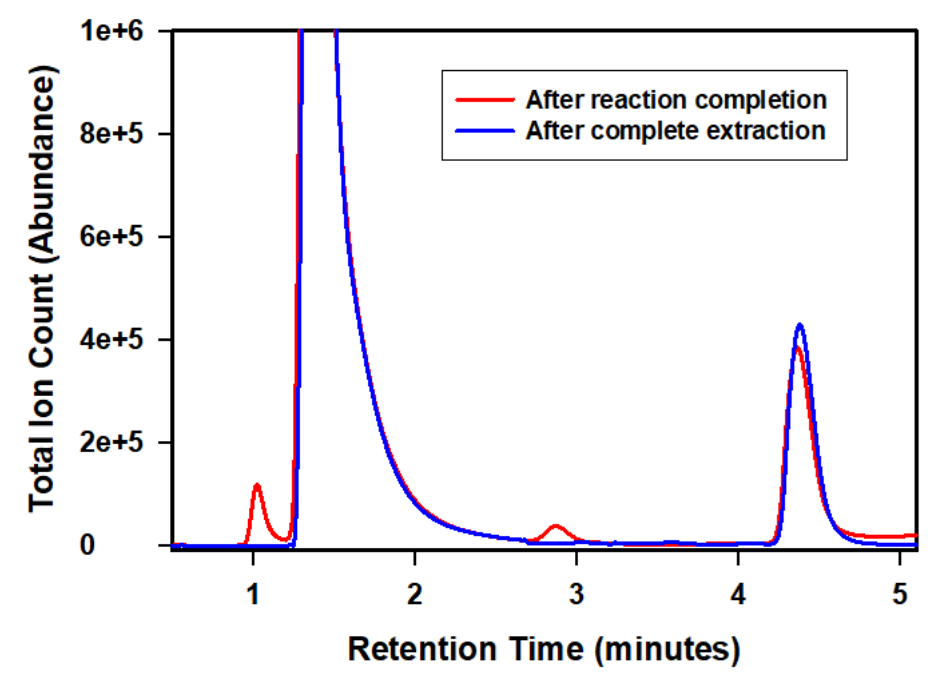

Figure 5.22. GC plots of the aqueous phase separated from the biphasic reaction mixture before and after extraction of the products (propanal and 1-propanol) with $\mathrm{CH}_{2} \mathrm{Cl}_{2} .4 .0$ $\mathrm{mL}$ of $\mathrm{CH}_{2} \mathrm{Cl}_{2}$ was used to extract the products, and the process was repeated 2 more times. 


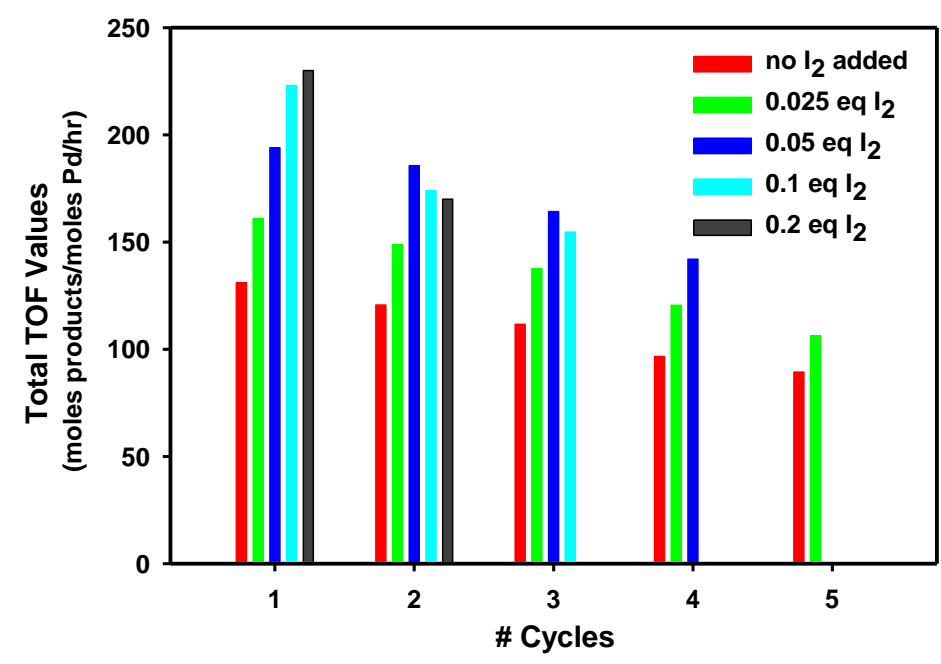

Figure 5.23. Plot of total TOF values vs. number of reaction cycles for hydrogenation/isomerization of allyl alcohol using catalytic PdSG MPCs with and without added $\mathrm{I}_{2}$. Successful cycle completion required that $100 \%$ of allyl alcohol be transformed into products within 60 minutes. 


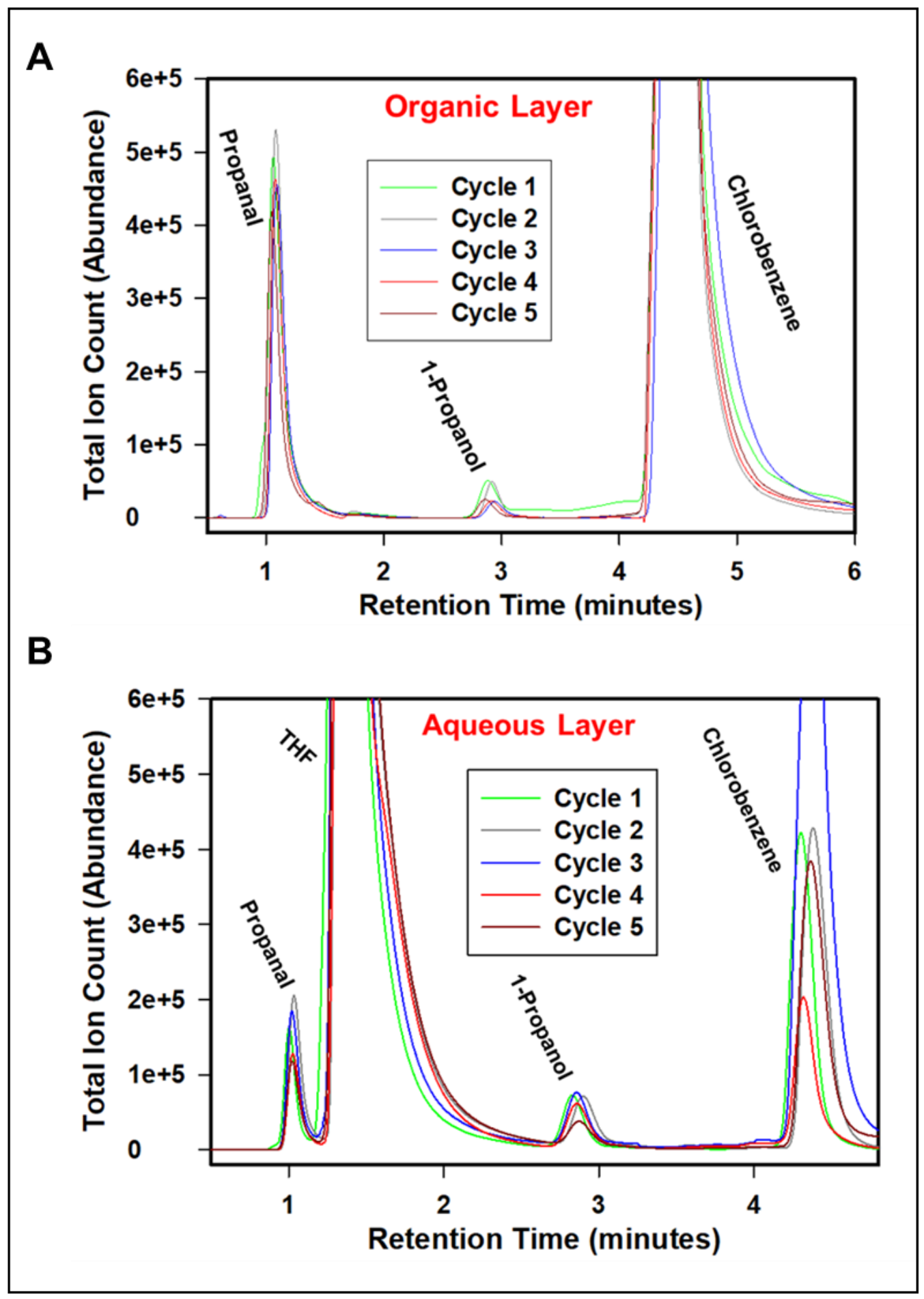

Figure 5.24. Gas chromatograms of cycle 1 to cycle 5 using PdSG MPCs as a catalyst for allyl alcohol hydrogenation/isomerization (no $\mathrm{I}_{2}$ added); A: organic layer, and B: aqueous layer. 

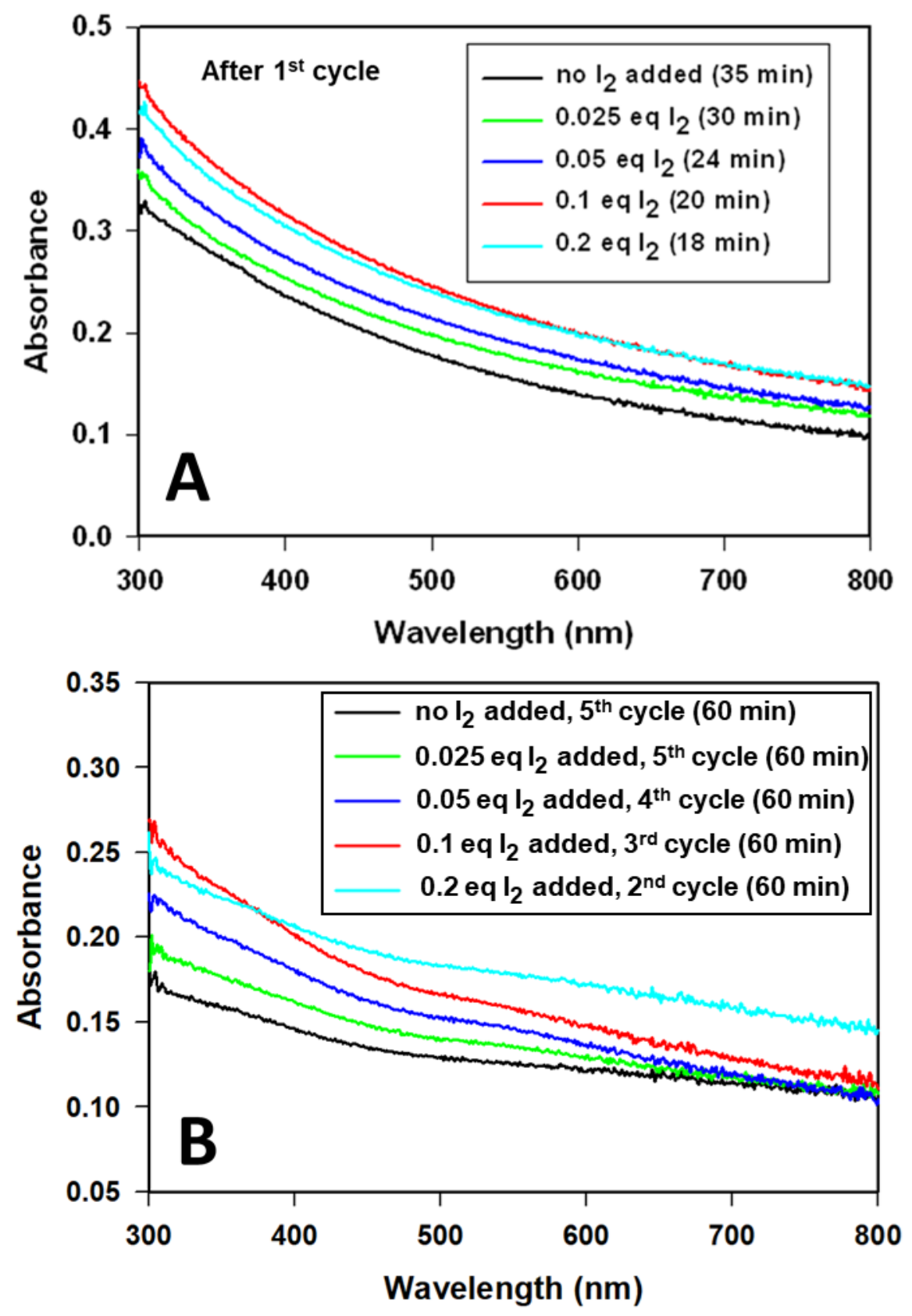

Figure 5.25. UV-Vis spectra showing the stability of PdSG MPCs after (A) completion of the first cycle and (B) completion of the last cycle (with no $\mathrm{I}_{2}$ and different equivalents of $\mathrm{I}_{2}$ addition) in the hydrogenation/isomerization of allyl alcohol (\# cycles shown in $\mathbf{B}$ ). 


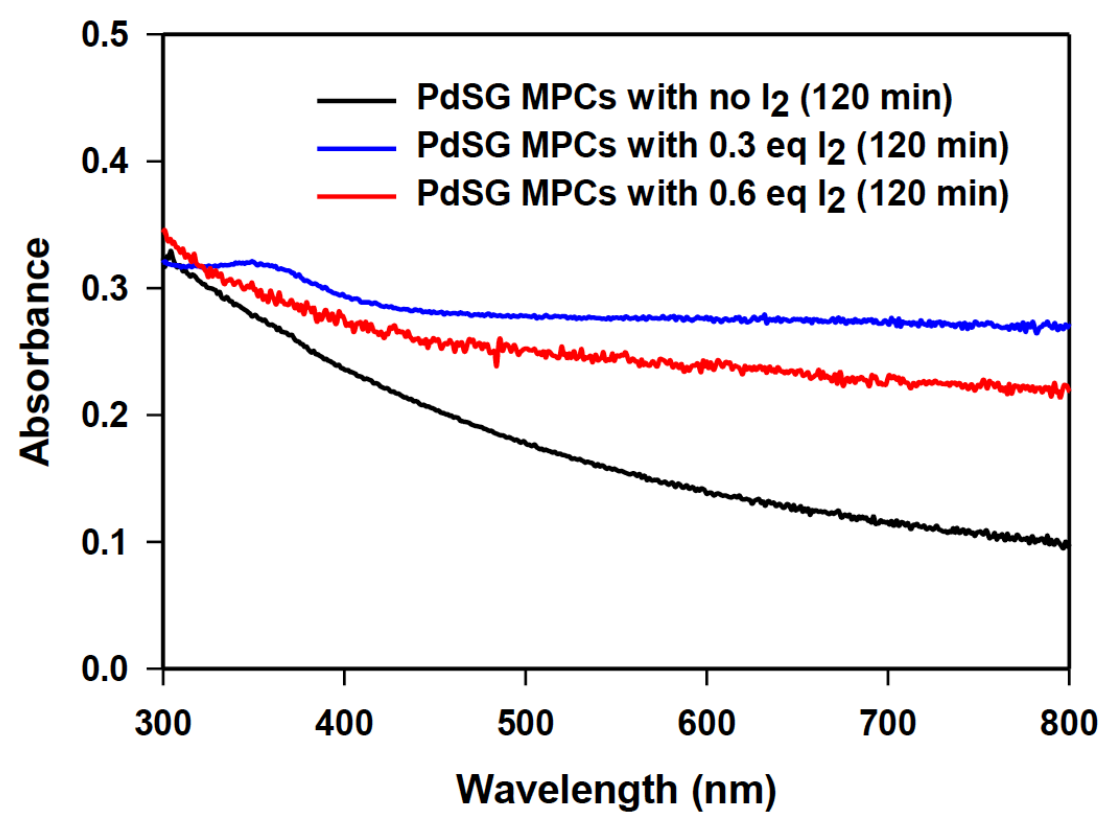

Figure 5.26. UV-Vis spectra of PdSG MPCs treated with 0.3 eq $\mathrm{I}_{2}$ and 0.6 eq $\mathrm{I}_{2}$. (compared with that of no $\mathrm{I}_{2}$ added). The high absorbance at higher wavelengths is a clear indication of aggregation.

\subsubsection{NMR Studies of $I_{2}$ Addition to PdSG MPCs}

The activity for hydrogenation/isomerization increases with increasing $I_{2}$ added, but the number of equivalents needed for activity and MPC stability was much lower than that observed for AuSG MPCs. This led to questions about whether the activation mechanism was the same for AuSG and PdSG MPCs. The smaller equivalents of $\mathrm{I}_{2}$ needed for enhanced catalytic activity of PdSG compared to AuSG MPCs may be related to the iodide-catalyzed oxidation of Pd, as reported by Soriaga and co-workers. ${ }^{183}$ Also, studies conducted by Biffis et al. suggested that $\mathrm{Pd}^{\mathrm{II}}$ species leach into solution during a cross-coupling reaction, where they catalyze the reaction and may form the cluster again. ${ }^{184}$ Shon and co-workers examined the leaching of $\mathrm{Pd}^{\mathrm{II}}$ from octanethiolate $\mathrm{Pd}$ 


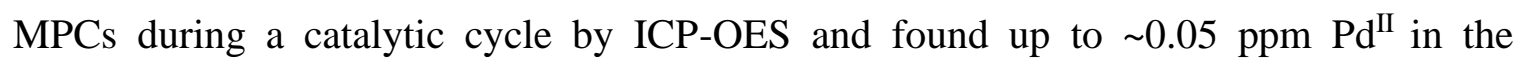
solution. ${ }^{185}$ We conducted NMR studies on the addition of 0.05 to 0.6 eq of $\mathrm{I}_{2}$ to PdSG MPCs in $\mathrm{D}_{2} \mathrm{O}$ using a $t$-butanol internal standard as described for AuSG MPCs (Figure 5.27). Surprisingly, while we found that some signals in the NMR spectra sharpened with increasing amounts of $\mathrm{I}_{2}$, indicating removal of SG ligands from the MPCs, we did not observe the characteristic GS-SG signals at $82.93-2.99,3.27-3.32$, and 4.72-4.76 ppm corresponding to disulfide formation. This suggests that the SG ligands dissociate from the PdSG MPCs in another form, possibly as Pd(II)-SG complexes (oligomers, clusters, or polymers), which might be expected to produce poorly resolved signals. In fact, NMR spectra of $\mathrm{Pd}^{\mathrm{II}} \mathrm{Cl}_{4}{ }^{2-}$ intentionally added to GSH and GSH plus $\mathrm{I}_{2}$ also did not show wellresolved peaks that are characteristic of GS-SG formation (Figure 5.28). This is also consistent with previous observations of Pd(II) leaching and the strong ability of iodide to catalyze Pd oxidation compared to $\mathrm{Au}$. With small amounts of added $\mathrm{I}_{2}$, we believe that small amounts of Pd(II)-SG species dissociate from the PdSG MPCs to expose bare Pd sites for enhanced catalytic activity. While this mode of catalyst activation is not conclusive, it is clear that SG ligands somehow dissociate from the PdSG MPCs in a manner that does not lead to detectable GS-SG or GSH. Without disulfide formation, it was difficult to quantify ligand loss by NMR. Using TGA, however, we measured the \% organic composition after treatment with various amounts of $I_{2}$, which revealed that the organic weight percent decreased by 5 to $38 \%$ for 0.025 to 0.2 eq of $\mathrm{I}_{2}$ added, respectively (Figure 5.29). This confirms that ligand loss did occur. 


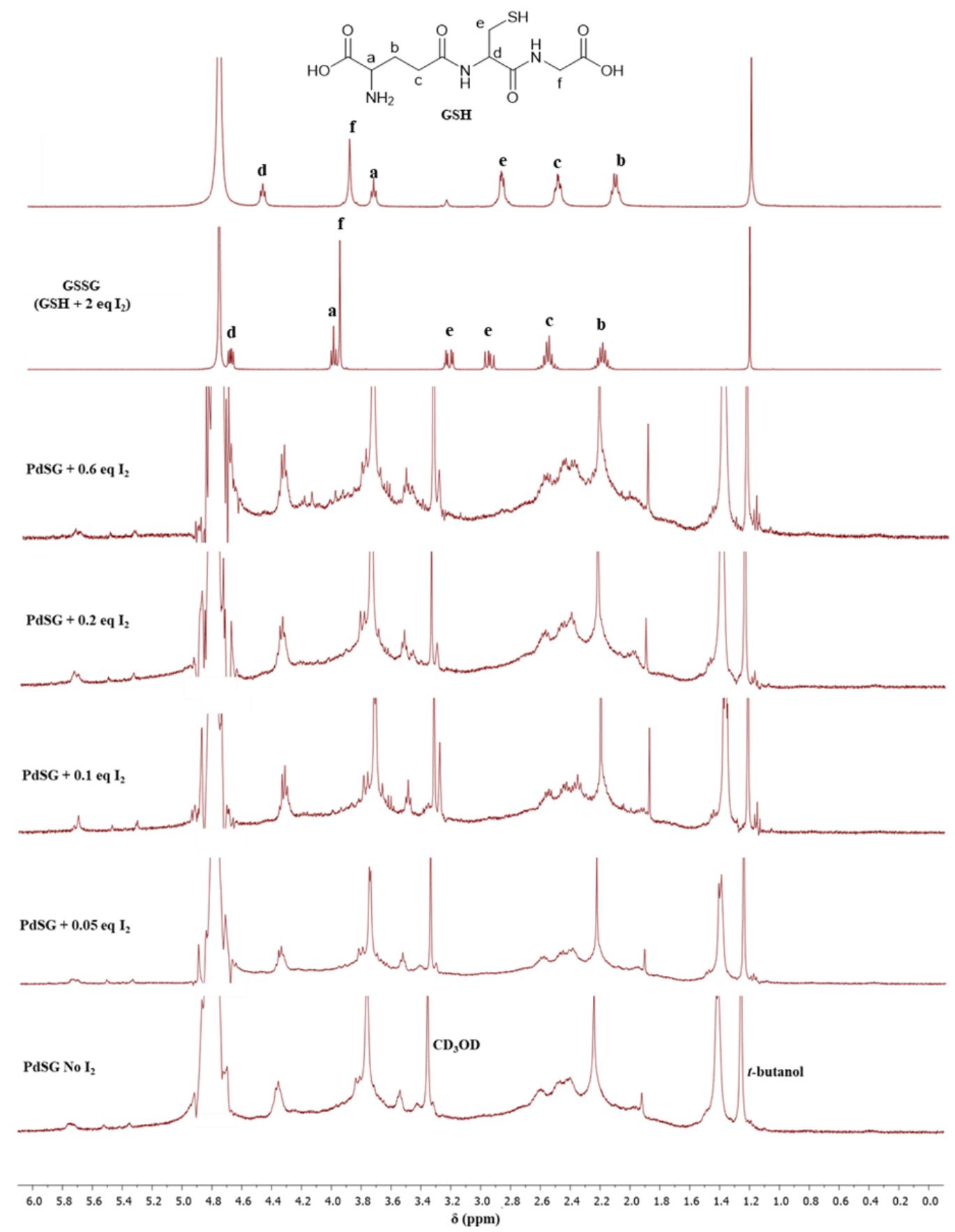

Figure 5.27. ${ }^{1} \mathrm{H}$ NMR $\left(400 \mathrm{MHz}, \mathrm{D}_{2} \mathrm{O}\right)$ spectra of particle stability studies of PdSG MPCs upon $\mathrm{I}_{2}$ activation. Each spectrum was obtained on a separate sample after 40 minutes of reaction with $I_{2}$ except for 0.1 equivalents $I_{2}$, which was obtained on the sample with 0.05 equivalents $I_{2}$ after adding a second 0.05 equivalents $(0.1$ total $)$ and obtaining the spectrum after another 40 minutes. 


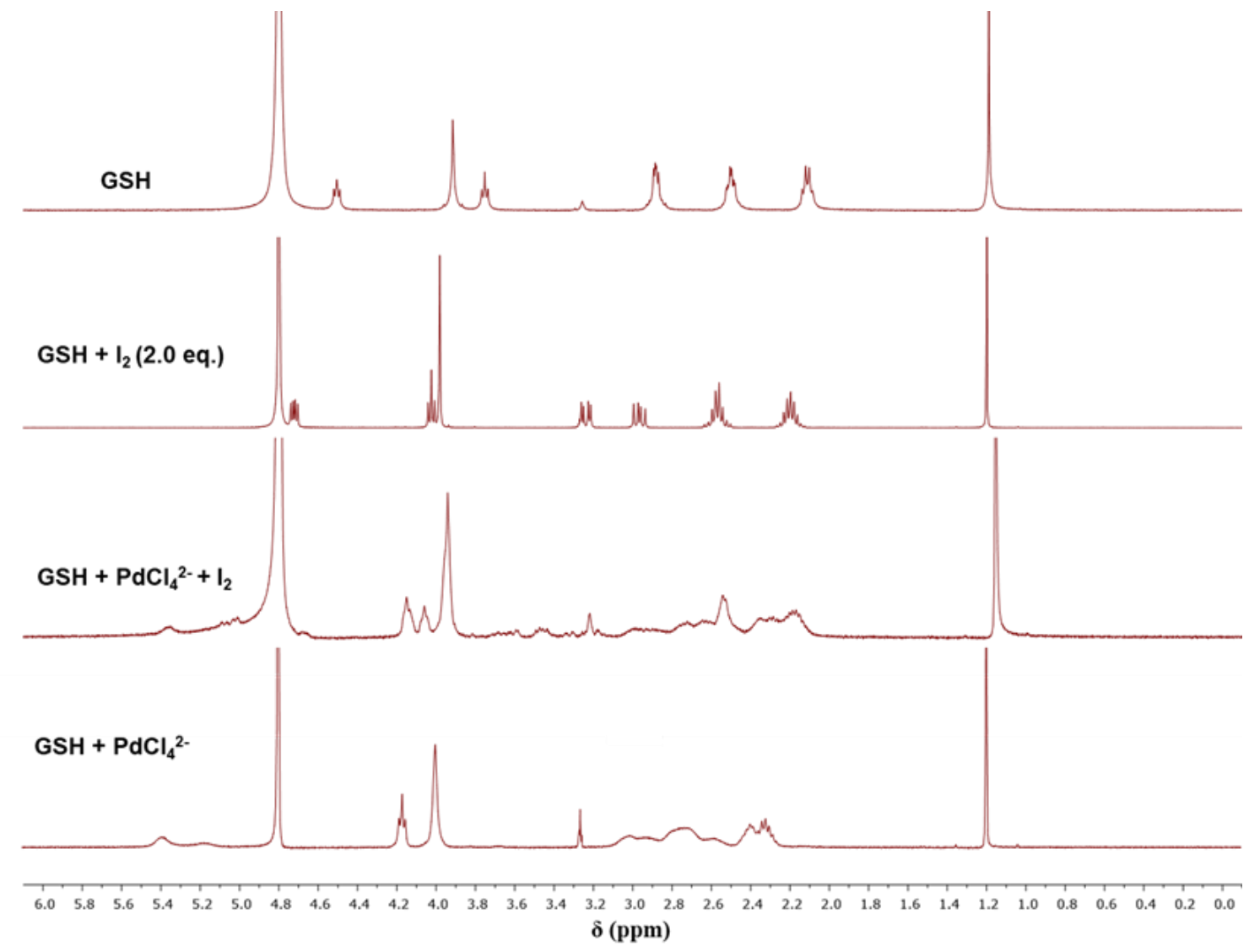

Figure 5.28. ${ }^{1} \mathrm{H}$ NMR spectra of GSH only and GSH treated with 2.0 equivalents $\mathrm{I}_{2}, 1.0$ equivalent of $\mathrm{K}_{2} \mathrm{PdCl}_{4}$, and both $\mathrm{I}_{2}$ and $\mathrm{K}_{2} \mathrm{PdCl}_{4}$ after 40 min. 


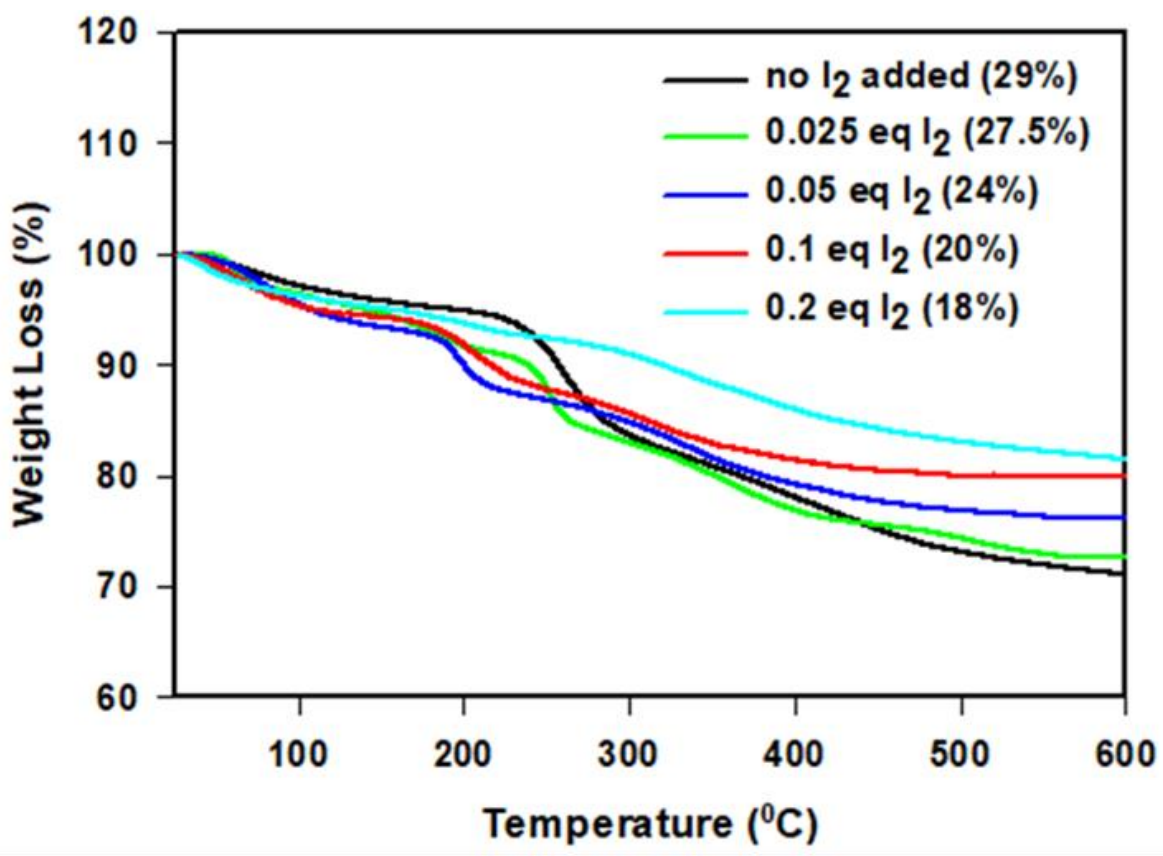

Figure 5.29. TGA of PdSG MPCs obtained after reaction with varying equivalents of $I_{2}$ addition for $60 \mathrm{~min}$. There is less organic weight percent as the amount of $\mathrm{I}_{2}$ increased. The estimated ligand loss is $5,17,31$, and $38 \%$ for the 0.025 eq, 0.05 eq, 0.1 eq, and 0.2 eq, respectively.

\subsubsection{Activation of AuSG and PdSG MPCs}

Our data is consistent with two different $\mathrm{I}_{2}$ activation mechanisms for AuSG and PdSG MPCs. With Au, the NMR data very clearly show ligand dissociation by disulfide formation as shown in Scheme 5.3. Small amounts of $I_{2}$ decrease the ligand density while the Au MPCs remain stable. Larger amounts of $\mathrm{I}_{2}$ lead to destabilization of the Au MPCs through aggregation and even complete dissolution of Au with the largest amounts of $\mathrm{I}_{2}$ studied ( $\left.2.0 \mathrm{eq}\right)$ over longer times. Optimal ligand removal for improved catalytic rates and good recyclability is in the $50-80 \%$ removal range. For PdSG MPCs, the $\mathrm{I}_{2}$ also removes SG ligands, but in a very different way not consistent with Scheme 5.3. First, ligand removal ranged from 5 to $38 \%$ for much smaller amounts of added $\mathrm{I}_{2}$. Second, the 
ligands are not removed as disulfides. While not conclusive, the data suggests that $\mathrm{Pd}$ oxidation occurs and the ligands are removed as $\mathrm{Pd}(\mathrm{II})$-thiolate species. There may also be $\mathrm{Pd}(\mathrm{II})$ iodide complexes formed. Pd oxidation is likely an issue since adding 0.3 and $0.6 \mathrm{I}_{2} \mathrm{eq}$, which work fine for $\mathrm{Au}$, does not result in any catalytic activity for Pd. This shows major changes in the Pd MPCs likely associated with Pd aggregation, oxidation, and dissolution.

An interesting question in both systems concerns the fate of the $I_{2} / I^{-}$species. $I_{2}$ becomes reduced to $2 \mathrm{I}^{-}$during disulfide formation or metal oxidation. The XPS data obtained on AuSG and PdSG MPCs after treatment with various eq $\mathrm{I}_{2}$ under catalytic reaction conditions show that $\mathrm{I}^{-}$adsorbs to the $\mathrm{Au}$ and $\mathrm{Pd}$ surfaces (Figure 5.30 A and 5.30 B, respectively). The signal was weak in some cases and we were not able to quantify it reliably, making the extent of $\mathrm{I}^{-}$coverage difficult to confirm. We can confidently say that a measurable amount of $\mathrm{I}^{-}$adsorbs to both $\mathrm{Au}$ and $\mathrm{Pd}$, however. While $\mathrm{I}^{-}$may still inhibit active sites on the Au and Pd surfaces, it is apparently not as poisoning as the original glutathione ligands, so increased reactivity still occurs. It is also likely that some $\mathrm{I}^{-}$dissolves into solution or forms $\mathrm{Au}(\mathrm{I}) / \mathrm{Au}(\mathrm{III})$ iodide or $\mathrm{Pd}(\mathrm{II})$ iodide ionic complexes, based on the evidence of metal dissolution. Despite some evidence of metal dissolution, the metallic ions are not the active catalysts, since deactivation is associated with larger amounts of $\mathrm{I}_{2}$. XPS spectra of the Au region shows primarily $\mathrm{Au}(0)$ while the Pd shows a mixture of Pd and Pd(II) species (Figure 5.31). More work will be required in the future to better understand the full details of the MPC activation mechanism and catalyst activation site. 

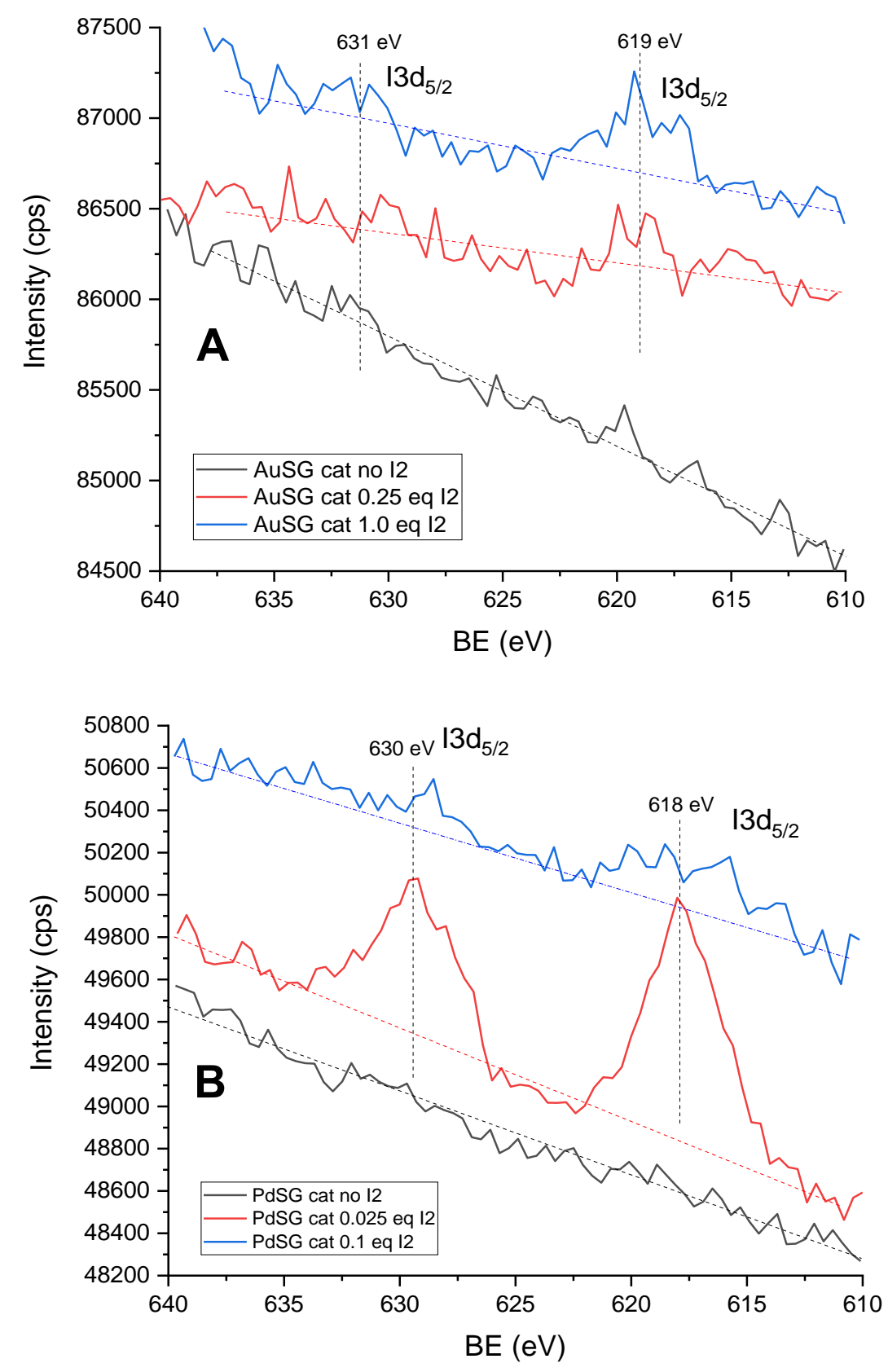

Figure 5.30. XPS survey spectra of the iodide region (BE 610-640 eV) of a sample of (A) AuSG MPCs and (B) PdSG MPCs after one reaction cycle with no $I_{2}$ and various $I_{2}$ additions as indicated. 

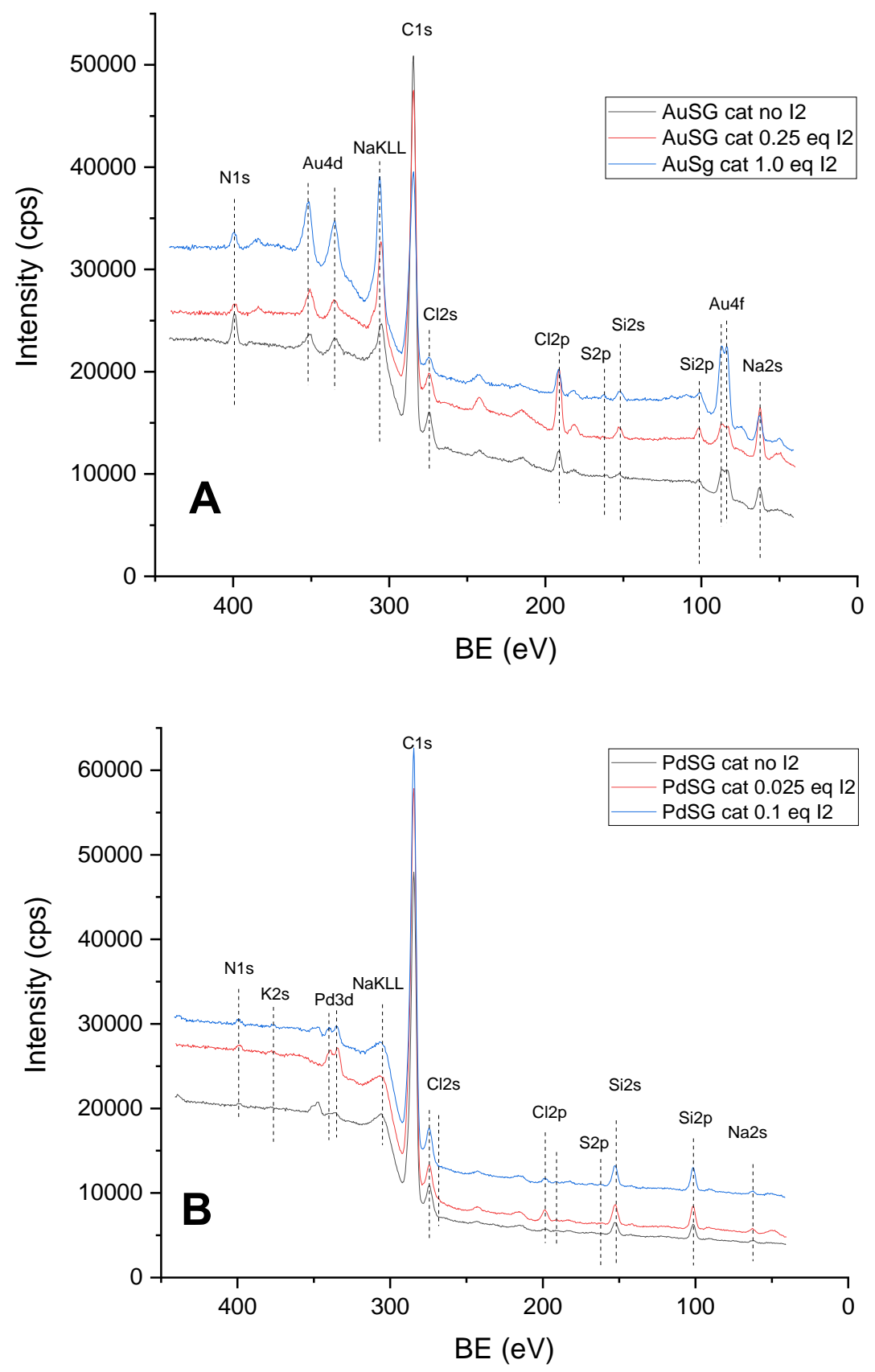

Figure 5.31. XPS survey spectra from 50-450 eV BE of a sample of (A) AuSG MPCs and (B) PdSG MPCs after one reaction cycle with no $I_{2}$ and various $I_{2}$ additions as indicated. 


\subsubsection{Heterogeneous Catalytic Reactions}

We described the synthesis of heterogenous MPC catalyst using two types of beads. The syntheses of Au cluster-functionalized PS beads and Pd cluster-functionalized agarose beads are explained in Chapter 4 (experimental details in sections 6.3 .7 and 6.3.8). In our first heterogenous catalytic reaction, we attempted to use Au clusterfunctionalized PS beads as a heterogenous catalyst to test the 4-NP reduction reaction following the previous conditions studied under the homogenous system. Unfortunately, this reaction did not work. We attributed this to either passivation of the Au MPCs by the thiol ligands or the high degree of PS bead cross-linking, which made the beads nonsuspendable. We exploited the same reaction under the same conditions with $\mathrm{I}_{2}$ activation (different equivalents), but the reaction still did not proceed. This suggests that high bead cross-linking is the main reason for the inactivity. This presumably leads to low surface area and inaccessibility of the Au surface for catalysis, even after ligand removal by $\mathrm{I}_{2}$.

Allyl alcohol hydrogenation/isomerization, a model reaction already studied using homogenous catalysis, was tested using the Pd-cluster functionalized agarose bead as a catalyst under heterogenous condition (Figure 5.32). The ratio of 1:10 of AOT:CHO was used during the functionalization of this catalyst. First, a monophasic condition was used to test the catalytic activity of the catalyst, which unfortunately did not yield the expected products; propanal and 1-propanol (Figure 5.33). Only a small amount of conversion was achieved after 2 hours of $\mathrm{H}_{2}$ flow ( $\left.\sim 20 \mathrm{~mL} / \mathrm{min}\right)$. Therefore, we turned our attention to the biphasic system that was studied previously. ${ }^{53}$ 


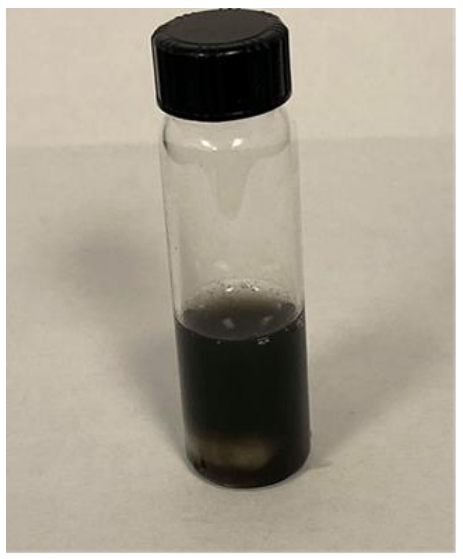

Catalyst suspension

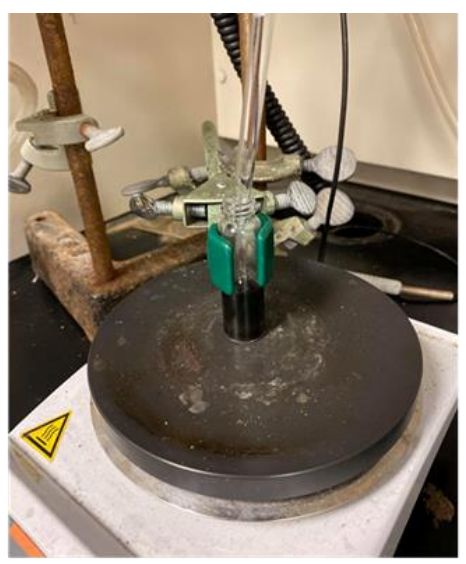

Reaction set-up

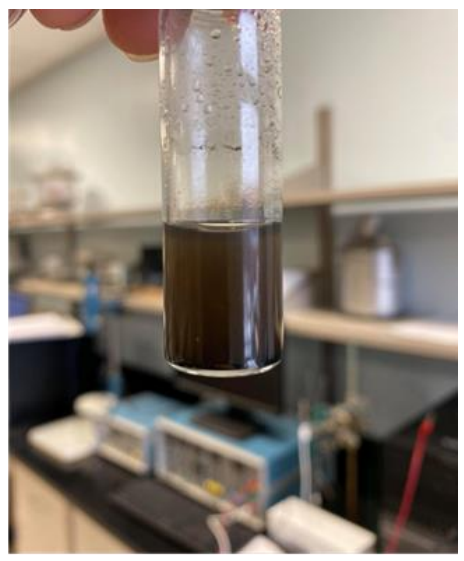

Catalyst settled over time

Figure 5.32. Pd-cluster functionalized agarose bead as a heterogenous catalyst for allyl alcohol hydrogenation/isomerization reaction (biphasic condition: $\mathrm{H}_{2} \mathrm{O} / \mathrm{EtOAc}$ ).

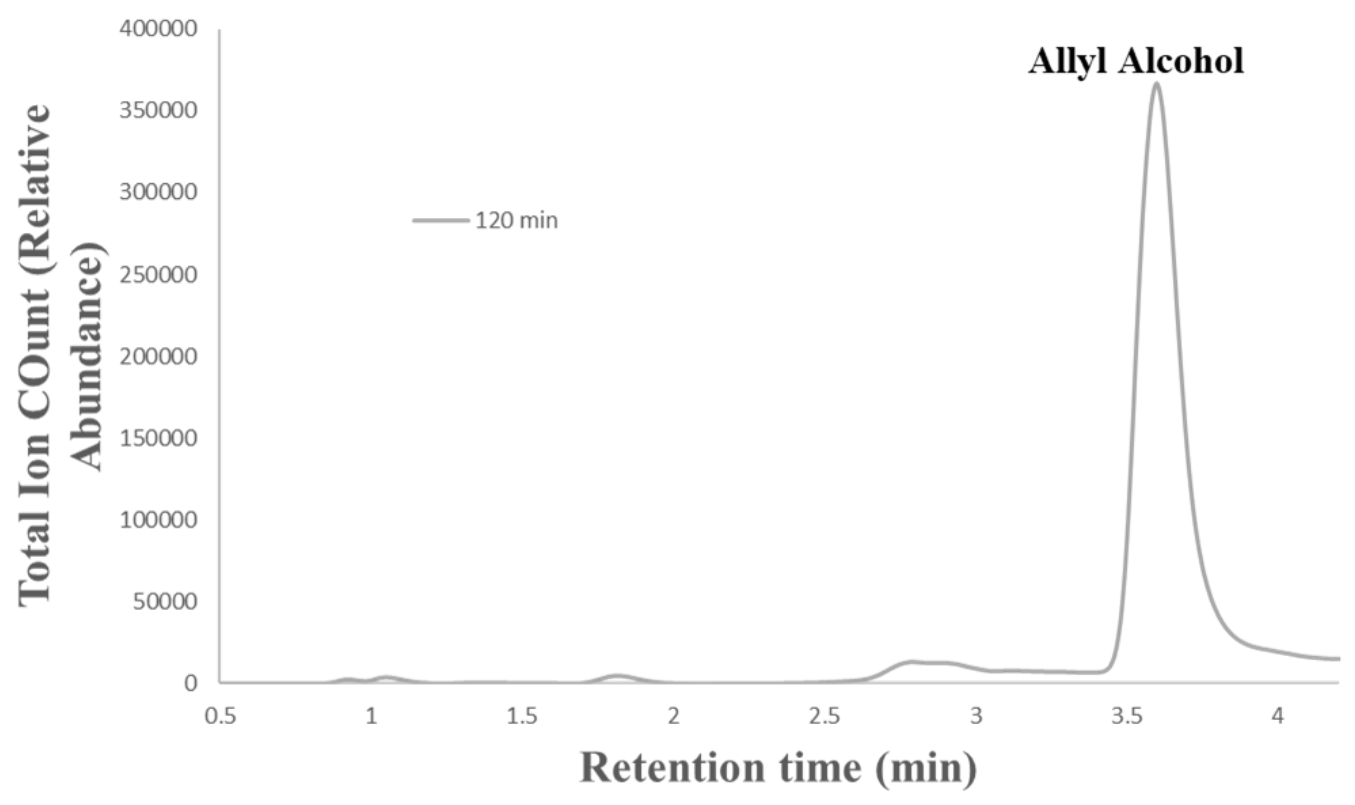

Figure 5.33. GC-FID chromatogram of allyl alcohol hydrogenation/isomerization reaction (Pd functionalized agarose bead catalyst) under monophasic condition $\left(\mathrm{H}_{2} \mathrm{O}\right)$. 
In a biphasic system, we chose to use ethyl acetate (EtOAc) and water. Indeed, the catalytic performance was improved, as GC-FID analysis showed $\sim 25 \%$ products formation after 1 hour with a selectivity of 60:40 hydrogenated:isomerized products in the organic layer (Figure 5.34). The reason for reactivity in the biphasic mixture is not well-understood at this time. More optimization of the catalyst preparation and reaction conditions is needed for successful catalysis.

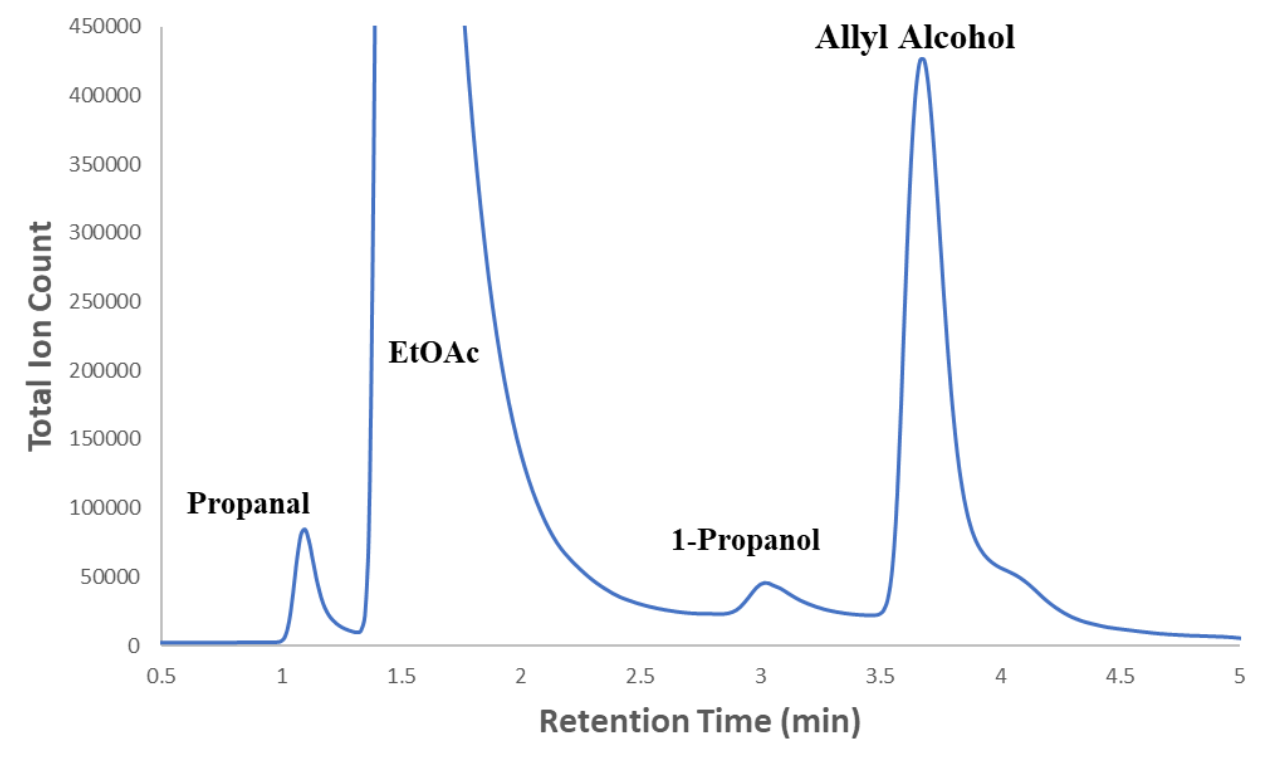

Figure 5.34. GC-FID chromatogram of allyl alcohol hydrogenation/isomerization reaction (Pd- cluster functionalized agarose bead catalyst) under biphasic condition (EtOAc and $\mathrm{H}_{2} \mathrm{O}$ ).

We observed catalytic enhancement on $\mathrm{I}_{2}$ activated PdSG MPCs for this reaction under homogenous conditions. Therefore, we attempted $I_{2}$ activation in heterogenous catalysis, which unfortunately did not yield catalytic efficiency. In the future, further optimization and investigation is required to improve the TOF and recyclability of such catalyst 


\subsection{Conclusions}

Using two organic reactions as representative models, our results demonstrate that the catalytic activity of thiolate-protected metal nanoclusters can be increased by the addition of low levels of iodine. The improvement in catalytic activity likely is the result of decreased ligand surface density that occurs on $I_{2}$ addition. The method is straightforward relative to other ligand removal strategies used to expose metal cores and enhance metal-substrate interactions. The analytical techniques that we employed $\left({ }^{1} \mathrm{H}\right.$ NMR, TGA, UV-Vis, XPS, and SEM) to characterize AuSG and PdSG MPCs before and after addition of $I_{2}$ collectively indicate that the $I_{2}$ functions to promote ligand dissociation through disulfide formation in the case of $\mathrm{Au}$ and through $\mathrm{Pd}(\mathrm{II})$-thiolate formation in the case of $\mathrm{Pd} .{ }^{99}$ Both the catalyzed reduction of 4-nitrophenol by AuSG MPCs and hydrogenation/isomerization of allyl alcohol by PdSG MPCs were accelerated by addition of $\mathrm{I}_{2}$ (7-fold and 2-fold rate enhancement for $\mathrm{Au}$ and $\mathrm{Pd}$, respectively). A judicious choice in the amount of added $\mathrm{I}_{2}$, however, is required to prevent particle (catalyst) instability, an important consideration for catalyst recyclability. In both cases examined, a threshold was observed for $\mathrm{I}_{2}$-induced removal of too many surface thiolates resulting in catalyst deactivation due to aggregation, precipitation, and even dissolution. Thiolate-protected metal cluster immobilization on solid supports may circumvent problems associated with particle aggregation, yet still allow catalytic enhancements afforded by exposure to $\mathrm{I}_{2}{ }^{87}$ The present strategy also may find utility in amplifying responses of thiolate monolayer-protected metal-based chemiresistors, where electron transport properties are ligand-dependent. The results from homogeneous metal MPC catalysis is promising based on the results presented herein. 
CHAPTER 6

EXPERIMENTAL PROCEDURES

\subsection{Experimental Procedures for Chapter 2}

\subsubsection{Materials and Methods}

All chemicals were purchased either from Sigma-Aldrich (Saint Louis, MO), Bean Town Chemical (Hudson, NH) or Alfa Aesar (Tewksbury, MA) unless otherwise noted. All solvents were freshly distilled before use. $\mathrm{HAuCl}_{4} \cdot 3 \mathrm{H}_{2} \mathrm{O}$ was synthesized from metallic bulk gold according to the literature procedure. ${ }^{186}$ Thin layer chromatography was performed using silica plates (silica gel 60G F254). UV active compounds were visualized by UV light (254 nm). The TLC plates were stained using either iodine or $p$ anisaldehyde. Water was purified using a Barnstead water ultra-purification system (ThermoFisher, resistivity of $18.2 \mathrm{M} \Omega \bullet \mathrm{cm}$ ) and used whenever required.

\subsubsection{Spectroscopic Measurements}

${ }^{1} \mathrm{H}$ and ${ }^{13} \mathrm{C}$ NMR spectra were recorded either on a Varian $400 \mathrm{MHz}$ or Varian Inova 500 MHz spectrometer. Infrared spectra were recorded on a Perkin-Elmer Spectrum FT-IR spectrophotometer with an attenuated total reflectance attachment. UV-Vis spectra were recorded on a Varian instrument model CARY 50 Bio UV-Visible spectrophotometer. All scans were obtained between $300 \mathrm{~nm}$ to $900 \mathrm{~nm}$ wavelength range at a fast scan rate in a glass cuvette and the background was subtracted using $\mathrm{CHCl}_{3}$ or nanopure $\mathrm{H}_{2} \mathrm{O}$ as the blank. High resolution mass spectrometry (HRMS) of new compounds was performed using a Thermo Scientific Q Exactive Focus Orbitrap LC-MS/MS system. 


\subsubsection{Thermogravimetric Analysis (TGA)}

Material compositions of the synthesized clusters were determined by measuring the weight changes using a thermogravimetric analyzer (TA Instruments, TA 2050) under a nitrogen atmosphere from 25 to $800{ }^{\circ} \mathrm{C}$ with a heating rate of $5{ }^{\circ} \mathrm{C} \mathrm{min}{ }^{-1}$. This provides an approximate cluster molecular formula when combined with the known cluster diameter.

\subsubsection{Synthesis Procedures for Aminooxy-Thiol Ligands (AOT $b$ bis)}

6.1.4.1. 2-[(2-Hydroxyethyl)- $N$-10-undecenylamino] ethanol (2)

A solution of diethanolamine $(\mathbf{1})(0.5 \mathrm{~mL}, 5.20 \mathrm{mmol})$ and 11-bromo-1-undecene $(1.25$

$\mathrm{mL}, 5.72 \mathrm{mmol})$ in a $30: 70 \mathrm{mixture}$ of methanol:acetonitrile $(20 \mathrm{~mL})$ was heated $48 \mathrm{~h}$ at reflux. The reaction mixture was cooled to $\mathrm{rt}$ and the solvents were removed by rotary evaporation. The residue was triturated with diethyl ether $(3 \times 20 \mathrm{~mL})$ and the solids dried under high vacuum to obtain the intermediate ammonium bromide $(1.02 \mathrm{~g}, 3.00$ $\mathrm{mmol})$ as a yellow viscous oil, which then was dissolved in water (20 $\mathrm{mL})$ and followed by addition of $2 \mathrm{~N}$ ammonium hydroxide solution $(20 \mathrm{~mL})$. The solution was transferred to a separatory funnel and was extracted with $\mathrm{Et}_{2} \mathrm{O}(3 \times 20 \mathrm{~mL})$. The combined ether extract was concentrated by rotary evaporation and the residue placed under high vacuum overnight to afford diol 2 (1.18 g, $88 \%$ ) as a waxy solid; IR (neat) 3351, 2924, 2853, 1640, $1464 \mathrm{~cm}^{-1} ;{ }^{1} \mathrm{H}$ NMR (400 MHz, $\left.\mathrm{CDCl}_{3}\right) \delta$ 5.75-5.83 (m, 1H), 4.90-5.00 (m, 2H), $2.49-2.53(\mathrm{t}, J=12.0 \mathrm{~Hz}, 2 \mathrm{H}), 2.63-2.66(\mathrm{t}, J=4.0 \mathrm{~Hz}, 4 \mathrm{H}), 3.59-3.62(\mathrm{t}, J=8.0 \mathrm{~Hz}$, 4H), 2.02-2.05 (m, 2H), 1.26-1.43( m, 14H) ppm; ${ }^{13} \mathrm{C} \mathrm{NMR}\left(100 \mathrm{MHz}, \mathrm{CDCl}_{3},\right) \delta 139.1$, 
$114.0,59.5,56.0,54.8,33.7,29.5,29.3,29.0,28.8,27.3,26.9 \mathrm{ppm} ; \mathrm{HRMS} m / z[\mathrm{M}+\mathrm{H}]^{+}$ calcd for $\mathrm{C}_{15} \mathrm{H}_{32} \mathrm{NO}_{2}{ }^{+} 258.2428$, found 258.2419 .

6.1.4.2. $2-(2-\{[2-(3-O x o-2-$ isoindolinoyloxy)ethyl]- $N$-10-undecenylamino $\}$ ethoxy)-1,3isoindolin-dione (3).

To a solution of $N$-hydroxyphthalimide $(0.456 \mathrm{~g}, 2.80 \mathrm{mmol})$ and triphenylphosphine $(0.734 \mathrm{~g}, 2.80 \mathrm{mmol})$ in toluene $(40 \mathrm{~mL})$ at $0{ }^{\circ} \mathrm{C}$ was added dropwise a solution of diol 2 $(0.30 \mathrm{~g}, 1.17 \mathrm{mmol})$ dissolved in toluene $(5 \mathrm{~mL})$. After stirring 1 hour at $0{ }^{\circ} \mathrm{C}$, diisopropyl azodicarboxylate $(0.551 \mathrm{~mL}, 2.8 \mathrm{mmol})$ was added slowly via syringe. The reaction mixture was stirred an additional 1 hour at $0{ }^{\circ} \mathrm{C}$ and followed by stirring $16 \mathrm{~h}$ at rt. The solvent then was removed by rotary evaporation and the residue was dissolved in ethyl acetate $(20 \mathrm{~mL})$ and the resultant solution was washed with saturated aq. $\mathrm{NaHCO}_{3}$ $(5 \times 20 \mathrm{~mL})$, water $(20 \mathrm{~mL})$ and brine $(2 \times 20 \mathrm{~mL})$. The organic layer was concentrated, and the crude material was then cooled to $0{ }^{\circ} \mathrm{C}$. Cold $5 \%$ aqueous $\mathrm{HCl}(5 \mathrm{~mL})$ was added followed by stirring the mixture 45 minutes at rt. The aqueous slurry was extracted with diethyl ether $(5 \times 20 \mathrm{~mL})$ and then cooled to $0{ }^{\circ} \mathrm{C}$ before adjusting the $\mathrm{pH}$ to $7-8$ by slow addition of saturated aq. $\mathrm{NaHCO}_{3}$. The alkaline aqueous layer was extracted with $\mathrm{CH}_{2} \mathrm{Cl}_{2}\left(3 \times 20 \mathrm{~mL}\right.$ ). The combined organic extract was dried (anhydrous $\mathrm{Na}_{2} \mathrm{SO}_{4}$ ), filtered, and concentrated by rotary evaporation to afford $3(0.60 \mathrm{~g}, 95 \%)$ as a light yellow solid that was used in the next step without further purification; mp $86-88^{\circ} \mathrm{C}$; IR (solid) 2921, 2850, 1789, 1733, 1466, $1378 \mathrm{~cm}^{-1} ;{ }^{1} \mathrm{H}$ NMR (400 MHz, $\left.\mathrm{CDCl}_{3}\right) \delta 7.73-$ $7.80(\mathrm{~m}, 8 \mathrm{H}), 5.77-5.85(\mathrm{~m}, 1 \mathrm{H}), 4.91-5.00(\mathrm{~m}, 2 \mathrm{H}), 4.31-4.33(\mathrm{t}, J=4.0 \mathrm{~Hz}, 4 \mathrm{H}), 3.06-$ $3.08(\mathrm{t}, J=4.0 \mathrm{~Hz}, 4 \mathrm{H}), 2.63-2.67(\mathrm{t}, J=8.0 \mathrm{~Hz}, 2 \mathrm{H}), 2.02-2.05(\mathrm{t}, J=6.0 \mathrm{~Hz}, 2 \mathrm{H}), 1.62$ (m, 2H) 1.25-1.42 (m, 12H) ppm; ${ }^{13} \mathrm{C}$ NMR $\left(100 \mathrm{MHz}, \mathrm{CDCl}_{3}\right) \delta 163.2,139.1,134.3$, 
$128.9,123.4,113.9,76.6,54.8,52.0,33.7,29.6,29.3,29.0,28.8,27.1$ ppm; HRMS m/z $[\mathrm{M}+\mathrm{H}]^{+}$calcd for $\mathrm{C}_{31} \mathrm{H}_{38} \mathrm{~N}_{3} \mathrm{O}_{6}{ }^{+}$548.2755, found 548.2740.

6.1.4.3. $\quad S$-(11-(Bis(2-((1,3-dioxoisoindolin-2-yl)oxy)ethyl)amino)undecyl)ethanethioate (4).

To a solution of bis(phthaloyloxy)amine $\mathbf{3}(0.465 \mathrm{~g}, 0.849 \mathrm{mmol})$ in THF $(10 \mathrm{~mL})$ at $0{ }^{\circ} \mathrm{C}$ was added camphorsulfonic acid $(0.296 \mathrm{~g}, 1.27 \mathrm{mmol})$ to form the corresponding ammonium salt and stirred at $0{ }^{\circ} \mathrm{C}$ for $2 \mathrm{~h}$ before allowing it to warm to $\mathrm{rt}$ for 30 minutes while degassing. The reaction mixture was heated at $40{ }^{\circ} \mathrm{C}$ for 15 minutes before slow addition of a solution of AIBN (182 $\mathrm{mg}, 0.594 \mathrm{mmol})$ dissolved in dry THF (2 mL) via syringe and followed by addition of thioacetic acid $(131 \mu \mathrm{L}, 1.86 \mathrm{mmol})$ via syringe. The resultant mixture was degassed for 20 min using a stream of argon before heating the solution at reflux for $20 \mathrm{~h}$. The reaction mixture was then cooled to rt, quenched with diethyl ether $(10 \mathrm{~mL})$ and concentrated by rotary evaporation to afford the crude product, which was dissolved in $\mathrm{CHCl}_{3}(20 \mathrm{~mL})$. The $\mathrm{CHCl}_{3}$ solution was washed three times with saturated aq. $\mathrm{NaHCO}_{3}(20 \mathrm{~mL})$, followed by washing with brine $(20 \mathrm{~mL})$, and the organic layer was dried with anhydrous sodium sulfate. The solvents were removed by rotary evaporation to afford a light-yellow solid residue that then was triturated with hexane $(3 \times 20 \mathrm{~mL})$ to give 4 as a pale yellow solid $(0.41 \mathrm{~g}, 78 \%)$; mp $96-98{ }^{\circ} \mathrm{C}$; IR (solid) 2919, 2849, 2795, 1788, 1736, 1690, $\left.1466 \mathrm{~cm}^{-1} ;{ }^{1} \mathrm{H} \mathrm{NMR} \mathrm{(500} \mathrm{MHz,} \mathrm{CDCl}_{3},\right) \delta$ 7.70-7.80 (m, 8H), 4.30-4.32 (t, $J=6.0 \mathrm{~Hz}, 4 \mathrm{H}), 3.05-3.07(\mathrm{t}, J=5.5 \mathrm{~Hz}, 4 \mathrm{H}), 2.83-2.86$ (t, $J=7.5 \mathrm{~Hz}, 2 \mathrm{H}), 2.62-2.65(\mathrm{t}, J=7.5 \mathrm{~Hz}, 2 \mathrm{H}), 2.30(\mathrm{~s}, 3 \mathrm{H}), 1.52-1.55$ (qui, $J=7.0 \mathrm{~Hz}$, 2H), 1.23-1.41 (m, 16H) ppm; ${ }^{13} \mathrm{C}$ NMR (125 MHz, $\left.\mathrm{CDCl}_{3}\right) \delta$ 195.9, 163.2, 134.2, 129.2, 
123.3, 76.6, 54.7, 52.2, 30.5, 29.4, 29.0, 28.7, $27.5 \mathrm{ppm}$; HRMS $\mathrm{m} / z[\mathrm{M}+\mathrm{H}]^{+}$calcd for $\mathrm{C}_{33} \mathrm{H}_{42} \mathrm{~N}_{3} \mathrm{O}_{7} \mathrm{~S}^{+}, 624.2738$, found 624.2731 .

6.1.4.4. 11-(Bis(2-(aminooxy)ethyl)amino)undecane-1-thiol (AOT bis $_{\text {). }}$.

To thioester $4(0.546 \mathrm{~g}, 0.876 \mathrm{mmol})$ in $\mathrm{CH}_{2} \mathrm{Cl}_{2}(10 \mathrm{~mL})$ at $0{ }^{\circ} \mathrm{C}$ was added slowly hydrazine monohydrate $(0.260 \mathrm{~mL}, 5.25 \mathrm{mmol})$ via syringe. The resultant reaction mixture was stirred at $0{ }^{\circ} \mathrm{C}$ for $30 \mathrm{~min}$. before stirring at $\mathrm{rt}$ for $12 \mathrm{~h}$. The precipitated phthalyl hydrazide was removed by filtration and the filtrate was concentrated by rotary evaporation to afford crude compound AOT bis. The residue was purified with silica gel column chromatography using $\mathrm{CH}_{2} \mathrm{Cl}_{2} /$ methanol (9.5:0.5) to give the bis(aminooxy) thiol AOT $_{\text {bis }}$ as a viscous oil $(0.230 \mathrm{~g}, 82 \%)$; IR (neat) $3306,2922,2852,1590,1464 \mathrm{~cm}^{-1} ;{ }^{1} \mathrm{H}$ NMR (400 MHz, $\left.\mathrm{CDCl}_{3}\right) \delta 5.30(\mathrm{~m}, 4 \mathrm{H}), 3.70-3.73(\mathrm{t}, J=4.0 \mathrm{~Hz}, 4 \mathrm{H}), 2.67-2.70(\mathrm{t}, J=$ $4.0 \mathrm{~Hz}, 4 \mathrm{H}), 2.61-2.64(\mathrm{t}, J=4.0 \mathrm{~Hz}, 2 \mathrm{H}), 2.44-2.48(\mathrm{~m}, 3 \mathrm{H}), 1.53-1.61(\mathrm{~m}, 2 \mathrm{H}), 1.41$ (m, 2H), 1.21-1.32 (m, 14H) ppm; ${ }^{13} \mathrm{C}$ NMR (100 MHz, $\left.\mathrm{CDCl}_{3}\right) \delta$ 73.4, 55.1, 52.9, 39.0, 33.9, 29.4, 29.1, 28.9, 28.4, 27.3, 26.7, $24.5 \mathrm{ppm}$; HRMS $\mathrm{m} / z[\mathrm{M}+\mathrm{H}]^{+}$calcd for $\mathrm{C}_{15} \mathrm{H}_{35} \mathrm{~N}_{3} \mathrm{O}_{2} \mathrm{~S}$ 322.2530, found 322.2518; $[\mathrm{M}+\mathrm{Na}]^{+}$calcd for $\mathrm{C}_{15} \mathrm{H}_{35} \mathrm{~N}_{3} \mathrm{NaO}_{2} \mathrm{~S}^{+}$ 344.2347, found 344.2336 .

\subsubsection{Synthesis Procedures for Water-Soluble Bis-Aminooxy-Thiol Ligand} $\left(\mathrm{AOT}_{\text {bis }}{ }^{+}\right)$

6.1.5.1. [11-(Acetylsulfanyl)undecyl $]$ bis(\{2-[(1,3-dioxo-2,3-dihydro-1H-isoindol-2yl)oxy]ethyl\} methylammonium iodide (6).

A threaded pressure tube was flushed with argon and then charged with thioester $4(0.355$ $\mathrm{g}, 0.561 \mathrm{mmol})$ and a solution of methyl iodide $(0.350 \mathrm{~mL}, 5.61 \mathrm{mmol})$ in $\mathrm{CH}_{2} \mathrm{Cl}_{2}(10$ 
$\mathrm{mL}$ ). The tube was sealed and heated at $50{ }^{\circ} \mathrm{C}$ for $24 \mathrm{~h}$. Upon cooling, the tube was opened, and the reaction solvent was concentrated by rotary evaporation. The crude residue was triturated with $\mathrm{Et}_{2} \mathrm{O}(5 \times 10 \mathrm{~mL})$ and the remaining solid then was dried to afford ammonium iodide $6(0.378 \mathrm{~g}, 88 \%)$ as a light yellow solid; mp $135-138{ }^{\circ} \mathrm{C}$; IR (solid) 2926, 2855, $1730 \mathrm{~cm}^{-1} ;{ }^{1} \mathrm{H}$ NMR $\left(500 \mathrm{MHz}, \mathrm{CDCl}_{3}\right) \delta$ 7.79-7.86 (m, 8H), 4.784.80 (br s, 4H), 4.43 (br s, 4H), 3.98-4.01 (t, J= $8.5 \mathrm{~Hz}, 2 \mathrm{H}), 3.70$ (s, 3H), 2.81-2.84 (t, $J$ $=7.0 \mathrm{~Hz}, 2 \mathrm{H}), 2.30(\mathrm{~s}, 3 \mathrm{H}), 1.93(\mathrm{~m}, 2 \mathrm{H}), 1.77(\mathrm{~m}, 2 \mathrm{H}), 1.20-1.53(\mathrm{~m}, 14 \mathrm{H}) \mathrm{ppm} ;{ }^{13} \mathrm{C}$ NMR (125 MHz, $\left.\mathrm{CDCl}_{3}\right) \delta 163.0,135.1,128.4,124.0,72.2,65.4,60.9,50.1,30.6,30.5$, 28.7-29.4, 26.1, $22.8 \mathrm{ppm}$; HRMS $\mathrm{m} / \mathrm{z}$ calcd for $\mathrm{C}_{34} \mathrm{H}_{44} \mathrm{~N}_{3} \mathrm{O}_{7} \mathrm{~S}^{+}$638.2894, found 638.2892 .

6.1.5.2. $N, N$-Bis(2-(aminooxy)ethyl)-11-mercapto- $N$-methylundecan-1-ammonium iodide $\left(\mathbf{A O T}_{\text {bis }}{ }^{+}\right)$.

To a solution of $6(0.251 \mathrm{~g}, 0.327 \mathrm{mmol})$ in anhydrous $\mathrm{CH}_{2} \mathrm{Cl}_{2}(10 \mathrm{~mL})$ at $0{ }^{\circ} \mathrm{C}$ was added hydrazine monohydrate $(159 \mu \mathrm{L}, 3.27 \mathrm{mmol})$ via syringe. The reaction mixture was stirred $1 \mathrm{~h}$ at $0{ }^{\circ} \mathrm{C}$ before allowing the reaction mixture to warm to rt. After stirring $12 \mathrm{~h}$, the reaction mixture was filtered, and the filtrate was concentrated by rotary evaporation (note: all workup operations are best performed under a nitrogen atmosphere to prevent undesired reaction of the aminooxy groups) to furnish bis-(aminooxy) thiol AOT $_{\text {bis }}{ }^{+}$as a light yellow amorphous solid $(0.127 \mathrm{~g}, 84 \%)$; mp 101-103 ${ }^{\circ} \mathrm{C}$; IR (solid) 3244, 2244, 2926, $2854 \mathrm{~cm}^{-1}$; ${ }^{1} \mathrm{H}$ NMR (500 MHz, D $\left.2 \mathrm{O}\right) \delta$ 4.11-4.13 (t, J=4.5 Hz, 4H), 3.66-3.67 (t, J= 5.5 Hz, 4H), 3.39-3.42 (t, J=4.5 Hz, 2H), 3.14(s, 3H), 2.54-2.57 (t, J=7.0 $\mathrm{Hz}, 2 \mathrm{H}), 1.70(\mathrm{~m}, 2 \mathrm{H}), 1.62(\mathrm{~m}, 2 \mathrm{H}), 1.27-1.37(\mathrm{~m}, 14 \mathrm{H}) \mathrm{ppm} ;{ }^{13} \mathrm{C} \mathrm{NMR}(125 \mathrm{MHz}$, 
DMSO) $\delta 68.4,63.6,60.5,53.9,49.3,42.8,34.0,33.1,25.5,24.4,21.2,19.7$ ppm;

HRMS $m / z$ calcd. for $\mathrm{C}_{16} \mathrm{H}_{38} \mathrm{~N}_{3} \mathrm{O}_{2} \mathrm{~S}^{+} 336.2679$, found 336.2670.

\subsubsection{Synthesis Procedures for Aminooxy-Hexane Thiol Ligand (11)}

6.1.6.1. 2-(hex-5-en-1-yloxy)isoindoline-1,3-dione (9).

To a stirred solution of 5-hexene-1-ol (4.50 g, $45 \mathrm{mmol})$ in THF $(240 \mathrm{~mL})$ was added triphenylphosphine (14.1 g, $54 \mathrm{mmol})$ and N-hydroxyphthalimide $(8.8 \mathrm{~g}, 54 \mathrm{mmol})$. The mixture was cooled to $0^{\circ} \mathrm{C}$ for $30 \mathrm{~min}$ and DIAD $(10.6 \mathrm{~mL}, 0.3 \mathrm{~mol})$ was added slowly over a period of 30 minute. The reaction mixture was allowed to stir further at $0^{\circ} \mathrm{C}$ for 30 min, followed by continuous stirring at room temperature for 12 hours. The reaction mixture was diluted with diethyl ether and concentrated to near dryness by rotary evaporation to afford yellowish oil compound. The oil was dissolved in $\mathrm{CH}_{2} \mathrm{Cl}_{2}$ and washed with water; saturated $\mathrm{NaHCO}_{3}$ Solution followed by a saturated $\mathrm{NaCl}$ solution. The organic layer was filtered through a column plugged with silica to remove $\mathrm{Ph}-\mathrm{P}=\mathrm{O}$ as much as possible. Then obtained filtrate was concentrated and purified by silica gel chromatography (hexane: EtOAc 4:1, $R_{\mathrm{f}}=0.45$ ). After purification, the title compound (9) was isolated as a yellow waxy solid (yield $82 \%) .{ }^{1} \mathrm{H} \mathrm{NMR}:\left(400 \mathrm{MHz}, \mathrm{CDCl}_{3}\right) \delta 7.73-$ $7.83(\mathrm{~m}, 4 \mathrm{H}), 5.77-5.84(\mathrm{~m}, 1 \mathrm{H}), 4.94-5.00(\mathrm{~m}, 2 \mathrm{H}), 4.18-4.21(\mathrm{t}, J=6.4 \mathrm{~Hz}, 2 \mathrm{H}), 2.11-$ $2.15(\mathrm{t}, J=7.2 \mathrm{~Hz}, 2 \mathrm{H}), 1.76-1.83(\mathrm{~m}, 2 \mathrm{H}) 1.58-1.64(\mathrm{~m}, 2 \mathrm{H}) \mathrm{ppm} ;{ }^{13} \mathrm{C} \mathrm{NMR}:(100 \mathrm{MHz}$, $\left.\mathrm{CDCl}_{3}\right) \delta 163,138,134,129,124,123,114,33,27,24 \mathrm{ppm}$.

6.1.6.2. S-(6-((1,3-dioxoisoindolin-2-yl)oxy)hexyl) ethanethioate (10).

To a solution of compound 9 (3.24 g, $13.20 \mathrm{mmol})$ in THF $(66 \mathrm{~mL})$ was slowly added of a solution of AIBN (325 mg, $1.98 \mathrm{mmol})$ dissolved in dry THF (2 mL) via syringe, 
followed by addition of thioacetic acid $(1.86 \mathrm{~mL}, 26.4 \mathrm{mmol})$ via syringe. The resultant mixture was degassed for 15 min using a stream of argon before heating the solution at reflux for $16 \mathrm{~h}$. The reaction mixture was then cooled to rt, quenched with diethyl ether $(10 \mathrm{~mL})$ and concentrated by rotary evaporation to afford the crude product, which was purified by silica gel chromatography (hexane: EtOAc 8.5:1.5, $\mathrm{R}_{\mathrm{f}}=0.43$ ). After purification, the thioester compound $\mathbf{1 0}$ was isolated as a white solid powder (yield 88\%) ; m.p 37-39 ${ }^{\circ} \mathrm{C} ;{ }^{1} \mathrm{H}$ NMR $\left(400 \mathrm{MHz}, \mathrm{CDCl}_{3}\right.$,) $\delta$ 7.72-7.83 (m, 4H), 4.16-4.20 (t, $J=6.8$ $\mathrm{Hz}, 2 \mathrm{H}$ ), 2.84-2.89 (t, $J=7.6 \mathrm{~Hz}, 2 \mathrm{H}$ ), 2.31 (s, 3H), 1.75-1.79 (qui, $J=7.2 \mathrm{~Hz}, 2 \mathrm{H}$ ), 1.40$1.61(\mathrm{~m}, 6 \mathrm{H}) \mathrm{ppm} ;{ }^{13} \mathrm{C}$ NMR (100 MHz, $\left.\mathrm{CDCl}_{3}\right) \delta 196,164,134,129,123,27-30,25$ ppm.

6.1.6.3. 6-(aminooxy)hexane-1-thiol (11).

To compound $10(1.021 \mathrm{~g}, 3.11 \mathrm{mmol})$ in $\mathrm{CH}_{2} \mathrm{Cl}_{2}(15 \mathrm{~mL})$ at $0{ }^{\circ} \mathrm{C}$ was added slowly hydrazine monohydrate $(1.2 \mathrm{~mL}, 24.9 \mathrm{mmol})$ via syringe. The resultant reaction mixture was stirred at $0{ }^{\circ} \mathrm{C}$ for 30 minutes before stirring at $\mathrm{rt}$ for $12 \mathrm{~h}$. The precipitated phthaloyl hydrazide was removed by filtration and the filtrate was concentrated by rotary evaporation under argon to afford crude compound 11. The residue was dissolved again in $\mathrm{CH}_{2} \mathrm{Cl}_{2}$ (5 mL), filtered again, and concentrated to obtain pure aminooxy hexanethiol as viscous pale liquid $(0.421 \mathrm{~g}, 91 \%) ;{ }^{1} \mathrm{H}$ NMR $\left(400 \mathrm{MHz}, \mathrm{CDCl}_{3}\right) \delta 5.31(\mathrm{~m}, 2 \mathrm{H}), 3.60-$ $3.63(\mathrm{t}, J=6.4 \mathrm{~Hz}, 2 \mathrm{H}), 2.46-2.52(\mathrm{~m}, 2 \mathrm{H}), 1.51-1.61(\mathrm{~m}, 4 \mathrm{H}), 1.28-1.41(\mathrm{~m}, 4 \mathrm{H}) \mathrm{ppm}$; ${ }^{13} \mathrm{C}$ NMR $\left(100 \mathrm{MHz}, \mathrm{CDCl}_{3}\right) \delta 73.4,55.1,52.9,39.0,33.9,29.4,29.1,28.9,28.4,27.3$, 26.7, $24.5 \mathrm{ppm}$. 


\subsubsection{Synthesis of $\mathrm{AuC}_{6} \mathrm{~S}$ MPCs}

Hexanethiolate-functionalized monolayer-protected gold clusters ( $\mathrm{AuC}_{6} \mathrm{~S}$ MPCs) with a $\sim 1.6 \mathrm{~nm}$ diameter was prepared using a 3:1 molar ratio of 1-hexanethiol: $\mathrm{HAuCl}_{4} \bullet 3 \mathrm{H}_{2} \mathrm{O}$. The synthesis was carried out following the conditions reported by Brust $e t$ al. with slight modifications. ${ }^{30}$ In a typical reaction, tetraoctylammonium bromide $(0.305 \mathrm{~g}, 0.559$ mmol) was dissolved in toluene $(60 \mathrm{~mL})$ and stirred for $15 \mathrm{~min}$. before addition of a solution of $\mathrm{HAuCl}_{4} \cdot 3 \mathrm{H}_{2} \mathrm{O}(0.200 \mathrm{~g}, 0.507 \mathrm{mmol})$ in water $(20 \mathrm{~mL})$. The biphasic mixture was stirred vigorously for 30 minutes to ensure full transfer of $\mathrm{HAuCl}_{4} \bullet 3 \mathrm{H}_{2} \mathrm{O}$ from the water to the toluene phase. The mixture then was transferred to a separatory funnel and the aqueous layer was removed. The organic layer was transferred to a clean flask and 1hexanethiol $(216 \mu \mathrm{L}, 1.52 \mathrm{mmol})$ was added followed by stirring 45 minutes at $\mathrm{rt}$, whereupon a color change from orange to colorless was observed. The reaction mixture was cooled to $0{ }^{\circ} \mathrm{C}$ using an ice bath and a solution of sodium borohydride $(0.192 \mathrm{~g}, 5.07$ mmol) in water $(20 \mathrm{~mL})$ was rapidly added to form a black solution, indicative of cluster formation. The reaction mixture was further stirred at $0{ }^{\circ} \mathrm{C}$ for 30 minutes and then stirred at $\mathrm{rt}$ for $4 \mathrm{~h}$, whereupon it was transferred to a separatory funnel and the aqueous layer was removed. The organic layer was concentrated to near dryness by rotary evaporation and acetonitrile $(c a .40 \mathrm{~mL})$ was added. The solid black MPCs $(0.140 \mathrm{~g})$ were collected after $12 \mathrm{~h}$ by vacuum filtration using a fritted glass funnel after washing with acetonitrile and finally drying in air $(0.140 \mathrm{~g})$. The clusters were characterized by UV-VIS, TGA, FTIR and ${ }^{1} \mathrm{H}$ NMR spectroscopy, which was consistent with pure $\mathrm{C}_{6} \mathrm{~S}$ protected Au MPCs with an approximate composition of $\mathrm{Au}_{144}\left(\mathrm{C}_{6} \mathrm{~S}\right)_{60}$ as described in the literature. ${ }^{139}$ 


\subsubsection{Synthesis of AuSG MPCs}

Water-soluble glutathione (SG)-functionalized Au MPCs were synthesized using a 3:1 molar ratio of glutathione to $\mathrm{HAuCl}_{4} \cdot 3 \mathrm{H}_{2} \mathrm{O}$ as previously described. ${ }^{175}$ The synthesis was carried out under a nitrogen atmosphere. Glutathione $(120 \mathrm{mg}, 0.380 \mathrm{mmol})$ was added to $400 \mathrm{~mL}$ of a methanolic $\mathrm{HAuCl}_{4} \cdot 3 \mathrm{H}_{2} \mathrm{O}$ solution $(50.1 \mathrm{mg}, 0.127 \mathrm{mmol})$ in a $1-\mathrm{L}$ single-necked round-bottom flask. The bright yellow $\mathrm{HAuCl} 4$ solution turned into a light-yellow solution upon addition of glutathione and the mixture was purged with $\mathrm{N}_{2}$ for 1 hour. The reaction mixture was cooled to $0{ }^{\circ} \mathrm{C}$ using an ice bath and stirred for 30 minutes to form a cloudy white color indicative of an $\mathrm{Au}(\mathrm{I})-\mathrm{SG}$ complex. To the reaction mixture was rapidly added a freshly prepared, chilled methanolic $\mathrm{NaBH}_{4}$ solution (125 $\mathrm{mL}$ of a $0.21 \mathrm{M}$ solution, $0.0264 \mathrm{~mol}$ ) to yield a black reaction mixture indicating the formation of Au SG MPCs. The flow of $\mathrm{N}_{2}$ was stopped at this stage. The reaction mixture was stirred at room temperature for 12 hours whereupon the Au MPCs precipitated and were isolated by discarding the supernatant. The precipitate was dissolved in nanopure $\mathrm{H}_{2} \mathrm{O}(10 \mathrm{~mL})$ and an excess amount of methanol $(60 \mathrm{~mL})$ was added to again precipitate the clusters, which were collected via centrifugation. The same process was repeated once more to remove any remaining unbound glutathione as well as diborane or disulfide species that may have formed during the synthesis. Finally, the Au SG MPCs were air-dried to afford AuSG MPCs $(42.0 \mathrm{mg})$ as a black powder.

\subsubsection{Synthesis of PdSG MPCs}

Water-soluble glutathione (SG)-capped Pd MPCs were synthesized using a 0.35:1 molar ratio of glutathione to $\mathrm{K}_{2} \mathrm{PdCl}_{4}$, as previously described. ${ }^{137}$ The synthesis was carried out 
under a $\mathrm{N}_{2}$ atmosphere in a three-necked round bottom flask. First, $\mathrm{K}_{2} \mathrm{PdCl}_{4}(0.205 \mathrm{~g}$, $0.628 \mathrm{mmol})$ was dissolved in nanopure water $(20 \mathrm{~mL})$ and the resultant solution was purged with $\mathrm{N}_{2}$ for 1 hour. Reduced-L-glutathione $(0.066 \mathrm{~g}, 0.221 \mathrm{mmol})$ was dissolved in nanopure water $(10 \mathrm{~mL})$ and purged with $\mathrm{N}_{2}$ for 1 hour. These two solutions were then combined at room temperature and stirred under $\mathrm{N}_{2}$ atmosphere until $\mathrm{PdCl}_{4}{ }^{2-}$ formed a complex with glutathione, as indicated by the change in color from yellow to wine red (ca. $5 \mathrm{~min}$ ). The reaction mixture was further stirred for 1 hour and then cooled to $0{ }^{\circ} \mathrm{C}$ using an ice-bath. A fresh aqueous solution of $\mathrm{NaBH}_{4}(0.232 \mathrm{~g}, 6.12 \mathrm{mmol})$ in nanopure water $(10 \mathrm{~mL})$ was purged with $\mathrm{N}_{2}$ for 1 hour before rapidly adding to the reaction mixture with vigorous stirring to result in an immediate color change from wine red to black. The solution was further stirred for $6 \mathrm{~h}$ at room temperature before an equal volume of methanol $(40 \mathrm{~mL})$ was added. The resultant suspension was stirred 15 minutes and then centrifuged 15 minutes to precipitate the MPCs. The supernatant was discarded and the precipitated MPCs were collected by dissolving in nanopure water to aid the transfer into a round bottom flask. The water was then removed by using a rotary evaporator. The obtained black MPCs were suspended in methanol (100 mL) overnight and collected by vacuum filtration using a glass fritted Büchner funnel. The MPCs were thoroughly washed with methanol $(2 \mathrm{x})$, ethanol $(2 \mathrm{x})$, acetone $(2 \mathrm{x})$, and then air-dried to afford PdSG MPCs (112 mg) as a black powder.

\subsection{Experimental Procedures for Chapter 3}

\subsubsection{Spectroscopic Measurements}

${ }^{1} \mathrm{H}$ NMR spectra were recorded either on a Varian $400 \mathrm{MHz}$ or Varian Inova $500 \mathrm{MHz}$ spectrometer. Infrared spectra were recorded on a Perkin-Elmer Spectrum FT-IR 
spectrophotometer with an attenuated total reflectance attachment. UV-Vis spectra were recorded on a Varian instrument model CARY 50 Bio UV-Visible spectrophotometer. All scans were obtained between $300 \mathrm{~nm}$ to $900 \mathrm{~nm}$ wavelength range at a fast scan rate in a glass cuvette and the background was subtracted using $\mathrm{CHCl}_{3}$ or nanopure $\mathrm{H}_{2} \mathrm{O}$ as the blank.

\subsubsection{Electrochemical Measurements}

The redox potential of ferrocene $(\mathrm{Fc})$-attached clusters was measured by cyclic voltammetry (CV) using a $\mathrm{CH}$ Instruments $\mathrm{CHI}$ 660A electrochemical workstation (Austin, TX) with the following electrode configurations: working electrode $-2 \mathrm{~mm}$ diameter Pt disk; counter electrode - Pt wire; and reference electrode - Ag wire (QRE). The supporting electrolyte solution was prepared using tetrabutylammonium hexafluorophosphate (TBAHFP). The Fc-coupled Au cluster $(10 \mathrm{mg}$ ) was added to a 0.1 M solution of TBAHFP $(0.39 \mathrm{~g}, 1.00 \mathrm{mmol})$ in $\mathrm{CH}_{2} \mathrm{Cl}_{2}(10 \mathrm{~mL})$ to yield a solution for CV measurements (1.70 $\mathrm{mM}$ total Fc concentration).

\subsubsection{Fluorescence Measurements}

Fluorescence measurements were performed using a Perkin Elmer LS55 fluorescence spectrometer with $\mathrm{CHCl}_{3}$ as a blank in a quartz cuvette. For the study with 1pyrenecarboxaldehyde (1-PyCHO), intensity measurements were performed at [1PyCHO $]=1.0 \times 10^{-7} \mathrm{M}$ with an excitation wavelength of $375 \mathrm{~nm}$. The $\mathrm{Au}\left(\mathrm{C}_{6} \mathrm{~S}\right)\left(\mathrm{AOT}_{\text {bis }}\right.$ pyrene) MMPCs $(2.0 \mathrm{mg})$ were added to $\mathrm{CHCl}_{3}(20 \mathrm{~mL}) .8 .5 \mu \mathrm{L}$ of this solution then was added to $10 \mathrm{~mL} \mathrm{CHCl} 3$ to yield a solution for fluorescence measurements $(1.0 \mathrm{x}$ $10^{-7} \mathrm{M}$ total pyrene concentration). 


\subsubsection{MALDI-TOF MS Measurements}

Spectral data were obtained using a Voyager Biospectrometry DE Workstation (Applied Biosystems, Foster City, CA, USA) outfitted with a nitrogen laser emitting at $337 \mathrm{~nm}$. Fragment ions were accelerated at $20 \mathrm{kV}$ after $75 \mathrm{nsec}$ extraction delay time. $70 \pm 5 \mathrm{~mJ}$ laser energy was used at a pulse rate of $3 \mathrm{~Hz}$. Laser intensity used was 1687 . The $50-900$ $\mathrm{m} / \mathrm{z}$ value range was explored with 50 shots averaged per spectrum in positive and negative-ion reflectron modes. The data shown is in positive mode. $2 \mathrm{mg}$ of ureaaldehyde adduct was dissolved in methanol, and $50 \mu \mathrm{L}$ of $26 \mathrm{mg}$ of p-nitroaniline (26 $\mathrm{mg}$ ) which was separately dissolved in $1 \mathrm{~mL}$ of methanol and sonicated for $5 \mathrm{~min}$ was added to the solution, and mixed together in a Eppendorf tube followed by sonication for 5 minutes. $0.75 \mu \mathrm{L}$ of the obtained solution was then spotted in a MALDI plate followed by air dry for 30 minutes for MALDI-TOF MS measurements.

\subsubsection{Place Exchange Reaction to Prepare $\mathrm{Au}\left(\mathrm{C}_{6} \mathrm{~S}\right)\left(\mathrm{AOT}_{\text {bis }}\right) \mathrm{MMPCs}$}

We prepared $\mathrm{Au}$ mixed monolayer-protected clusters (MMPCs) functionalized with mixtures of hexane-thiolate and $\mathrm{AOT}_{\text {bis }}$ by performing place exchange reactions on the $\mathrm{Au}_{144}\left(\mathrm{C}_{6} \mathrm{~S}\right)_{60}$ MPCs. In a typical procedure, the $\mathrm{AOT}_{\text {bis }}$ ligand $(96.7 \mathrm{mg}, 0.301 \mathrm{mmol})$ and the $\mathrm{Au}_{144}\left(\mathrm{C}_{6} \mathrm{~S}\right)_{60}$ MPCs $(80.1 \mathrm{mg}, 0.150 \mathrm{mmol})$ were mixed under argon in dry THF and followed by stirring at $\mathrm{rt}$ for $3 \mathrm{~d}$. The resultant, crude $\mathrm{Au}\left(\mathrm{C}_{6} \mathrm{~S}\right)\left(\mathrm{AOT}_{\text {bis }}\right)$ MMPCs were concentrated by rotary evaporation, suspended in acetonitrile $(10 \mathrm{~mL})$, and then purified by repeated centrifugation and supernatant removal (x3). Characterization by UV-VIS,

IR (solid), and ${ }^{1} \mathrm{H}$ NMR revealed that the composition of the Au MMPCs was approximately $\mathrm{Au}_{144}\left(\mathrm{C}_{6} \mathrm{~S}\right)_{30}\left(\mathrm{AOT}_{\text {bis }}\right)_{30}$. 


\subsubsection{Synthesis Procedures for Urea-Aldehyde 15}

6.2.6.1. 8-((Trimethylsilyl)oxy)octan-1-amine (13).

To a solution of 8-amino-1-octanol (12) $(0.94 \mathrm{~g}, 6.47 \mathrm{mmol})$ in THF $(30 \mathrm{~mL})$ at $0{ }^{\circ} \mathrm{C}$ was added successively trimethylsilyl chloride $(0.985 \mathrm{~mL}, 7.76 \mathrm{mmol})$ and triethylamine $\left(\mathrm{Et}_{3} \mathrm{~N}\right)(1.53 \mathrm{~mL}, 10.9 \mathrm{mmol})$ via syringe while under argon. The reaction mixture was stirred at $0{ }^{\circ} \mathrm{C}$ for $2 \mathrm{~h}$ and then allowed to warm to rt. After $12 \mathrm{~h}$, the reaction was diluted with diethyl ether $(30 \mathrm{~mL})$ and the precipitated solids were filtered. The filtrate was concentrated, dissolved in ethyl acetate $(50 \mathrm{~mL})$, successively washed with sat'd aq. $\mathrm{NaHCO}_{3}(3 \times 50 \mathrm{~mL})$ and brine $(2 \times 50 \mathrm{~mL})$, and then dried $\left(\mathrm{Na}_{2} \mathrm{SO}_{4}\right)$. The solvents were removed by rotary evaporation to afford amine $7(1.18 \mathrm{~g}, 85 \%)$ as a pale yellow waxy solid suitable for use without further purification; mp 60-62 ${ }^{\circ} \mathrm{C}$; IR (solid) 3393, 3356, 2922, 2851, 1561, 1445, $\mathrm{cm}^{-1},{ }^{1} \mathrm{H}$ NMR (400 MHz, $\left.\mathrm{CDCl}_{3}\right) \delta 3.55$ (s, br, 2H), 2.69 (s, br, 2H), $1.70(\mathrm{~s}, b r, 2 \mathrm{H}), 1.44-1.51(\mathrm{~m}, b r, 4 \mathrm{H}), 1.30(\mathrm{~m}, 8 \mathrm{H}), 0.10(\mathrm{~s}, 9 \mathrm{H}) \mathrm{ppm} ;{ }^{13} \mathrm{C} \mathrm{NMR}$ $\left(100 \mathrm{MHz}, \mathrm{CDCl}_{3}\right) \delta 62.8,48.9,42.1,33.6,32.7,29.6,29.3,26.7,25.6$ ppm; HRMS m/z $[\mathrm{M}+\mathrm{H}]^{+}$calcd for $\mathrm{C}_{11} \mathrm{H}_{28} \mathrm{NOSi}^{+}$218.1935, found 218.1932.

6.2.6.2. 1-(tert-Butyl)-3-(8-hydroxyoctyl)urea (14).

To amine $13(0.955 \mathrm{~g}, 4.39 \mathrm{mmol})$ in dry $\mathrm{CH}_{2} \mathrm{Cl}_{2}(20 \mathrm{~mL})$ under argon at $0{ }^{\circ} \mathrm{C}$ was added slowly triethylamine $(672 \mu \mathrm{L}, 4.83 \mathrm{mmol})$ and then tert-butyl isocyanate $(508 \mu \mathrm{L}, 4.39$ mmol) via syringe. The resultant reaction mixture was stirred at $0{ }^{\circ} \mathrm{C}$ for 30 min before stirring at $\mathrm{rt}$ for $18 \mathrm{~h}$. The crude solution so obtained was concentrated by rotary evaporation and used directly in the next step without further purification. To a stirred solution of crude urea intermediate dissolved in dry THF $(25 \mathrm{~mL})$ under argon at $0{ }^{\circ} \mathrm{C}$ 
was added tetrabutylammonium fluoride $(5.6 \mathrm{~mL}$ of $1.0 \mathrm{M}$ solution in $\mathrm{THF}, 5.27 \mathrm{mmol}$ ) via syringe. The resultant reaction mixture was stirred at $0{ }^{\circ} \mathrm{C}$ for $30 \mathrm{~min}$ before stirring at $\mathrm{rt}$ for $5 \mathrm{~h}$. The crude mixture was diluted with deionized water $(25 \mathrm{~mL})$, extracted with $\mathrm{CH}_{2} \mathrm{Cl}_{2}(2 \times 20 \mathrm{~mL})$ and the combined organic layer then was dried $\left(\mathrm{Na}_{2} \mathrm{SO}_{4}\right)$ before concentrating via rotary evaporation. The residue was purified by $\mathrm{SiO}_{2}$ flash column chromatography eluting with a 3:1 mixture of $\mathrm{CH}_{2} \mathrm{Cl}_{2}$ : EtOAc $\left(\mathrm{R}_{\mathrm{f}}=0.42\right)$ to give ureaalcohol 14 as a white solid (0.740 g, 69\%); mp 78-80 ${ }^{\circ} \mathrm{C}$; IR (solid) 3361, 3230, 2967, 2923, 2851, 1652, 1563, 1478, $1463 \mathrm{~cm}^{-1} ;{ }^{1} \mathrm{H} \mathrm{NMR}\left(400 \mathrm{MHz}, \mathrm{CDCl}_{3}\right) \delta 3.63(\mathrm{t}, J=6.0$ $\mathrm{Hz}, 2 \mathrm{H}), 3.09(\mathrm{t}, J=7.2 \mathrm{~Hz}, 2 \mathrm{H}), 1.53-1.57(\mathrm{~m}, 2 \mathrm{H}), 1.40-1.47(\mathrm{~m}, 4 \mathrm{H}), 1.30-1.32(\mathrm{~m}$, $17 \mathrm{H}) ;{ }^{13} \mathrm{C} \mathrm{NMR}\left(100 \mathrm{MHz}, \mathrm{CDCl}_{3}\right) \delta(\mathrm{ppm}), 157.9,63.3,50.7,40.7,33.1,30.6,29.9$, 29.6, 29.5, 27.1, 25.9; HRMS $m / z[\mathrm{M}+\mathrm{H}]^{+}$calcd for $\mathrm{C}_{13} \mathrm{H}_{29} \mathrm{~N}_{2} \mathrm{O}_{2}{ }^{+}$245.2224, found 245.2218; $[\mathrm{M}+\mathrm{Na}]^{+}$calcd for $\mathrm{C}_{13} \mathrm{H}_{28} \mathrm{~N}_{2} \mathrm{NaO}_{2}{ }^{+}$267.2043, found 267.2039.

6.2.6.3. 1-(tert-Butyl)-3-(8-oxooctyl)urea (15).

A suspension of alcohol 14 (0.720 g, $2.94 \mathrm{mmol})$, pyridinium chlorochromate (PCC) $(2.22 \mathrm{~g}, 10.3 \mathrm{mmol})$, silica gel $(2.90 \mathrm{~g})$, and sodium acetate $(0.846 \mathrm{~g}, 10.3 \mathrm{mmol})$ in dry THF $(20 \mathrm{~mL})$ at $0{ }^{\circ} \mathrm{C}$ was stirred for $1 \mathrm{~h}$ before warming to $\mathrm{rt}$. After $4 \mathrm{~h}$, the reaction mixture was diluted with diethyl ether $(30 \mathrm{~mL})$ and the resultant solution was filtered through a short column of celite. The filtrate was dried $\left(\mathrm{Na}_{2} \mathrm{SO}_{4}\right)$ and the solvents then removed by rotary evaporation. The residue was purified by $\mathrm{SiO}_{2}$ flash column chromatography eluting with 3:1 mixture of $\mathrm{CH}_{2} \mathrm{Cl}_{2}$ : EtOAc $\left(\mathrm{R}_{\mathrm{f}}=0.52\right)$ to afford ureaaldehyde 15 (0.450 g, 63\%) as a pale yellow viscous liquid; IR (neat) 3362, 2928, 2857 , 1723, 1632, 1555, $1451 \mathrm{~cm}^{-1} ;{ }^{1} \mathrm{H}$ NMR $\left(500 \mathrm{MHz} \mathrm{CDCl}_{3}\right) \delta 9.73(\mathrm{~s}, 1 \mathrm{H}), 4.60-4.66(\mathrm{NH}$, 2H), $3.07(\mathrm{~m}, 2 \mathrm{H}), 2.40(\mathrm{~m}, 2 \mathrm{H}), 1.60(\mathrm{~m}, 2 \mathrm{H}), 1.42(\mathrm{~m}, 2 \mathrm{H}), 1.23-1.35(\mathrm{~m}, 17 \mathrm{H}) \mathrm{ppm}$; 
${ }^{13} \mathrm{C}$ NMR $\left(125 \mathrm{MHz}, \mathrm{CDCl}_{3}\right) \delta 203.2,158.1,50.3,44.0,40.3,30.4,29.8,29.2,26.9,22.1$

ppm; HRMS $m / z[\mathrm{M}+\mathrm{H}]^{+}$calcd for $\mathrm{C}_{13} \mathrm{H}_{27} \mathrm{~N}_{2} \mathrm{O}_{2}{ }^{+} 243.2067$, found 243.2065.

\subsubsection{Oximation Reactions on $\mathrm{Au}\left(\mathrm{C}_{6} \mathrm{~S}\right)\left(\mathrm{AOT}_{\text {bis }}\right)$}

Both non-polar and aqueous oximation reactions were performed on $\operatorname{Au}_{144}\left(\mathrm{C}_{6} \mathrm{~S}\right)_{30}\left(\mathrm{AOT}_{\text {bis }}\right)_{30}$ MMPCs with a panel of aldehydes. The overall reaction design and conditions are depicted in Scheme 3.2.

6.2.7.1. Benzaldehyde adduct. To a solution of $\mathrm{Au}_{144}\left(\mathrm{C}_{6} \mathrm{~S}\right)_{30}(\mathrm{AOT})_{30} \mathrm{MMPCs}(25.6 \mathrm{mg}$, $0.0185 \mathrm{mmol}$ of $\left.\mathrm{AOT}_{\text {bis }}\right)$ dissolved in dry THF $(2.0 \mathrm{~mL})$ was added benzaldehyde $(7.55$ $\mu \mathrm{L}, 0.0740 \mathrm{mmol}$ ) followed by stirring at $\mathrm{rt}$ for $12 \mathrm{~h}$. The reaction mixture was concentrated by rotary evaporation and the residue was suspended in acetonitrile (10 $\mathrm{mL}$ ). The oxime ether-modified MMPCs then were isolated by centrifugal precipitation. The process of washing using acetonitrile followed by centrifugation was repeated two times. The isolated $\mathrm{Au}\left(\mathrm{C}_{6} \mathrm{~S}\right)\left(\mathrm{AOT}_{\text {bis-benzaldehyde })}\right.$ MMPCs $(20.5 \mathrm{mg})$ were characterized by IR and ${ }^{1} \mathrm{H}$ NMR spectroscopy.

6.2.7.2. Ferrocene carboxaldehyde adduct. Ferrocene carboxaldehyde $(31 \mathrm{mg}, 0.14$ mmol) was added to the $\mathrm{Au}\left(\mathrm{C}_{6} \mathrm{~S}\right)\left(\mathrm{AOT}_{\text {bis }}\right) \mathrm{MMPCs}\left(50.5 \mathrm{mg}, 0.0365 \mathrm{mmol}^{\circ} \mathrm{AOT}_{\text {bis }}\right)$ dissolved in dry THF $(2.5 \mathrm{~mL})$ at $\mathrm{rt}$ with stirring. After $12 \mathrm{~h}$, the reaction mixture was concentrated by rotary evaporation and the residue was suspended in acetonitrile (10 $\mathrm{mL}$ ). The oxime ether adduct was isolated by centrifugal precipitation. The process of washing using acetonitrile followed by centrifugation was repeated two times. The isolated $\mathrm{Au}\left(\mathrm{C}_{6} \mathrm{~S}\right)\left(\mathrm{AOT}_{\text {bis }}\right.$ ferrocene) MMPCs $(43.6 \mathrm{mg})$ were characterized by $\mathrm{IR}$ and ${ }^{1} \mathrm{H}$ 
NMR spectroscopy. The redox behavior of the cluster was studied by cyclic voltammetry measurements.

6.2.7.3. 1-Pyrenecarboxaldehyde adduct. 1-Pyrenecarboxaldehyde ( $40 \mathrm{mg}, 0.17 \mathrm{mmol})$ was added to the $\mathrm{Au}\left(\mathrm{C}_{6} \mathrm{~S}\right)(\mathrm{AOT}$ bis) MMPCs $(60.5 \mathrm{mg}, 0.0437 \mathrm{mmol}$ of AOT bis) dissolved in dry THF $(3.0 \mathrm{~mL})$ at $\mathrm{rt}$ while stirring. The reaction mixture was purged with argon and stirred at $\mathrm{rt}$ for $18 \mathrm{~h}$. Upon completion, the reaction mixture was concentrated by rotary evaporation and the residue was suspended in acetonitrile $(10 \mathrm{~mL})$. The oxime ether adduct was isolated by centrifugal precipitation. The process of washing using acetonitrile followed by centrifugation was repeated two times. The isolated $\mathrm{Au}\left(\mathrm{C}_{6} \mathrm{~S}\right)\left(\mathrm{AOT}_{\text {bis }}\right.$-pyrene) $\mathrm{MMPCs}(50.5 \mathrm{mg})$ were dried under high vacuum overnight and characterized by IR, ${ }^{1} \mathrm{H}$ NMR, and fluorescence spectroscopy.

6.2.7.4. Propanal adduct. To a stirred solution of the $\mathrm{Au}\left(\mathrm{C}_{6} \mathrm{~S}\right)\left(\mathrm{AOT}_{\text {bis }}\right)$ MMPCs $(20.5$ $\left.\mathrm{mg}, 0.0148 \mathrm{mmol} \mathrm{AOT}_{\text {bis }}\right)$ in $\mathrm{pH} 4.0$ acetate buffer solution $(5.0 \mathrm{~mL})$ at $\mathrm{rt}$ was added propanal $(6.5 \mu \mathrm{L}, 0.0888 \mathrm{mmol})$. The mixture was stirred at $\mathrm{rt}$ for $18 \mathrm{~h}$ before addition of pH 8.0 phosphate buffer $(10 \mathrm{~mL})$. The resultant suspension was extracted using THF (3 $\mathrm{x} 10 \mathrm{~mL}$ ). The combined organic extract was concentrated by rotary evaporation and the residue was dried under high vacuum overnight. The $\mathrm{Au}\left(\mathrm{C}_{6} \mathrm{~S}\right)\left(\mathrm{AOT}_{\text {bis-propanal) MMPCs }}\right.$ (16.9 mg) were characterized by IR and ${ }^{1} \mathrm{H}$ NMR spectroscopy.

6.2.7.5. Glyceraldehyde adduct. To a stirred solution of the $\operatorname{Au}\left(\mathrm{C}_{6} \mathrm{~S}\right)\left(\mathrm{AOT}_{\mathrm{bis}}\right) \mathrm{Au}$ MMPCs $\left(45.0 \mathrm{mg}, 0.0325 \mathrm{mmol} \mathrm{AOT}_{\text {bis }}\right)$ in $\mathrm{pH} 4.0$ acetate buffer solution $(10 \mathrm{~mL})$ was added DL-glyceraldehyde $(29.3 \mathrm{mg}, 0.325 \mathrm{mmol})$. After stirring at rt for 1d, a pH 8.0 phosphate buffer solution $(20 \mathrm{~mL})$ was added. The resultant suspension was freeze dried and then lyophilized. The obtained residue was suspended in acetonitrile $(10 \mathrm{~mL})$ for $1 \mathrm{~d}$ 
followed by removal of the supernatant. Centrifugal precipitation using successive acetonitrile washings $(3 \times 10 \mathrm{~mL}$ ) followed by drying under high vacuum overnight afforded $\mathrm{Au}\left(\mathrm{C}_{6} \mathrm{~S}\right)\left(\mathrm{AOT}_{\text {bis }}\right.$-glyceraldehyde) MMPCs $(35.9 \mathrm{mg})$. The MMPCs were characterized by FTIR and ${ }^{1} \mathrm{H}$ NMR spectroscopy.

6.2.7.6. Urea-aldehyde 15 adduct. To a solution of the $\mathrm{Au}\left(\mathrm{C}_{6} \mathrm{~S}\right)\left(\mathrm{AOT}_{\text {bis }}\right)$ MMPCs (76.8 $\mathrm{mg}, 0.0555 \mathrm{mmol}$ of $\left.\mathrm{AOT}_{\text {bis }}\right)$ dissolved in dry THF $(3.0 \mathrm{~mL})$ was added urea-aldehyde 9 $(53.8 \mathrm{mg}, 0.222 \mathrm{mmol})$ followed by stirring $\mathrm{rt}$ for $22 \mathrm{~h}$. The reaction mixture was concentrated by rotary evaporation and the residue was suspended in acetonitrile $(10 \mathrm{~mL})$ for 1 day. The oxime ether-modified MMPCs then were washed by repeated centrifugal precipitation and supernatant removal steps using acetonitrile $(3 \times 10 \mathrm{~mL})$. The isolated $\mathrm{Au}\left(\mathrm{C}_{6} \mathrm{~S}\right)\left(\mathrm{AOT}_{\text {bis }}\right.$-urea-aldehyde) MMPCs $(70.2 \mathrm{mg})$ were characterized by $\mathrm{IR}$ and ${ }^{1} \mathrm{H}$ NMR spectroscopy.

\subsubsection{Place Exchange Reactions to Prepare $\mathrm{Au}(\mathrm{SG})\left(\mathrm{AOT}_{\text {bis }}{ }^{+}\right)$MMPCs}

Synthesis of AuSG MPCs is described in section 6.1.8. To prepare gold mixed monolayer-protected clusters (MMPCs) functionalized with mixtures of glutathiolate and $\mathrm{AOT}_{\text {bis }}{ }^{+}$, we performed the place exchange reactions on the Au(SG) MPCs. In a typical procedure, the $\mathrm{AOT}_{\text {bis }}{ }^{+}$ligand $(103 \mathrm{mg}, 0.221 \mathrm{mmol})$ and the AuSG MPCs $(51.5 \mathrm{mg}$, $0.055 \mathrm{mmol} \mathrm{SG}$ based on $33 \%$ TGA) were mixed under argon in nanopure $\mathrm{H}_{2} \mathrm{O}(2 \mathrm{~mL})$ and followed by stirring at $\mathrm{rt}$ for $4 \mathrm{~d}$. The resultant, crude $\mathrm{Au}(\mathrm{SG})\left(\mathrm{AOT}_{\text {bis }}{ }^{+}\right)$MMPCs were concentrated by rotary evaporation, suspended in acetonitrile $(10 \mathrm{~mL})$, and then purified by repeated centrifugation and supernatant removal (x3). Characterization by UV-VIS, IR (solid), and ${ }^{1} \mathrm{H}$ NMR revealed that the composition of the Au MMPCs was approximately $\mathrm{Au}_{\mathrm{x}}(\mathrm{SG})_{0.5}\left(\mathrm{AOT}_{\mathrm{bis}}{ }^{+}\right)_{0.5}$. 


\subsubsection{Oximation Reactions on $\mathrm{Au}(\mathrm{SG})\left(\mathrm{AOT}_{\text {bis }^{+}}{ }^{+}\right) \mathrm{MMPCs}$}

6.2.9.1. Ferrocenecarboxaldehyde adduct. Ferrocene carboxaldehyde $(31 \mathrm{mg}, 0.14$ mmol) was added to a solution of $\mathrm{Au}(\mathrm{SG})\left(\mathrm{AOT}_{\mathrm{bis}}{ }^{+}\right) \mathrm{MMPCS}(42.8 \mathrm{mg}, 0.0360 \mathrm{mmol}$ of $\left.\mathrm{AOT}_{\text {bis }}{ }^{+}\right)$dissolved in a 1:1 $\mathrm{H}_{2} \mathrm{O}: \mathrm{CH}_{2} \mathrm{Cl}_{2}$ mixture $(2.0 \mathrm{~mL})$ at $\mathrm{rt}$ with stirring. After $12 \mathrm{~h}$, the reaction mixtures were concentrated by rotary evaporation and the residues were suspended in acetonitrile $(10 \mathrm{~mL})$. The oxime ether adducts were isolated by centrifugal precipitation. The process of washing using acetonitrile followed by centrifugation was repeated two times. Then isolated $\mathrm{Au}(\mathrm{SG})\left(\mathrm{AOT}_{\text {bis }}{ }^{+}\right.$-ferrocene) MMPC $(43.6 \mathrm{mg})$ was characterized by FTIR and ${ }^{1} \mathrm{H}$ NMR spectroscopy.

\subsection{Experimental Procedures for Chapter 4}

\subsubsection{Materials and Methods}

$\mathrm{HAuCl}_{4} .3 \mathrm{H}_{2} \mathrm{O}$ was synthesized in-house to prepare $\mathrm{AuC}_{6} \mathrm{~S}$ MPC. $\mathrm{K}_{2} \mathrm{PdCl}_{4}$, glutathione, and 1-hexanethiol (Sigma-Aldrich), polystyrene beads (SupraSciences), amino-link coupling resin (ThermoFisher scientific, known as 'agarose bead') were purchased and used as received. ${ }^{1} \mathrm{H}$ NMR, FTIR, TGA, UV-Vis, and optical microscopy are the techniques used for the characterization of the synthesized materials.

\subsubsection{Optical Microscopic Image}

In order to take an optical image of the clusters, a thin glass cover slip was used. PSaldehyde beads (dissolved in THF), and agarose-aldehyde beads (dissolved in water) were simply added on top of the slip to take their corresponding images. Au or Pd cluster functionalized beads were also dissolved in the suitable solvent, sonicated as required and 
drop-cast deposited over the glass slip, followed by air drying for $30 \mathrm{~min}$. Optical images were taken at 10X magnification using Nikon Eclipse Ti-E inverted optical microscope with a halogen lamp light source.

\subsubsection{FTIR Measurements}

Infrared spectra were recorded on a Perkin-Elmer FTIR spectrophotometer with an attenuated total reflectance attachment. Approximately $5.0 \mathrm{mg}$ solid powder of PS aldehyde beads, $\mathrm{AuC}_{6} \mathrm{~S}$ reacted PS bead, Au cluster-functionalized PS beads and Pd cluster-functionalized agarose bead, and a pipette drop of agarose beads were used for recording the FTIR spectral measurements.

\subsubsection{UV-Vis Spectroscopy Measurements}

UV-Vis spectra were recorded on a Varian UV-Visible spectrophotometer, model CARY 50 Bio. All spectra were scanned from $300 \mathrm{~nm}$ to $800 \mathrm{~nm}$ at a fast scan rate using aqueous solutions or suspension of the obtained clusters or direct supernatants obtained after centrifugation. The background was subtracted using nanopure $\mathrm{H}_{2} \mathrm{O}$ as the blank.

\subsubsection{Thermogravimetric Analysis}

Material compositions of the synthesized Au or Pd clusters functionalized beads were determined by measuring the weight changes using a TA Instruments thermogravimetric analyzer, model TA 2050 , under a nitrogen atmosphere from $25{ }^{\circ} \mathrm{C}$ to $600{ }^{\circ} \mathrm{C}$ with a heating rate of $5{ }^{\circ} \mathrm{C} \mathrm{min}^{-1}$. TGA also was used to determine the change in the $\%$ weight loss of the $\mathrm{Pd}(\mathrm{SG})\left(\mathrm{AOT}_{\mathrm{bis}}{ }^{+}\right)$MMPCs.

\subsubsection{Place Exchange Reactions to Prepare Pd(SG)(AOT $\left.{ }_{\text {bis }}{ }^{+}\right)$MMPCs}

Synthesis of PdSG MPCs is described in section 6.1.9. To prepare Pd mixed monolayerprotected clusters (MMPCs) functionalized with mixtures of glutathiolate and $\mathrm{AOT}_{\mathrm{bis}}{ }^{+}$, 
we performed the place exchange reactions on the $\mathrm{Pd}(\mathrm{SG}) \mathrm{MPCs}$. In a typical procedure, the $\mathrm{AOT}_{\text {bis }}{ }^{+}$ligand $(298 \mathrm{mg}, 0.644 \mathrm{mmol})$ and the PdSG MPCs (170 mg, $0.161 \mathrm{mmol} \mathrm{SG}$ based on $29 \%$ TGA) were mixed under argon in nanopure $\mathrm{H}_{2} \mathrm{O}(3 \mathrm{~mL})$ and followed by stirring at rt for $7 \mathrm{~d}$. The resultant, crude $\mathrm{Pd}(\mathrm{SG})\left(\mathrm{AOT}_{\text {bis }}{ }^{+}\right) \mathrm{MMPCs}$ were concentrated by rotary evaporation, suspended in acetonitrile $(10 \mathrm{~mL})$, and then purified by repeated centrifugation and supernatant removal (x3). Characterization by UV-VIS and ${ }^{1} \mathrm{H}$ NMR revealed that the composition of the synthesized Pd MMPCs was approximately $\mathrm{Pd}_{\mathrm{x}}(\mathrm{SG})_{0.5}\left(\mathrm{AOT}_{\text {bis }}{ }^{+}\right)_{0.5}$.

\subsubsection{Synthesis of Au Cluster Functionalized Polystyrene Beads}

Synthesis of $\mathrm{Au}\left(\mathrm{C}_{6} \mathrm{~S}\right)\left(\mathrm{AOT}_{\text {bis }}\right)$ MMPCs is described in 6.2.5. We prepared $\mathrm{Au}$ cluster functionalized polystyrene beads by straightforward addition of $\mathrm{Au}\left(\mathrm{C}_{6} \mathrm{~S}\right)\left(\mathrm{AOT}_{\text {bis }}\right)$ MMPCs to PS-CHO beads. In a typical reaction, $\mathrm{Au}\left(\mathrm{C}_{6} \mathrm{~S}\right)\left(\mathrm{AOT}_{\mathrm{bis}}\right)(30 \mathrm{mg}, 0.0217$ mmol $\mathrm{AOT}_{\text {bis }}$ based on the composition of $\left.\mathrm{Au}_{144}\left(\mathrm{C}_{6} \mathrm{~S}\right)_{30}\left(\mathrm{AOT}_{\text {bis }}\right)_{30}\right)$ and the PS-CHO beads $(10.5 \mathrm{mg}, 0.0105 \mathrm{mmol}$ based on $1.0 \mathrm{mmol}-\mathrm{CHO}$ per $\mathrm{g}$ of bead) were mixed under argon in dry THF $(1 \mathrm{~mL})$ and followed by stirring at $\mathrm{rt}$ for $12 \mathrm{~h}$. The resultant, crude Au cluster functionalized PS beads were centrifuged by adding excess THF, and the resultant precipitate was further washed with THF $(10 \mathrm{~mL}, 3 \mathrm{x})$ to remove any unreacted MMPCs, and then purified by repeated centrifugation and supernatant removal (x3). Characterization by UV-VIS revealed that this Au cluster functionalized bead is indeed aggregated. However, the characterization data from FTIR (solid) confirmed that the oximation reaction was successful. As a control study, we reacted non-aminooxy $\mathrm{AuC}_{6} \mathrm{~S}$ MPCs with the same beads in the same 2:1 ratio $\left(\mathrm{C}_{6} \mathrm{~S}: \mathrm{CHO}\right) . \mathrm{Au}_{6} \mathrm{~S}$ MPCs $(30.0 \mathrm{mg}, 54$ $\mu \mathrm{mol} \mathrm{C}_{6} \mathrm{~S}$ based on TGA data of $21 \%$ organic species) and the PS-CHO beads $(27.5 \mathrm{mg}$, 
$27 \mu$ mol-CHO based on $1.0 \mathrm{mmol}-\mathrm{CHO}$ per $\mathrm{g}$ of bead) were mixed under argon in dry THF ( $1 \mathrm{~mL})$ and followed by stirring at $\mathrm{rt}$ for $12 \mathrm{~h}$.

\subsubsection{Synthesis of Pd Cluster Functionalized Agarose Beads}

We prepared Pd cluster functionalized polystyrene beads by simple mixing $\mathrm{Pd}(\mathrm{SG})\left(\mathrm{AOT}_{\mathrm{bis}^{+}}{ }^{+}\right)$MMPCs and agarose-CHO beads. In a typical reaction, $\mathrm{Pd}(\mathrm{SG})\left(\mathrm{AOT}_{\mathrm{bis}}{ }^{+}\right) \mathrm{MMPCs}\left(2.0 \mathrm{mg}, 1.082 \mu \mathrm{mol} \mathrm{AOT}_{\mathrm{bis}}{ }^{+}\right.$based on $\mathrm{TGA}$ data of $50 \%$ organic species) and the agarose-CHO beads $(0.27 \mathrm{~mL}, 10.8 \mu \mathrm{mol}$ based on $40 \mu \mathrm{mol}-$ $\mathrm{CHO}$ per $\mathrm{mL}$ of bead) were mixed under argon in nanopure water $(2 \mathrm{~mL})$ and followed by stirring at rt for $12 \mathrm{~h}$. Any unreacted $\mathrm{AOT}_{\text {bis }}{ }^{+}$that may have remained on the MPC surface was passivated by adding acetone $(10 \mu \mathrm{L})$ to the reaction mixture and further stirred for 1 hour. The resultant, crude Pd cluster functionalized agarose beads could be isolated by settling down the precipitate, and supernatant removal. To completely remove the unreacted MMPCs, it was centrifuged by adding excess $\mathrm{H}_{2} \mathrm{O}$. The UV-VIS of both supernatant and precipitate were measured. Characterization by UV-VIS, FTIR (solid), and ${ }^{1} \mathrm{H}$ NMR confirmed that the oximation reaction was successful. This functionalized bead was soluble in water in a form of suspension. Varying ratios of $\mathrm{AOT}_{\text {bis }}{ }^{+}$present on MPC surface: CHO (such as 1:1, 1:100) were also used to prepare Pd cluster bead with different MPCs concentration on the surface. As a control study, PdSG MPC only was reacted with agarose aldehyde beads in the same 1:10 ratio (SG: $\mathrm{CHO}$ ). In this reaction, PdSG MPCs (2.0 mg, $1.89 \mu \mathrm{mol}$ SG based on TGA data of $29 \%$ organic species $)$ and the agarose-CHO beads $(0.47 \mathrm{~mL}, 18.9 \mu \mathrm{mol}$ based on $40 \mu \mathrm{mol}-\mathrm{CHO}$ per $\mathrm{mL}$ of bead) were mixed under argon in nanopure water $(2 \mathrm{~mL})$ and followed by stirring at $\mathrm{rt}$ for $12 \mathrm{~h}$. 


\subsection{Experimental Procedures for Chapter 5}

\subsubsection{Materials}

$\mathrm{HAuCl}_{4} \cdot 3 \mathrm{H}_{2} \mathrm{O}$ was synthesized from metallic bulk gold according to the literature. Sodium borohydride ( $\geq 98.5 \%$ reagent grade), 4-nitrophenol solution (10 mM in THF), Lglutathione reduced $(\geq 98.0 \%)$, iodine $(\geq 99.8 \%)$, ACS grade methanol, tetrahydrofuran, ethanol, acetone, 2-propanol (99.9\%), allyl alcohol (99\%), propionaldehyde (99\%), potassium tetrachloropalladate(II) (99\%), chlorobenzene (98\%), and sodium hydroxide were purchased and used as received. Deuterium oxide and methanol- $\mathrm{d}_{4}$ were purchased from Cambridge Isotope Laboratories. $\mathrm{N}_{2}$ and $\mathrm{H}_{2}$ gas cylinders were purchased from Welders Supply (Louisville, KY). Indium-tin-oxide-coated glass slides with a resistivity Rs $=8-12 \Omega$ were purchased from Delta Technologies, Limited (Loveland, CO). Water was purified using a Barnstead water ultra-purification system (ThermoFisher; Rs $=18.2$ $\mathrm{M} \Omega \bullet \mathrm{cm})$.

\subsubsection{Electron Microscopy Measurements}

Transmission electron microscopy (TEM) images were obtained using a FEI Tecnai F20 FEG operated in TEM mode with an acceleration voltage of $200 \mathrm{kV}$. TEM data analysis was carried out using digital micrograph software (version 3.11.0) and ImageJ software for particles size analysis. TEM samples of water-soluble glutathione-capped $\mathrm{Au}$ and $\mathrm{Pd}$ MPCs were prepared by dissolving in water $(0.5 \mathrm{mg} / 1 \mathrm{~mL})$ and then drop casting the aqueous solution $(10 \mu \mathrm{L})$ on a 400 mesh Formvar/carbon-coated copper grid, letting it air-dry for at least 4 hours. Scanning electron microscopy (SEM) images were taken using FEI, Nova600 FEG-SEM model with acceleration voltage of $12 \mathrm{kV}$. The average 
particle size was determined by using ImageJ software. SEM samples were prepared by soaking the ITO slides in solutions of the iodine-activated AuSG MPCs for 15 minutes, drying with $\mathrm{N}_{2}$, and then imaging the conductive side of the ITO slides for size analyses.

\subsubsection{Thermogravimetric Analysis}

Material compositions of the synthesized AuSG and PdSG MPCs were determined by measuring the weight changes using a TA Instruments thermogravimetric analyzer, model TA 2050, under a nitrogen atmosphere from $25^{\circ} \mathrm{C}$ to $800{ }^{\circ} \mathrm{C}$ with a heating rate of

$5{ }^{\circ} \mathrm{C} \mathrm{min}{ }^{-1}$ for AuSG MPCs and from $25{ }^{\circ} \mathrm{C}$ to $600{ }^{\circ} \mathrm{C}$ with a heating rate of $5{ }^{\circ} \mathrm{C} \mathrm{min}{ }^{-1}$ for PdSG MPCs. TGA also was used to determine the change in the \% weight loss upon treatment of PdSG MPCs with different equivalents of $\mathrm{I}_{2}$. For this study, the MPCs were prepared as follows: PdSG MPCs $(20.5 \mathrm{mg})$ were dissolved in nanopure water $(2 \mathrm{~mL})$ followed by addition of varying equivalents of $\mathrm{I}_{2}$ (e.g., $24 \mu \mathrm{L}$ of a $20 \mathrm{mM}$ methanolic $\mathrm{I}_{2}$ solution for addition of $0.025 \mathrm{eq}$ ). The mixture was reacted at room temperature for 3 hours, whereupon the MPCs were precipitated by addition of THF $(2 \mathrm{~mL})$ followed by sedimentation via centrifugation (4000 rpm, $10 \mathrm{~min}$ ). The supernatant was removed and fresh THF ( $2 \mathrm{~mL}$ ) was added to rinse the MPCs followed by centrifugation. The MPCs were rinsed using this process one additional time for complete removal of disulfide species prior to analysis by TGA.

\subsubsection{X-ray Photoelectron Spectroscopy (XPS) Measurements}

X-ray photoelectron spectroscopy (XPS) analyses were performed using a VG Scientific MultiLab 3000 ultra-high vacuum surface analysis system that is equipped with a dualanode $(\mathrm{Mg} / \mathrm{Al}) \mathrm{X}$-ray source and a CLAM4 hemispherical electron energy analyzer. The 
measurements were conducted using non-monochromatized $\mathrm{Al} \mathrm{K}_{\alpha} \mathrm{X}$-ray radiation ( $\mathrm{h} v$ $\approx 1486.6 \mathrm{eV})$ as the $\mathrm{X}$-ray source and at a base pressure in the $10^{-9}$ Torr range. XPS spectra were collected at an electron emission angle of $54.7^{\circ}$ relative to the surface normal. Aqueous solutions $(200 \mu \mathrm{L})$ of AuSG $(4 \mathrm{mg} / \mathrm{mL})$ and PdSG $(3 \mathrm{mg} / \mathrm{mL})$ were drop-cast deposited on top of black carbon tape and air-dried for 2 hours before loading. Survey spectra were recorded between 50-450 eV BE for N, S, Pd and Au and between 610-640 eV BE for I.

\subsubsection{FTIR Measurements}

Infrared spectra were recorded on a Perkin-Elmer FTIR spectrophotometer with an attenuated total reflectance attachment. $10 \mathrm{mg}$ solid powder of PdSG or AuSG MPCs powder were used for their respective measurements.

\subsubsection{UV-Vis Spectroscopy Measurements}

UV-Vis spectra were recorded on a Varian UV-Visible spectrophotometer, model CARY 50 Bio. All spectra were scanned from $300 \mathrm{~nm}$ to $800 \mathrm{~nm}$ at a fast scan rate using aqueous solutions of AuSG MPCs in a quartz cuvette. The background was subtracted using nanopure $\mathrm{H}_{2} \mathrm{O}$ as the blank. First, AuSG MPCs (2.1 mg) were dissolved in nanopure $\mathrm{H}_{2} \mathrm{O}(20 \mathrm{~mL})$. Then, $1.5 \mathrm{~mL}$ of this stock solution was added to a cuvette and diluted to $3.0 \mathrm{~mL}$ final volume (diluted with $\mathrm{H}_{2} \mathrm{O}$ and/or methanolic $\mathrm{I}_{2}$ solutions) for the UV-Vis recordings. Based on the TGA data (33\% organic composition, $1.7 \times 10^{-4} \mathrm{mmol}$ thiolates), $1.43 \mathrm{mM}$ methanolic $\mathrm{I}_{2}$ solution was prepared, and $30 \mu \mathrm{L}$ to $240 \mu \mathrm{L}$ of the prepared solution was added to the cuvette to afford 0.25 eq to 2.0 eq $\mathrm{I}_{2}: \mathrm{SG}$ ratios, respectively. For PdSG MPCs, $10 \mu \mathrm{l}$ aliquots of the aqueous solution from the reaction 
mixture were diluted to $3 \mathrm{~mL}$ using nanopure water for their measurements after consecutive cycles for each $\mathrm{I}_{2}$ addition.

\subsection{7. ${ }^{1}$ H NMR Measurements}

${ }^{1} \mathrm{H}$ NMR spectra were recorded on a Varian $400 \mathrm{MHz}$ spectrometer using $\mathrm{D}_{2} \mathrm{O}$ as the solvent. AuSG MPCs (40 mg) or PdSG MPCs (40 mg) were dissolved in $450 \mu \mathrm{L} \mathrm{D} \mathrm{D}_{2} \mathrm{O}$ and the resultant solution was added to an NMR tube. To the MPCs solution in the NMR tube was added a solution of $t$-butanol (internal standard) in $\mathrm{D}_{2} \mathrm{O}(50 \mu \mathrm{L}$ of a $0.38 \mathrm{M}$ solution, $0.019 \mathrm{mmol})$. Deuterated methanol $(100 \mu \mathrm{L})$ containing different amounts of $\mathrm{I}_{2}$ was added to the MPCs solution and the mixtures were allowed to react for 40 minutes before measuring spectra. For example, the NMR tube reaction of AuSG MPCs with 0.25 eq $\mathrm{I}_{2}$ contained $40 \mathrm{mg}$ of AuSG MPCs in $450 \mu \mathrm{L} \mathrm{D} \mathrm{D}_{2} \mathrm{O}, 50 \mu \mathrm{L}$ of a $0.38 \mathrm{M} t$-butanol solution in $\mathrm{D}_{2} \mathrm{O}$, and $100 \mu \mathrm{L}$ of a $0.106 \mathrm{M} \mathrm{I}_{2}$ solution in $\mathrm{CD}_{3} \mathrm{OD}$ (0.0106 mmol). Stoichiometric ratio of 1:2 was used in the reaction between GSH and $\mathrm{I}_{2}$, while for additions of $\mathrm{K}_{2} \mathrm{PdCl}_{4}$, the stochiometric ratios between salt and $\mathrm{GSH}$ was kept at 1:1.

\subsubsection{Electrochemical Measurements}

The oxidation potential of Au SG MPCs after iodine activation was measured by anodic stripping voltammetry (ASV) in $40 \mathrm{~mL}$ of $0.01 \mathrm{M} \mathrm{KBr}+0.1 \mathrm{M} \mathrm{KClO}_{4}$ electrolyte solution using a $\mathrm{CH}$ Instruments (Austin, TX) electrochemical workstation, model CHI 660A, with a glass/ITO/Au SG MPCs working electrode, Pt wire counter electrode, and $\mathrm{Ag} / \mathrm{AgCl}(3 \mathrm{M} \mathrm{KCl})$ reference electrode. Indium-tin-oxide (ITO)-coated glass slides were sonicated for $30 \mathrm{~min}$ each in acetone, ethyl alcohol, and 2-propanol before drying under $\mathrm{N}_{2}$. Solutions of $\mathrm{Au}$ SG MPCs without added iodine or with varying equivalents $(0.25$, 
0.5, 1.0 and 2.0) of added iodine were freshly prepared and allowed to react for $3 \mathrm{~h}$. Cleaned ITO slides were soaked in the respective solutions for 30 minutes before measuring the ASV. Each ASV scan was performed from $0 \mathrm{~V}$ to $1.2 \mathrm{~V}$ at a scan rate of $0.01 \mathrm{~V} / \mathrm{s}$.

\subsubsection{Gas Chromatography Measurements}

The progress of the PdSG MPCs catalyzed reaction was monitored by gas chromatography (GC). The GC data was recorded on a Buck Scientific model 910 GC equipped with a 1/8th inch packed column (10\% Carbowax 20M on silica 80/100 mesh, 6 foot) using a flame ionization detector (FID) and helium as the carrier gas. The method developed for GC includes injecting $1 \mu \mathrm{L}$ of sample solution and using a temperature gradient for elution: hold at $80{ }^{\circ} \mathrm{C}$ for $2 \mathrm{~min}$, ramp at $25^{\circ} \mathrm{C} / \mathrm{min}$ to $135^{\circ} \mathrm{C}$ and then hold for $1 \mathrm{~min}$. The pressure was varied from a start of 12 psi helium, holding for $3 \mathrm{~min}$, to 14 psi helium for $2 \mathrm{~min}$.

\subsubsection{4-Nitrophenol Reduction}

A solution of 4-nitrophenol (4-NP) (0.5 mL of $0.3 \mathrm{mM}$ aq. solution, $0.00015 \mathrm{mmol})$ was added to nanopure $\mathrm{H}_{2} \mathrm{O}\left(4.14 \mathrm{~mL}\right.$; this volume varies based on whether an $\mathrm{I}_{2}$ solution is added at a later stage). Then, $\mathrm{NaOH}(60 \mu \mathrm{L}$ of $20 \mathrm{mM}$ aq. solution, $0.0012 \mathrm{mmol}, 8$ eq. $)$ was added to give a bright yellow solution and followed by addition of $\mathrm{NaBH}_{4}(150 \mu \mathrm{L}$ of $0.1 \mathrm{M}$ aq. solution, $0.015 \mathrm{mmol}, 100 \mathrm{eq})$. Finally, a solution of AuSG MPCs $(150 \mu \mathrm{L}$ of a stock solution, prepared by dissolving $2.0 \mathrm{mg}$ AuSG MPCs in $20 \mathrm{~mL}$ nanopure water, $5.1 \times 10^{-5} \mathrm{mmol} \mathrm{Au}, 0.34$ eq of $\mathrm{Au}$ ) was added to the reaction mixture to obtain a total reaction mixture volume of $5.0 \mathrm{~mL}$. The reaction mixture was maintained at room 
temperature for 30 seconds before removing an aliquot $(3 \mathrm{~mL})$ for $\mathrm{UV}$ absorption measurements in a clean quartz cuvette, recording the absorption spectrum between 250 to $750 \mathrm{~nm}$. Absorption measurements were taken every 1 min or longer as required.

For additions of $\mathrm{I}_{2}$ to the reaction mixture, a methanolic stock solution of $\mathrm{I}_{2}(0.0539 \mathrm{mM})$ was first prepared and the corresponding $\mathrm{I}_{2}$ equivalents $(75 \mu \mathrm{L}$ of stock solution $=0.25$ equivalents, based on glutathiolate calculated using an organic composition determined for the AuSG MPCs by TGA of 33\%) were then added to the reaction vial. In each reaction examined, the final total reaction volume was maintained at $5.0 \mathrm{~mL}$ by adjusting the amount of water used in the first step.

To assess the recyclability of the Au SG MPCs, fresh 4-NP ( $0.015 \mathrm{~mL}$ of $10 \mathrm{mM}$ solution in THF, $0.00015 \mathrm{mmol}$ ) was added to the final reduction mixture of the first cycle in the cuvette within 2 minutes after the completed 4-NP reduction. UV-vis measurements then were resumed until the added 4-NP was completely reduced. This cycle was repeated until the 4-NP absorbance remained unchanged. A product removal approach was used to assess the effect of product accumulation on the recyclability of the catalyst. In this experiment, to a solution of 4-NP $(4.8 \mathrm{~mL}$ of a $0.0042 \mathrm{M}$ aq. solution, $0.02 \mathrm{mmol})$ were added successively $\mathrm{NaOH}$ ( $50 \mu \mathrm{L}$ of $3.2 \mathrm{M}$ aq. solution, $0.16 \mathrm{mmol}, 8$ eq.), $\mathrm{NaBH}_{4}$ (150 $\mu \mathrm{L}$ of $13.3 \mathrm{M}$ aq. solution, $2.00 \mathrm{mmol}, 100 \mathrm{eq}$ ), and AuSG MPCs (2.0 mg AuSG MPCs, $6.8 \times 10^{-3} \mathrm{mmol} \mathrm{Au}, 0.34$ eq of $\left.\mathrm{Au}\right)$. A methanolic stock solution of $\mathrm{I}_{2}(11.5 \mathrm{mM})$ was first prepared and the corresponding $\mathrm{I}_{2}$ equivalents $(75 \mu \mathrm{L}$ of stock solution $=0.5$ equivalents), were then added to the reaction mixture. Within a minute, an aliquot (22.5 $\mu \mathrm{L}$ ) of the reaction mixture was added into a quartz cuvette and diluted with nanopure water $(3 \mathrm{~mL})$ for $\mathrm{UV}$ absorption measurement. Upon the completion of first reaction 
cycle, the products were removed via centrifugation (2-fold THF, $10 \mathrm{~mL}$ ) for $15 \mathrm{~min}(3 \mathrm{x})$ and the precipitated AuSG MPCs were immediately reused for the next cycle.

\subsubsection{Allyl Alcohol Hydrogenation/Isomerization (Homogenous System)}

The hydrogenation/isomerization reaction was performed by dissolving PdSG MPCs (6.0 $\mathrm{mg}$ ) in nanopure water $(2 \mathrm{~mL})$ in a $10 \mathrm{~mL}$ glass vial fitted with a stir bar. To the solution was added chlorobenzene $(2 \mathrm{~mL})$ and allyl alcohol $(200 \mu \mathrm{L})$ to form a biphasic reaction mixture. The reaction mixture was then stirred at $800-1000 \mathrm{rpm}$ at room temperature. Hydrogen gas was purged into the reaction mixture at a flow rate of $20.0 \pm 0.5 \mathrm{~mL} / \mathrm{min}$ through a glass pipette. Stirring was ceased and aliquots $(20 \mu \mathrm{L})$ were removed from both the aqueous and organic phase at 5 min intervals or as required. MPCs in the aqueous phase aliquot were precipitated by adding THF $(40 \mu \mathrm{L})$ and then centrifuged at $4000 \mathrm{rpm}$ for $10 \mathrm{~min}$. For additions of $\mathrm{I}_{2}$ to the reaction mixture, a fresh methanolic stock solution of $\mathrm{I}_{2}(5 \mathrm{mM})$ was first prepared and the corresponding $\mathrm{I}_{2}$ equivalents (e.g., $28 \mu \mathrm{L}$ of $5 \mathrm{mM}$ stock solution $=0.025$ equivalents relative to $\mathrm{SG}$ ligands) were then added to the reaction vial. For the $\mathrm{I}_{2}$-catalyzed reactions, the final total reaction volume was kept at $4.0 \mathrm{~mL}$ by adjusting the $2.0 \mathrm{~mL}$ of water used in the first step.

For recycling experiments, the products were extracted into chlorobenzene $(4.0 \mathrm{~mL})$ and the organic layer was then removed. This process was repeated two more times to ensure full removal of the products. UV-Vis and GC analyses of the aqueous layer were performed to confirm that no products remained in the aqueous phase. For each consecutive cycle, fresh substrate $(200 \mu \mathrm{L})$ and chlorobenzene $(2 \mathrm{~mL})$ were added. To

compensate the volume loss, nano-pure water also was added as required in the 
consecutive cycles. The cycles were repeated until the reaction required more than 60 minutes to complete.

\subsubsection{Allyl Alcohol Hydrogenation/Isomerization (Heterogenous System)}

In a typical hydrogenation/isomerization reaction, $2 \mathrm{~mL}$ aqueous suspension of the $\mathrm{Pd}$ cluster functionalized beads ( $2 \mathrm{mg} \mathrm{Pd}$ content), and $2 \mathrm{~mL}$ chlorobenzene were added (total $4.0 \mathrm{~mL}$ nanopure $\mathrm{H}_{2} \mathrm{O}$ in a monophasic condition) in a $5 \mathrm{~mL}$ scintillation glass vial. To the biphasic mixture was added $200 \mu \mathrm{L}$ of allyl alcohol and let the reaction mixture stirred at 800-1000 rpm at room temperature. Hydrogen gas was purged into the reaction mixture at a flow rate of $20.0 \pm 0.5 \mathrm{~mL} / \mathrm{min}$ through a glass pipette. Stirring was ceased in a particular time interval to withdraw the aliquots $(20 \mu \mathrm{L})$ from both the aqueous and organic phase (or one aliquot for monophasic condition). For MPCs in the aqueous phase aliquot, it was allowed to settle down at room temperature and clear supernatant was used for the purpose of the analysis. For experiment with $\mathrm{I}_{2}$ additions to the reaction mixture, a fresh methanolic stock solution of $\mathrm{I}_{2}(5 \mathrm{mM})$ was first prepared and the corresponding $\mathrm{I}_{2}$ equivalents (e.g., $224 \mu \mathrm{L}$ of $5 \mathrm{mM}$ stock solution $=0.2$ equivalents relative to $\mathrm{SG}$ ligands) were then added to the reaction vial.

For recycling experiments, the products were extracted into organic phase and the obtained organic layer was then removed. This process was repeated two more times to ensure full removal of the products. For each consecutive cycle, fresh substrate $(200 \mu \mathrm{L})$ and chlorobenzene $(2 \mathrm{~mL})$ were added. All the collected aliquots were subjected to the GC-FID measurements to monitor the progress of the reactions. 


\section{CHAPTER 7 \\ SUMMARY AND FUTURE DIRECTIONS}

\subsection{Summary}

In summary, this dissertation presents strategies to synthesize aminooxy MMPCs, nanomaterial characterization techniques, particle stability studies, and catalytic activation of MPCs. We synthesized several classes of aminooxy thiol ligands for incorporation onto $\mathrm{Au}$ or $\mathrm{Pd}$ clusters. First, a trifunctional bis-aminooxy amino thiol $\left(\mathrm{AOT}_{\text {bis }}\right)$ ligand was prepared to exploit its functionalization on thiolated-MPCs via ligand-place exchange as discussed in Chapters 2 and 3. Au and Pd MPCs were synthesized by following the literature with slight modification using a suitable stabilizer. The $\mathrm{AOT}_{\text {bis }}$ ligand was used to coat $\mathrm{AuC}_{6} \mathrm{~S}$ MPCs to afford $\mathrm{Au}\left(\mathrm{C}_{6} \mathrm{~S}\right)(\mathrm{AOT})_{\text {bis }}$ MMPCs, followed by the subsequent oximation reactions with various commercially available aldehydes. All the adducts were fully characterized by using several techniques such as ${ }^{1} \mathrm{H}$ NMR, FTIR, UV-Vis, CV, MALDI-TOF-MS, and fluorescence. Proton NMR and FTIR spectra of all the obtained adducts confirmed the successful oximation reactions, demonstrating the usefulness of "click-chemistry" in adorning the surface of nanomaterials by simple mixing operations. The unique properties of the $\mathrm{Au}\left(\mathrm{C}_{6} \mathrm{~S}\right)\left(\mathrm{AOT}_{\text {bis }}\right)$ were investigated to understand the tunability of such MMPCs. Protonation of the amine group resulted in water-soluble MMPCs. This study demonstrated that the synthesis of a water-soluble ligand, $\mathrm{AOT}_{\text {bis }}{ }^{+}$, or water-soluble MPC (for example, AuSG) is not essential. AOT itself can be tuned to be organic or watersoluble by controlling $\mathrm{pH}$. Overall, the studies discussed in Chapter 2 and 3 describes the 
feasible functionalization of aminooxy ligands on the MPC surface and the chemoselective reactions of aminooxylated MPCs with various aldehydes/ketones.

In addition to demonstrating the simple click reaction with various small molecule aldehydes, we also showed in Chapter 4 that aminooxylated MMPCs react with aldehyde-functionalized microbeads, both polystyrene-CHO and agarose-CHO. For this work, aldehyde-activated polystyrene and agarose beads were reacted with $\mathrm{Au}\left(\mathrm{C}_{6} \mathrm{~S}\right) \mathrm{AOT}_{\text {bis }}$ MMPCs and $\mathrm{Pd}(\mathrm{SG})\left(\mathrm{AOT}_{\text {bis }}{ }^{+}\right)$MMPCs, respectively to obtain the $\mathrm{Au}$ cluster and Pd cluster functionalized bead, which could have potential applications in heterogenous catalysis as described in Chapter 5. The initial studies suggested that the attachment of MMPCs on the solid-support was successful based on visual observation, microscopy, UV-vis spectroscopy, and FTIR spectroscopy.

Aminooxylated MPCs have potential applications in both catalysis and sensing. Pd-cluster functionalized agarose beads, synthesized by oxidation reaction of aminooxyfunctionalized Pd MMPCs with agarose-CHO microbeads, were tested for catalyzing the hydrogenation/isomerization of allyl alcohol. While some reactivity occurred, the rates were very slow, which we attributed to passivation of the Pd surface with the thiolate ligands. To address this, we demonstrated catalytic activation of thiolate-coated MMPCs by addition of $I_{2}$, as described in Chapter 5 . First, we studied the homogeneous catalytic activity of non-aminooxylated MPCs (AuSG and PdSG) to understand the thiol activation chemistry. Our studies ${ }^{53}$ show some initial catalysis, however, we hypothesized that the catalytic activity could be enhanced further by partial thiolate ligand removal from the passivated MPC surface. For this, we introduced the addition of iodine $\left(\mathrm{I}_{2}\right)$ as a novel activation concept for catalytic enhancement, as discussed in Chapter $5 .{ }^{102}$ Low levels of 
$\mathrm{I}_{2}$ were used to 'knock-off' bound thiol ligands from the surface of MPCs via disulfide formation as indicated by the ${ }^{1} \mathrm{H}$ NMR studies conducted by Murray and coworkers and confirmed in our own studies for Au. In a different mechanism, the bound ligands dissociated from Pd MPCs by forming Pd(II)-thiolates. In our studies, we utilized the ligand removal to improve catalytic performance by testing two model reactions: (1) 4nitrophenol (4-NP) reduction by $\mathrm{NaBH}_{4}$ catalyzed by AuSG MPCs, and (2) allyl alcohol hydrogenation/isomerization by PdSG MPCs. Different equivalents of $I_{2}$ (based on the organic weight $\%$ obtained from TGA analyses) were added at the beginning of the catalytic cycle. We found that $\mathrm{I}_{2}$ enhances the catalytic activity 7 -fold in the case of the AuSG MPCs and 2-fold for PdSG MPCs. Although the respective rate constants (k) or TOF values improved, the $\mathrm{I}_{2}$-promoted enhancement approach suffered from poor recyclability when higher equivalents of $I_{2}$ were added, mostly due to the aggregation or dissolution of the particles. Aggregation could potentially be prevented by attaching the catalytic metal clusters to microbeads and then performing $I_{2}$ activation, but this was not optimized.

\subsection{Scientific Impact}

In this dissertation, we present our findings on the ease of Au MPC functionalization with aminooxy thiol ligands and discussed the strategy to prepare solidsupported catalysts. A ligand removal strategy ( $\mathrm{I}_{2}$ activation) was introduced for the first time as a means to enhance the catalytic activity on metal MPC surfaces. Overall, our research provides a functional nanomaterial that has applications in catalysis and potentially in sensing. Our effort could potentially reduce the cost of catalyst preparation in industry and also provide insight into the development of sensors for VOCs detection. 
Possible ultrasensitive detection of VOCs could be useful in medical and environmental applications, such as monitoring air quality and diagnosing early stage lung cancer.

\subsection{Future Directions}

The next step of this research would have been to test $I_{2}$ activation on the agarose microbeads functionalized with aminooxy-functionalized Pd or Au MMPCs. Both metals could have been used and the beads tested as a heterogeneous catalytic material for the reduction of 4-NP or hydrogenation/isomerization of allyl alcohol, both with and without $\mathrm{I}_{2}$ activation. This would require optimization of MPC loading and $\mathrm{I}_{2}$ activation conditions with thorough characterization of the MMPC-functionalized beads. The goal would-be long-term recyclability with high catalytic activity. As discussed in Chapter 2, other types of aminooxy ligands, such as straight chain non-amino thiol ligand (aminooxy hexanethiol), were synthesized for potential use in sensing applications. Our group has a strong interest in developing sensors for the detection of VOCs with high sensitivity and selectivity. Previous works from the Nantz and Fu groups showed that aminooxyfunctionalized MPC-based chemiresistors are promising for the detection of carbonyl VOCs, such as acetone. ${ }^{140}$ Aminooxy chemistry is also promising for breath analysis of carbonyl-containing molecules by mass spectrometry. ${ }^{17}$ The Zamborini group has interest in the development of metal cluster or nanoparticle-based SERS sensors. Previous studies from our group showed strong Raman scattering signals for molecules in the vicinity of electrodeposited Ag nanoneedles on the surface of Au films. ${ }^{187}$ 


\section{REFERENCES}

1. Kolb, H. C.; Finn, M. G., Sharpless K. B. Angew. Chem. Int. Ed. 2001, 40, 20042021.

2. Thygesen, M. B.; Sørensen, K. K.; Cló, E.; Jensen, K. J. Chem. Commun., 2009, 42, 6367-6369.

3. Nagahori, N., Abe, M.; Nishimura, S. I. Biochemistry 2009, 48, 583-594.

4. $\quad$ Moses, J. E.; Moorhouse, A. D. Chem. Soc. Rev. 2007, 36, 1249-1262.

5. Huisgen, R. Angew. Chem. Int. Ed. 1963, 2, 565-598.

6. Sibakoti, Tirtha Raj. "Synthesis and characterization of carborane-appended biomolecules." (2015). Dissertation and Theses. Paper 12047.

7. Amantini, D.; Fringuelli, F.; Piermatti, O.; Tortoioli, S.; Vaccaro, L. ARKIVOC 2003, 2002, 293-311.

8. Hoyle, C. E.; Bowman, C. N. Angew. Chem. Int. Ed. 2010, 49, 1540-1573.

9. (a) Glover, S. A.; Mo, G. J. Chem. Soc., Perkin Trans. 2002, 2, 1728-1739; (b) Apfel, C.; Banner, D. W.; Bur, D.; Dietz, M.; Hirata, T.; Hubschwerlen, C.; Malcolm, H. L.; Page, M. G. P.; Pirson, W.; Specklin, J.-L. J. Med. Chem. 2000, 43, 2324-2331; (c) Langlois, N.; Moro, A. Eur. J. Org. Chem. 1999, 1999, 34833488; (d) Duan, J. J.-W.; Lu, Z.; Xue, C.-B.; He, X.; Seng, J. L.; Roderick, J. J.; Wasserman, Z. R.; Liu, R.-Q.; Covington, M. B.; Magolda, R. L.; Newton, R. C.; Trzaskos, J. M.; Decicco, C. P. Bioorg. Med. Chem. Lett. 2003, 13, 2035-2040. 
10. Edwards, J. O.; Pearson, R. G. J. Am. Chem. Soc. 1962, 84, 16-24.

11. Kool, E, T.; Crisalli, P.; Chan, K. M. Org Lett. 2014, 16, 1454-1457.

12. Rose, K. J. Am. Chem. Soc. 1994, 116, 30-33.

13. Pifferi, C; Daskhan, G. C; Fiore, M, Shiao, T.C; Roy, R; Renaudet, O. Chem. Rev. 2017, 117, 9839-9873

14. Dirksen, A.; Dawson, P. E. Bioconjugate Chem. 2008, 19, 2543-2548.

15. Bahta, M.; Liu, F.; Kim, S-E.; Stephen, A. G.; Fisher, R. G.; Bruke, Jr, T. R. Nat. Protoc. 2012, 7, 686-702.

16. Laulhe, Sebastien, "Aminooxy reagents for synthesis and analysis: expanding the role of oximation." (2013). Electronic Theses and Dissertations. Paper 796.

17. Li, M.; Biswas, S.; Nantz, M.H.; Higashi, R.M.; Fu, X-A. Anal. Chem 2012, 84, 1288-1293.

18. Grochowski, E.; Jurczak, J. Synthesis 1976, 682-684.

19. Varnavski, O.; Ramakrishna, G.; Kim, J.; Lee, D.; Goodson III, T. ACS Nano 2010, 4, 3406-3412.

20. Schwartzberg, A. M.; Zhang, J. Z. J. Phys. Chem. C 2008, 112, 10323-10337.

21. Lee, D.; Donkers, R. L.; Wang, G.; Harper, A. S.; Murray, R. W. J. Am. Chem. Soc. 2004, 126, 6193-6199.

22. Hicks, J. F.; Zamborini, F. P.; Murray, R. W. J. Phys. Chem. B 2002, 106, 77517757. 
23. Gréget, A.; Nealon, G. L.; Vileno, B.; Turek, P.; Mény, C.; Ott, F.; Derory, A.; Voirin, E.; Riviére, E.; Rogalev, A.; Wilhelm, F.; Joly, L.; Knafo, W.; Ballon, G.; Terazzi, E.; Kappler, J-P.; Donnio, B.; Gallani, J-L. Chem Phys Chem 2012, 13, 3092-3097.

24. Bönnemann, H.; Richards, R. Eur. J. Inorg. Chem. 2001, 2001, 2455-2480.

25. Pankhurst, Q. A.; Thanh, N. T. K.; Jones, S. K.; Dobson, J. J. Phys. D: Appl. Phys. 2009, 42, 224001.

26. Ibañez, F. J.; Zamborini, F. P. Small 2012, 8, 174-202.

27. Ohara, S.; Hatakeyama, Y.; Umetsu, M.; Sato, K.; Naka, T.; Adschiri, T. J. Power Sources 2009, 193, 367-370.

28. Narayanan, R.; El-Sayed, M. A. J. Phys. Chem. B 2005, 109, 12663-12676.

29. a) Pattadar, D. K.; Sharma, J. N.; Mainali, B. P.; Zamborini, F. P., Curr. Opin. Electrochem. 2019, 13, 147-156. b) Pattadar, D. K.; Zamborini, F. P. J. Phys. Chem. C 2019, 123, 9496-9505. c) Pattadar, D. K.; Sharma, J. N.; Mainali, B. P.; Zamborini, F. P. J. Phys. Chem. C 2019, 123, 24304-24312. d) Pattadar, D. K.; Zamborini, F. P. Langmuir 2019, 35, 16416-16426.

30. Brust, M.; Walker, M.; Bethell, D.; Schiffrin, D. J., Whyman, R. J. Chem. Soc., Chem.Commun. 1994, 801-802. 
31. Sardar, R.; Funston, A. M.; Mulvaney, P.; Murray R. W. Langmuir 2009, 25, 13840-13851.

32. Zamborini, F, P.; Gross, S. M.; Murray, R. W. Langmuir, 2001, 17, 481-488.

33. Moores, A.; Goettmann, F. New J. Chem., 2006, 30, 1121-1132.

34. Majumder, C. Langmuir. 2008, 24, 10838-10842.

35. Xue, Y.; Li, X.; Zhang, W. Nat Commun. 2014, 4348, 1-9.

36. Templeton, A. C.; Wuelfing, W. P.; Murray, R. W. Acc. Chem. Res. 2000, 33, 2736.

37. Kassam, A.; Bremner, G.; Clark, B.; Ulibarri, G.; Lennox, R. B. J. Am. Chem. Soc. 2006, 128, 3476-3477.

38. Hostetler, M. J.; Templeton, A. C.; Murray, R. W. Langmuir 1999, 15, 37823789.

39. Zhang, J.; Liu, Y.; Lv, J.; Li, G. Nano Res. 2015, 8, 920-930.

40. Thygesen, M. B.; Sauer, J.; Jensen, K. J. Chem. Eur. J. 2009, 15, 1649-1660.

41. Mancini, R. J.; Li, R. C.; Tolstyka, Z. P.; Maynard, H. D. Org. Biomol. Chem. 2009, 7, 4954-4959.

42. Chan, E. W. L.; Yu, L. Langmuir 2002, 18, 311-313.

43. Sharma, J. N.; Pattadar, D. K.; Mainali, B. P.; Zamborini, F. P. Anal. Chem. 2018, 90, 9308-9314. 
44. Pattadar, D. K.; Mainali, B. P.; Zamborini, F. P. ChemElectroChem 2020, 7, 800809.

45. Segev-Bar, M.; Haick, H. ACS Nano 2013, 7, 8366-8378.

46. Lee, J. E.; Lee, N.; Kim, J.; Hyeon, T. Acc. Chem. Res., 2011, 22, 893-902.

47. Salata, O. J. Nanobiotechnol. 2004, 2, 3.

48. Astefanei, A.; Nunez, O.; Galceran, M. T. Anal. Chim. Acta, 2015, 882, 1-21.

49. Jollans, T.; Baaske, M. D.; Orrit, M. J. Phys. Chem. C 2019, 123, 14107-14117.

50. Hisatomi, T.; Kubota, J.; Domen, K. Chem. Soc. Rev., 2014, 43, 7520-7535.

51. Kharel, P. L., Cuillier, P. M., Fernando, K., Zamborini, F. P., Alphenaar, B. W. J. Phys. Chem. C 2018, 122, 15090-15096.

52. Kharel, P. L., Zamborini, F. P.; Alphenaar B. W. J. Electrochem. Soc. 2018, 165, H52-H56.

53. Bhama, S.; Sibakoti, T. R.; Jasinski, J. B.; Zamborini, F. P. ChemCatChem., 2020, 12, 2253-2261.

54. Biswas, M.; Saha, A.; Dule, M.; Mandal, T. K. J. Phys. Chem. C 2014, 118, 22156-22165.

55. Feng E, X-T.; Zhang, Y.; Zou, J-J.; Wang, L.; Zhang, X. Ind. Eng. Chem. Res. 2014, 53, 12312-12318.

56. Shende, V. S.; Saptal, V. B.; Bhanage, B. M. Chem. Rec. 2019, 19, 1-23.

57. Gursel, I. V.; Noel, T.; Wang, Q.; Hessel, V. Green. Chem., 2015, 17, 2012-2026.

58. Hayes, K. Appl. Catal. A: General, 2001, 221, 187-195. 
59. Srabovic, M.; Huremovic, M.; Catovic, B.; Kulic, S.; Taletovic, A. J. Chem. Bio. Phy. Sci. Sec. A, 2017, 7, 218-230.

60. Satoh, T.; Suzuki, S.; Suzuki, Y.; Miyaji, Y.; Imai, Z. Tetrahedron Lett. 1969, 10, $4555-4558$.

61. Pradhan, N.; Pal, A.; Pal, T. Colloids Surfaces A Physicochem. Eng. Asp., 2002, $196,247-257$.

62. Wunder, S.; Polzer, F.; Lu, Y.; Mei, Y.; Ballauff, M. J. Phys. Chem. C 2010, 114, 8814-8820.

63. Xu, W.; Kong, J. S.; Yeh, Y. T.; Chen, P. Nat. Mater. 2008, 7, 992-996.

64. Ciganda, R.; Li, N.; Deraedt, C.; Gatard, S.; Zhao, P.; Salmon, L. Chem. Commun. 2014, 50, 10126-10129.

65. Haldar, K. K.; Kundu, S.; Patra, A. ACS. Appl. Mater. Interfaces 2014, 6, 2194621953.

66. Dasog, M.; Hou, W.; Scott, R. W. J. Chem. Commun. 2011, 47, 8569-8571.

67. Kawasaki, H.; Yamamoto, H.; Fujimori, H.; Arakawa, R.; Iwasaki, Y.; Inada, M. Langmuir, 2010, 26, 5926-5933.

68. Yamamoto, H.; Yano, H.; Kouchi, H.; Obora, Y.; Arakawa, R.; Kawasaki, H. Nanoscale, 2012, 4, 4148-4154.

69. H. Yamamoto et al. (ref. 68) noted that the layer of adsorbed DMF prohibited the 
reactants from penetrating to the surface of the Au nanoclusters at the onset of the reaction, resulting in an induction time before 4-NP reduction could begin.

70. He, J.; Razzaque, S.; Jin, S.; Hussain, I.; Tan, B. ACS. Appl. Nano Mater. 2019, 2, 546-553.

71. Musolino, M. G.; Maio, P.; Donato, a.; Pietropaolo, R. J. Mol. Catal. A: Chem. 2004, 208, 219-224.

72. Bhattacharjee, S.; Dotzauer, D. M.; Bruening, M. L. J. Am. Chem. Soc. 2009, 131, 3601-3610.

73. Maung, M. S.; Dinh, T.; Salazar, C.; Shon, Y.-S. Colloids Surf., A 2017, 513, 367372.

74. Sadeghmoghaddam, E.; Lam, C.; Choi, D.; Shon, Y.-S. J. Mater. Chem. 2011, 21, $307-312$.

75. Sadeghmoghaddam, E.; Gaïeb, K.; Shon, Y.-S. Appl. Catal., A 2011, 405, 137-141.

76. Eklund, S. E.; Cliffel, D. E. Langmuir 2004, 20, 6012-6018.

77. Oh, S.-K.; Niu, Y.; Crooks, R. M. Langmuir 2005, 21, 10209-10213.

78. Scott, R. W. J.; Datye, A. K.; Crooks, R. M. J. Am. Chem. Soc. 2003, 125, 37083709.

79. Sadeghmoghaddam, E.; Gu, H.; Shon, Y.-S. ACS Catal., 2012, 2, 1838-1845.

80. Uma, R.; Crévisy, C.; Grée, R. Chem. Rev. 2003, 103, 27-51. 
81. Moreno, M.; Ibanez, F. J.; Jasinski, J. B.; Zamborini, F. P. J. Am. Chem. Soc. 2011, 133, 4389-4397.

82. Moreno, M.; Kissell, L. N.; Jasinski, J. B.; Zamborini, F. P. ACS Catal., 2012, 2, $2602-2613$.

83. Bai, Y.; Huang, H.; Wang. C.; Long, R.; Xiong, Y. Mater. Chem. Front., 2017, 1, 1951-1964.

84. Rossi, L. M.; Fiorio, J. L.; Garcia, M. A. S.; Ferraz, C. P. Dalton Trans., 2018, 47, $5889-5915$.

85. Lu, L.; Lou, B.; Zou, S.; Kobayashi, H.; Liu, J.; Xiao, L.; Fan, J. ACS Catal., 2018, 8, 8484-8492.

86. Elliott III, E. W.; Glover R. D.; Hutchison, J. E. ACS Nano, 2015, 9, 3050-3059.

87. Lopez-Sanchez, J. A.; Dimitratos, N.; Hammond, C.; Brett, G. L.; Kesavan, L.; White, S.; Miedziak, P.; Tiruvalam, R.; Jenkins, R. L.; Carley, A. F.; Knight, D.; Kiely C. J.; Hutchings, G. J. Nat. Chem., 2011, 3, 551-556.

88. Cademartiri, L.; Ghadimi, A.; and Ozin, G. A. Acc. Chem. Res., 2008, 41, 18201830.

89. Niu, Z.; Li, Y. Chem. Mater., 2014, 26, 72- 83.

90. Brimaud, S.; Coutanceau, C.; Garnier, E.; Léger, J. M.; Gerard, F.; Pronier, S.; Leoni, M. J. Electroanal. Chem., 2007, 602, 226-236.

91. Collins, G.; Davitt, F.; O’Dyer C.; Holmes, J. D. ACS Appl. Nano Mater., 2018, 1, 7129-7138. 
92. Henckel, D. A.; Lenz O.; Cossairt, B. M. ACS Catal., 2017, 7, 2815-2820.

93. Grass, M. E.; Joo, S. H.; Zhang Y.; Somorjai G. A. J. Phys. Chem. C 2009, 113, 8616-8623.

94. Baker, L. R.; Kennedy, G.; Krier, J. M.; Van Spronsen, M.; Onorato, R. M.; Somorjai, G. A. Catal. Lett., 2012, 142, 1286 - 1294.

95. Cargnello, M.; Chen, C.; Diroll, B. T.; Doan-Nguyen, V. V. T.; Gorte R. J.; Murray C. B. J. Am. Chem. Soc., 2015, 137, 6906-6911.

96. Villa, A.; Wang, D.; Veith, G. M.; Vindigni, F.; Prati, L. Catal. Sci. Technol., 2013, 3, 3036-3041.

97. Zhong, R. Y.; Sun, K. Q.; Hong, Y. C.; Xu, B. Q. ACS Catal., 2014, 4, 39823993.

98. Elliott, E. W.; Glover, R. D.; Hutchison, J. E. ACS Nano, 2015, 9, 3050-3059.

99. Templeton, A. C.; Hostetler, M. J.; Kraft C. T.; Murray, R. W. J. Am. Chem. Soc., 1998, 120, 1906-1911.

100. Sun, L.; Crooks, R. M.; Chechik, V. Chem. Commun., 2001, 4, 359-360.

101. Kim, J. -B.; Bruening, M. L.; Baker, G. L. J. Am. Chem. Soc., 2000, 122, 76167617.

102. Sibakoti, T. R.; Jasinski J. B.; Zamborini F. P.; Nantz, M. H. Nanoscale, 2020, DOI: $10.1039 / \mathrm{d} 0 \mathrm{nr} 00844 \mathrm{c}$. 
103. Liang, S.; Hammond, G. B.; Xu, B. Chem. Commun., 2015, 51, 903-906.

104. Wang, Y.; Zhong, H.; Li, L.; Wang, R., ChemCatChem, 2015, 8, 2234-2240.

105. Lee, J-H.; Mahmoud, M. A.; Sitterle, V.; Sitterle, J.; Mederith, J. C. J. Am. Chem. Soc. 2009, 131, 5048-5049.

106. Wang, N.; Sun Q.; Bai. R.; Li, X. G. Guo, G.; Yu, J. J. Am. Chem. Soc., 2016, $138,7484-7487$.

107. Choi, K.; Na, K.; Somorjai, G.; Yaghi, O. J. Am. Chem. Soc., 2015, 137, 78107816

108. Wang, P.; Zhao, J.; Li, X.; Yang, Y.; Yang, Q.; Li, C. Chem. Commun., 2013, 49, 3330-3332.

109. Wang, Z.; Zhang, H.; Li, L.; Miao, S.; Wu, S.; Hao, X.; Zhang, W.; Jia, M. Catal. Commun. 2018, 114, 51-55.

110. Shen, C.; Wang, Y. J.; Xu, J. H.; Lu, Y. C.; Luo, G. S. Chem. Eng. J. 2011, 173, 226-232.

111. Rossi, L. M.; Costa, N. J. S.; Silva, F. P.; Wojcieszak, R. Green Chem., 2014, 16, 2906-2933.

112. Huang, J.; Lin, L.; Liang, H.; Lu, J. Polymer Chem. 2015, 6, 4020-4029.

113. Ulrich, S.; Boturyn, D.; Marra, A.; Renaudet, O.; Dumy, P. Chem. Eur. J. 2014, 20, 34-41. 
114. Grover, G. N.; Lam, J.; Nguyen, T. H.; Segura, T.; Maynard, H. D. Biomacromolecules 2012, 13, 3013-3017.

115. Yuan, W.; Edwards, J. L.; Li, S. Chem. Commun. 2013, 49, 11080-11082.

116. Mattingly, S. J.; Xu, T.; Nantz, M. H.; Higashi, R. M.; Fan, T. W. Metabolomics 2012, 8, 989-996.

117. Perraud, V.; François, S.; Wortham, H.; Jourdain, B.; Houdier, S.; Kardos, N. Talanta 2008, 76, 824-831.

118. Houdier, S.; Perrier, S.; Defrancq, E.; Legrand, M. Anal. Chim. Acta 2000, 412, 221-233.

119. Knipp, R. J.; Li, M.; Fu, X-A.; Nantz, M. H. Anal. Methods 2015, 7, 6027-6033.

120. Zeng, Y.; Ramya, T. N. C.; Dirksen, A.; Dawson, P. E.; Paulson, J. C. Nat. Methods 2009, 6, 207-209.

121. Zhang, Y.; Yu, M.; Zhang, C.; Ma, W.F.; Zhang, Y. T.; Wang, C. C.; Lu, H. J. Anal. Chem. 2014, 86, 7920-7924.

122. Algar, W. R.; Prasuhn, D. E.; Stewart, M. H.; Jennings, T. L.; Blanco-Canosa, J. B.; Dawson, P.E.; Medintz, I. L. Bioconjugate Chem. 2011, 22, 825-858.

123. Biswas, S.; Gordon, L. E.; Clark, G. J.; Nantz, M. H. Biomaterials 2011, 32, 2683-2688.

124. Li, N.; Zhao, P.; Astruc, D. Angew. Chem. 2014, 126, 1784; Angew. Chem. Int. Ed. 2014, 53, $1756-1789$.

125. Vericat, C.; Vela, M. E.; Benitez, G; Carro, P.; Salvarezza, R. C. Chem. Soc. Rev. 2010, 39, 1805-1834.

126. Zhang, J. Lv, J. Wang, X. Li, D. Wang, Z. Li, G. Nano Res. 2015, 8, 3853-3863. 
127. Mitsudome, T.; Kaneda, K. Green Chem. 2013, 15, 2636-2654.

128. Liu, L.; Corma, A. Chem. Rev. 2018, 118, 4981-5079.

129. Wohltjen, H.; Snow, A. W. Anal. Chem. 1998, 70, 2856-2859.

130. Abdelhamid, H. N.; Wu, H. F. Anal. Bioanal. Chem. 2016, 408, 4485-4502.

131. Gholipourmalekabadi, M.; Mobaraki, M.; Ghaffari, M.; Zarebkohan, A.; Omrani, V. F.; Urbanska, A. M.; Seifalian, A. Curr. Pharm. Des. 2017, 23, 2918-2929.

132. Elahi, N.; Kamali, M.; Baghersad, M. H. Talanta 2018, 184, 537-556.

133. Kool, E. T.; Crisalli, P.; Chan, K. M. Org. Lett. 2014, 16, 1454-1457.

134. Mitsunobu, O.; Yamada, Y. B CHEM SOC JPN 1967, 40, 2380-2382.

135. Battistuzzi, G.; Giannini, G. Curr. Bioact. Compd. 2016, 12, 282-288.

136. Wu, Z.; Chen, J.; Jin, R. Adv. Funct. Mater., 2011, 21, 177-183.

137. Sharma, S.; Kim, B.; Lee, D. Langmuir, 2012, 28, 15958-15965.

138. Sibakoti, T. R.; Stinger, C. R.; Adhihetty, P. K.; Zamborini, F. P.; Nantz, M. H. Part. Part. Syst. Charact., 2019, 36, 1900093.

139. Chaki, N. K.; Negishi, Y.; Tsunoyama, H.; Shichibu, Y.; Tsukuda, T. J. Am. Chem. Soc. 2008, 130, 8608-8610.

140. Xie, Z.; Mandapati, R.; Stewart, A. C.; Nantz, M. H.; Fu, X. A. RSC Adv. 2018, $62,35618-35624$. 
141. Stiles, R. L.; Balasubramanian, R.; Feldberg, S. W.; Murray, R. W. J. Am. Chem. Soc. 2008, 130, 1856-1865.

142. Beasley, C. A. Sardar, R. Barnes, N. M. Murray, R. W. J. Phys. Chem. C 2010, $114,18384-18389$.

143. Battistini, G.; Cozzi, P. G.; Jalkanen, J. P.; Montalti, M.; Prodi, L.; Zaccheroni, N.; Zerbetto, F. ACS Nano 2007, 2, 77-84.

144. Wang, Z.; Zhang, H.; Li, L.; Miao, S.; Wu, S.; Hao, X.; Zhang, W.; Jia, M. Catal. Commun., 2018, 114, 51-55.

145. Munnik, P.; de Jongh, P. E.; de Jong, K. P. Chem. Rev. 2015, 115, 6687-6718.

146. Carabineiro, S. A. C. Front. Chem., 2019, 7, 702.

147. Corma, A.; Garcia, H. Chem. Soc. Rev., 2008, 37, 2096-2126.

148. Min, H.; Lee, S.; Park, M.; Hwang, J.; Jung, H. M.; Lee, S. J. Organomet. Chem. 2014, 755, 7-11.

149. Murugan, E.; Jebaranjitham, J. M.; Usha, A. Appl. Nanosci. 2012, 2, 211-222.

150. Liu, L.; Corma, A. Chem. Rev. 2018, 118, 4981-5079.

151. Turner, J. L., Wooley, K. L. Nano Lett. 2004, 4, 683-688.

152. Goswami, N.; Bright, R.; Visalakshan, R. M.; Biswas, B.; Zilm, P.; Vasilev, K. Nanoscale Adv., 2019, 1, 2356-2364.

153. Qui, L.; McCaffrey, R.; Jin, Y.; Gong, Y.; Hu, Y.; Sun, H.; Park, W.; Zhang, W. Chem. Sci., 2018, 9, 676-680. 
154. Zhang, Y.; Xiong, Y.; Ge, J.; Lin, R.; Chen, C.; Peng, Q.; Wang, D.; Li, Y. Chem. Commun., 2018, 54, 2796-2799.

155. Jencks, W. P. J. Am. Chem. Soc. 1959, 81, 475-481.

156. Zhao, H.; Heindel, N.H. Pharmaceutical Research. 1991, 8, 400-402.

157. Contado, C.; Mehn, D.; Gilliland, D.; Calzolai, L. J. Chromatogr. A, 2019, 1606, 460383.

158. Lee, J-H.; Mahmoud, M-A.; Sitterle, V.; Sitterle, J.; Meredih, J. C. J. Am. Chem. Soc. 2009, 131, 5048-5049.

159. Geckeler, K.; Zheng, H.; Bayer, E. Journal of Polymer Science: Polymer Chemistry Edition, 1983, 21, 3541-3549.

160. Singh, S.; Akher, M. S., Dodt, J.; Volkers, P.; Reuter, A.; Reinhart, C.; Krettler, C.; Oldenburg, J.; Biswas, A. Int. J. Mol. Sci. 2019, 20, 2682-2694.

161. Zamborini, F.P; Gross, S. M; Murray, R.W. Langmuir 2001, 17, 481-488.

162. Hughes, M. D.; Xu, Y. J.; Jenkins, P.; McMorn, P.; Landon, P.; Enache, D. I.; Carley, A. F.; Attard, G. A.; Hutchings, G. J.; King, F.; Stitt, E. H.; Johnston, P.; Griffin K.; Kiely, C. J. Nature, 2005, 437, 1132-1135.

163. Li G.; Jin, R. Acc. Chem. Res., 2013, 46, 1749-1758.

164. Dasog, M.; Hou, W.; Scott, R. W. J. Chem. Commun., 2011, 47, 8569-8571.

165. Gavia D. J.; Shon, Y.-S. Langmuir, 2012, 28, 14502-14508.

166. San, K. A.; Chen V.; Shon, Y.-S. ACS Appl. Mater. Interfaces, 2017, 9, 98239832. 
167. Wand, P.; Kratzer, E.; Heiz, U.; Cokoja M.; Schurl, M. T. Catal. Commun., 2017, $100,85-88$.

168. Lu, F.; Ruiz J.; Astruc, D. Tetrahedron Lett., 2004, 45, 9443-9445.

169. Lu Y.; Chen, W. Chem. Soc. Rev., 2012, 41, 3594-3623.

170. Parker, J. F.; Fields-Zinna, C. A.; Murray, R. W. Acc. Chem. Res., 2010, 43, $1289-1296$.

171. Kang, X.; Chong, H.; Zhu, M. Nanoscale, 2018, 10, 10758-10834.

172. Rossi, L. M.; Fiorio, J. L.; Gracia, M. A. S.; Ferraz, C. P. Dalton Trans., 2018, 47, $5889-5915$.

173. Lohse, S. E.; Dahl, J. A.; Hutchison, J. E. Langmuir, 2010, 26, 7504-7511.

174. Ju-Nam, Y.; Abdussalam-Mohammed W.; Ojeda, J. J. Faraday Discuss., 2016, 186, 77-93.

175 Wu, Z.; Chen, J.; Jin, R. Adv. Funct. Mater., 2011, 21, 177-183.

176. Jin, R. Nanoscale, 2010, 2, 343-362.

177. Ringe, E.; DeSantis, C. J.; Collins, S. M.; Duchamp, M.; Dunin-Borkowski, R. E.; Skrabalak, S. E.; Midgley, P. A. Sci Rep., 2015, 5, 17431.

178. Herves, P.; Perez-Lorenzo, M.; Liz-Marzan, L. M.; Dzubiella, J.; Lu, Y.; Ballauff, M. Chem. Soc. Rev., 2012, 41, 5577-5587.

179. Ngene, P.; van den Berg, R.; Verkukuijlen, M. H. W.; de Jong K. P.; de Jongh, P. E. Energy Environ. Sci., 2011, 4, 4108-4115. 
180. Feng, Z. V.; Lyon, J. L.; Croley, J. S.; Crooks, R. M.; Vanden Bout D. A.;

Stevenson, K. J. J. Chem. Educ., 2009, 86, 368-372.

181. Qi, P. H.; Hiskey, J. B. Hydrometallurgy, 1991, 27, 47-62.

182. Bhattacharjee, S.; Bruening, M. Langmuir, 2008, 24, 2916-2920.

183. Sashikata, K.; Matsui, Y.; Itaya, K.; Soriaga, M. P. J. Phys. Chem., 1996, 100, 20027- 20034.

184. Biffis, A.; Zecca, M.; Basato, M. Eur. J. Inorg. Chem., 2001, 2001, 1131-1133.

185. T-A. Chen and Y-S. Shon. Catal. Sci. Technol., 2017, 7, 4823-4829.

186. Block, B. P. Inorg. Synth. 1953, 4, 14-17.

187. Dasari, R.; Zamborini, F. P. Anal. Chem. 2016, 88, 675-681. 
APPENDIX-A

\section{LIST OF ACRONYMS AND ABBREVIATIONS}

\begin{tabular}{|c|c|}
\hline NPs & Nanoparticle \\
\hline NCs & Nanoclusters \\
\hline MNPs & Metal Nanoparticles \\
\hline MPCs & Monolayer Protected Clusters \\
\hline MMPCs & Mixed Monolayer Protected Clusters \\
\hline $\mathrm{Au}$ & Gold \\
\hline $\mathrm{Pd}$ & Palladium \\
\hline GSL & Glycosphingolipids \\
\hline NHP & N-hydroxyphthalimide \\
\hline $\mathrm{PPh}_{3}$ & Triphenylphosphine \\
\hline DIAD & Diisoproylazodicarboxylate \\
\hline AIBN & Azoisobutyronitrile \\
\hline AOT & Aminooxy-Thiol \\
\hline $\operatorname{AOT}(\mathrm{aq})$ & Aminooxy-Thiol (aqueous) \\
\hline $\mathrm{OE}$ & Oxime Ether \\
\hline TMSCl & Trimethylsilyl chloride \\
\hline t-BuNCO & Tert-Butyl isocyanate \\
\hline TBAF & Tetra- $n$-butylammonium fluoride \\
\hline PCC & Pyridinium chlorochromate \\
\hline GSH & Glutathione \\
\hline SG & Glutathiolate \\
\hline $\mathrm{C}_{6} \mathrm{~S}$ & Hexanthiolate \\
\hline 4-NP & 4-Nitrophenol \\
\hline 4-AP & 4-Aminophenol \\
\hline $\mathrm{I}_{2}$ & Iodine \\
\hline SAM & Self-Assembled Monolayers \\
\hline DMDO & Dimethyl dioxirane \\
\hline TOABr & Tetra-octlyammonium bromide \\
\hline TBAHFP & Tetrabutylammonium hexafluorophosphate \\
\hline $\mathrm{CV}$ & Cyclic Voltammetry \\
\hline ASV & Anodic Stripping Voltammetry \\
\hline NMR & Nuclear Magnetic Resonance \\
\hline TGA & Thermogravimetric Analysis \\
\hline XPS & X-ray Photoelectron Spectroscopy \\
\hline UV-Vis & Ultraviolet- Visible \\
\hline TLC & Thin Layer Chromatography \\
\hline GC & Gas Chromatography \\
\hline FID & Flame Ionization Detector \\
\hline FTIR & Fourier Transform Infrared Spectroscopy \\
\hline
\end{tabular}




$\begin{array}{ll}\text { TEM } & \text { Transmission Electron Microscopy } \\ \text { SEM } & \text { Scanning Electron Microscopy } \\ \text { HRMS } & \text { High Resolution Mass Spectrometry } \\ \text { MALDI-TOF } & \text { Matrix Assisted Laser Desorption Ionization- Time of Flight } \\ \text { VOC } & \text { Volatile Organic Compounds } \\ \text { SERS } & \text { Surface Enhanced Raman Spectroscopy } \\ \text { LHMDS } & \text { Lithium bis(trimethylsilyl) amide } \\ \text { IDA } & \text { Interdigitated Arrays } \\ \text { ATP } & \text { 4-Aminothiophenol } \\ \text {R}_{\mathrm{f}} & \text { Retention Factor } \\ \text { TOF } & \text { Turnover Frequency } \\ \text { M.P } & \text { Melting Point } \\ \text { P.S } & \text { Polystyrene } \\ \text { SAR } & \text { Structural Activity Relationship } \\ \text { SPR } & \text { Surface Plasmon Resonance } \\ \text { FTICR } & \text { Fourier Transform Ion Cyclotron Resonance } \\ \text { ATM } & \text { 2-(aminooxy)ethyl]-N,N,N-trimethylammonium iodide } \\ \text { pAPG } & \text { 4-aminophenyl- } \alpha \text {-D-glucopyranoside } \\ \text { PDBA } & \text { 1,4-phenylenediboronic acid } \\ \text { QRE } & \text { Quasi-Reference Electrode } \\ \text { ITO } & \text { Indium Tin Oxide } \\ \text { EtOAC } & \text { Ethyl Acetate } \\ \text { HCP-TPMT } & \text { Hyper-cross-linked polymers-triphenylmethanethiol } \\ & \end{array}$


APPENDIX-B

SPECTRA $\left({ }^{1} \mathrm{H}\right.$ NMR, ${ }^{13} \mathrm{C}$ NMR, FTIR, and HRMS $)$

B.1. Index of Spectra

B.2. Spectra from Chapter 2

B.3. Spectra from Chapter 3

\section{B.1. Index of Spectra}

Section B.2.

Page

Figure B.2.1. ${ }^{1} \mathrm{H} \mathrm{NMR}\left(400 \mathrm{MHz}, \mathrm{CDCl}_{3}\right)$ spectrum of compound 2

Figure B.2.2. ${ }^{13} \mathrm{C}$ NMR $\left(100 \mathrm{MHz}, \mathrm{CDCl}_{3}\right)$ spectrum of compound 2

Figure B.2.3. FTIR spectrum of compound 2

Figure B.2.4. HRMS spectrum of compound 2

Figure B.2.5. ${ }^{1} \mathrm{H}$ NMR spectrum $\left(400 \mathrm{MHz}, \mathrm{CDCl}_{3}\right)$ of compound $\mathbf{3} 202$

Figure B.2.6. ${ }^{13} \mathrm{C}$ NMR spectrum $\left(100 \mathrm{MHz}, \mathrm{CDCl}_{3}\right)$ of compound 3

Figure B.2.7. FTIR spectrum of compound 3

Figure B.2.8. HRMS spectrum of compound $3 \quad 204$

Figure B.2.9. ${ }^{1} \mathrm{H}$ NMR spectrum $\left(500 \mathrm{MHz}, \mathrm{CDCl}_{3}\right)$ of compound 4

Figure B.2.10. ${ }^{13} \mathrm{C}$ NMR spectrum $\left(125 \mathrm{MHz}^{\mathrm{CDCl}_{3}}\right)$ of compound 4

Figure B.2.11. FTIR spectrum of compound 4

Figure B.2.12. HRMS spectrum of compound 4

Figure B.2.13. HRMS spectrum of compound 4 in methanol (ring opening) 206 
Figure B.2.14. FTIR spectrum of compound $\mathbf{A O T}_{\text {bis }}$

Figure B.2.15. HRMS spectrum of compound AOT bis

Figure B.2.16. ${ }^{1} \mathrm{H}$ NMR spectrum $\left(400 \mathrm{MHz}, \mathrm{CDCl}_{3}\right)$ of compound 6

Figure B.2.17. ${ }^{13} \mathrm{C}$ NMR spectrum $\left(100 \mathrm{MHz}, \mathrm{CDCl}_{3}\right)$ of compound 6

Figure B.2.18. FTIR spectrum of compound 6

Figure B.2.19. HRMS spectrum of compound 6

Figure B.2.20. FTIR spectrum of compound AOT bis $^{+}$

Figure B.2.21. HRMS spectrum of compound $\mathbf{A O T}_{\text {bis }^{+}}$

210

Figure B.2.22. ${ }^{1} \mathrm{H}$ NMR spectrum of compound 9

Figure B.2.23. ${ }^{13} \mathrm{C}$ NMR spectrum of compound 9 211

Figure B.2.24. ${ }^{1} \mathrm{H}$ NMR spectrum of compound 10 212

Figure B.2.25. ${ }^{13} \mathrm{C}$ NMR spectrum of compound 10

Section B.3.

Figure B.3.1. ${ }^{1} \mathrm{H}$ NMR spectrum $\left(500 \mathrm{MHz}, \mathrm{CDCl}_{3}\right)$ of compound 13

Figure B.3.2. ${ }^{13} \mathrm{C}$ NMR spectrum $\left(125 \mathrm{MHz}, \mathrm{CDCl}_{3}\right)$ of compound 13

Figure B.3.3. FTIR spectrum of compound 13

Figure B.3.4. HRMS spectrum of compound 13

Figure B.3.5. ${ }^{1} \mathrm{H}$ NMR spectrum ( $400 \mathrm{MHz}, \mathrm{CDCl}_{3}$ ) of compound 14

Figure B.3.6. ${ }^{13} \mathrm{C}$ NMR spectrum $\left(400 \mathrm{MHz}, \mathrm{CDCl}_{3}\right)$ of compound 14 
Figure B.3.7. FTIR Spectrum of compound 14

Figure B.3.8. HRMS spectrum of compound 14

Figure B.3.9. FTIR Spectrum of compound 15

Figure B.3.10. HRMS spectrum of compound 15

Figure B.3.11. ${ }^{1} \mathrm{H}$ NMR spectrum $(400 \mathrm{MHz}, \mathrm{CDCl} 3)$ of $\mathrm{Au}\left(\mathrm{C}_{6} \mathrm{~S}\right)\left(\mathrm{AOT}_{\text {bis }}-\right.$ ferrocene) MMPCs

Figure B.3.12. ${ }^{1} \mathrm{H}$ NMR spectrum $(400 \mathrm{MHz}, \mathrm{CDCl} 3)$ of $\mathrm{Au}\left(\mathrm{C}_{6} \mathrm{~S}\right)\left(\mathrm{AOT}_{\text {bis }}-\right.$ pyrene) MMPCs

Figure B.3.13. FTIR spectrum of $\mathrm{Au}\left(\mathrm{C}_{6} \mathrm{~S}\right)\left(\mathrm{AOT}_{\text {bis }}\right.$-benzaldehyde) MMPCs 219

Figure B.3.14. FTIR spectrum of $\mathrm{Au}(\mathrm{C} 6 \mathrm{~S})\left(\mathrm{AOT}_{\mathrm{bis}}-\right.$ ferrocene) MMPCs 220

Figure B.3.15. FTIR spectrum of $\mathrm{Au}(\mathrm{C} 6 \mathrm{~S})\left(\mathrm{AOT}_{\text {bis }}-\right.$ pyrene) MMPCs 220

Figure B.3.16. FTIR spectrum of $\mathrm{Au}(\mathrm{C} 6 \mathrm{~S})\left(\mathrm{AOT}_{\text {bis }}\right.$-propanal) MMPCs 221

Figure B.3.17. FTIR spectrum of $\mathrm{Au}(\mathrm{C} 6 \mathrm{~S})\left(\mathrm{AOT}_{\mathrm{bis}}\right.$-glyceraldehyde) MMPCs 221

Figure B.3.18. FTIR spectrum of $\mathrm{Au}(\mathrm{C} 6 \mathrm{~S})\left(\mathrm{AOT}_{\mathrm{bis}}-\right.$ urea-aldehyde 15) MMPCs 222

Figure B.3.19. ${ }^{1} \mathrm{H}$ NMR spectrum of $\mathrm{Au}(\mathrm{SG}) \mathrm{MPCs}$

Figure B.3.20. ${ }^{1} \mathrm{H}$ NMR spectrum of $\mathrm{Au}(\mathrm{SG})\left(\mathrm{AOT}_{\mathrm{bis}^{+}}{ }^{+}\right) \mathrm{MMPCs} 223$

Figure B.3.21. ${ }^{1} \mathrm{H}$ NMR spectrum of $\mathrm{Au}(\mathrm{SG})\left(\mathrm{AOT}_{\mathrm{bis}}{ }^{+}-\right.$ferrocene) MMPCs 223 


\section{B.2. Spectra from Chapter 2}

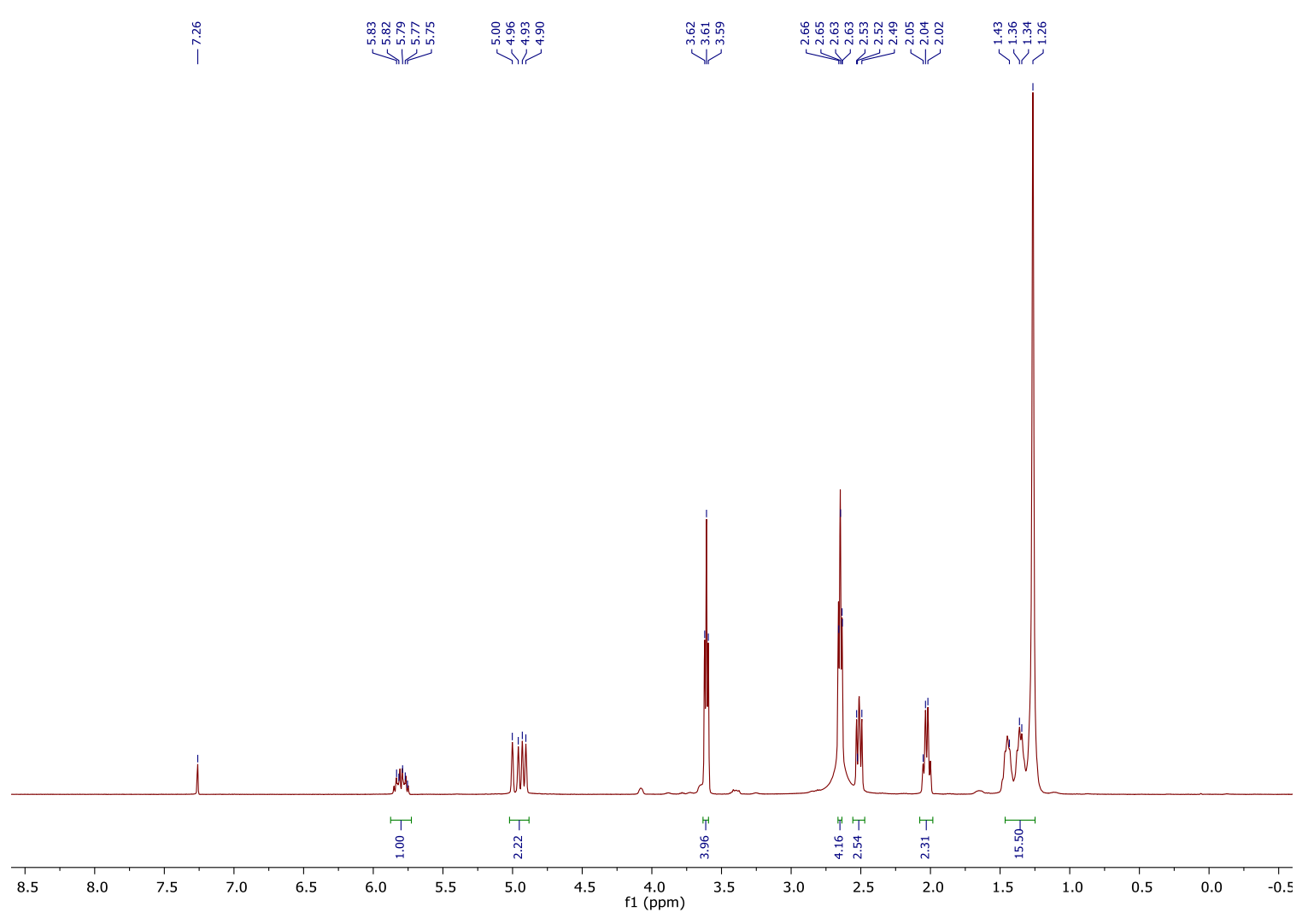

Figure B.2.1. ${ }^{1} \mathrm{H} \mathrm{NMR}\left(400 \mathrm{MHz}, \mathrm{CDCl}_{3}\right)$ spectrum of compound 2 


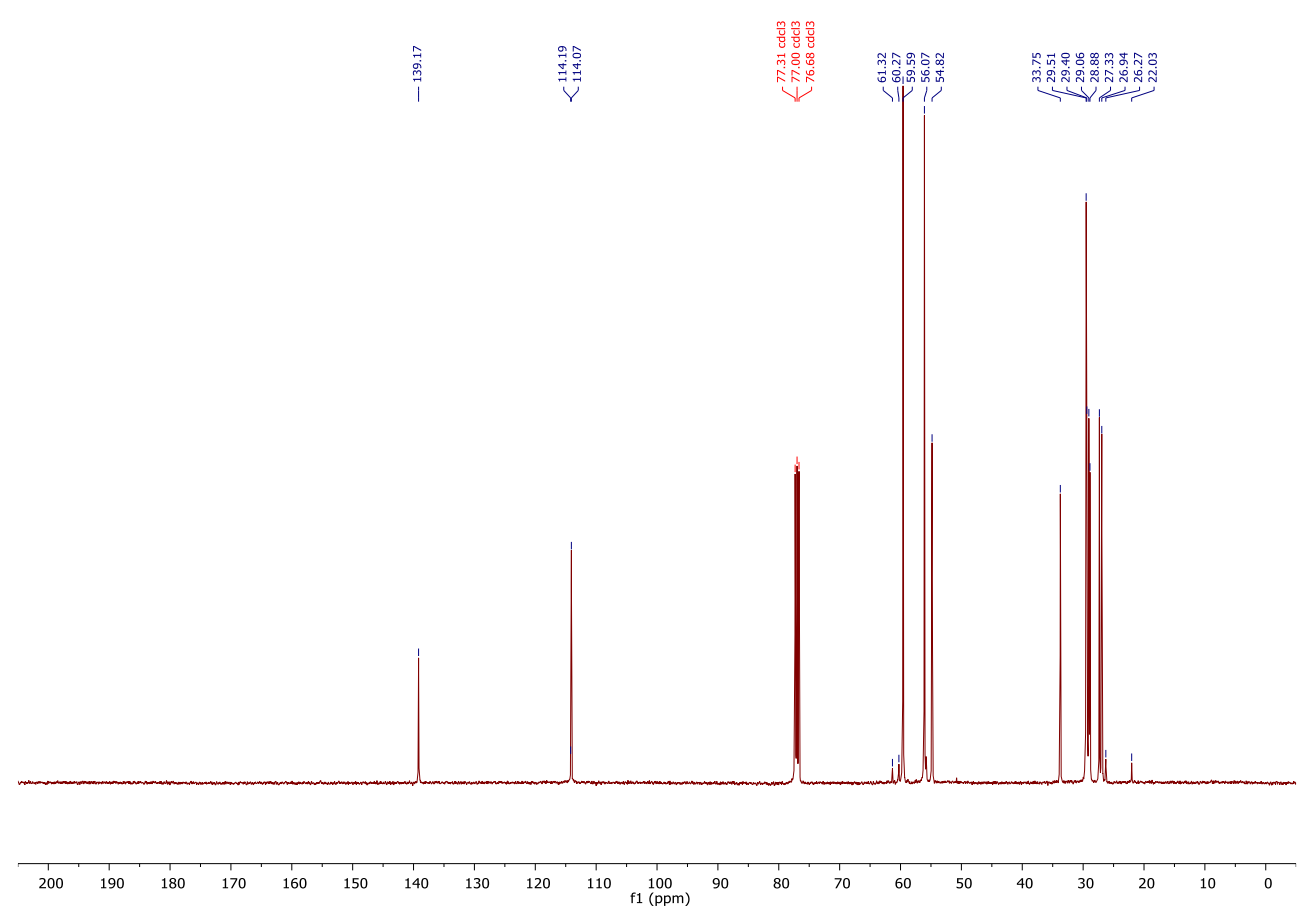

Figure B.2.2. ${ }^{13} \mathrm{C} \mathrm{NMR}\left(100 \mathrm{MHz}, \mathrm{CDCl}_{3}\right)$ spectrum of compound 2

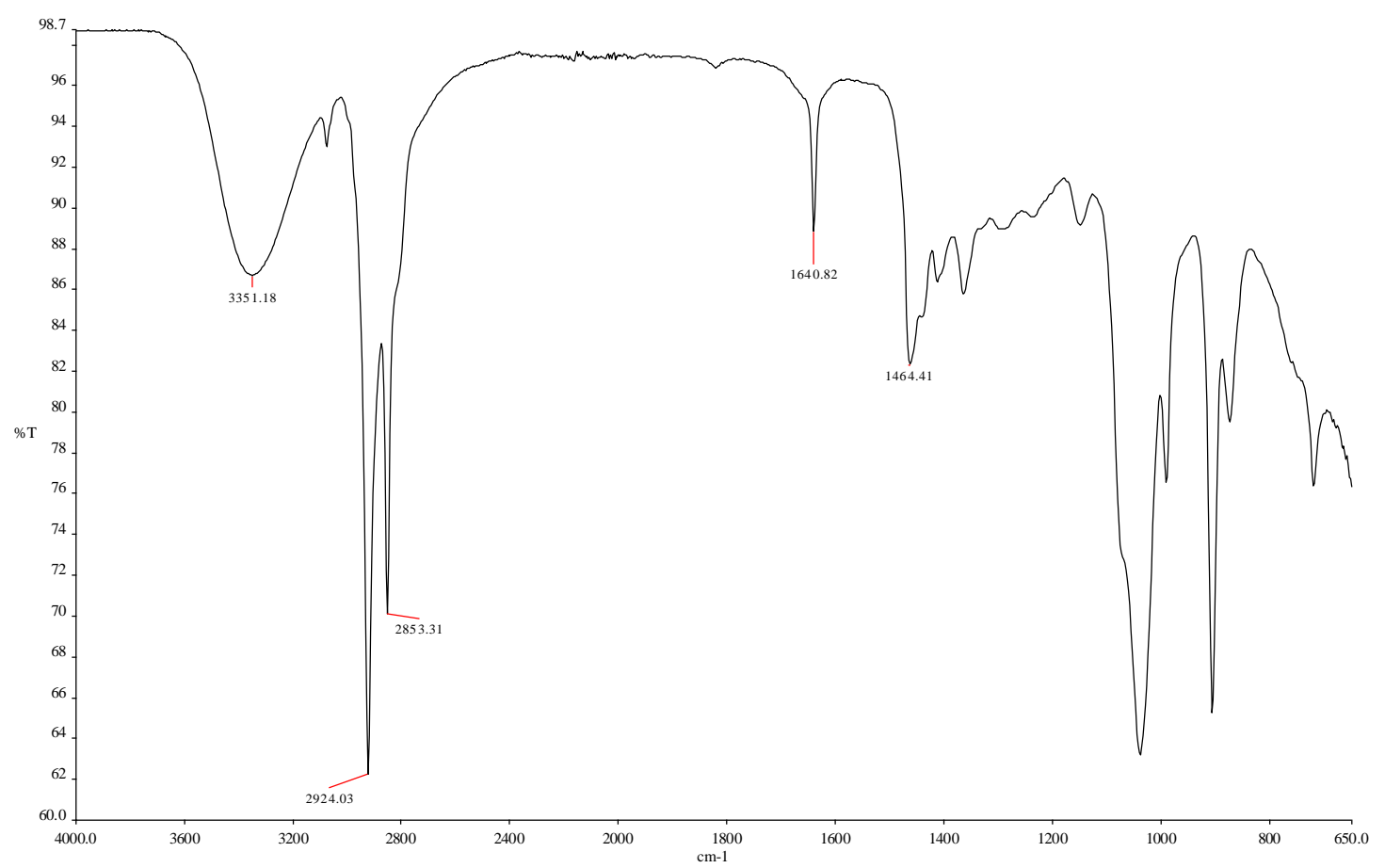

Figure B.2.3. FTIR spectrum of compound 2 


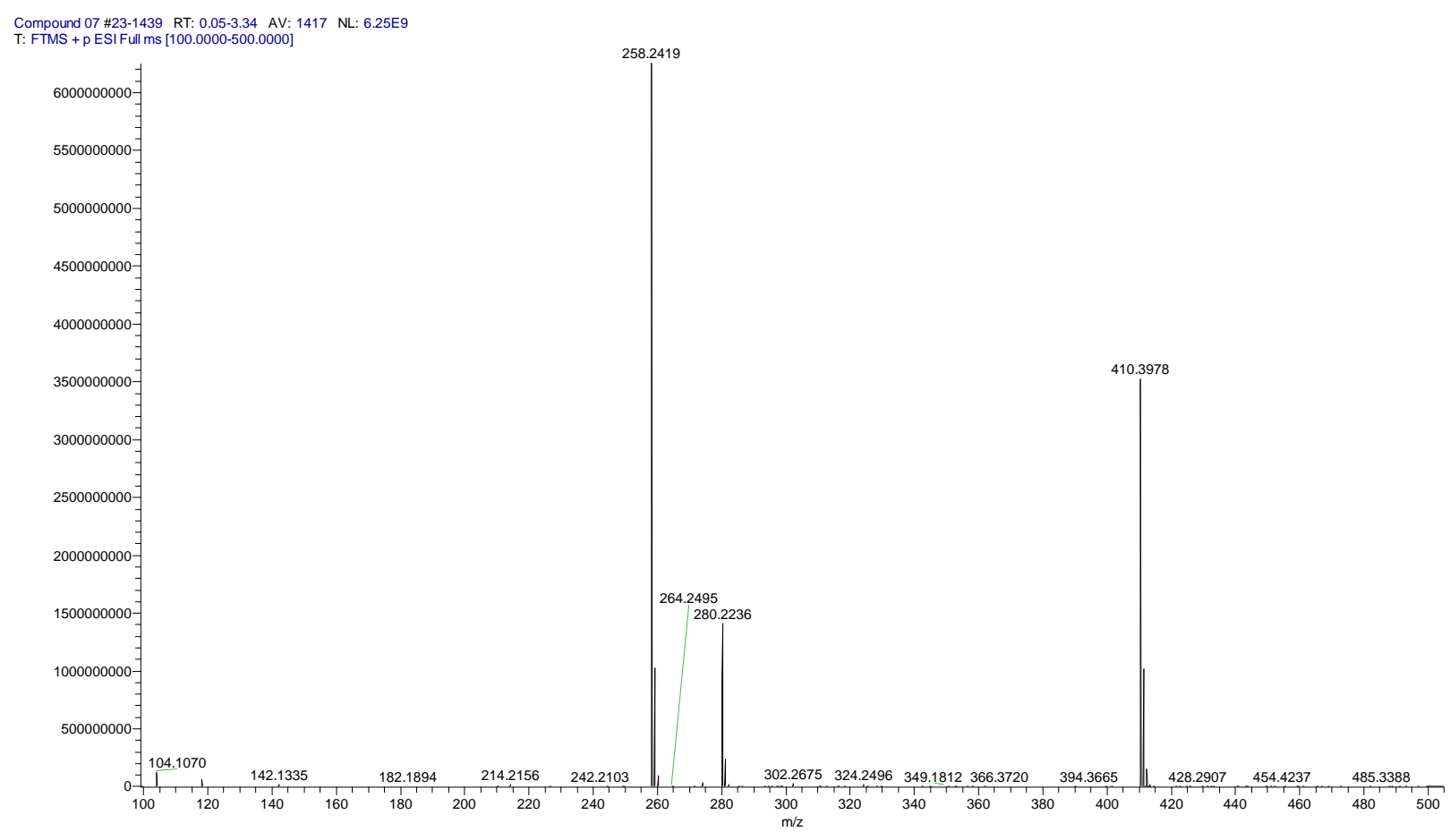

Figure B.2.4. HRMS spectrum of compound 2

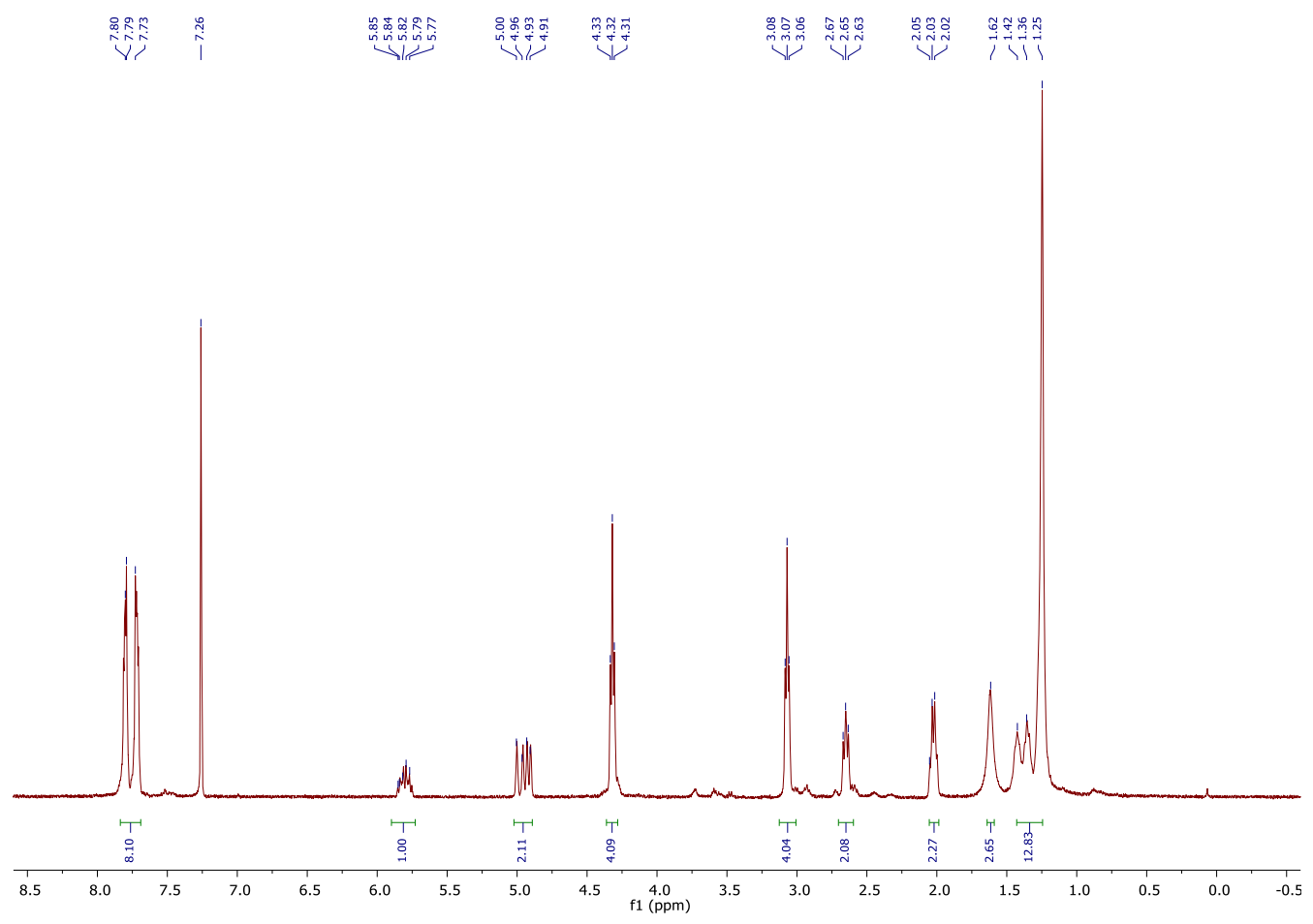

Figure B.2.5. ${ }^{1} \mathrm{H} \mathrm{NMR}$ spectrum $\left(400 \mathrm{MHz}, \mathrm{CDCl}_{3}\right)$ of compound 3 


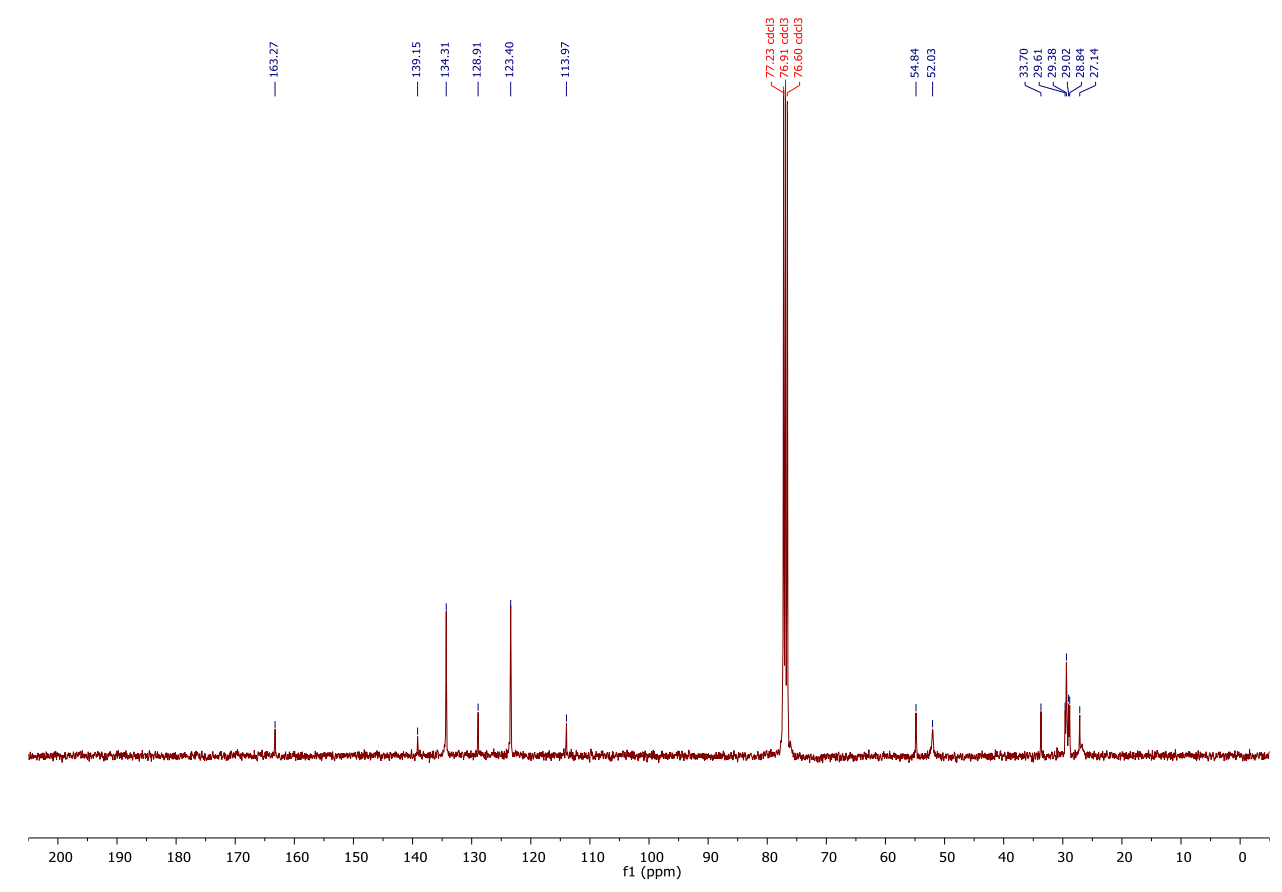

Figure B.2.6. ${ }^{13} \mathrm{C}$ NMR spectrum $\left(100 \mathrm{MHz}, \mathrm{CDCl}_{3}\right)$ of compound 3

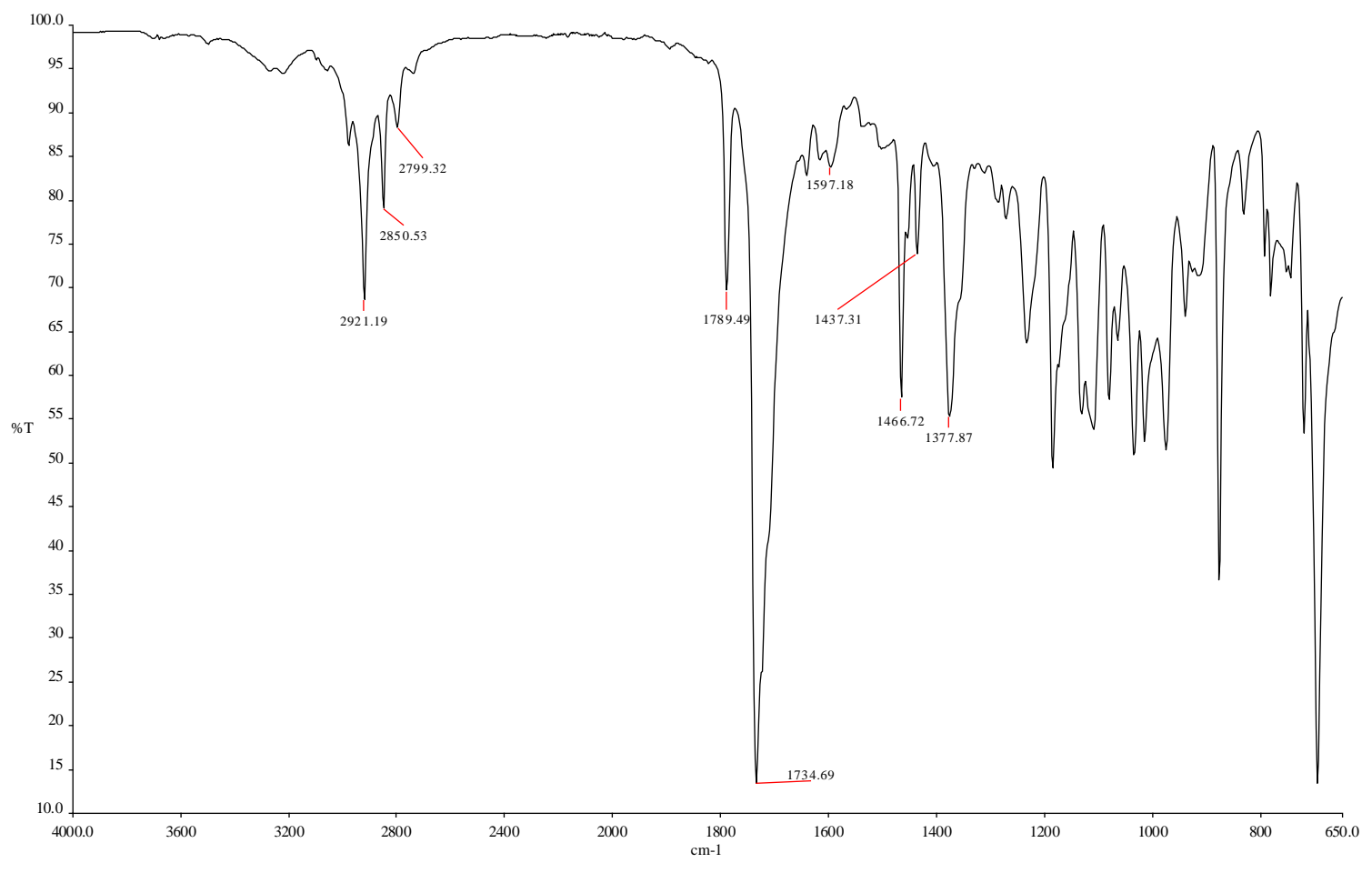

Figure B.2.7. FTIR spectrum of compound 3 


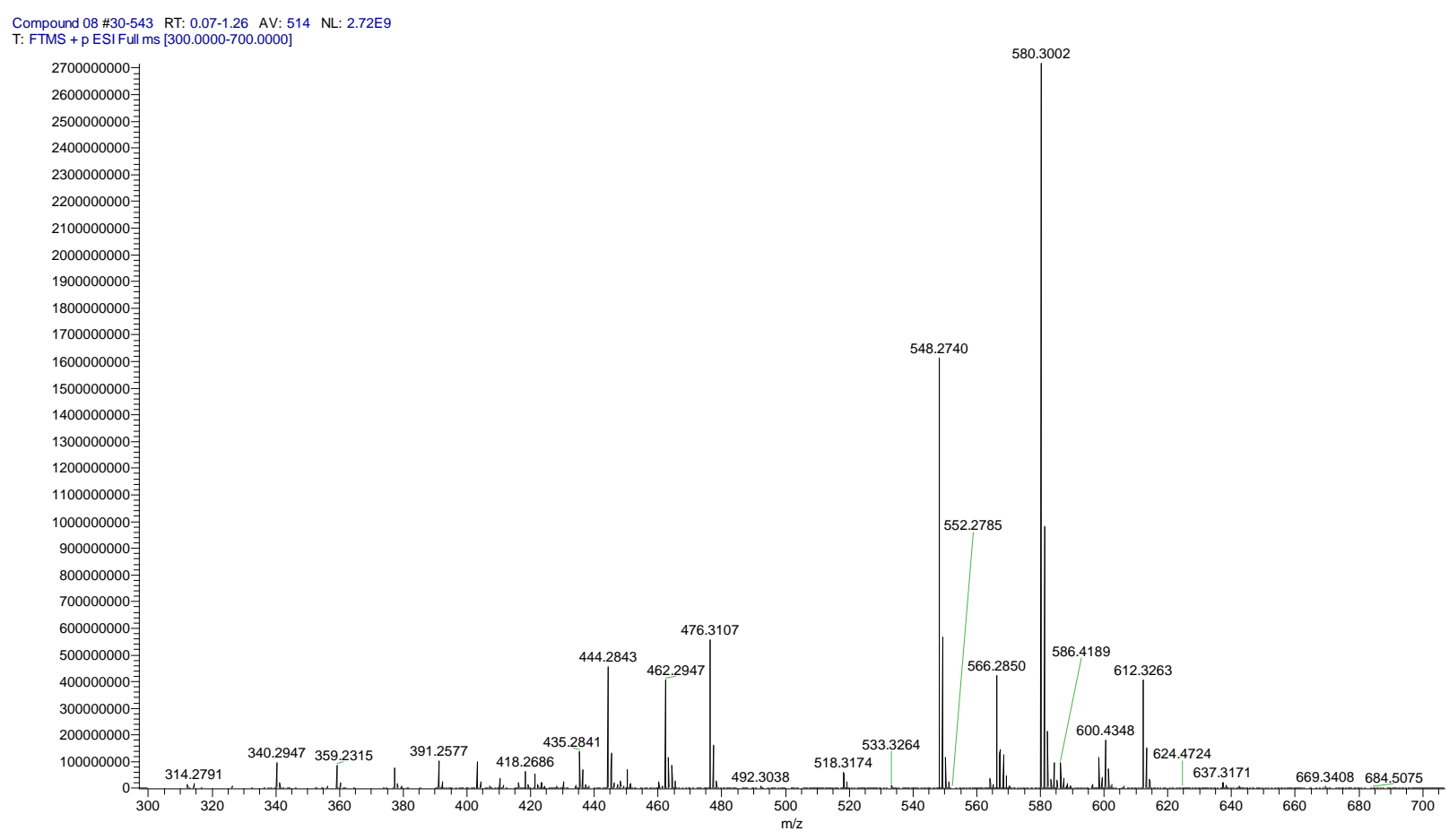

Figure B.2.8. HRMS spectrum of compound 3

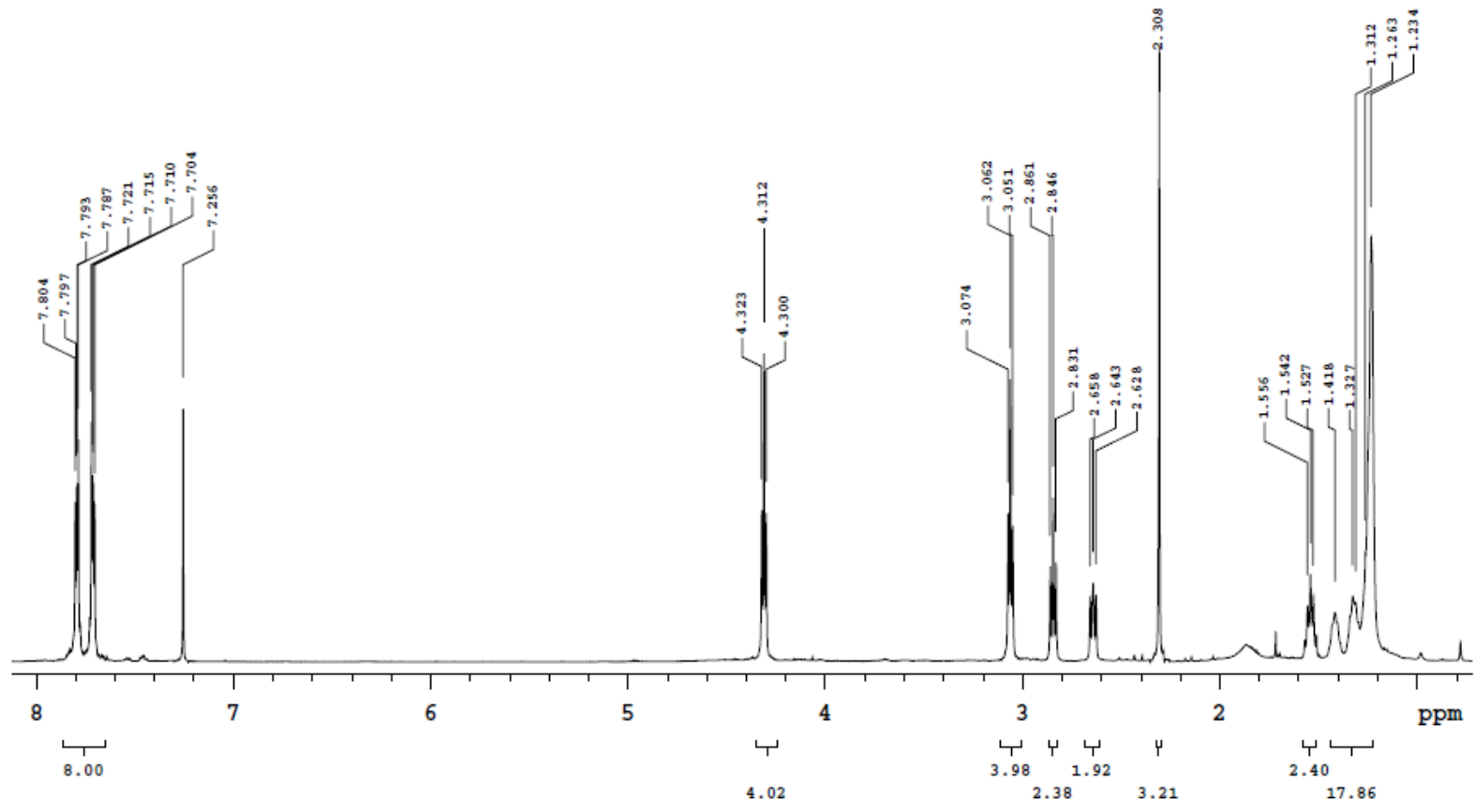

Figure B.2.9. ${ }^{1} \mathrm{H} \mathrm{NMR}$ spectrum $\left(500 \mathrm{MHz}, \mathrm{CDCl}_{3}\right)$ of compound 4 


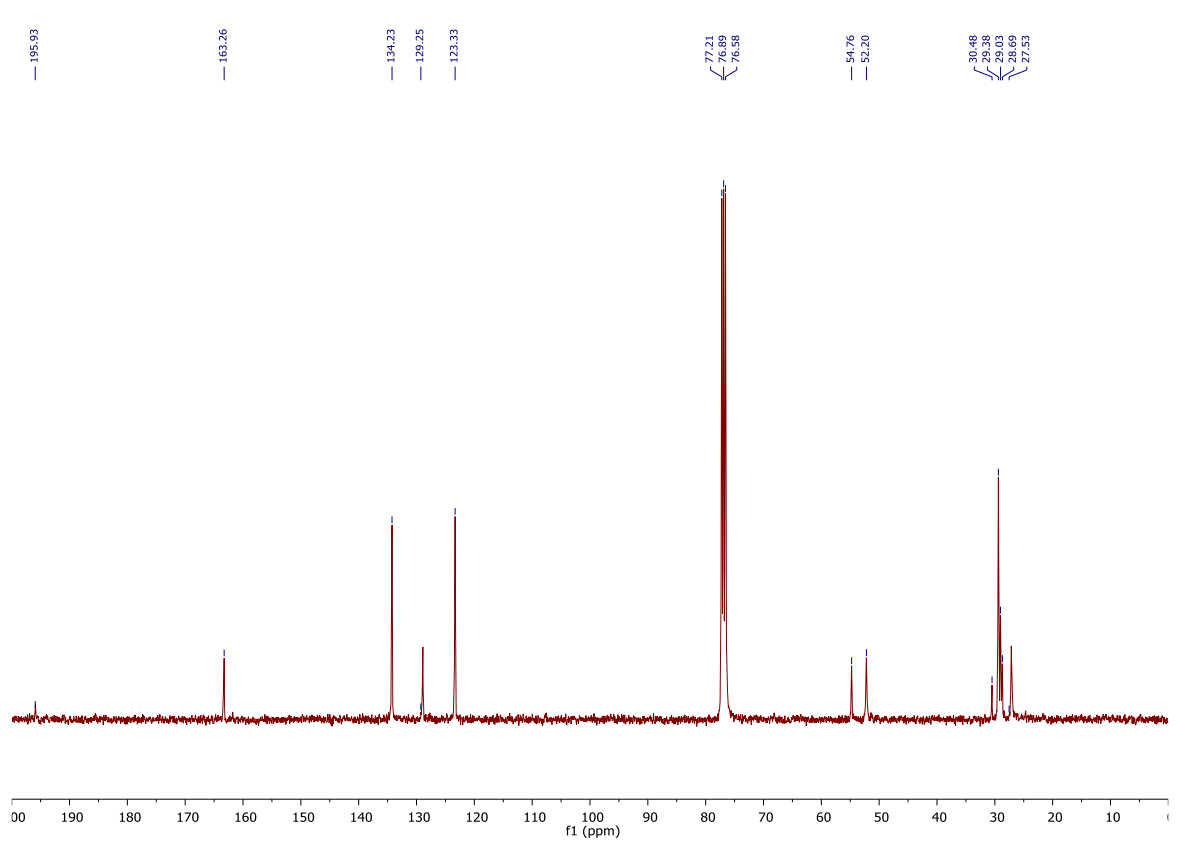

Figure B.2.10. ${ }^{13} \mathrm{C}$ NMR spectrum $\left(125 \mathrm{MHz}, \mathrm{CDCl}_{3}\right)$ of compound 4

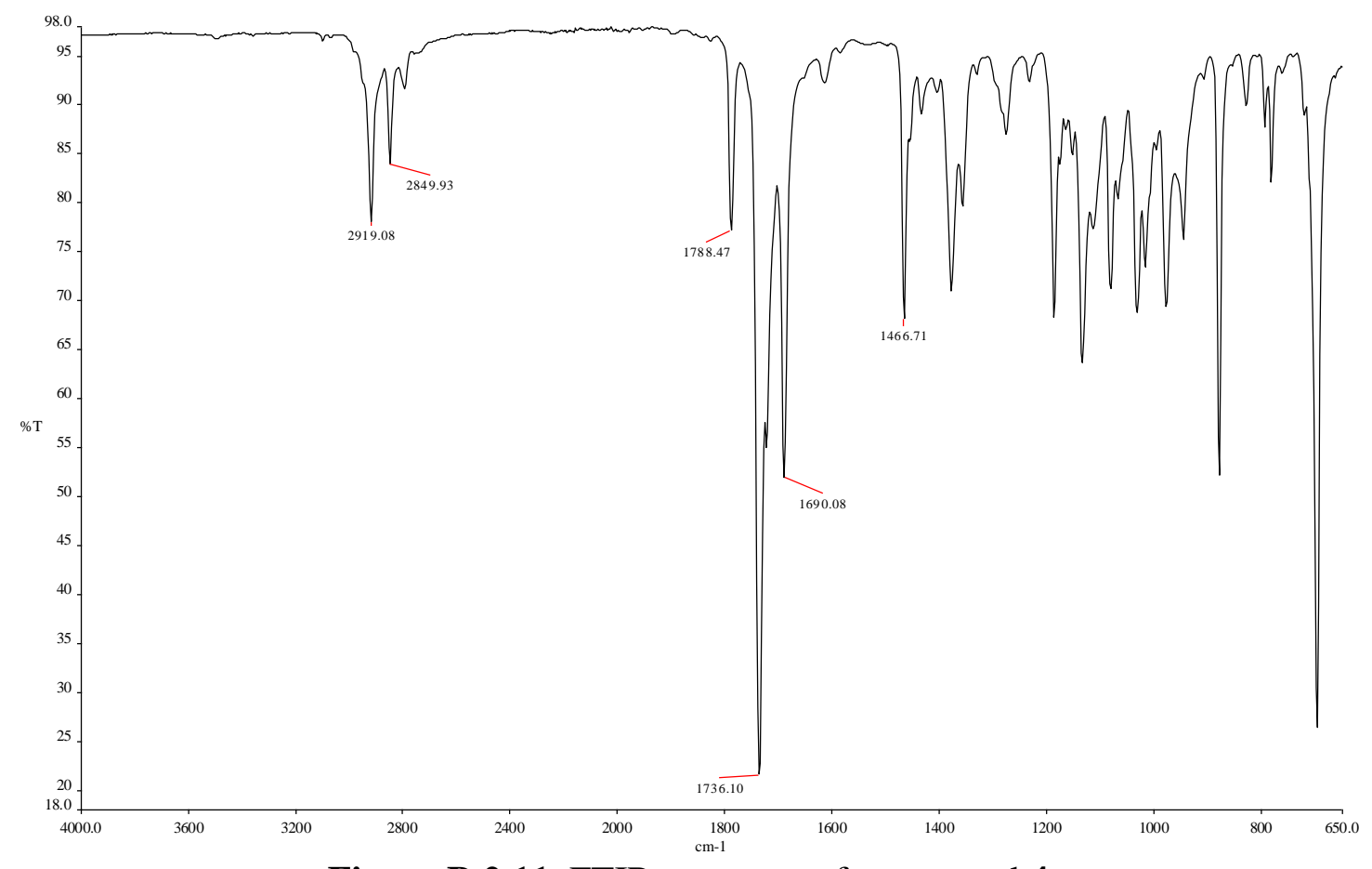

Figure B.2.11. FTIR spectrum of compound 4 


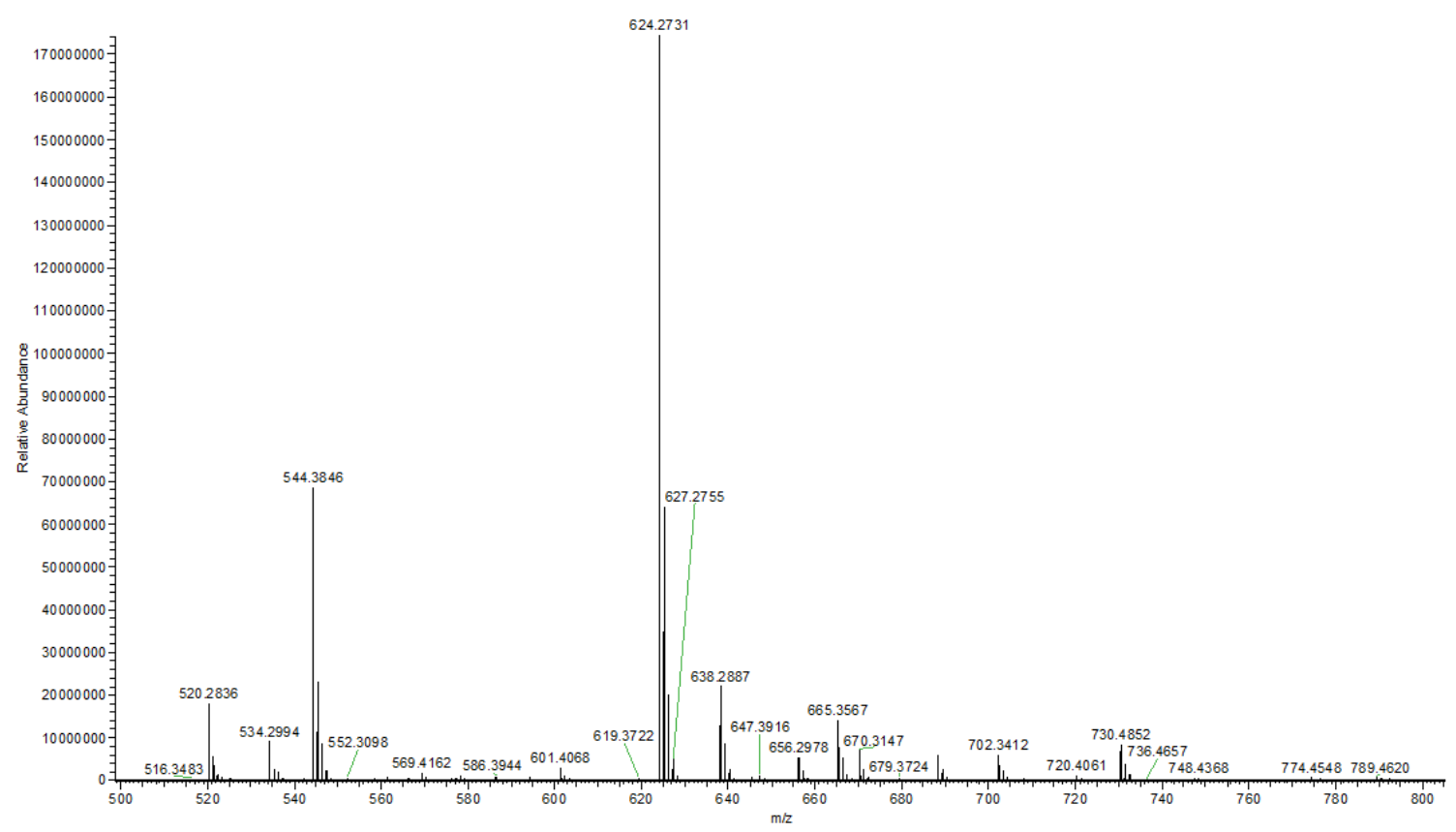

Figure B.2.12. HRMS spectrum of compound 4

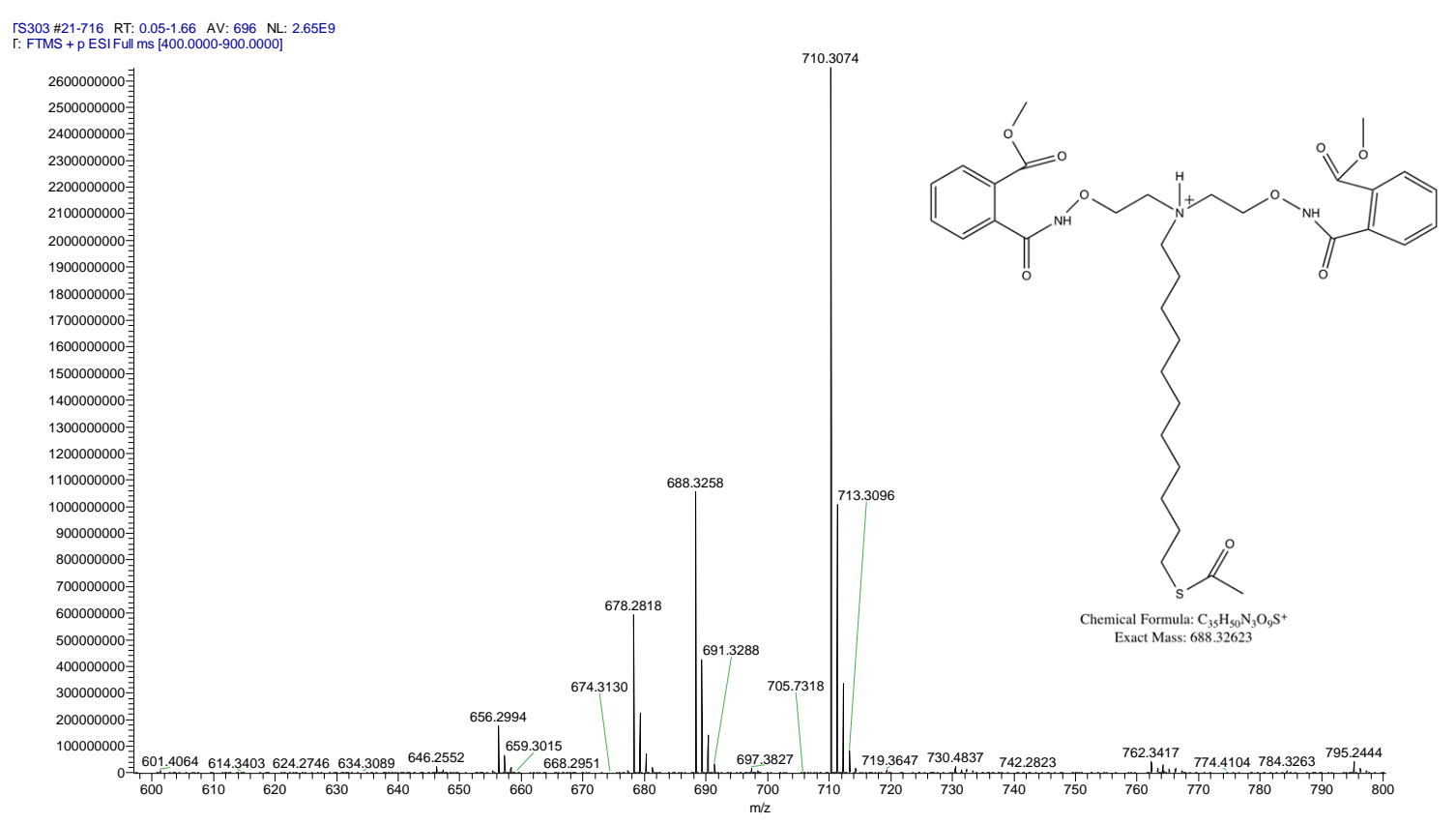

Figure B.2.13. HRMS spectrum of compound 4 in methanol (ring opening) 


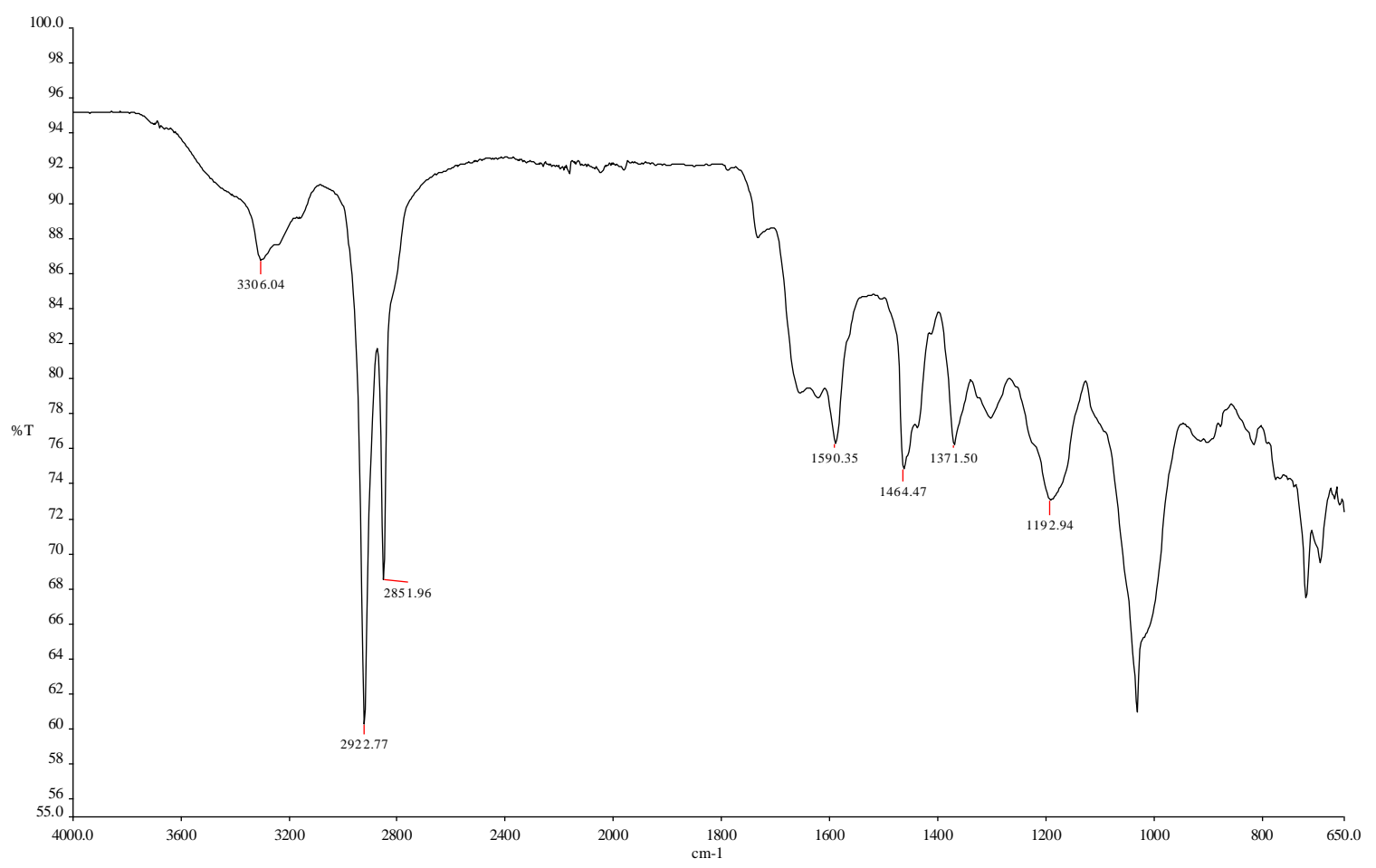

Figure B.2.14. FTIR spectrum of compound AOT bis $_{\text {b }}$

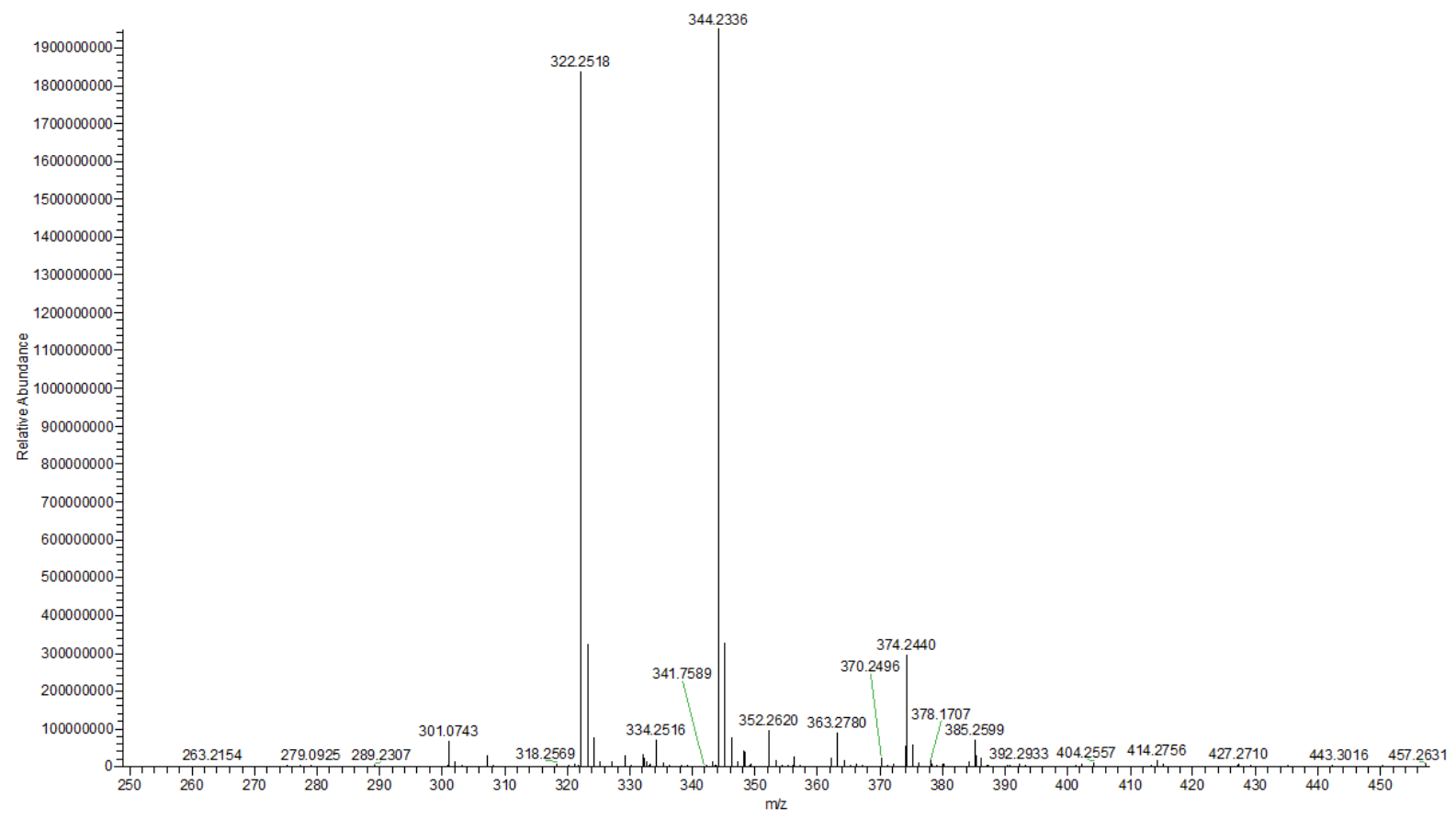

Figure B.2.15. HRMS spectrum of compound AOT $\mathbf{A i s}_{\text {bis }}$ 


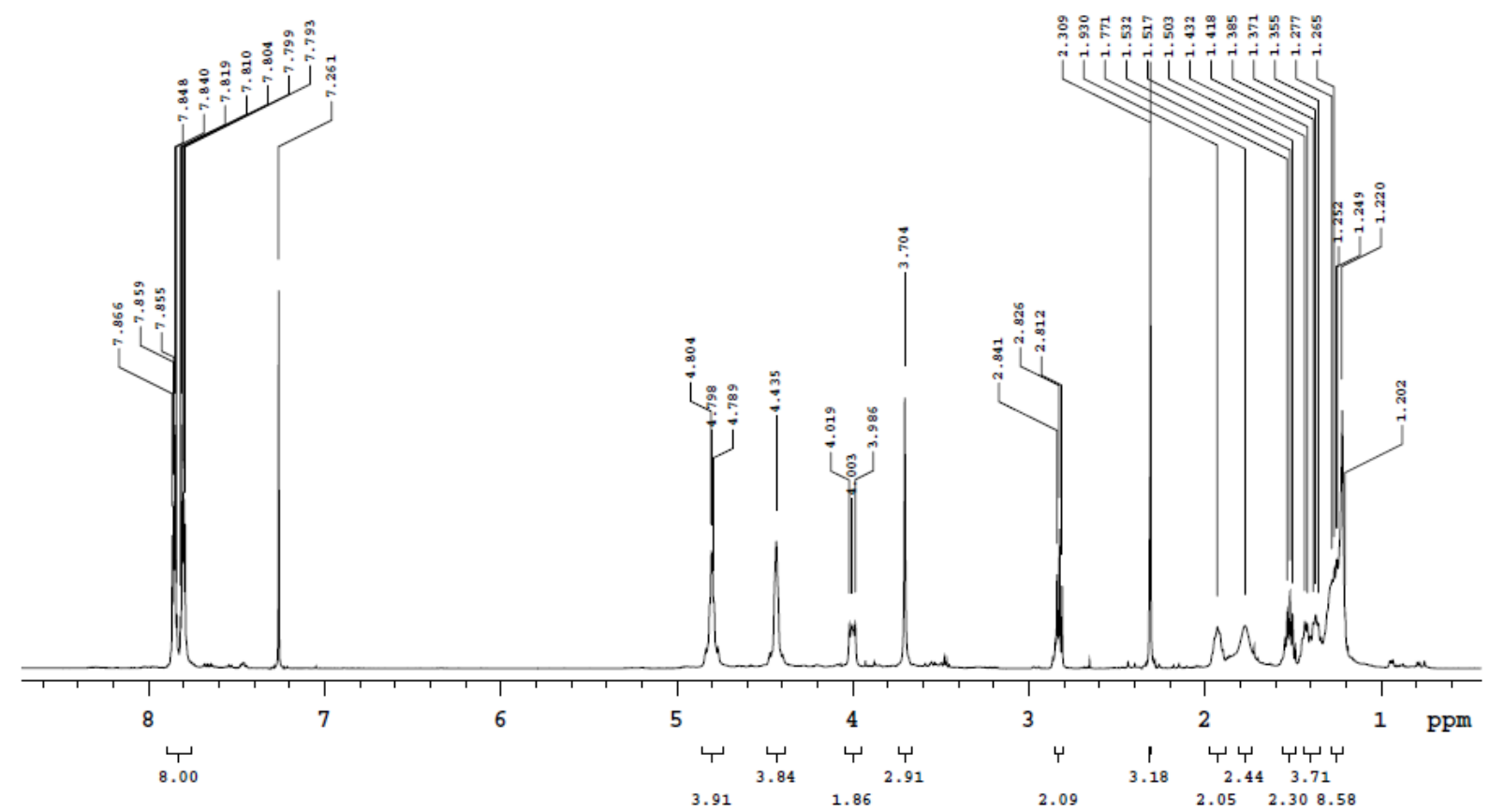

Figure B.2.16. ${ }^{1} \mathrm{H}$ NMR spectrum $\left(400 \mathrm{MHz}, \mathrm{CDCl}_{3}\right)$ of compound 6

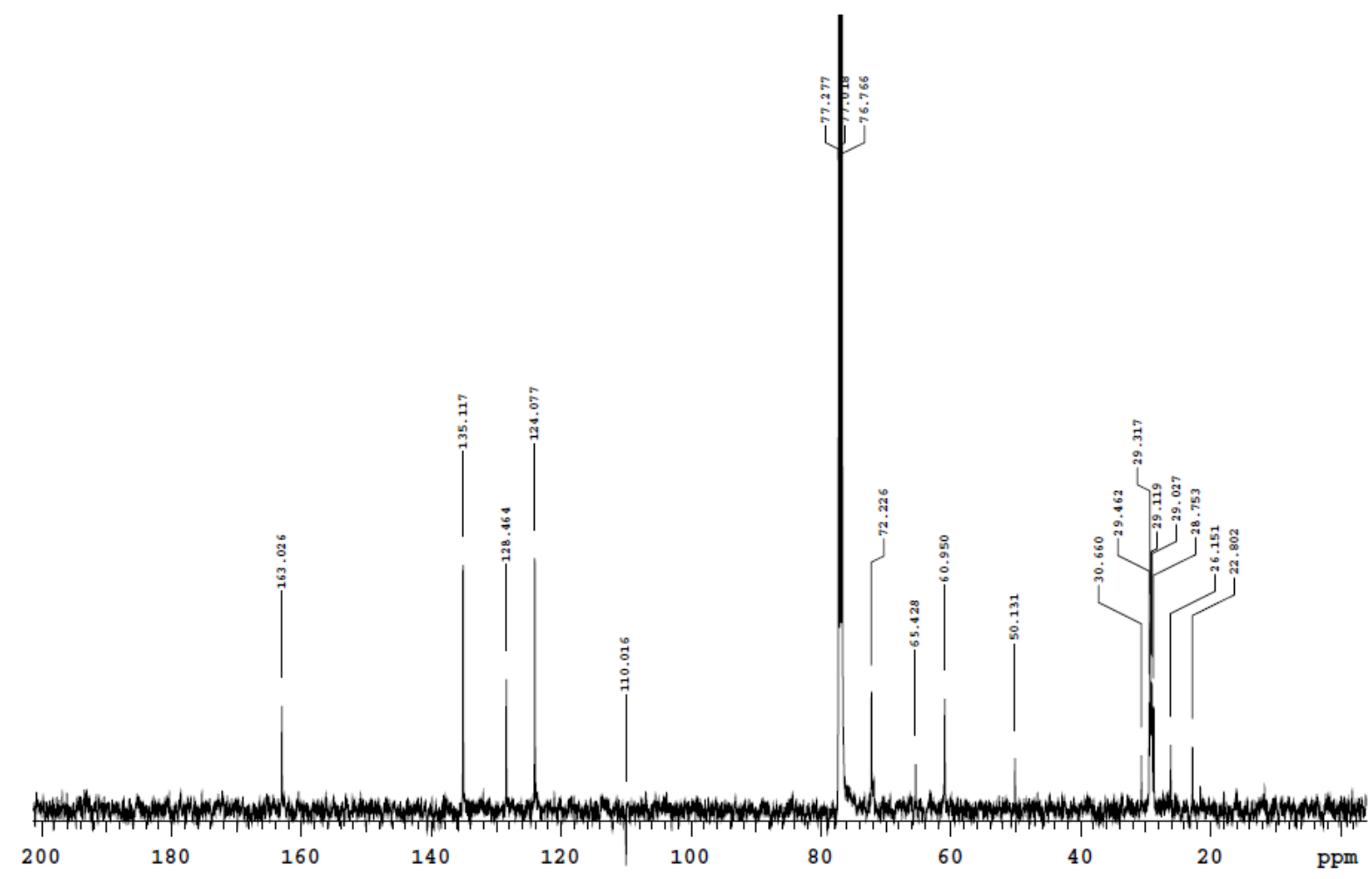

Figure B.2.17. ${ }^{13} \mathrm{C}$ NMR spectrum $\left(400 \mathrm{MHz}, \mathrm{CDCl}_{3}\right)$ of compound 6 


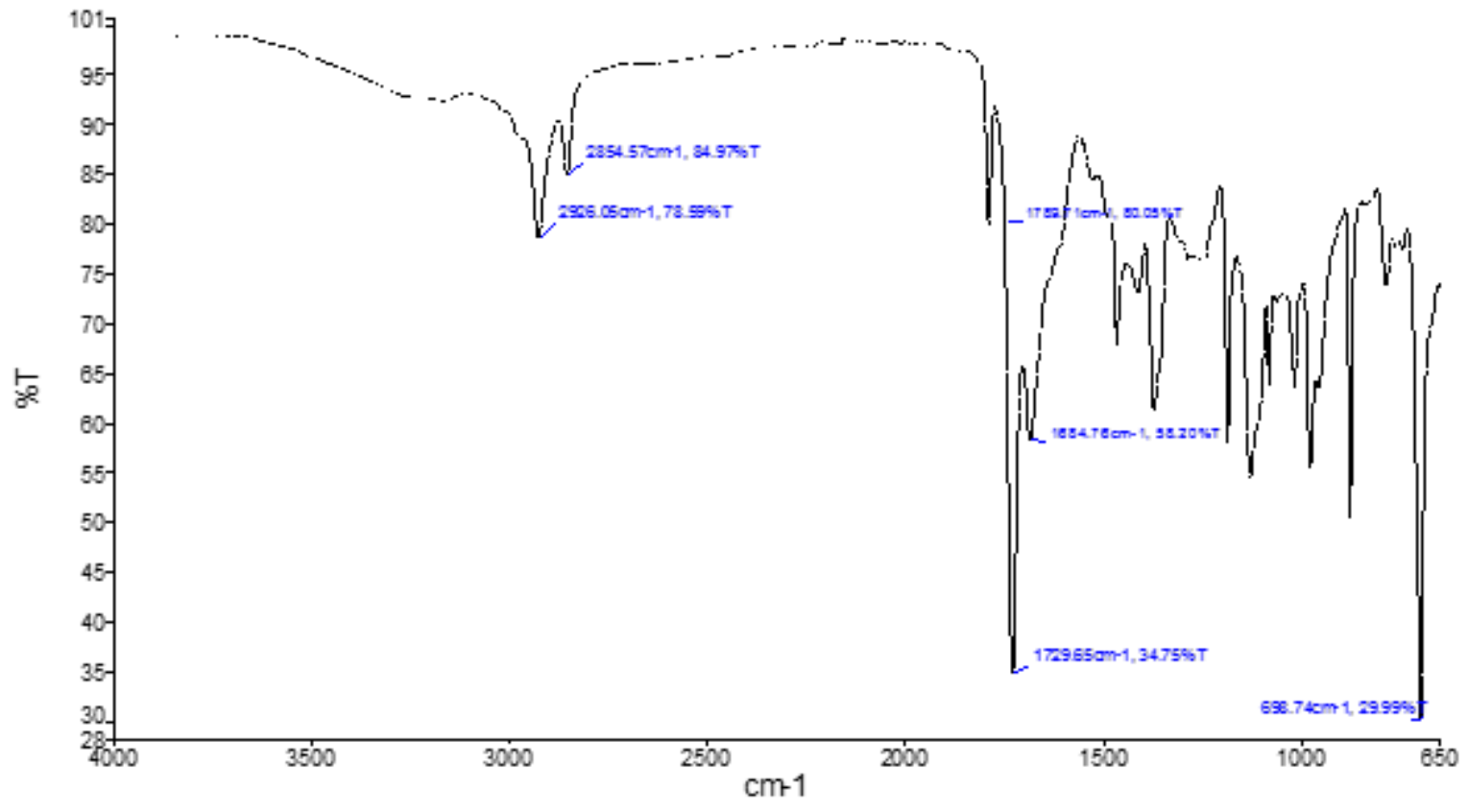

Figure B.2.18. FTIR Spectrum of compound 6

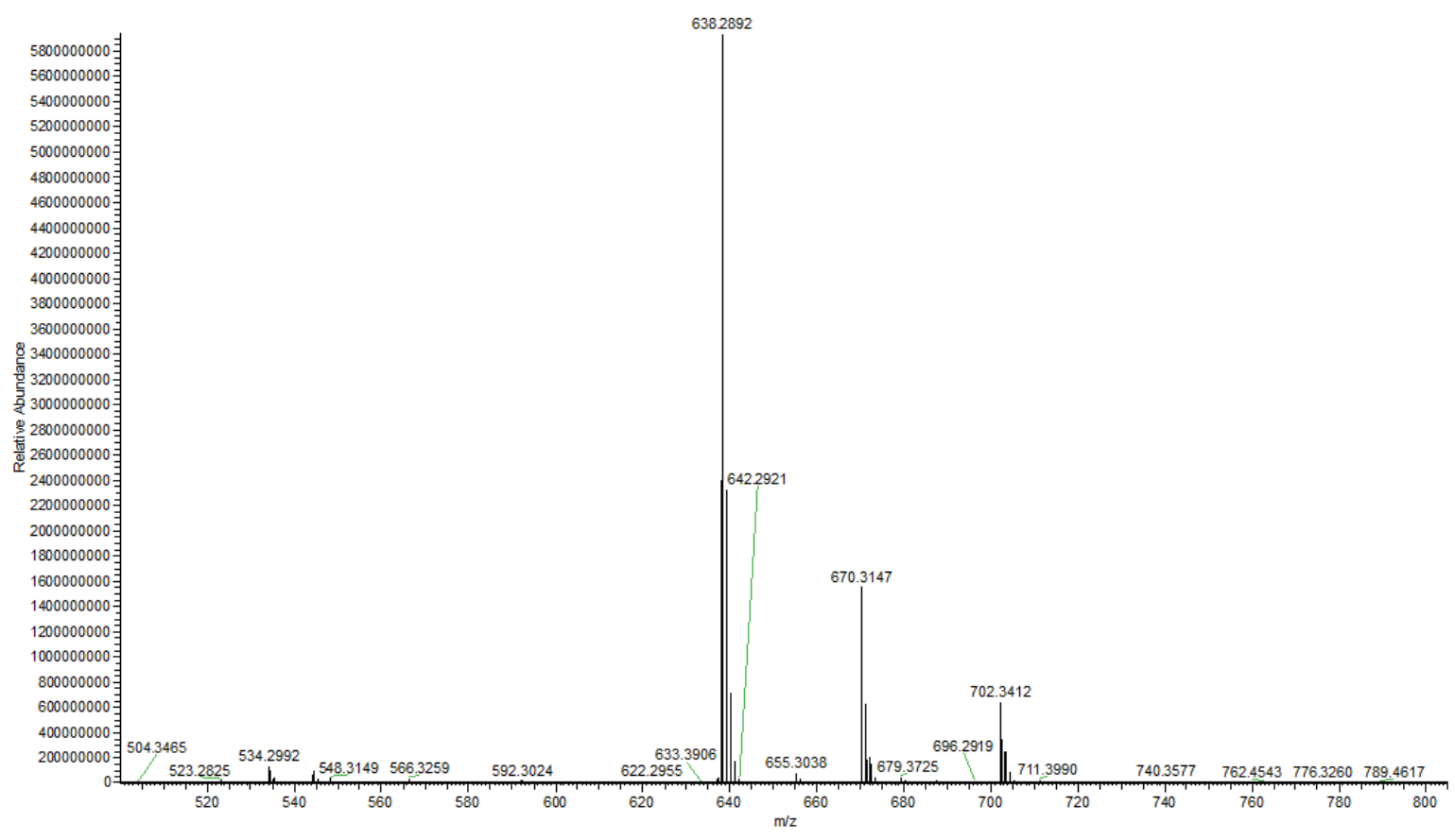

Figure B.2.19. HRMS spectrum of compound 6 


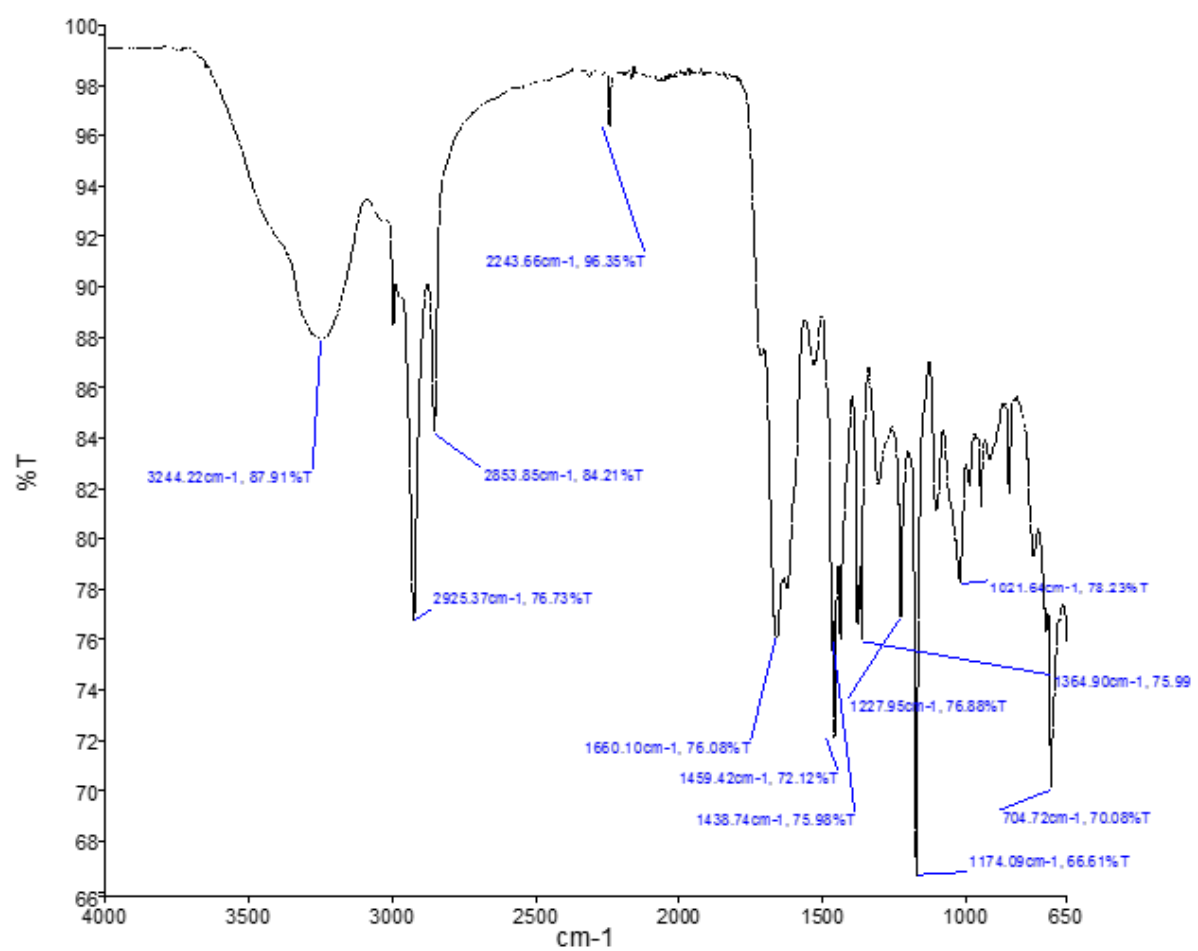

Figure B.2.20. FTIR spectrum of compound AOT bis $^{+}$

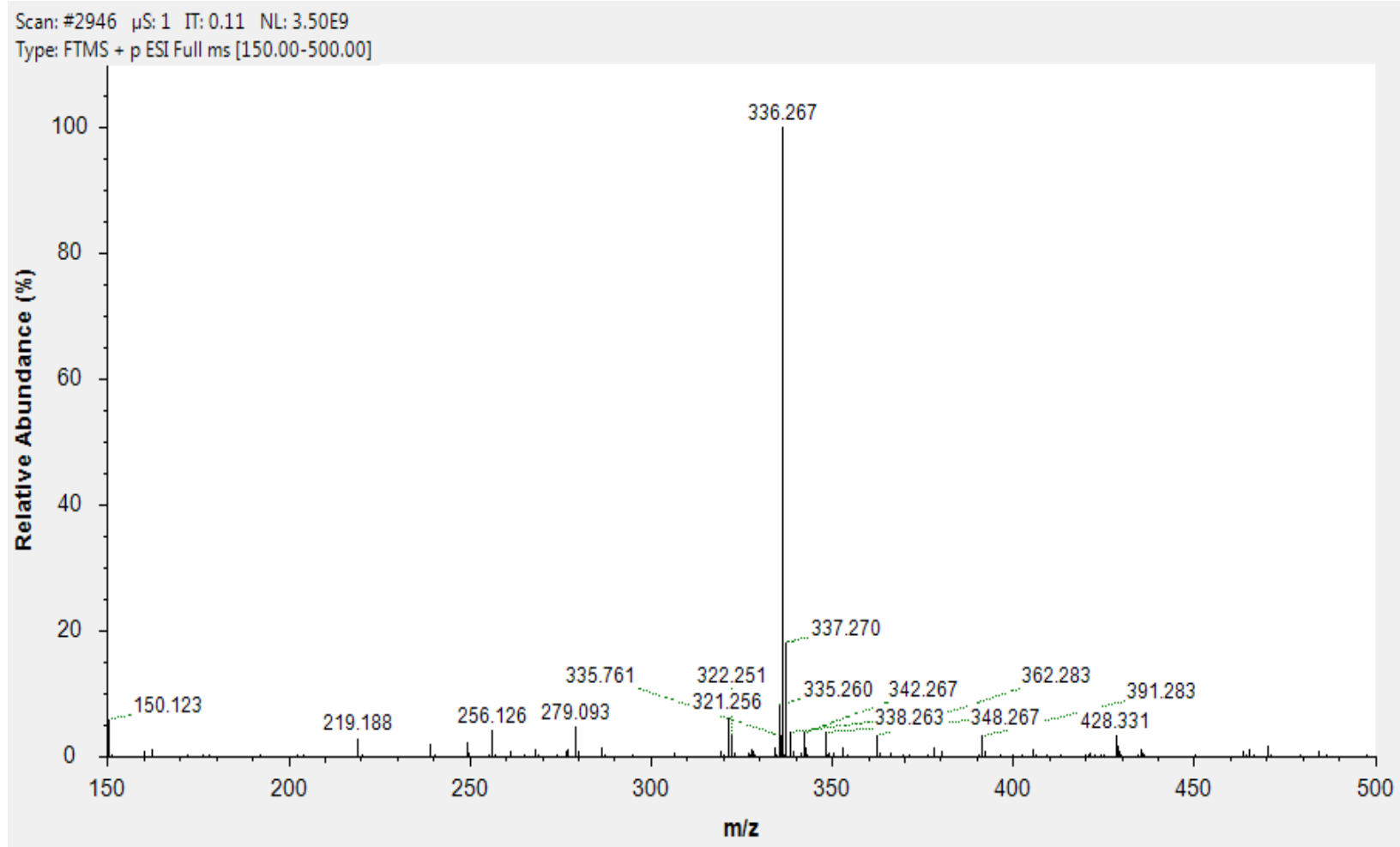

Figure B.2.21. HRMS spectrum of compound AOT bis $^{+}$ 


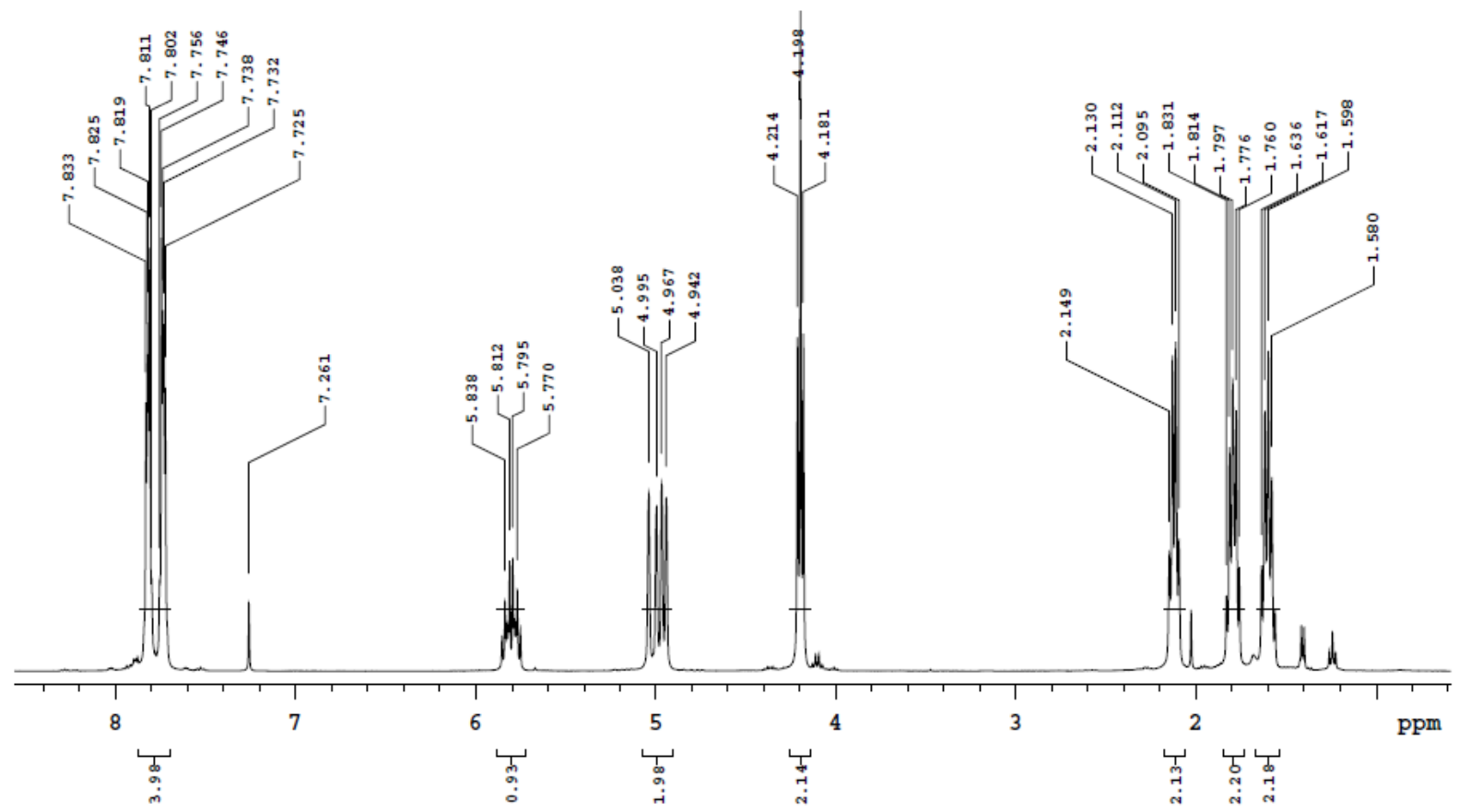

Figure B.2.22. ${ }^{1} \mathrm{H}$ NMR spectrum of compound 9

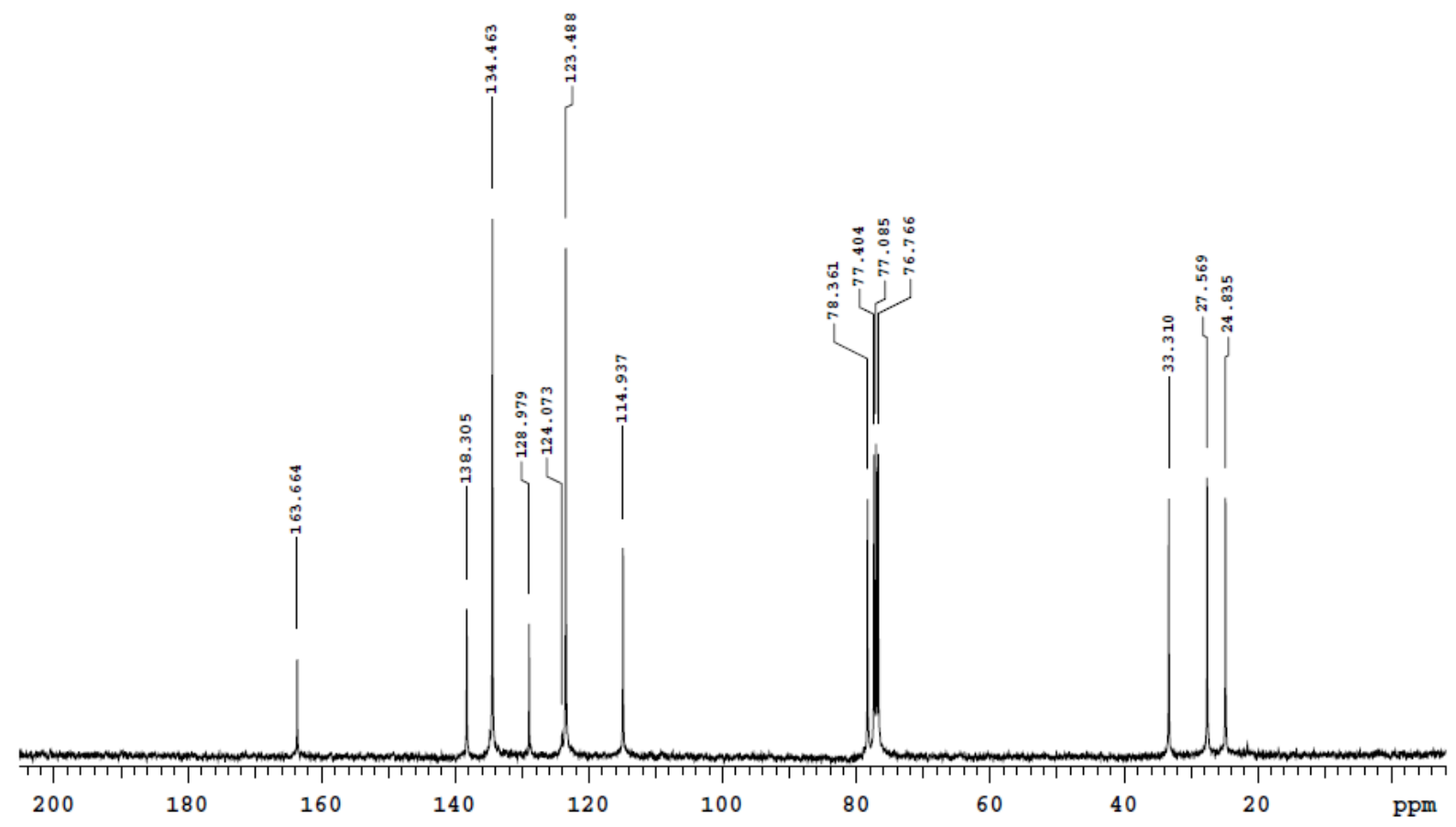

Figure B.2.23. ${ }^{13} \mathrm{C}$ NMR spectrum of compound 9 


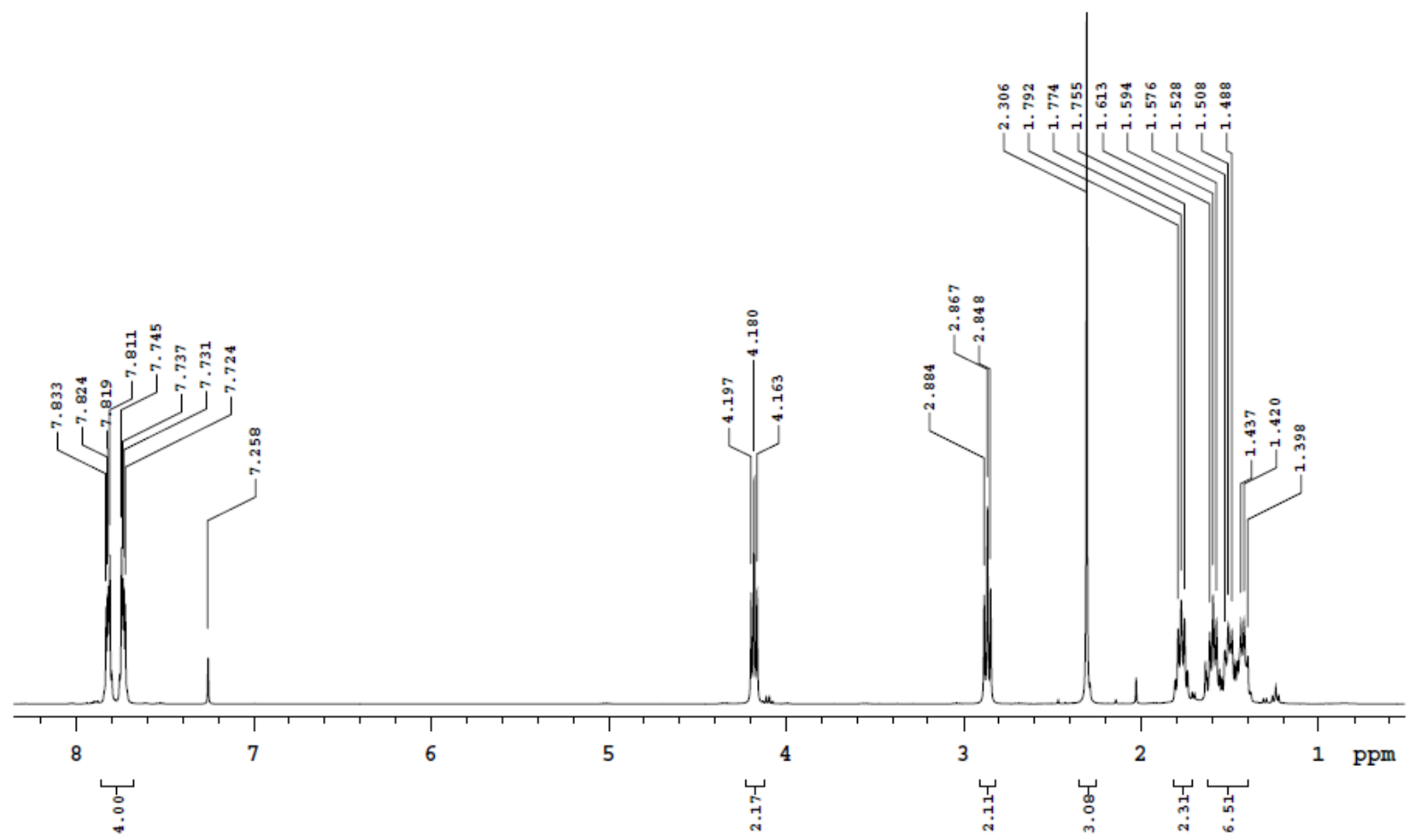

Figure B.2.24. ${ }^{1} \mathrm{H}$ NMR spectrum of compound $\mathbf{1 0}$

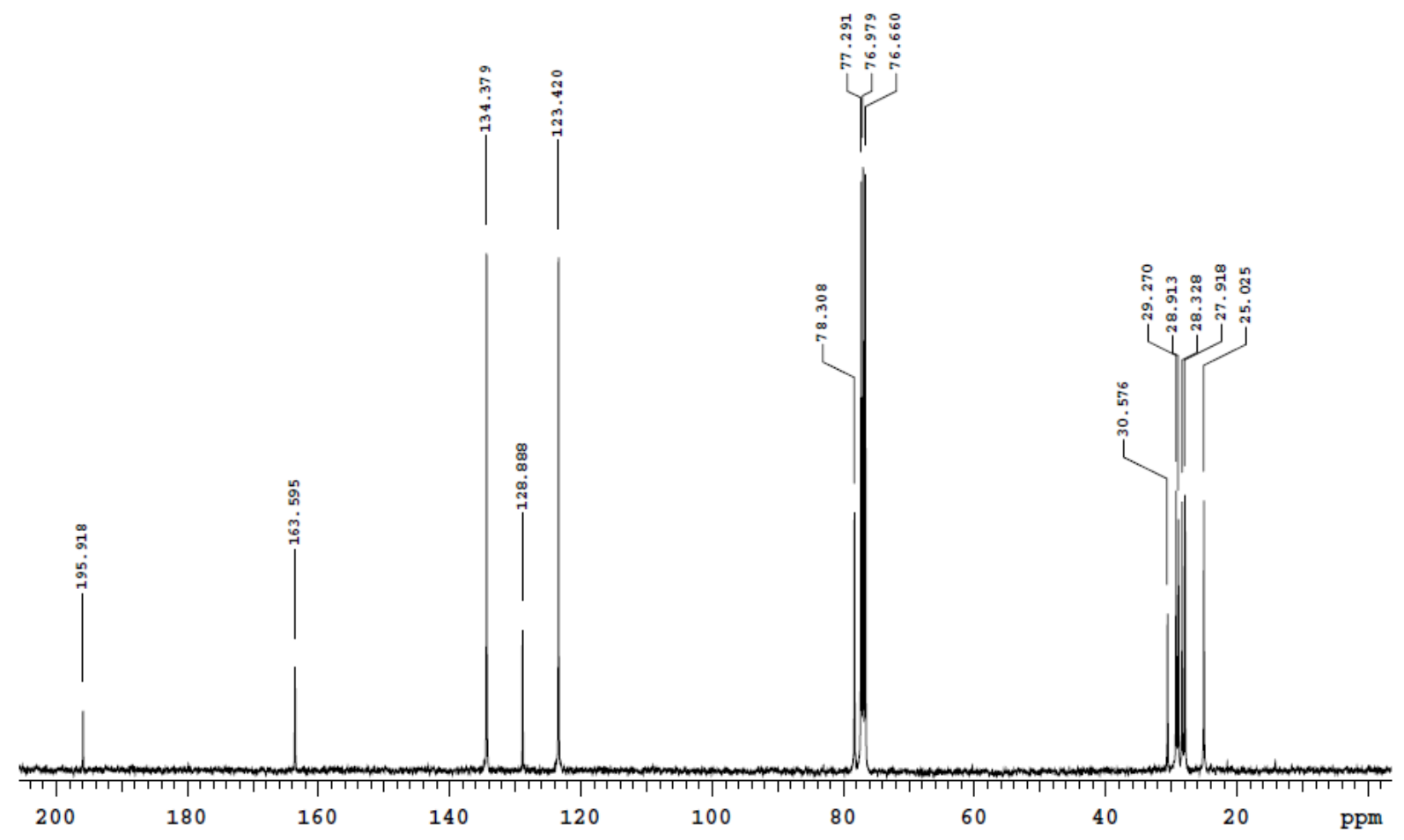

Figure B.2.25. ${ }^{13} \mathrm{C}$ NMR spectrum of compound 10 


\section{B.3. Spectra from Chapter 3}

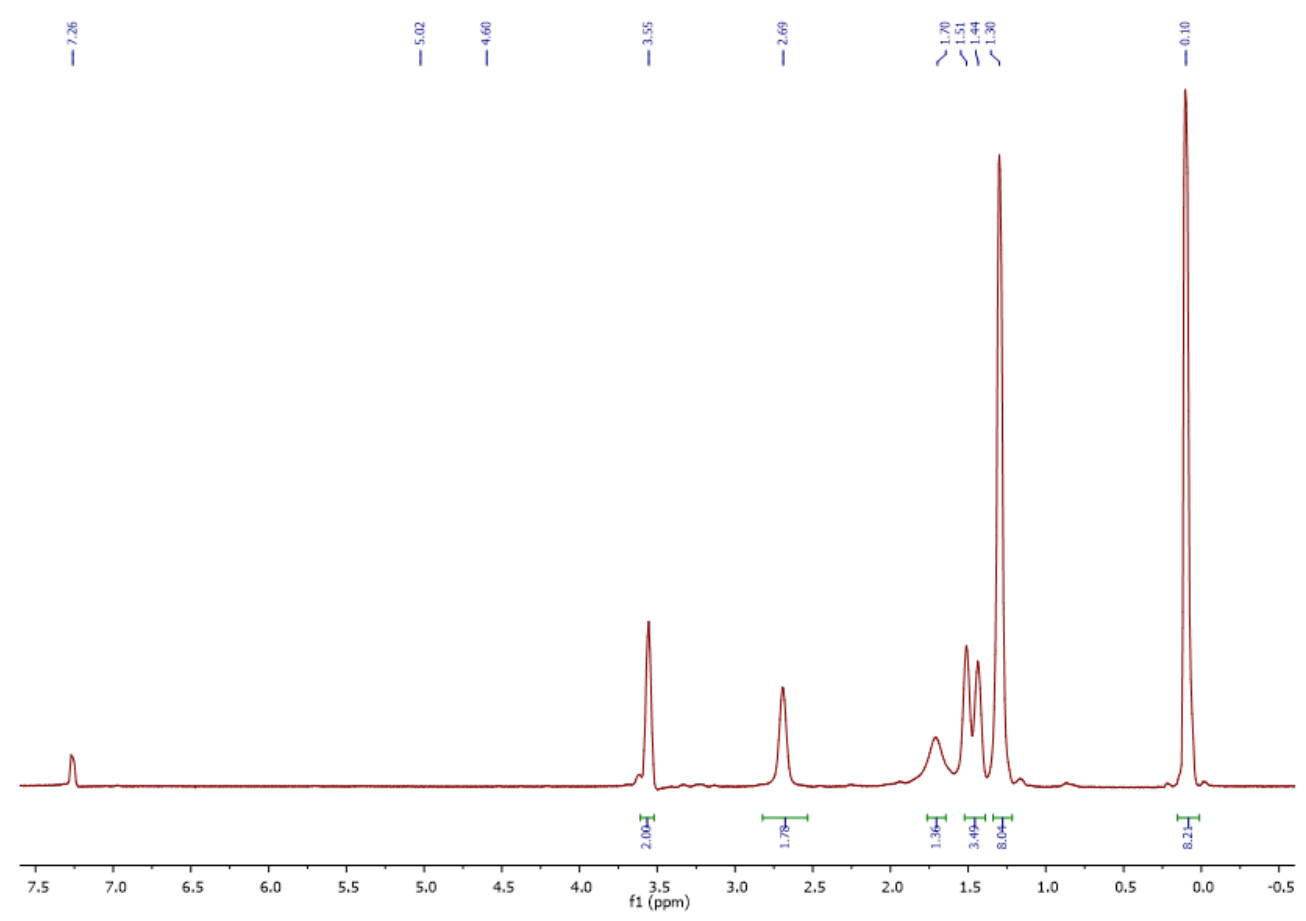

Figure B.3.1. ${ }^{1} \mathrm{H}$ NMR spectrum $\left(400 \mathrm{MHz}, \mathrm{CDCl}_{3}\right)$ of compound 13 


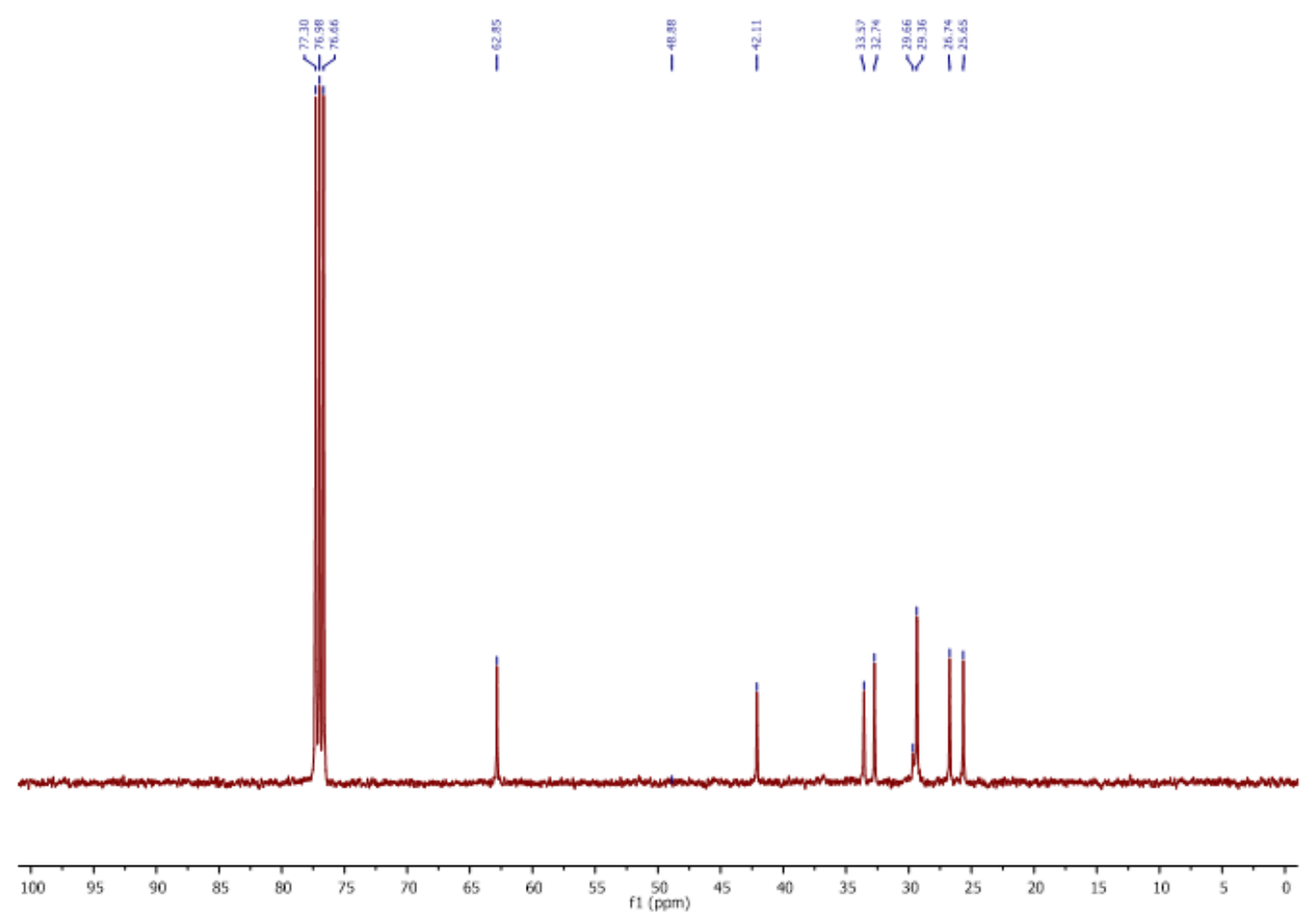

Figure B.3.2. ${ }^{13} \mathrm{C}$ NMR spectrum $\left(100 \mathrm{MHz}, \mathrm{CDCl}_{3}\right)$ of compound 13

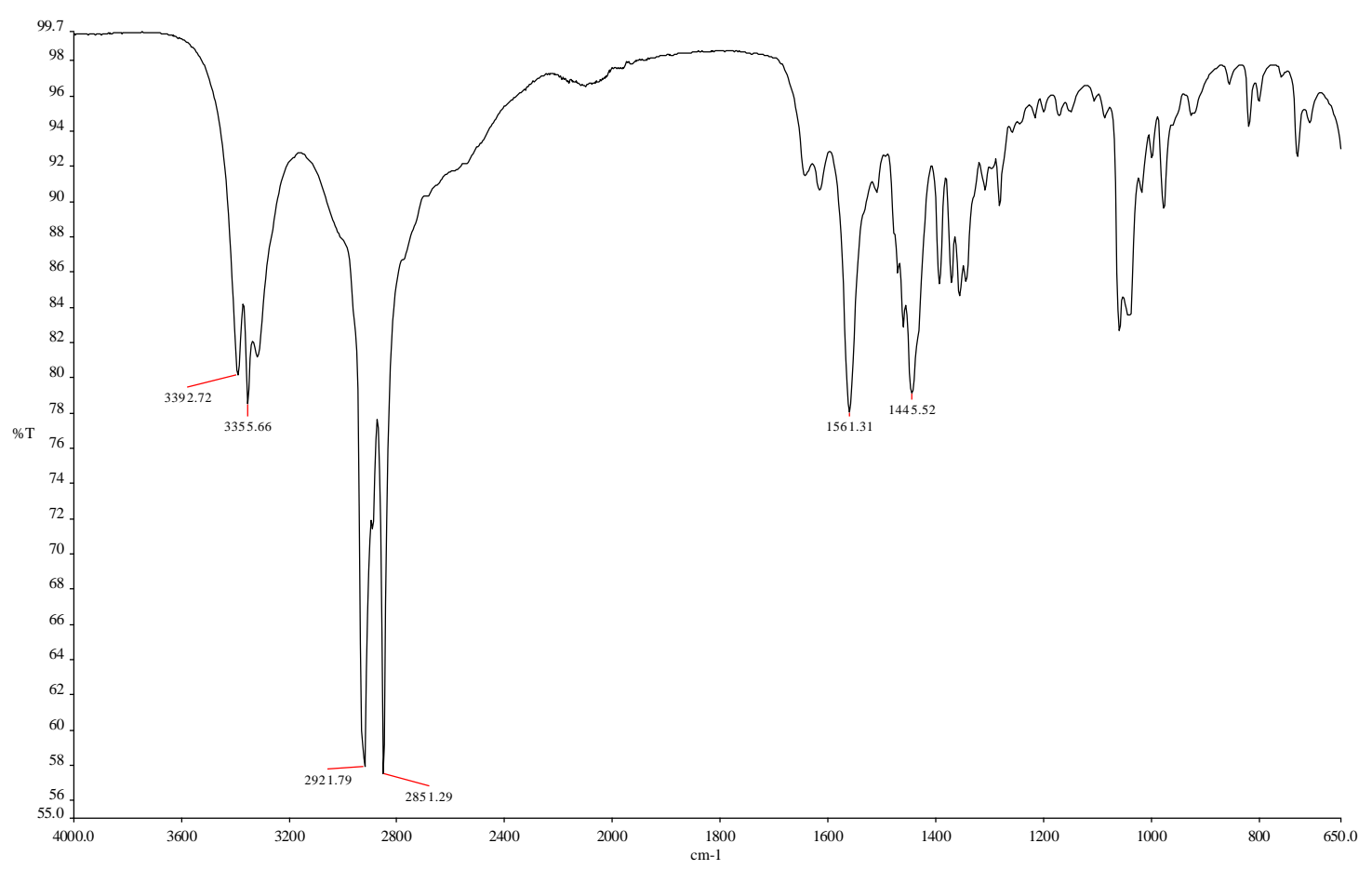

Figure B.3.3. FTIR spectrum of compound 13 


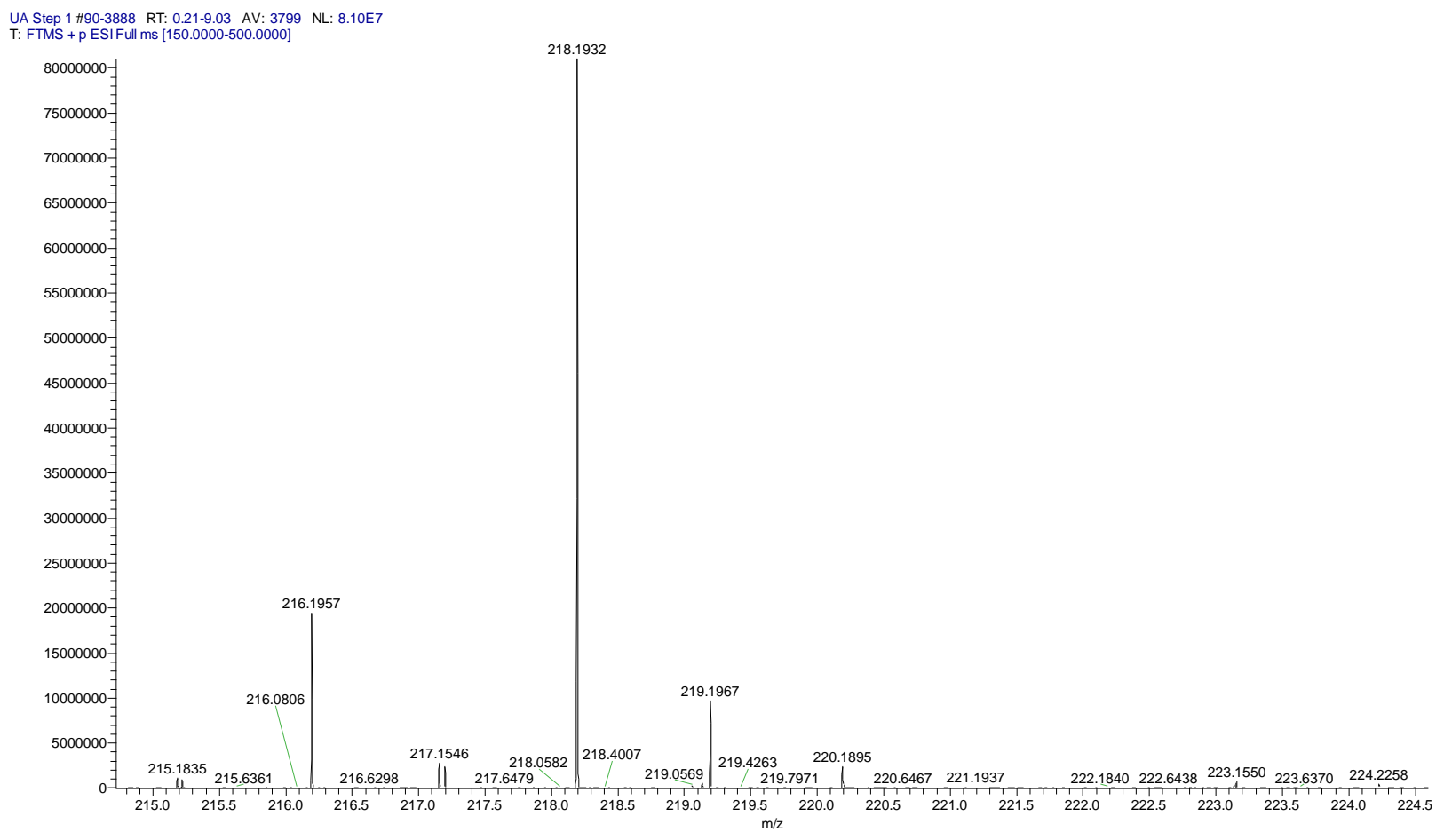

Figure B.3.4. HRMS spectrum of compound 13

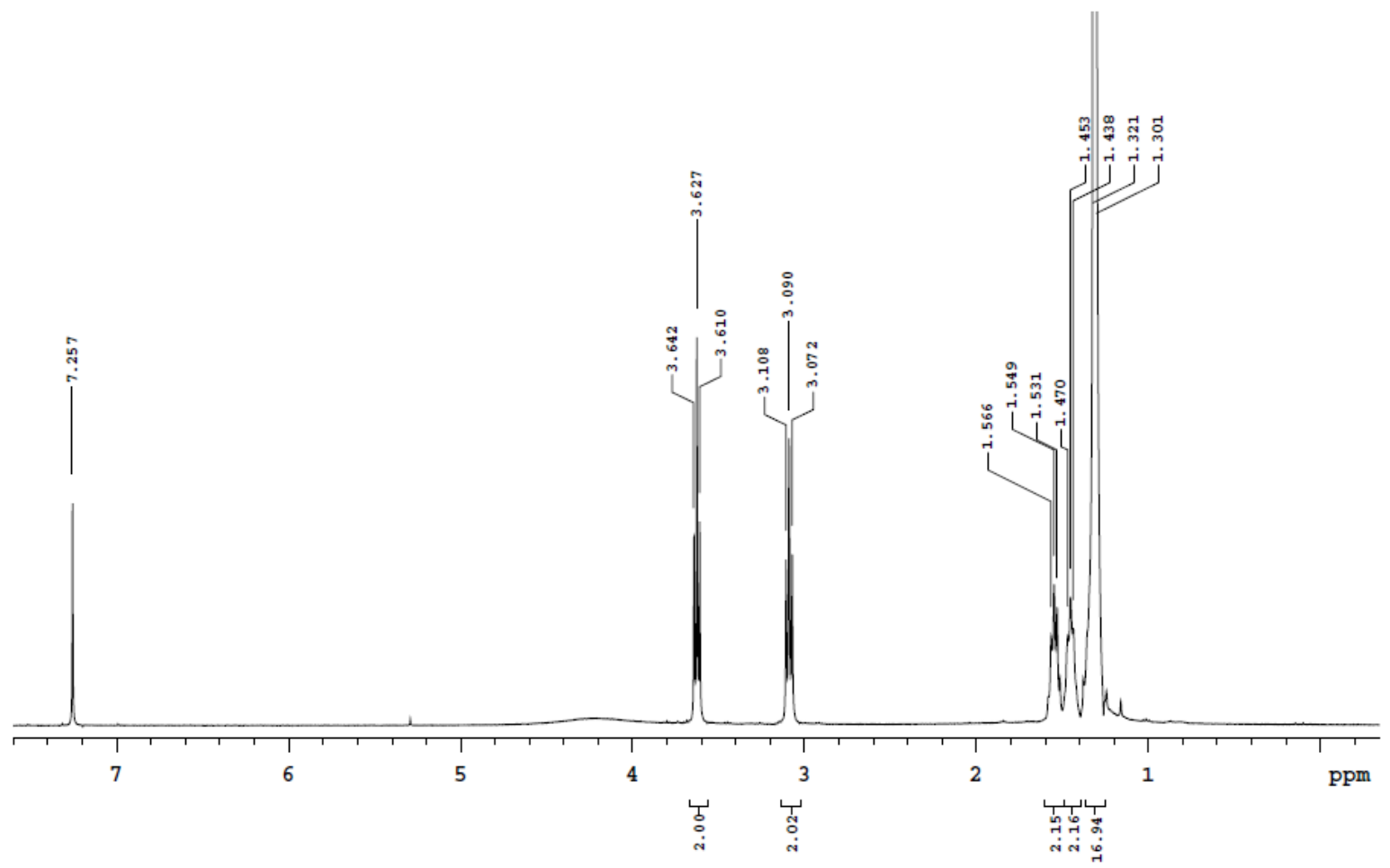

Figure B.3.5. ${ }^{1} \mathrm{H}$ NMR spectrum $\left(400 \mathrm{MHz}, \mathrm{CDCl}_{3}\right.$ ) of Compound 14 


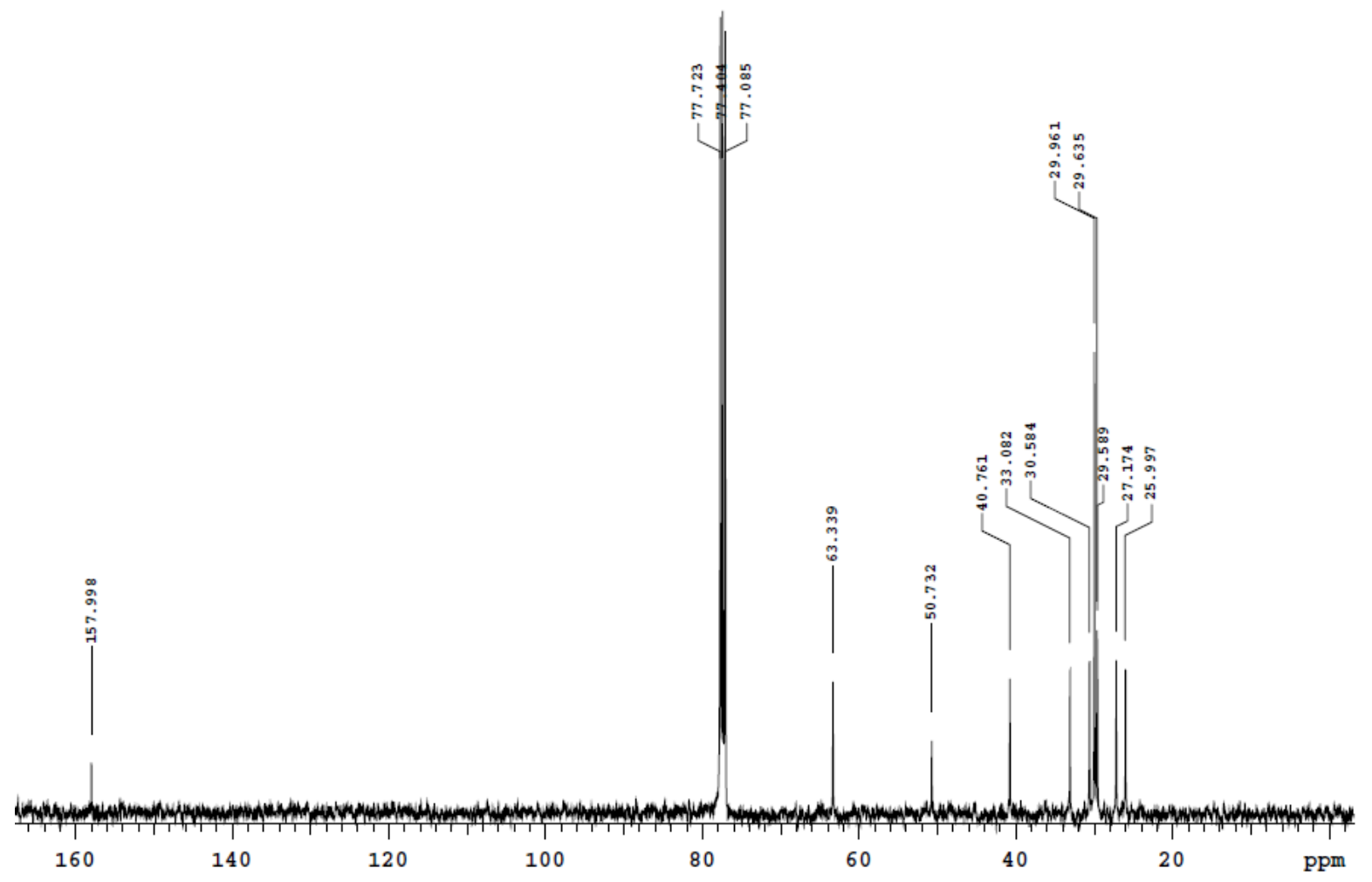

Figure B. $3.6{ }^{13} \mathrm{C}$ NMR spectrum $\left(100 \mathrm{MHz}, \mathrm{CDCl}_{3}\right)$ of compound 14

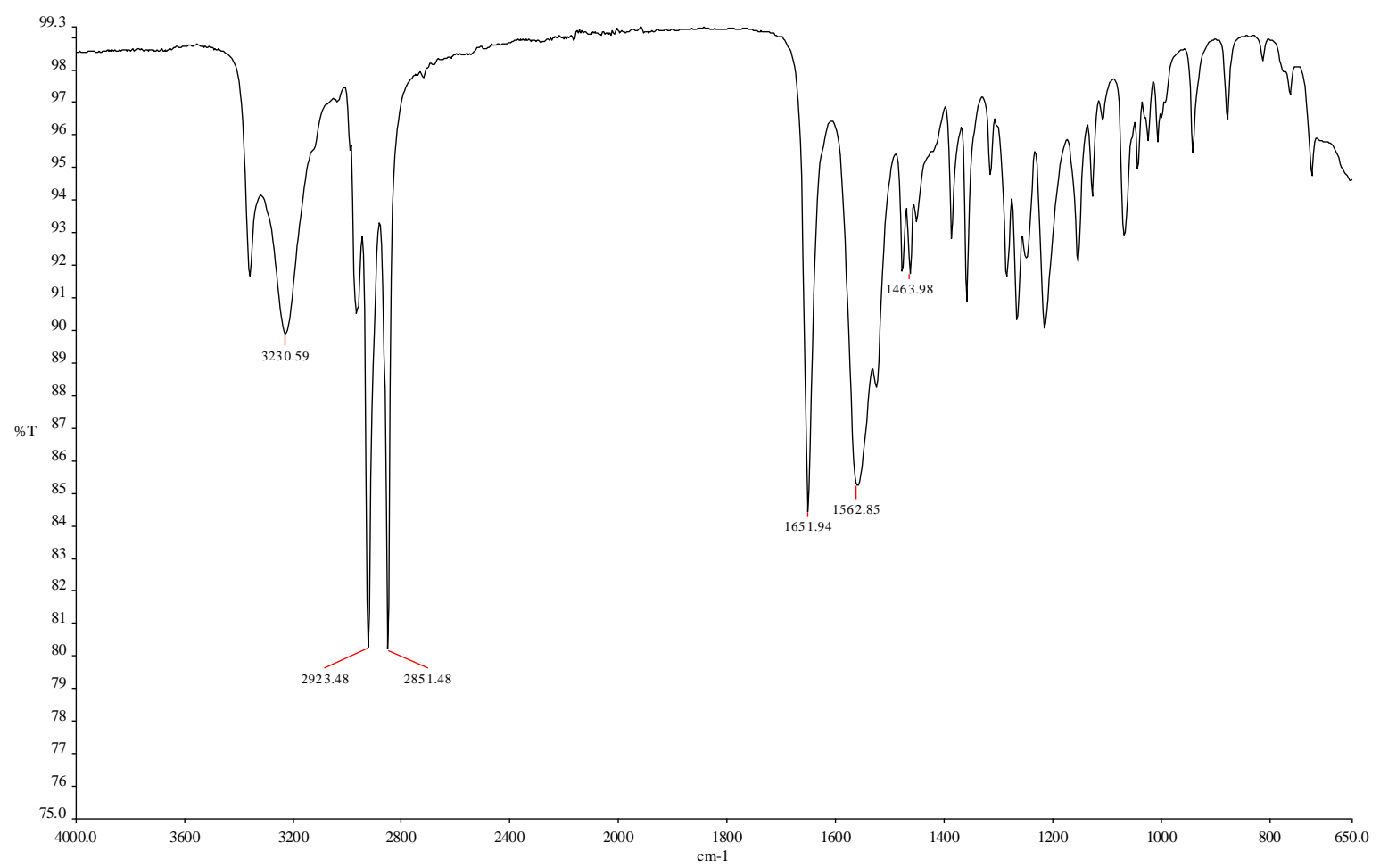

Figure B.3.7. FTIR spectrum of compound 14 


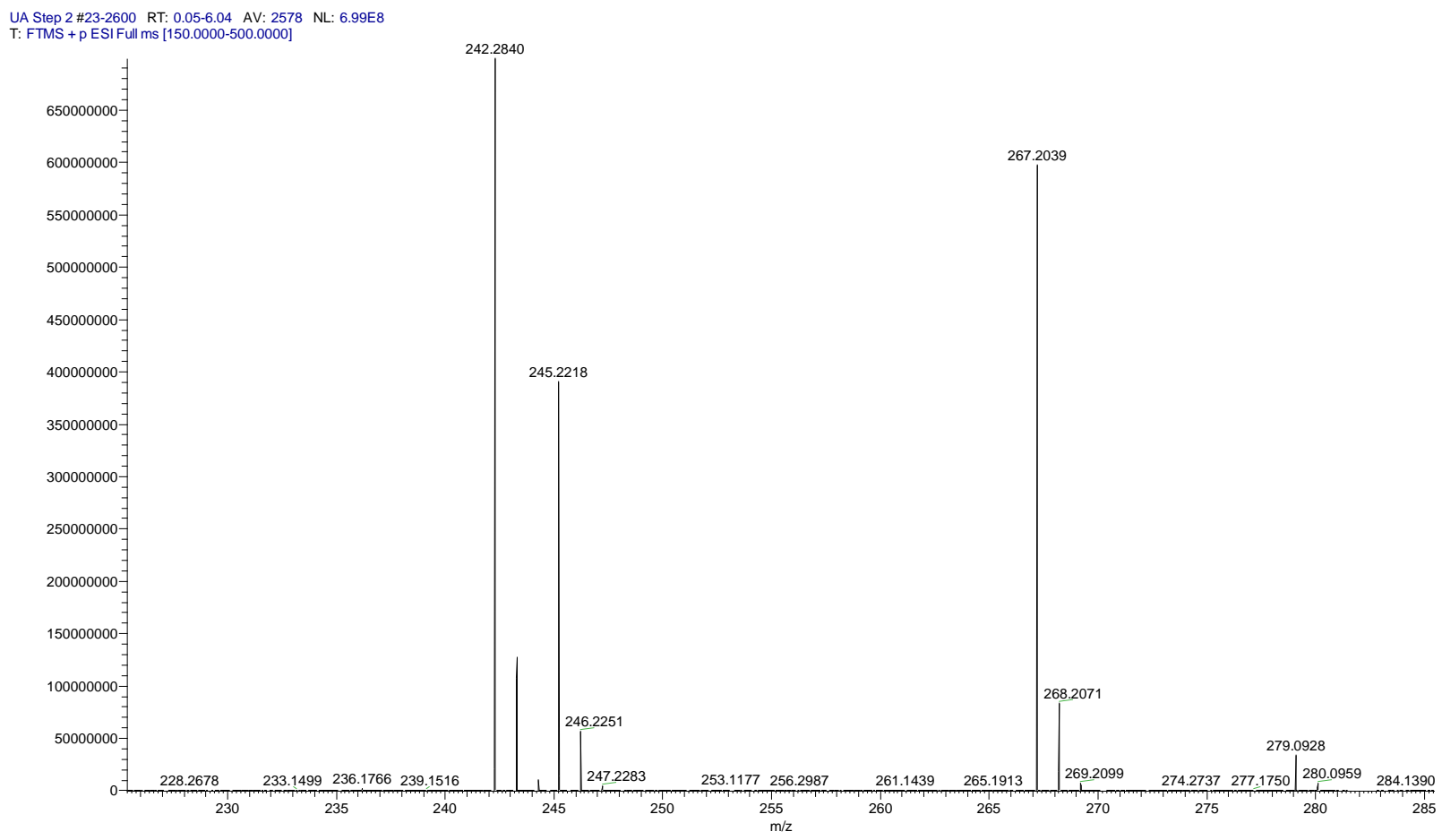

Figure B.3.8. HRMS spectrum of compound 14

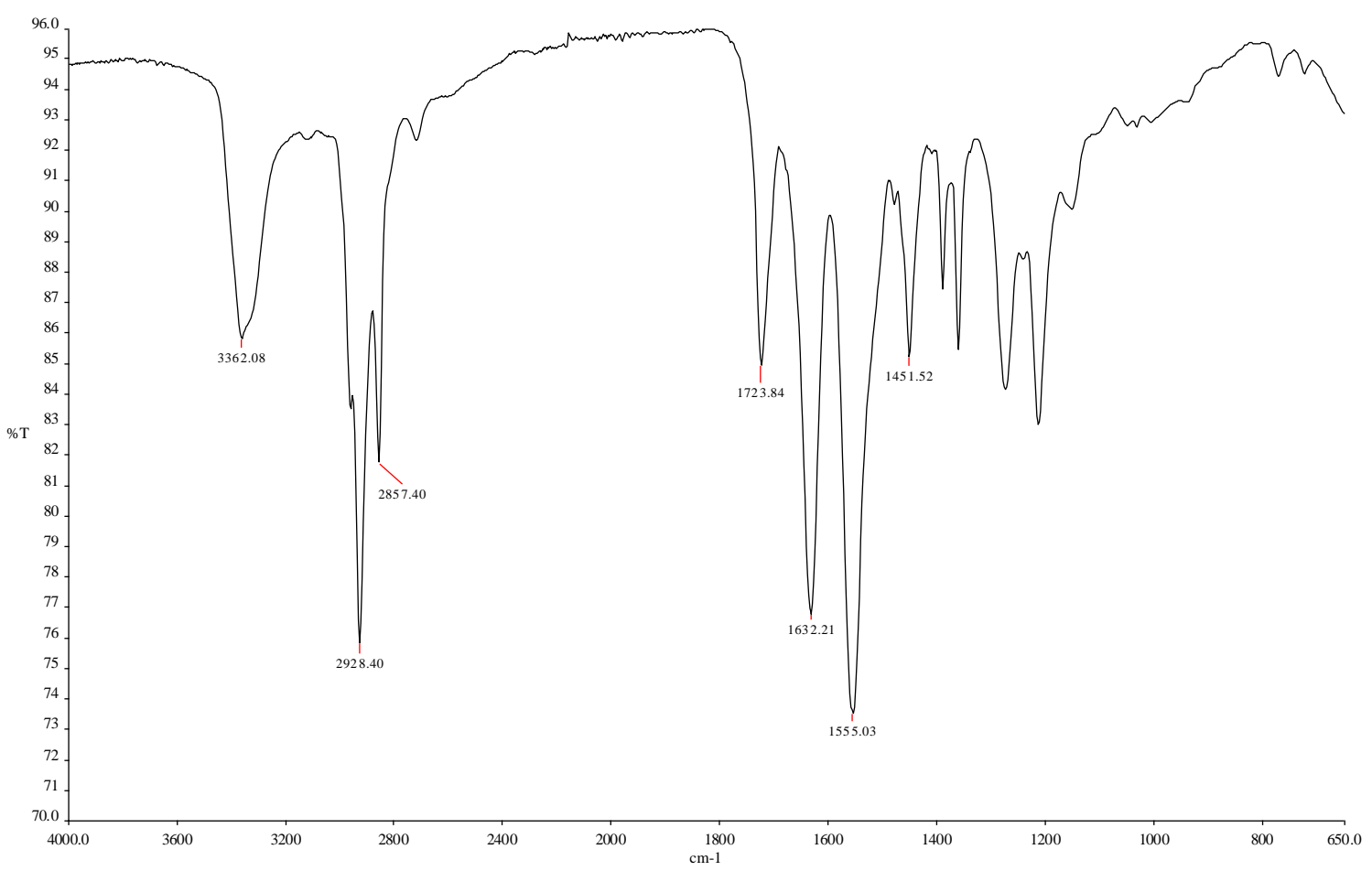

Figure B.3.9. FTIR spectrum of compound 15 (urea-aldehyde) 


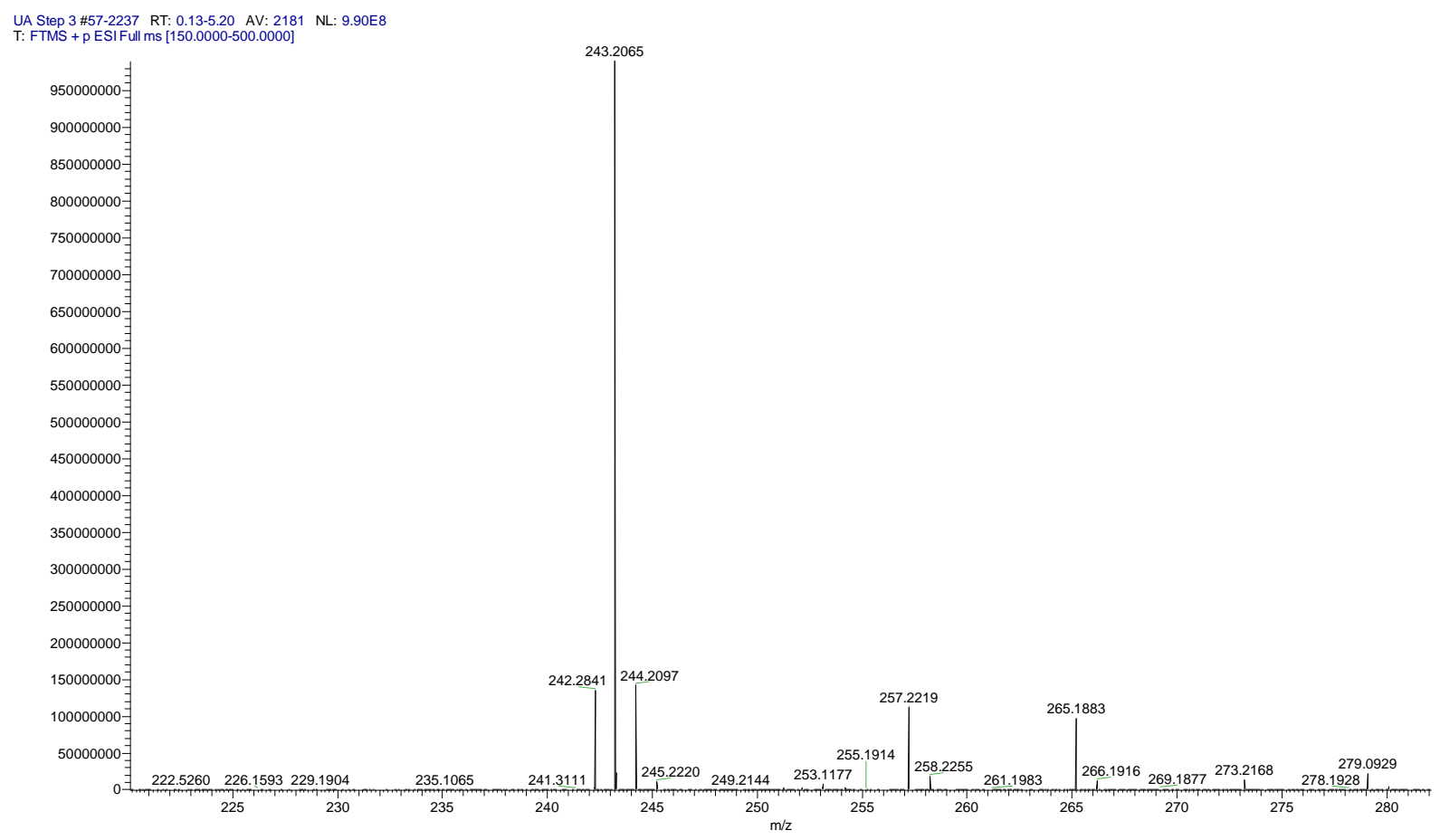

Figure B.3.10. HRMS spectrum of compound 15 (urea-aldehyde)

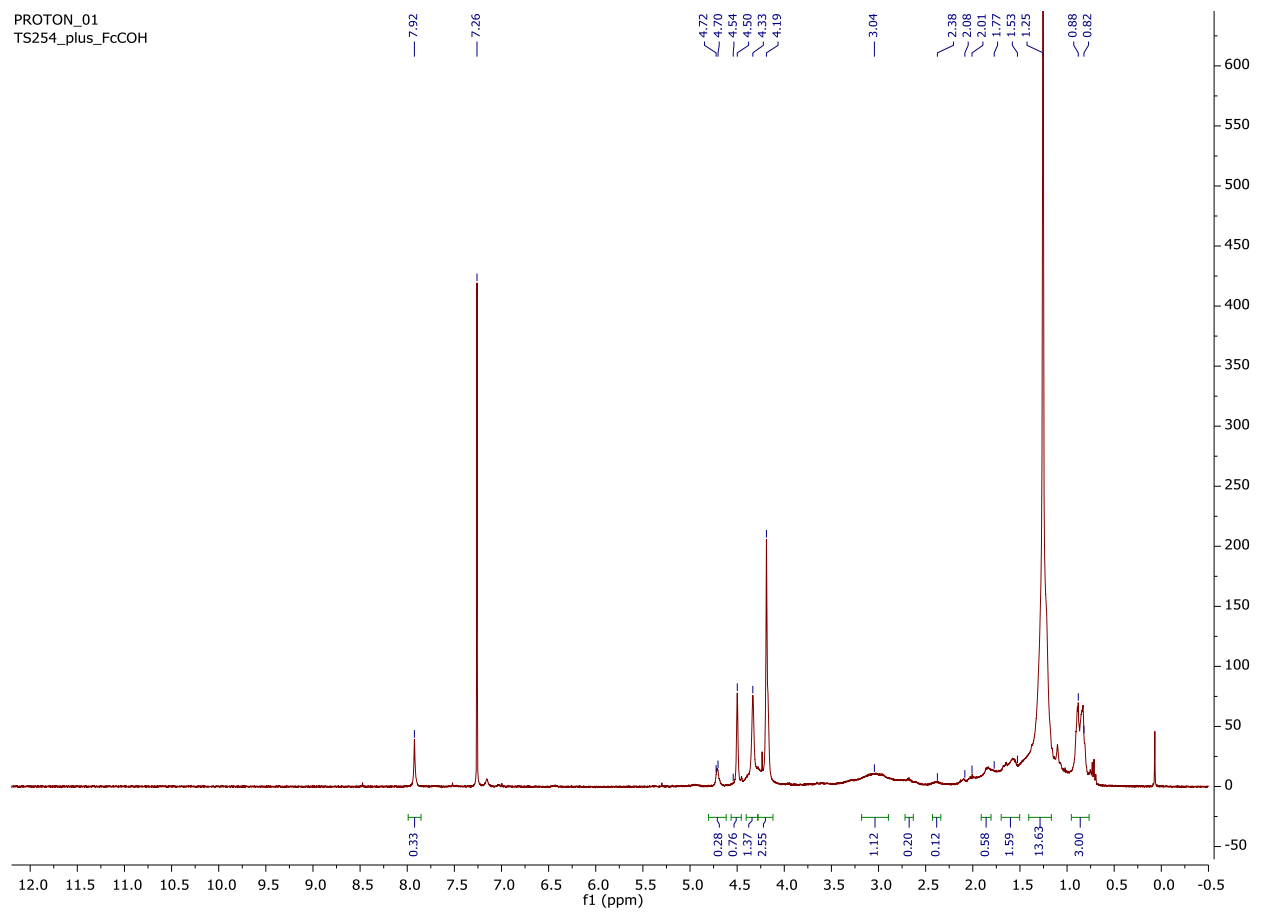

Figure B.3.11. ${ }^{1} \mathrm{H}$ NMR spectrum of $\mathrm{Au}\left(\mathrm{C}_{6} \mathrm{~S}\right)\left(\mathrm{AOT}_{\text {bis }}-\right.$ ferrocene) MMPCs 


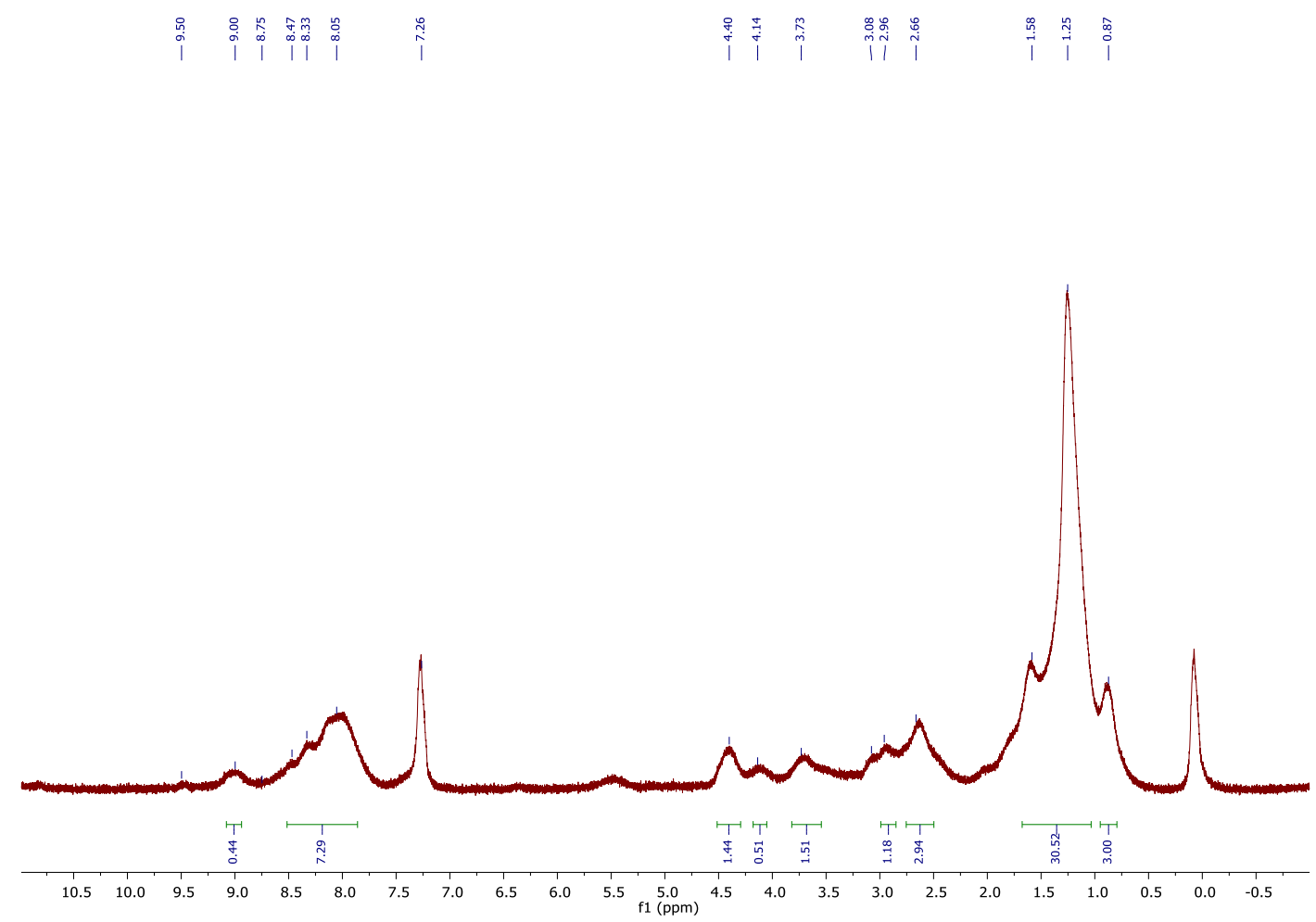

Figure B.3.12. ${ }^{1} \mathrm{H}$ NMR spectrum of $\mathrm{Au}\left(\mathrm{C}_{6} \mathrm{~S}\right)\left(\mathrm{AOT}_{\text {bis }}\right.$-pyrene) MMPCs

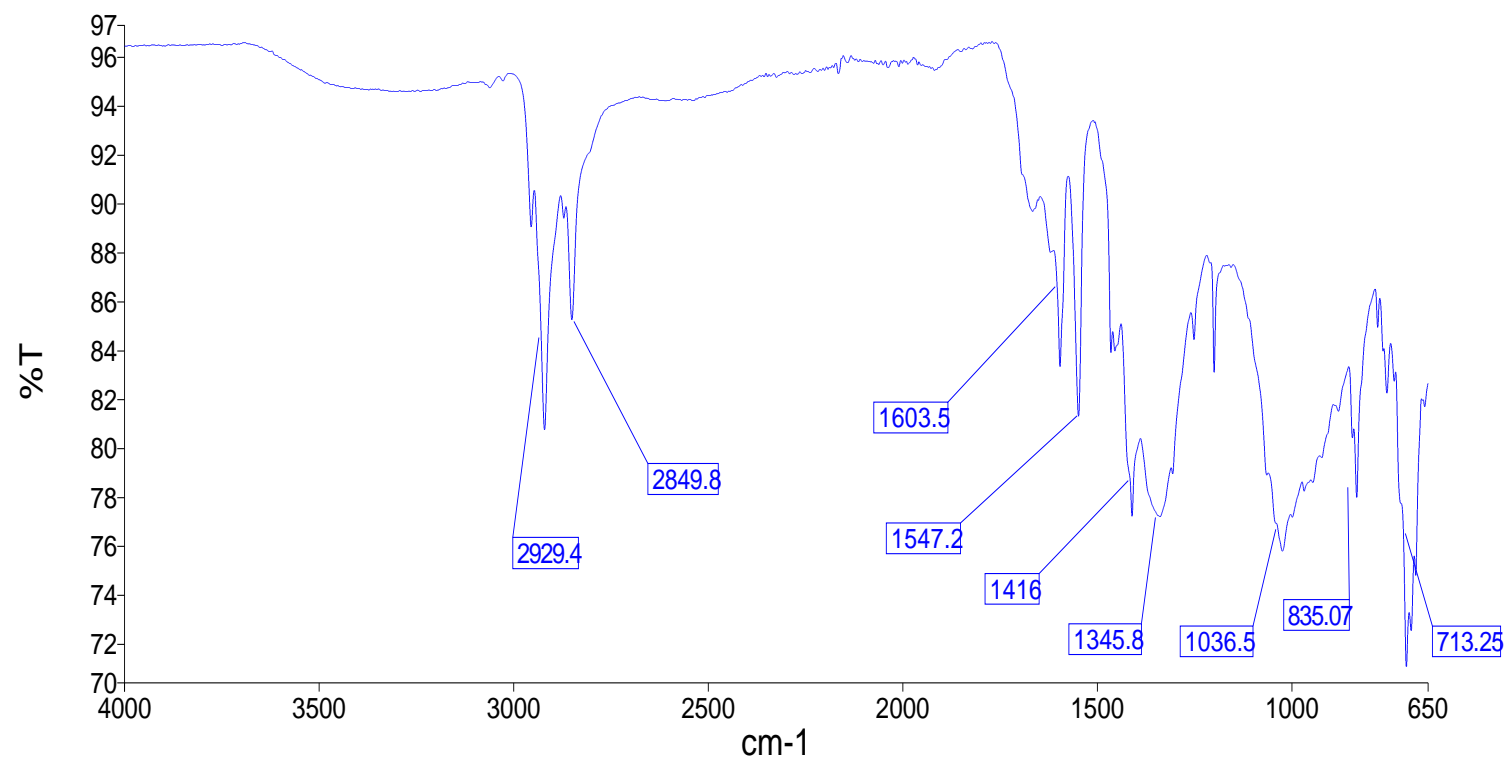

Figure B.3.13. FTIR spectrum of $\mathrm{Au}\left(\mathrm{C}_{6} \mathrm{~S}\right)\left(\mathrm{AOT}_{\mathrm{bis}}-\right.$ benzaldehyde) MMPCs 


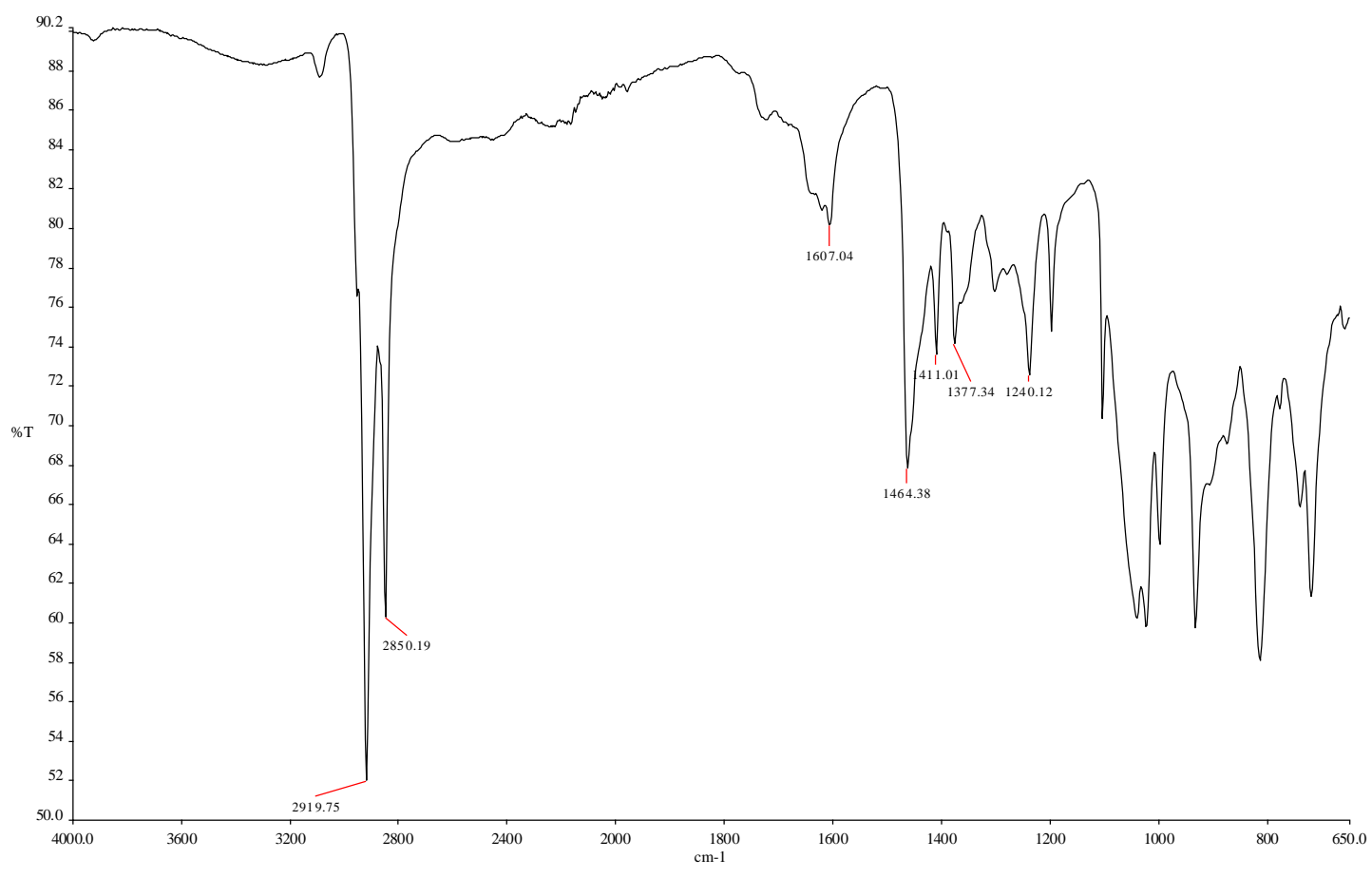

Figure B.3.14. FTIR spectrum of $\mathrm{Au}\left(\mathrm{C}_{6} \mathrm{~S}\right)\left(\mathrm{AOT}_{\text {bis }}\right.$-ferrocene) MMPCs

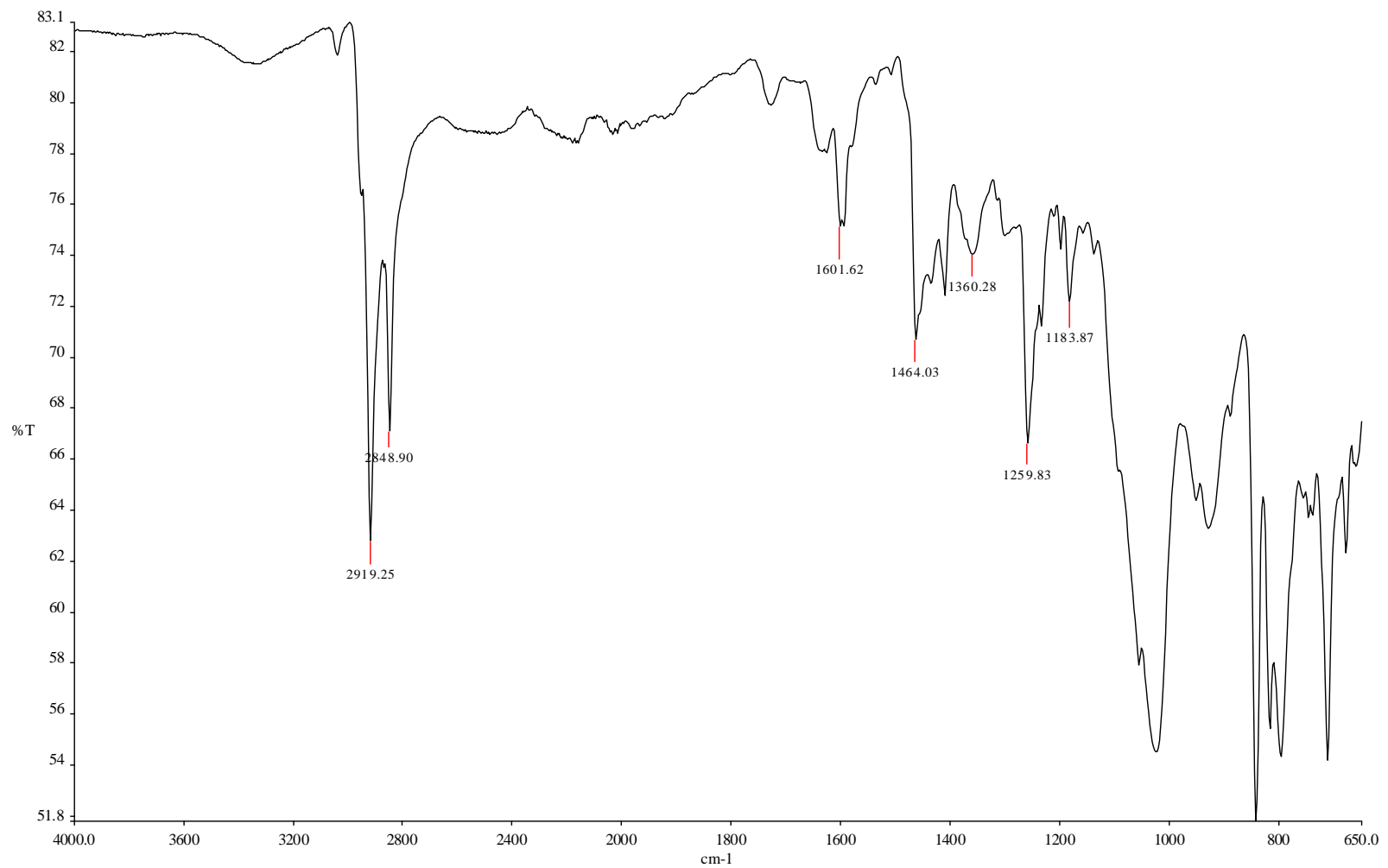

Figure B.3.15. FTIR spectrum of $\mathrm{Au}\left(\mathrm{C}_{6} \mathrm{~S}\right)\left(\mathrm{AOT}_{\mathrm{bis}}\right.$-pyrene) MMPCs 


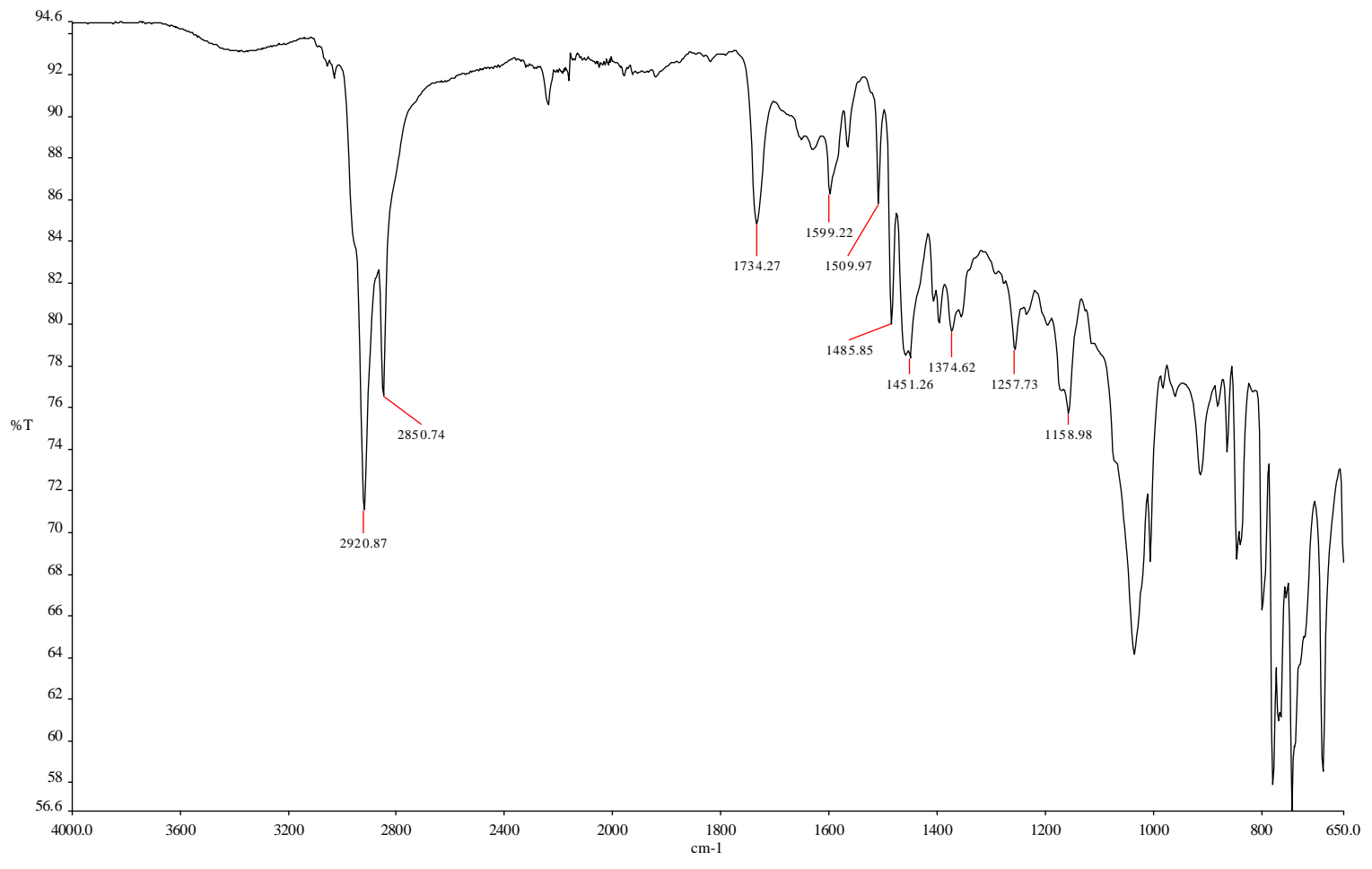

Figure B.3.16. FTIR spectrum of $\mathrm{Au}\left(\mathrm{C}_{6} \mathrm{~S}\right)\left(\mathrm{AOT}_{\text {bis }}\right.$-propanal) MMPCs

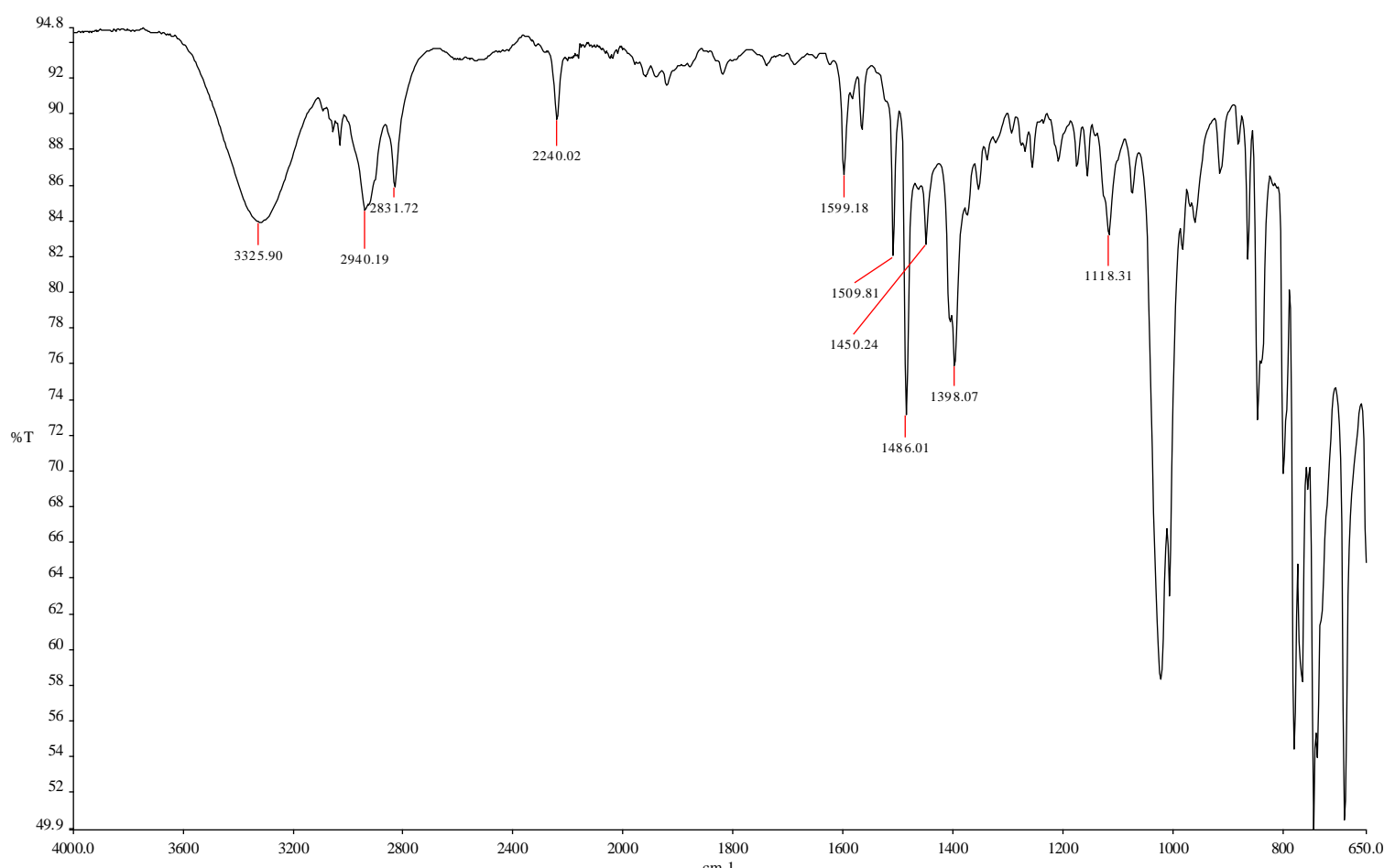

Figure B.3.17. FTIR spectrum of $\mathrm{Au}\left(\mathrm{C}_{6} \mathrm{~S}\right)\left(\mathrm{AOT}_{\text {bis }}-\right.$ glyceraldehyde) MMPCs 


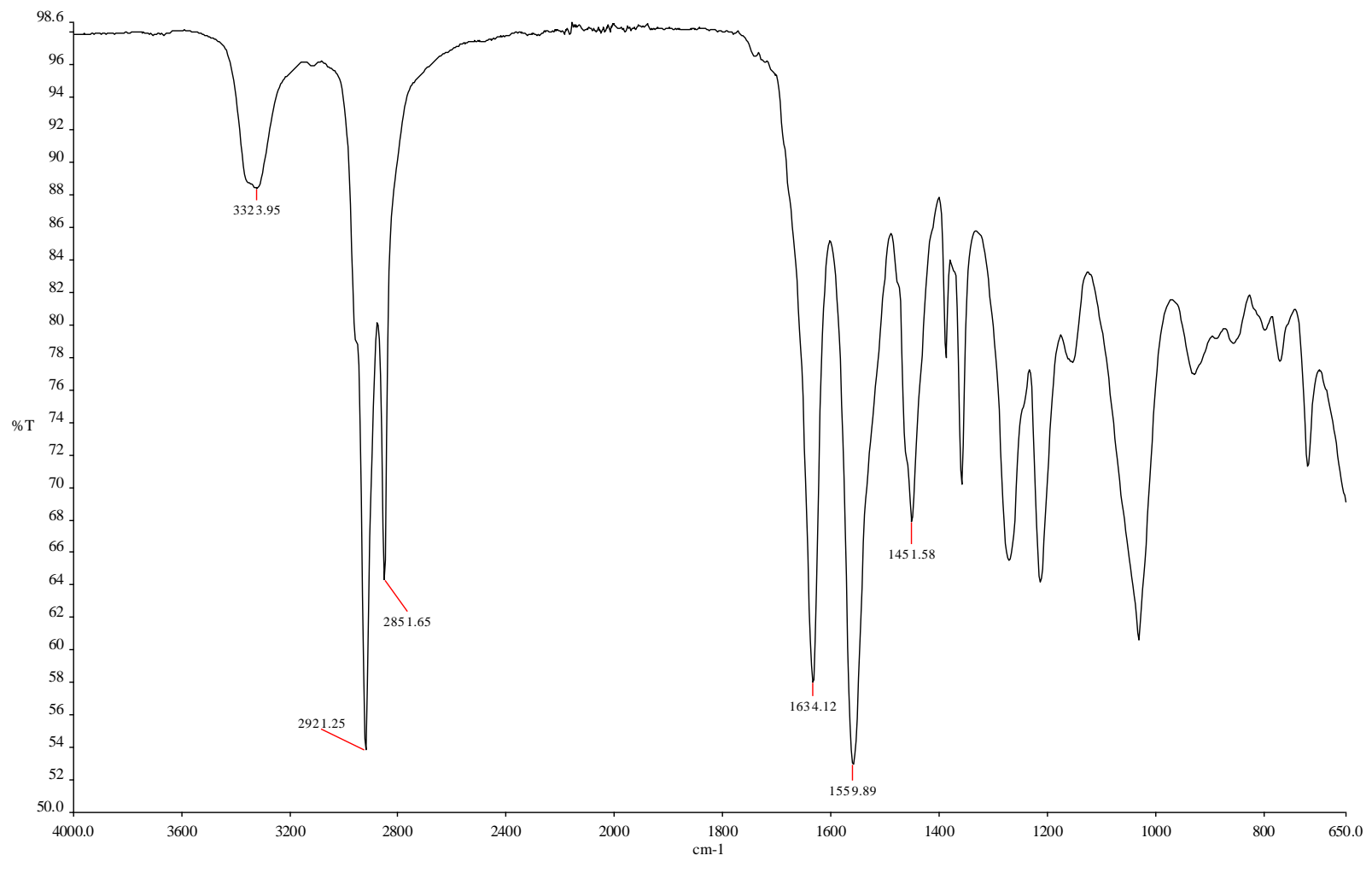

Figure B.3.18. FTIR spectrum of $\mathrm{Au}\left(\mathrm{C}_{6} \mathrm{~S}\right)\left(\mathrm{AOT}_{\text {bis }}\right.$-urea-aldehyde 31) MMPCs.

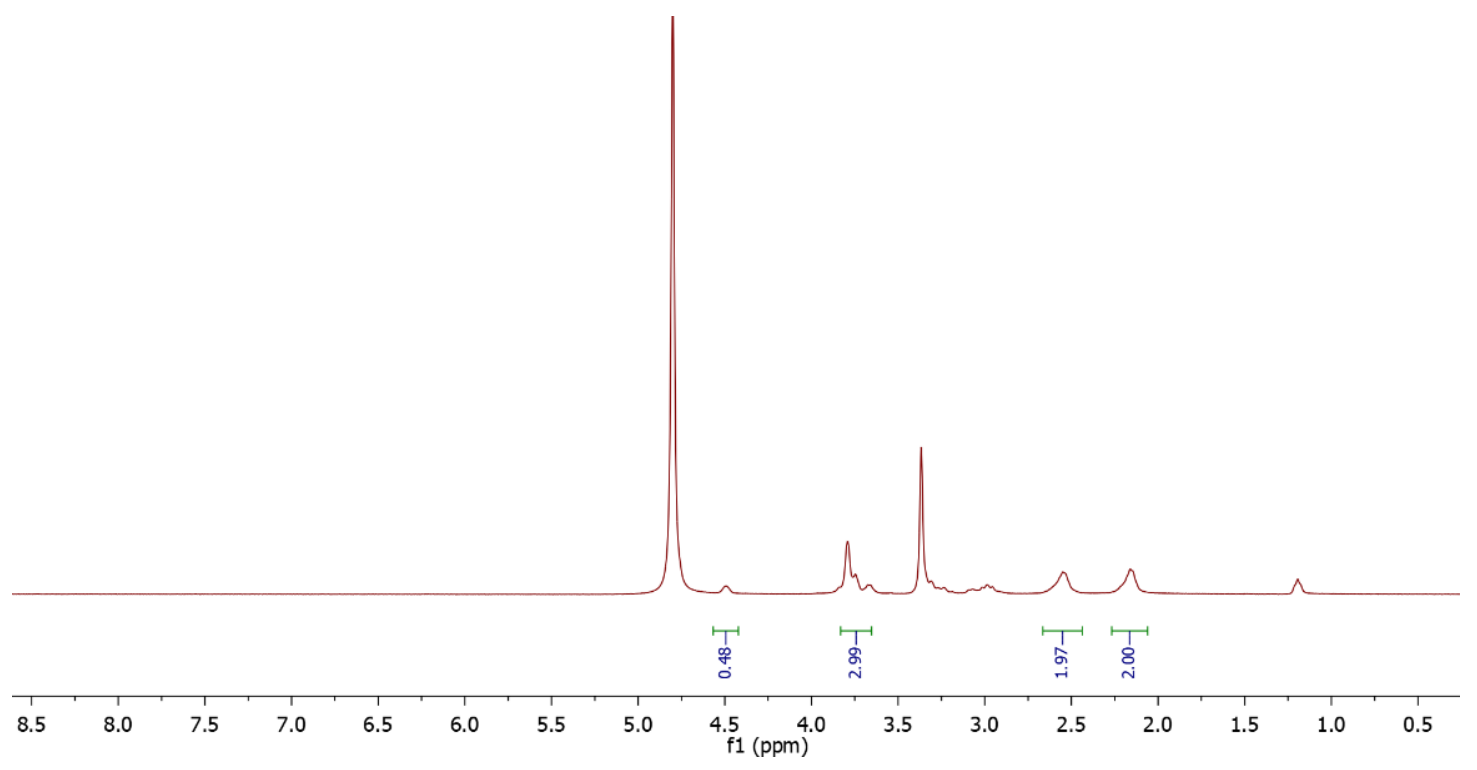

Figure B.3.19. ${ }^{1} \mathrm{H}$ NMR spectrum of $\mathrm{Au}(\mathrm{SG}) \mathrm{MPCs}$ 


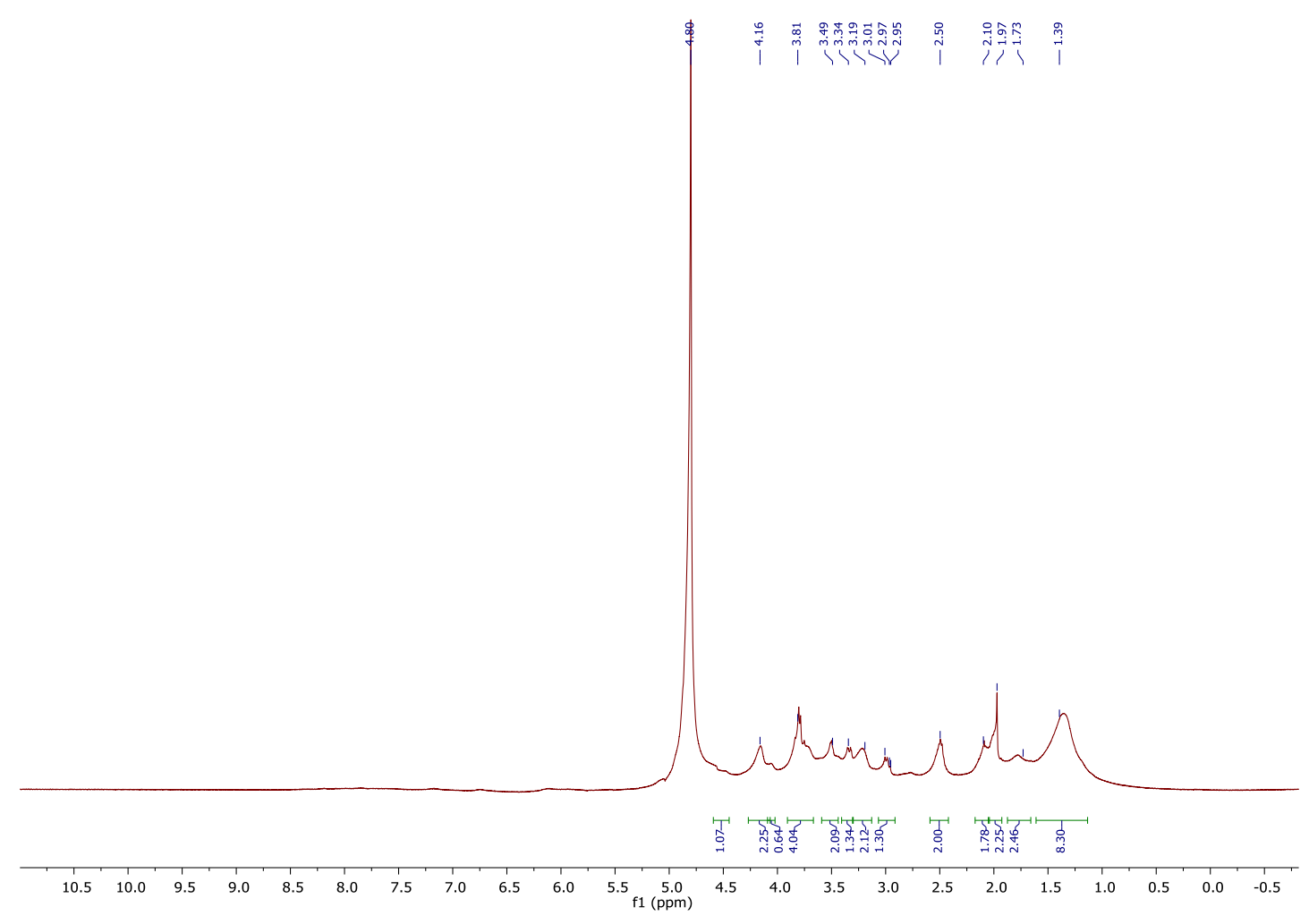

Figure B.3.20. ${ }^{1} \mathrm{H}$ NMR spectrum of $\mathrm{Au}(\mathrm{SG})\left(\mathrm{AOT}_{\mathrm{bis}}{ }^{+}\right) \mathrm{MMPCs}$

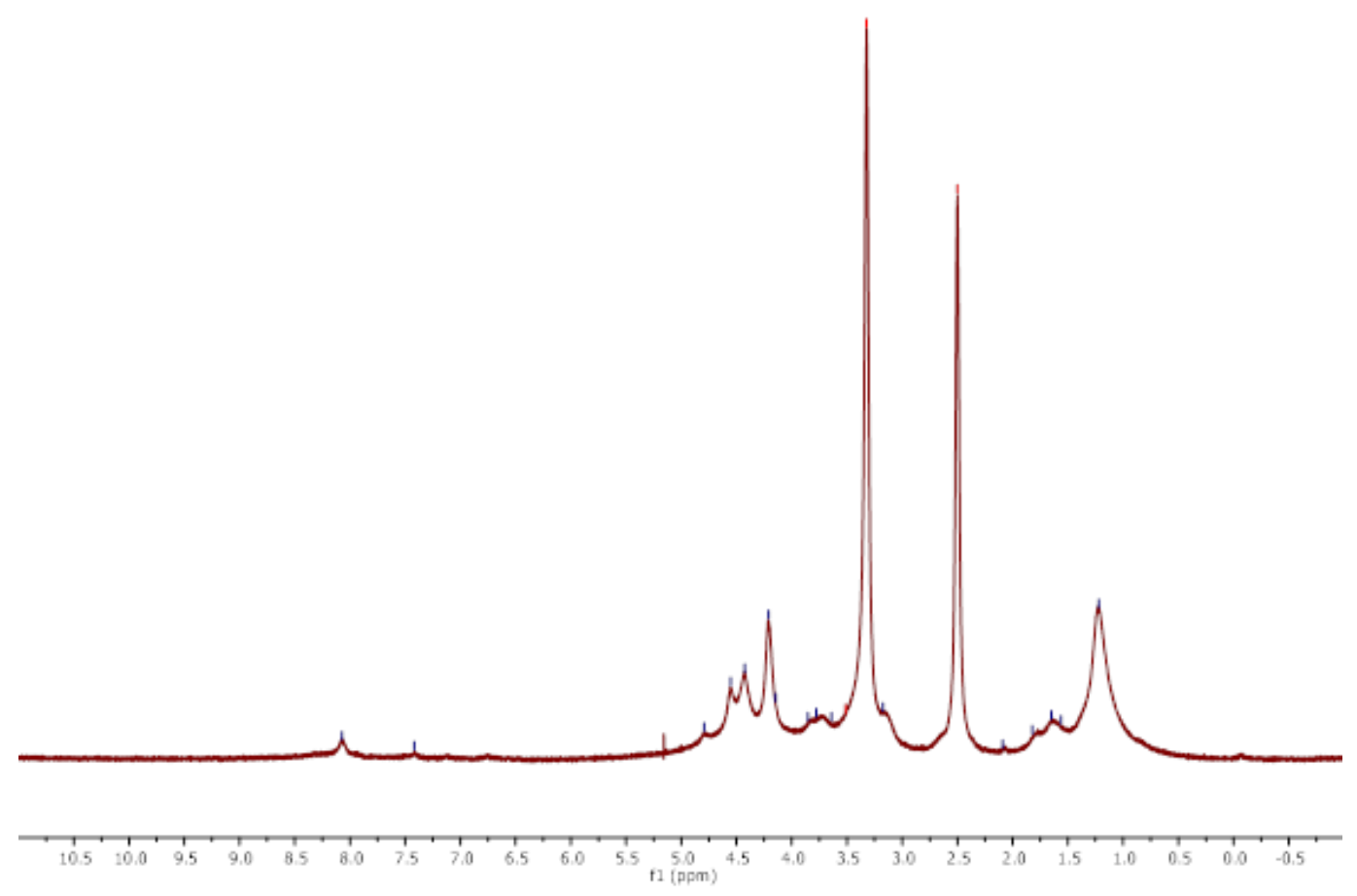

Figure B.3.21. ${ }^{1} \mathrm{H}$ NMR spectrum of $\mathrm{Au}(\mathrm{SG})\left(\mathrm{AOT}_{\mathrm{bis}}{ }^{+}\right.$-ferrocene) MMPCs 


\section{APPENDIX-C}

C.1. List of publications

C.2. Published papers and previous researches title pages

\section{C.1. LIST OF PUBLICATIONS}

1. Sibakoti, T. R.; Nantz, M. H.; Zamborini, F. P. Iodine Activation: A General Method for Catalytic Enhancement of Thiolate Monolayer-Protected Metal Clusters and Nanoparticles. Nanoscale, 2020, DOI: 10.1039/d0nr00844c.

2. Bhama, S.; Sibakoti, T. R.; Zamborini, F. P. Highly Active, Selective, and Recyclable Water-Soluble Glutathione-Stabilized $\mathrm{Pd}$ and $\mathrm{Pd}$-Alloy Nanoparticle Catalysts in Biphasic Solvent. ChemCatChem. 2020, 12, 22532261.

3. Sibakoti, T. R.; Stinger, C.; Adhihetty, P.; Zamborini, F. P.; Nantz, M. H. Tunable Aminooxy-Functionalized Monolayer-Protected Gold Clusters for Non-Polar or Aqueous Oximation Reactions. Particle \& Particle Systems Characterization 2019, 36 (7), 1900093.

4. Sibakoti, T. R. Synthesis and characterization of Carborane-appended Biomolecules. Retrieved from the Northern Illinois University (2015), https://commons.lib.niu.edu/handle/10843/18857.

5. Sibakoti, T. R.; McCurdy, D. L. Determination of Lead (Pb) in Freshwater Fish Sample by HG-AAS. Retrieved from the Truman State University (2011), http://osr.truman.edu/grants-in-aid-of-scholarship-and-research/fall-2011funded-projects/\#Sabakoti. 


\title{
C.2. PUBLISHED PAPERS AND PREVIOUS RESEARCHES TITLE PAGES
}

\author{
Nanoscale \\ ROYAL SOCIETY \\ OF CHEMISTRY
}

\section{PAPER}

Cite this: DOI: $10.1039 / d 0$ nroD844c
Received 31st January 2020 Accepted 12 th May 2020

DOI: $10.1039 / \mathrm{dD}$ rr 00844C

rsclinanoscale

\section{lodine activation: a general method for catalytic enhancement of thiolate monolayer-protected metal clusters $\dagger$}

\author{
Tirtha R. Sibakoti, Jacek B. Jasinski, (5) Michael H. Nantz ${ }^{* a}$ and \\ Francis P. Zamborini(6*a
}

\begin{abstract}
To enhance catalytic activity, the present study details a general approach for partial thiolate ligand removal from monolayer-protected clusters (MPCs) by straightforward in situ addition of iodine. Two model reactions are examined to illustrate the effects on the catalytic activity of glutathione (SG)-capped Au MPCs serving as a catalyst for the $\mathrm{NaBH}_{4}$ reduction of 4-nitrophend to 4-aminophenol and SGcapped Pd MPCs serving as a catalyst for the hydrogenation/isomerization of allyl alcohol. lodine addition promoted partial thiolate ligand removal from both MPCs and improved the catalytic properties, presumably due to greater surface exposure of the metal cores as a result of ligand dissociation. The rate of 4-nitrophenol reduction increased from $0.066 \mathrm{~min}^{-1}$ in the absence of $\mathrm{k}$ to $0.505 \mathrm{~min}^{-1}$ in the presence of 2.0 equivalents $I_{2}$ (equivalents based on total ligated glutathione). The reaction of allyl alcohol to produce 1-propanol and propanal was similarly accelerated as indicated by the increase in tumover frequency from 131 to 230 moles products per moles catalyst per h by addition of 0.2 equivalents $\mathrm{I}_{2}$. In both reactions, as the amount of $\mathrm{l}_{2}$ added increases the catalyst recyclability decreases due to catalyst instability. Low equivalents of $\mathrm{l}_{2}$ are optimal when considering both reaction rate and catalyst recyclability.
\end{abstract}

\section{Introduction}

Metal monolayer-protected clusters (MPCs) stabilized by organothiol monolayers have been used broadly as homogeneous and heterogeneous catalysts in numerous organic transformations, ${ }^{1,2}$ including nitro group reduction, ${ }^{3}$ alkene isomerization, ${ }^{4}$ alkyne hydrogenation, ${ }^{5,6}$ and Suzuki-Miyaura coupling reactions. The great advantage in using metal MPCs is the excellent synthetic control over the metal cluster size and composition, which allows strong control over the metal structural and electronic properties for tuning catalytic activity. The use of a wide variety of thiol-containing capping ligands enables high stability, tuning of the solubility properties, potential molecular gating properties, and the ability to treat these molecular metals as normal chemical reagents, thereby allowing characterization by standard analytical spectroscopic

"Department of Chanisty, University of Louisvili, Louisville, Kontuchy 40292, LSA.

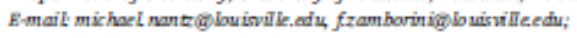

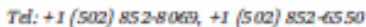

"Cann Center for Renewable Energy Resench, University of Louivilik, Lo uisuille, Kentudily 40292 , USA

† Hectronic supplementary information (ESI) available: Characterization of synthesized chusters inchudes TCA, UV-vis, TEM, SEM, ${ }^{2} \mathrm{H}$ NMR, UV-VIS, XPS, and GC data (14 pages). See DOR: 10.1039/donroos44c tools of the organic chemist (e.g., nuclear magnetic resonance spectroscopy (NMR), Fourier-transform infrared spectroscopy (FTIR), mass spectrometry (MS), and UV-vis). . Additionally, improvements in separation methods have led to syntheses of highly uniform MPCs of one composition, also known as atomically-precise clusters, such as the widely studied $\mathrm{Au}_{2 s}(\mathrm{SR})_{18}$ cluster product. ${ }^{9,10}$ While use of thiol ligands as capping agents affords tremendous synthetic control, they also strongly passivate the metal duster surface and poison (or inhibit) the catalytic properties. As a result, there is much interest in developing new ligand systems or incorporating strategies to increase MPC catalytic activity without sacrificing stability. This is a tremendous challenge as the two-ligandimparted stability vs. ligand-diminished activity - work directly against each other.

One main strategy for balancing the stabilizing properties of a given ligand while increasing reactant access to the metal core, essential for catalytic activity to occur, ${ }^{11}$ is the use of lower coverage thiol ligands, such as those derived from Bunte salts, ${ }^{12,13}$ or branched, bulky thiol-containing ligands, so as to decrease ligand surface density. ${ }^{14}$ Bhama et al. recently demonstrated the use of branched, water-soluble glutathione (SG)-capped Pd MPCs, which catalyzed the hydrogenation/isomerization of allyl alcohol with a turnover frequency (TOF) roughly 5 times greater than linear chain hexanethiol-stabil- 


\title{
Highly Active, Selective, and Recyclable Water-Soluble Glutathione-Stabilized Pd and Pd-Alloy Nanoparticle Catalysts in Biphasic Solvent
}

\author{
Shekhar Bhama, ${ }^{[a]}$ Tirtha R. Sibakoti, ${ }^{\left[{ }^{[]]}\right.}$Jacek B. Jasinski, ${ }^{[b]}$ and Francis P. Zamborini* ${ }^{\left[{ }^{[a]}\right.}$
}

Glutathione-protected Pd, PdAu and PdPt nanoparticles (NPs) show greatly increased reactivity and stability towards the hydrogenation/isomerization of allyl alcohol in a biphasic organic/aqueous solvent mixture compared to single-phase alkanethiol-protected Pd-based NPs. NPs synthesized under aerobic conditions have higher activity compared to those synthesized under nitrogen, with the highest TOF of 676 mole product/mole metal/hour for 75:25 PdAu NPs at $20 \mathrm{~mL} / \mathrm{min} \mathrm{H}_{2}$ flow. 75:25 PdAu NPs prepared under nitrogen have a

\section{Introduction}

Recent developments in nanotechnology have led to the synthesis of advanced materials with desirable reactivity as well as optical and electronic properties that have use in many applications, such as $\mathrm{H}_{2}$ storage, drug delivery, sensing, and catalysis. $^{[n-\pi]}$ The unique electronic structure and properties of nanoparticles (NPs) are largely due to the high surface area-tovolume ratio along with low coordination number of the surface atoms of NPs. This leads to size-dependent properties very different from the bulk materials. These properties brand NPs as a suitable candidate for the catalysis of a variety of organic reactions. The key issue is to obtain maximum catalytic efficiency by controlling the NP size, shape, composition and functionality, which can be accomplished by using various organic stabilizers, such as polymers, ${ }^{100}$ dendrimers, ${ }^{151}$ peptides, ${ }^{101}$ and ligands. ${ }^{[/ 1}$ The big challenge is to find a protecting stabilizer that prevents irreversible aggregation under the reaction conditions, but also allows high catalytic activity and controlled selectivity.

Transition metal NPs in solution have been frequently used over a solid support for catalyzing a variety of organic and inorganic reactions. ${ }^{[-10]}$ While it is easy to separate the catalyst

[a] Dr. S. Bhama, T. R. Sibakoti, Prot. F. P. Zamborini Department of Chemistry University of Lowiswils

Louiville, $K Y-40292$ (USA)

Email framborinizioviswille.ed

[b] Dr. J.R. Jasinski

Cann Center for Renewable Energy Research

University of Lowisvils Lovisvile, $K \gamma-40292$ (USA)

$\square$ Supporting information for this article is svailsble on the WWW under https//doi.org/10.1002/cctc 201901968

This manuscript is part of the Special lssue on the Sustainable and Affordable Chemistry to Meet Future Challenges in the Pharmaceutical Industry. maximum TOF of 507 at $20 \mathrm{~mL} / \mathrm{min} \mathrm{H}_{2}$ flow, but can be recycled 4 times ( 9 times at $8 \mathrm{~mL} / \mathrm{min} \mathrm{H}_{2}$ flow). Ethyl acetate is a better organic phase solvent compared to dichloromethane and chlorobenzene in terms of TOF and recyclability. All of the NPs studied show $>80 \%$ selectivity for the isomer product (propanal). The branched, open structure of the glutathione and use of the biphasic solvent provides high catalytic activity. high selectivity, and easy removal of the products in the organic phase for good recyclability of the water-soluble catalysts.

ChemCarchom $2020,12,1-10$

from the reaction products, heterogeneous systems often suffer from low turnover frequency (TOF), limited lifetime and poor selectivity, primarily due to the principle of diffusion and the involvement of two different phases in the catalytic system. ${ }^{[11}$ Also, their complex method of preparation and poor particle morphological control ${ }^{[1,17]}$ has redirected studies on homogeneous systems. Homogeneous systems have recently drawn great interest due to their potential for higher activity and selectivity ${ }^{113,10}$ Some of the current problems include leaching of delicate core metals and imperfect recycling and recovery. Homogeneous NPs with weak stabilizers often have high activity but become unstable in solution, leading to changes in size and morphology, dissolution, aggregation, or precipitation.

These stability issues can be addressed by synthesizing metal NPs with dendrimers, polymers or surfactants. ${ }^{\text {Is.16. }}$ Another option is the use of organic ligand stabilizers, such as thiols, ${ }^{[1]}$ phosphines, ${ }^{[1]}$ amines ${ }^{[11]}$ or ammonium salts. ${ }^{[1]}$ The ligands usually form a monolayer coating on the NP surface and provide control over the core size of the NPs ${ }^{[20]}$ and the solubility and catalytic activity. ${ }^{[21]}$ The NPs are usually highly stable and can be stored as solid powders, but often suffer significantly from lower catalytic activity due to the blocking of reactive sites ${ }^{[2]}$ Crooks and co-workers reported the synthesis and substrate size-selective catalytic activity of various Pd NPs encapsulated within poly(amidoamine) dendrimers of different generations and different end groups. ${ }^{|2|}$ Steric crowding on the dendrimer periphery led to lower TOF for larger substrates. Also, PdPt and PdAu dendrimer-encapsulated alloy NPs had higher catalytic activity (TOF $=200-1500$ mole $\mathrm{H}_{2} /$ mole metal/ hour) than dendrimer-encapsulated $\mathrm{Pd}, \mathrm{Au}$, or $\mathrm{Pt}$ single metal NPs (TOF $=0-800$ mole $\mathrm{H}_{2} /$ mole metal/hour) for the hydrogenation of allyl alcohol. The work of Sadeghmoghaddam et al. represents an example of thiol ligand-stabilized Pd NPs for catalysis, where they focused on understanding the mechanism and regioselectivity of hexanethiolate- and dodecanethiolate- 


\title{
Tunable Aminooxy-Functionalized Monolayer-Protected Gold Clusters for Nonpolar and Aqueous Oximation Reactions
}

\author{
Tirtha R. Sibakoti, Colton R. Stinger, Prasadanie K. Adhihetty, Francis P. Zamborini,* \\ and Michael H. Nantz*
}

Aminooxy $\left(-\mathrm{ONH}_{2}\right)$ groups are well known for their chemoselective reactions with carbonyl compounds, specifically aldehydes and ketones. The versatility of aminooxy chemistry has proven to be an attractive feature that continues to stimulate new applications. This work describes application of aminooxy click chemistry on the surface of gold nanoparticles. A trifunctional aminecontaining aminooxy alkane thiol ligand for use in the functionalization of gold monolayer-protected clusters (Au MPCs) is presented. Diethanolamine is readily transformed into an organic-soluble aminooxy thiol (AOT) ligand using a short synthetic path. The synthesized AOT ligand is coated on $\leq 2$-nm-diameter hexanethiolate- $\left(\mathrm{C}_{6} \mathrm{~S}\right)$-capped $\mathrm{Au}$ MPCs using a ligandexchange protocol to afford organic-soluble $A O T / C_{6} S$ (1:1 ratio) Au mixed monolayer-protected clusters (MMPCs). The synthesis of these $A u\left(C_{6} S\right)$ (AOT) MMPCs and representative oximation reactions with various types of aldehyde-containing molecules is described, highlighting the ease and versatility of the chemistry and how amine protonation can be used to switch solubility characteristics.

\section{Introduction}

Aminooxy-based click[1] chemistry has proven to be a versatile means of ligation, as evidenced by broad application in the fields of material science, biology, biochemistry, analytical chemistry, and nanoscience. ${ }^{[2-4]}$ Aminooxy groups ( $\left.\mathrm{RONH}_{2}\right)$ react chemoselectively with aldehydes and ketones under mild conditions to form highly stable oxime ether adducts. The chemoselectivity of the oximation reaction has stimulated efforts to exploit oxime ether formation not only as a straightforward, efficient coupling approach, but also as a means for identification and quantification of carbonyls in complex mixtures. For example, aminooxy derivatization reagents have been used to selectively tag and/or sequester carbonyl substrates in biological extracts, ${ }^{[5,6]}$ environmental air

\footnotetext{
T. R. Sibakoti, C. R. Stinger, P. K. Adhihetty, Prof. F. P. Z amborini, Prof. M. H. Nantz

Department of Chemistry

University of Louisville

Louisville, KY 40208, USA

E-mail: fzamborini@louisville.edu; michael.nantz@louisville.edu

The ORCID identification number(s) for the author(s) of this article can be found under https://doi.org/10.1002/ppsc.201900093.
}

DOI: 10.1002/ppsc.201900093 and water, 7,8$]$ exhaled breath, ${ }^{9,10]}$ and even living organisms. $]^{[1]}$

As the usefulness of oximation chemistry has become apparent, several approaches exploiting nanoparticles fitted with aminooxy surfaces have been reported.[12-14] The ease of functionalization of gold surfaces, clusters, and nanoparticles with a monolayer of thiol- or dithiol-containing molecules ${ }^{[15,16]}$ has guided some efforts toward developing and applying thiol ligands containing aminocxy functionality.[17] For example, Nagahori et al. prepared aminooxy-functionalized gold nanoparticles (NPs) for capture and enrichment of glycosphingolipid (GSL)-generated aldehydes as a means to characterize whole GSLs in living cells. [18] Thygesen et al. developed a bifunctional thiol-aminooxy oligo(ethylene glycol) ligand for preparation of aminooxy-conjugated gold glycanoparticles. ${ }^{[19]}$ Maynard and co-workers synthesized a photocaged aminooxy alkane thiol for conjugating carbonyl substrates to gold surfaces following photolysis. [20] Given the rapidly growing interests in using gold monolayer-protected clusters (Au MPCs) and Au $\mathrm{NP}_{\mathrm{s}}$ for applications in catalysis, ${ }^{[21-23]}$ sensing volatile organic compounds, ${ }^{[2,25]}$ analyzing small molecule mixtures using NP-mediated Raman and laser desorption/ionization spectroscopy, ${ }^{[26]}$ and drug delivery, ${ }^{[27,28]}$ the development of aminooxyfunctionalized thiol ligands to improve ease of carbonyl ligation as well as to provide flexibility in addressing solubility and adduct loading considerations would enable new applications. With this in mind, we designed a trifunctional amine-containing aminooxy thiol (AOT) ligand for use in monolayer functionalization of hexanethiolate $\left(\mathrm{C}_{6} \mathrm{~S}\right)$-protected $\mathrm{Au}$ MPCs to produce $\mathrm{Au}\left(\mathrm{C}_{6} \mathrm{~S}\right)(\mathrm{AOT})$ mixed monolayer protected clusters (MMPCs) (Figure 1). Incorporation of the amine group was motivated by several considerations: (a) The solubility properties of the cluster may be adjusted by control over the amine protonation or alkylation state; (b) the amine moiety enables convenient structural bifurcation to increase pendant aminooxy density; and (c) an ammonium NH could potentially accelerate oximation reactions.[29] Herein, we describe the synthesis of such a trifunctional thiol ligand and its use in the formation of Au MMPCs. We also present our findings on representative 


\title{
NORTHERN ILLINOIS UNIVERSITY \\ DE KALB, ILLINOIS
}

AUGUST 2015

\author{
SYNTHESIS AND CHARACTERIZATION OF \\ CARBORANE-APPENDED BIOMOLECULES
}

A THESIS SUBMITTED TO THE GRADUATE SCHOOL

IN PARTIAL FULFILLMENT OF THE REQUIREMENTS

FOR THE DEGREE

MASTER OF SCIENCE

DEPARTMENT OF CHEMISTRY AND BIOCHEMISTRY

Thesis Director:

Narayan S. Hosmane 


\title{
Hydride Generation Atomic Absorption Spectrophotometry (HGAAS) for the Determination of Lead $(\mathrm{Pb})$
}

\author{
Authors: Dr. David L. McCurdy* and Tirtha R. Sibakoti
}

\section{Introduction}

Hydride generation atomic absorption spectrophotometry (HG-AAS) is a common technique for the determination of hydride forming metals. In HG-AAS, metals such as Se, As, $\mathrm{Sn}, \mathrm{Pb}$ in solution are reacted with trace metal grade acid $\left(\mathrm{HNO}_{3}\right)$ along with sodium peroxysulfate $\left(\mathrm{Na}_{2} \mathrm{~S}_{2} \mathrm{O}_{8}\right.$, an oxidizing reagent) and sodium borohydride $\left(\mathrm{NaBH}_{4}\right.$, a reducing reagent) to form volatile metal-hydride compounds that can be removed from solution by purging with an inert gas, such as Argon. The volatile hydride is then swept by the inert gas into a quartz-tube, mounted in a flame atomic absorption spectrophotometer. There, the metalhydride compound is atomized and measured. The apparatus to be used for doing this experiment is vapor generator accessory (VGA-77). HG-AAS improves the limits of detection by a factor of 100 or more (into sub part-per-billion range) compared to flame AA, removes the analyte from most common matrix interferences present in the original sample, and speeds up the time of the measurement, offering advantages not found in the other techniques.

This technique is a vapor phase sample introduction method for AAS. In this method, lead hydride will be formed by the oxidation of aqueous $\mathrm{Pb}$ solution to $\mathrm{Pb}^{+4}$ in suitable acidic conditions and then further reduced in to $\mathrm{PbH}_{4}$ using $\mathrm{NaBH}_{4}$. Thus formed volatile plumbium is swept in to a quartz tube sitting over AA's flame using argon gas where $\mathrm{PbH}_{4}$ is atomized to $\mathrm{Pb}^{0}$ and hence the free gaseous lead atoms are measured by AA. The reaction is shown below.

1. $\mathrm{Pb}^{+2}$ (aq) $\stackrel{\text { acid }+ \text { sodium perosysulfate }}{\longrightarrow} \mathrm{Pb}^{+4}$

2. $\mathrm{Pb}^{+4}+\mathrm{BH}_{4} \stackrel{\text { sodium borohydride }}{\longrightarrow} \mathrm{PbH}_{4}$ (g)

The anticipated advantages of this method are:

1. Quicker approach than GFAAS

2. Comparable LODs to GFAAS

3. Less interferences issues 


\title{
CURRICULUM VITAE
}

\author{
Tirtha Raj Sibakoti \\ 5252 Brindisi Ct, Apt 4, Middleton, WI 53562 \\ Email: trsiba01@louisville.edu. Phone: 502-202-8674
}

\section{SUMMARY}

An interdisciplinary doctoral candidate with experiences in the development of analytical methods, multi-step organic synthesis, compound purification and spectral characterization, nanoparticle synthesis and functionalization, and nanoparticles-based sensing and catalysis. My research training across organic and analytical chemistry has provided me experiences in operating and troubleshooting instruments such as NMR, GC-MS, UV-Vis among others. My experience in the design of chemiresitors sensor for detecting volatile organic compounds (VOCs) has enhanced my technical skills. My problem-solving skills, equipment maintenance and troubleshooting abilities have prepared me to begin my career as an independent scientist.

\section{EDUCATION}

Ph.D. Chemistry, University of Louisville, Louisville, KY, May 2020

Dissertation Title: Aminooxy- Functionalized Monolayer- Protected Clusters: Synthesis \& Applications

Advisors: Michael H. Nantz, PhD and Francis P. Zamborini, PhD

M.S. Chemistry, Northern Illinois University, DeKalb, IL, Aug 2015

Thesis Title: Synthesis and Characterization of Carborane-Appended Biomolecules

Advisor: Narayan S. Hosmane, PhD

B.S. Chemistry, Biology minor, Truman State University, Kirksville, MO, Dec 2012

Advisor: David L. McCurdy, PhD

Higher Secondary School, Science, Kathmandu Valley College, Tahachal, Kathmandu Graduated May 2007, Advisor, Swagat Shrestha, PhD 


\section{PROFESSIONAL EXPERIENCE}

Graduate Research Assistant, University of Louisville, Louisville, KY January 2016present

- Synthesis and characterization of aminooxy functionalized gold clusters using ${ }^{1} \mathrm{H}$ NMR, FTIR, HRMS, UV-Vis, TGA, TEM, SEM, CV and fluorescence

- Study of reaction catalysis using Au/Pd-clusters in homogenous and heterogenous system

- Collaboration with chemical engineers for the development of gold nanoparticles chemiresistors based benzene sensor

- Synthesis of chemoselective reagents with different counterions for selective detection of different volatile aldehydes and ketones

- Troubleshoot laboratory instruments, ordering of chemical reagents/solvents for group

- Organization of laboratory notebooks

Graduate Research Assistant, Northern Illinois University, DeKalb, IL May 2013-July 2015

- Synthesis and characterization of boronated biomolecules suitable for boron neutron capture therapy (BNCT) applications

- Development of a new methodology to attach carborane cages to organic compounds

- Biological distribution studies (cytotoxicity) of synthesized compounds

- Supervision and training of undergraduate students in research

Summer Internship, Truscholars Summer Research Program, Truman State University, Kirksville, MO May 2011- Aug 2011

- Exploration of hydride generation atomic absorption spectroscopy sample introduction technique (HGAAS) for ppb level detection of $\mathrm{Pb}$

- Optimization of experimental conditions and analysis of the collected data

- Development of lab manual on HGAAS technique for the instrumental analysis course

- Documentation and presentation of research work in Truscholar symposium 
Graduate Teaching Assistant, 2011-2019

- University of Louisville (Aug 2015- Dec 2019): CHEM 207, 208, 209, 210, 344, 527

- Northern Illinois University (Jan 2013- May 2015): CHEM 111, 212 and 213

- Truman State University (May 2011- Aug 2011): CHEM 333

\section{PUBLICATIONS}

- Sibakoti, T. R.; Nantz, M. H.; Zamborini, F. P. Iodine Activation: A General Method for Catalytic Enhancement of Thiolate Monolayer-Protected Metal Clusters and Nanoparticles. Nanoscale, 2020, DOI: 10.1039/d0nr00844c.

- Bhama, S.; Sibakoti, T. R.; Zamborini, F. P. Highly Active, Selective, and Recyclable Water-Soluble Glutathione-Stabilized Pd and Pd-Alloy Nanoparticle Catalysts in Biphasic Solvent. ChemCatChem. 2020, 12, 2253-2261.

- Sibakoti, T. R.; Stinger, C.; Adhihetty, P.; Zamborini, F. P.; Nantz, M. H. Tunable Aminooxy-Functionalized Monolayer-Protected Gold Clusters for NonPolar or Aqueous Oximation Reactions. Particle \& Particle Systems Characterization 2019, 36 (7), 1900093.

- Sibakoti, T. R. Synthesis and characterization of Carborane-appended Biomolecules. Retrieved from the Northern Illinois University (2015), https://commons.lib.niu.edu/handle/10843/18857.

- Sibakoti, T. R.; McCurdy, D. L. Determination of Lead $(\mathrm{Pb})$ in Freshwater Fish Sample by HG-AAS. Retrieved from the Truman State University (2011), http://osr.truman.edu/grants-in-aid-of-scholarship-and-research/fall-2011funded-projects/\#Sabakoti.

\section{CONFERENCES ATTENDED AND PRESENTATIONS}

- Pittcon 2020, March 03, 2020. McCormick Place, Chicago, IL. Title: AminooxyFunctionalized Gold and Palladium Monolayer-Protected Clusters for Catalysis Applications. Type: Poster

- Graduate Network in Arts \& Sciences (GNAS) Scholarship Symposium, Feb 27, 2020. University of Louisville, Louisville, KY. Title: Iodine Activation: A General Method for Catalytic Enhancement of Thiolated Monolayer Protected Clusters. Type: Oral

- Graduate Network in Arts \& Sciences (GNAS) Scholarship Symposium, Nov 06, 2019. University of Louisville, Louisville, KY. Title: Iodine Activation of Au MPCs. Type: Oral

- The $71^{\text {st }}$ Southeastern Regional Meeting of American Chemical Society (SERMACS), Oct 20-Oct 23, 2019. Savannah Riverfront Marriott, Savannah, GA. Title: Aminooxy-Functionalized Metal Monolayer-Protected Clusters (MPCs) for Catalysis Applications. Type: Oral 
- NIEHS Superfund Research Program 2018 Annual Meeting, Nov 28-30, 2018. Sacramento, CA. Title: Aminooxy-Functionalized Aminothiols as Ligands for Gold Nanoparticles Chemiresitors. Type: Poster

- The $70^{\text {th }}$ Southeastern Regional Meeting of American Chemical Society (SERMACS), Oct 31-Nov 3, 2018. Augusta convention center, Augusta, GA. Title: Tunable Aminooxy Functionalized Gold Monolayer-Protected Clusters for Non-polar and Aqueous Oximation Reactions. Type: Oral

- $255^{\text {th }}$ ACS National Meeting, March 18-22, 2018. New Orleans, LA. Title: Nonpolar and Aqueous Oximation Reactions on Aminooxy Functionalized Monolayer-Protected Clusters. Type: Poster

- Graduate Student Regional Research Conference (GSRRC), March 02, 2018. University of Louisville, Louisville, KY. Title: Non-polar and Aqueous Oximation Reactions on Aminooxy Functionalized Monolayer-Protected Clusters. Type: Poster

- ACS regional meeting (JGLCRM), May 28-30, 2015. Devos Place, Grand Rapids, MI. Title: Synthesis and characterization of carborance-appended biomolecules for BNCT applications. Type: Poster

\section{ACTIVITIES AND MEMBERSHIP}

- American Chemical Society (member, Jan 2018- Jan 2019), Society of Applied Spectroscopy (member, Aug 2008- Dec 2012)

- Chemistry Graduate Student Association, University of Louisville (vicepresident, Aug 2017- Sept 2018), Nepalese Student Association, University of Louisville (treasurer and secretary, Aug 2016- Aug 2011), Namaste Nepal, Truman State University (member, 2008-2012)

\section{AWARDS AND HONORS}

- Doctoral Dissertation Completion Award, College of Arts \& Science (UofL, Spring 2020)

- Graduate Student Council (GSC), Graduate Network in Arts and Sciences (GNAS) and Chemistry Department Travel Awards (UofL, 2018-2020)

- Department of Chemistry and Biochemistry Travel Award (NIU, Summer 2015)

- Scientists Prepared, Enriched, Challenged Through Research-based Activities (SPECTRA) Grant, Truscholars Summer Research Program Grant, Grants-in-Aid of Scholarship and Research (GIASR) (awardee, Truman State University, 2011)

- Presidential Scholarship, International Scholarship (recipient, Truman State University, 2009) 


\section{REFERENCES}

Prof. David McCurdy

Prof. Francis Zamborini

Department of Chemistry

Department of Chemistry

University of Iowa

University of Louisville

Iowa City, IA 52242

Louisville, KY 40292

david-mccurdy@uiowa.edu

319-335-4867

f.zamborini@louisville.edu

502-852-6550

Prof. Michael Nantz

Ass. Prof. Amartya Chakrabarti

Department of Chemistry

Department of Physical Sciences

University of Louisville

Dominican University

Louisville, KY 40292

RiverForest, IL 60305

michael.nantz@louisville.edu

achakrabarti@dom.edu

$502-852-8148$

312-375-7654 